\title{
Common Ground:
}

\section{Multivalent Communal Space In Medium Density Housing}

by

Stephen John Pattinson

\begin{abstract}
A thesis
submitted to the Victoria University of Wellington in fulfilment of the requirements for the degree of Master of Architecture
\end{abstract}

Victoria University of Wellington 


\section{Abstract}

The combined effect of shrinking average household size and steady urban population growth places considerable stress on existing land supply within urban limits, creating significant challenges for meeting new housing demand in a sustainable way. One option, intensification, is occurring rapidly in New Zealand's main urban centres. Intensive housing in Auckland, for example, represents $35 \%$ of Auckland's total housing market (2007), and “...indications are that the size of the intensive housing market will increase ... it is possible that in the future intensive housing will be the housing market rather than a segment within it." It is therefore important and urgent to consider ways of achieving higher density housing efficiently on less available land. One design strategy that may contribute significantly towards achieving this goal is the use of 'multivalent' communal space.

In New Zealand, little consideration is given to the provision of 'multivalent' communal space in higher density housing. Consequently, opportunities are being missed to utilise urban land more efficiently in ways that enhance the quality of higher density living environments.

This thesis research suggests that 'multivalent' communal space has significant potential for better utilisation of urban land and the enhancement of residents' quality of life in intensive urban environments. 
(C) 2012 Stephen Pattinson $\quad$ Preface M. Arch Thesis

\section{Preface}

Victoria University of Wellington (VUW) Faculty of Architecture and Design (FAD) recommends the following reading for architecture and design students who are carrying out post-graduate thesis research:

“... the list of books ... recommended:

- Wang and Groat's 'Architectural research methods'

- The Dissertation by Borden and Ruedi - See chapter 3.

- Evans 'How to write a better thesis'.

- Booth et al, 'The Craft of Research'."

(E-mail 17.6.10 to FAD post-graduate students, Rebecca Mclaughlan, pp Dr Robin Skinner, Associate Dean of Research, VUW School of Architecture)

From the above references, I have found Groat and Wang 'Architectural Research Methods' (2002) and Evans and Gruba 'How to Write a Better Thesis', $2^{\text {nd }}$ Ed. (2002) particularly helpful. Also helpful is material from a Research Colloquium at VUW FAD on $19^{\text {th }}$ October 2010 presented by Dr Anne Galloway (Senior Lecturer, Design Research, School of Design) and Dr Robin Skinner (Associate Dean of Research, School of Architecture). References to this latter material are attributed as “Galloway and Skinner, 2010". This preface explains how I have used general guidelines from these various sources to plan this research and structure the thesis.

Terminology

In planning my research, I have had to think through conflicting research terminology even in the above short list of references. The following discussion makes explicit my understanding of the research terminology and how I have used various terms in describing my research methodology. 
I begin this discussion on terminology with Groat and Wang (2002):

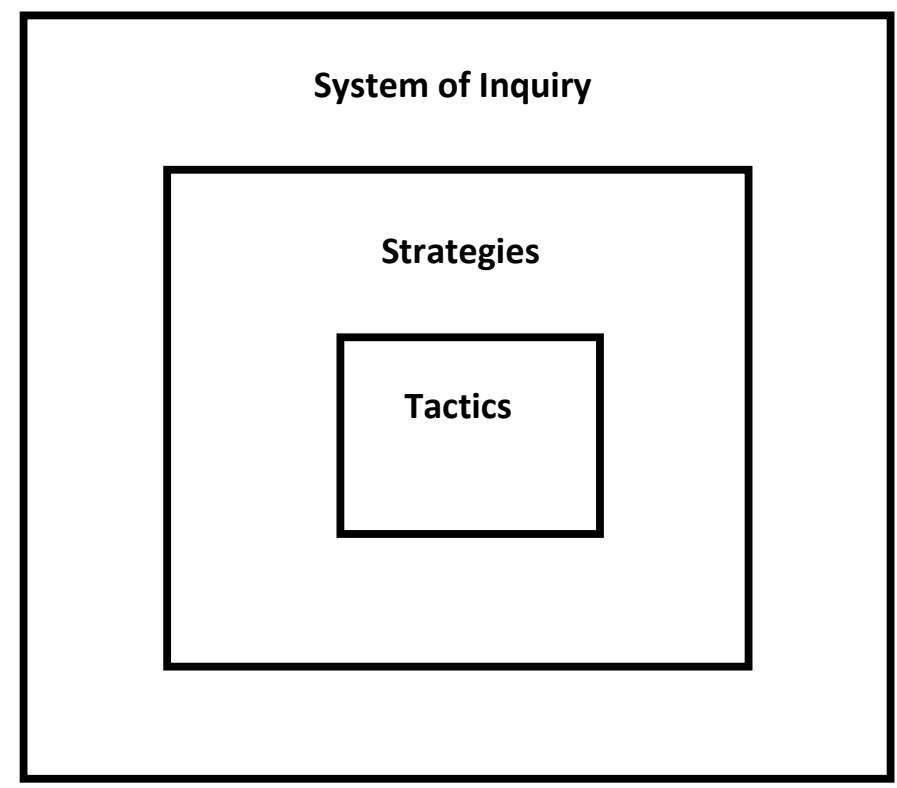

Figure P1: A system of enquiry frames both strategies and tactics.

(Groat and Wang, 2002:10, Fig.1.4)

In the above diagram (Figure P1), Groat and Wang (2002) illustrate three levels of their proposed overall framework for architectural research:-

1. A system of inquiry (Groat and Wang, 2002:10)

“... a particular research design is necessarily framed by the researcher's own assumptions about the nature of reality [ontological assumptions] and how we can come to apprehend it [epistemological assumptions]. We have used the term system of inquiry to describe these sets of assumptions; another term that is frequently used to describe such assumptions is paradigm. Both terms convey the notion of a world view..." (2002:21, cf 26)

2. Groat and Wang use the term methodology "...for 'mid-range' aspects of the research process ..." which they label "... strategies (methods) ...", using the terms "method and methodology" interchangeably $(2002: 8,10)$ 
3. Finally in Groat and Wang's framework come 'tactics': "... tactics are the specific techniques used, such as data collection devices, response formats, archival treatment, and analytical procedures." (2002:11)

It was noted above that Groat and Wang use the terms method and methodology interchangeably. Others, however, make a distinction between these two terms:

\begin{tabular}{|c|c|c|c|}
\hline & $\begin{array}{l}\text { Groat and Wang } \\
(2002)\end{array}$ & $\begin{array}{l}\text { Evans and Gruba } \\
(2002)\end{array}$ & $\begin{array}{l}\text { Galloway and Skinner } \\
\text { (2010) }\end{array}$ \\
\hline $\begin{array}{l}\text { Ontological and } \\
\text { epistemological } \\
\text { assumptions }\end{array}$ & $\begin{array}{l}\text { system of inquiry (10) } \\
\text { paradigm (21) } \\
\text { world view (21) } \\
\text { Examples: positivism, } \\
\text { post-positivism, } \\
\text { structuralism, post- } \\
\text { structuralism, critical } \\
\text { theory (10) }\end{array}$ & $\begin{array}{l}\text { 'methodology' = 'how } \\
\text { we gain knowledge of } \\
\text { the world' (89) } \\
\text { Examples: neo-Marxist, } \\
\text { post-modernist, } \\
\text { feminist, liberal ... 'a } \\
\text { particular philosophical } \\
\text { stance' }(89,90)\end{array}$ & $\begin{array}{l}\text { world-view (Doing Res.I) } \\
\text { 'ontological and } \\
\text { epistemological } \\
\text { positions’ (Doing Res.II) }\end{array}$ \\
\hline $\begin{array}{l}\text { Overall research } \\
\text { approach / plan }\end{array}$ & $\begin{array}{l}\text { methodology }(8,10) \\
\text { strategy/ies }(10,11) \\
\text { method/s }(10) \\
\text { research design }(11)\end{array}$ & $\begin{array}{l}\text { 'methodology' = 'the } \\
\text { general stance that the } \\
\text { researcher is taking' eg } \\
\text { 'as objective observer', } \\
\text { 'as participant' (89) } \\
\text { 'research design' (88) }\end{array}$ & $\begin{array}{l}\text { 'methodology ... is a } \\
\text { justification for an } \\
\text { approach ... presents a } \\
\text { storyboard ... provides a } \\
\text { blueprint or recipe that } \\
\text { includes methods for } \\
\text { doing the work' } \\
\text { (Design Research IV - VI) }\end{array}$ \\
\hline $\begin{array}{l}\text { Research } \\
\text { methods }\end{array}$ & $\begin{array}{l}\text { methodology }(8,10) \\
\text { strategy/ies }(10,11) \\
\text { method/s }(10) \\
\text { research design }(11) \\
\text { Examples - “... the full } \\
\text { range of research } \\
\text { methods ... applicable } \\
\text { to ... architectural } \\
\text { research" (3); "Part 2: } \\
\text { Seven Research } \\
\text { Strategies" (133 ff) }\end{array}$ & $\begin{array}{l}\text { 'method or methods } \\
\text { appropriate for testing } \\
\text { your hypotheses or } \\
\text { answering your } \\
\text { questions' (85) as } \\
\text { distinct from the term } \\
\text { 'methodology' (89) }\end{array}$ & $\begin{array}{l}\text { strategies (Doing Res.III) } \\
\text { 'methods or strategies' } \\
\text { (Doing Research V) }\end{array}$ \\
\hline $\begin{array}{l}\text { Research } \\
\text { techniques }\end{array}$ & $\begin{array}{l}\text { tactics (10) } \\
\text { techniques (10) } \\
\text { Examples: interviewing } \\
\text { archival searches, data } \\
\text { collection, analysis (10) }\end{array}$ & $\begin{array}{l}\text { 'instruments or } \\
\text { procedures' (96) }\end{array}$ & $\begin{array}{l}\text { tactics (Doing Res.III) } \\
\text { 'techniques or tactics' } \\
\text { (Doing Research VI) }\end{array}$ \\
\hline
\end{tabular}

Figure P2: Research terminology: comparisons from three sources 
C 2012 Stephen Pattinson $\quad$ Preface M. Arch Thesis

Because Evans and Gruba (2002) and Galloway and Skinner (2010) differentiate between methodology and method I have found it helpful, for clarification, to modify Groat and Wang's research framework as follows:

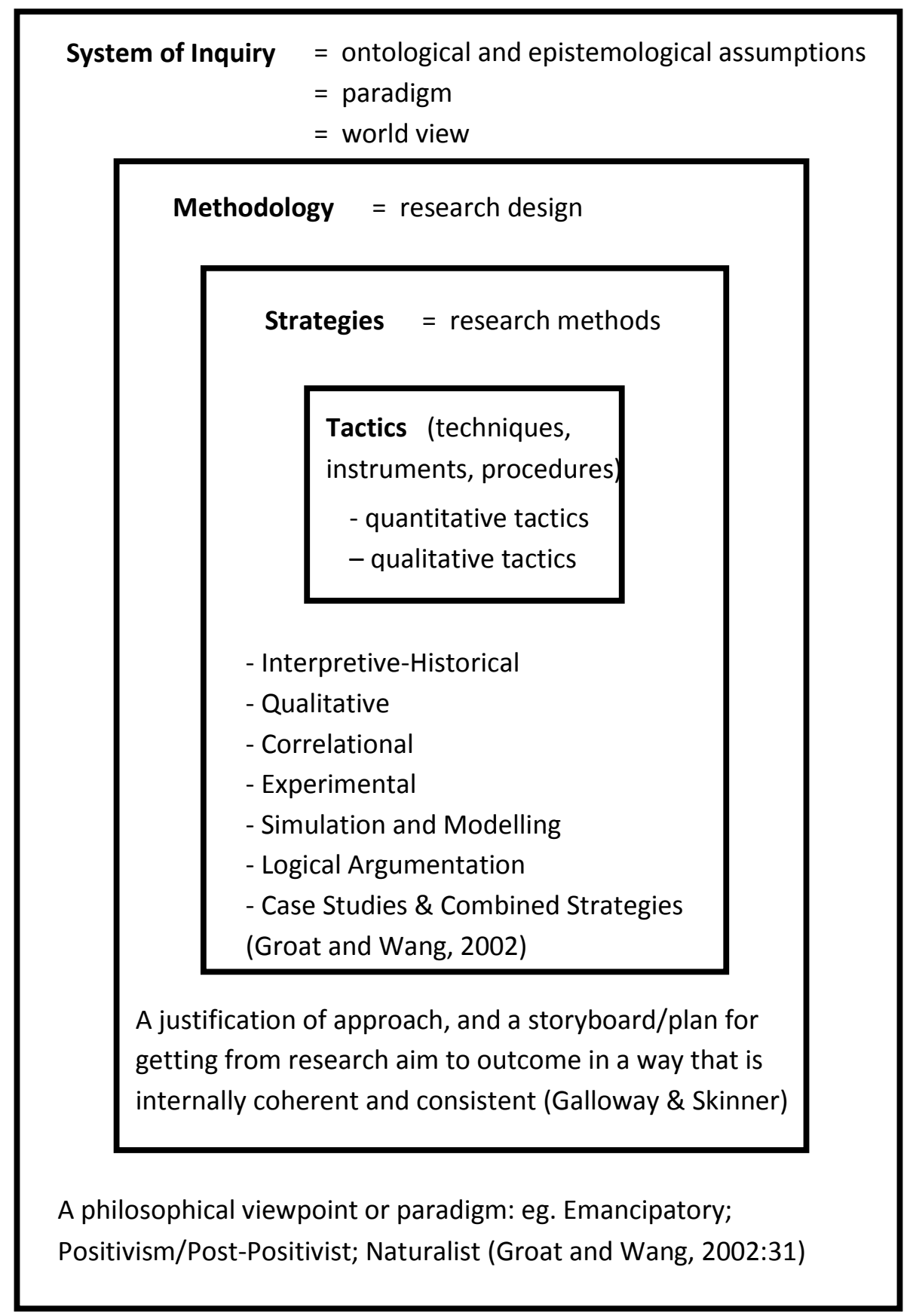

Figure P3: A modified research framework that distinguishes 'methodology' from 'methods'. $\quad$ (adapted from Groat and Wang, 2002:10, Fig.1.4) 
(C) 2012 Stephen Pattinson $\quad$ Preface M. Arch Thesis

Note that in the above diagrams (Figures P2 and P3) Evans and Gruba (2002) and Groat and Wang (2002) use the term 'research design' as follows:

- Evans and Gruba suggest that the thesis chapter 'Research Method' or 'Research Methodology' should be titled 'Research Design', which “... should start with a drawing together of reviews of previous work and preliminary studies to formulate research hypotheses or research questions. The methods we select are ways of testing the hypotheses or answering the questions." (Evans and Gruba, 2002:88, 89)

- 'A research design is 'an action plan for getting from here to there', where here describes the investigator's research question(s), and there describes the knowledge derived from the research. In between the here and the there are a set of steps and procedures that may be highly prescribed or emerge as the research proceeds." (Groat and Wang, 2002:11)

Based on the foregoing discussion, I distinguish between 'methodology' (research design) and 'methods' (research strategies). Accordingly, I have titled my research methodology chapter 'Research Design', following Evans and Gruba (2002:88). In this chapter, I identify my research questions which must be answered to achieve the overall research aim. I review possible research strategies (methods) to address these questions, and make explicit my choice of strategies and why I chose them.

Also following Evans and Gruba (2002:91), I am using a triangulation of several complementary perspectives (see this thesis, 17, Fig. 2.2) to throw light on different aspects of the multi-faceted research topic (multivalent communal space). The logic flow in my thesis is explained in the following discussion. 

(C) 2012 Stephen Pattinson
Preface
M. Arch Thesis

Evans and Gruba (2002:12) advise that there is no one definition for a thesis, but suggest there is a 'standard thesis structure ... that ... works well for theses in the physical, biological and social sciences ..." (58). Their suggested structure consists of four parts as illustrated in the following diagram (Figure P4). The arrows in the diagram indicate the logic flow between the four parts. The writer has generally followed this structure, adapting it as required to suit the research design.

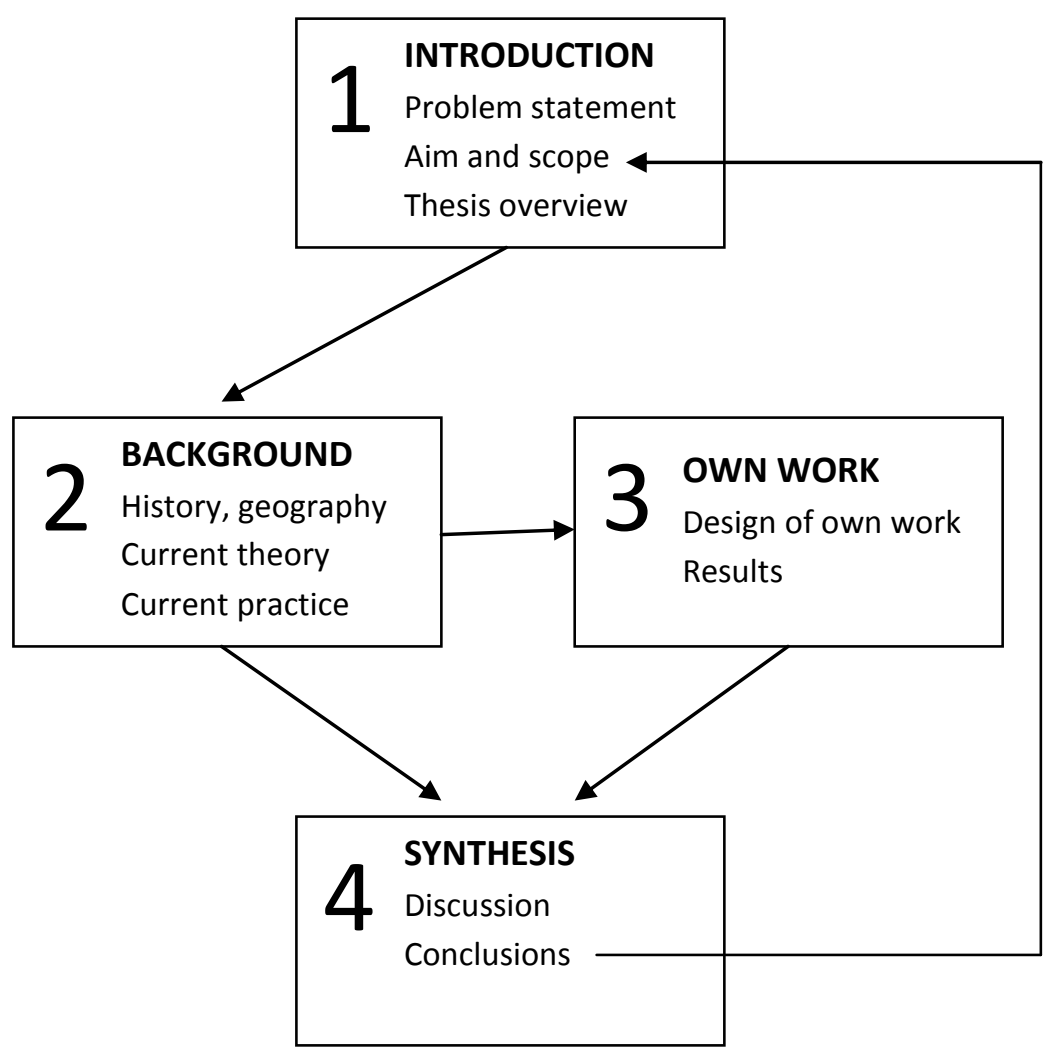

Figure P4: Structure of theses in the physical, biological and social sciences. (Evans and Gruba, 2002:13, Fig.1) 
The writer started out on this linear tack ...

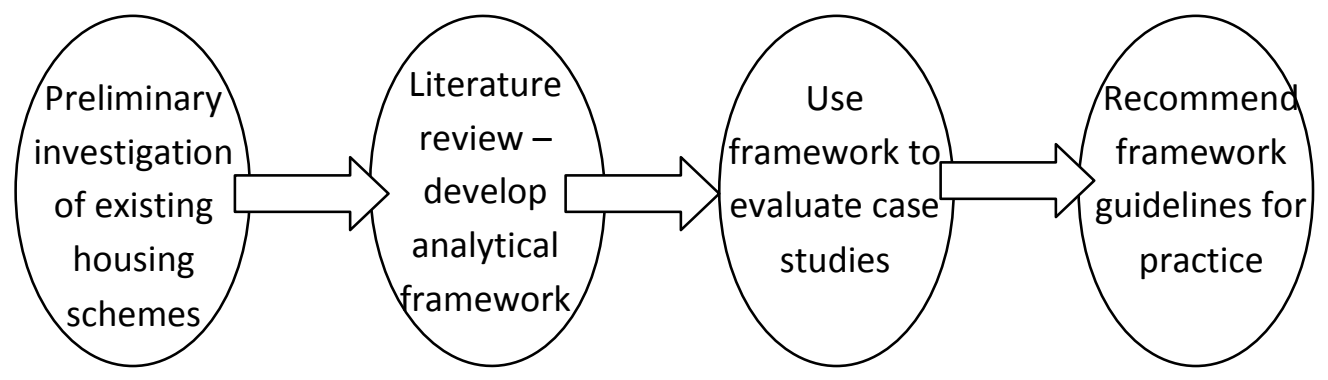

See Figure 6.2 Linear Theory-to-Practice Approach

S.J. Pattinson

... but along the way the research developed into a more integrated approach:

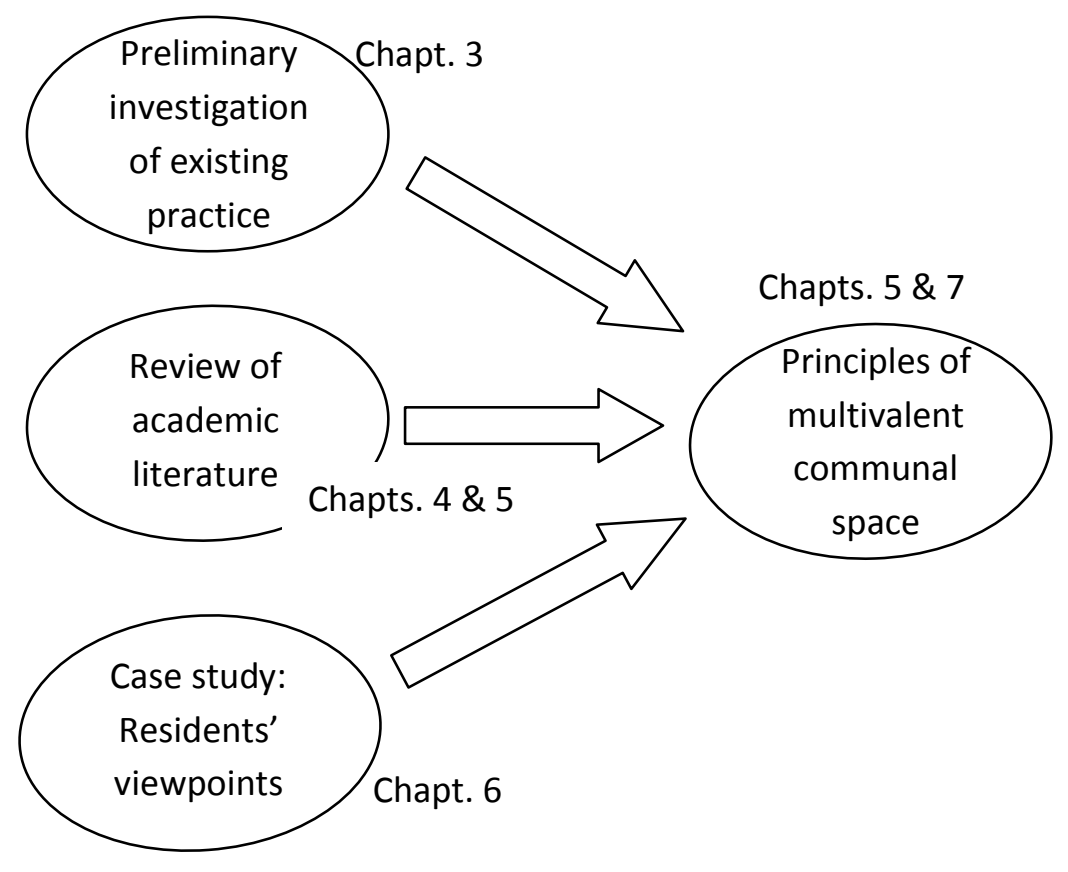

See Figure 6.3 Participatory Approach: Dialectical Relationship Between Theory and Practice.

S.J. Pattinson

The attempt at a more participatory approach is further explained in Chapters $2 \& 6$. 
C 2012 Stephen Pattinson $\quad$ Preface M. Arch Thesis

\section{Dedication}

This research was inspired by Cameron McClean's vision of medium density, low rise, low energy settlements, in which two and three storeyed timber dwellings, linked and grouped closely together, achieve a compact, walkable urban form:

"The energy studies indicated houses which would be two or three storeys high, with narrow frontages and with considerable depth. This proportion diminishes the exterior wall to common wall ratio and consequently the heating and cooling requirements. The ground floor might contain the small scale industry or commercial business of its occupiers and the upper floors the living rooms and bedrooms. The roof could well be flat, grassed and open to the sun and sky. A group of twenty or thirty of these houses could be connected in a ring to form and contain a sheltered courtyard. This interior area could be grassed and planted as a social place. The outside of the houses would open onto pedestrian lanes or roofed arcades and onto paved piazzas surrounded by the shops and overlooked by balconies."

Cameron McClean (13)

As part of undergraduate study in a student team supervised by Cameron McClean, the writer explored housing ...

"linked together ... and arranged in ... three sided rectangles ... or in bay forms ... [to provide] a common open space in the centre, visible and available to all [residents] for a great many social and recreational purposes [for example] ...

"recreational and physiological (active exercise, sunshine, fresh air);

"psychological (a change from the internal environment);

"socio/cultural (meeting friends and making new contacts);

"occupational (outdoor domestic tasks, for example repairing a push bike or drying clothes).

"A recognition of the many co-existing uses that can be made of a single space will ... [facilitate] richness in the housing environment. This principle of multiplicity-of-use comes from Alexander's description of the city as a semi-lattice of many interacting and overlapping sets of activities (Alexander, in Bell and Tyrwhitt, 401)."

S J Pattinson, in Mclnnes, Pattinson and de Lisle $(123,129)$ 
In this post-graduate research, the writer re-visits the "many interacting and overlapping sets of activities" and "co-existing uses that can be made of a single space" for efficiency and "richness in the housing environment".

This research is dedicated to Cameron McClean, a visionary academic with a do-able vision, to the residents of the terrace housing at Pinehill Park who participated in the Case Study in this research, and to all who want better living environments in medium density housing.

"Every corner and every space must be programmed for multiple roles."

Hertzberger, in Jencks (318)

"People do not only live in[side] houses." Marc Fried

"An ordinary day in an ordinary street. Pedestrians are passing on the sidewalks, a few people are sitting on benches and stairs, children are playing around the front doors, a yell and a greeting exchanged between two pedestrians, two mechanics are repairing a car, groups are chatting on the sidewalk. Altogether a diverse and complex pattern of outdoor activities, each being influenced by a number of factors, the quality of the physical environment being one factor...

"The life between the houses is to a very high degree influenced by physical planning. ... By improving the quality of the environment it has often been proved [sic] that this tends to have a very positive influence on the outdoor activities. ... The quality of physical environment ... can influence the amount and character of the activities in public [or shared] spaces."

Jan Gehl (1980: 2,3) 


\section{Acknowledgements}

For support throughout this research I especially thank:

- $\quad$ my wife Sue who unfailingly supported me and assisted with insightful and constructive comment throughout the entire research project

- my family and friends

I also gratefully acknowledge assistance from:

- Susan Pattinson, Holly Spiers and Rebecca Grimmer for their assistance with the Pinehill Crescent Case Study survey and interviews;

- special thanks to all the terrace house residents at Pinehill Park who participated in the Case Study survey and interviews;

- David Evans and Paul Gruba - "How to Write a Better Thesis" 2 $2^{\text {nd }}$ ed. (2009);

- Hugh Kearns for his transformational "How to Turbo-charge your Writing"

- VUW architecture staff - Julie Baga (library - literature search skills); Michael Dudding (MLA Citation Style Guide); and John Gray (critical enquiry);

- Various Regional and Local Council planning and urban design staff, particularly John Stenberg, Gabrielle Seo, Matt Riley, Andrew Trevalyan, Brenna Waghorn, Fred Baxton and Robert Graham;

- Architects - Peter Beaven, John Chaplin, John McClean, Nigel Bond and Crispin Schurr;

- Developers and builders, especially John Sax and Dean Spiers;

- Stuart Bracey at Housing New Zealand Corporation;

- NZ Centre for Sustainable Cities for financially supporting this research;

- VUW School of Architecture for the opportunity to carry out this research;

- Also thanks to Taylor and Francis Books (Routledge) for permission to reproduce Hildebrand Frey's tables summarising criteria for a sustainable city form and region (see this thesis, 120,121 Tables 5.1a, b). 
(C) 2012 Stephen Pattinson Contents M. Arch Thesis

\section{Contents}

Title Page

Abstract ii

Preface iii

Dedication, Acknowledgements $\quad x-x i i$

List of Figures, Tables $\quad$ xvi - xix

$\begin{array}{lll}\text { Chapter } 1 & \text { Introduction } & 1\end{array}$

- An introductory explanation of the problem 2

$\begin{array}{ll}\text { - A brief clarification of key terms } & 7\end{array}$

- What is 'medium density' housing? 7

- What is communal space? 8

- What is multivalence? 8

- What is multivalent communal space in medium density housing? 9

- Scope and Limitations

- A brief overview of this thesis

\section{Chapter 2 Research Design}

○ Research Questions; Research Design

15,16

- Investigating practitioners' viewpoints through current practice

- Exploring academics' viewpoints in the Literature Review

- Exploring community viewpoints through Pinehill Park Case Study

- Collaboration between practitioners, researchers and the community 21

- Strategic, incremental innovation 25

- An analytical framework for exploring communal space (see also App. 8) 26

Chapter 3 Preliminary Investigations

- Urban Design Case Studies $\quad 29$

- The Good Solutions Guide for Medium Density Housing 38

- The Good Solutions Guide for Apartments 38

- Good Solutions Guide for Mixed Use Developments in Town Centres 41

- Viewpoints of Practioners: Current Practice 43

- Car-dominated housing environments 45

- Private outdoor space- often compromised 47

- Landscape-dominated environments 48

- Summary of main points and observations 49

○ Conclusion $\quad 50$ 
Chapter $4 \quad$ Current Theory $\quad 51$

○ Multivalent communal space in medium density housing 51

- Communal space: potential for multivalence 57

- Can enhance neighbourhood identity 57

- Can foster neighbourhood attachment 64

- Can invite walking, recreation and exercise 65

- Can foster social interaction, inclusion and heterogeneity 66

- Can enhance mental health 76

- Can foster inter-generational interaction 77

- Can provide places for children's play 80

- Can provide places for teenagers to hang out 88

- Territorial space, safety and crime prevention 90

- Can provide opportunities for community participation 98

- Can enhance the environment and improve property values 100

- Can foster urbanity 102

- Urbanity thresholds 102

- Walkable urbanism; drivable sub-urbanism; neverlands 105

- Archetypal bay-structure for community connectedness 107

- Conclusion 116

$\begin{array}{lll}\text { Chapter } 5 & \text { Proposed Principles for Multivalent Communal Space } & 117\end{array}$ In Medium Density Housing

$\begin{array}{ll}\text { O Introduction } & 117\end{array}$

- Sustainable Cities: An over-arching theme 119

○ Principle \#1: Walkable Neighbourhoods 123

- Walkable Urbanism 124

- Diversity of house size and type 128

- Living sustainably 130

- Principle \#2: Housing Clustered to Create Communal Space 131

- Principle \#3: Communal Green Open Space 137

- Principle \#4: Communal Space Fit for Children 140

- Principle \#5: Communal Space to Foster 'Sense of Community' 146

- Conclusion 150 
Chapter 6 Case Study: Pinehill Park $\quad 151$

○ Part 1: The Case Study 152

1.1. An explanation of the approach taken for this Case Study 153

1.2. Background information about Pinehill Park 158

1.3. Observations of the existing use of Pinehill Park 172

1.4. Interviews with residents of terrace houses adjoining the Park 173

○ Part 2: Results of The Case Study 174

2.1. Observations of Pinehill Park 174

2.2. Comments relating to the observations of Pinehill Park 177

2.3. Interviews with residents of terrace houses adjoining the Park 181

2.4. Responses correlated with the Five Principles 182

○ Conclusion 200

Chapter 7 Discussion: Multivalent Communal Space 201

O Introduction 201

- Five Principles for Multivalent Communal Space in Medium Density 204

- A Correlation of Best Practice, Theory and Community Input 206

- The Five Principles for multivalence are inter-dependent 213

- Resolving 'incompatible' mixes 214

- Application of the Five Principles for Multivalent Communal Space $\quad 215$

$\begin{array}{lll}\text { Chapter } 8 & \text { Conclusion } & 227\end{array}$

$\begin{array}{ll}\text { Bibliography } & 231\end{array}$

\section{Appendices}

Appendix 1: Floor space and household size

(12 pages)

Appendix 2: Government Press Release - Pinehill Crescent (1 page)

Appendix 3: Template of observation sheet (1 page)

Appendix 4: Observations of Pinehill Park (4 pages)

Appendix 5.1: Survey / Interview Forms (5 pages)

Appendix 5.2: Analysis - Survey / Interview Responses (23 pages)

Appendix 6: Ethics Approval Information Sheet \& Consent Form $\quad$ (2 pages)

Appendix 7.1: List of Sites Visited for thesis research (14 pages)

Appendix 7.2: Field Notes - Melbourne (41 pages)

Appendix 7.3: Preliminary Investigations (47 pages)

Appendix 8: Analytical Framework (Wellington schemes) (56 pages) 


\section{List of Figures}

Figure

Page

\section{Chapter 1}

1.1

1.2

1.3

1.4

1.5

1.6

1.7

1.8

1.9

1.10

1.11

1.12

1.13

1.14

1.15

1.16

1.17

1.18

Chapter 2

2.1

2.2
Intensive Housing - Grafton, Auckland 2

Double-decker flats Mt Eden 2

"...pack 'em in" Mt Eden 2

Beaumont Quarter. Auckland Centre 2

$4 \times 1000 \mathrm{~m}^{2}$ lots on 0.4 ha 4

$24 \times 140 \mathrm{~m}^{2}$ lots on 0.4 ha 4

Zabeel Crescent, Addison, Auckland 5

122 Rintoul Street, Wellington 5

15 Warwick Street, Christchurch 5

10 Park Avenue, Lower Hutt 6

Grafton, Auckland 6

Alverna Heights, Gulf Harbour 6

Visualizing dwelling density $\quad 7$

Site of open-air library, Salbke, Magdeburg, Germany 9

Open-air library, Salbke, Magdeburg, Germany 9

Open-air library, Salbke, Magdeburg, Germany 10

View of stage and café over 10

$\begin{array}{ll}\text { Open-air library, street view } & 10\end{array}$

The bellbird and flax plant 13

A model for a participatory approach in the development of 17

theory and practice

\section{Chapter 3}

$3.1 \mathrm{a}-\mathrm{c}$

Beaumont Quarter, Auckland: Design variety 28

3.2

3.3

3.4

The Park, Viaduct Harbour

28

Communal gardens, Talbot Park, Glen Innes, Auckland 28

Public seating, Harbourview, Waitakere 28

Indoor pool and fitness gym, Beaumont Quarter, Auckland 28

Beaumont Quarter, raised mounds $\quad 29$

Lancewood Courts, communal outdoor area 29

Takapuna Terrace Housing $\quad 30$

Communal Courtyard, Takapuna Terrace Housing 30

Communal greens, Freemans Bay, Auckland 31

Communal green rarely used, Freemans Bay, Auckland 31

A car dominated central courtyard, Krisley Court, New Lynn 32

Picnic area in car dominated courtyard, Krisley Court 32

Part of Addison development, Takanini, Papakura 33

Bruce Pulman Park, Addison development, Takanini 34 
Addison - car access via rear lanes 35

3.16

Addison terrace housing - front door faces pocket park 36

$3.17 a$

First floor plan - Addison terrace housing

$3.17 \mathrm{~b}$

Ground floor plan - Addison terrace housing

Linear flats - 1960s

Contemporary linear flats

The bay-form structure

Merivale Mews, Christchurch

Mt Eden, Auckland

Greenwich Park, Grafton

Green open space and tennis court at Tuscany Towers 
4.21 Albany, Auckland. Leinberger's 'neverlands' 106

$\begin{array}{lll}4.22 & \text { The bay-structure } & 107\end{array}$

4.23 The edge defines the bay-structure 108

$4.24 \quad$ Piazetta of St Mark's Square 108

$4.25 \quad$ St Mark's Square Venice 108

4.26 Cooperative housing: N. Bond \& G. Horne student project 110

4.27 Model of cooperative housing project: N. Bond \& G. Horne 110

4.28 a - c Community Centre for Hebrew Congregation, Auckland $\quad 111$

4.29 a - c University School of Music, Auckland 112

4.30 Primary School at 126 Oteha Valley Road, North Shore 112

$4.31 \quad$ Kerr Street, Primary School, Devonport 112

$4.32 \mathrm{a}, \mathrm{b} \quad$ Central courtyard. IHC Home, Hendersons Rd, Christchurch 113

$4.33 \quad$ Courtyard House Types. 113

4.34 a - c Housing for singles with AIDS, Bay Bridge Housing, Oakland, CA 113

Chapter 5

5.1

5.2

5.3

5.4

5.5

5.6

'Walkable Urbanism' - Hammarby-Sjostad, Sweden 123

A multi-generational household 128

Clustered eco-housing, Lecthworth, UK 131

Positive open spaces between buildings 134

Shared private open space $\quad 135$

Families with children living in Auckland's high rise apartments 141

Chapter 6

6.1

6.2

6.3

6.4

6.5

6.6

6.7

$6.8 \mathrm{a}, \mathrm{b}$

$6.9 a, b$

$6.10 a, b, c$

6.11

6.12

6.13

$6.14 \mathrm{a}-\mathrm{d}$

$6.15 \mathrm{a}-\mathrm{f}$

6.16

6.17

Chapter 7

7.1

7.2

7.3
Aerial View of Pinehill Crescent subdivision, Upper Hutt

152 Linear theory to practice approach

153

Participatory Approach

154

Survey Plan of the proposed new reserve, Pinehill Crescent 160

Site Plan of the proposed 14 new terrace houses 161

Aerial View of Pinehill Park 162

Plan View of terrace house group 163

Plan and Elevations of two terrace houses 164

Plan and Elevations of 7 terrace houses 165

Typical Floor Plans and Cross Section of a terrace house 167

Pinehill Park viewed from roadside 168

Pinehill Park viewed from eastern end 169

Pinehill Park viewed from western end 169

Pinehill Park playground $\quad 170$

Views outdoors from inside one of the terrace houses 171

Users of Pinehill Park during observation period 175

Heretaunga Drain 176

The 'bay' structure 201

A participatory approach to developing theory and practise 203

Five principles for multivalent communal space 204 
7.4

Pinehill Crescent subdivision as existing

7.5

Same density: two types of development

7.6

120 detached houses

219

7.7

Detached vs. cluster housing with open space

220

7.8

Cluster housing with open space - Stage 1 and 2

7.9

Cluster housing with open space - Stage 3

\section{List of Tables}

Table

Page

$1.1 \quad$ Falling average household size 3

$1.2 \quad$ Dwelling floor area/person 4

$2.1 \quad$ Case Study Design 23

4.1 Erikson's Life Cycle Developmental Stages 78

$4.2 \quad$ Design patterns for children 82

4.3 Densities by Residential Type 102

$5.1 \mathrm{a}, \mathrm{b} \quad$ Criteria for a more sustainable city form (from Frey) 120-121

5.2 Children in New Zealand's four largest urbanised areas 140

$\begin{array}{lll}6.1 & \text { Chronology of Pinehill Park } & 158-159\end{array}$

$\begin{array}{llr}6.2 & \text { Traffic passing Pinehill Park } & 179\end{array}$

7.1 Principle \#1: Sustainable Neighbourhoods 207

7.2 Principle \#2: Clustered Housing 208

7.3 Principle \#3: Green Open Space 209

7.4A Principle \#4a: Places for Children 210

7.4B Principle \#4b: Places for Teenagers 211

$\begin{array}{ll}7.5 & 212\end{array}$

$\begin{array}{lll}7.6 & \text { Comparison of conventional vs. cluster housing development } & 225\end{array}$ 


\section{CHAPTER 1 - Introduction}

A social trend towards lower average household size is reflected in the growing demand for smaller dwellings. The minimum floor space required per individual in a one- or two-person dwelling is more than the minimum floor space required to house an individual in a five- or six-person dwelling - two or three times more, in fact (see Appendix 1). With household sizes falling, this effect, multiplied several thousand times in the population of a city, results in a dramatic increase in the built floor space required to house a population, without any increase in population size.

At the same time, lot sizes are diminishing in New Zealand's main urban centres. A few decades ago a typical suburban house lot was around $800 m^{2}-1000 m^{2}$. Now, in intensified residential localities introduced about the mid-1990s typically in the form of terrace housing, $150 \mathrm{~m}^{2}-350 \mathrm{~m}^{2}$ lots are common (Turner et al. 2004:2, 12, 22).

The combination of increasing floor space and diminishing lot size is resulting in a significant reduction in communal and public open space. This is evidenced in tiny front and rear yards, narrower streets, fewer footpaths and smaller parks (pocket parks) (Trancik 2007). Communal areas in medium density housing developments are typically univalent spaces, having only a single purpose. In New Zealand housing developments, this purpose is usually the parking and moving of cars.

With the reduction in open space, especially private outdoor space, more thought needs to be given to communal (shared) space in medium density housing.

\section{Research Problem}

Individualism demands more floor space per person; urban intensification supplies less site space per dwelling. The result is less open space. The problem is how to deliver more amenity within less open space. 
To compensate for smaller yards and less open space, better use must be made of communal space for residents. The aim of this research is to develop principles for making communal space in medium density housing more 'multivalent'.

\section{Aim of the Research}

Develop principles of multivalent communal space that achieve more efficient use of urban land and enhance the living environment for residents in medium density housing

\section{An introductory explanation of the problem:}

Medium density housing is a growing market in New Zealand. Mead \& McGregor reported in 2007 that 'intensive housing', which they define as 'terrace or apartment type housing' (62), made up $35 \%$ of Auckland's total housing market, and that it is likely to become the housing market in Auckland in the future, not just a segment within it (62).

Bill MacKay (70) referring to developers of medium density housing in New Zealand, comments:

"...most developers seem to be in it for the quick buck ... Houses ... are ... built cheap and sold fast ... the role of the architect is to rack 'em, stack 'em, and pack 'em in."

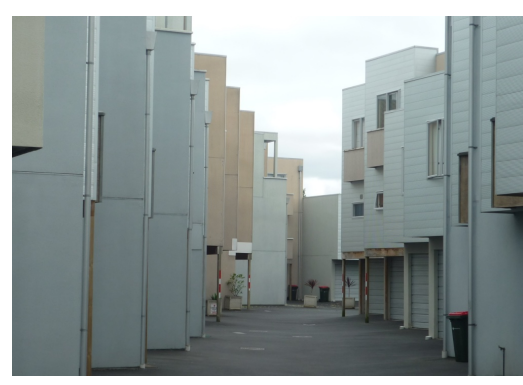

Fig. 1.3 “...pack 'em in" Mt Eden, Auckland (Photo: S.J. Pattinson)

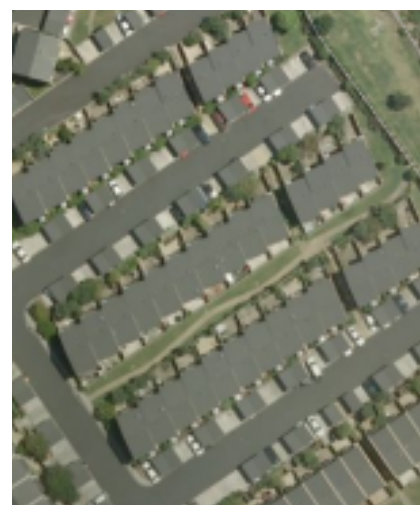

Fig. 1.1 Grafton, Auckland Top right is motorway embankment (Alggi map)

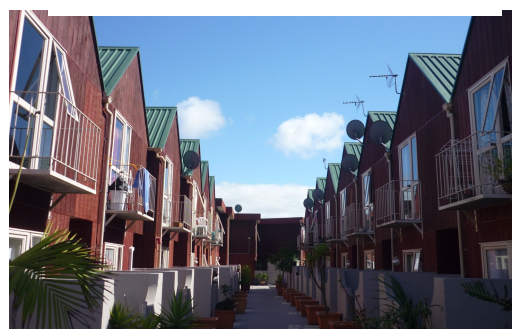

Fig. 1.2 Double-decker flats on top of carpark building, Mt Eden, Auckland (Photo: S.J.Pattinson)

Fig. 1.4 Dwelling units lack personalisation. Beaumont Quarter, Auckland Central (Photo: S.J. Pattinson)

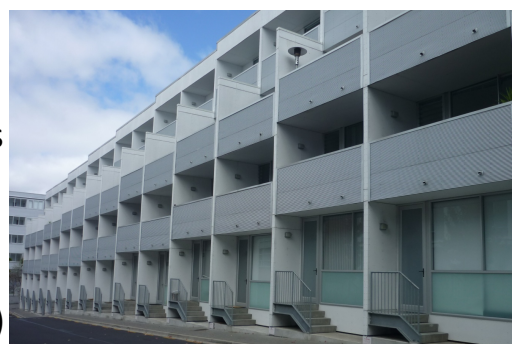


The anthropologist Edward T. Hall asserts culture shapes perceptions of the built environment, but culture itself is shaped by the environment. Hall endorses Churchill's comment that "[w]e shape our buildings and they shape us" (106). The dwellings shown in Figures 1.3 and 1.4 lack personalisation. The access streets lack social space. What do we understand about the effects of such environments on their occupants? Robert Sommer, described by Hall as a perceptive psychologist, notes how little is known about the influence of the built environment on human development (108) and comments:

"We are in the midst of reshaping the environment on an unprecedented scale, but we do not know what we are doing to ourselves." (7)

\begin{tabular}{|l|l|l|l|l|l|l|l|l|l|}
\hline & 1821 & 1881 & 1900 & 1950 & 1961 & 1981 & 1991 & 2000 & 2006 \\
\hline UK & 5.75 & 5.38 & 5.2 & & 3.0 & & 2.5 & & 2.4 \\
\hline USA & & & 4.60 & 3.38 & & & & 2.59 & \\
\hline NZ & & & & 3.7 & & 3.0 & & & 2.6 \\
\hline
\end{tabular}

Table 1.1 Falling average household size (Turner et al. 8; UK Focus on People and Migration, 2005:12; UK Office for National Statistics 37 Social Trends 2007:14; US Census Bureau)

A significant part of housing demand today is driven by the falling average household size. A couple of hundred years ago the average number of people living together in each house in the United Kingdom, in the United States of America and in New Zealand would have been about 5 or 6; today in these countries there are on average about 2.5 people in each household (Table 1.1). Madanipour refers to this fall in average household size as a long process of individualism, referring to the change from living in large, shared dwellings to private living in smaller households (77-87). Hall and Ward attribute this to...

"... many more young people leaving home for higher education or first jobs, many more divorces and separations, more old people living longer but eventually getting widowed. ... 79 per cent [of the increasing housing demand in the UK] will be one-person households, and more than half of these will be never-married people living alone." (111) 
Auckland Council notes:

"In 2006, almost half (49.2 per cent) of all households in the Auckland region were one or two person households ... Only a small proportion of households (15.2 per cent) had five or more members. ..." http://monitorauckland.arc.govt.nz/our-community/households-andfamilies/household-size.cfm (Retrieved 06.11.2010)

The trend toward smaller average household size is being accompanied by a trend toward smaller lot size. Four traditional $1000 \mathrm{~m}^{2}$ (quarter-acre) lots can convert to $24 \times 140 \mathrm{~m}^{2}$ terrace house lots on the same overall land area (see Figs. 1.5 \& 1.6):

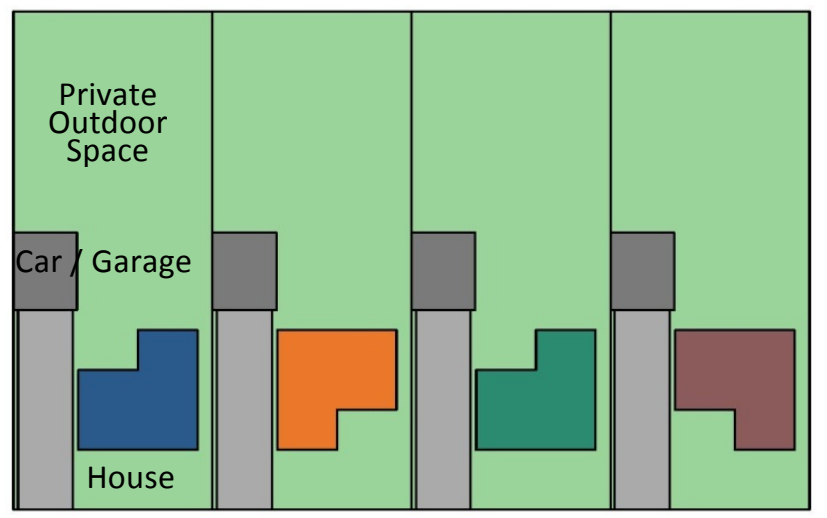

4 Dwellings on 0.4 ha (See also Appendix 1:11) Scale 1:1000

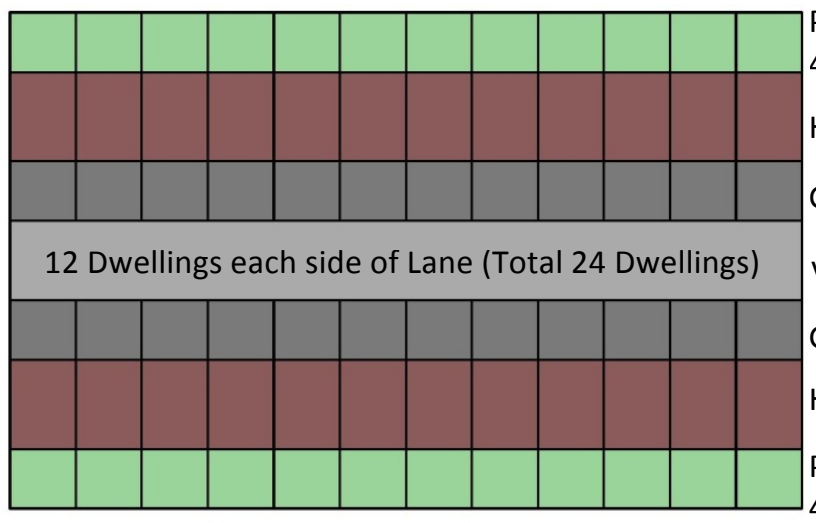

$0 \mathrm{~m} 10 \mathrm{~m}$ لسلسلسا
24 Dwellings on $\mathbf{0 . 4}$ ha (See also Appendix 1:12) Scale 1:1000
Fig. 1.5. $4 \times 1000 \mathrm{~m}^{2}$ lots $=4,000 \mathrm{~m}^{2}$. Each lot measures about $20 \mathrm{~m} \times 50 \mathrm{~m}$. Dwellings are about $120 \mathrm{~m}^{2}$ each, plus garage/workspace of about $60 \mathrm{~m}^{2}$. Allowing $100 \mathrm{~m}^{2}$ for driveway, there is a net area of about $720 \mathrm{~m}^{2}$ of private outdoor space per lot. This provides about $20 \mathrm{~m}^{2}$ of house and about $120 \mathrm{~m}^{2}$ of private outdoor space per person, assuming 6 persons per household. (Source: S.J. Pattinson)

\begin{tabular}{|c|c|c|c|c|c|c|c|c|c|c|c|}
\hline & & $\begin{array}{l}\text { Block } \\
\text { area } \\
\left(\mathrm{m}^{2}\right)\end{array}$ & $\begin{array}{c}\text { No. } \\
\text { of } \\
\text { Lots }\end{array}$ & $\begin{array}{l}\text { Lot } \\
\text { area } \\
\left(\mathrm{m}^{2}\right)\end{array}$ & $\begin{array}{c}\text { House } \\
\text { area } \\
\left(\mathrm{m}^{2}\right)\end{array}$ & $\begin{array}{l}\text { No. of } \\
\text { storeys }\end{array}$ & $\begin{array}{l}\text { Persons/ } \\
\text { dwelling } \\
\text { (average) }\end{array}$ & $\begin{array}{c}\text { Floor } \\
\text { area }\left(\mathrm{m}^{2}\right) \\
\text { /person }\end{array}$ & $\begin{array}{c}\text { Outdoor } \\
\text { area }\left(\mathrm{m}^{2}\right) \\
\text { /person }\end{array}$ & $\begin{array}{l}\text { Total } \\
\text { resi- } \\
\text { dents }\end{array}$ & $\begin{array}{c}\text { Total } \\
\text { vehi- } \\
\text { cles }\end{array}$ \\
\hline 1 & Fig. 1.5 & 4,000 & 4 & 1,000 & 120 & 1 & 6 & 20 & 120 & 24 & $4-8$ \\
\hline 2 & Fig. 1.6 & 4,000 & 24 & 140 & 120 & 2 & 4 & 30 & 10 & 96 & $24-48$ \\
\hline 3 & Fig. 1.6 & 4,000 & 24 & 140 & 120 & 2 & 2 & 60 & 20 & 48 & $24-48$ \\
\hline 4 & Fig. 1.6 & 4,000 & 24 & 140 & 60 & 1 & 1 & 60 & 40 & 24 & 24 \\
\hline
\end{tabular}

Table 1.2 Dwelling floor area per person increases as number of persons per dwelling decreases on smaller lots, along with reduced outdoor space per person, and significantly more vehicles (See App.1) (S.J. Pattinson) 
As the number of persons per household reduces, the dwelling floor space per person increases (see Table 1.2). This is confirmed by an analysis by the writer of house floor plans (see Appendix 1) which reveals that the floor space required to house an individual in a one-person dwelling is at least twice the floor space required to house an individual in a six-person household.

Many more houses are required as individuals seek larger private realms in smaller households without any increase in population size, e.g. on Auckland's North Shore:

"Even if population growth was to fall to nil, there would be a demand for over 6,000 $d$ wellings during the next 20 years, due to changes in the structure of the population and resulting household trends." (Section 6.2, p.6-2, North Shore /Auckland Council - District Plan, 2009)

Madanipour's 'individualisation process' (86) is a significant driver of intensification. But the enlargement of individual private realms in smaller households occurs at the expense of open outdoor space. Minimum private outdoor space of about $40 \mathrm{~m}^{2}$ per dwelling (as little as $10 \mathrm{~m}^{2}$ per occupant - see Line 2, Table 1.2) is typical in New Zealand medium density housing, e.g. Addison, Auckland (Fig. 1.7); 122 Rintoul Street, Wellington (Fig. 1.8); 15 Warwick Street, Richmond, Christchurch (Fig. 1.9).

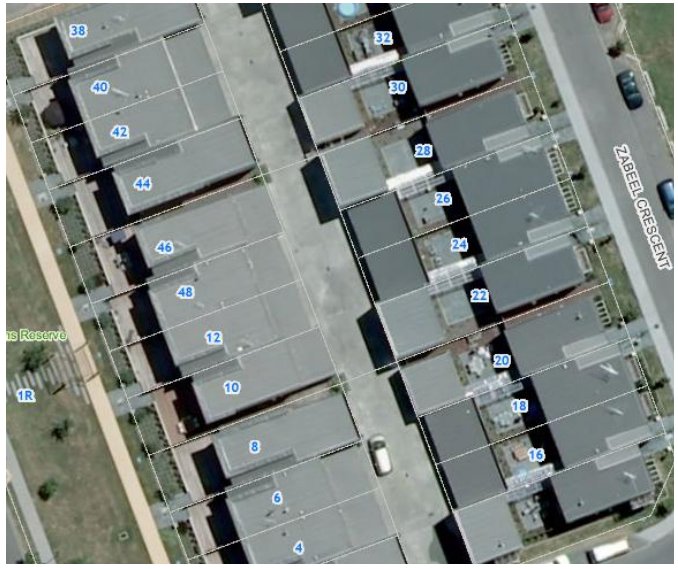

Fig. 1.7 Zabeel Crescent, Addison, Auckland Sources: TA GIS viewers and Google Maps. Scales approximately 1:1,000 (at A4 size)

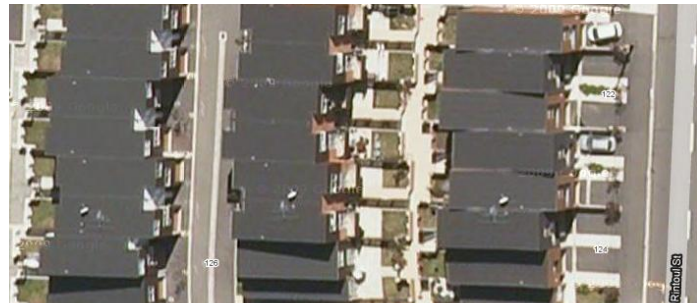

Fig. 1.8 122 Rintoul Street, Wellington

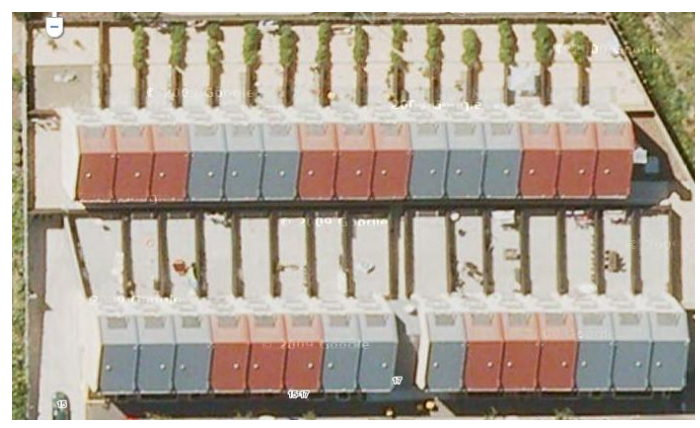

Fig. 1.9 15 Warwick Street, Christchurch 


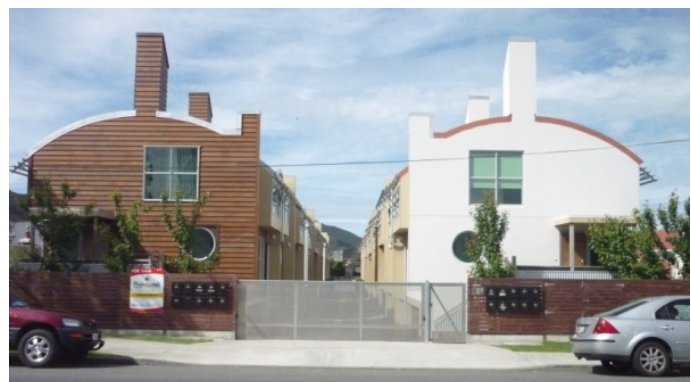

Fig. 1.10a. 10 Park Ave., Lower Hutt (Photo: TradeMe Property) Motorised entry gate.

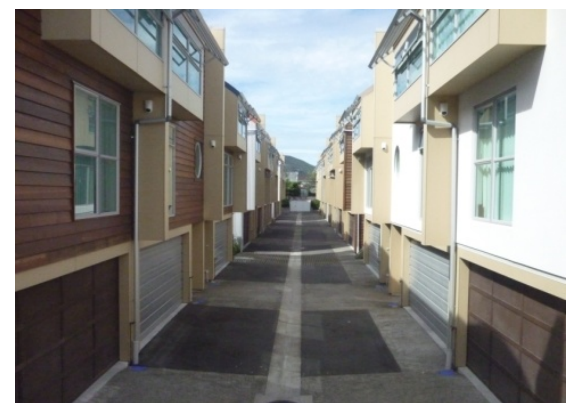

Fig. 1.10b. 10 Park Ave., Lower Hutt (Photo: S. J. Pattinson) Main access.

The housing layout at 10 Park Ave (Fig. 1.10a,b) follows the site layout in Fig. 1.6,

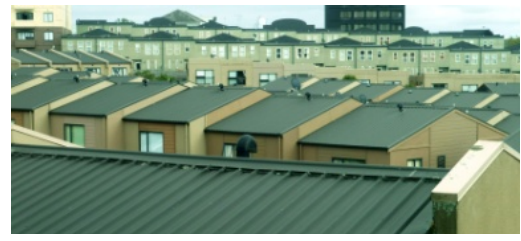

Fig. 1.11 Grafton, Auckland (Photo: S.J.Pattinson) and the shared space from motorised entry gate to 20 remote-controlled garage doors is narrow, linear and univalent, for vehicles.

Fig. 1.11 illustrates a typical medium density housing development in New Zealand - repetitive and linear terrace housing on small individual lots.

Sometimes the linear form is continued around the perimeter of a rectangular residential block, enclosing some shared open space in the centre (e.g. Fig. 3.10).

At Alverna Heights, Whangaparaora, the block shape is triangular. The housing layout on each side of the triangle is linear and repetitive. The open space in the centre of this triangular block is undeveloped and appears un-used (Fig. 1.12).

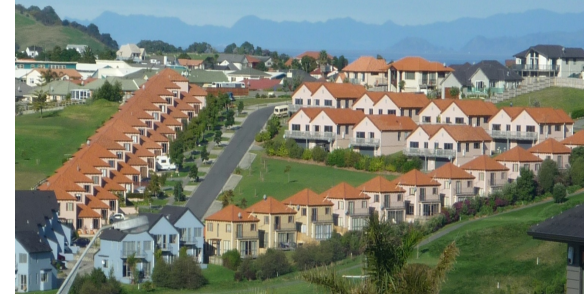

Fig. 1.12 Alverna Heights, Gulf Harbour, It could be shared by the people living around it. Whangaparaora (Photo: S.J.Pattinson) contractors. It is sloping and planted with a few trees, having little use other than as univalent, passive green space. Meanwhile, small private yards around this open space try to accommodate trampolines, swings and basketball hoops for children.

Shared open space (i.e. communal space) was selected for study because of its potential to be multivalent, to support multiple uses, meanings and values. It is hypothesized that multivalent communal space will make more efficient use of limited urban land and enhance the quality of residents' living environments. 


\section{A brief clarification of key terms}

The following briefly clarifies the meanings of key terms as used in this thesis 'medium-density', 'communal space', and 'multivalence'.

\section{What is 'medium density' housing?}

'Medium density' is a relative term indicating a broad range of housing between low density detached housing and high density high rise. Biddulph (2) describes medium density as being anywhere in the range from 30 to 450 dwellings units per hectare (Fig 1.13). In New Zealand, according to Turner et al. (22), medium density housing is at the low end of this range, i.e. at about 30-66 dph (dwellings per hectare).

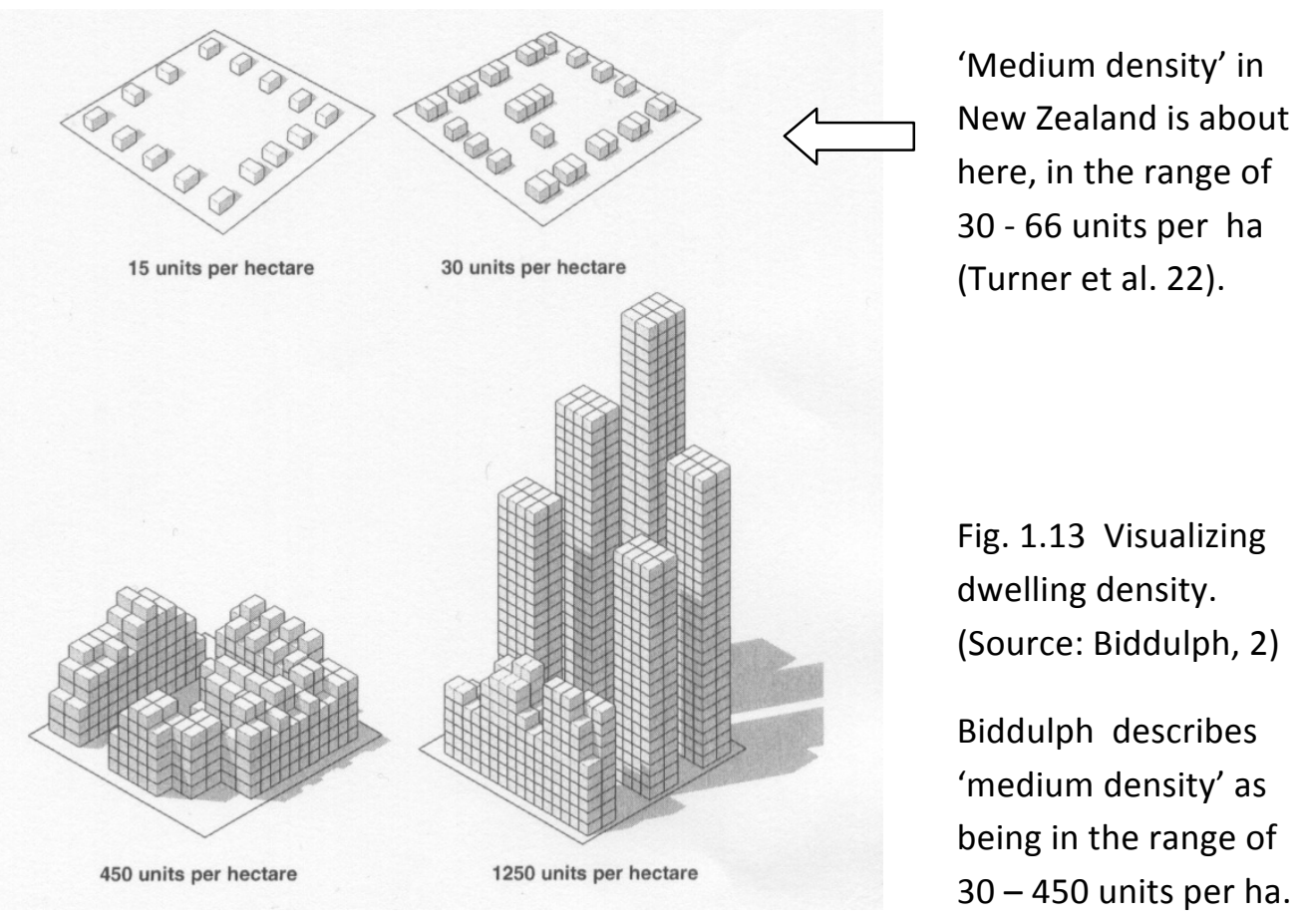

Alexander (191) notes there are many forms of medium density including terrace housing, cluster housing, low-rise walk-up (up to four storeys), and medium-rise apartments (up to about six or eight stories). In New Zealand it is mainly in the form of terrace housing, i.e. typically narrow, two-storey dwellings attached in a row. 


\section{What is communal space?}

David Levitt, in "The Housing Design Handbook: A Guide to Good Practice" describes the key characteristics of communal space:

"There is a difference between true public open space, to which anyone and everyone has access, and shared communal space - such as a central courtyard, garden or pocket park enclosed by terraces of houses or flats - access to which is restricted to certain groups of residents." (110)

'Communal space' is shared by a group of residents. There may be varying degrees of enclosure, and of pedestrian permeability and connectedness to the public realm, but the key point in Levitt's definition is that communal space is for the benefit of 'certain groups of residents'. This definition distinguishes 'communal space' (shared by a certain group) from 'private space' (the realm of the individual) on the one hand, and 'public space' (which is for everyone) on the other.

\section{What is multivalence?}

According to Charles Jencks (14), multivalence is imaginative architectural fusion:

"Certain buildings have a richness and density of meaning which make them more enjoyable to inhabit, view and visit than others.... We return to them again and again because of the exciting and deep way in which the meanings are ... fused together into a powerful pattern.

"For this quality I have adopted the general term multivalence because it points to the presence of multi-valued levels of meaning ... multivalence consists of four distinct qualities:

- imaginative creation, or the putting together of parts in a new way,

- the amount of parts so transformed,

- the linkage between the parts which is the cause of this creation

- and which allows the parts to modify each other"

Janz and Beckley (7) use the term 'multivalence' in urban design to "embrace many values ... [from] the context and the environment (people, objects, events, patterns, etc.). It is concerned with the process as well as the product of design." 
According to Janz and Beckley, univalent design "expresses a single value, a single idea, a single approach ... most often ... the value of the designer", whereas a multivalent approach expresses a synthesis of political, cultural and historical phenomena that are unique to a particular place $(7,8)$.

\section{What is 'multivalent communal space in medium density housing'?}

Multivalent communal space is layered with many uses, meanings and values. The 'in-between' place, the common ground, is enriched, making it more enjoyable to inhabit. Abstract 'space' and 'time' become memorable 'place' and 'occasion'.

A good example is an open-air library in Salbke, Magdeburg, awarded the 2010 European prize for urban public space. It demonstrates creative, integrated use of shared outdoor space which any medium density housing community could emulate, with professional assistance, to shape its own 'best-fit' living environment.

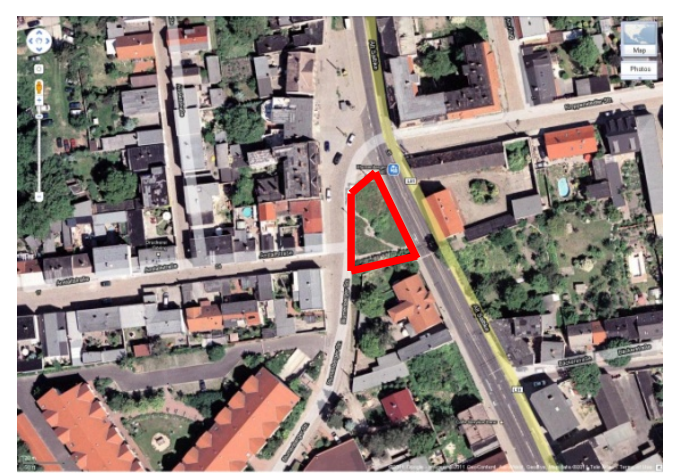

Fig. 1.14 Site of open-air library, Salbke, Magdeburg, Germany (Google map)

The site is described as being in a "difficult district... derelict and shrinking... marked by ...high unemployment [20\%]" (CCCB 55). Many properties are vacant and vandalised by bored youth-gangs. The library on this site burned down in the 1980s.

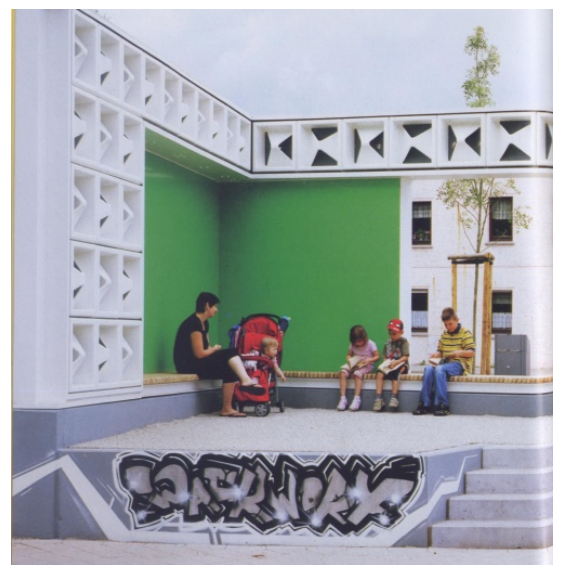
The local community decided to build a new library on the site. In 2005, facilitated by a collaborative of architects, workshops were held and concepts discussed. Full scale models of possible designs were built on site out of beer crates. Local residents donated books $(20,000)$. The final scheme built in 2009 closely follows the first beer-crate model (CCCB 55-57). Fig. 1.15 Open-air library, Magdeburg, in former Eastern Germany (Source: CCCB 58) 


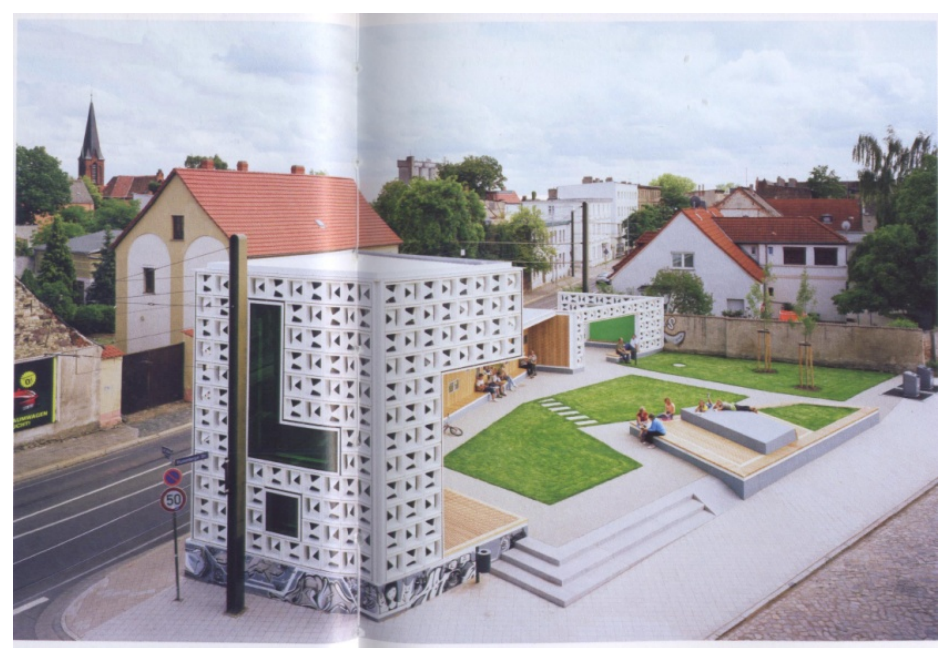

Fig. 1.16 Open-air library, Magdeburg, Germany

24-hr free library, café (upper floor), green open space and covered stage used by local residents including young people for hanging out, youth band concerts, public meetings, primary school plays, book and poetry reading festivals - all managed by residents. (Source: CCCB 56, 57)

The façade panels are recycled from a 1960's chain-store and can "be read as a reuse of a typical Western consumerism symbol for Eastern social action" (CCCB 56).

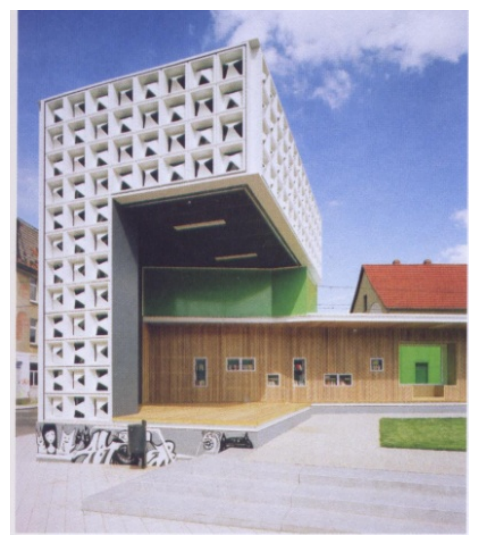

Fig. 1.17 View of stage and café over. (CCCB 57)

Fig. 1.18 Open-air library, street view. (CCCB 58)
"... this project was not generated by a top-down approach that could later easily fall prey to negligence and vandalism - just the opposite. It was the result of a patient social intervention process ... When the first attack of vandalism occurred ... the people were aware of its meaning and defended their space. They organised the repairs and thus propagated a strong signal, that they would not tolerate such actions." (CCCB 55, 56)

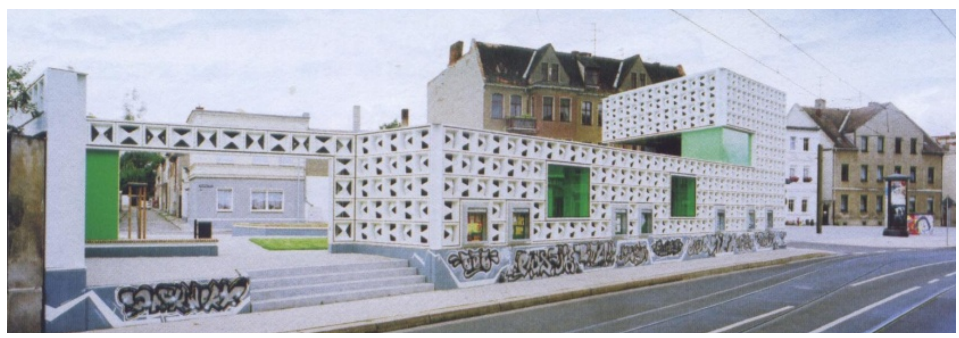

This project synthesizes local politics, culture, history and physical site in new ways, enriching the neighbourhood with many shared values integrated on this 'common ground'. Although a 'public' rather than 'communal' space, this project exemplifies the potential of multivalence for communal space in medium density housing. "... it is ... possible for [communal] space to work as a place of meeting and contact, indispensable for mutual recognition, which is the basis for any form of truly possible coexistence ... given the imperative need for different peoples to learn to live together ..." 


\section{Scope and Limitations}

Although drawing on overseas examples, this research is limited to the study of communal space in the context of medium density housing in New Zealand. The focus of the study is on principles and patterns of multivalence for communal space, rather than on lists of social activities, or on site planning or landscaping details for site works. Nor does it address building and construction issues in medium density housing such as sound control or fire resistance for inter-tenancy walls or floors. Information on these technical issues can be found elsewhere.

This research does not address cultural perspectives on the uses and meanings of communal space. One recent study in this area is an investigation by John Gray of the possible meshing of Polynesian and European settlement patterns as a way of intensifying the suburbs for more sustainable suburban forms. Gray's study (2010) compares cultural differences and speculates on the benefits of mixing cultural perspectives for more sustainable suburban patterns. 


\section{A brief overview of this thesis}

This thesis argues that multivalent communal space will make more efficient use of urban land and improve the quality of living environments for residents compared with current observations of typical medium density housing in New Zealand. The aim of the research is to find principles for making communal space multivalent.

Chapter 2 describes the research methodology to achieve the research aim. The research begins by looking at current practice from the perspectives of practitioners (Chapter 3) and academic researchers (Chapter 4). Emerging from current best practice and theory, principles for achieving multivalent communal space in medium density housing are proposed (Chapter 5).

These principles are further explored in a case study of terrace housing at Pinehill Park (Chapter 6) which investigates community perspectives through the uses, attitudes, meanings and values which the terrace house residents associate with the park as shared neighbourhood space.

Chapter 7 draws together the research findings from the viewpoints of practitioners, academics and the community in a discussion of the proposed principles of multivalent communal space and applies these principles to a medium density subdivision to investigate the effectiveness of these principles for making more efficient use of urban land and enhancing the living environment for residents.

Chapter 8 presents the conclusion of this research and its general applicability.

The Appendices include an investigation of floor space related to household size (App. 1), Case Study data for Pinehill Park housing (Apps. 2 -6), a list of the 227 housing sites visited (App. 7.1), including field notes (App. 7.2) and first impressions (App. 7.3), and an analytical framework and detailed analysis of communal space in several medium density housing developments in the Wellington region (App. 8). 


\section{CHAPTER 2 - Research Design}

Chapter 1 introduced the research problem and aim which is to find principles for multivalent communal space in medium density housing. When applied, these principles are to make more efficient use of urban land and enhance the living environment for residents. This chapter explains the methodology for carrying out the research aim, beginning with some preliminary comments on:

- the writer's world view, focusing on quality living environments for people;

- becoming more collaborative in practice, involving the community in a more participatory approach to shape its own 'best-fit' living environments, and

- making strategic incremental steps towards these goals.

The writer will then discuss the research design, outlining the research strategies (i.e. methods), explaining why they have been selected, and noting the tactics (techniques) that will be used for collecting data for this research.

\section{People and their environment matter: one shapes the other}

Hutia te rito o te harakeke

Kei hea te kōmako e kō

Ki mai ki ahau

He aha te mea nui i te ao

Maku e ki atu

He tangata, he tangata, he tangata.

If the center shoot of the flax is pulled out (and the flax dies)

Where will the bellbird sing

If you were to ask me

What is the most important thing in the world?

I would reply

It is a person, a person, a person

(that is, each and every person).

(http://maaori.com/misc/raranga.htm)

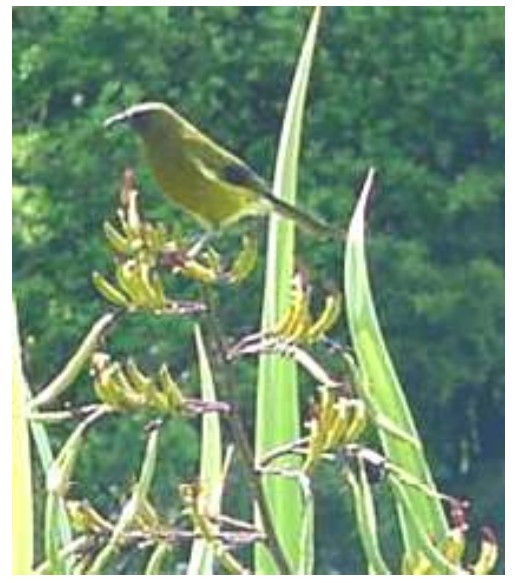

Fig. 2.1 The bellbird (te kōmako) and flax plant (te harakeke)

http://www.nzbirds.com/birds/k orimako.html 
"[The above] whakatauki [Māori proverb] ... is quoted often in speeches and whaikōrero to emphasize the mana and tapu of humankind. It also acknowledges human fragility, interdependence and dependence on other parts of the creation: if you pluck out the heart of the flax bush where would the bellbird sing?"

Source: http://christchurchcitylibraries.com/TiKoukaWhenua/JanetStewart/

This whakatauki is rich with imagery. One meaning it could have which is relevant to this research is to liken the harakeke to medium density housing environments. Homes are woven together in a way that nurtures shared or communal space at the heart, the communal space in turn nurturing a sense of community within the local neighbourhood. Without shared space, where will the social interaction occur that builds a sense of community in a compact urban neighbourhood?

Both the people and their living environment matter. As Edward T. Hall points out, the one shapes the other (106). The principles for achieving multivalent communal space will be attractive to caring communities who have regard both for the quality of their living environment and for the role it plays in nurturing social interaction and a sense of community.

This research is based on the premise that people and the quality of their collective living environments matter. Clare Cooper Marcus \& Wendy Sarkissian (1986) in “Housing As If Peopled Mattered: Site Design Guidelines for the Planning of Medium-Density Family Housing" exemplify this viewpoint that people and their living environments matter. In her later book with Carolyn Francis titled "People Places" (1998), Clare Cooper Marcus comments on creating spatial environments that reflect caring about people:

"She [Carolyn] and I believe strongly in our field of study [social and psychological factors in the design of people places] and the importance of considering the human use of designed spaces. First, we have assumed that the providers and designers of the kinds of spaces we describe in this book care about people and want to create places that are socially appropriate ..." (viii) （Writer's emphasis) 
The built environment can express caring (or not) about people. Robert Sommer (2007) asserts that the built environment communicates this care (or lack of it) to the people who live in or use it. He comments that there is much in the built environment that expresses a lack of caring about people, and that this has repercussions on the type of communities that develop in uncaring environments.

Through the quality of the built environment, designers and developers can demonstrate care about people, recognising that the environment contributes to human well-being and can facilitate the development of caring communities. It is incumbent on designers to care about people and think carefully about the impact the environments they create may have on human and community well-being.

\section{Research Questions}

The aim of this research is to develop principles of multivalent communal space in medium density residential environments to make more efficient use of urban land and enhance the living environment for residents. The research questions are:

\section{Research Questions}

1. "What is the nature of communal space in existing medium density housing developments in New Zealand?"

2. "What is the potential multivalence of communal space in medium density housing?"

3. "What are the principles for achieving multivalent communal space in medium density housing?"

4. "Will the application of these principles for multivalent communal space result in more efficient use of urban land and enhance the living environment for residents?" 


\section{Research design:}

The intention is to find principles for achieving multivalent communal space in medium density housing from current practice (Chapter 3 ) and from a review of academic literature (Chapter 4). Chapter 5 draws from Chapters 3 and 4 to propose principles from multivalent communal space in medium density housing in New Zealand. These principles will be further explored in the Pinehill Park case study (Chapter 6), discussed from various viewpoints and applied to the overall Pinehill subdivision in Chapter 7. Chapter 8 discusses the general applicability of the conclusions of the research.

According to Hillier and Rooksby (3-6), Pierre Bourdieu challenges the traditional assumption that practice is the application of academic theory. The writer explicitly declares the influence of architectural design theory regarding the 'bay form' on the writer's perspective in this research. The reader will be introduced to architectural design theory on the 'bay form' and how it relates to communal space in Chapter 3. It is sufficient to say at this point that the writer regards the 'bay' and 'courtyard' as natural architectural forms for expressing and supporting 'community'. This declaration of the writers' perspective follows Bourdieu on the point of 'reflexive' research. Webster, quoting Bourdieu, explains reflexive research as:

"investigation in which the researcher is aware of their contribution to the construction of meanings throughout the research process", a process in which "researchers ... explicitly recognise (or declare) ... their perspectives on the objects of their research" (Webster 6, 102)

The following diagram (Fig 2.2) expresses an ideal which will not be fully implemented in this research, but towards which some first steps are made. This research tries to avoid a completely linear academic-theory-to-practice approach and investigates multivalent communal space dialectically from three different perspectives - practitioners (primarily expressed in current practice - see Chapter 
3), academics (from the literature of various disciplines - see Chapters 4 and 5) and the community (represented in a case study by the terrace house residents at Pinehill Park, Upper Hutt, Wellington - see Chapter 6). From these three perspectives, the writer will attempt to meet the aim of this research which is to develop principles for achieving multivalence in communal space in medium density housing. The principles for achieving multivalence will primarily be derived from current theory and practice, and from the academic literature. These principles will then be tested for their validity in the case study, which will allow the writer to explore an example of communal space in its natural setting, that is, in the everyday environment of an existing medium density housing development.

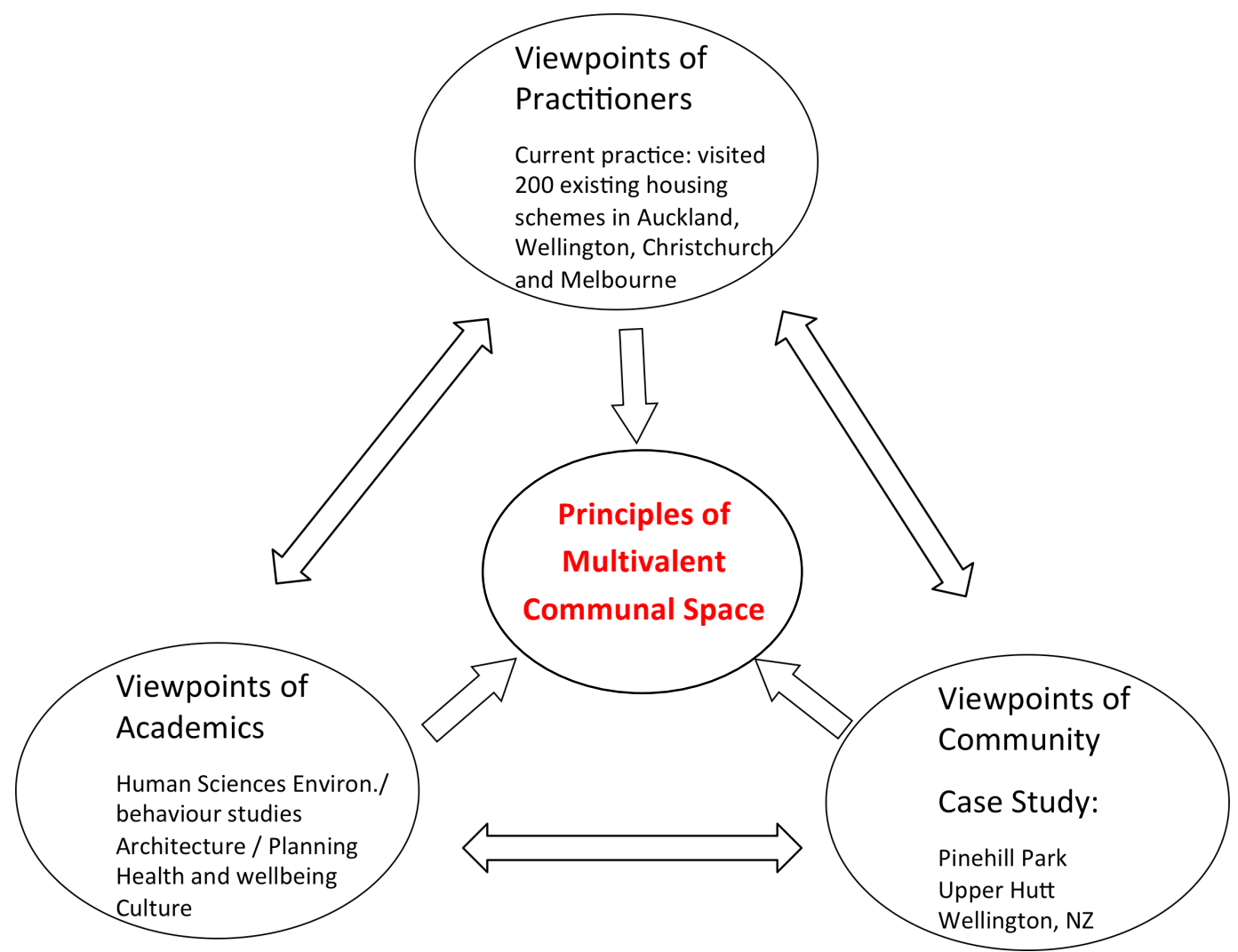

Fig. 2.2 A model for a participatory approach, including the viewpoints of practitioners, academics and community in the development of theory and practice. (S.J. Pattinson) 


\section{Investigating practitioners' viewpoints through current practice}

The following outlines the scope of activities carried out by the writer to become familiar with past and current practice regarding communal space in medium density housing (salient issues from these sources are discussed in Chapter 3):-

- reviewed District Plans for Auckland, Wellington and Christchurch;

- reviewed the Urban Growth Strategy for the Auckland region;

- met with Council planners/urban designers at Rodney, North Shore, Auckland, Manukau, Waitakere, Papakura, Wellington, Porirua, Upper Hutt and Christchurch (August - November 2009);

- reviewed the "Report and Recommendations of the Urban Taskforce 2009" on urban intensification commissioned by the Minister for Building and Construction, NZ Government;

- reviewed NZ Ministry for the Environment Case Studies:-

- Chester Courts, Auckland (draft Case Study)

- The Altair, Wellington (draft Case Study)

- Stonefields, Christchurch (draft Case Study)

- Beaumont Quarter, Auckland

- Sacamento, Botany Downs, Auckland

○ Eastpark, Botany Downs, Auckland

- Harbourview, Waitakere, Auckland

- Lancewood Courts, Christchurch

- Northwood Residential Area, Christchurch

- Terraced Housing (cnr Hurstmere Rd \& The Promenade) Takapuna

- Talbot Park, Glen Innes, Auckland, and

- Addison Housing, Papakura, Auckland;

- reviewed six case studies in 'Good Solutions Guide for Apartments' (Q City; Beaumont Quarter; ACC Housing Freemans Park; Scene One Apartments; Trinity Apartments; Waitakere Gardens); 
- reviewed four case studies in 'Good Solutions Guide for Mixed Use Development in Town Centres (Hemisphere Apartments; The Lofts; Parnell Road Apartments; 2 Queens Parade, Devonport);

- reviewed the UNITEC report "Best Practice in Medium Density Housing Design: for Housing New Zealand Corporation" by Turner et al. (2004), and the follow-up report by Turner and Su (2005);

- reviewed judging criteria and entries in Housing New Zealand Corporation's 2005 “Design competition for state housing - Housing New Zealanders 100 Years On" for a site at Aurora Crescent, Sandringham, Auckland, regarding the requirement "for public space - streets or green, shared, open spaces";

- $\quad$ reviewed specific Design Guides - AMCORD (1997); Essex Design Guide (2005), HNZC Design Guides (Urban; Site; Architecture; Maori Housing Solutions; Pacific Housing); Good Solutions Guides (Medium Density Housing 2007, Apartments 2001, Mixed Use Town Centres 2005);

- reviewed books on medium density housing practice by Judd and Dean (1983), Hermanuz et al. (1988), Colquhoun and Fauset (1991a, 1991b), Polyzoides et al (1992), Judd (1993), Jingmin Zhou (2005), French (2006), Forster (2006), Mozas and Per (2006), Crosbie (2003), Crosbie (2007), London and Anderson (2008), Murray et al (2008), Levitt (2010);

- reviewed general design guides by Greater London Council (1965), Alexander et al. (1977), Lynch and Hack (1984), Biddulph (2007);

- reviewed street design - A. Jacobs (1993), Crawford (2002), Southworth and Ben-Joseph (2003);

- reviewed various planning, urban, landscaping and architectural design journals and magazines

- attended a symposium on medium density housing in Melbourne titled "Beyond Greenfield Development and the Expanding Metropolis Housing Symposium" (Office of the Victoria Government Architect, 7 Sept 2009); 
- carried out preliminary field trips to 227 medium density developments (August 2009 - July 2010) in the following main centres to view communal space first hand (see Appendix 7 for a full list, field notes and impressions):

$\begin{array}{lr}\text { Auckland (region), New Zealand } & 114 \text { sites } \\ \text { Wellington, New Zealand } & 47 \text { sites } \\ \text { Christchurch, New Zealand } & 54 \text { sites } \\ \text { Melbourne, Australia } & 12 \text { sites }\end{array}$

From the above investigations, the writer discovered little evidence to date of practitioners considering communal space a priority in medium density housing.

\section{Exploring academics' viewpoints in the literature review}

The following outlines the scope of a literature search in various disciplines carried out by the writer to become familiar with the views and theories of academic researchers regarding communal space in medium density housing (salient issues from these sources are discussed in Chapters 4 and 5):-

- reviewed books regarding theory and/or research on medium/high density housing by Chermayeff and Alexander (1965), Newman (1973) Unterman and Small (1977), Hester (1975, 1984), Hatch (1984), Cooper Marcus and Sarkissian (1986), Davis (1995), Cooper Marcus and Francis (1998);

- reviewed urban planning/design - Jacobs (1961), Alexander et al. (1987), Calthorpe (1993), Duany et al. (2000), Calthorpe and Fulton (2001), Tibbalds (2001), NZ Ministry for the Environment (2002), Carmona et al. (2003), Mehrotra (2005), Fishman (2005), Strickland (2005), Thomas (2006), Bentley et al. (2007), Hinshaw (2007), Krier (2009);

- reviewed social and community planning - Hillier and Hanson (1984), Lozano (1990), McCammant and Durrett (1994), Hall and Ward (1998), Harriot and Matthews (1998), Walters (2007). 
- reviewed urban /social space and place - Gehl (1977), Whyte (2004), Dovey et al. (1985), Trancik (1986), Hiss (1990), Gehl (1996), Ford (2000), Madanipour (2003), Di Palma et al. (2009), CCCB (2010);

- $\quad$ reviewed 'placemaking' - Walljasper (2007); Hamdi (2010);

\section{Exploring community viewpoints through Pinehill Park Case Study}

The reasons for selecting Pinehill Park in Trentham, Upper Hutt, for the case study are discussed in Chapter 6. A qualitative approach will be used to explore the case study in depth using observations, a survey and interviews. The survey and interview questions will be open-ended to give respondents the opportunity to contribute their viewpoints. Insights gained from residents' viewpoints in the case study may have applicability to medium density housing generally, and this will be discussed in Chapter 8.

\section{Collaboration between practitioners, researchers and the community:}

Collaboration between practitioners, researchers and the community could assist in the creation of a more caring built environment. An example of collaboration between practitioners, researchers and the community from modern medical research illustrates the potential benefits of such collaborative research. The example is reported by Richard Faull, distinguished New Zealand brain researcher, regarding break-through discoveries being made at the Centre for Brain Research (October, 2010). Faull comments that research at the Centre has proven that the adult brain contains stem cells, and indications are strong that, given a stimulating environment, exercise, and exciting things to do, the adult brain can create new brain cells. Faull acknowledges the contribution of many collaborators in medical research and practice and the participation of the community (families of patients) in this break-through. Such exemplary collaboration could be emulated in the building industry to make housing shemes "stimulating environments" to live in. 
By contrast, Graeme McIndoe (urban design practitioner) in a lecture, 2010, Victoria University of Wellington School of Architecture attributed the "abject failure" of modernist town planning to the lack of professional collaboration. He pointed out that the authors of the 'Athens Charter' published in 1943 (an influential document in the shaping of modern cities) never consulted with any urban geographers, sociologists, environmental psychologists, or city mayors - people who might have known more about how cities actually work. The charter was formulated by artistplanner-architect idealists who, McIndoe suggests, were out of touch with reality.

Katharina Borsi (138-141) describes the Berlin urban residential block as being developed through much professional interdisciplinary collaboration ...

"The 'Familian Haus' (family house) of 1823... [was] the focus of intense discussion among health experts, municipal councillors, and both the municipal and building police ... [over] detailed accounts of its individual elements, its structure, its materials, the size of its plot, the volume of air inside the building and in its courtyards, its relation to the space of the street and its connectivity to the city....

The significance of these debates lay ...[in the] diversity of disciplines that coalesced around the building ... [examining] issues ranging from structural integrity and construction techniques to hygiene and the safety of the city beyond the block itself, from the grouping of individuals within the block to its position on the site, and from inhabitants' conduct ... to the block's implications for the urban economy .... [bringing] together economists, doctors, sanitary experts, engineers, architects and municipal leaders."

According to Borsi (2009) the Berlin block survives today as an archetypal model of good urban design. McIndoe (urban design lecture, 2010) comments that cities today are too big, too multi-faceted, and their maladies too complex, for any one discipline to resolve alone. The New Zealand Centre for Sustainable Cities is an example of emerging multi-disciplinary collaboration on urban environments. Participants are from a range of disciplines and practices, fostering holistic enquiry into human well-being in New Zealand's urban environments. 
In this research, preliminary field trips to existing medium density housing developments, and discussions with practitioners (town planners, urban and landscape designers, architects, developers, and builders will inform the writer on current practice (Chapter 3). Other disciplinary perspectives (e.g. sociology, environmental psychology/environment-behaviour studies, etc) will be investigated (Chapter 4). In the Case Study (Chapter 6), residents of the terrace housing at Pinehill Park will be surveyed for their viewpoints on communal space and their responses will be analysed for correlation with proposed principles for multivalence.

\begin{tabular}{|c|c|c|c|}
\hline \multicolumn{4}{|c|}{ Chapter 6} \\
\hline \multicolumn{4}{|c|}{ Pinehill Park terrace housing as a Case Study } \\
\hline \multicolumn{4}{|c|}{ (See Evans and Gruba, 94, Figure 4, for a case study outline on which this is based) } \\
\hline Title & \multicolumn{3}{|c|}{ Communal space at Pinehill Park } \\
\hline Aim & \multicolumn{3}{|c|}{$\begin{array}{l}\text { To test tentative principles of multivalence for communal space (the } \\
\text { public park) used by residents of the adjoining terrace housing }\end{array}$} \\
\hline Background & \multicolumn{3}{|c|}{$\begin{array}{l}\text { Provide a brief history of the development and a detailed } \\
\text { description of the terrace housing and park in context }\end{array}$} \\
\hline \multicolumn{2}{|c|}{ Research Questions } & Methods & Tactics \\
\hline \multicolumn{2}{|c|}{$\begin{array}{l}\text { 1. What is the nature, } \\
\text { quality and use of existing } \\
\text { communal space }\end{array}$} & \multirow{3}{*}{ Case Study (Chapter 6) } & $\begin{array}{l}\text { Description of site and } \\
\text { existing communal space } \\
\text { in historical and physical, }\end{array}$ \\
\hline \multicolumn{2}{|c|}{$\begin{array}{l}\text { 2. What is the potential } \\
\text { multivalence of communal } \\
\text { space in this setting }\end{array}$} & & $\begin{array}{l}\text { existing use of communal } \\
\text { space; neighbourhood } \\
\text { survey }\end{array}$ \\
\hline \multicolumn{2}{|c|}{$\begin{array}{l}\text { 3. What are the principles } \\
\text { for achieving multivalent } \\
\text { communal space }\end{array}$} & & $\begin{array}{l}\text { User interviews to obtain } \\
\text { residents' viewpoints }\end{array}$ \\
\hline \multicolumn{2}{|c|}{$\begin{array}{l}\text { 4. Will the application of } \\
\text { these principles make } \\
\text { more efficient use of land } \\
\text { and enhance residents' } \\
\text { living environment? }\end{array}$} & See Chapter 7 & \\
\hline
\end{tabular}

Table 2.1 Case Study Design

(S.J. Pattinson) 
Initially, grounded theory was considered for this research but was rejected in favour of the case study approach. The reason for considering grounded theory was because it allows the flexibility of both inductive and deductive reasoning (Groat and Wang 182 ). The writer did not want to be constrained by a choice between objective or subjective approaches, nor between deductive (general to particular) or inductive (particular to general) reasoning. Grounded theory is a subjective approach, unashamedly admitting the researcher's interpretation of what is observed. But it also claims to not bring any pre-conceived ideas, perspectives or theories to the research task (Groat and Wang 180-181). This seems a difficult stance to assume, which is why grounded theory has not been used in this research.

Groat and Wang discuss how both deductive and inductive reasoning can be employed in a research project in a cyclic fashion. This seems appropriate to the interdisciplinary nature of this research topic. At times, deductive reasoning is helpful, and at times inductive reasoning is equally as helpful. In fact, Groat and Wang comment that "...there is a fair amount of philosophical literature that addresses just how complex deduction/induction is, to wit, that we cannot have one without the other" (317). Similarly, both objective and subjective viewpoints can complement each other in a cyclic process of discovery. Therefore the research approach adopted here allows for exploration to occur in a cyclic fashion from a range of viewpoints, rather than within a linear dichotomy.

The suggestion of combining deductive and inductive approaches was endorsed by Anne Galloway and Robin Skinner at a colloquium on research design ("Research-led Design; Design-led Research" Faculty of Architecture and Design, Victoria University Wellington, 19 October, 2010). The case study approach, using qualitative survey and interview techniques, provides this flexibility. 


\section{Strategic, incremental innovation:}

Creating caring built environments for people requires commitment and determination. Commercial realities and political power imbalances are not easily overcome (Hamdi, 2010). Naïve idealism and good intentions are not enough where the economic organisation of cities prevails over social well-being.

Some have found a positive way forward through strategic, incremental interventions. Diane Brand (Head of School of Architecture, Victoria University of Wellington, Lecture 25 May 2009) sees incremental interventions in places like a favela of Rio de Janeiro as being on the cutting edge of international urban design. Brand explains that incremental interventions can be simple (a new concrete footpath or soccer field in a Brazillian favela) but community-enhancing.

Sommer relates how as an incrementalist he is content with small, progressive improvements in the built environment. Sommer relates how efforts that began 30 years ago in his home city of Davis, California, with safe cycle ways and then other incremental innovations (rubbish recycling, better public transport, solar water heating, solar power) have resulted in Davis now being a model 'green' city (203-5).

Hamdi (2001) is an example of an architectural incrementalist who works collaboratively with the community. Hamdi describes the architect's role as one of enabling communities to find their own 'best fit' for their living environments. Hamdi advocates community participation in 'placemaking' to create their own environments, rather than having the built environment 'done to them' by design professionals and the construction industry. For Hamdi, an architect, strategic incremental intervention is about architects providing background frameworks and supports rather than foreground objects. 'Architecture-as-object' means the spaces in between the buildings often get overlooked. This research focuses on communal in-between spaces and their potential multivalence for enhancing local community. 


\section{An Analytical Framework for exploring communal space:}

An analytical framework for exploring quantatative data on communal space is included in Appendix 8. Refer to Appendix 8 for the following:

1. An analytical framework for quantatively researching communal space in medium density housing;

2. Metrics - private and communal outdoor space per person;

3. A trend towards a qualitative approach.

Appendix 8 uses outdoor space metrics related to numbers of bedspaces to investigate communal spaces in the following medium density housing developments in the Wellington region:

1. Existing detached housing on $400 \mathrm{~m}^{2}$ lots at Pinehill Crescent, Upper Hutt;

2. Detached housing on hypothetical $300 \mathrm{~m}^{2}$ lots, Pinehill Crescent, Upper Hutt;

3. Existing terrace housing at Pinehill Park, Upper Hutt;

4. Pinehill Crescent - hypothetical concept with clustered housing and multivalent communal open spaces;

5. "The Boundary" townhousing in Karori, Wellington;

6. "Maidstone Quarter", as yet unbuilt proposal in Blenheim Street, Upper Hutt;

7. "The Altair", townhousing at Rintoul Street, Newtown, Wellington;

8. "Monterey" townhousing at 232 Middleton Road, Glenside, Wellington;

9. 10 Park Avenue, Lower Hutt - townhousing.

Appendix 8 measures and compares quantatative data on communal spaces in the above developments, as a starting point to understanding communal space. It notes, however, a trend towards a qualitative approach to describing and providing open space in medium density housing, which is the approach taken in this thesis research toward investigating and understanding communal space. 


\section{CHAPTER 3 - Current practice}

Question 1: What is the nature of communal space in existing medium density housing in New Zealand?

Creative communal spaces can be found in New Zealand's medium density housing. Preliminary investigations, however, find wide variability in quality, with much communal space being univalent.

Given the complexities of dealing with dense living environments, the default position for practitioners is generally to reduce housing programmes into systems of circulation, housing and open space, and to resolve the housing using standard typologies such as terrace houses, apartment buildings and high-rise towers. These standard 'solutions' oversimplify dense living environments by reducing them to three main issues: convenient vehicle access (circulation), privacy (housing), and landscaping (open space).

Within this context, communal space receives little consideration and tends to be univalent. In many developments its sole purpose is for moving and parking vehicles. In some developments, communal space is included in the landscaping programme but it is still univalent, functioning primarily as 'landscaped outlook'. This chapter investigates these issues, to bring to light the nature of communal space in existing medium density developments, and the views of practitioners.

"One of [our] aims ... is to create housing that fosters the development of caring communities. The main catalyst for a caring community is a "community initiator" someone who can get people together for things like street $B-B-Q s$, mentoring schemes or hobby groups to help develop a sense of neighbourhood community.

"The architecture plays a part in the creation of caring communities. The architecture can help or hinder social interaction between residents. We try to create housing developments where neighbourly interaction can happen easily." 
Southpark is currently developing Kensington Park, Orewa, with spacious communal facilities to foster a sense of community. Other quality intensive housing schemes are identified in Auckland's Regional Growth Strategy (RGS):

"There are some great examples of quality residential intensification, for example, Beaumont Quarter (Victoria Park), the Parc (Viaduct), Harbour View Estate (Te Atatu), Addison (Takinini), Talbot Park (Glen Innes), and in Takapuna, Parnell, Ponsonby and Newmarket". http://www.arc.govt.nz/auckland/aucklands-growth/residential-intensification.cfm

A common characteristic of the examples mentioned above is that they all comprise a variety of designs, which seems to contribute to their appeal:
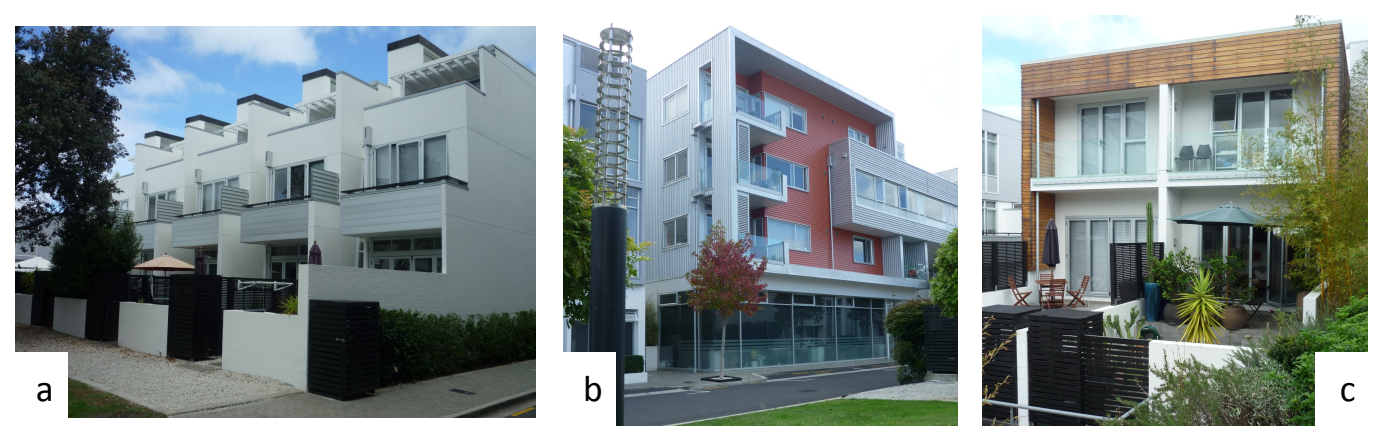

Figures 3.1 a,b,c - Beaumont Quarter, Auckland: Design variety. (Photos: S.J.Pattinson)

Another common characteristic of the above-mentioned "great examples of quality residential intensification" is quality communal space, e.g.:

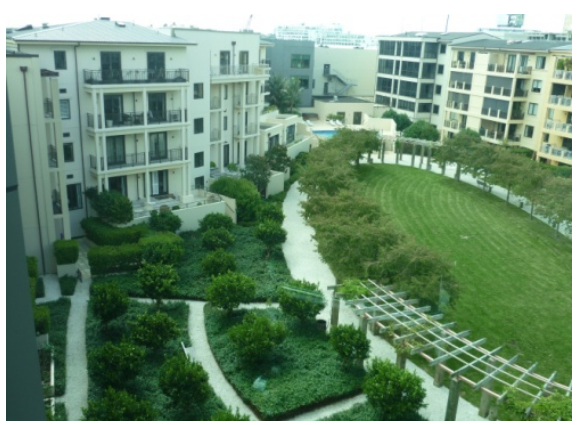

Fig. 3.2 The Parc, Viaduct Harbour

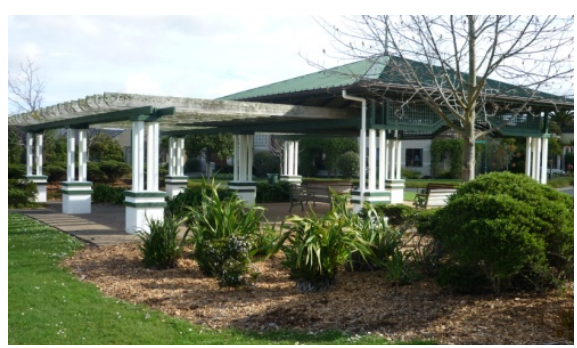

Fig. 3.4 Public seating, Harbourview, Waitakere (All photos: S.J. Pattinson)

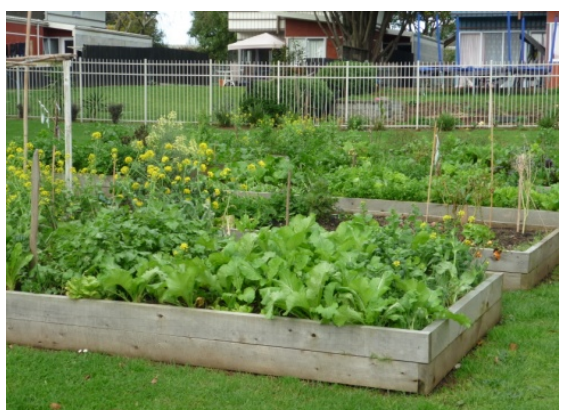

Fig. 3.3 Communal gardens, Talbot Park

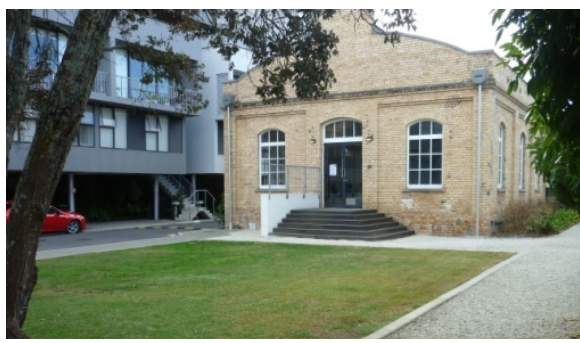

Fig. 3.5 Indoor pool and fitness gym, Beaumont Quarter, Auckland 
Urban Design Case Studies NZ Ministry for the Environment (MfE)

Not all the MfE case study housing developments have communal spaces. The following discusses the character and quality of such spaces in those which do.

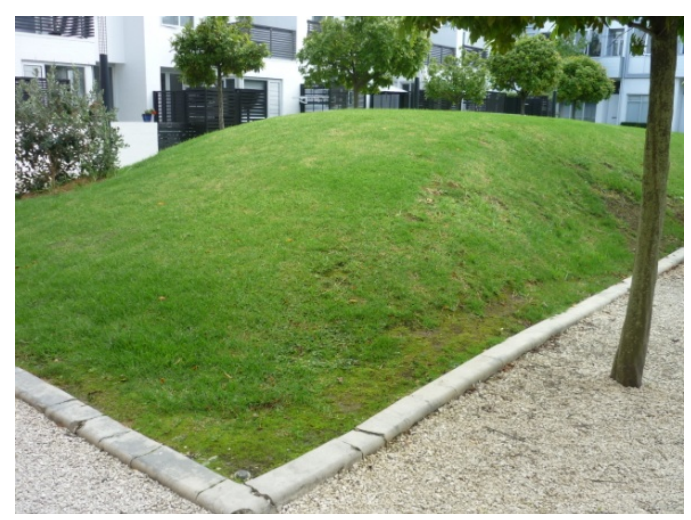

The Beaumont Quarter case study mentions bunded [humped] lawns. These humps might not be ideal for children to "kick a ball around" (16), but the case study describes them as "lovely outdoor areas ... [g]reat for children of all ages" (17).

Fig. 3.6 Beaumont Quarter - Raised mounds: 'challenging the assumption that public open space [is] where you can kick a ball around' (Urban Design Case Studies, 16). (Photo: S.J. Pattinson)

The Lancewood Courts case study describes these 11 units as catering "for the needs of some marginalised people ... with a mix of disabilities, both emotional and physical" (57).

The complex provides " $a$ healing environment through architecture, design, landscaping and a sense of community ... A common open space of $619 \mathrm{~m}^{2}$ at the rear of the site faces north and provides ... a sunny, enclosed court and garden ...

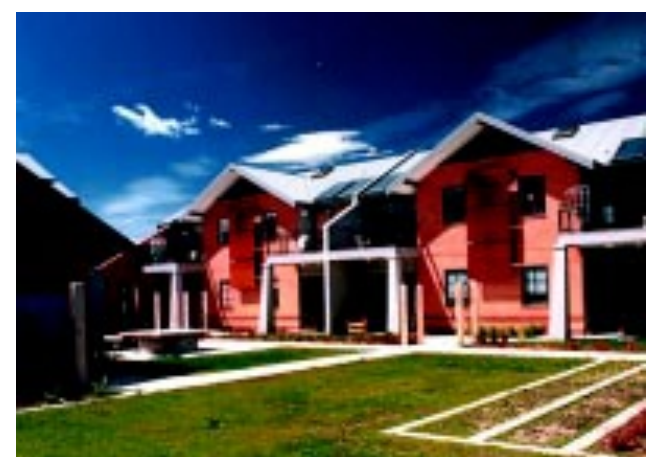

Fig. 3.7 Lancewood Courts communal outdoor area (MfE Case Studies, 56) The shared open space facilitates socialising to encourage mutual support. The oversized stair access becomes a meeting space, and the letterbox is a deliberately accented meeting spot, not just an after-thought" $(57,58)$.

There may be lessons here for medium density design generally. Other intensive living environments, not just those for housing disabled and marginalised people, might be enhanced by these kind of design considerations. 
The Takapuna Terrace Housing (MfE, 2005) is on the northern edge of the Takapuna town centre. The case study comments that:

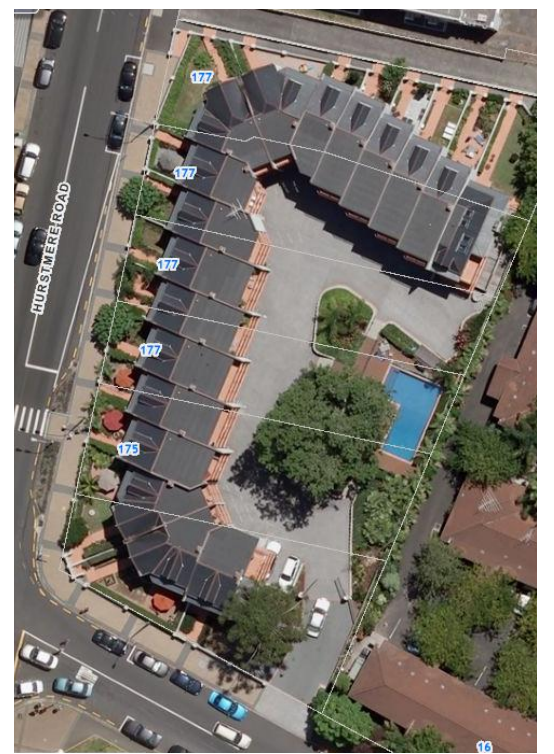

Fig. 3.8 Takapuna Terrace Housing (Source: Auckland Council GIS viewer)
"Regional policy initiatives to deal with population growth encourage intensification close to new or existing town centres ... This development provides a good example of how these initiatives can be implemented... The development forms a perimeter block with the units facing out to the ...streets on three sides.

"Each unit has a double aspect, facing both the street and the inner communal courtyard. The arrangement of the units around the site perimeter provides a good relationship to the street while also allowing for generous communal open space within the site ... retaining three large existing trees ... [and a central barbeque area and swimming pool] ..."

The develoment forms a perimeter block, with communal open space and access to internal garaging sharing the central courtyard. The case study notes that this form of development (cars and communal facilities sharing the central open space of a perimeter block) is not common in New Zealand.

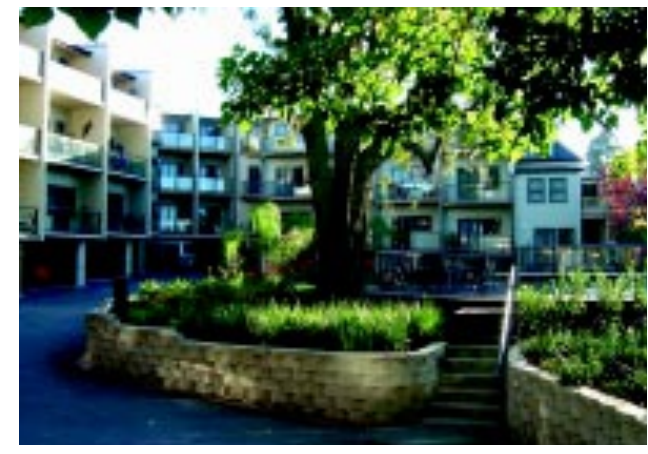

Fig. 3.9 Communal courtyard. Takapuna Terrace Housing (MfE Urban Design Case Studies, 84)

The case study describes this perimeter block form at Takapuna (where cars and communal facilities share the central open space) as very successful, noting the overall high quality of the design. It notes that the development is "generally well regarded ... for its contribution to the Takapuna town centre", that marketing went "exceptionally well" and that the developer was "very happy with the outcome ... in terms of both the built form and fiscal return" $(88,89)$. 


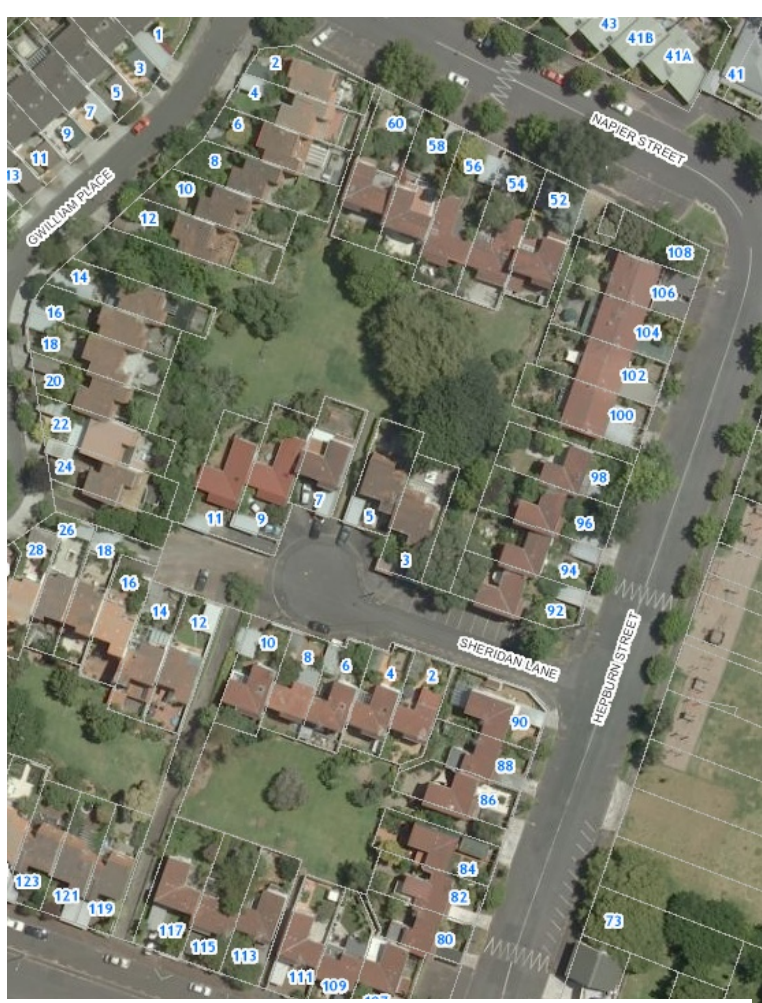

Fig. 3.10 Communal greens at the centre of terrace house perimeter blocks, Sheridan Lane Freemans Bay, Auckland (Council GIS viewer)
A different version of perimeter block form, with green open space in the centre and cars on perimeter ring roads, was visited by the writer at Sheridan Lane, Freemans Bay, Auckland (see Fig. 3.10). The greens are publicly accessible via pedestrian ways from the surrounding streets. The day the writer visited, a mother and her primary-school aged daughter [who do not live in this area but had heard about this 'park' from a friend] were preparing a 'treasure hunt' for the girl's birthday party the next day.

A resident of the terrace housing at

Sheridan Lane commented to the writer that this green space is very rarely used. Instead, the local residents tend to meet and interact with their neighbours on the street side of their houses, for example, in the cul-de-sac of Sheridan Lane.

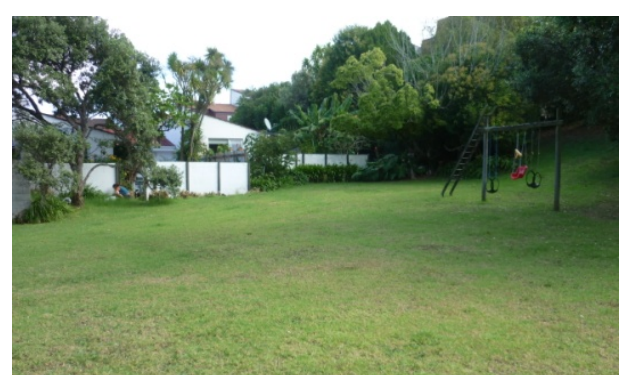

Fig. 3.11 Communal green rarely used. Sheridan Lane Freemans Bay, Auckland (Photo: S.J. Pattinson)

The Takapuna terrace house development mentioned above differs from Sheridan Lane in that residents' cars at the Takapuna scheme are garaged not on the street side but on the central courtyard side. The arrangement at Takapuna of mixing cars and people in the central courtyard may account for the success of the central communal space at Takapuna compared with the usually deserted, Council-owned and maintained 'green commons' in Freemans Bay. 
But mixing cars and people in the central courtyard of a perimeter block does not automatically guarantee the success of the courtyard space, nor the social and financial success of this form of housing development. The success of the Takapuna perimeter block is attributed in the MfE case study to the quality of the overall design (89), including "generous communal open space" (85).

An example of the likely outcome for this perimeter block form when overall design quality is not a priority can be seen in the terrace housing at Krisley Court, Ambrico Place, New Lynn, Auckland. Like the Takapuna scheme, cars access the central courtyard space. But a case study of Krisley Court by Turner et al. (73) describes its central courtyard as "congested" and "lacking any significant pedestrian domain". The authors comment (73) that ...

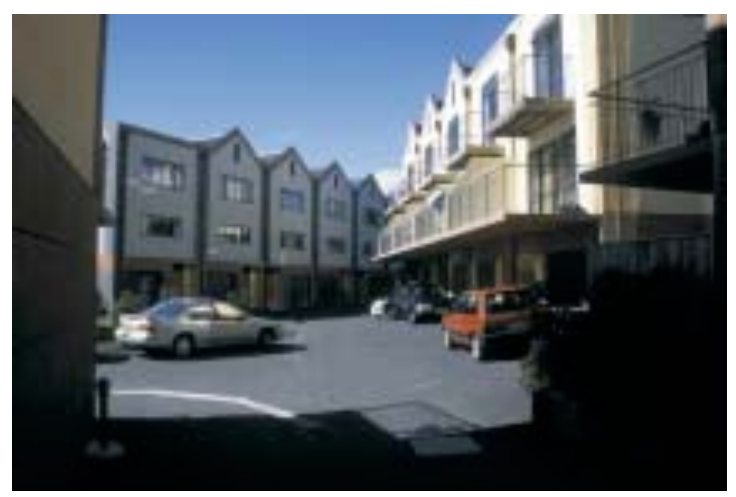

"... with few exceptions ... developments of this type are characterised by poor standards of privacy, car-dominated access, poor or non-existent public space, and impractical internal planning. At the time of development these [Krisley Court] houses were the lowest priced new units in the area."

(Turner et al. 73)

Fig. 3.12 A car-dominated central courtyard. Krisley Court, 6 Ambrico Place, New Lynn (Photo: Turner et al.

'Left-over' spaces between blocks often become token landscaping or picnic areas, such as in the example illustrated opposite at Krisley Court, New Lynn.

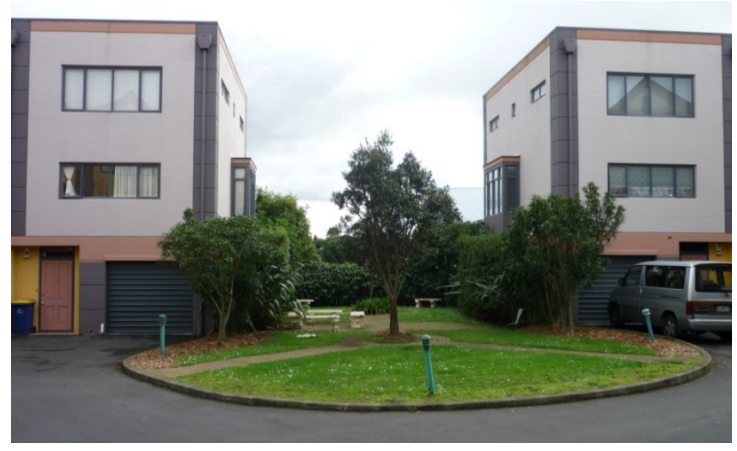

Fig. 3.13 Picnic area in the car-dominated central courtyard, Krisley Court, Ambrico Place, New Lynn, Auckland (Photo: S.J. Pattinson) 
The MfE Case Study of the "Master Planned Community - Addison, Papakura" notes that one of the "key design elements of the masterplan" includes "dwellings that front onto the street or communal open space". Following Crime Prevention Through Environmental Design (CPTED) principles, houses front onto "commons and neighbourhood parks [to] encourage good, passive surveillance of these spaces" (91). The development is based on ...

"...defined neighbourhoods, each with its own neighbourhood-scale reserve $\left(1,500 \mathrm{~m}^{2}\right)$. A concept of 'neighbourhood commons' was also developed ... small neighbourhood parks or open spaces of $500 \mathrm{~m}^{2}$ to $800 \mathrm{~m}^{2}$, fronted by a small cluster of houses." (93).

The case study does not explain what constitutes a "defined neighbourhood", nor the criteria used for determining the size of a "neighbourhood-scale reserve $\left(1,500 \mathrm{~m}^{2}\right)$ " or of the "small neighbourhood [pocket] parks... of $500 \mathrm{~m}^{2}$ to $800 \mathrm{~m}^{2}$ ". The writer calculates that some of the pocket parks are actually less than $500 \mathrm{~m}^{2}$.

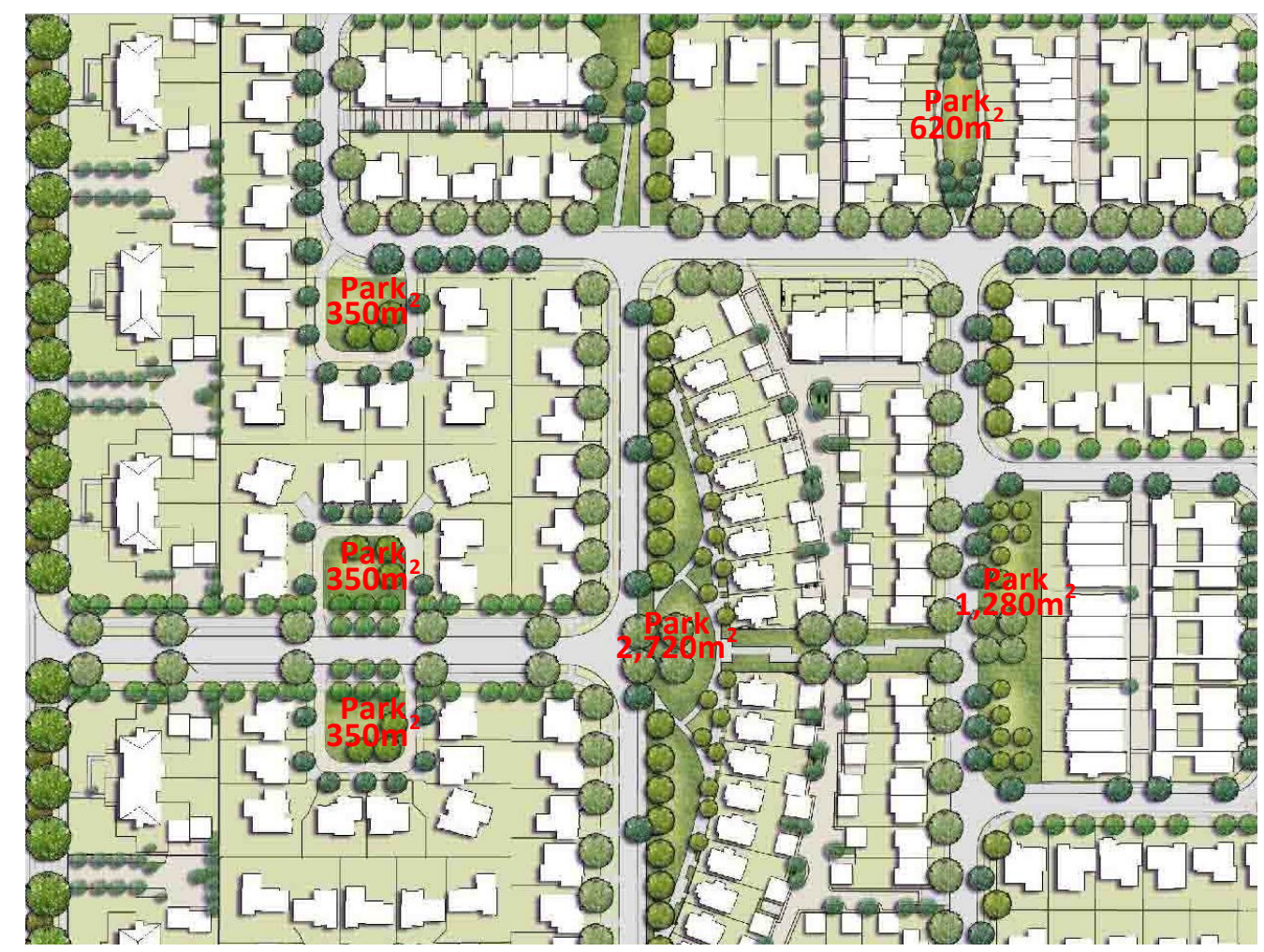

Fig. 3.13 Part of Addison development, Takanini, Papakura, Auckland. Figures in red show approx. areas of neighbourhood reserves and communal open spaces. http://www.addison.co.nz/uploads/images/Addison/Addisontour/08masterplanlarge.jpg

Accessed 29.10.10 (Park areas added by the writer from measurements taken off scale drawings) 
"Emphasis has been placed on the streetscape and open spaces, to create a sense of place, an intimate scale of houses clustered around open spaces and a hierarchy of parks and reserves that includes ... a series of neighbourhood parks of a size that enables the opportunity for active recreation and play $\left(\mathbf{1 , 5 0 0 \mathrm { m } ^ { 2 } )}\right.$..." (96). (Emphasis by writer)

It is not clear how the $2,720 \mathrm{~m}^{2}$ narrow, landscaped and aesthetically attractive reserve in the centre of the plan (Fig. 3.13) supports "active recreation and play".

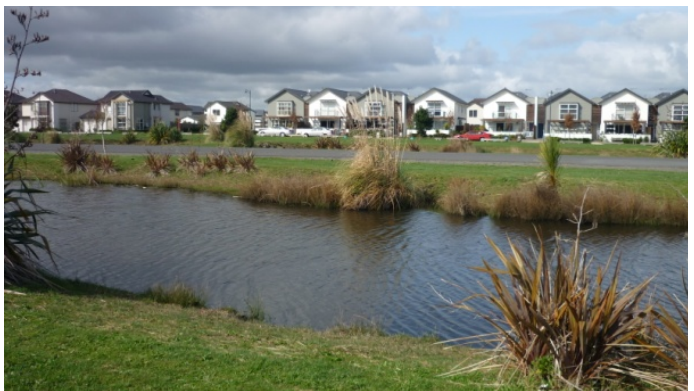

Fig. 3.14 Bruce Pulman Park recreational area beside the housing at Addison. (Photo: S.J. Pattinson)
There are recreational areas and sports fields close to Addison at Bruce Pulman Park. The case study notes Addison was "deliberately developed adjacent to Bruce Pulman Park with a [perimeter] road ... creating interaction ... between the park and private dwellings" (94). The mention

of a perimeter road creating the interaction between the dwellings and Bruce Pulman Park is interesting. It reflects the car-dependent nature of Addison, despite claims about its "good connectivity and permeability" as a "pedestrianoriented development" (93).

The study notes that public walking routes (owned by Council) connect the neighbourhood reserves and pocket parks (also vested in Council) throughout the development (91). But what are the nature and usage of the neighbourhood reserves and pocket parks within Addison?

A promotional brochure about Addison states: "The park-like nature of the community commons not only provides the perfect environment for getting together, but also creates an attractive outlook for the surrounding homes." Landscaped outlook is probably the main function because for Addison to be truly pedestrian-oriented several factors would need to be included.

One important factor for pedestrianism is to achieve a "transit-oriented development (TOD)" (93). This was intended at Addison with the construction of 
a new rail station within walking distance at Glenora. However, the case study notes the "matrix of parks to link the development with ... a proposed [new rail] station at Glenora, [is] now unlikely to happen" $(91,94)$. A Council planner confirmed to the writer that the proposed Glenora station has been deleted.

A second important factor for pedestrianism would be a good bus service. The study notes that "bus connections are ... poor. Thus, while the intent was to be transit oriented, this aspect of the development has not materialised" $(94,95)$.

A third missing factor for a pedestrian-oriented environment is local shops and entertainment. Having noted how the Addison masterplan departs from the Council's original structure plan for Takanini in a number of ways, the case study notes that the "most noticeable departure, however, is that there is no mixed-use nodal area in the masterplan" (93).

These missing ingredients run counter to a "pedestrian-oriented development" (93) as a "concept ... to create community" (91). Residents at Addison are dependent on private vehicles to get to schools, work, and shops. Although well connected, if few residents/pedestrians use the pocket parks and walking routes then they become univalent - primarily aesthetic - to create "an attractive outlook for the surrounding homes" (Addison promotional brochure).

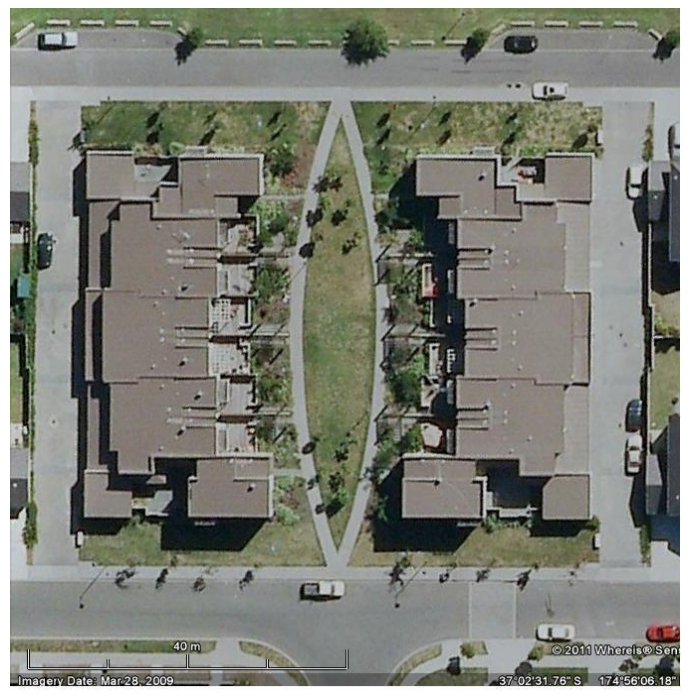

Regarding "terraces that front onto pocket parks, with access lanes at the rear", the Addison Case Study notes:

"... the compact terrace dwellings front onto open space to create amenity within this higher density environment ... [with vehicle] access through rear lanes. ...thus allowing narrow site frontage" $(94,95)$

Fig. 3.15 Addison; car access is via rear The Addison fase study notes that

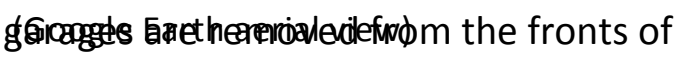
terrace houses "thus allowing narrow site frontage" (95). Why is a narrow site 
frontage desirable? Colquhoun \& Fauset (1991b, 272-273) note that the only advantages of narrow-frontage terrace houses is that they are relatively cheap to build and more can be fitted on to a site:

"... more houses can ... be accommodated ... therefore higher densities can be achieved. Because the area of external walling is generally smaller when narrow-frontage terrace houses are used, this house plan is the most economical of all house types."

Colquhoun \& Fauset point out that while more houses and cheaper construction costs "may be an important advantage to the developer, it is offset by serious disadvantages for the occupants", such as: narrow and deep floor plans requiring large windows for light, resulting in lack of privacy (i.e. overlooking) to rear gardens; internal bathrooms with no natural light (or natural ventilation); and difficulty to get vehicle access or garaging to the front of the house $(272,273)$.

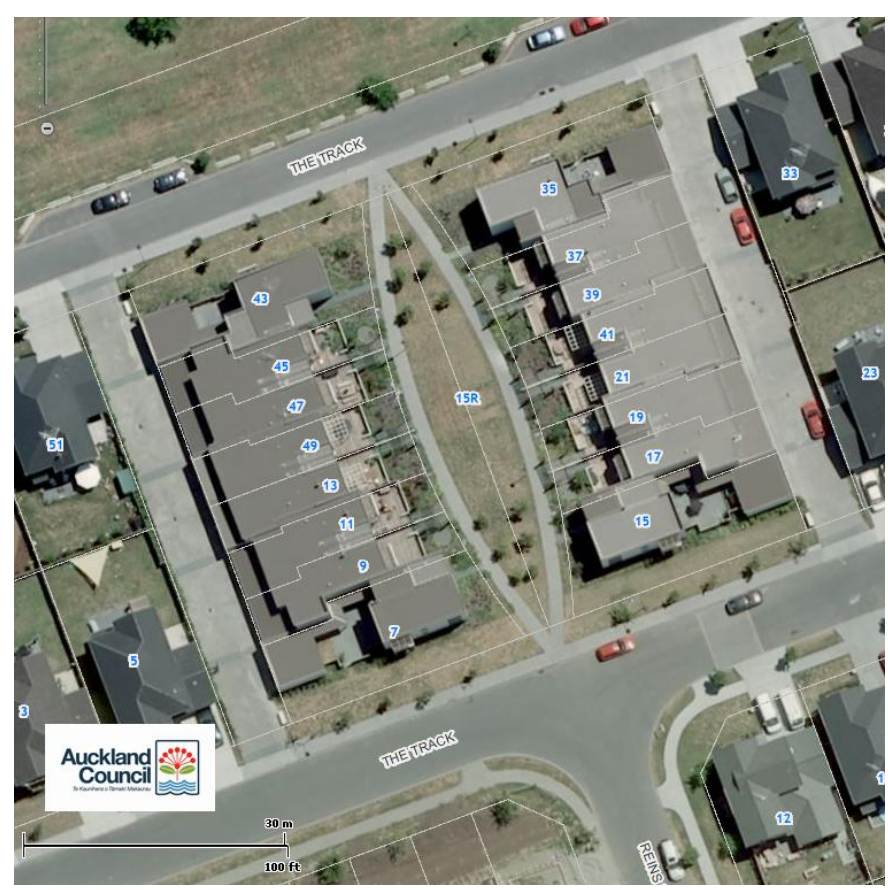

Fig. 3.16 Addison terrace housing: Front door faces pocket park, but main entry to house for occupants is through the double garage at the rear of the house. (Source: Auckland Council GIS aerial view)
At Addison, the terrace houses in the example opposite (Fig. 3.16) have no rear yard - the garages at the rear are attached directly to the houses. This means that although these houses have front doors facing the pocket park, it is more likely that the main coming and going by the occupants will be via the back doors (i.e. internal access from the double gargage - see Fig. 3.17). 
Fig. 3.17a First Floor Plan, Addison terrace housing. (Source: Auckland Council Resource Consent)

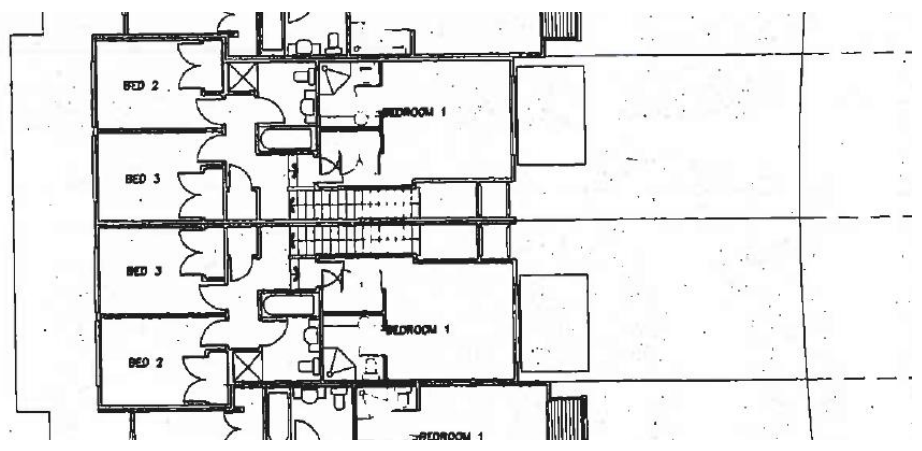

Fig. 3.17b Ground Floor Plan Addison terrace housing. (Source: Auckland Council Resource Consent)

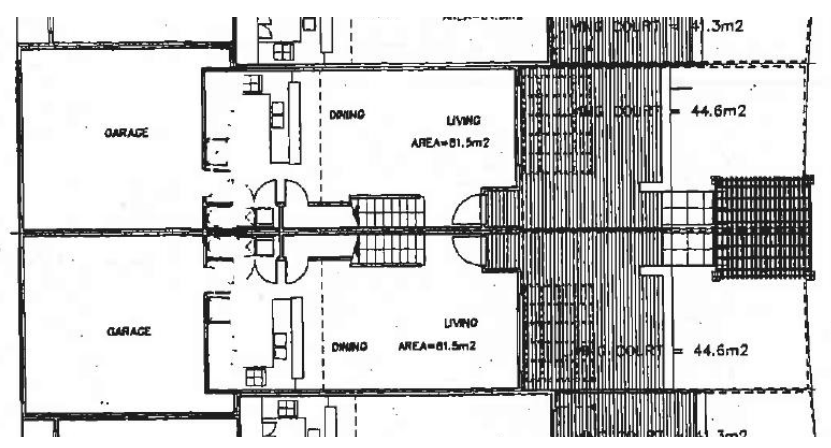

Because occupants use the internal access through the garage (Fig. 3.17b), they are less likely to use the 'front' door facing the pocket park for their regular coming and going. Opportunities exist for low intensity contact (seeing, hearing, occasional greeting) between residents in their front courtyards and passersby in the pocket park. But if there are few walkers or activities in the pocket park and it is not maintained by residents but by Council contractors, and if neighbours come and go by car, and residents use their front yards infrequently, then it is likely that the pocket park is univalent, being primarily for landscaped outlook.

The writer observed a basketball hoop in the communal vehicle access lane. This suggests that residents are using a communal driveway serving eight houses for this recreational activity, rather than the pocket park at the front of the units.

For comments on the MfE case study for Talbot Park, Glen Innes, Auckland, see Chapter 4. For the six case studies in Good Solutions Guide For Apartments, see later in this chapter (39-40). For the four case studies in Good Solutions Guide For Mixed Use Development in Town Centres, see later in this chapter (42). 


\section{The Good Solutions Guide for Medium Density Housing}

(North Shore / Auckland Council) (2007)

This guide recommends that communal open space should be:

- easily accessible for residents

- located on flat land

- have some seating, shade, attractive landscape planting

- have a play area for small children

\section{The Good Solutions Guide for Apartments}

(North Shore / Auckland Council) (2002):

Open space can be public, communal or private. Communal open spaces (shared by residents):

- can foster a sense of identity for residents, which can occur on several levels - a small number of apartments, a single floor, or a whole building

- should be "consolidated, configured and designed to be accessible, useable and attractive for all users"

- should be "configured, sized, furnished and located so that they are suitable for children of different ages" (17).

The notes mention that "better design practice" for communal open space means:

- that not so much of it need be required where site sizes are small and higher building coverage is permitted;

- that where it is difficult to accommodate communal open space on site it will be important that there be adequate existing public open space nearby; 
- that communal open space may be located on a building podium or roof top;

- that communal open space maybe reduced if private open spaces are increased (17)

The notes mention that where communal open space is provided it should:

- receive direct sunshine

- have adequate shading

- be well defined as communal ownership

- be of a suitable size and "appropriately landscaped and contain necessary facilities"

- should not be polluted by basement car park ventilation outlets

- be designed for both daytime and night time use with good lighting

- be accessible to all members of the community (18)

It is suggested that, when required, communal open space should be about 25$30 \%$ of the site area, but on larger or brown field sights it could be more than $30 \%$. The guide comments that when adequate areas of communal open space cannot be achieved, e.g. in dense inner city areas, then this should be compensated by an increase in private open space and access to nearby public open space. The guide notes that the minimum area for private open space for each apartment at ground level is $25 \mathrm{~m} 2$ with a minimum dimension of four metres. The guide suggests thinking of open space 'as an outdoor room' and designing it accordingly. It notes that public open space should be overlooked by houses or other activity areas and that it should itself generate activity (but it doesn't suggest how). The guide notes give suggestions for landscaping and furnishing open outdoor spaces, including both 'soft' and 'hard' surfaced areas for various functions, eg children's outdoor play (19-24).

Of the six case studies included in The Good Solutions Guide for Apartments, only two could be said to meet the objectives outlined above for communal 
open space, viz. Trinity Apartments, Parnell, and Waitakere Gardens, Henderson. The L-shaped Trinity Apartments building is described as an "extremely wellconsidered development" (128), running along the two road boundaries of its corner site, shielding the enclosed communal outdoor space from the streets. The outdoor space includes gardens, hard and soft landscaping, a reflecting pond and a communal lap pool (127).

Waitakere Gardens, a retirement village, has a "generous provision of common areas" (132). These include indoor areas - a central atrium that acts as an internal street and social space, restaurant dining, library, gym and pool (131) and outdoor areas - landscaped gardens, lawns, pergolas/gazebos, seating, petanque, and communal seed raising/vegetable gardening areas.

Beaumont Quarter, although " $a$ coherent, well-considered development ... [with] landscaping generally ... [of] high quality, ... [has] no play areas specifically designed for children" (115).

At Freemans Park, "no distinction exists between public and communal areas. Public footpaths run straight onto common grassed areas ... The development creates the impression of an open arrangement of individual buildings in a parklike landscape ... There could ... be greater definition of boundaries between public and private open space ... Outdoor areas provide a pleasant outlook from apartments, without providing many functional open spaces for residents" (118, 119).

Of the remaining two case studies, neither provides any significant communal spaces.

Overall, of the six apartment case studies, none provide outdoor open space specifically for children. Where communal space is provided it tends to be mainly for landscaped outlook. 


\section{Good Solutions Guide for Mixed Use Development in Town Centres}

(North Shore / Auckland Council) (2005):

Mixed-use offers "a more diverse, complex and dense arrangement" than the division into separate zones such as residential, commercial or industrial (2).

Mixed-use development, located close to town centres, provides easy access to:

- shops, banks, grocery stores

- public transport

- schools and healthcare facilities

- open space / recreation areas

- off-street and on-street parking

- an existing residential population

- offices

- cafes, restaurants

- educational and institutional facilities

- libraries, community centres

- crèches $(03,11,13)$

"Compatibility of use is essential to the success of a mixed-use development. The majority of business and commercial activity today is compatible with residential development" (13). These are "... often within walking distance of the development. A more vibrant urban realm results from the intensification of use and increased activity that a mixed-use development brings to a town centre ... development in central locations helps to concentrate and contain growth, thereby reducing urban sprawl" (11).

"Courtyards can provide either quiet, private spaces or public, commercial spaces within a [mixed-use] development." (16) 
"[C]ourtyard buildings may allow more intensive development than other building forms" and provide "private, open space for occupants"

"Because courtyards offer a unique opportunity for creating peaceful or bustling gathering places within the city, special attention should be paid to their planting and landscaping." (37)

"Landscaping, including the provision of open space, is one of the most important elements of a development's design. ...Good landscaping creates a pleasant environment for occupants and greatly enhances the development's image, thereby increasing its marketability and value.

"Landscaping includes a wide range of elements such as:

- trees / plants of an appropriate type and scale

- public and private open space

- driveways and entrance areas

- hard landscaping including paths and walkways

- planting along streets" (44)

The above comments reflect a generally passive, univalent view of open space primarily as landscaped outlook provided by the landscape architect.

Of the four mixed-use development Case Studies, only one includes open space in the site layout. The development is at 2 Queens Parade, Devonport, Auckland, adjacent to the Esplanade Hotel (72-75). The development is U-shaped, consisting of five small shops on the Ground Floor fronting the main street, ground level car parking along the secondary street, and a courtyard in the centre of the site. "[T]he courtyard allows for communal and private open spaces within the interior" (72). Two floors of apartments are located above the shops and car parking area, with double aspect (i.e. a view down into the courtyard on the interior side, and the street on the other). 


\section{Viewpoints of Practitioners: Current Practice}

Medium density development arranges groups of houses together in some way. This research advocates grouping houses in ways that create collective, positive open space which is meaningful and beneficial to the residents who share it.

The typical linear forms (Figs. $3.18 \& 3.19$ ) of medium density housing place houses in very close proximity but without relating them to each other. In a linear arrangement there is generally no collective open space other than the space required for shared vehicle access - a distinctive characteristic of the 'sausage flats' of the 1960's and 70's which is still

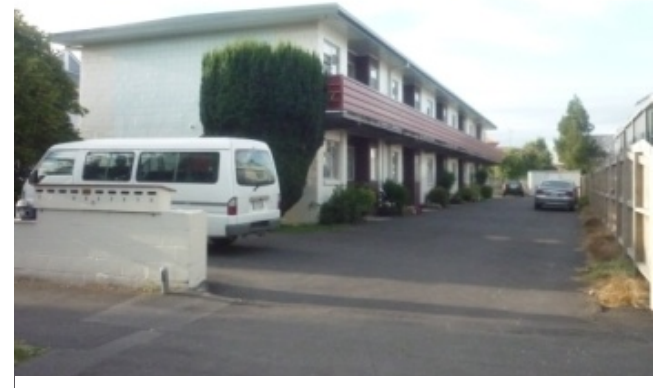

Fig. 3.18 Linear flats - 1960's (Photo: S.J. Pattinson)

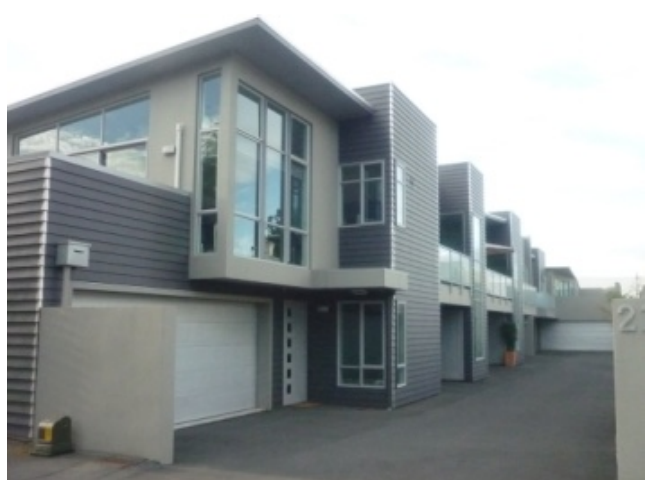

Fig. 3.19 Contemporary linear flats (Photo: S.J. Pattinson) noticeable in contemporary linear arrangements.

The architectural form for 'communal space' is well known in New Zealand. There is a clear tradition in this country of teaching, learning and practice that acknowledges the 'bay' or 'courtyard' form as the archetypal form for community or communal space.

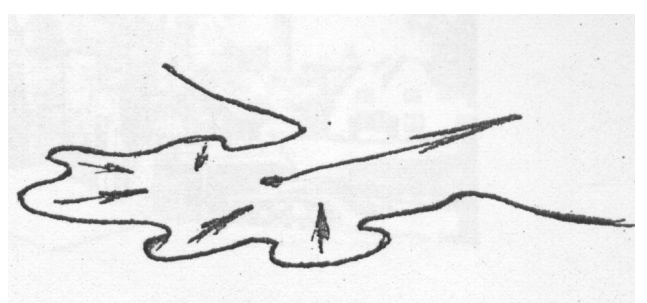

Fig. 3.20 The bay-form structure (Dr Richard Toy)

Although well known, the 'bay' form is generally not applied to medium density housing to create collective, positive open space. 
Currently, the linear form prevails as the stereotype solution for New Zealand's medium density housing, despite many alternative forms for medium density being well known (Diamond 1976; Alexander 1993; Levitt 2010). In “At Home: A Century of New Zealand Design", Douglas Lloyd Jenkins notes a flourish of alternative designs for village communities in the 1970s by New Zealand's leading architects $(226,227)$. Projects regarded by the writer as seminal in New Zealand for mixed-use, multivalent, medium density design include Merivale Mews and retail complex which includes a small lake and village green (cnr Papanui and Office Roads, Christchurch) by architect Peter Beaven; Ropata Village (cnr High St. and Ropata Cres. Lower Hutt) by architect Roger Walker; and architect lan Athfield's mixed-use of own home and office in Amritsar Street, Khandallah, Wellington. Projects like these demonstrate the imaginative use of various non-linear forms to create multiple layers of shared open space and a sense of community.

Linear terrace house forms can also create positive, shared open space when they enclose several sides of a pocket park or courtyard, for example, The Domain Terraces in George Street, Newmarket, Auckland and the terraces on the corner of 177 Hurstmere Road and The Promenade at Takapuna, Auckland.

However the stereotype linear solution seldom features positive, shared open space, giving priority instead to other exterior design issues, viz. vehicle access, private outdoor space and landscaping.

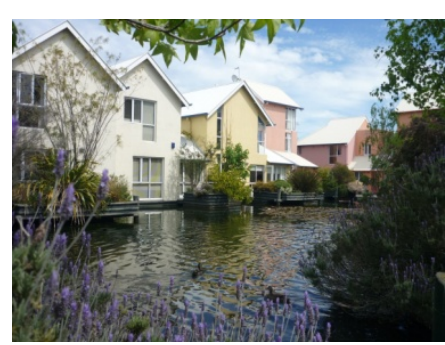

Fig. 3.21 Merivale Mews Christchurch

(Photo: S.J. Pattinson)

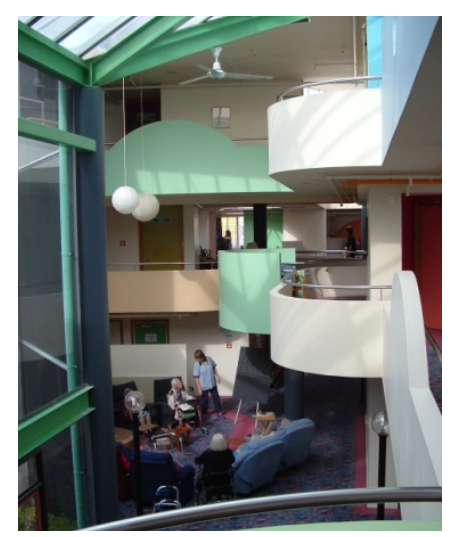

Fig. 3.22 Ropata Village Lower Hutt (Photo: S.J. Pattinson)

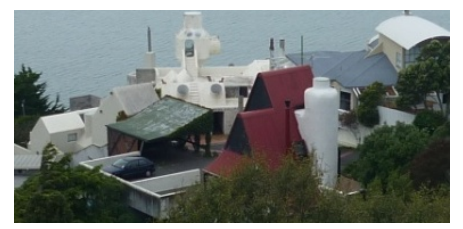

Fig. 3.23 Athfield residence Wellington

(Photo: S.J. Pattinson)

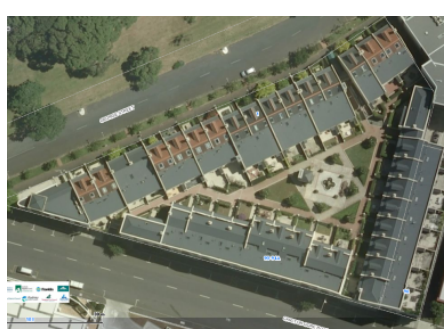

Fig. 3.24 Domain Terraces Auckland

(Auckland Council GIS) 


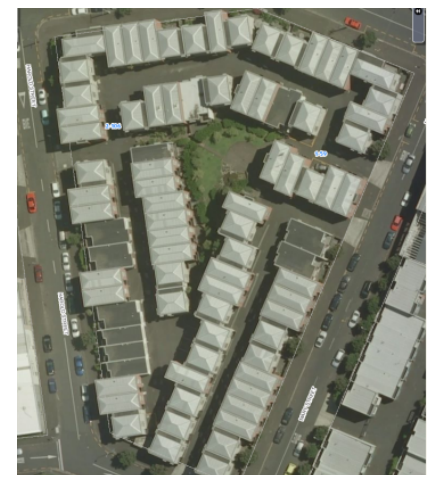

Fig. 3.25 Mt Eden

Auckland

(Auckland Council GIS)

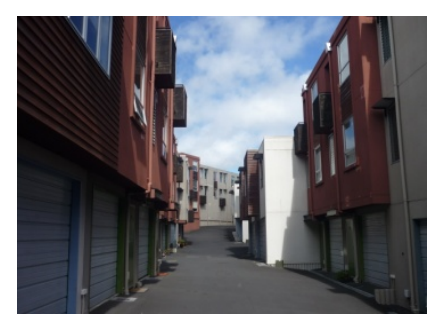

Fig. 3.26 Mt Eden

Auckland

(Photo: S.J. Pattinson)

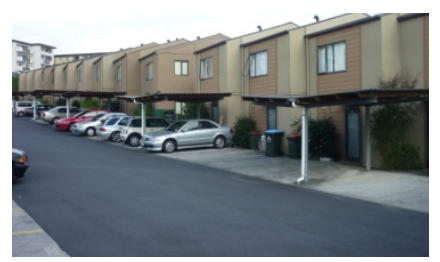

Fig. 3.27 Greenwich Park Grafton, Auckland

(Photo: S.J. Pattinson)

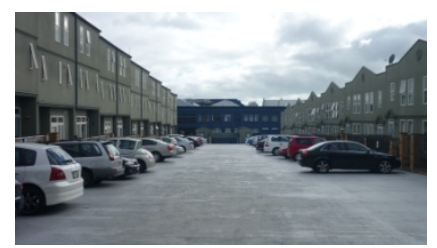

Fig. 3.28 150 Symonds St Auckland

(Photo: S.J. Pattinson)

\section{Car-dominated housing environments}

Looking firstly at vehicle access, many medium density projects give high priority to this requirement. Because of the importance placed on vehicle access by the market, Turner et al. (2004) use four different vehicle access solutions as the basis of their classification system for medium density housing typologies. Their four housing types more or less align with increasing levels of housing density (32):

1. Vehicle access to the front of the house;

2. Vehicle access to the rear of the house;

3. Vehicle 'remote' from the house and parked in a public parking area; and

4. The three-storey house type with internal garaging occupying the ground floor level.

Turner et al. note that "no single design factor determines best practice" in medium density housing (2), but they acknowledge that "[t]he vehicular environment has a dominant role in many examples of the typology" (25), and that in New Zealand medium density housing is characterised as " $a$ 'cardominated' environment" (84). This is in contrast to 'building-dominant' and 'landscape-dominant' distinctions made by the Essex Design Guide. Alexander et al. (122) suggest that car-dominated environments inhibit residents from going outdoors. Many schemes visited by the writer were notable for their garaging, car parks and lack of people outdoors. 
The HNZC Report "Best Practice in Medium Density Housing Design" (Turner et al, 2004) studied predominantly two-storey terrace housing in Auckland, under four classifications based on vehicle access and proximity of the car to the house:
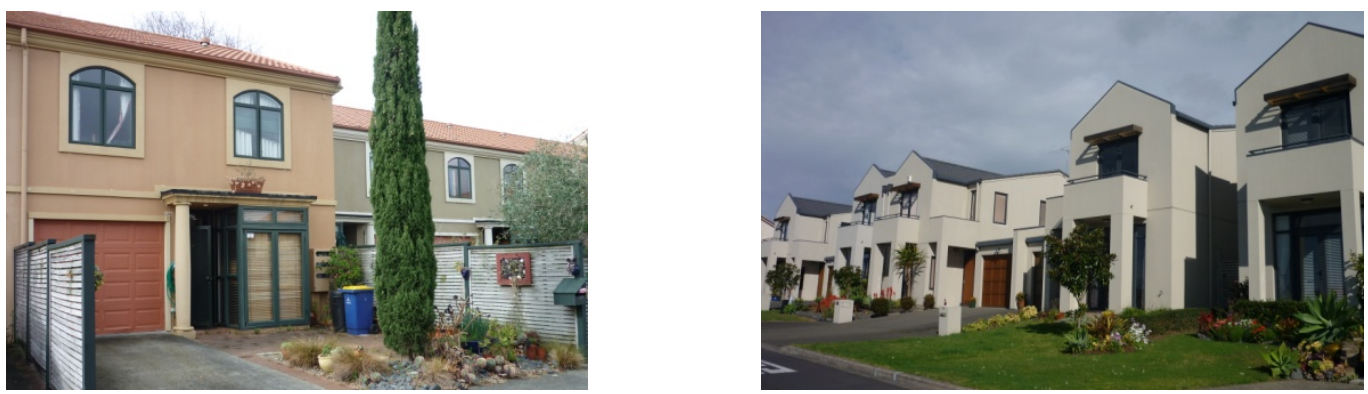

Type 1: front access (garage, carport or parking within property boundary);
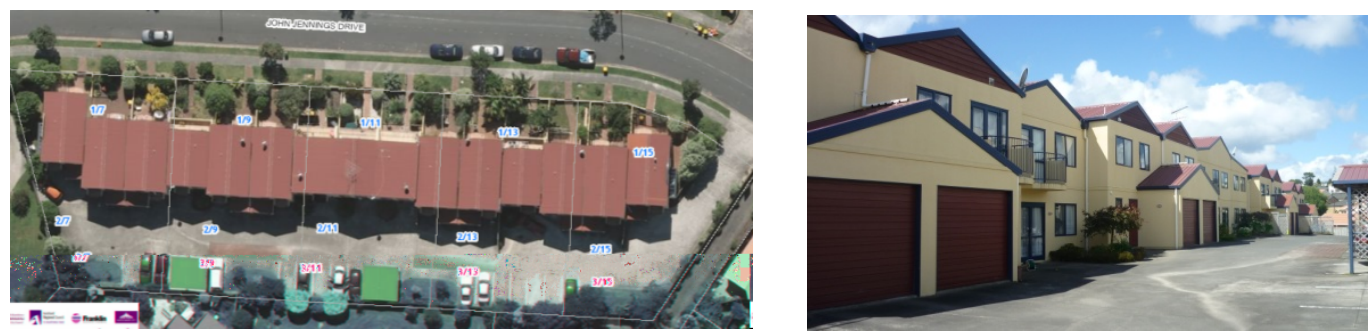

Type 2 - rear access (garage, carport or parking within property boundary);
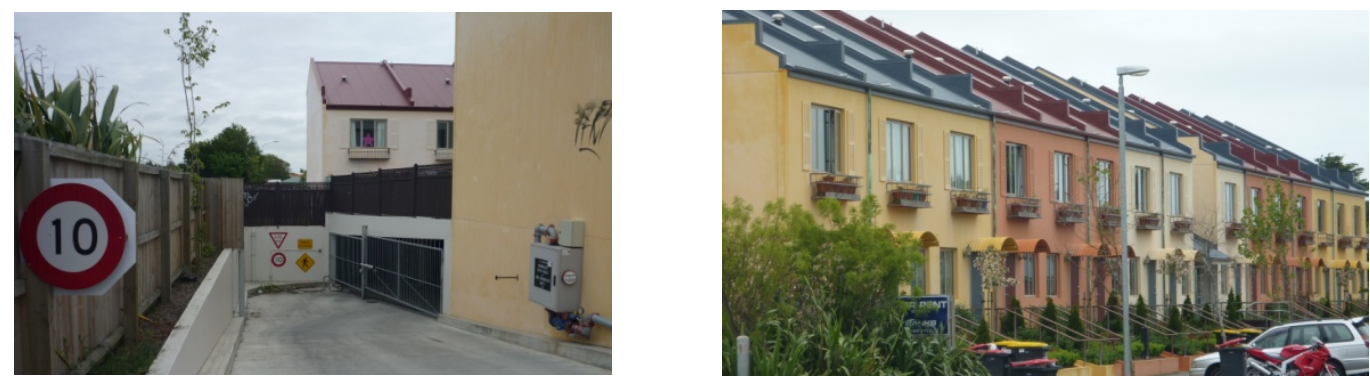

Type 3 - Remote parking -in this case, basement (Warwick St, Christchurch);
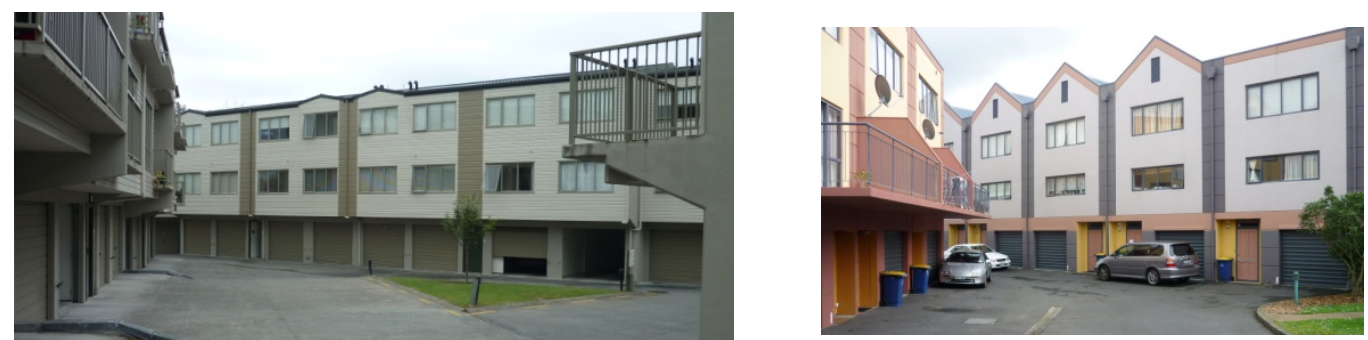

Type 4 - three-storey house with living area elevated above internal garage.

Fig. 3.29 - Medium density classified by vehicle access Types 1 - 4 (Turner et al. 2004) (All photos: S. J. Pattinson) 


\section{Private Outdoor Space - often compromised}

Secondly, privacy is a high priority requirement in medium density housing, yet often compromised. Of the seven principal criteria identified in 2004 by Turner et al. for assessing the quality of medium density residential environments, three relate to privacy - the provision of adequate private open space; the degree of 'overlooking' and perceptions of crowdedness; and the convenience of private vehicle parking (41).

In their follow-up report in 2005, Turner and Su note:

"... over one hundred developments of more than 16 units ... in Auckland ... intended to meet demand at the lower end of the market for new housing... [now] define the characteristics of medium density housing in the New Zealand context. These characteristics include limited private external space, ... reducing standards of privacy, ... and as density increases progressively greater compromises in the quality of residential environment offered" (6).

Figs. 30 - 33 show examples of "limited private external space ... [and] reducing standards of privacy". These projects, and others like them, offer no shared outdoor space (other than carpark) to compensate for low private outdoor space standards.

The "Good Solutions Guide for Medium Density Housing" notes that "access to high quality, private open space [meaning spacious, sunlit, and private, with good access to indoor living space] is a vital component of good residential developments. ... Avoid... creat[ing] private open spaces... that are ... narrow ... inevitably dark, often damp and provide little or no usable outdoor space" (18).

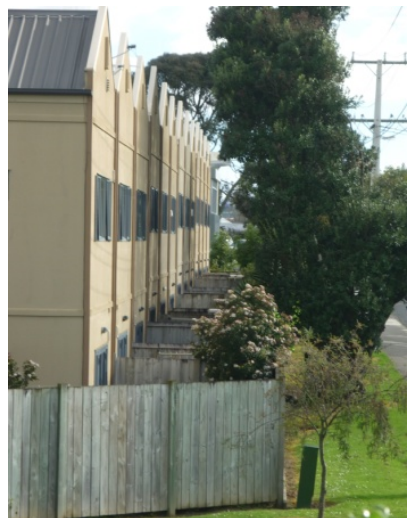

Fig. 3.30 Mt Wellington Auckland (S.J. Pattinson)

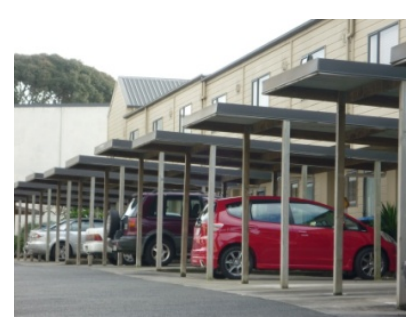

Fig. 3.31 Mt Wellington Auckland (S.J. Pattinson)

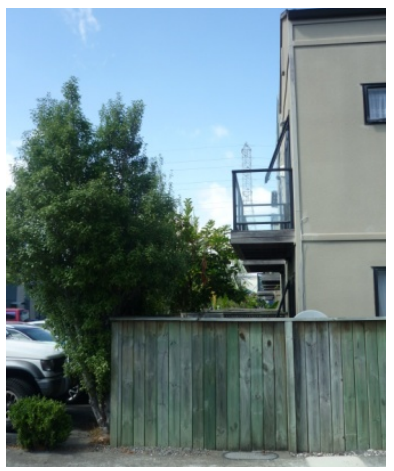

Fig. 3.32 Mays Rd Onehunga (S.J. Pattinson)

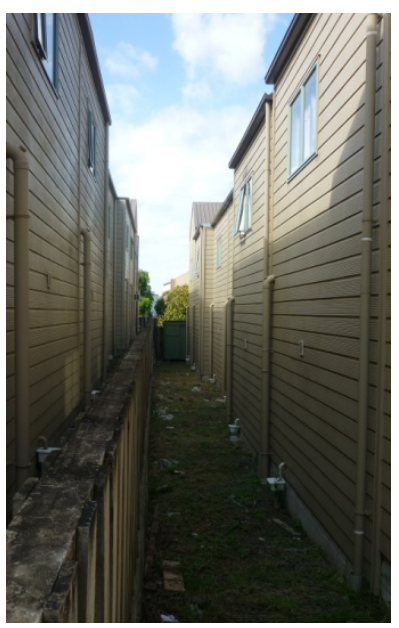

Fig. 3.33 Mays Rd

Onehunga (S.J. Pattinson) 


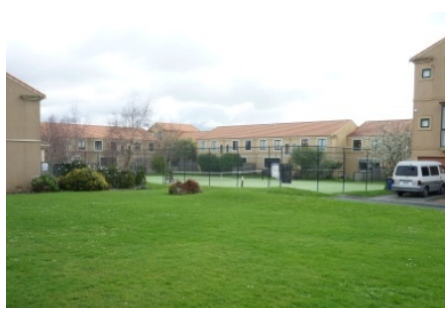

Fig. 3.34 Green open space and tennis court at Tuscany Towers Ambrico Place, New Lynn (Photo: S.J.Pattinson)

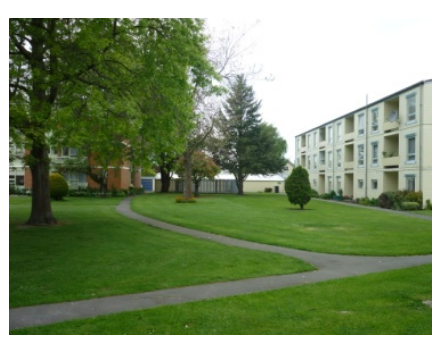

Fig. 3.35 Conference St Christchurch (Photo: S.J.Pattinson)

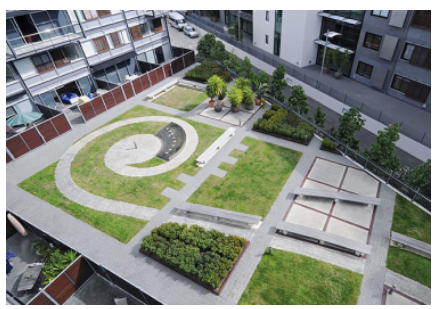

Fig. 3.36 The Point Viaduct Harbour, Auck. (promotional website)

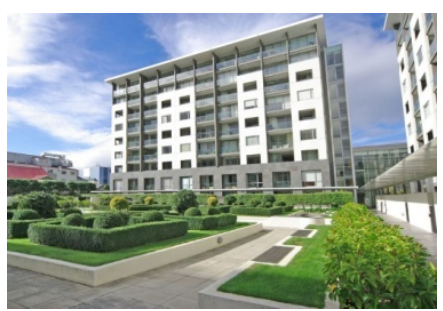

Fig. 3.37 Sanctum Apartments, Wellington (promotional website)

\section{Landscape-dominated environments}

Thirdly, landscaping is a high priority item in medium density housing practice. Turner et al. (41) include landscaping at the top of their list of seven criteria for quality medium density residential environments. But in assessing the quality of "open space (public)" in 30 case studies in Auckland, Turner et al. rate only 5 positively. Interestingly, all 5 have communal outdoor spaces including shared facilities such as tennis and petanque courts, swimming pools and changing pavilions, gardens and green open spaces. Clearly, communal space, done well, enhances the quality and value of medium density environments.

Communal green spaces, however, often seem to be for landscaped outlook rather than for recreation and social interaction. The central lawn at Tuscany Towers (Fig. 3.34), for example, appears to have no defined use. Similarly, the generous green open space at Conference Street flats (Fig. 3.35) is under-defined except for some paths. The creative central space at Viaduct Point (Fig. 3.36) may be over-defined primarily for an aesthetic experience.

The social value of green open spaces is minimised if used merely for landscaped outlook, maintained by contractors. Kazmierczak and James conclude that shared green open space has many benefits for all ages - social, recreational, physical health and mental well-being - which are most likely to be realised by resident participation in the planning, development, maintenance and use of the shared spaces (361). 


\section{Summary of main points and observations}

To summarise the viewpoints of practitioners, it can be said that communal space is currently not well defined nor considered a priority in medium density housing. Yet case studies examined by Turner et al. reveal that communal outdoor space, done well, contributes significantly to the quality and value of medium density living environments.

Currently, priority is given to vehicle access (often creating car-dominated environments), private outdoor space (which reduces as density increases), and landscaping (which is more notable in up-market developments where it is primarily used for aesthetics and outlook).

The Bush Road scheme in Albany, consisting of 105 small units (average size $59 \mathrm{~m} 2$ ) and "aiming to provide low cost housing for younger buyers", is one of the few schemes assessed by Turner et al. as having positive open space. Its central open space "has social significance" and includes a tennis court and swimming pool surrounded by gardens (62). This scheme demonstrates that quality communal facilities and open space is not confined to up-market schemes only, but has relevance to affordable housing schemes as well.

In summary it would be fair to say that practitioners generally need educating on the benefits of quality communal space in all types of medium density housing development, and training in the principles and strategies for making such communal facilities and spaces multivalent so as to enhance these living environments for residents.

\section{Other observations about current practice}

Appendix 7.3 records the writer's first impressions and comments about communal spaces in medium density housing from preliminary field visits in New Zealand's three main cities and in Melbourne, Australia, organised under the following points a) to q) listed below: 
a) residents appear to spend little time outdoors in medium-density housing;

b) "location, location, location" can mean "motorway, railway and industry";

c) provision for cars can dominate a housing development;

d) outdoor recreational areas are limited to 'left-over' space;

e) cramming can squeeze out open space;

f) averaging does not work with landscaping, i.e. high quality landscaping in one small part of a housing development does not make barren areas in the rest of the development more liveable;

g) picnic areas and playgrounds may be just token gestures;

h) pools and playgrounds can be inaccessible to residents;

i) playgrounds can be unimaginative;

j) communal space can be ambiguous;

k) communal space may just be 'landscaped outlook' rather than social space;

I) communal facilities can be inadequate;

m) the needs of children and teenagers are often overlooked;

n) the needs of the elderly may be overlooked;

o) neighbourhoods are fragmented;

p) fragmentation of neighbourhoods may be a reflection of 'silo' thinking;

q) holistic, integrated, multivalent thinking is needed.

See Appendix 7.3 for comments and examples of the above observations.

\section{Conclusion}

Some creative communal spaces can be found in New Zealand's medium density housing. However, the key finding from investigations of current practice is that communal space in medium density housing is generally univalent in nature. It tends to be either car-dominated (typically in low-status schemes), or landscapedominated (typically for aesthetic value and outlook) in high-status locations. Sommer points out the close connection between space and status (32). 


\section{CHAPTER 4 - Current Theory}

\section{Multivalent Communal Space in Medium Density Housing}

Question 2: What is the potential multivalence of communal space in medium density housing?

After extensive literature searching, the writer found very little research specifically addressing 'multivalent communal space in medium density housing'. Cooper Marcus and Sarkissian comment that research on medium density housing generally is difficult to find; they refer to it as 'research in hiding' (5). Furthermore, it is acknowledged there is little research on 'density' generally (Rapaport 141; Alexander 182; Churchman 399, 404, 408). Rapoport notes there has been much research on crowding but it focuses on building interiors - people inside rooms whereas housing density, although related to crowding, focuses on people's perceptions of the exterior built environment $(133,134)$.

Forsyth notes in 2003 there is still "a surprising lack of clarity about what counts when considering density" (2) and "there are no agreed-upon definitions of density ... rather each location and profession has come up with an idiosyncratic view" (3). Research on perceived density by Zacharias and Stamps (2004) uses a method that is limited to two dimensional aesthetic judgements. An approach proposed by Berghauser Pont and Haupt (2007) of defining three dimensional density using 'Spacemate', a composite measure using five physical variables, appears to still only deal with objective urban form and not the subjective personal and cultural aspects of perceived density which Rapoport drew attention to in 1975.

Clearly, an interdisciplinary view of issues relating to dense living environments is needed, and in 1997 Moudon reported that urban morphology was emerging as an interdisciplinary field "to establish common theoretical foundations" about urban 
development (3). Conzen (2010) reflects on challenges still affecting progress. An interdisciplinary approach to an integrated understanding of the benefits of urban green space appears to be developing a useful framework for urban green space research (James, P. et al. 2009).

Southworth and Ben-Joseph trace the gradual development of a multidisciplinary approach to street design, a seemingly benign topic but one which has an enormous influence on urban form (3). This interdisciplinary effort is resulting in a change from vehicular-dominated streets (univalent urban elements) to streets that recognise other uses and users - cyclists, pedestrians, plants, children's play, and adult social interaction, as well as vehicles (multivalent streets) $(117-128)$. Southworth and Ben-Joseph call for "an interdisciplinary approach to street design and planning" for "flexible design guidelines" that "advance new concepts in lieu of rigid standards" $(155,156)$. They point out the potential for "new urban forms" through the principles of integrated streets being applied not just to linear arrangements but to any configuration of clustered housing (123).

A similar approach needs to be taken to the design of communal areas in medium and higher density housing to transform them from univalent 'spaces' to multivalent 'places'. To date, communal facilities in housing developments have received little attention. Kevin Lynch and Gary Hack, writing in 1984, comment on this lack:

"Until recently, communal provisions have been relatively neglected in this country [USA]. We favour the individual lot ... But the ... grounds of a housing site are, for many of its residents, the most important setting for social life. Children are not only the most committed group of users but also the brokers for many adult friendships ...

"A common solution bundles all the stereotyped activities of children below adolescence into a single facility, tucked away in an unusable corner of the ground. Teenagers are usually forgotten altogether. The remainder of the ground is then designed for its appearance to the adult eye. A few hours on the site will uncover the unreality of this approach." 
Cooper Marcus and Sarkissian offer comprehensive guidelines for the design of quality medium density family housing. Their guidelines "address the quality of housing environments from a social perspective, as defined by the residents" (5), and is based on about one hundred Post Occupancy Evaluation (POE) case studies.

Cooper Marcus and Sarkissian comment that though most designers acknowledge environment and behaviour research, few use this research in their work. Some reject environment and behaviour research, linking it with architectural determinism. Regarding architectural determinism the authors comment:

"We reject determinism on a macro, societal scale, but we believe that on the micro scale in and around the home the environment very much influences behaviour. Design cannot cause behaviour, but it can offer the possibility of certain activities taking place. The physical environment of a housing development, for example, can encourage, discourage, or be neutral to its residents' behaviours ...

"The environment ... is laden or encoded with symbolic dimensions and messages, which [people] read or decode according to [their] role, expectations, motivation, and other factors (Becker, 1977; Rapoport, 1978). The environment facilitates (or inhibits) behaviour simultaneously by several modes of physical, social and symbolic communication."

Cooper Marcus and Sarkissian (10)

Cooper Marcus and Sarkissian note the limitations of design with regard to human behaviour and quality of life:

"We have learnt enough now to know that a move to better housing in a 'good' environment cannot, by itself, improve the economic circumstances of deprived families, the emotional circumstances of disturbed families, or the general happiness of 'normal' families. But we have also learned that the design of environments affects people in a multitude of ways, and that in terms of their well-being, it matters deeply."

\section{Cooper Marcus and Sarkissian (7)}

Cooper Marcus and Sarkissian address the needs of all age groups in medium density housing developments, including the social needs of adults. However, they comment that communal space in medium density housing is primarily for the 
benefit of children. This appears to be a widely held view which this thesis challenges. Multivalent communal space should attract and benefit all age groups.

Cooper Marcus and Sarkissian suggest that because people spend a lot of time in and around their homes, these living environments need to be made as life enhancing as possible $(\mathrm{x})$. For higher density housing to become socially acceptable to middle- and higher-income groups it will need to provide a high quality of living. This can be enhanced by good access to shared recreational outdoor space and an appropriate range of local services and facilities (Frey, 341).

As noted above, current literature is sparse on multivalent shared recreational space and communal facilities in medium density housing. The following comments are from literature that alludes to the necessity for multivalent communal space.

Jacobs, in a chapter titled "The Uses of Neighbourhood Parks" (89 - 111), records her observations about the qualities of successful parks. Her comments are relevant to the idea of multivalent, shared neighbourhood spaces. She notes that more than just landscaping and 'magnificent views' are required to attract lots of people to use a park. The physical design factors Jacobs identifies that, in her view, distinguish 'useful' from 'useless' neighbourhood parks, include ...

- concentrated diversity of uses around the edge of the park to ensure its use throughout different times of the day and night;

- intricacy (at eye-level) in the variety of uses the park has to offer;

- a centre 'stage setting' for people as a pausing or focal point

- lots of sunshine (and summer shade);

- definition and enclosure - 'positive' rather than left-over space. (101-111)

Where appropriate, Jacobs also suggests that possible attractions such as swimming pools, fishing, sports fields, carnivals, a band shell, music, live theatre, plays, and 
cultural life can also add to the vibrancy of parks. But Jacobs emphasises the importance of locating parks where there are people, with active streets around the edges of parks ensuring a spill-over of people and activity into the parks:

"The more successfully a city mingles everyday diversity of uses and users in its everyday streets, the more successfully, casually (and economically) its people thereby enliven and support well-located parks that can thus give back grace and delight to their neighbourhoods instead of vacuity." (Jacobs 111)

Tibbalds notes that towns and cities are essentially for and about people. Oversimplification of land-uses makes them dull; mixed uses and activities are essential for making towns and cities lively and people-friendly. He comments that:

"The concept of mixed uses should apply to a whole city centre, an urban block, an individual site or a building" (27) ... The re-creation of a rich and diverse public environment is one of the urban designer's most important tasks ... environments are necessarily complex, intricate and lively and are undermined by the over-simplification of land uses or activities and the dull uniformity of some built development. We must aim to produce environments which are of a mixed-use nature and are of a deliberately rich and varied character" (28) Jacobs emphasises the importance of correctly locating neighbourhood parks so that they benefit from the diverse activities of surrounding streets. William $\mathrm{H}$. Whyte discusses the content of successful small urban spaces, what makes them work, what gives them life, what attracts people to them and concludes that: "The sun is important. So are trees and water and food, and most of all, seats..." (7).

Project for Public Spaces (PPS) advocate in the publication "How to Turn a Place Around: A Handbook for Creating Successful Public Spaces" (2005) principles for 'place-making' and a guide to improving public spaces in cities. Building on Whyte's research, the book emphasises the creation of 'places', not 'designs'. It points out that what attracts people to successful 'places' is other people. A successful place has more things for people to do, attracts people of different ages from preschool children to seniors and gives occasion for more expressions of affection - people smiling, embracing, holding or shaking hands. The framework for creating such 
places requires four essential factors - a diversity of uses and activities; comfort (grouped seating, sunshine and shade, shelter, etc) and image (clean, secure, and well maintained); easy and safe access and linkages (to streets, shops and transportation options); and sociability (places where people choose to meet with friends and neighbours, and be able to mix comfortably with strangers) (80-93). Jay Walljasper (2007) provides guidelines for creating great neighbourhood places, and also includes the important idea of 'third places', i.e. places to socialise other than home or work - coffee shops, cafés or other spots where everyone feels welcome.

There is a hint at multivalence in AMCORD where it discusses community participation in the design and management of shared open space, noting:

"... opportunities for integration, multiple use and sharing between compatible activities."

(AMCORD PND5) [emphasis by writer]

Alexander refers to pioneering studies by Martin and March on urban form and efficiency of land use: "They found court building forms more efficient than ... tower types in enabling higher density development while preserving site amenities (light penetration, open space, etc.)" (Alexander 190). Martin and March point out the importance of seeing 'the relatedness of things':

"This question of the relatedness of things is central to the consideration of ... housing, schools, open space or the roads by which they are served. All these things are aspects of the main problem of relationships: and by looking at a question in this way the old barriers created by zoning are immediately removed." (Martin and March 40)

Martin and March theorize that 'the relatedness of things' suggests the possible overlapping of land uses, resulting in more efficient use of land and the creation of more open space, without any loss of built floor space.

The remainder of this chapter looks at 'the relatedness of things' potentially relevant to communal space to explore possible overlapping uses that could make such space multivalent. 


\section{Communal Space: Potential for Multivalence}

Given that there is very little research on 'multivalent communal space in medium density housing', the question remains -

Question 2: What is the potential multivalence of communal space in medium density housing?

To explore the potential multivalence of communal space, the writer reviewed a range of literature relating to the following possible uses, meanings and values for communal space in medium density housing:

\section{Communal Open Space Can Enhance Neighbourhood Identity}

Territorial Authority District Plans typically aim to foster a 'sense of community'. Auckland's Residential Design Guide, for example, seeks, as one of its principal aims, "to foster a sense of community and strong local identity in neighbourhoods." It therefore emphasizes "walkable neighbourhoods clustering around centres of compatible mixed uses in order to reduce vehicle dependence for access to employment, retail and community facilities" (4).

Clear definition of a neighbourhood contributes to neighbourhood identity. The 'neighbourhood unit' was developed by Clarence Perry but its roots were in Ebenezer Howard's 'garden city' concept. Howard sought to provide the ideal living environment with employment, shopping, schooling and recreation all within walking distance of the home. Howard's scheme used medium density housing to preserve green open space (Thorns 28-30, Howard 1898; 1902, Hall \& Ward 1998).

Raymond Unwin and Barry Parker applied Howard's ideas to Letchworth, the first town designed on 'garden city' principles. Inherent in Howard's concepts were 
economic, social and environmental sustainability goals for a polycentric city form, being reconsidered today for contemporary cities (e.g. Hall and Ward 1998).

In the 1920s, Clarence Perry re-interpreted Howard's ideas "in a distinctly American version of the garden suburb" in the form of the 'neighbourhood unit' with the following characteristics:-

a. Community facilities (e.g. schools, shopping, recreation, community building) within easy walking distance of home - in the suburban situation this was taken as an area with a 5 minute walking radius (about $400 \mathrm{~m}$ ) from the centre to the edge;

b. Clearly identifiable neighbourhood boundaries - natural topography (e.g. river, ridgeline); railway track; main arterial roads (street patterns within the neighbourhood are designed to prevent through-traffic);

c. A population sufficient to support a primary school, which would be the focus of the neighbourhood community. It was considered that the ideal primary school size was about $800-1,200$ children which would therefore require a neighbourhood population of about $5-6,000$ people;

d. The neighbourhood would be a residential environment - people would generally work elsewhere;

e. Small neighbourhood shops, usually located on neighbourhood boundaries to form local commercial centres with adjoining neighbourhoods;

f. Small parks and recreation spaces scattered throughout the neighbourhood;

g. Each neighbourhood would have its own "character". Perry asserted that "... the district [neighbourhood] in which a person lives tells something about him. Since he chose it, ordinarily, it is an extension of his personality. One individual can do but little to create it. It is strictly a community product."

(Clarence Perry, "The Neighbourhood Unit", in The Urban Design Reader, eds. Michael Larice and Elizabeth Macdonald, $3^{\text {rd }}$ Ed., 2007) 
Randolph Hester (1975), in "Neighbourhood Space", illustrates the classic 'neighbourhood unit' as applied at Columbia, Maryland, USA, noting that ...

"the town is ... subdivided into neighbourhoods, providing ... an elementary school and a neighbourhood centre" (9).

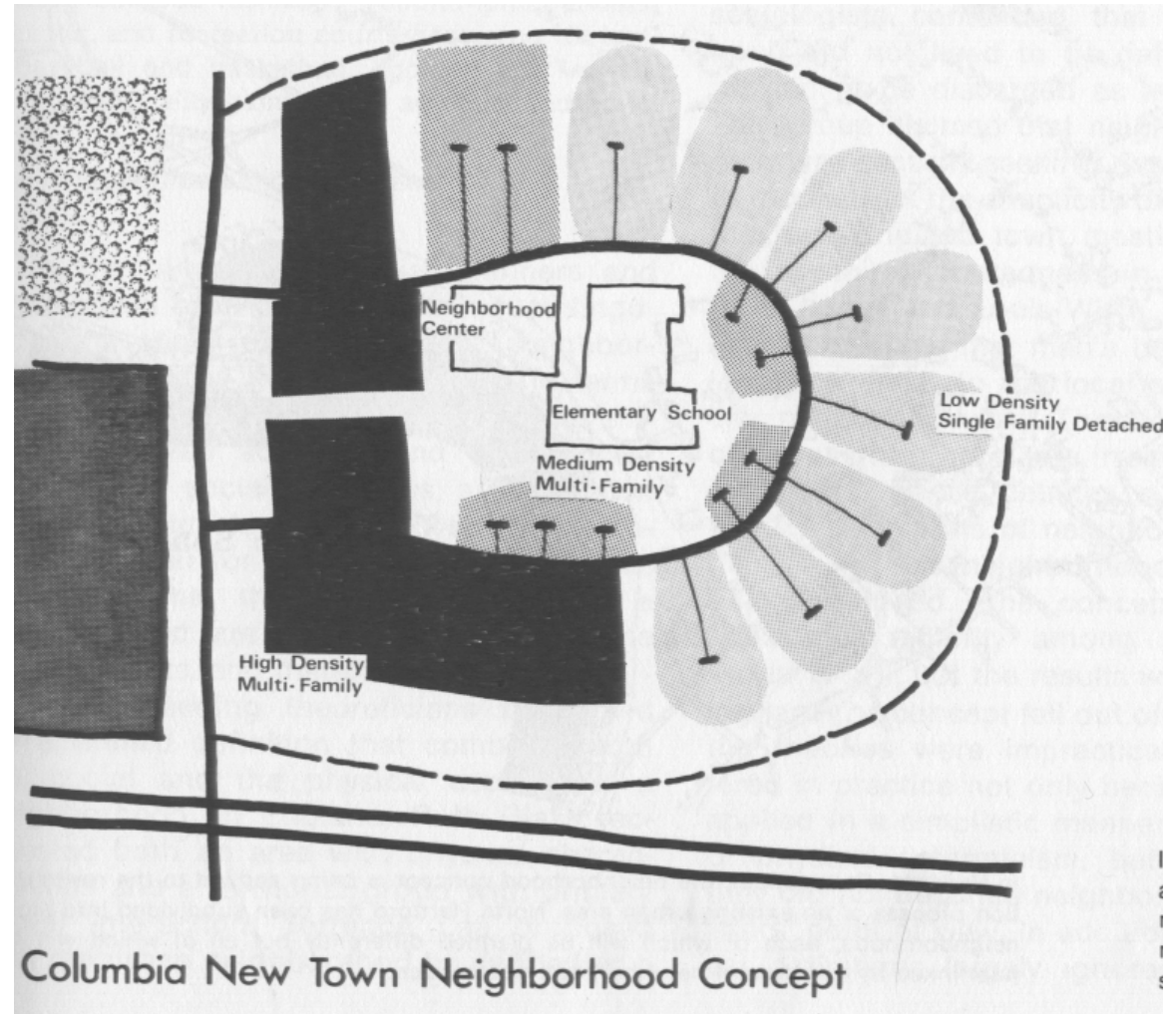

Figure 4.1 Conceptual diagram of a "Neighbourhood Unit" (Source: Hester 9)

The above diagram (Figure 4.1) is of a neighbourhood unit planned for a population of $5-6,000$ people, sufficient to support a primary school. Open space for the medium density housing is provided by the school playing fields.

Perry applied his neighbourhood unit principles to four different levels of density:-

- Low density suburban

- Medium density housing bordering industrial areas

- Low- to mid-rise apartment blocks

- High-rise towers up to 33 storeys (Perry in Larice and Macdonald eds. 2007) 
As a planning tool, the 'neighbourhood unit' has been applied mostly at suburban densities - in Radburn NJ (the first application of these ideas in the USA, designed by Clarence Stein and Henry Wright in 1928), and Weston, VA, USA; British New Towns; Vallingby (Sweden); Sabende (Guinea); American suburbs generally; and, most recently, in Traditional Neighbourhood Developments (TNDs) and New Urbanism developments (USA) and in Urban Villages (UK, Europe).

The 'neighbourhood unit' was widely criticized. Lynch thought 'neighbourhoods' of 5,000 too big (Lynch 1981:246). Keller thought likewise and noted that most people only know 3 or 4 neighbours "living in houses either side of and directly opposite one's own" $(37,39,49)$.

Keller distinguished 'neighbour' (a role distinct from friend or family), 'neighbouring' (activities shared in varying degrees and intensities between neighbours, including exchanging help, material items, and information), and 'neighbourhood' (a geographical area that can be either clearly or vaguely defined) $(10,12,24,30)$. She thought it unrealistic to expect people to cohere locally in big cities because ...

"... in big urban centres ... friends tend to replace neighbours as sources of assistance and sociability and, as self-sufficiency increases, the need for neighbourly assistance diminishes... "...self-contained ... neighbourhood units ... deprive ... people of some notable advantages of urban life. Some individuals and groups may indeed welcome the intimate small town atmosphere and face-to-face contacts such units intend to foster, but others, truer to the urban ethos, prefer to use the whole city and to travel to their social contacts and work ..."

(Keller 118,119,132)

Contemporary New Urbanist planning supports the idea of the neighbourhood unit:

"Neighbourhoods should be compact, pedestrian-friendly, and mixed-use ... Many activities of daily living should occur within walking distance ... Within neighbourhoods, a broad range of housing types and price levels can bring people of diverse ages, races and incomes into daily interaction, strengthening the personal and civic bonds essential to an authentic community ...

"Is the plan divided into neighbourhoods, where each neighbourhood has the following characteristics: 
- "Is it roughly a five minute walk ... from edge to centre? ...

- "Is there a civic space such as a plaza or green at the neighbourhood centre?

- “... [and] at least one prominent ... civic building?

- "Are there small parks distributed evenly through the neighbourhood...?

- "Are elementary schools ... sized accordingly, and ... accessible on foot?

- "Is the neighbourhood edge [defined] ... ? etc"

("The Charter of The New Urbanism" in "Suburban Nation", Duany, Plater-Zyberk and Speck 247, 248, 259)

Churchman reports criticism of the New Urbanism approach: "The emphasis of the new urbanism movement is on small towns ... New urbanism has come in for criticism, for example, for its emphasis on shape and form rather than substance." (Churchman 393)

A comprehensive criticism of the new urbanist "Traditional Neighbourhood Development" in the USA and its counterpart in the UK and Europe, the "urban village', can be found in Mike Biddulph's “Villages Don't Make a City” (2000). Biddulph's main criticism is that whilst the established principles of good urban design in the urban village movement are to be welcomed, alternative city forms should also be considered. Biddulph writes:

"... people identify with local streets ... [and] use local shops, but ... they also want to ... use shops elsewhere in the city... [and] find other communities of interest. Rather than encouraging greater atomisation ... we could be promoting greater overlapping of functional, social, economic and environmental systems. This would provide people with choice, whilst giving the city humanity and richness. Making the city a better place should not mean turning it into a group of villages. Life in, and subsequently the planning of, a city could clearly transcend such a parochial vision." (Biddulph 80)

Despite the criticism, the neighbourhood concept is defended on the basis that whilst physical form cannot determine social interaction and community, it is a necessary framework for it: 
"We recognise that physical solutions by themselves will not solve social and economic problems, but neither can economic vitality, social stability and environmental health be sustained without a coherent and supportive physical framework."

(The Charter of The New Urbanism in "Suburban Nation" Andres Duany, Elizabeth Plater-Zyberk and Jim Speck 256)

To give legibility to a community, the Essex Design Guide (2005:29) proposes 'the core', a principle of spatial organisation which connects community facilities with the residential buildings they might be related to, rather than having community facilities in isolated locations. The idea of 'the core' is that community facilities become the focus in the centre or on the edge of a cluster of houses, encouraging a high concentration of people in the vicinity of the community facilities and an easy connection between the facilities and the houses. A 'nodal point', such as a café, childcare centre, or convenience store, can provide a focus for a cluster of houses giving it a point of local identity (2005:30).

An alternative to using nodal buildings for neighbourhood identity is to use open space by giving it distinctive character. The 'Good Solutions Guide for Apartments' (Auckland Council) notes:

"Open space plays an important role in fostering and maintaining a sense of identity for the residents of a development. Providing many communal open spaces within a residential development allows community identity to develop on many levels. For example, communal open space can be shared by residents of a small number of apartments, by residents on a single floor, or by residents in a whole building or development" (17).

The Urban Place Supplement (UPS) to the Essex Design Initiative (EDI) addresses communal space. It advises that in "[m]ore compact developments ... [e]very home shall have the benefit of some individual private or communal private amenity space" and includes small communal gardens as one way of achieving this (74). 
Cooper Marcus and Sarkissian, in Pattern \#5 'Community Identity', state:

"A sense of community and security is likely to be enhanced when access to the site by outsiders is discouraged" (40)

They comment that residents of a housing development are more likely to identify with it and take a sense of ownership and responsibility over it if it is contained in a way that strangers are less likely to intrude through it. In Pattern \#21 'Sub-unit Identity', Cooper Marcus and Sarkissian comment that in large housing schemes, smaller parts need to have something unique about them for a sense of place and identity. They comment this could be provided through the unique design of ...

"... the common open space of each cluster [of houses] so that the size, dimensions, grading, planting, site furniture, and play equipment of each are different ... where the residents themselves can jointly create unique milieus" (57).

Jencks would seem to be in agreement with this comment that unique milieus can contribute to a sense of place and identity when he says ...

"... multivalent architecture is one way of establishing place and ... identity ..." (318) 


\section{Communal Open Space Can Foster Neighbourhood Attachment}

Cooper Marcus and Sarkissian state that when common open space is the indisputable territory of a group of residents it provides them with a needed sense of place and belonging. They note that a greater sense of territory develops from residents having reason to walk through communal spaces on a regular basis, e.g. to parking, laundry or recreational facilities so they begin to perceive the communal space as an extension to the dwelling place (Pattern No. 72 'Group Territory', 119120).

Furthermore Cooper Marcus and Sarkissian note that residents develop a protective attitude towards communal open space that they grow comfortable with and this can result in significantly lower maintenance costs because residents willingly take responsibility for the maintenance and upkeep of communal space (120).

Cooper Marcus and Sarkissian state that maintenance of communal open space is strongly linked with residents' satisfaction (133) with amenities, management, neighbours, housing appearance and perceived value (42), and co-ownership of defined group territory which they use regularly and enjoy (119-120).

Morgan notes that for many people "childhood place experience plays an important role in adult identity" (12). Place attachment emerges from patterns of positive childhood place experiences so that a person will "often develop feelings of affection for, and a sense of belonging or being of that place, so that place becomes 'one anchor of his or her identity'" which endures in adult remembrances (12). Morgan presents a case for a significant transactional link between human attachment and place attachment. "[B]oth ... stimulate human development, give rise to long-term affective bonds, and contribute to adult identity (21). It seems important from this study to provide children with living environments that offer opportunities for positive 'place' experiences - exploration, fascination, adventure. 


\section{Communal Open Space Can Invite Walking, Recreation and Exercise}

Green open space can provide opportunities for recreation and physical exercise (Kazmierczak and James 355). Recreational facilities and open space should be equitably distributed and accessible to the communities they serve (AMCORD PND5). The 'Good Solutions Guide for Medium Density Housing' (Auckland Council) notes "that communal open space should be provided as part of any medium density housing development if there is not easy access and close proximity to a public open space. Communal open space should be easily accessible for residents, located on flat land and have some seating, shade, attractive landscaping and a play area with apparatus for small children. It must not be used for parking." (7)

For communal spaces to be inclusive and walkable for the elderly, they need to provide "flat or gently sloping pathways with slip free, well-drained but reasonably level surfaces with a good standard of lighting" (AMCORD PND21 / see also Burton and Mitchell 2006).

Elizabeth Wood comments that from time to time people need to get 'out', to go outside or go somewhere, but that during times of inclement weather it may not be possible to go outside (328). The need to 'get out' in such circumstances could be met through the provision of 'indoor' communal or public spaces - for example, music rooms, gyms, community centres, club rooms, indoor sports facilities. Alternatively, getting 'out' might mean going to a suitably enclosed (or semienclosed) indoor/outdoor space - the idea of the 'outdoor room' - for example, a private outdoor room attached to the house, a shared indoor/outdoor communal kitchen/dining/B-B-Q area, or a public sidewalk café that provides some shelter from the wind or rain and a convivial atmosphere.

Leinberger relates walkability to dense (FAR 0.8+), diverse, mixed-use urban development (113-137). 


\section{Communal Open Space Can Foster Social Interaction, Inclusion and Heterogeneity}

Social interaction and a sense of community can be facilitated in housing developments by providing a mix of compatible and complementary activities and uses necessary for living, working and recreation (AMCORD PND5).

In any housing development it is important to consider "social well-being, community identity and quality of life". This is particularly important for those "who spend a considerable part of their time at home ... women, children, the elderly and people with disabilities." (AMCORD PND5)

Opportunities for socialising with neighbours can be provided by including shared recreational facilities within housing developments, for example, swimming pools, gyms, coffee rooms, workshops etc. (AMCORD PND21)

Communal open space can also contribute significantly to social interaction. Kazmierczak and James note a survey which finds that $85 \%$ of urban dwellers believe green open spaces improve their quality of life. They conclude from a literature review that urban green space also contributes to inclusion and community cohesion:

- by being freely accessible to all - the authors note, however, that access can be limited by the uneven distribution of green space in urban areas, and that neglected parks can attract anti-social behaviour (357);

- by being social arenas - open spaces offer opportunities to relax and enjoy social contacts and short-duration outdoor conversations and greetings. The authors mention that urban green spaces provide a beneficial social setting particularly for single-parents, adolescents, youngsters and the elderly (357). Conversely, the authors note that parks separating starkly contrasting urban 
neighbourhoods may serve as deserted 'green walls', and that poorly laid out or poorly managed green space is unlikely to function as a social arena (358);

- by relieving the stress and mental fatigue often associated with living in urban environments. One study finds that the more green open space there is near high density housing, the more residents become familiar and socialise with neighbours and the greater is their sense of community (358);

- by offering opportunities for resident participation in the design, management and stewardship of green open space, thereby increasing social cohesion and strengthening local communities. (359).

Kazmierczak and James sum up their review on green open space by commenting:

"[W] hat separates the 'successful' neighbourhood from the 'unsuccessful' one is the level of social cohesion ... Therefore, any mechanism that may improve social interaction and increase sense of belonging to place and community should be implemented ...

"This paper has presented the evidence [that] ... urban green spaces can improve social inclusion and community cohesion ... [by being] ... free and accessible ... stress-relieving ... social arenas .... [providing] opportunities for [voluntary community] participation" (361).

Communal outdoor spaces can function as 'third places' - social places other than home or work where everyone feels welcome (Oldenburg 1989). Jacobs notes that public streets can provide this kind of place for people to socialise away from their homes, thereby preserving the privacy of their home-life. She comments that if faced with the choice of sharing much (i.e. their private home-life) or sharing nothing (i.e. because there is no suitable public place to socialise) people generally choose to share nothing:

"The more common outcome in cities, where people are faced with the choice of sharing much or nothing, is nothing. In city areas that lack a natural and casual social life, it is common for residents to isolate themselves from each other ..." (Jacobs 65) 
Elizabeth Wood comments about the importance of providing communal outdoor space that fosters the informal, casual development of social fabric:

"The first goal of housing design is to satisfy 'with richness and imagination' the needs of people for activities outside their dwelling units. The second goal is to so design for peoplein-aggregate that out of the fulfilment of these needs there can come into being this thing called social fabric.

"People begin life in a housing project as an aggregation of strangers ... Design should help this aggregation of strangers become less strange, more familiar to each other. Out of this familiarity can come the informal communication, the informal grouping that constitutes social fabric. This ... has particular relevance for families with children."

Elizabeth Wood in Bell and Tyrwhitt, Eds (328, 329)

Cooper Marcus and Sarkissian advise a relatively high degree of homogeneity is required for a population to engage in neighbouring and develop a sense of community, the crucial similarities being "life-style, education, income, and childrearing practices" (42). They also advise against forcing 'togetherness' with 'unlike' neighbours, recommending clustering families at similar stages in the life-cycle (42). Thorns observes people tend to sort themselves into homogenised neighbourhoods, that is 'people like us' ... 'who live in houses like ours', but that this works against the egalitarian ideal and social balance (86-87).

While some people may prefer to live near others of similar lifestyle, others prefer the stimulation of a mixed community (AMCORD PND21). According to Keller (80, 82, 83):

"... social differences need not work against friendly or neighbourly interaction ... [S]hared attitudes toward neighbouring irrespective of social affiliations are the crucial determinants of social relations within a given physical space. ...individuals may have similar attitudes to privacy, open space, entertaining and sociability despite differences in cultural and social characteristics." 
A concept that facilitates neighbourly interaction which is proving popular in some countries is the concept of the 'shared street'. Southworth and Ben-Joseph note surveys that show "that shared streets foster encounters and communication between neighbours" (125). They comment this is particularly so when residents are involved in the planning, implementation and management of shared streets. This is not only crucial to the acceptance of the shared street concept (128) but where this is carried out the shared street system proves highly popular, and a high percentage of residents voluntarily maintain street planters and landscaping (125).

There are several names for the shared street concept including the woonerf in the Netherlands, the wohnstraßen or living street in Germany, the shared street or mixed court in England, the community doro or community street in Japan, and the rehov meshulav or integrated street in Israel. The overall term now used to include all of these is the unified street system (Southworth and Ben-Joseph 122).

"To design ... residential streets solely for the convenience of easy automobile movement overlooks the many overlapping uses of a residential street. ... Residential streets serve many functions beyond vehicular access - they are settings for social activity including children's play and adult recreation, the framework for pedestrian and bicycle circulation, and the space that provides an entry to homes. They should be designed for all of these activities"

(Southworth and Ben-Joseph 6-7).

Shared streets answer Jacobs' call for wider sidewalks:

"If sidewalks on a lively street are sufficiently wide, [children's] play flourishes ... right along with other uses... Sidewalks thirty or thirty-five feet [about 10m] wide can accommodate virtually any demand of incidental play put upon them - along with trees to shade the activities, and sufficient space for pedestrian circulation and adult public sidewalk life ...

Twenty-foot [6m] sidewalks, which usually preclude rope jumping but can feasibly permit roller skating and the use of other wheeled toys can still be found ... the livelier and more popular a sidewalk and the greater the number and variety of its users, the greater the total width needed for it to service its purposes pleasantly $(86,87)$. 
Southworth and Ben-Joseph note that conventional sidewalks typically range in width from 3.5 to 5 feet ( 1 to $1.5 \mathrm{~m}$ ), might be provided on one side of the street only, and are sometimes not provided at all $(98,102,129)$. With narrow sidewalks, the roadway becomes "a play area (whether one likes it or not) for ... children" (6).

The benefit of the shared street concept is that "[p]edestrians, children at play, bicyclists, parked cars and moving cars all share the same street space" (Southworth and Ben-Joseph 117). Research on shared streets in Germany, before and after their conversion from conventional streets, reveals a significant increase in children's play activity after street redesign, as well as a greater variety of activities:

"Children stayed longer, and without adult supervision, and play became more complex. Games requiring more space and the use of bicycles and toy vehicles also increased. Most notable was the shift in play location from narrow street sidewalks to the ... entire width [of the street], including the former traffic lane" (Southworth and Ben-Joseph 124).

The research results are illustrated in Figure 4.2 below which shows the increased play activity in the street after the redesign.

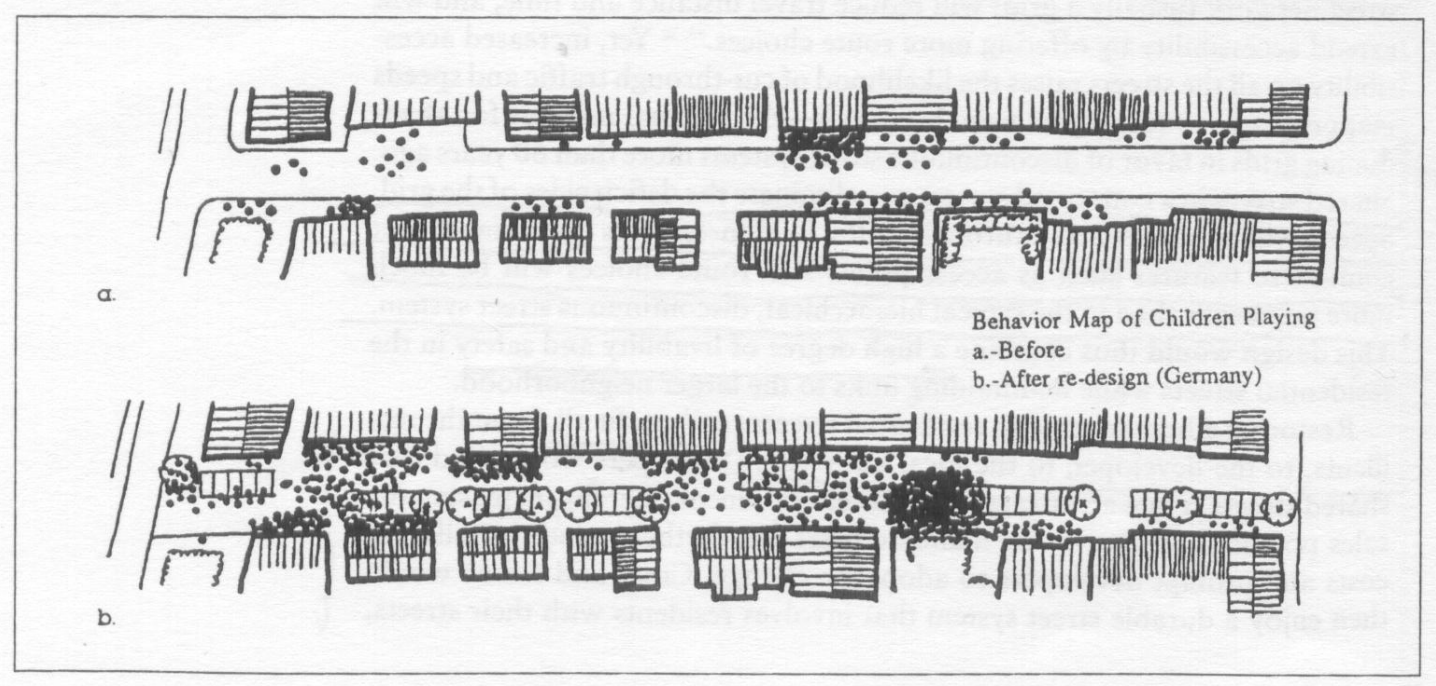

Fig. 4.2 Before and after conversion to shared street (Southworth \& Ben-Joseph 124) 


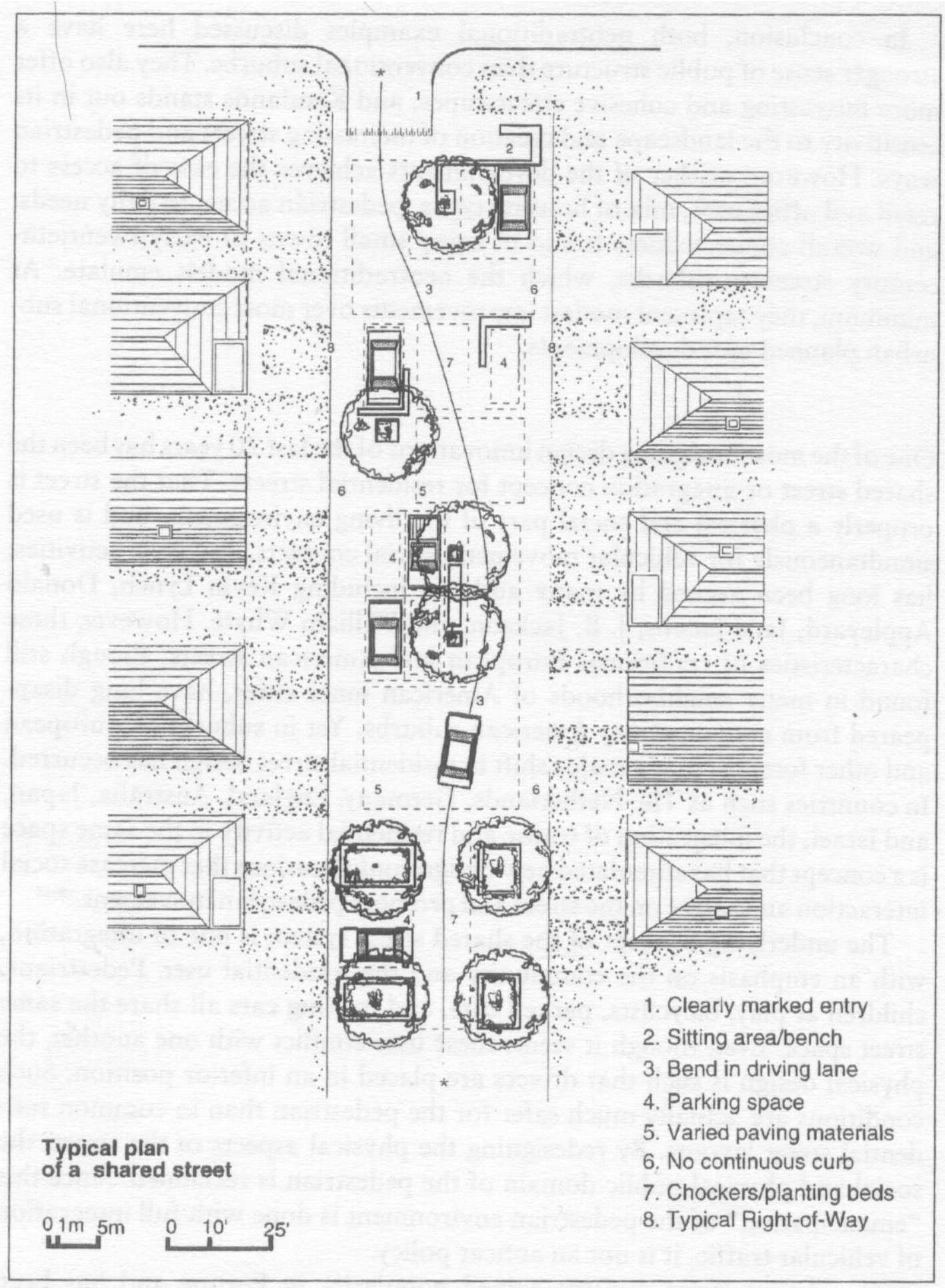

Fig. 4.3 Typical plan of a shared street (Southworth \& Ben-Joseph 118) 
Southworth and Ben-Joseph note that the characteristics of a shared street are:

- It is public space;

- It is not intended for through traffic;

- Pedestrians have priority over the entire street for walking or playing;

- It can be linear or square, or some other form or combination of connected spaces;

- The entrance is clearly marked;

- There are no curbs. Road carriageway and footpath merge into one surface. Paving materials blend pedestrian and car use into the same areas;

- Car speeds and movements are restricted by the layout of extensive planting, landscaping and street furnishing;

- Residents' vehicles have access to the fronts of their dwellings (122).

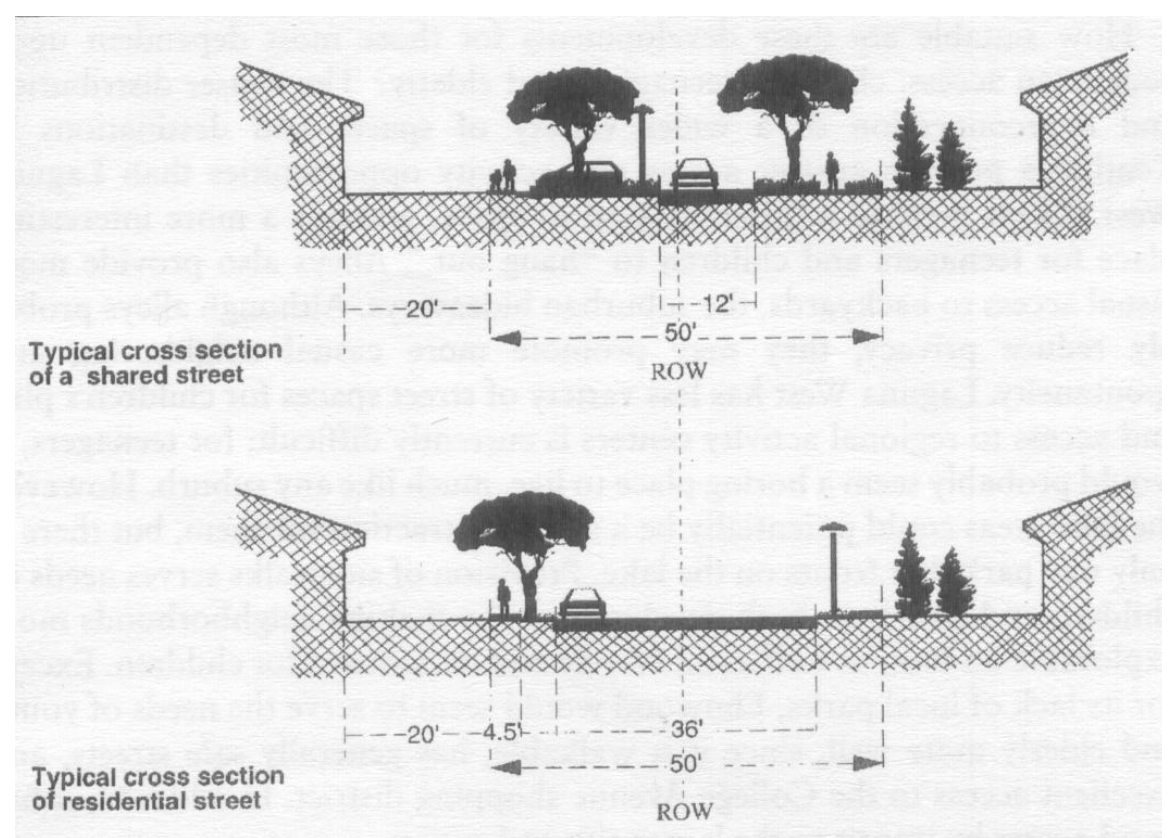

Fig. 4.4 Typical cross-section of a shared street (Southworth \& Ben-Joseph 119)

Within the shared street, driver behaviour changes significantly because of the realisation that this is primarily a pedestrian zone. Vehicles typically drive at about walking pace. Experience in several countries has shown that these are very safe environments for pedestrians and children (Southworth and Ben-Joseph 125, 126). 
Southworth and Ben-Joseph comment that unified street principles are usually applied to linear streets, but could be applied to any configuration and therefore have the potential to enable the development of new urban forms in which "pedestrians and drivers reach ... clusters of houses across a shared undemarcated surface. This arrangement has freed designers to develop new spatial patterns, unconstrained by the regularity of linear streets" (123).

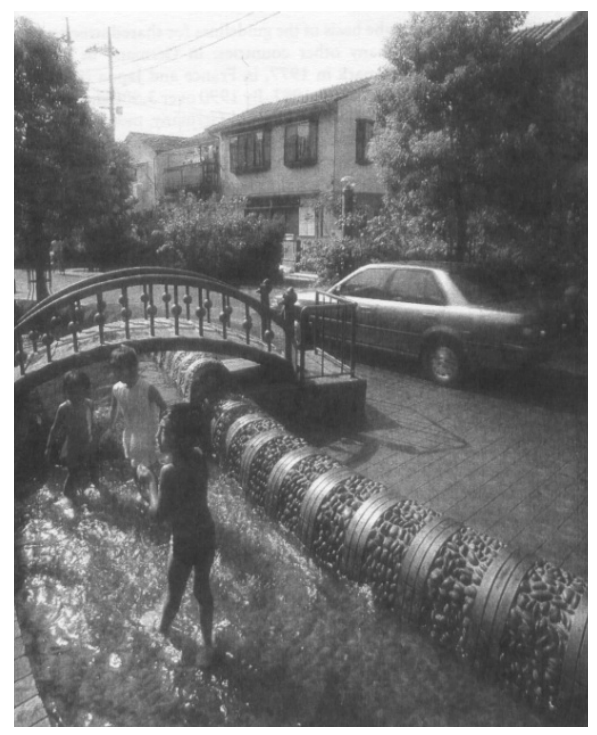

Fig. 4.5 A shared street in Tokyo (Southworth \& Ben-Joseph 121)

Fig. 4.6 A shared street in Israel (Southworth \& BenJoseph 119)

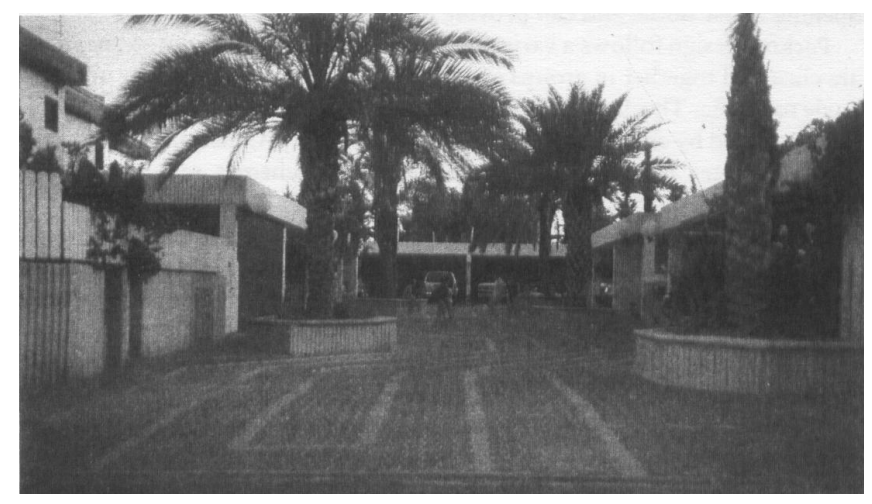

Southworth and Ben-Joseph state that unified street systems would be particularly appropriate in multi-family medium density housing developments. The shared street concept in housing developments would increase the area of usable space for residents, provide suitable outdoor play areas for children and improve the appearance of vehicle access and parking areas (127). 
Gehl (1996) classifies activities that bring life to outdoor spaces. These classes are:

- optional activities

- recreational activities, and

- social interactions (from passive low intensity to active high intensity)

Low-intensity social interactions are the natural, small starting points for larger, more engaging interactions, therefore it is important to provide optimum conditions for enabling, rather than impeding, the occurrence of long-stay, low-intensity contacts. Much street life would disappear if the only events in the street were simply the brief comings and goings of residents. A few long-lasting activities produce as much life as many short activities. Focus should be on providing opportunities for stopping and/or doing something in a purpose-designed, semiprivate realm on the street side of the houses (185).

Gehl provides detailed design guidelines for providing optimum conditions for small, semi-private front yards between indoor private space and outdoor public streets. These are transitional realms where opportunities occur daily for low-intensity contacts, assisting the development of more recreationally and socially active streets. Outdoor activities are influenced by, and very sensitive to, the quality of outdoor space (131). Therefore attention to detail is important for the activities of:

Walking (135f)

- Consider spatial sequences -

o winding streets, alternating street spaces and small squares psychologically make the distance seem shorter

- the experience of a large space is greatly enriched when approached through a small space (143)

- although people like shortcuts, there are advantages to moving along the edge of a large space rather than traversing through the middle of it

- from the edge it is possible to experience both the large space and the details of the boundary (e.g. building facades, niches, shelter, shade) 
Standing (149f)

- at the edges - alongside facades, in niches, beside columns (150). In residential areas, recessed entrances, porches, verandahs, and plantings in front yards serve the same purpose -psychological and physical protection, but still a good view (153)

- at the edge, one's back is protected, one is less exposed than out in the middle of a space, one can survey the space and move further into the space later if desired

- activities grow from the edge into the middle

- "If the edge fails, then the space never becomes lively" (Gehl citing C. Alexander)

- supports to stand in/by/against, e.g. recesses, corners, gateways, columns, trees, street lamps, bollards ...

- good cities for staying out in have irregular facades and a variety of supports in their outdoor spaces (155)

Sitting (157f)

- $\quad$ Sitting paves the way for other activities - reading, eating, sunbathing, watching people, talking ....

- Sitting generally only happens where external conditions are favourable

o on the edges of open spaces

- a local quality, e.g. a small space within a space, a niche, a corner ...

o intimacy, security

- a view of surrounding activities

- Primary seating - benches and chairs

- Secondary seating - steps, ledges, façade details, low walls, planter boxes, a fountain with a wide base ...

- Some primary and lots of secondary seating means a place will not look abandoned when only a few people are there

Seeing (165f)

- Spatial dimensions should correspond with the social field of vision

- maximum up to $70 \mathrm{~m}$ or $100 \mathrm{~m}$, for seeing events

- maximum up to $20 \mathrm{~m}-25 \mathrm{~m}$ for seeing facial expressions

- $\quad$ warm, friendly lighting - on people, faces, tables, signs ... not just on streets

Hearing and talking (169f)

O in pedestrian streets, the noise of cars is replaced by the sounds of voices, running water, children playing, music, footsteps ...

○ conversation (it helps if there's something in the social space to talk about) 


\section{Communal Open Space Can Enhance Mental Health}

Research findings show that accessible green space provides an affiliation with nature, which can enhance the mental health and vitality of city people (Kazmierczak and James 355)

A leisurely walk in green open space can have a positive impact on behaviour and social interaction by relieving the stress and mental fatigue often associated with living in urban environments (Kazmierczak and James 358). Note, however, that the restorative and social potential of green open space can be affected by conflicts between user groups e.g. dog-walkers versus parents with children, therefore becoming a potential generator rather than reliever of stress (359).

There can be positive benefits of working with plants (horticulture therapy) which can enhance self-esteem, aid recovery from depression and reduce aggression, allowing better social interaction (Kazmierczak and James 359). James et al (2009), a large interdisciplinary team, are developing a framework for a more integrated understanding of people's relationships with urban green space. 


\section{Communal Open Space Can Foster Inter-Generational Interaction}

Communal space in housing environments can play a supportive role in various developmental stages of the life cycle. Christopher Alexander et al. (1977) note Erik Erikson's seminal work in identifying developmental tasks associated with each stage of the human life cycle:

"Here is a summary of the stages in Erikson's scheme, adapted from his charts:

1. Trust vs. mistrust: the infant; relationship between the infant and the mother; the struggle for the confidence that the environment will nourish.

2. Autonomy vs. shame and doubt: the very young child; relationship between the child and parents; the struggle to stand on one's own two feet, to find autonomy in the face of experiences of shame and doubt as to one's capacity for self-control.

3. Initiative vs. guilt: the child; relationship to the family, the ring of friends; the search for action; to make and eagerly learn, checked by the fear and guilt of one's own aggressions.

4. Industry vs. inferiority: the youngster; relationship to the neighbourhood, the school; adaption to the society's tools; the sense that one can make things well, alone, and with others, against the experience of failure, inadequacy.

5. Identity vs. identity diffusion: youth, adolescence; relationship to peers and 'outgroups' and the search for models of adult life; the search for continuity in one's own character against confusion and doubt; a moratorium; a time to find and ally oneself with creeds and programs of the world.

6. Intimacy vs. isolation: young adults; partners in friendship, sex, work; the struggle to commit oneself concretely in relations with others; to lose and find oneself in another, against isolation and the avoidance of others.

7. Generativity vs. stagnation: adults; the relationship between a person and the division of labour, and the creation of a shared household; the struggle to establish and guide, to create, against the failure to do so, and the feelings of stagnation.

8. Integrity vs. despair: old age; the relationship between a person and his[/her] world, his[/her] kind, [human]kind; the achievement of wisdom; love for oneself and one's kind; to face death openly, with the forces of one's life integrated vs. the despair that life has been useless."

Erikson's life cycle developmental stages, adapted in Alexander et al. $(141,142)$ 
A stage in Erikson's scheme omitted by Alexander et al. is 'Middle Age' - 'creativity vs. stagnation' - the time when children leave home and important relationships become more of a focus with one's life partner and in serving society. Note also, children are brought up by others but also impact on the development of others:

"Babies control and bring up their families as much as they are controlled by them; in fact, we may say that the family brings up a baby by being brought up by him [her]."

Erikson, in Alexander et al. (143)

In Table 4.1 below, Alexander et al. list environmental settings which can support the life-challenges at each stage of the life cycle.

\section{STAGE}

1. INFANT

Trust

2. YOUNG CHILD Autonomy

3. CHILD Initiative

4. YOUNGSTER Industry

5. YOUTH Identity

6. YOUNG ADULT Intimacy

7. ADULT Generativity

8. OLD PERSON Integrity
IMPORTANT SETTINGS

Home, crib, nursery, garden

Own place, couples' realm, children's realm, commons, connected play

Play space, own place, common land, neighbourhood, animals

Children's home, school, own place, adventure, play, club, community

Cottage, teenage society, hostels, apprentice, town and region

Household, couples' realm, small work group, the family, network of learning

Work, community, town hall, a room of one's own

Settled work, cottage, the family, independent regions

\section{RITES OF PASSAGE}

Birth place, setting up the home ... out of the crib, making a place

Walking, making a place, special birthday

First ventures in town ... joining

Puberty rites, private entrance, paying your way

Commencement, marriage, work, building

Birth of a child, creating social wealth ... building

Special birthday, gathering, change in work

Death, funeral, grave sites

Table 4.1 Erikson's life cycle developmental stages, and supportive environmental settings, adapted in Alexander et al. (144) 
Environmental settings, including shared space, can facilitate inter-generational interaction. However, McKnight and Block note the vital role of 'connectors', i.e. people who take the initiative to bring others together, people who themselves are active in the community and well-connected. In "The Abundant Community", McKnight and Block give an example of two women who, over a two year period, initiated many individual and associational connections in their neighbourhood. One of the women sums up their community-building with these comments:

"What we have done is broken all the [dividing] lines. We broke the lines between the men. We broke the lines between the women. Then the lines were broken between the men and the women. And best of all, the lines were broken between the adults and the children and between all of us and our seniors. All the [dividing] lines are broken; we're all connected, and we're a real community now."

(Jackie Barton, in McKnight and Block 148)

Jackie Barton is referring to the ripple effect of mentoring schemes in her neighbourhood which began with one elderly man showing a teenage boy how to do lathe and forge work, and eventually giving him some paid work to do. They met when the boy noticed from the street that the man was doing some interesting metalwork in his garage. Other people began connecting over shared interests and skills - for the benefit of children learning from adults and also for adults learning such things as computer skills from children (McKnight and Block 145 - 148).

Dividing lines are borders within communities. Swanson notes young people using their resourcefulness to overcome borders (2010). In New Zealand, an organisation overcoming borders is 'Big Brothers Big Sisters' which provides mentoring for young people by appropriately skilled adults (Tindall Foundation 2010).

Garages and workshops (private or communal) facing public streets or communal outdoor spaces could facilitate spontaneous interaction between neighbours (adults and children) to share skills and interests, as in the Jackie Barton story above. 


\section{Communal Open Space Can Provide Places for Children's Play}

In "COHOUSING", McCamant and Durrett (1994) describe typical examples of families and groups of people, mainly in Denmark (from where the concept of cohousing originates), who have chosen to live together 'cooperatively':

"Each household has a private residence, but also shares extensive common facilities with the larger group, such as kitchen and dining hall, children's playrooms, workshops, guest rooms, and laundry facilities... a dark room for photography, a television room ... and a music room [for] teenagers ..." $(12,27)$.

Note the mention in the above quote of a range of indoor communal facilities for children of various ages - playrooms for young children; workshops; a photography darkroom; and a music room for adolescents. This section discusses potential use of communal space by children; the next section discusses spaces for adolescents.

Elizabeth Wood lists what she believes to be the basic needs of people, including children, living in housing developments:

1. There is a need for active exercise ... felt by all children ... the more limited the space within the dwelling, the more acute the need ...

2. There is a need for sunshine and fresh air ... felt by children, all mothers and babies, all old people...

3. There is the need to get 'out' ... felt by all housebound people, especially mothers with preschool children, all other children and the old. This need is felt no less when getting 'out' cannot mean getting 'outdoors' because the weather is cold and stormy.

4. There is the need to go somewhere; to shops, to church, to a movie ... the list is endless. It is felt by every human over the age of three or thereabouts.

5. There is the need to do some household chores ... outdoors ... washing the car, sunning the woollens, repairing the bike, drying the baby's clothes ... 
Wood suggests three design goals - design for visibility, design for leisurely activity, and design for the formation of informal groups. Her suggestions include:

- wide corridors, (preferably external for fresh air and sunshine)

- large, glazed lobbies (with public toilets attached)

- play-sitting areas for parents of preschool children (located close to building entrances)

- playgrounds for 5-12 year olds, close enough to the building so parents can overlook them

Elizabeth Wood, in Bell and Tyrwhitt, Eds. (329-333)

Alexander et al. assert that the built environment cannot come alive unless it is created by all members of the society who use it, and for this to happen requires a shared language for the built environment - a 'pattern language' (1977: x). 'Patterns', the authors explain, describe possible solutions to recurring problems or human needs in the built environment, solutions which, although applied differently in every unique situation, are intelligible to all.

Alexander et al. (1977) offer a comprehensive range of overlapping patterns, from the regional scale to building construction details, for assisting communities to develop languages for meaningful urban forms. The authors note that 'patterns' can be used prosaically or poetically (1977: xli). How 'patterns' are used depends on the skill of the designers. This suggests a role for professionals in assisting communities to imagine and develop their own 'best fit', poetic built environments.

There are many aspects of the city that affect children. Patterns offered by Alexander et al. which are particularly relevant to children are shown in Table 4.2. 


\begin{tabular}{|c|c|}
\hline Patterns for children in the city generally & Patterns for children in communal space \\
\hline \#14 - Neighbourhood Identity & \#37- House Cluster \\
\hline \#15 - Boundary (for urban legibility) & \#60 - Accessible Green \\
\hline \#25 - Access to Water & \#67-Common Land \\
\hline \#56-Bike Paths & \#68 - Connected Play \\
\hline \#57-Children in the City & \#73 - Adventure Playground \\
\hline \#59-Quiet Backs & \#86 - Children's Home \\
\hline \#64 - Pools and Streams & \#106 - Positive Outdoor Space \\
\hline \#71 - Still water & \#114 - Hierarchy of Open Space \\
\hline \#74 - Animals & \#115 - Courtyards Which Live \\
\hline \#92-Bus Stop & \#125 - Stair Seats \\
\hline \#93 - Food Stands & \#129 - Common Areas at the Heart \\
\hline \#120 - Paths and Goals & \#137 - Children's Realm \\
\hline \#123 - Pedestrian Density & \#147 - Communal Eating \\
\hline \multirow[t]{8}{*}{ \#124 - Activity Pockets } & \#163-Outdoor Room \\
\hline & \#165 - Opening to the Street \\
\hline & \#167-Six-Foot (1.8m) Balcony \\
\hline & \#168 - Connection to the Earth \\
\hline & \#172 - Garden Growing Wild \\
\hline & \#192 - Windows Overlooking Life \\
\hline & \#203 - Child Caves \\
\hline & \#204 - Secret Place \\
\hline
\end{tabular}

Table 4.2 Design patterns for children - from "A Pattern Language" (Alexander et al. 1977)

Cooper Marcus and Sarkissian comment that 5-12 year olds will probably be the main users of communal outdoor space (this thesis challenges this conventional viewpoint, proposing that communal spaces be of significant value to all residents). They recommend that children be included as planners of outdoor play spaces, and that these spaces be designed for both boys and girls, and for different seasons of the year (135-184). 
Cooper Marcus and Sarkissian (1986) provide comprehensive design guidelines for accommodating the needs of children in clustered housing developments, including:

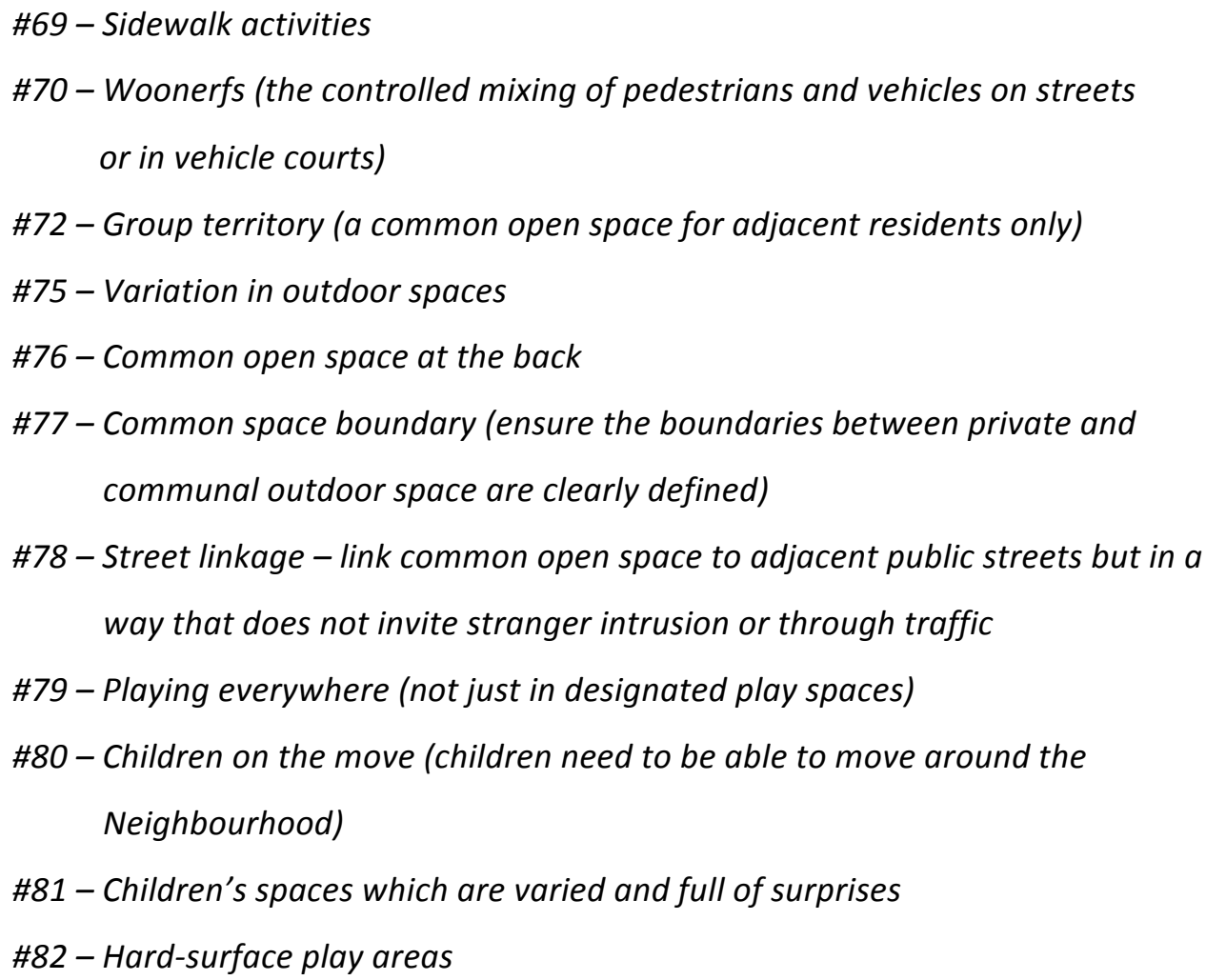

"Common Open Space and the Needs of Children" (107-134)

- For Pre-school Children - Cooper Marcus and Sarkissian provide comprehensive advice on a wide range of children's needs, and facilities to accommodate them, including yards, balconies, doorsteps, tot lots, sand, water play etc. (138-150)

- For Five to Twelve Year Olds - Again, there is extensive advice on providing opportunities for mobility, biking, ball games, equipment variety, flow of play, lookouts, adventure playgrounds, huts and self-made enclosures etc. (151-179)

$$
\text { "Purpose built play areas for children" (135-179) }
$$

(Note: For teenagers' recreational needs, see the next section.) 
Children's playgrounds designed by Aldo van Eyck provide good examples of multivalent outdoor spaces. Jencks credits van Eyck's work with high levels of multivalence $(13,313,316)$. Children's playgrounds formed a significant part of Aldo van Eyck's work (although they receive no attention from Jencks).

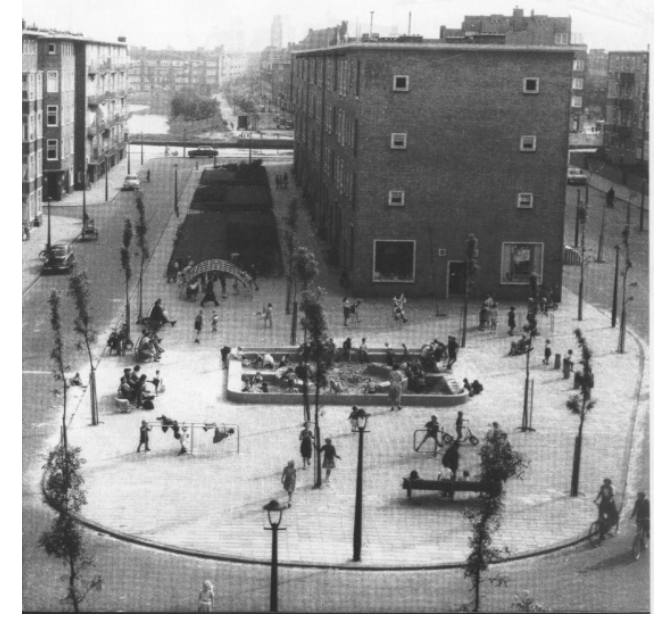

Fig. 4.7 Van Eyck - street playground, Amsterdam (Lefaivre and de Roode, 21)

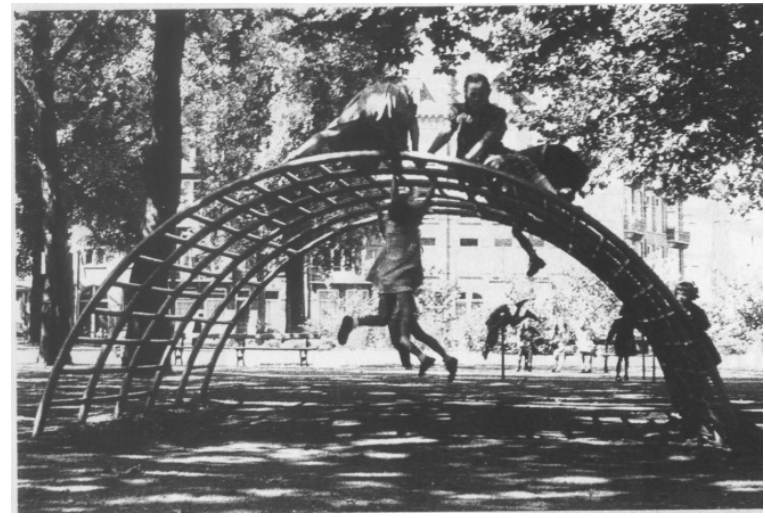

Fig. 4.8 Van Eyck - Play frame, Amsterdam (Lefaivre and de Roode, 68)

In "Aldo van Eyck: The Playgrounds and the City" (c2002: frontispiece) the editors Liane Lefaivre and Ingeborg de Roode write that Aldo van Eyck (1918 - 1999), throughout his architectural career, produced many designs for children's playgrounds for Amsterdam, The Netherlands. Van Eyck estimates he designed about 700 playgrounds, but more than 860 are on record as being built (Lefaivre and de Roode 25). A synonym Jencks uses for multivalence is 'multi-meaning' (316). Van Eyck's playgrounds can be considered multivalent because they can be read with several layers of meaning, including: 
- that 'children belong in the city'

- that children are valued participants in the creation of the city (van Eyck's own children assisted him in the design of playgrounds)

- that playground equipment can serve as territory markers in city streets for children, ensuring their welcome involvement in city vitality and signalling to drivers of vehicles the need to reduce speed

- that well designed playgrounds can serve as public sculpture and art

- that given the simplest of props children are capable of much creativity and social cooperation

- that bodily movement and coordination are recognised as important to children's health and physical development

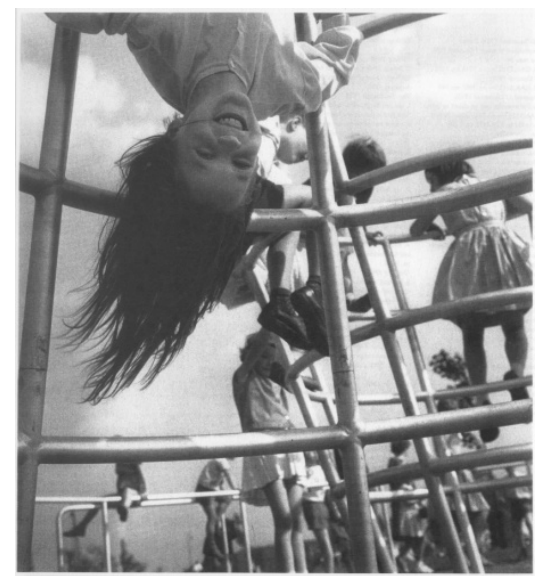

Fig. 4.9 Van Eyck - Play frame (Lefaivre and de Roode, 102)

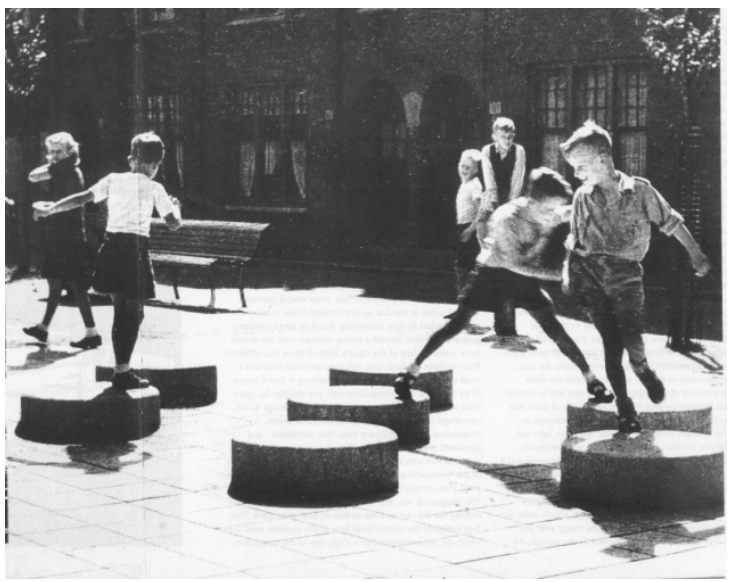

Fig. 4.10 Van Eyck - Stepping stones, street in Amsterdam (Lefaivre and de Roode, 69)

Rudi Fuchs, recalling his own childhood delight in Amsterdam in the 1950s playing on the playground equipment designed by van Eyck, comments that:

"The playgrounds were fantastic because the objects were simple: rectangular and round frames for climbing (the latter like an igloo), a sandpit, a group of circular concrete blocks for jumping from one to another. ...

"A child sits still on a slide or a swing: it is the object that produces the movement. Van Eyck's objects do not move, but they allow a child to move ..." (Lefaivre and de Roode 7) 
Child obesity is a significant contemporary issue. It is important to find ways to get children outdoors and moving about more (see, for example, Kyttä 2004; Woodward 2010).

The design of playground equipment can facilitate movement. Van Eyck's simple playground objects - sculptured concrete discs (used as seats, play tables or jumping / stepping stones) and tubular climbing frames (arches, domes and funnels) - allow the child to do the moving. Many contemporary playgrounds consist of equipment that produce movement for the child - swings, seesaws, roundabouts, and rockers mounted on springs - rather than allowing the child to do the moving.

Most of Van Eyck's playgrounds were located on city streets and within courtyards of housing developments. Children will play everywhere; but it is important to create special places for children's play and socialising (AMCORD PND21).

Facilities for children should be located within sight and calling distance of most dwellings. They should be located so as not to cause annoyance to nearby dwellings but contained in an area and able to be observed from the work areas of the home (AMCORD PND11 - 6). It is especially important to consider the visibility of children under supervision by a single parent in the layout of both indoor and outdoor areas (AMCORD PND21).

Cooper Marcus and Sarkissian (107) note that early Radburn-type schemes, which separated pedestrians from traffic, were criticized. This was because ill-defined green-belt areas at the back of houses meant for children's play remained virtually unused. Instead, many children played on the roads and culs-de-sac. Parents worried about their safety. Later layouts improved on earlier Radburn-type schemes in the following ways:

- $\quad$ private and communal spaces were clearly demarcated; 
- communal spaces in the green-belts behind houses comprised interesting sequences of large and small open spaces with paths suitable for playing on, equipped play areas, and safe linkages to neighbourhood shops and schools.

The authors note that at an improved Radburn-type layout at Pin Green, Stevenage, UK, 85 percent of the children used these communal open spaces for play instead of the roads and parking areas. The child accident rate was about half that of a nearby conventional street-oriented layout. The authors comment that the key to the improved layouts is the purposeful design of the communal spaces. 


\section{Communal Open Space Can Provide Places for Teenager's to 'Hang-out'}

"COHOUSING" authors McCamant and Durrett (1994) note that communal facilities for teenagers can include “... workshops, guest rooms, ... a dark room for photography, a television room ... a music room where teenagers can 'jam' on drums and electric guitars ..." $(12,27)$. It is advisable to locate spaces for youth so they don't cause nuisance to neighbourhood dwellings (AMCORD PND11 - 7).

Teenagers need privacy and personal space indoors, plus access to public outdoor space "where they can watch others and be seen. They need areas to sit and socialise as well as spaces where they can be physically active" (AMCORD PND21). Wood suggests that places for teenagers to 'hang out' be located away from residential buildings but where there is plenty of pedestrian activity (333).

Cooper Marcus and Sarkissian note:

"Teenagers have many of the same needs as adults - places to get together, to have fun, to compete in sports - yet unlike many adults, they are dependent on public transport. Hence if these places are not within easy access of home, teenagers are likely to become bored and frustrated; some may even begin to engage in vandalism to relieve boredom" (180).

Cooper Marcus and Sarkissian offer suggestions for a range of facilities for teenagers, including space for ball games, hanging out (informal gathering places), a teen centre, teen entertainment, and access to off site facilities. For specific design guidelines oriented to teenager's needs, see the patterns suggested by Cooper Marcus and Sarkissian (180-184 "Recreation Opportunities for Teenagers").

Cooper Marcus and Sarkissian point out that different age groups can use common outdoor spaces at different times of the day. For example, parents may bring infants out into communal outdoor spaces to enjoy the morning sun, followed by school children in the afternoon, followed by teenagers hanging out in the evenings. 
Patterns by Alexander et al (1977) relevant to teenagers include:

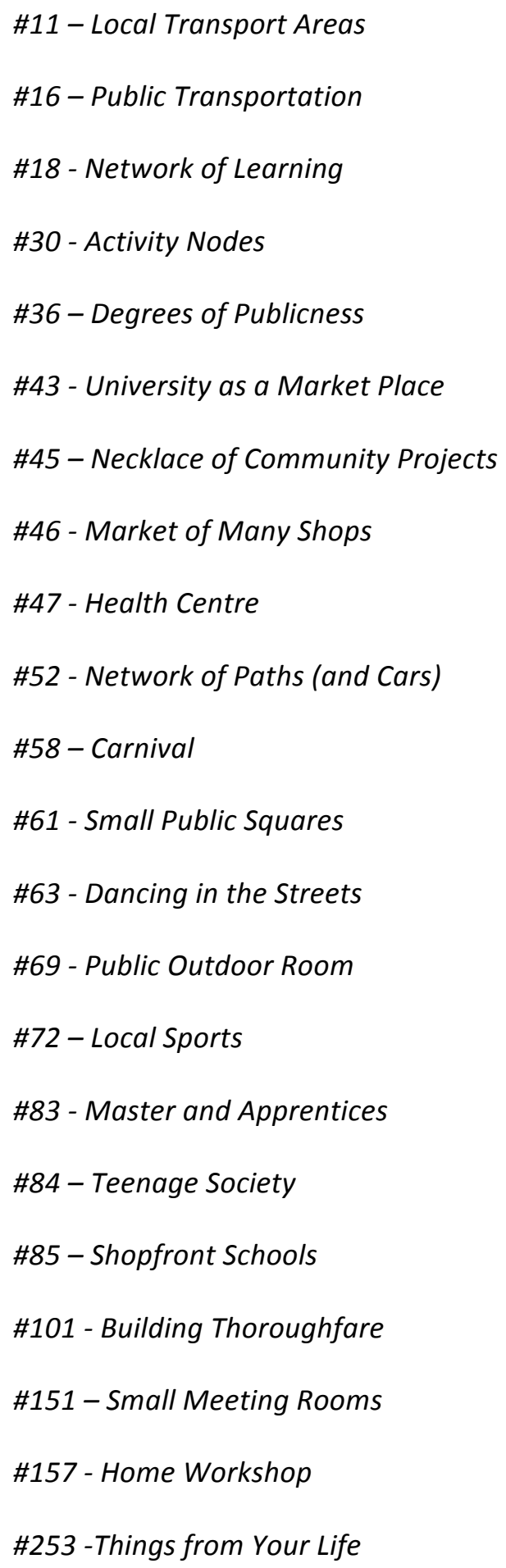




\section{Territorial Communal Space, Safety and Crime Prevention}

Le Corbusier's 1947 Unité d' Habitation in Marseilles, France, is rated by Jencks as a great multivalent work (14-20). This housing megastructure for 1,600 inhabitants meets all Jencks' criteria for multivalence. Jencks praises Le Corbusier's 'creative' inversion of the diverse parts of the Unité - the elevation of everyday domesticity to the level of public monument, raised above ground level on massive columns that taper downwards instead of upwards; the gardens and landscaping high up in the air on the roof of the Unité; the 'streets in the air' inside the building instead of outside on the ground; a shopping centre on the $7^{\text {th }}$ floor instead of connected to the commercial centre of Marseilles.

The Unité d' Habitation may provide a popular mode of living for professional singles and couples. However, it is the direct antithesis of the aim of this research. Le Corbusier's housing towers, stripped down for economical solutions to social housing, have created social problems. Towers at Pruiit Iggoe, St Louis, USA, were demolished because of the high levels of vandalism and crime associated with them.

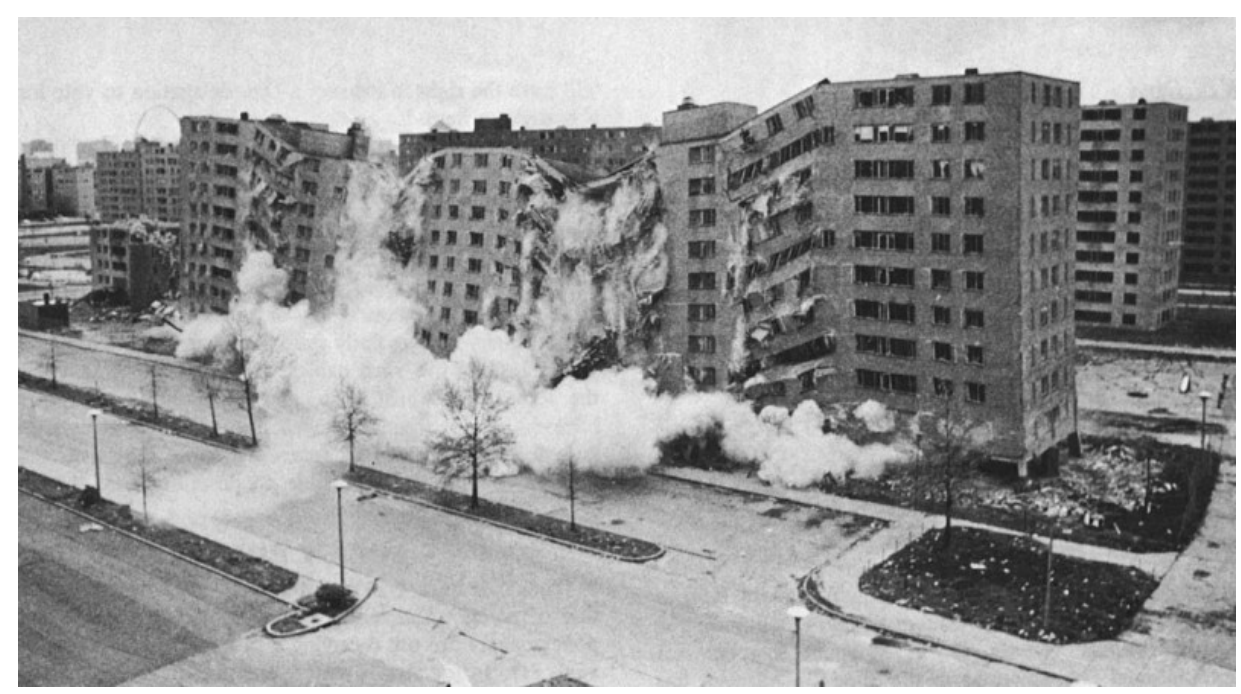

Fig. 4.11 Demolition of Pruitt-Igoe housing super-block development in 1972 in St Louis, Missouri, USA, following a history of persistent crime and vandalism (built 1954-55). Source: http://en.wikipedia.org/wiki/Pruitt-lgoe 


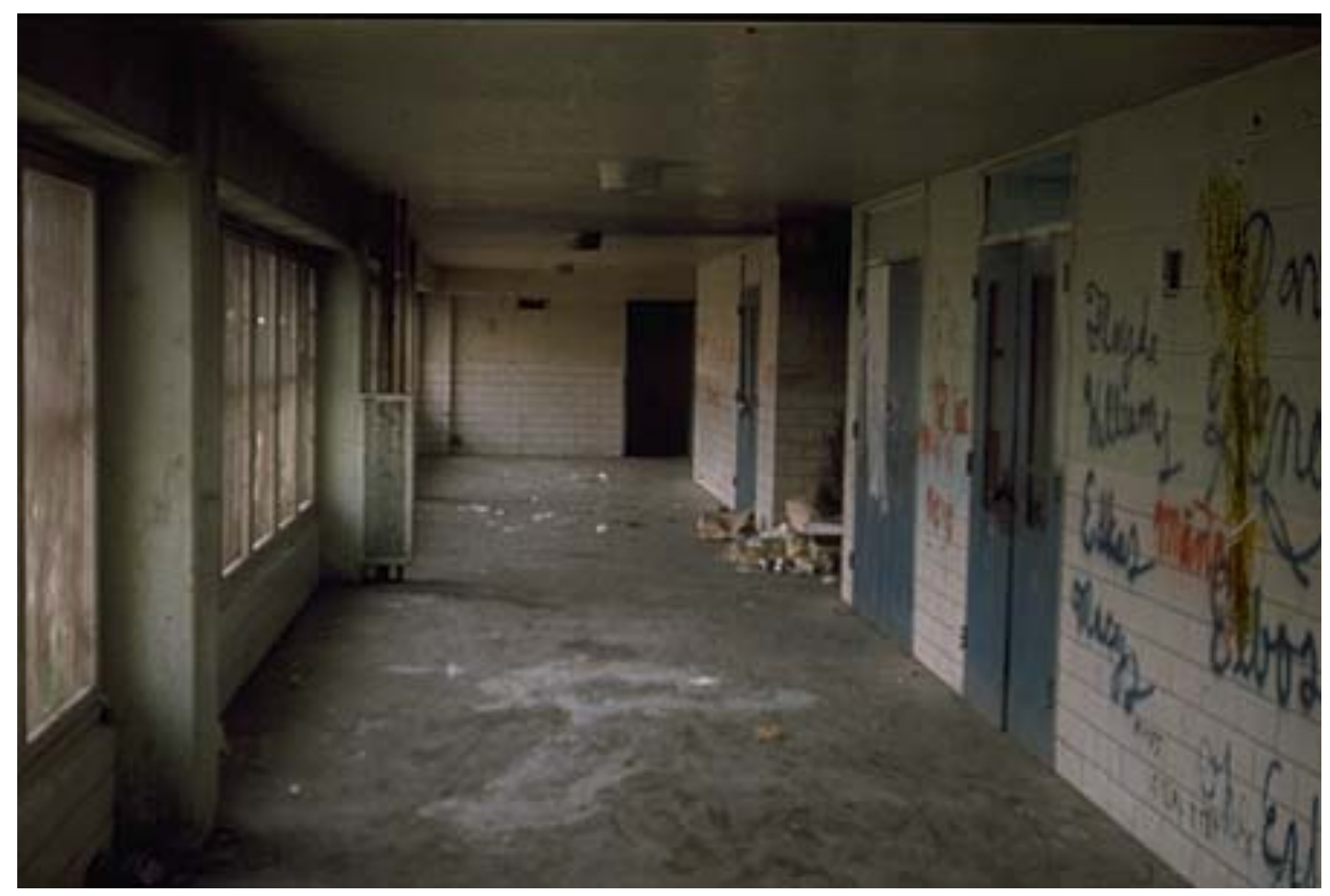

Fig. 4.12 A vandalized interior public lobby in Pruitt-Igoe housing, St Louis, Missouri, USA. http://en.wikipedia.org/wiki/Pruitt-Igoe

Oscar Newman (1972) concludes that ill-defined territoriality of public, private and communal space (external and internal), is a basic design flaw of tower developments such as Pruitt-Igoe, resulting in unmanageable social problems (Figs. 4.11 \& 4.12). Newman comments that:

"Defensible space is a model for residential environments which inhibits crime by creating the physical expression of a social fabric that defends itself."(3)

According to Newman, four design elements which make space defensible are:

1) The territorial definition of space in developments reflecting the areas of influence of the inhabitants. This works by subdividing the residential environment into zones toward which adjacent residents easily adopt proprietary attitudes;

2) The positioning of apartment windows to allow residents to naturally survey the exterior and interior public areas of their living environment; 
3) The adoption of building forms and idioms which avoid the stigma of peculiarity that allows others to perceive the vulnerability and isolation of the inhabitants;

4) The enhancement of safety by locating residential developments in functionally sympathetic urban areas immediately adjacent to activities that do not provide continued threat. (9)

Newman illustrates the idea of territorial definition through schematic sketches:

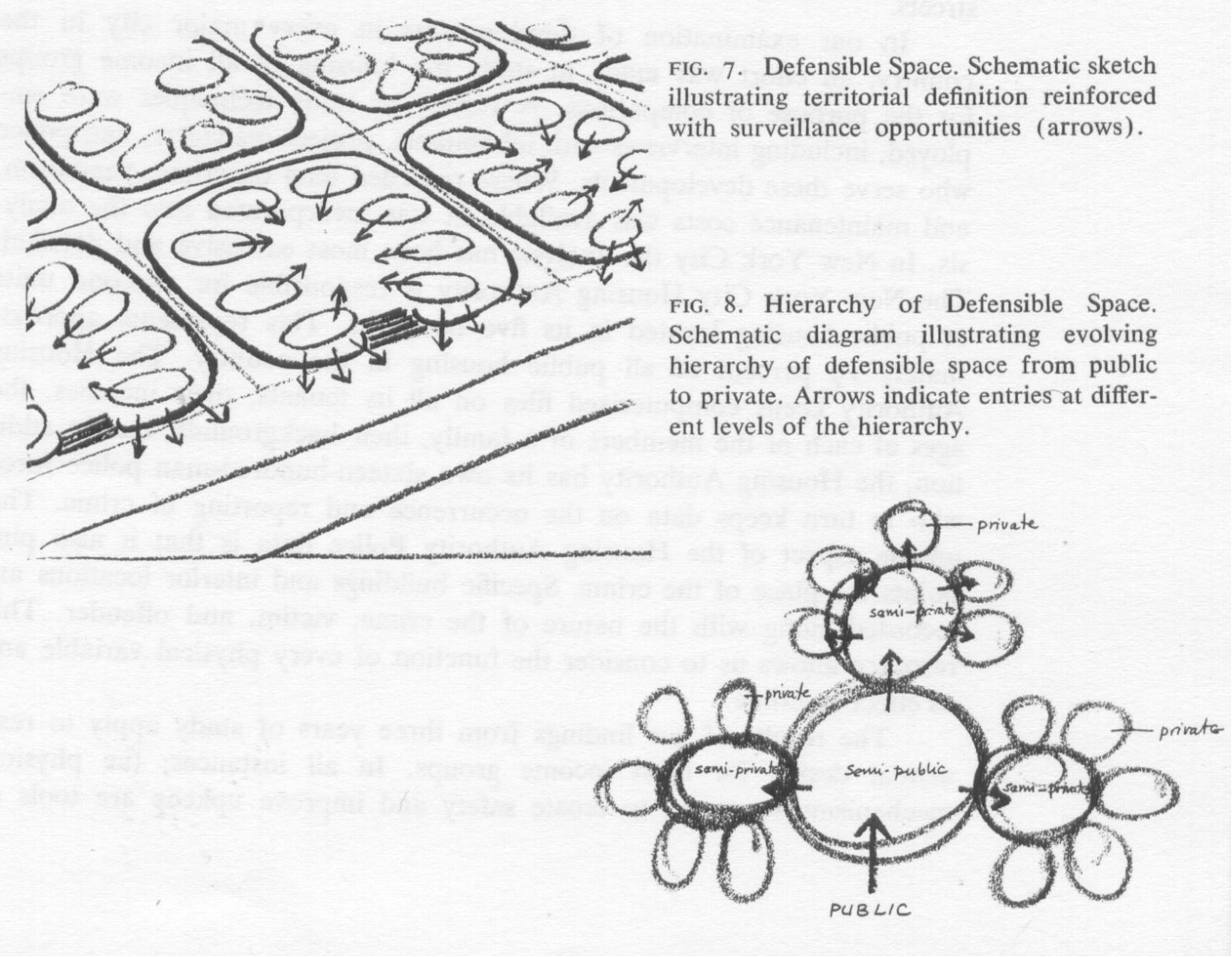

Fig. 4.13 Hierarchy of Defensible Space (Newman 9)

Sommer describes how defence of territory is dependent on visible boundaries and markers (63-64). Altman discusses the importance of personalisation and ownership of territory to regulate social interaction (107). 


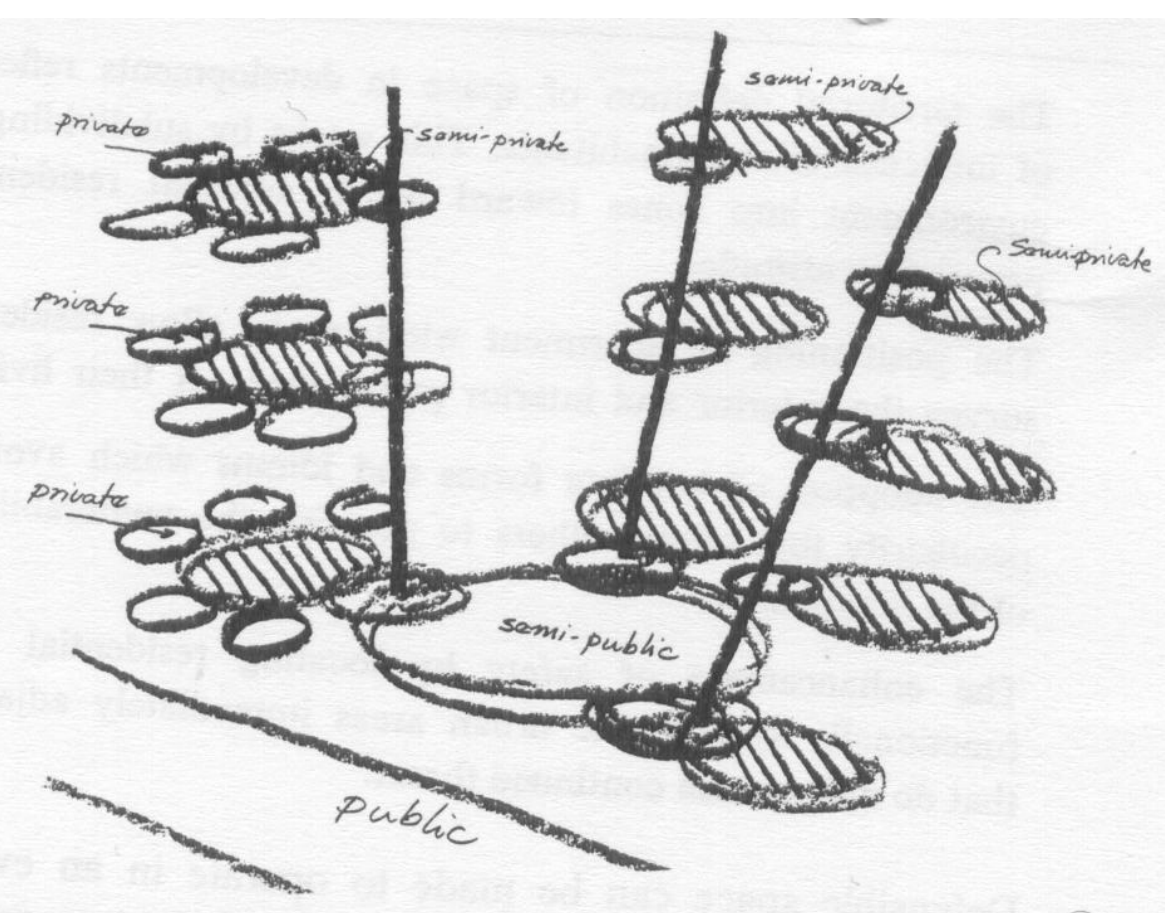

Fig. 4.14 Defensible space hierarchy in a multi-level building (Newman 10)

Guidelines for crime prevention address a number of design issues for public safety in multi-unit housing developments. For example, AMCORD, the Australian Model Code for Residential Development, advises clear boundary delineation of public and private spaces to discourage intrusion; casual surveillance of open spaces from adjacent dwellings; and making open space attractive to legitimate users so that the presence of people will discourage anti-social activities (AMCORD PND17).

For multi-unit dwellings, the AMCORD guidelines recommend visually permeable fencing, and landscaping that is either low ground cover planting or high canopy trees so as to assist visual surveillance of communal areas for public safety. Other advice includes locating footpaths so they do not intrude on private areas, and selecting vandal resistant materials for common open spaces. (AMCORD PND11) 
The National Guidelines for Crime Prevention through Environment Design In New Zealand (Ministry of Justice 2005) cover similar considerations for safer places, including safe access and connections, seeing and being seen, logical layouts and orientation of buildings, mixing activities to ensure eyes on streets and public spaces, showing that a space is owned, maintained and cared for, and using active security measures.

Housing New Zealand Corporation's recent redevelopment project at Talbot Park, Glen Innes, serves as a good example of the application of CPTED principles to a social housing development. The Ministry for the Environment Urban Design Case Study (2008) on "Community Renewal - Housing New Zealand Corporation, Talbot Park, Auckland" comments that before the site was redeveloped residents felt unsafe in the internal public green space (68). The green space was an ill-defined strip of common land running through the middle of the five hectare site with housing along the edges of it, "based on the 'Radburn' concept" (68). Many such strips of common land can be found in the surrounding areas of Glen Innes and Pt England - grassed but generally deserted strips of public land running along natural gullies behind solid-fenced back yards of houses.

The Radburn Plan, so named because it was first developed in 1928 at Radburn, New Jersey, USA, modified the Garden Suburb in an arrangement that separated cars from pedestrians. It sought to achieve this by providing pedestrian green-belts at the rear of houses and reversing the houses to face the green-belts and back on to the streets (Untermann and Small 67). Although popular and widely adopted, early Radburn-type schemes were criticised for their deserted, unsafe green-belts resulting from lack of pedestrian use: children chose to play on the roads instead of in the green-belts (Cooper Marcus and Sarkissian 107, 269). 
Comments received by the writer from local people in Glen Innes confirm the Talbot Park Case Study findings that people in the area feel unsafe using the green-belts at the rear of houses. The Case Study notes that improving neighbourhood safety and reducing crime was one of the primary objectives of the Talbot Park Community Renewal programme (68). The internal green spine was removed and replaced with two new separate parks with improved urban relationships to residences - visual permeability, connectivity, dwellings overlooking the parks, and the provision of a variety of children's playgrounds and open space (69).

One resident in the redeveloped Talbot Park project commented to the writer that, by comparison, she feels much safer herself, and much happier about her children's safety, in the new Talbot Park scheme. She attributes this to the closeness of neighbours, who she feels would respond quickly to any calls for help, and the fact that people can see across streets, open spaces, car parking and children's play areas from their windows and entry doors [fences are visually permeable and plantings are low]. This resident's comments support the Case Study finding that the reconfiguration of the area has successfully addressed the main crime and safety concerns raised by residents (77). This is also borne out by the new low tenant turnover at Talbot Park - previously about 50\% per annum, it now sits at about 5\% (76). The Case Study notes:

"A fundamental endorsement for the project is that HNZC housing applicants are now specifically requesting to live in the Talbot Park project area." (78)

The Good Solutions Guide for Medium Density Housing (Auckland Council) mentions the importance of clearly defining public and private space. It states that:

"Houses should have clearly defined public fronts and private backs. ... windows enabling residents to see what is happening in their local community are also desirable. Ensuring house fronts face other house fronts across a public street, and backyards face other backyards is the best layout to achieve this". 
An urban designer at Auckland Council explained to the writer that the Council's current focus is on encouraging developers to buy several adjacent detached houses and amalgamate the titles for intensive housing development. The aim is to create active street frontages by facing terrace housing to the street, accessed by rear lanes, rather than facing housing to access ways perpendicular to the street.

Clear definition of open space is required for residents to feel a sense of ownership of it. Biddulph distinguishes four types of urban space - public (easily accessible to the general public at any time of the day or night); semi-public (public space with a greater degree of control over access, e.g. management may close a space to the public after hours or at night for security); semi-private (a member of the general public will only enter if they have a reason to, e.g. a front yard of a house); and private (space that is exclusively for the residents of a property) (43-44).

Levitt refers to "semi-private/semi-public shared space" (111). Such a phrase typifies a widespread lack of clarity on the nature of shared space. Chermayeff and Alexander offer a way of differentiating six domains of spatial hierarchy (121-122):

Urban-Public The places and facilities in public ownership: roads, paths etc.;

Urban-Semi-public The special areas of public use controlled by government or institutions: city halls, schools, hospitals, garages, theatres etc.;

Group-Public The meeting ground between public services and utilities and private property: e.g. mail box, rubbish collection, utilities;

Group-Private Various areas controlled by management for tenants or occupants: reception, community gardens, playgrounds etc.;

Family-Private Spaces within a private domain for communal activities: eating, entertainment, hygiene, and maintenance;

Individual-Private A 'room of one's own', the inner-most sanctum for individuals

Chermayeff and Alexander note that terms such as 'space' or 'zone' are abstract, but the above six domains are social as well as functional, and easily visualised. 
Biddulph describes shared communal courtyards as semi-private spaces, which he envisages as being at the back of a group of houses - typically the central open space of a peripheral block arrangement $(44,48)$. The surveillance of strangers is important in these communal areas (AMCORD PND21).

For security, the entries of most dwellings should be visible from inside other dwellings. Communal entries should be visible to passersby. Avoid "hidden space, overgrown areas or planting or fencing that may screen intruders". If public spaces are well designed they will attract more people who will use them, making public places even safer (safety in numbers) (AMCORD PND11 - 8).

The Good Solutions Guide for Apartments (Auckland Council) notes that open spaces should be safe and secure, incorporating casual surveillance (17):

"Open space may be public (accessible to members of the general public), communal (shared by residents) or private (associated with a single dwelling for the exclusive use of the occupants)." (17)

Furthermore, the 'Apartments' guide advises that a clear, well-defined hierarchy of outdoor spaces ensures there is no ambiguity or left over areas (17). Another important point is that private open space for dwellings should be "clearly defined and distinct from communal areas" (AMCORD PND11 - 4).

Elizabeth Wood (in Bell and Tyrwhitt) comments ...

"What has been completely lacking is ... design based on a theory of what kind of social structure is desirable in a [housing] project and how to use design to get it. Such a theory would be expressed almost exclusively in the design of the space outside the dwelling units.

"Most housing projects seem designed to minimize or to prevent accidental and casual communication between people and the informal gathering of people ...

"... [T]he rich fulfilment of the needs of people, of individuals and groups, is in itself a suitable design objective. Design to serve this objective serves also a larger purpose: it makes possible the development of a social structure, by means of which people can create their own social controls, and do their own self-policing." (327) 


\section{Communal Open Space Can Provide Opportunities for Community Participation}

"Community involvement in the design and management of recreational facilities and open space areas is essential. This helps to ensure high-quality safe and diverse recreational opportunities and activities that:

- meet the needs of current and future populations,

- are safe for users, and

- enhance opportunities for integration, multiple use and sharing between compatible activities."

(AMCORD PND5)

A key factor in the success of the redevelopment of Talbot Park (see p.95) is community participation "by involving local people and resources in addressing local problems" to "build social networks to facilitate residents supporting each other" (NZ Ministry for the Environment, Urban Design Case Studies 68). This case study exemplifies the role of architects, planners and other professionals in assisting communities to imagine, articulate and develop, among other things, multivalent communal spaces in housing environments.

David Walters promotes the idea of architects and urban designers working with individuals and communities to help them visualise their ideas in a participatory design and planning process. He notes that the value of this approach is that, in addition to considering physical aspects of the environment, it brings the social and cultural dimensions of community into the design process as well (79). Hamdi notes that community participation is ...

"... an integral part of making design and planning efficient and effective ... It cultivates ownership and, with it, a sense of belonging and responsibility, both of which are important to the health of a place and of community." (xvi)

Kazmierczak and James advocate resident participation in designing and caring for green open space for the benefit of social interaction and community cohesion. They mention there exists some 4,000 community groups in the UK involved in 
green space management, and that a CABE survey indicates that $75 \%$ of the population would like to be involved in improving their local area in some way (359).

Richard Toy describes the bay-form as the expression of community connectedness (see pp107-109). A New Zealand documentary, "A Place to Stay", explores the interaction of a bay-form (a cluster of houses around a tennis court) and the 'sense of community' at Salisbury Garden Court, Wadestown, Wellington, built in 19291930. A quote in the documentary refers to creating places of shared use:

"A primary task of all urban architecture and landscape design is the physical definition of streets and public spaces as places of shared use."

Charter of the New Urbanism (Quoted in 'A Place to Stay')

The documentary asks: "Is the heritage value of these houses in their architecture, or is there something special about their layout ...?"

Michael Donn, Victoria University of Wellington School of Architecture, responds: "What's special about the community is the grouping of the houses ... they sit well to the land and to this courtyard, and [socially] it appears to work." (in 'A Place to Stay')

That the central court layout can foster 'community' is confirmed by a resident:

"...the tennis court ... got a fair bit of use ... it was very much a community activity area. ... everyone looked after everyone else. There was a lot of comradeship built up ..."

Pat Bloomfield, former resident (in 'A Place to Stay')

Urban form can support community participation; community participation is essential to the creation of meaningful urban form. Regarding the move towards 'shared street' forms, Southworth and Ben-Joseph note that "public information about shared streets is non-existent, yet the involvement of neighbourhood residents ... is crucial for [its] acceptance" (128). Lozano sees designers and design activities having a significant role as "valid agents of change" $(67,78)$. 
Communal Outdoor Space Can Enhance the Environment and Improve Property Values

Kazmierczak and James note that urban green space improves property values:

"... good quality urban green space ... is characterised by ... physical access, ... facilities for all social groups, security measures ... cleanliness, visually pleasing layout, diverse vegetation structure and presence of wildlife ... Such spaces provide aesthetic experience, improve the quality of the urban environment and increase property values." (355)

Conserving trees and greenery and encouraging biodiversity in towns and cities enhances the quality of the urban environment (Simmons et al. 1990; TCPA 2004). Jensen et al. (2004) note there is a positive correlation between greenery in residential areas and median income and house values. New Zealand Case Studies show that quality communal open space in medium density housing can contribute significantly to the financial value of the housing. Two examples are indicative of this, one at the high end of the residential property market and the other aimed at housing for first time home buyers. The density of both schemes is exactly the same, at 56 dwelling units per hectare.

The "Terraced Housing in Takapuna - North Shore City" Urban Design Case Study (see p30) notes this development provides a good example of urban intensification. It consists of eighteen high quality terrace houses arranged around three sides of a quality central communal court yard (85). The development is ideally located by the beach and close to Takapuna town centre. The design deliberately preserves three mature trees on the site including a very large silky oak which is a prominent feature in the courtyard by the communal swimming pool. The study notes that the high quality of the design "makes a positive contribution to the urban environment" (88). The study reports that the developer "is very happy with the ... fiscal return", and that marketing "went exceptionally well". It also reports that: 
"subsequent ... resale value of the apartments increased significantly. While this has been driven in part by demand [reflecting, no doubt, the location of the development], the developer's investment in the design and construction quality has been a major contributing factor" (89).

A significant feature of this successful design is the "inner communal courtyard. The arrangement of the units around the site perimeter provides a good relationship to the street while also allowing for generous communal open space ..." (85).

The other Case Study is of a housing scheme at Bush Road in Albany. It is noted in Chapter 3 (23-24) that in assessing thirty medium density housing developments in Auckland, Turner et al. rated only five schemes positively regarding the quality of open space, this Bush Road scheme being one of them. All five schemes feature quality communal spaces and shared facilities, the Bush Road scheme including a tennis court and swimming pool surrounded by gardens. It has 105 small units and is aimed "to provide low cost housing for younger buyers" (62). The study notes "market price expectations at lower levels" for various reasons, but the scheme demonstrates the benefit of quality communal open space to affordable housing.

An important component of resident satisfaction is the maintenance of communal open spaces in medium density housing. Cooper Marcus and Sarkissian emphasise the importance of identifying early in the planning stage the responsibility for maintenance, which may follow one of four approaches: by the landlord; by Council (for large spaces accessible to the general public); by a 'special service district' (USA) with costs levied to residents; or by a home owner association (where dwellings are owner-occupied and shared open space is for the exclusive use of residents, not the general public). For home owner associations, the authors recommend they be set up before units are sold, membership be mandatory, responsibilities be permanent and include insurances, taxes and maintenance, that costs be assessed on a pro rata basis, and that these requirements be adjustable to meet changing needs (133-134). 


\section{Communal Open Space Can Foster Urbanity}

There are three important interrelated concepts to be considered:

i. urbanity thresholds

ii. 'walkable urbanism', 'driveable suburbia' and 'Neverlands'

iii. the bay structure as the archetypal form for community connectedness

\section{i. Urbanity thresholds}

Leinberger explains one way, called FAR, in which real estate is measured:

"...development patterns are defined by density; by how much is built on a particular area of land. Real estate professionals measure density through 'floor area ratio' (FAR), which is the ratio of the amount of building ... to the amount of structure on which the building sits. For example, if a 100,000-square-foot building is placed onto a 100,000-square-foot piece of land, it will have a FAR of 1.0. That building could be five stories high with each floor 20,000 feet square, or it could be one story and occupy the whole site; in both cases the FAR would be 1.0. Another example is 10,000 square feet of building on 100,000 square feet of land; the FAR is 0.10. This is regardless of whether it is five stories of 2,000 square feet per floor or one story of 10,000 square feet. Going the other direction toward higher densities, if the building is 1,000,000 square feet sitting on 100,000 square feet of land, the FAR is 10.0-a much higher density with at least 10 stories of 100,000 square feet per floor. " (113-114)

Lynch and Hack provide data for alternative measures of density by house type:

\begin{tabular}{|l|c|r|r|}
\hline & & \multicolumn{2}{|c|}{ Families [dwellings] per hectare (per acre) } \\
\hline & & & Neighbourhood \\
\hline & FAR & Net density & [gross] density \\
\hline Single-family [detached] & up to 0.2 & up to 20 (8) & up to $12(5)$ \\
\hline Zero lot line detached & 0.3 & $20-25(8-10)$ & $15(6)$ \\
\hline Two family detached & 0.3 & $25-30(10-12)$ & $18(7)$ \\
\hline Row houses & 0.5 & $40-60(16-24)$ & $45(12)$ \\
\hline Stacked townhouses & 0.8 & $60-100(25-40)$ & $50(20)$ \\
\hline 3 story walkup apartments & 1.0 & $100-115(40-45)$ & $75(30)$ \\
\hline 6 story elevator apartments & 1.4 & $160-190(65-75)$ & $100(40)$ \\
\hline 13 story elevator apartments & 1.8 & $215-240(85-95)$ & \\
\hline
\end{tabular}

Table 4.3 Densities by Residential Type (Lynch \& Hack, 1984, Table 5, 253) 
Leinberger explains that 'dwellings per hectare' (dph) or 'dwellings per acre' (dpa) may be an appropriate way to measure residential density but not commercial or industrial uses. The advantage of FAR is that it can be used to measure density for all real estate uses (191). This is particularly helpful for mixed-use developments.

Regarding 'net' and 'gross' densities, there are no universally agreed definitions for these terms. For FAR, Leinberger provides this distinction between 'gross' and 'net': "Gross FAR includes all the land used for transportation circulation (streets, sidewalks, transit lines, parks, stand-alone parking lots and decks, etc.) as well as the buildings and the parking lot or decks connected with the building. Net FAR includes only the buildings and the land under the parking lot or decks connected to the buildings in its calculation. Gross FAR will be a lower number than net FAR when measuring a particular building because the denominator (land) is larger than in the calculation for net FAR while the building square footage is the same. Gross FAR can be applied to a district because it includes the common area used for transportation and parks surrounding various buildings." (191)

James advocates the use of gross density measures for residential developments rather than net, because of his concern for residents "having associated with their homes and immediate neighbourhood all the facilities that a town can offer in the way of good schools and playing fields, and open spaces, shops and civic services", and for residential areas being "properly equipped with facilities within easy reach" of homes. Net densities are deceptive, he says, because they take no account of neighbourhood facilities and amenities, whereas gross densities do, "covering a whole district or sector of a town" (p553).

According to Lozano, density drives urbanity:

"Density determines the accessibility of people to people, of people to work, of people to services and recreation; in short, it allows urban relationships to flourish." (162-163)

Lozano advocates residential intensification around mixed-use local retail and community facilities and amenities to provide the necessary density to support a more urban way of life within walking distance of home (180). 
Lozano notes there are density thresholds for different levels of urbanity (165-166):

Sub-urban - Gross FAR $<0.3$ (Fig. 4.15)

Detached and semi-detached housing.

At this low density it is difficult to provide community amenities close to the dwellings.

First threshold - Gross FAR $=0.5$ (Fig. 4.16)

Row housing. All dwellings are in contact with the ground. It becomes possible to have community facilities close to the dwellings.

Second threshold - Gross FAR $=0.8 \quad$ (Fig. 4.17)

Stacked townhousing; low-rise walkup apartments. This is 'the watershed' where the nature of outdoor space changes radically as dwelling units become elevated above ground; dwellings also begin to lose individual identity as they merge into larger complexes, and common car parking areas are needed.

Third threshold - Gross FAR $=1.4 \quad$ (Fig. 4.18) Mid-rise 6 - 13 story elevator apartments. A wide range of facilities and activities are easily accessible from the dwellings, but provision of parking and open space become critical issues.

Fourth threshold - city centre (Fig. 4.19) High-rise central city apartments. Maximum accessibility, but limited open space. Attractive if good public transit and parks are provided.

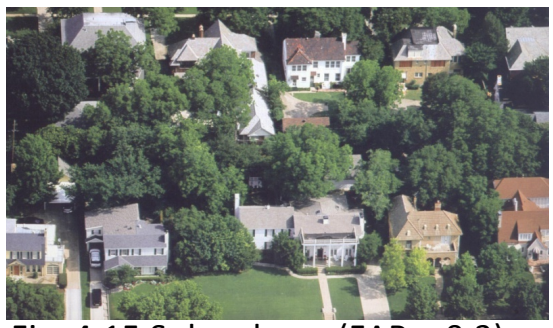

Fig. 4.15 Sub-urban (FAR $<0.3$ )

(Campoli \& MacLean 40)

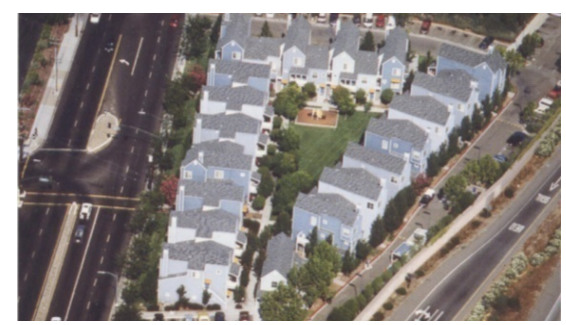

Fig. 4.16 Row housing (FAR =0.5) (Campoli \& MacLean 112)

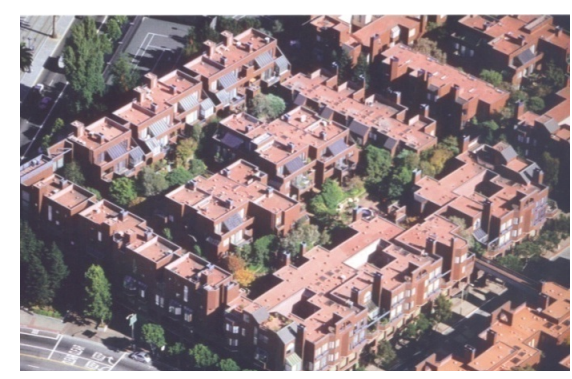

Fig. 4.17 Low-rise $\quad(F A R=0.8)$ (Campoli \& MacLean 126)

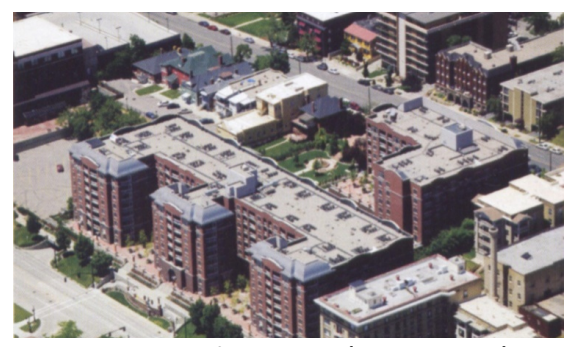

Fig. 4.18 Mid-rise $\quad(F A R=1.4)$

(Campoli \& MacLean 140)

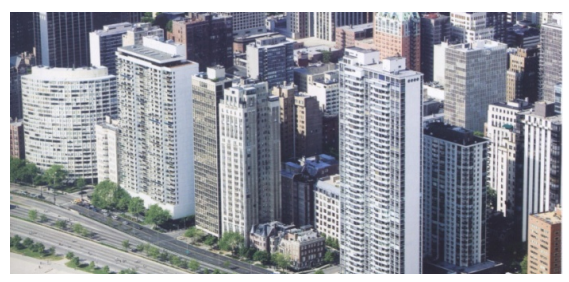

Fig. 4.19 High-rise

(Campoli \& MacLean 57) 


\section{ii. 'Walkable urbanism', 'drivable sub-urbanism' and 'neverlands'}

Leinberger characterises sub-urbanism as "drivable density" (113) and truly urban places as "walkable":

"... there are only two general development patterns: drivable sub-urbanism and walkable urbanism. ... Based on my experience in this country (USA) and Europe, drivable sub-urban and walkable urban places are at two ends of the density spectrum: drivable sub-urban development tends to have an FAR between 0.005 and 0.3 , and walkable urbanism's FAR tends to range between 0.8 and 40.0. The least dense walkable urban place is at least several times as dense as the most dense drivable sub-urban place." (114)

Typical low density housing in New Zealand (Leinberger's "drivable sub-urbanism”):

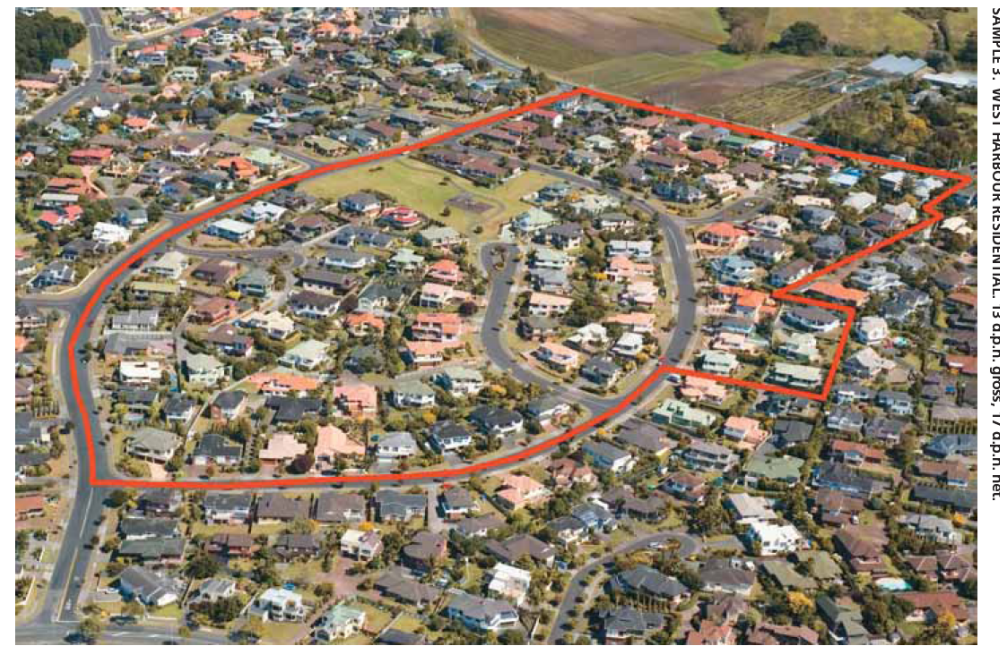

Figure 4.20

West Harbour, Auckland.

Density within the red border line is 13 dph gross

(FAR 0.2)

(Auckland Urban Density Study, 19)

Leinberger's

"drivable sub-urbanism"

Leinberger asserts that these two fundamentally different development patterns, 'drivable sub-urbanism' and 'walkable urbanism', are the "only two ... ways of constructing the built environment in a market-viable manner" (114). He then points out and describes a third development pattern which he calls 'neverlands':

"You'll notice that the two basic patterns leave out a third density range, from 0.3 to 0.8 FAR. This is an ill-fitting garment that is neither drivable sub-urban nor walkable urban. These places could be called 'neverlands'. Neverlands generally combine higher density residential with little in the way of street life. Most people have to rely on their cars for most trips from home. ... Whether a mistake or a transitional phase, the neverland development pattern is not a long-term viable condition." (114-115) 
Typical medium density housing in New Zealand (Leinberger's "neverlands"):

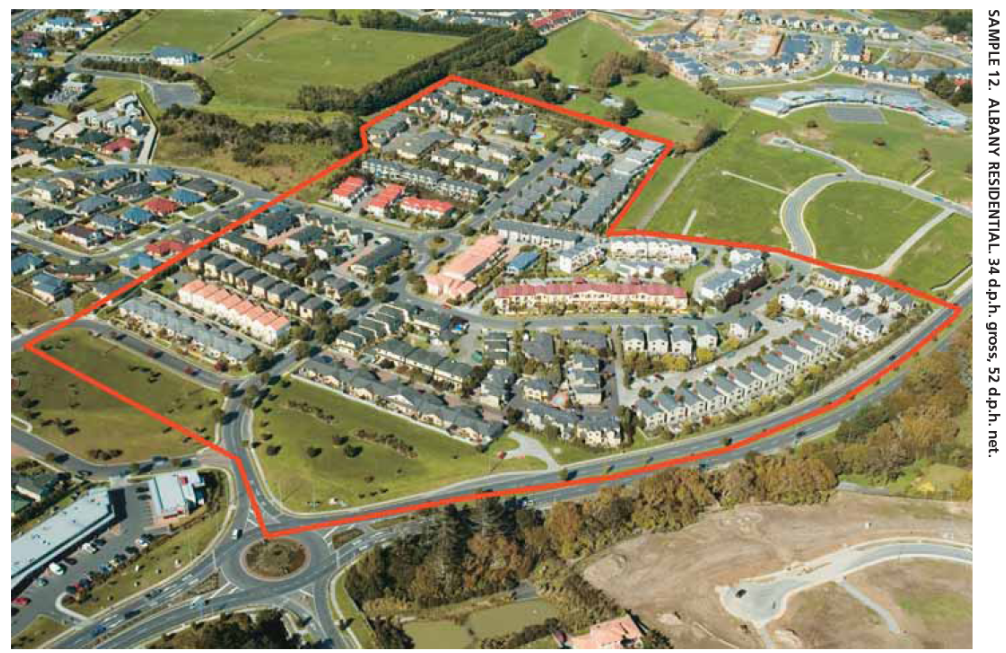

Figure 4.21

Albany, Auckland. Density within the red border line is 34 dph gross (FAR 0.5)

(Auckland Urban Density Study, 19)

Leinberger's

"neverlands"

New Zealand's medium density housing is in the range of 30-66 dph (Turner et al. 22), i.e. FAR 0.5. According to Lozano, this is the first level of urbanity because it is theoretically within easy walking distance of town facilities. This is seldom so for New Zealand's medium density housing, much of which could be described as 'neverlands' (Figs. 4.16 \& 4.21) - too dense (FAR 0.5) for suburbia (FAR $<0.3$, Fig. 4.20), but not dense enough to support public transport and the diversity of local shops and community facilities needed for 'walkable urbanism' (FAR 0.8+ Fig. 4.17).

The density threshold of FAR 0.8 for walkable urbanism has a significant impact on communal space in medium density housing. As mentioned above, this density threshold is 'the watershed' where the nature of outdoor space changes radically as dwellings become elevated above ground, lose individual identity, and common car parking areas become necessary. One way of addressing this is to employ Lozano's strategy of cluster zoning (179), i.e. clustering houses to create open space, and locating these clusters close to mixed-use nodes whereby ...

"... the number of dwelling units that can exist in a given area under normal zoning are concentrated in a much smaller area and the area remaining is devoted to open space."

An urban typology particularly suited to this strategy is the 'bay structure'. 


\section{iii. The bay-structure as the archetypal form for community connectedness}

Lozano discusses the development of 'urban typologies', explaining that urban patterns, being formed by repetitive elements, can be grouped conceptually into typologies - structures, facilities and spaces with strong similarities suggesting a 'family resemblance' (55). Lozano defines 'Type' as "the general form, structure, or character distinguishing a particular kind, group, or class of beings or objects hence a model after which something is made." (55)

"Prototype' and 'archetype' are practically interchangeable concepts, indicating the first or primary type of any thing, the original form from which copies are made. 'Stereotype' is defined as something continued or constantly repeated without change ... [even when] obsolete or ill-adjusted ..." $(55,56)$. Lozano states that "urban types are basically types of spatial organisation in settlements" and notes that "the more abstract the essence of a type is, the wider the range - and the richer the variation - of a typology will be." $(56,57)$

The 'bay' is the archetype for 'community'. Being abstract in essence, it yields rich variation. Professor Richard Toy (1977) explains the 'bay structure' as an archetypal form for community and connectedness. He describes the bay as " $a$ vital 'place' element latent with ... great complexity", giving expression to "an almost infinite variety of conditions and combinations of human belonging ...":

Dr Richard (Dick) Toy:

"One immediately noticeable characteristic of Auckland is its wateriness. There is more sea than land. ... Nature has thrust up volcanic cones ... From these, ridges of lava run down to the sea ... earth invades and embraces water-filled hollows ... These hollows open outwards to the even greater hollows of the Gulf and ... Pacific ...

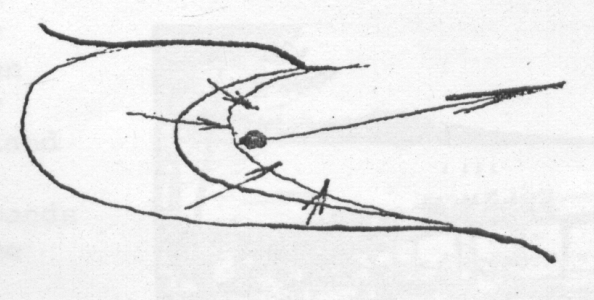

Fig. 4.22 The bay-structure Dr Richard Toy 
"Of these hollows the nearest to home and very like home is the bay; like home but at neighbourhood level the bay offers the opportunity and choice for haven or to venture out into a wider community. Because of its vertical closure behind and its openness to the sea in front the bay structure has a potential to accommodate an almost infinite variety of conditions and combinations of human belonging and withdrawal. Its pulsing tidal edge of land and sea marks the important direction of the bay and provides a transition for an alternation between inwardness and outwardness. The edge with its direction and alternation, is the essence of the structure.

"In this sense it is like the verandah of a house, or say, the Piazetta of St. Mark's Square. This living essence of the bay, a vital 'place' element latent with such great complexity, is precious but easily destroyed. Causeways which provide motor movement across a bay can do just this.

"From home to bay to harbour to ocean the water-filled hollows constitute an hierarchical structure connecting inward and outward, permeated through and through with this potential for human place, and connectedness too, and for fundamental social and psychological satisfaction."

Dr Richard Toy, 1975, "Auckland: WaterCity of the South Pacific" 3, 4 (lecture material). Published in "Auckland at Full Stretch: issues of the seventies" eds. Bush and Scott, 1977.

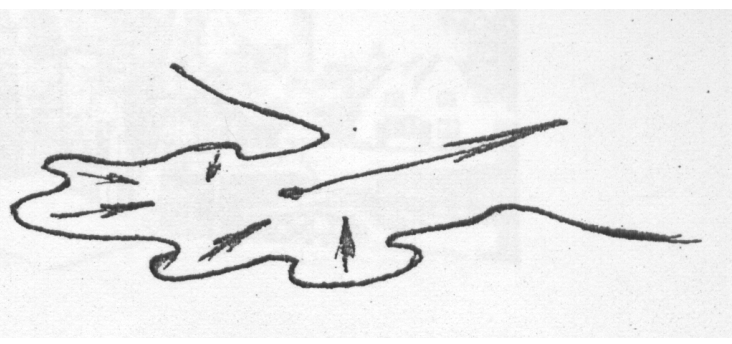

Fig. 4.23 The edge defines the bay-structure. Dr Richard Toy

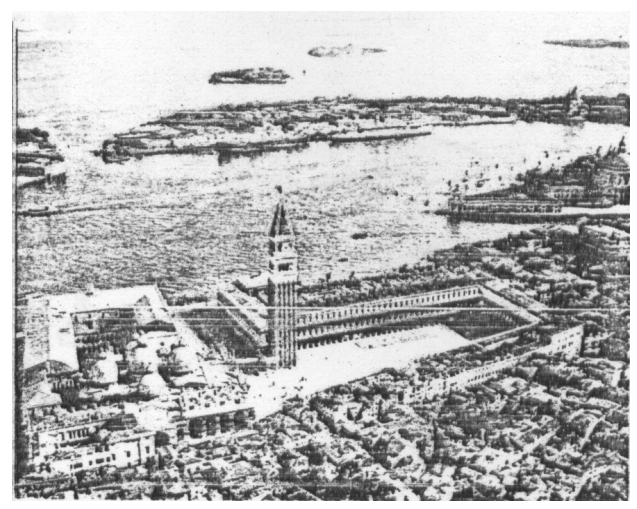

Fig. 4.24 Piazetta of St. Mark's Square Dr Richard Toy

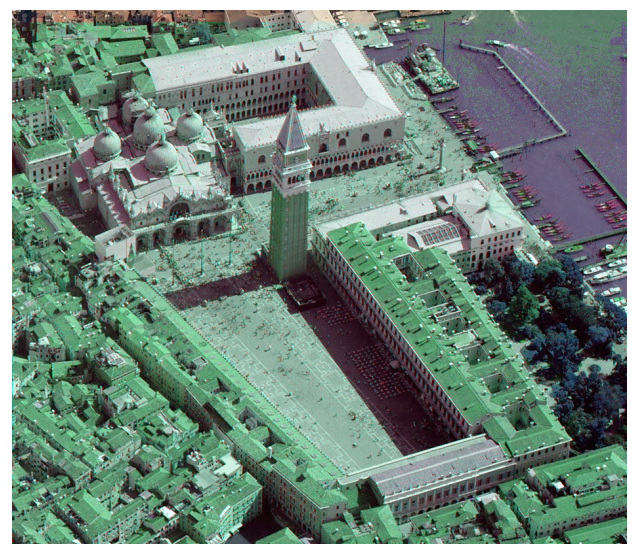

Figure 4.25 The bay-form - people gathering and dispersing, coming and going. St Mark's Square, Venice, Italy. Bing maps Accessed 29.10.10 
Dr Toy, reflecting on Norberg-Schulz's theories about architectural forms concretising varying degrees of spatial openness and closure to create stable systems of 'places', uses the example of St Mark's Square in Venice to illustrate the concretisation of the bay spatial form to express in architecture a place of alternating occasions, of gathering and dispersing, coming and going, of people at "the pulsating tidal edge of land and sea" - a space "latent with such great complexity ... an hierarchical [spatial] structure connecting inward and outward, permeated through and through with this potential for human place, and connectedness too, and for fundamental social and psychological satisfaction."

At a humbler scale, Dr Toy notes how the verandah of a house, a transitional zone between 'home' and 'neighbourhood' reflects "the pulsing tidal edge of land and sea ... an alternation between inwardness and outwardness ...".

Such is the nature of communal space, a transitional zone between private and public, "an alternation between inwardness and outwardness". The bay is the natural expressive form for communal space, offering "the opportunity and choice for haven, or to venture out into a wider community".

This thesis explores the nature of the 'pulsating tidal edge' between home and neighbourhood, and the alternating inward and outward flow of life between neighbourhood and the wider urban community. The thesis highlights the 'edges' between private and public space, the edges being 'the essence of the structure', the edges which developers, urban designers, architects and builders, participating with urban communities, will concretise to give expression to the multivalence of communal places. This thesis explores this multivalence, the many overlapping features, uses, meanings and values latent within these communal places. 


\section{Bay-structure: Examples A bay-structure for 'sense of community' is common in} architectural design. Nigel Bond and Geoff Horne apply it in crescent-shaped form:

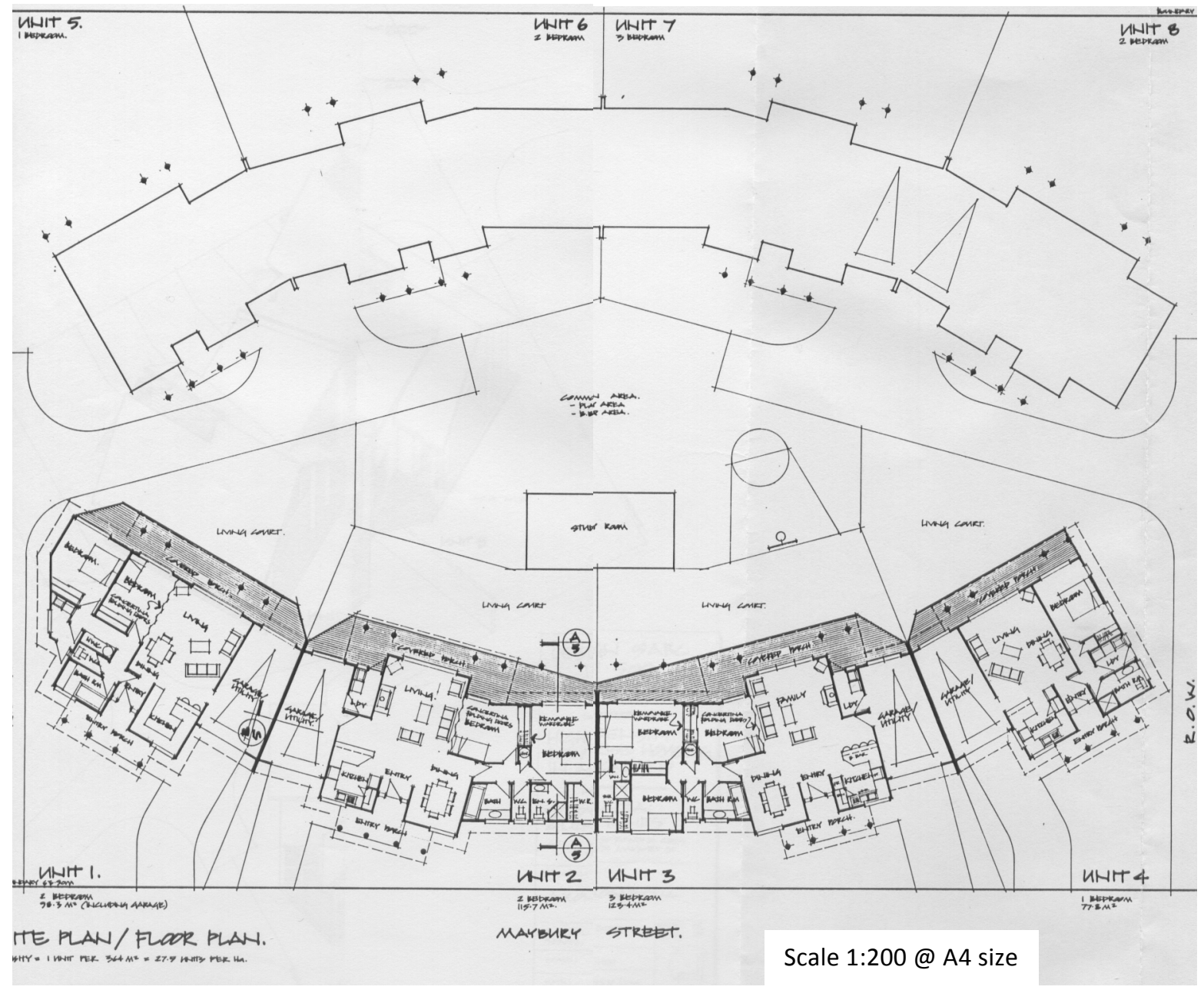

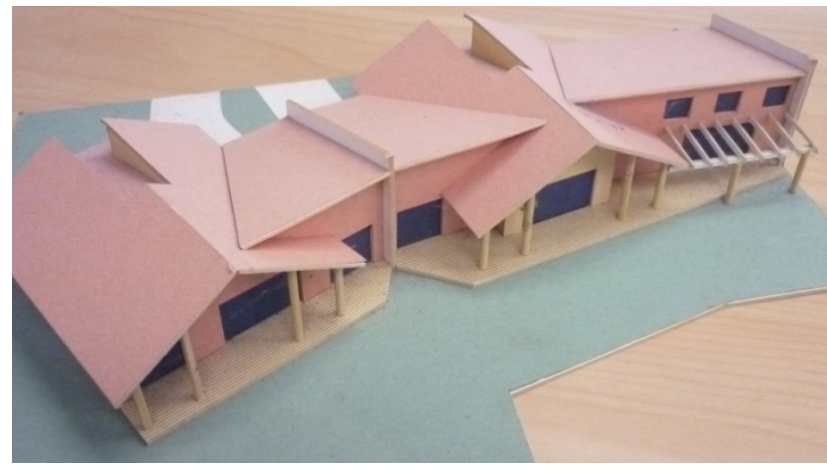

Fig. 4.27 Part-model of cooperative housing scheme, Nigel Bond and Geoff Thorne (1994) - University of Auckland School of Architecture.
Fig. 4.26 Housing at Glen Innes, Auckland. Final year student project by Nigel Bond and Geoff Horne, 1994. Their study challenges "the adequacy of ... 'conventional' housing types to satisfy the needs of extended family situations....and uses a double crescentshaped central communal space:

- $\quad$ to facilitate the concept of whanau/extended family housing...

- "to nurture a sense of community, safety, and belonging through cooperative housing types." 
For the Hebrew Congregation in Auckland, John Goldwater, architect, uses a courtyard, a closed bay-form, to give expression to 'community':

"The use of the courtyard form was novel in Auckland at the time and provided the inner-city building with a sense of security and privacy, yet also openness and freedom ... 'an unselfconscious piece of architecture embodying a sense of community' ..."

(Justine Goode, Architecture New Zealand, 2007, Issue 5, 92)

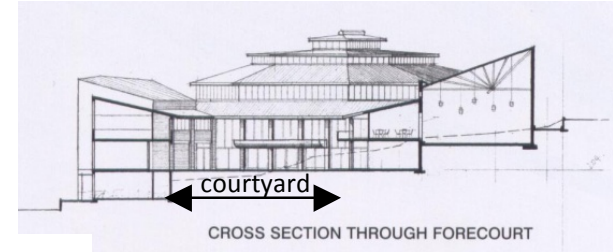

a

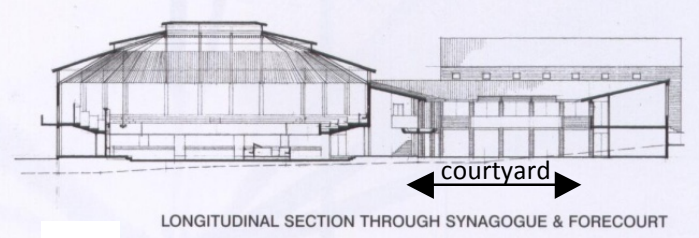

b

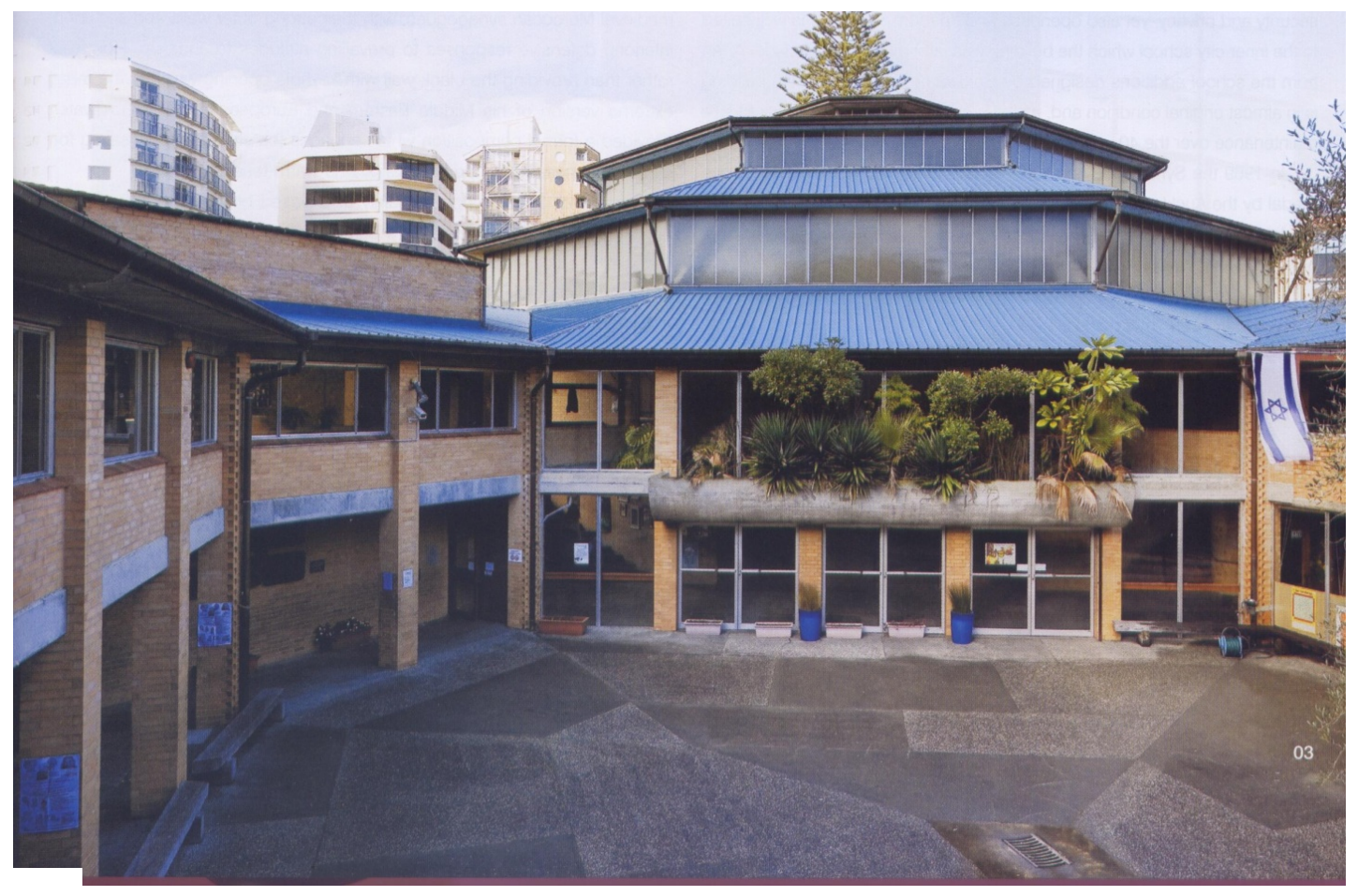

C

Fig. 4.28 a,b,c Community Centre for Hebrew Congregation, Greys Avenue, Auckland (1964-66) John Goldwater, Architect (Photo: Whit Preston, Architecture New Zealand, 2007, Issue 5, 91) 
David Mitchell uses a courtyard form to give expression to a community of music within a sheltered bay, withdrawn from the street, and protected from noisy traffic along its street edge by a high, free-form, undulating, ochre-coloured concrete wall:
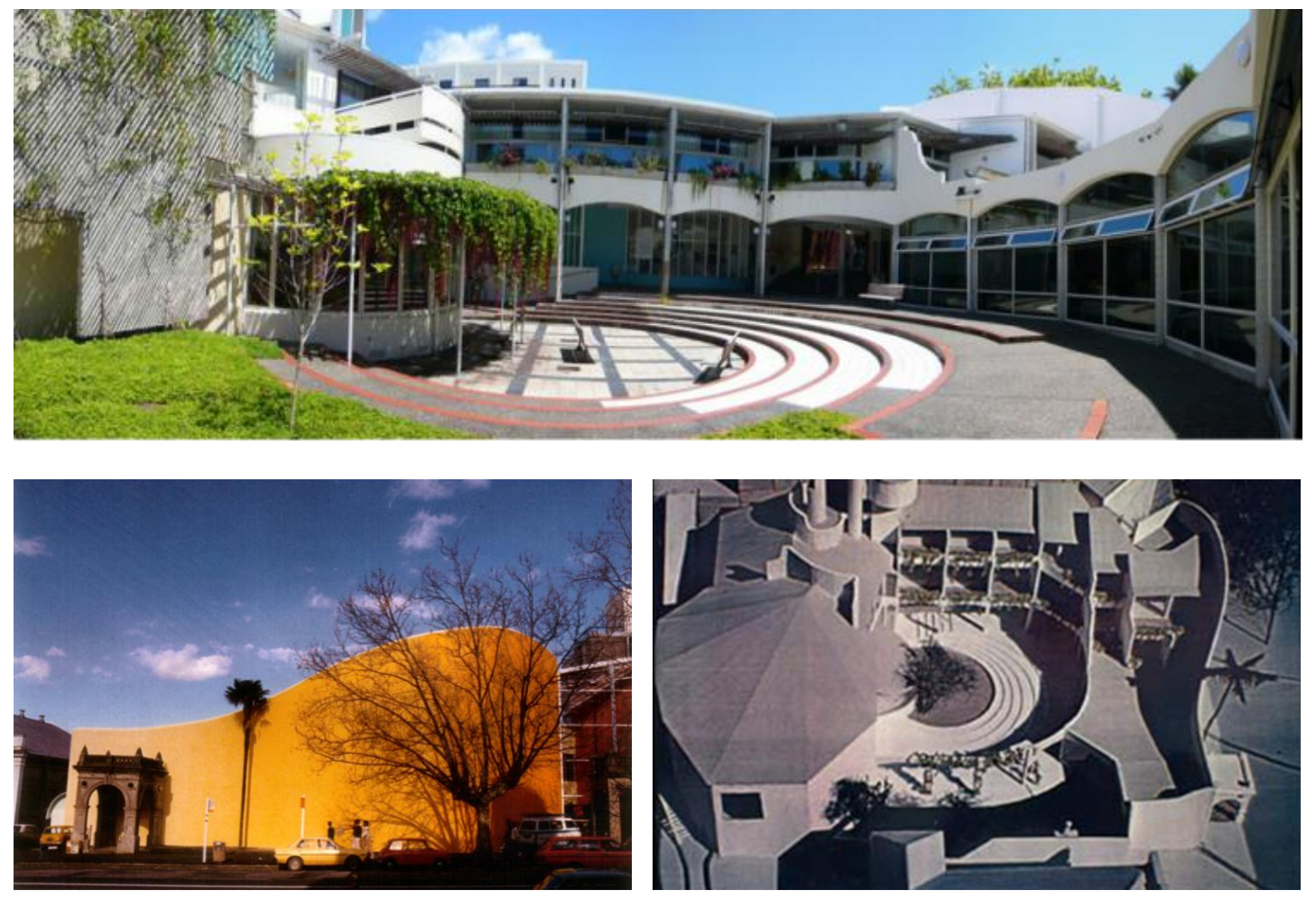

Fig. 4.29 a,b,c University of Auckland School of Music, Symonds Street, Auckland David Mitchell and Jack Manning of Hill Manning Mitchell Architects 1986 Photo source: http://www.mitchellstoutarchitects.co.nz/music-school/

Pure curved bay-form for an intentional community - a primary school:

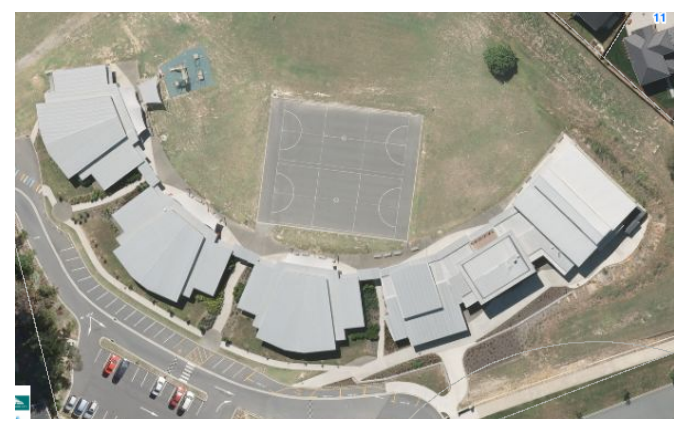

Figure 4.30 Primary School at 126 Oteha Valley Road, North Shore, Auckland.

(Alggi)
Pure rectangular bay-form for an intentional community - a primary school:

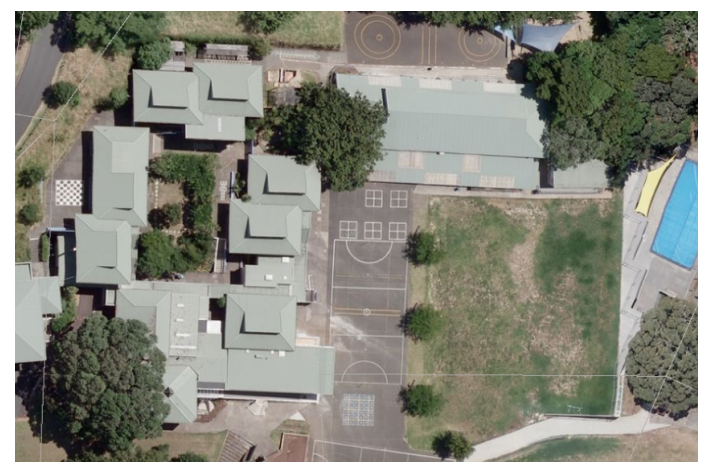

Figure 4.31 Kerr Street Primary School, Devonport, Auckland (Alggi map) 

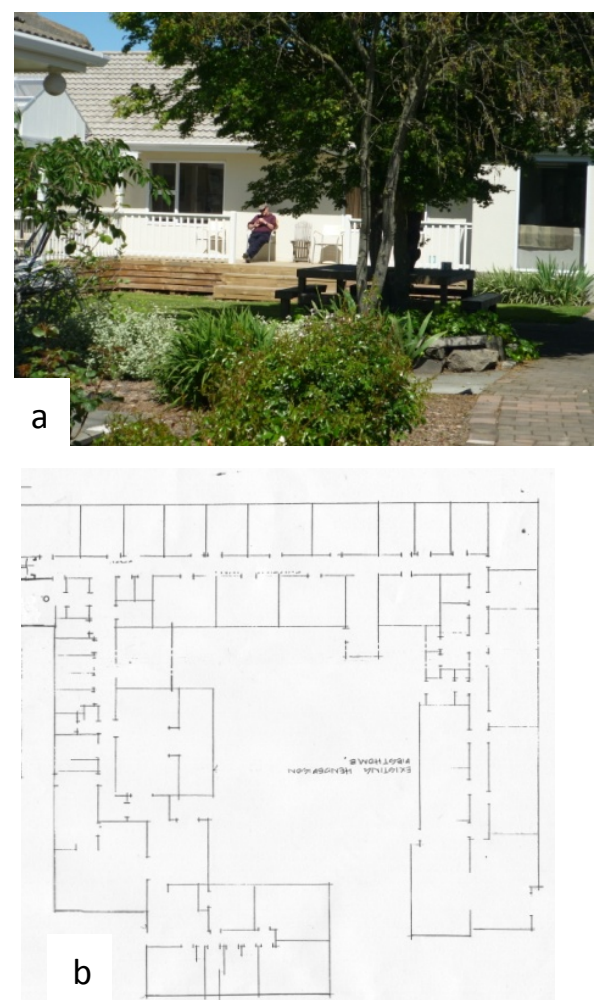

Fig. 4.32 a, b Central green communal courtyard. IHC Home, Hendersons Road, Christchurch (Photo: S.J. Pattinson)
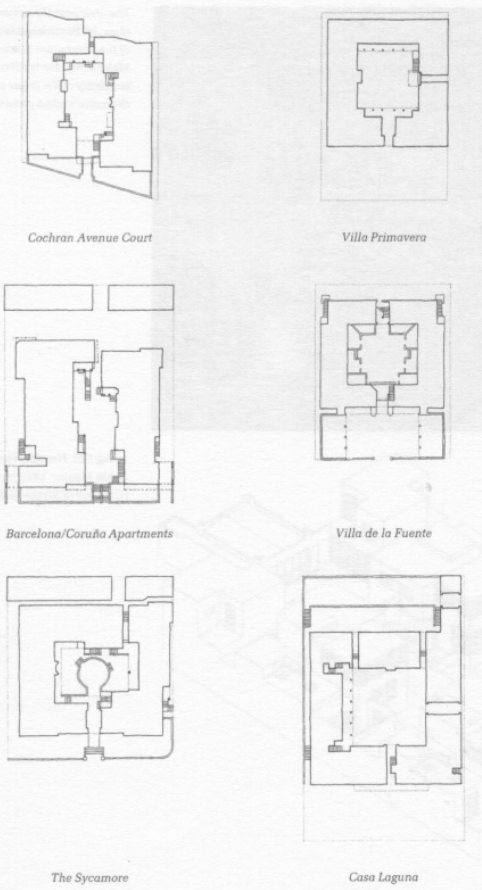
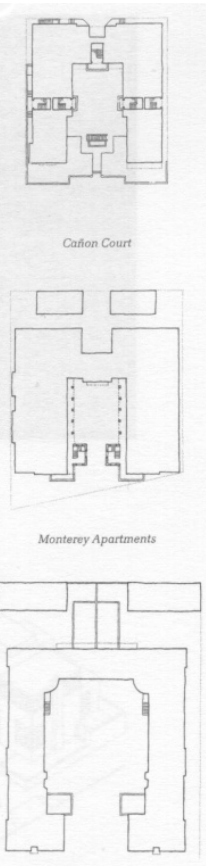

Fig. 4.33 Courtyard House Types

(Source: Polizoides et al. 8)

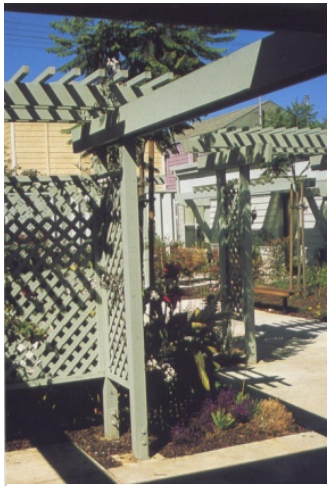

A Courtyard

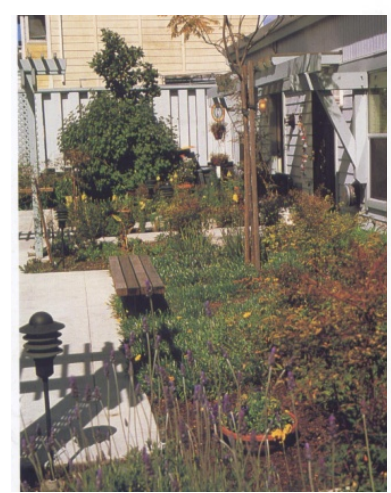

B Garden seat

Fig. 4.34 a,b,c Rental housing for low-income singles with AIDS, Bay Bridge Housing, Oakland, California (Crosbie 2003, 17)

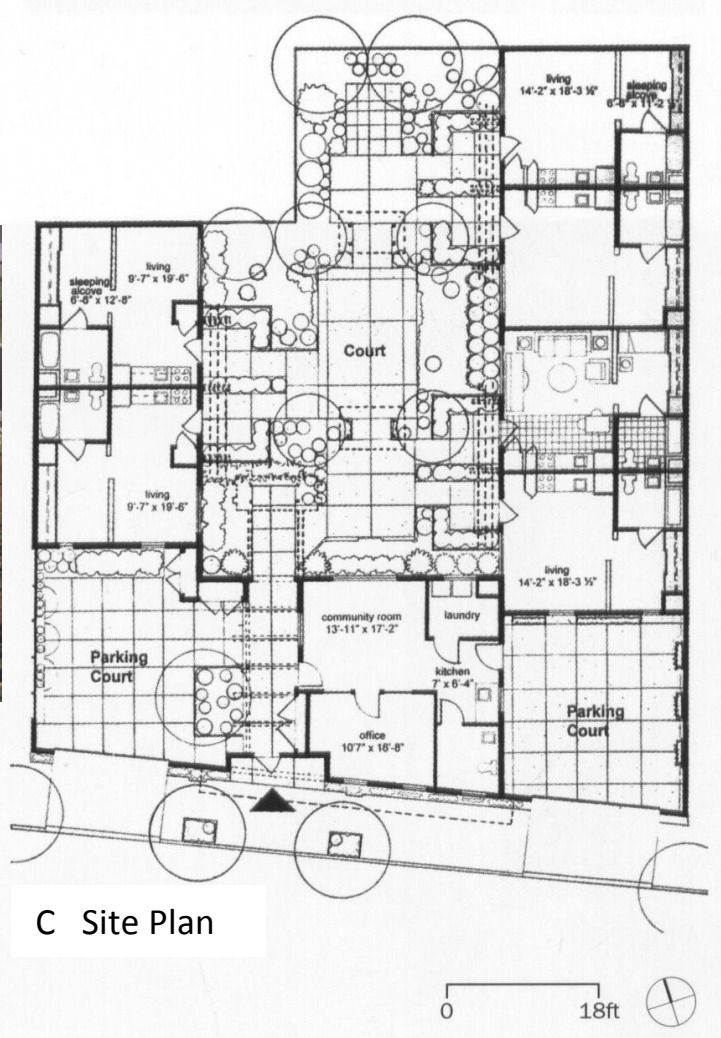


The recurrence of the theme of 'sense of community' in architecture and planning practice and theory suggests it is an important topic in the education and practice of architecture, urban design and planning. The above examples illustrate how the 'bay' form can be interpreted in a variety of ways - including curved forms and rectangular courtyards - to create communal space, being an archetypal form for community belonging and connectedness.

The Good Solutions Guide for Apartments (Auckland Council) notes that "Open space, also called outdoor space, is an important environmental resource. It mitigates the effects of living in small dwellings and improves the liveability of a development." (17)

The AMCORD (Australian Model Code for Residential Development 1997) design guide aims to achieve "a more integrated and holistic approach to neighbourhood design" following the principles of "... Traditional Neighbourhood Design, Transit Oriented Development, Pedestrian Pockets ...". These approaches aim to increase neighbourly sociability and reduce dependence on private vehicles for day to day getting around. Among many other issues, it addresses designing for different lifestyles and changing household structures, including the fact that households are getting smaller (AMCORD PND21).

Single person households "are rapidly becoming the norm" because "people are marrying later, experiencing separation and choosing to live alone". It is noted that many single person households are elderly people and that many of these have physical disability needs. Single person households need less living space, but because of a lack in the market for this type of household many end up occupying houses that exceed their spatial needs ... if our urban areas are to promote efficient use of space to suit the whole range of community needs, the development of better housing solutions for single people is a very high priority. The challenge is for designers to provide small dwellings which have a high level of internal amenity, 
attractive but low maintenance, open space, privacy, and an appearance which provides for a sense of personal identity (avoiding institutional connotations). (AMCORD PND21).

Regarding shared housing for unrelated groups, the notes mention "there are considerable economies to be achieved by single persons sharing accommodation rather than each maintaining their own dwelling unit. There is a market for well designed shared housing, but relatively few models exist." The notes list features that are appropriate to shared housing. Of interest are "large shared spaces including cooking, dining and sitting areas", complemented with areas that meet individual needs for privacy, for example, "large bed sitting rooms for personal privacy [with] ensuite bathrooms" (AMCORD PND21).

When recycling warehouses, industrial, commercial and office type buildings for residential accommodation, "where there are a large number of dwellings within the one building, an area of communal open space is desirable ... to accommodate various leisure activities (e.g. reading, board games, table tennis, snooker) or outdoor space. The outdoor area should preferably be provided at ground level, or alternatively the roof top space of multi-storey buildings could be utilised. If roof top space is used consideration has to be given to user safety, noise impacts on occupants of the building and neighbours, the visual impact of any structure and the maintenance for privacy of neighbouring activities" (AMCORD PND23).

The Good Solutions Guide for Apartments (Auckland Council) provides a solid and void diagram (16), noting that built space and open space are both important, and implying that open space should be deliberately designed and not just be left over space. The notes on open space include the following objectives: that open space should contribute to residents' sense of identity and ownership, have casual surveillance for safety and security and feature both planting and outdoor furniture. 
It notes that communal open space should be accessible and attractive to residents and have a pleasant outlook.

The Good Solutions Guide for Apartments (Auckland Council), in the solid and void diagram (19), also indicates three purposes for open space: recreation; a pleasant outlook for residents; and a sense of identity.

\section{Conclusion}

Van Eyck deliberately sought to transform abstract, neutral space and time into memorable 'place' and 'occasion'. Jencks quotes from van Eyck's design philosophy:

"Whatever space and time mean, place and occasion mean more ... Provide that place, articulate the inbetween ... make a welcome of each door and a countenance of each window ... The material slum has gone in Holland ... but what has replaced it? Just mile upon mile of organised nowhere, and nobody feeling [s] he is 'somebody living somewhere'."

Aldo van Eyck, in Jencks (311)

The aim of this research is to find many ways to transform neutral communal spaces in medium-density housing into welcoming and meaningful 'places' that create the feeling of being 'somebody living somewhere'...

... in other words, to transform communal univalent 'space' into multivalent 'place'. Multivalence creates 'places' that are varied in use, rich in meaning and enjoyable to inhabit (Jencks 14). "Every corner and every space must be programmed for multiple roles" (Hertzberger, in Jencks 318). 


\section{CHAPTER 5 - Proposed Principles for Multivalent Communal Space in Medium Density Housing}

\section{Introduction}

Question 3: What are the principles for achieving multivalent communal space in medium density housing?

To answer this question requires a new way of looking at and creating communal space, viz. not as a univalent spatial programme undertaken by either the traffic engineer (resulting in car-dominated communal space) or the landscape designer (resulting in 'landscaped outlook'), but as a multivalent programme carried out collaboratively by practitioners, researchers and residents resulting in the creation of rich and meaningful shared places for all age groups to live in and enjoy.

The key finding from Chapter 3 is that communal space in New Zealand's medium density housing is generally univalent in nature. It tends to have only one use - to be either car-dominated or landscape-dominated.

The key findings from Chapter 4 are that little consideration is being given to communal space in medium density housing, but that it has the potential to be highly multivalent. Twelve potential uses or meanings for communal space were explored. They seem to be interrelated. These uses and meanings are generally well-known. By integrating or overlapping these various uses, communal spaces have the potential to provide rich and meaningful living environments for residents.

To transform univalent into multivalent communal space, a new way of looking at things is needed to elevate the importance of communal space. Chapter 5 proposes five principles which encompass the twelve potential aspects of multivalence discussed in the previous chapter. These five principles will give impetus to the 
creation of sustainable neighbourhoods by shaping them around multivalent communal space. Because of the nature of the topic of this research, a fledgling interdisciplinary approach is attempted, under the broad, over-arching theme of 'sustainable cities' to locate the research in the overall context of environmental and social sustainability.

The twelve potential aspects for multivalent communal space (Chapter 4) are related here to five proposed principles from Frey's table of criteria (339-340) for a more sustainable city form as part of a sustainable region:

1. Walkable neigbourhoods

- Urbanity thresholds / Walkable urbanism

2. Clustered housing (that creates communal open space)

- Enhance the environment and property values

3. Open space

- Walking, recreation and exercise

- Mental health

- Inter-generational interaction

4. Needs of children

- Places for children

- Places for teenagers

5. Sense of community

- Neighbourhood identity

- Neighbourhood attachment

- Social interaction, inclusion and heterogeneity

- Safety and Crime Prevention

- Community participation 


\section{Sustainable Cities: An over-arching theme}

The editors of The Urban Design Reader 2007 (328) comment that, over the last few decades, there has been an ongoing debate regarding the most appropriate urban form for sustainable cities. Some advocate a centralised, dense core - contained, well-connected and mixed-use - for developed Western cities. Others argue for 'decentralised concentration', that is, separate dense urban nodes in a polycentric urban form connected by transportation corridors.

Frey, in The Urban Design Reader (2007), reviews the arguments for and against the Compact City. The editors note that ...

"[Frey's] analysis points to several important conclusions: that for any given city appropriate urban form should be determined as much by its specific structure, form, topography and socio-economic conditions; that the focus should always be on the city region, because cities are eco-systems in symbiosis with the country side; that an open space strategy must be part of any design framework for a sustainable city; and that density measures are only meaningful when related to built form variables.

Frey in The Urban Design Reader 2007 (328) (emphasis by writer)

This thesis proposes 'an open space strategy' for sustainable neighbourhoods in a more compact city, in the form of multivalent communal space.

"Frey also provides a very helpful table that relates commonly agreed sustainable criteria for city centres ... differences seem to lie in the degree in compacting of the urban fabric, and the degree of centralisation or decentralisation, rather than the principle." (328, 338-341)

Frey concludes that, despite differences of opinion, most agree cities need to be contained in some measure or form. There is little clarity at this point about what the most appropriate 'contained' form should be - centralised or poly-centric form. There is however, according to Frey, considerable consensus on basic principles. Frey's tables are reproduced here in full in Tables 5.1a and 5.1b, with five principles relevant to this thesis highlighted in yellow: 


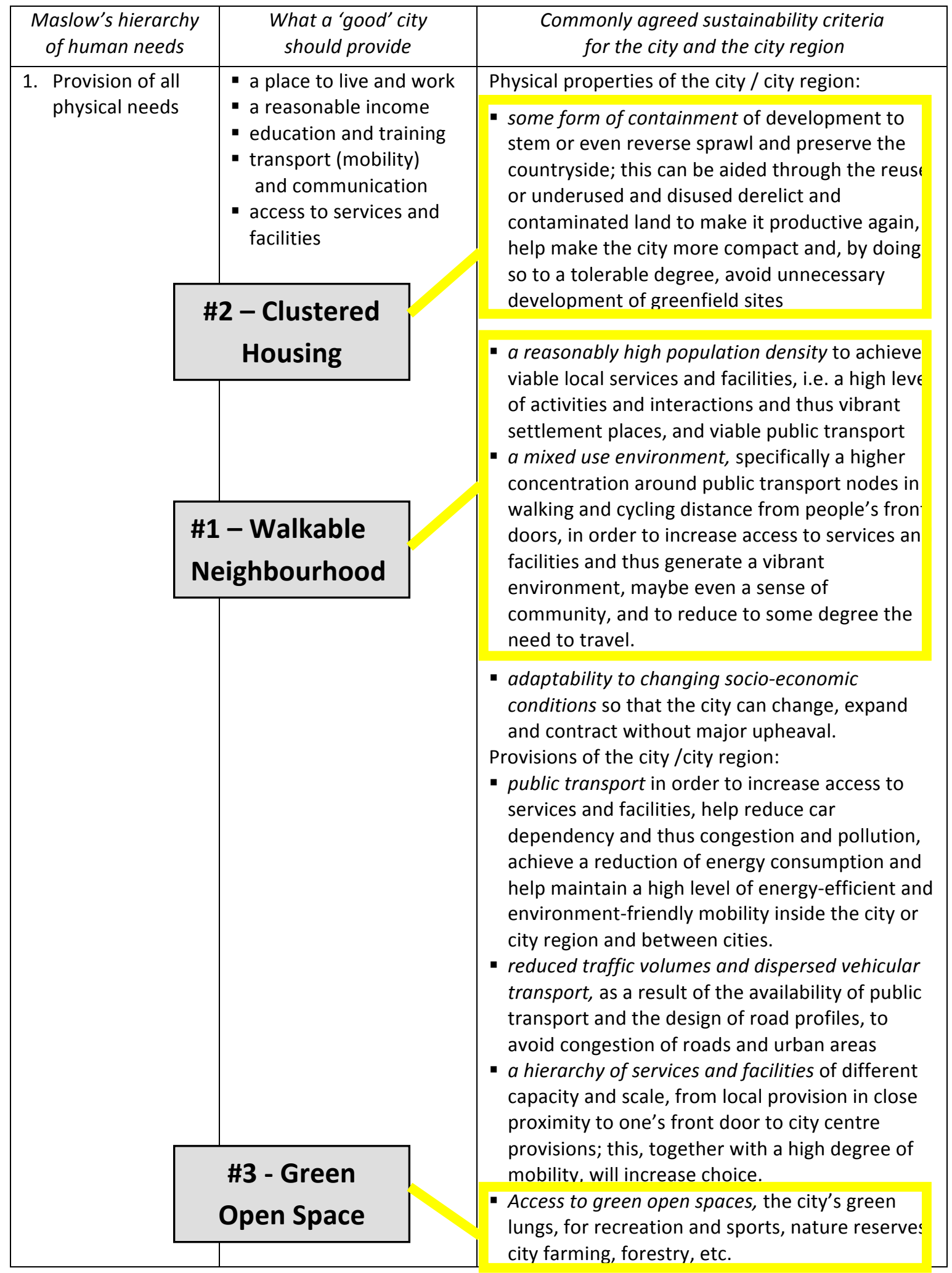

Table 5.1a Criteria for more sustainable city form - Frey, in Urban Design Reader 2007 (339-340) 
(C) 2012 Stephen Pattinson Chapter 5: Principles M. Arch Thesis

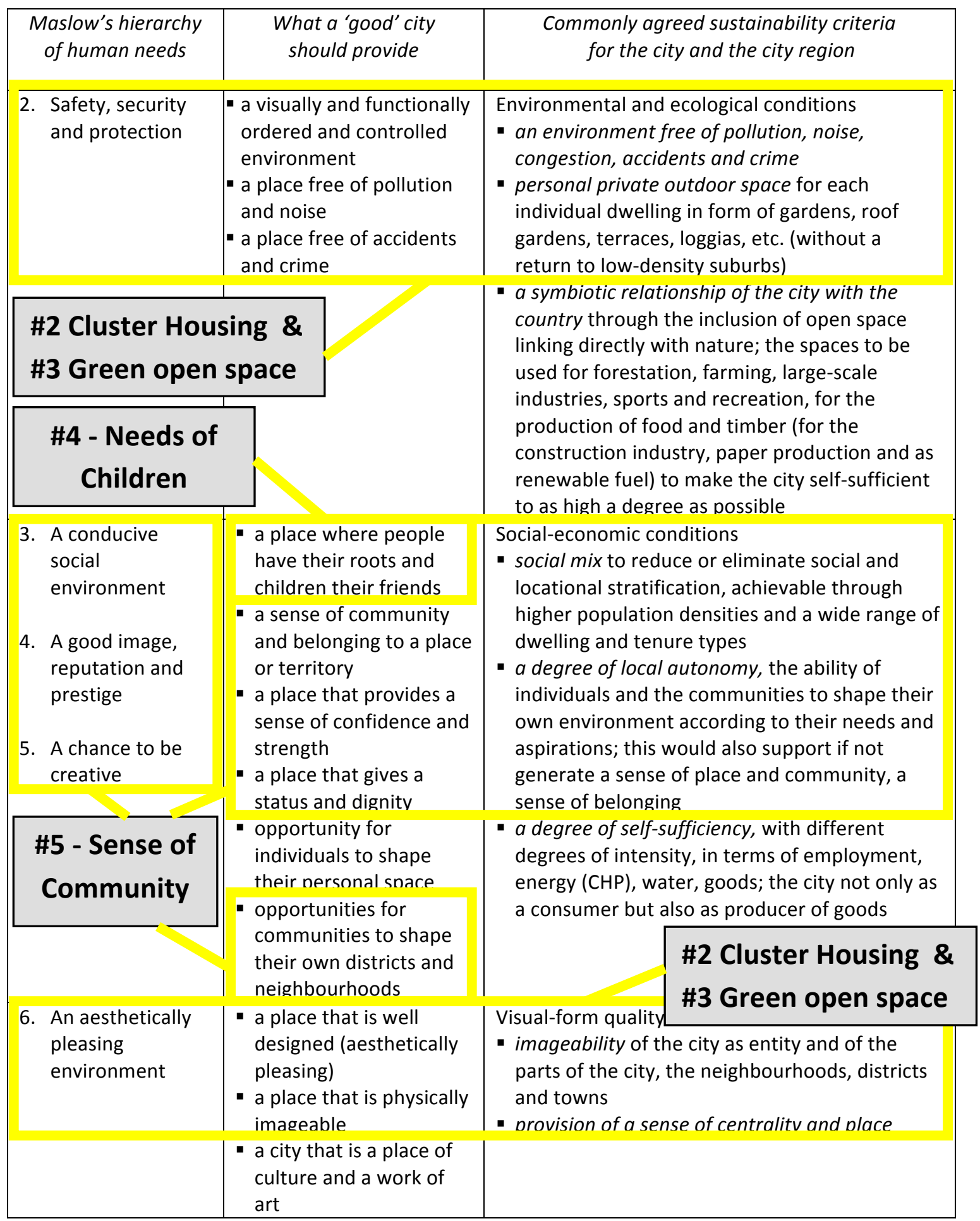

Table 5.1b Criteria for more sustainable city form. Frey, in Urban Design Reader 2007 (339-340)

Five principles for multivalent communal space correlate with various aspects of Frey's agreed criteria for a more sustainable urban form (numbered principles and yellow highlighting by writer). 


\section{Principle \#1: Walkable Neighbourhoods}

The issues this principle addresses are:

- Pedestrian-oriented, walkable urbanism

- Diversity of house sizes and types

- Living sustainably

Frey writes with respect to city form as a whole. A goal of this research on multivalent communal space in housing schemes is to enhance living environments for residents. Part of the answer to Frey's question about how to sustain the city will be found by addressing the question at the level of the neighbourhood. This research explores housing clustered around multivalent communal space as a potential solution to Frey's question of sustainability at the neighbourhood level.

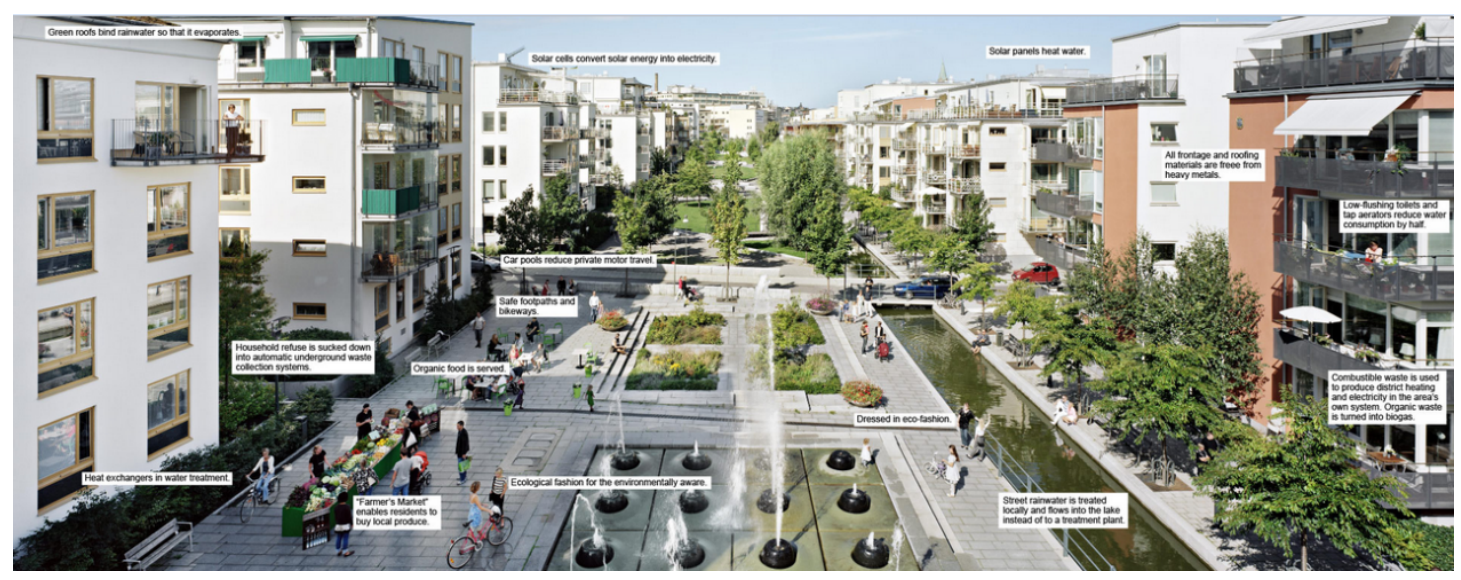

Fig. 5.1 Mid-rise 'walkable urbanism' - Hammarby-Sjostad, Sweden (Photo: Johan Topel) http://www.sweden.se/upload/Sweden_se/english/publications/SI/Facing\%20t he\%20future/Hammarby-sjostad.jpg Retrieved 09.09.2010

Hammarby-Sjostad is a recently constructed settlement for a population of 20,000 people, designed for environmental and social sustainability. It is of a density that would qualify as Leinberger's 'walkable urbanism', i.e. shopping, recreation and community facilities are within a walkable distance of apartments because of the concentrated mix of commercial, residential and recreational land uses. 


\section{Walkable urbanism}

Leinberger (2009) refers to mixed-use, well connected, compact urban development as 'walkable urbanism' in contrast to low-density 'driveable suburbia'. Mark Hinshaw (2007) simply calls this 'true urbanism - living in and near the centre'.

One argument opposing compact urban form claims that sophisticated communication technologies will allow a more dispersed urban form. People will have "the ability to work in and communicate from one's home" (329). However, the human need for 'dwelling' and physical propinquity will continue to exist irrespective of technologically improved mobility and virtual communication (Norberg-Schultz 35, 36; Lozano 189-192; Putnam 411).

Frey suggests that persuading suburbanites to accept "a compact city requires massive financial incentives, which are economically suspect, and a high degree of social control, which is politically unacceptable" (332). This may have been a fair comment in the mid-1990s, but consumer preferences are changing.

Leinberger (2009), for example, reports a significant shift, beginning about 1995, in Americans' attitudes away from 'driveable suburbia'. After WW2 the vast majority of Americans flocked to the newly formed suburbs. Since the mid-1990's, however, the attraction of the suburbs has started to give way to more compact, diverse and walkable urban development (following the influence of Smart Growth and New Urbanist design principles) so that now Leinberger calculates about a 50/50 split in public preference between 'walkable urbanism' and 'driveable suburbia'. Despite the inertia of economic and political policies still tipped in favour of 'driveable suburbia', Leinberger predicts the trend towards 'walkable urbanism' to continue.

Despite many still having a preference for low-density suburbia, others (and according to Leinberger the number is growing) want to live more centrally. This 
trend, coupled with the trend of smaller households, may escalate the demand for inner-city housing. Frey notes the argument that the compact city will "not be able to respond to the predicted increase in the number of households ... in the UK over the next 20 years an extra 3.3 million single households are expected to require accommodation" (332). Multivalent communal space is more efficient utilisation of urban land, meaning more households will be able to be accommodated.

Leinberger (2009) provides evidence there is already a large pent up demand in the USA for 'walkable urbanism' which he describes as a more environmentally and socially sustainable way of life. He notes this demand is steadily growing. Mead and McGregor report similar growth of demand for more intensive housing in Auckland (62). It is timely to consider the potential of multivalent communal space that will enhance medium density living is this growing New Zealand market.

Presently, housing for many New Zealanders is limited to dormitory suburbs, and as Lozano notes, is necessarily car-oriented:

"The separation of workplaces from residential areas... means that very few people can walk to work. ... because of the low density prevalent in many residential suburban areas, people have no way of getting to work except by automobile, since even buses are uneconomical to operate below certain thresholds. And to make the situation worse, the dispersion of employment in low-density outlying zones means that urban mass-transit service cannot be provided to those areas - forcing full reliance on the private automobile." (163-164)

The car-oriented lifestyle, which has been prevalent for the last sixty years or so, has only been made possible by cheap oil. As petrol prices continue to rise, a pedestrian-oriented urbanism, which is more compact than low density suburbia, may become an appealing alternative for more people.

In comparing low-density fringe urban growth with more compact development, Burchell et al. conclude that: 
(C) 2012 Stephen Pattinson Chapter 5: Principles M. Arch Thesis

"There appear to be more costs than benefits of sprawl growth... Benefits of sprawl are fewer .... In terms of costs, sprawl development consumes land and various types of infrastructure to a level that compact development does not." (Burchell et al. 21)

Burchell et al. note that 'smart growth' is a more sustainable form of urban growth than sprawl growth:

"It is possible to accommodate growth in ... compact development, or smart growth. ... Resources need not be as aggressively consumed ... That is the message of this study ... these benefits can be achieved through compact development with little loss of freedom of choice or housing value and with significant savings of man-made and natural resources. Smarter growth appears to be a reasonable approach ... "

(Burchell et al. 21)

Frey says "commonly agreed sustainability criteria for the city" includes ...

"...some form of containment of development to stem or even reverse sprawl and preserve the countryside ... to ... help make the city more compact and ... avoid unnecessary development of greenfield sites." (Frey, in Urban Design Reader 2007, 339)

Frey notes the necessity for a 'reasonably high population density' to make public transport and local services viable, and a mixed-use environment concentrated around transportation nodes within walking distance of people's homes (339) to reduce dependence on private vehicles for commuting. Burchell et al. note:

"A small portion of the public has been introduced to a different way of organizing ... neighborhoods. This new organization includes mixed land uses and housing types, new types of street ... and the provision of central places and open space to make neighborhoods meaningful. Those who have been introduced to it like the concept ... " (20)

(emphasis by the writer)

Leinberger (2009) refers to this new way of organising neighbourhoods as 'walkable urbanism', in contrast to 'drivable suburbanism'. Leinberger defines the required density for walkable urbanism as starting at around FAR 0.8 (i.e. Floor-Area-to-sitearea Ratio of 0.8:1) (114), and walkable distance as 1,500 - 3,000 feet (450m $900 \mathrm{~m})$ which as a radius covers an area of about $200-500$ acres ( $80-160$ ha) (103). 
Southworth and Ben-Joseph note that the distance Americans will walk for daily trips is 'quite limited'. The figures they give roughly agree with Leinberger's figures, although they note only $10 \%$ will walk $800 \mathrm{~m}$ for daily errands (115).

New Zealand 'medium density' environments are generally not compact enough or located close enough to local shopping, services and public transport nodes to be 'walkable'.

According to Turner et al. (2004), medium density housing in New Zealand is in the range of $30-66$ dwellings per hectare (dph), i.e. more than $150 \mathrm{~m}^{2} /$ unit and less than $350 \mathrm{~m}^{2} /$ unit (22), typically around a FAR 0.5 and in the form of two-storey terrace housing (dwellings attached in a row). According to Leinberger, this level of density is 'Neverlands' - too dense to be suburban (which is generally less than FAR $0.3)$, but not dense enough to support 'walkable urbanism' (114-115), or 'true urbanism' as Hinshaw puts it (2007).

Much of New Zealand's medium density housing is not located within easy walking distance of regular public transport services or local retail and community facilities. Therefore, many medium density living environments are car-oriented rather than pedestrian-oriented, explaining why Turner et al. describe much of the typology as "car-dominated" (84). This explains the univalence of much of the communal space in New Zealand medium density environments. 


\section{Diversity of house size and type}

Evidence is emerging of changes to the American lifestyle in the wake of the current economic recession in the USA. The trend is towards more inter-dependent living, as the following 'Impactlab' news article reports:

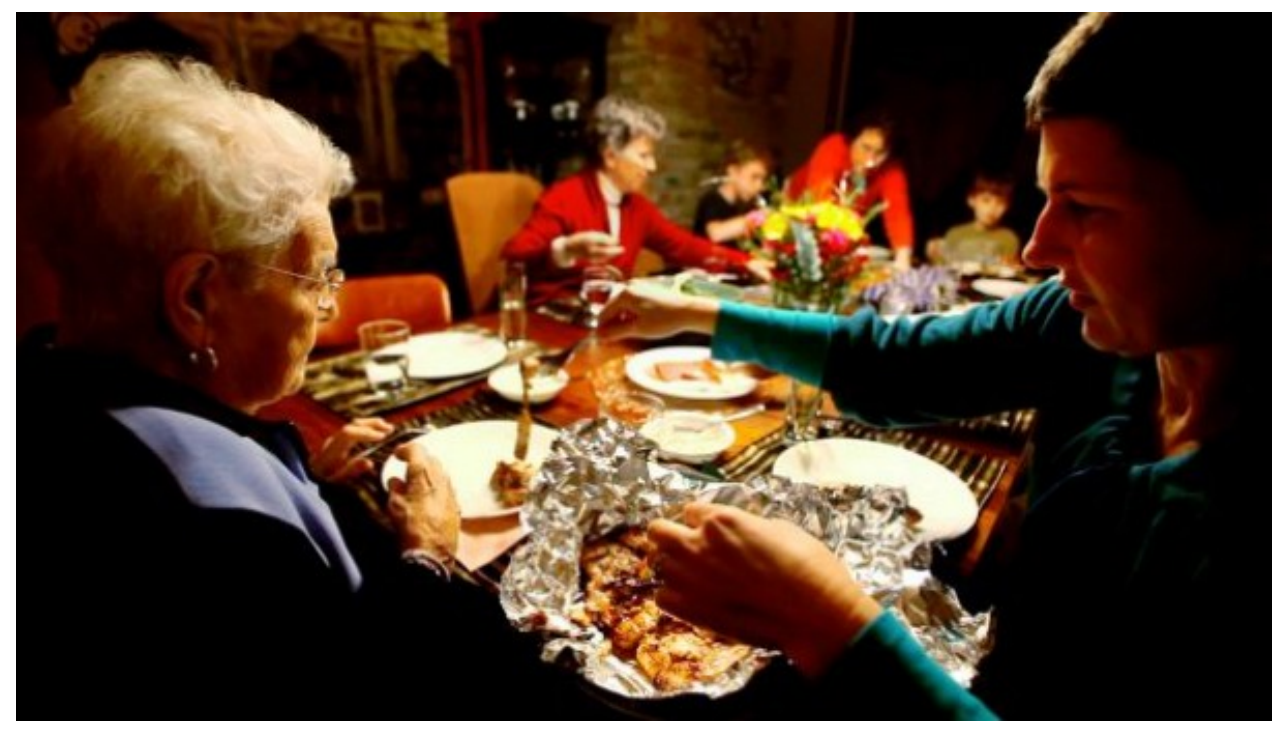

Figure 5.2 This family is part of a growing trend of Americans living in a multi-generational household.

"Multi-generational households are on the rise ... Household size began inching up in 2005, ... a trend that might have been driven by the real estate boom that made housing unaffordable to many. Now, it's more likely to be caused by the poor economy.

"It's going to have huge implications for the housing market," Nelson says.

Impactlab May 7th, 2010

No one is sure if this current trend in the USA towards larger, intergenerational households is a temporary or permanent shift. The Auckland Council reports a trend towards smaller households, but also reports a recent trend towards larger households: 
(C) 2012 Stephen Pattinson Chapter 5: Principles M. Arch Thesis

"In recent years there has been a growth in both smaller and larger households.

"... in the period 2001 to 2006, the rate of growth of larger households was ... noticeable. For example, households with eight or more members grew by 23.8 per cent during the last inter-censal period, compared with 11.3 per cent growth overall.

"Numbers of large households are relatively small however, and the numerical growth in one-person households has been significant during this period too.

http://monitorauckland.arc.govt.nz/our-community/households-andfamilies/household-size.cfm (Retrieved 06.11.2010)

It may be that current trends towards larger households are only temporary, until the current economic recession is over and job opportunities open up again. Nevertheless, with the significant rise in one- and two-person households (Chapter 1), it is clear that a diversity of house types and sizes are needed in future sustainable neighbourhoods. 


\section{Living sustainably}

Multivalent communal space at the neighbourhood level can include: rainwater harvesting, solar power generation and passive solar space heating; food production and local production of goods; neighbourhood systems of waste disposal, recycling and composting; and diverse, accessible, pedestrian-oriented environments that encourage walking and cycling, thereby reducing pollution and reducing health issues related to inactivity. Frey notes the argument that a compact city cannot also be a green city because of the loss of open space (331). This thesis argues that neighbourhoods can be both compact and green by the use of multivalent communal space.

Frey notes that " $\mathrm{t}] \mathrm{h}$ c city cannot exist on its own; it needs a hinterland to provide goods, food, raw materials, etc" (333). Multivalent communal space may increase the capacity of local neighbourhoods to produce some of their own goods, food, and recycled materials, becoming producers and not just consumers, enhancing the liveability of higher density, and protecting the hinterland from sprawl.

Frey emphasises the importance of preserving open space at the big scale in cities, whatever form those cities may take (337). The present research is limited to exploring mulitivalence at the small scale, the spaces in-between buidings, the potentially multivalent communal spaces in housing clusters, e.g. for gardening and "...the small-scale production of vegetables", which, says Frey, "is very much a thing of the present" (338). Small-scale 'green open space' is a key component of multivalent communal space in higher density housing.

Robert and Brenda Vale (129-37) provide a guide to all aspects of sustainable living. They suggest smaller buildings, or more shared facilities, would significantly reduce resource consumption, and they question the need for more floor space per person. 


\section{Principle \#2: Housing Clustered to Create Communal Space}

The following example at Letchworth, UK (the winning entry of a design competition for eco-housing) shows a small cluster of houses arranged around some shared recreational space. It illustrates well the principal of clustering housing to create communal open space:

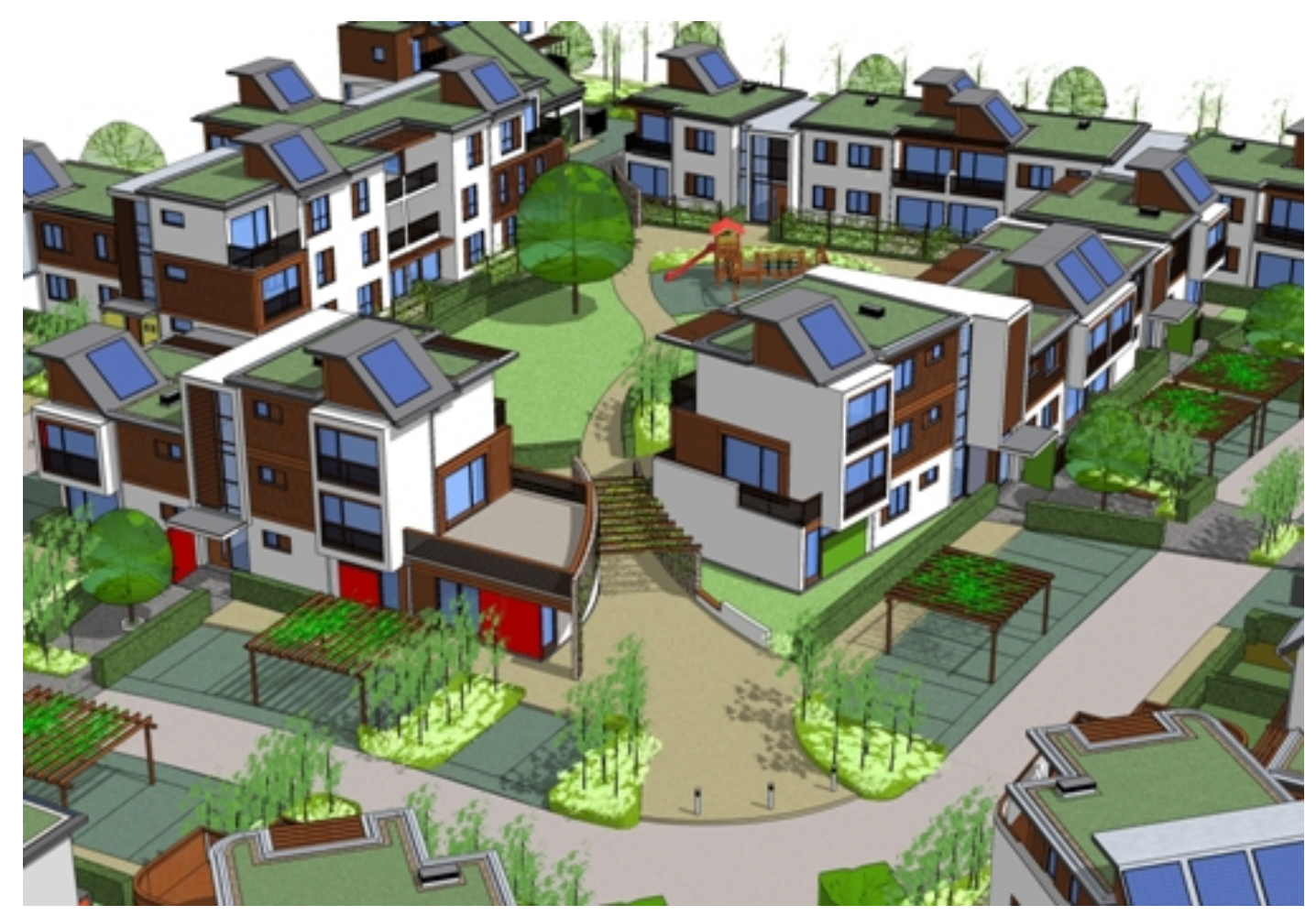

Figure 5.3 Clustered eco-housing - Lecthworth, UK

"Tomorrow's Garden City" Cade Close, Letchworth Garden City http://www.nhh.org.uk/development/about-our-development-programme/

Cooper Marcus and Sarkissian explain their preference for the term 'clustered housing' rather than 'medium-density housing'. The latter term is relative. What is considered medium-density in one country may be low density in another country. Therefore the authors avoid the term 'medium-density' wherever possible, preferring the term 'clustered housing', explaining that: 
"We have adopted the term clustered housing instead, by which we mean any arrangement whereby dwellings are clustered on a site (these could be single-family houses, row houses or apartments) so some of the site can be left free to create communal open space or shared recreational facilities. The guidelines deal only with low-rise housing forms - buildings without elevators (depending on local regulations, three to five stories)."

\section{Cooper Marcus and Sarkissian (12)}

The term 'clustering' is free of connotations of any particular density level. This suggests the possibility that the principles of multivalent communal space could be applied at any density level - low, medium or even high. Residents in housing at any of these densities can potentially benefit from the provision of multivalent communal space.

The grouping of houses into a cluster arrangement enables the creation of positive open space - deliberate outdoor space created as an attractive amenity for the use and benefit of the residents whose houses form the boundary edges of the space.

According to Cooper Marcus and Sarkissian (7- 12), well-designed clustered housing, i.e. housing arranged to create areas of shared/communal space, is an ideal housing form for many segments of the population, including traditional nuclear families with children, single-parent households, non-traditional family groups, working parents, and, an age-group often overlooked, adolescents.

Clustered housing, at say $3-5$ stories high, has the potential to achieve reasonably high densities. This point was eloquently asserted by Martin and March (1972) and supported with examples by Ernie Scoffham and Brenda Vale (1996), as noted earlier. Their point is that relatively high densities can be achieved by attaching and arranging housing around the periphery of a block while at the same time creating a generous amount of central open space. Higher density AND open space is achievable with 'clustered' housing - a building form that makes more efficient use of scarce urban land than conventional detached housing development. 
Scoffham and Vale (in Jenks et al 1996: 68) note that peripheral, three-storey attached housing with integral garages can achieve densities of at least 237 people per hectare (eg Merton), potentially with all dwellings having private gardens plus access and/or outlook to a generous shared open space (eg an unbuilt proposal for Eastfields).

Assuming an average of 2.5 people per household, typical medium density housing in New Zealand achieves densities of about 75-165 people per hectare. This is based on 30-66 dwellings per hectare (dph) which is $350 \mathrm{~m}^{2}$ to $150 \mathrm{~m}^{2}$ lot size per dwelling unit (Turner et. al. 22). This density range is typically achieved with row/terrace housing (Lynch \& Hack 253; Campoli \& MacLean 53). In New Zealand, this housing will typically have some open space, often for car parking, but sometimes for landscaped, ornamental space and/or outdoor recreational areas. Most Territorial Authorities (TAs) in New Zealand, i.e. City and District Councils, control the density of residential areas by applying rules for the minimum lot size per dwelling. For example, in the main residential areas (the suburban areas) of, say, Upper Hutt City the minimum lot size is $400 \mathrm{~m}^{2}$ for a single dwelling (18/4). This is a similar minimum lot size rule used by many TAs, such as Auckland Council's North Shore District Plan which states that the minimum lot size in the Main Residential Zone $4 \mathrm{~B}$ is $450 \mathrm{~m}^{2}$ (16-64). Upper Hutt City has another zone (Residential Conservation Zone) where the existing amenity of low density housing amongst established bush is protected by a minimum lot size of $750 \mathrm{~m}^{2}$ per dwelling (18/4).

The North Shore District Plan discusses the possibility of reducing the minimum lot size to $350 \mathrm{~m}^{2}$ to gain 6,000 or so 'infill' lots (for, say, the additional dwellings expected to be required as a result of smaller households - see Chapter 1), but the Plan comments that a survey clearly shows that this slightly higher density would not be acceptable to the majority of North Shore residents (6-3). 
There are other ways of controlling residential density. The Wellington City Council District Plan, for example, does not specify minimum lot sizes but relies on standard rules that control the bulk physical volume and location of buildings on a lot, principally by three controls - site coverage (the maximum percentage of site area that the footprint of the building may cover), car parking and open space requirements per dwelling unit. Other controls influencing density include maximum permitted heights (including heights related to lot boundaries to protect sun access to neighbouring lots) and minimum setbacks (front, side and rear yard dimensions). These rules directly control the building envelope on a lot, and hence indirectly control the overall density of development (4/7).

Medium density residential development in New Zealand is generally provided for by 'relaxing' the above-mentioned rules that protect low density, i.e. by permitting smaller lots $\left(150 \mathrm{~m}^{2}\right.$ to $350 \mathrm{~m}^{2}$ per house), shorter setbacks, greater site coverage and maybe taller height limits. These basic density, bulk and location rules are usually supplemented with special guidelines or rules for multi-unit housing that typically address site planning, building massing and design, and open space design.

Wellington City Council's (WCC) Residential Design Guide (2009) provides specific design guidelines for multi-unit housing covering such things as site planning, building design and open space design. Among other things, it recommends that spaces around and between buildings be 'positive open spaces', meaning sunny, useful areas (rather than left-over space) with outlook, and connecting directly to main living areas inside dwelling units.

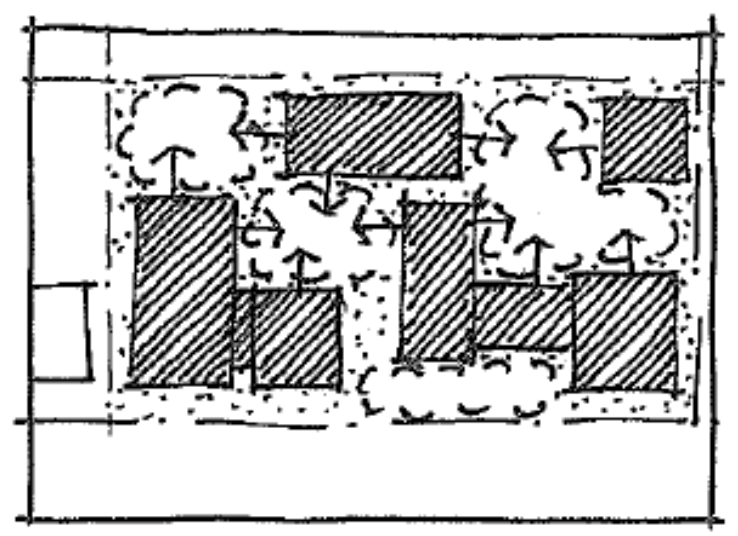

Figure 5.4 Positive open spaces between buildings (WCC Residential Design Guide July 2009: 4, G1.2 
The Design Guide notes that "apartment living may require relaxation or flexibility in the provision of outdoor open space" (WCC Residential Design Guide July 2009: 5, G1.3). This suggests that on inner city lots it may not always be possible to provide the required minimum $35 \mathrm{~m}^{2}$ private outdoor space for every apartment and some flexibility is recognised whereby private outdoor space can be partially traded off for some other compensatory amenity, for example a shared lap pool, tennis court, or high quality landscaped outlook. This hints at benefits that can accrue to residents by sharing some outdoor space, and there is a provision in the Guide for this.

The sharing of outdoor space is provided for in Wellington City Council's Residential Design Guide by allowing the aggregation of 'private open space' for each unit into 'shared private open space' for all contributing units, recommending that it will:

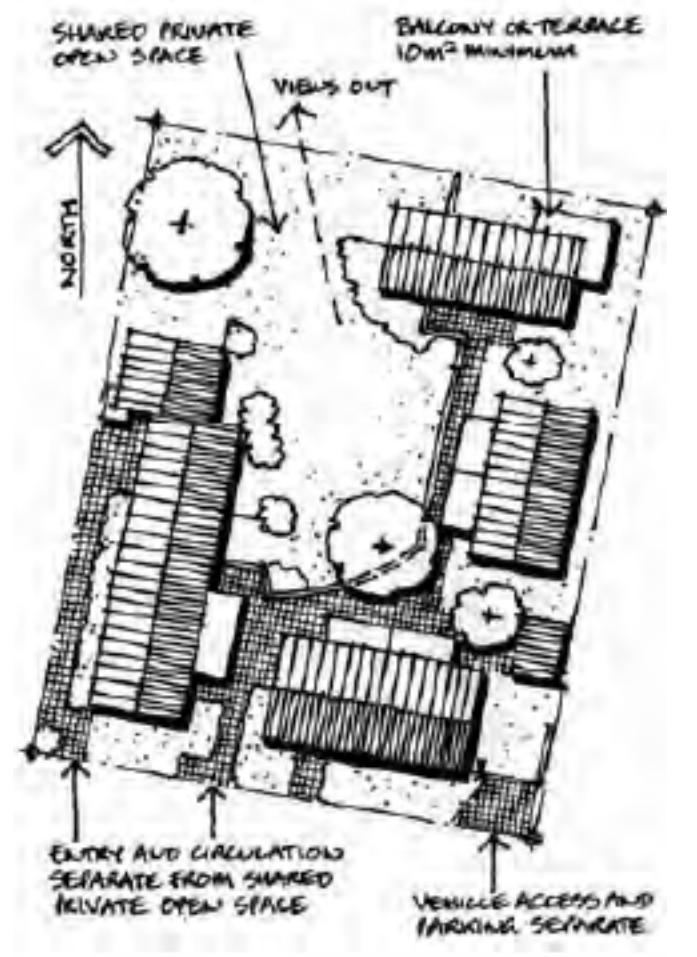

Figure 5.5 Shared private open space (Wellington City Council Residential Design Guide - July 2009: 16, G3.6)
- form the planning focus of the development;

- have direct or easy connection to all dwellings served;

- be access-controlled by its location, planning and design, and managed so it is available to the residents of the development only;

- be sunny and have a view beyond the site; and

- be generally flat, but may incorporate changes in level where these are designed to add to the visual and functional amenity of the shared space

(WCC Residential Design Guide July 2009: 16, G3.6) 
The Design Guide envisages 'shared private outdoor space' to be beneficial in dwellings designed for communal living, such as "housing for the elderly, student housing, and papakainga" (ibid. 17, G3.6). In such instances the Guide envisages shared outdoor space will have active edges by having entrances and windows of habitable rooms located around its edges. Wellington City's Residential Design Guide notes, however, that a "balanced approach that also provides reasonable privacy for dwellings is anticipated." This suggests that dwellings located around a communal outdoor space might also have a measure of private outdoor space of their own, as well as connecting with the communal outdoor space.

Frey notes there is general consensus for more compact housing, open space, safe areas for children, walkability, social interaction, attachment and identity, efficient use of resources:

"...an urban form that responds to the sustainability criteria [listed in Frey's Table above], a city that is people-friendly, works efficiently and has a sustainable relationship with the regional and global hinterland"

Frey (1999) in The Urban Design Reader (2007: 342)

Clustered housing with multivalent communal space fulfils many of the criteria summarised by Frey in the above quote - to provide living environments that are people-friendly, housing that is sustainable and that works efficiently, and that has a symbiotic relationship with (micro-scale multivalent) green open space. Clustered housing, according to Cooper Marcus and Sarkissian (1986) will have an increasingly significant role in accommodating families within the city. 


\section{Principle \#3: Communal Green Open Space}

Frey, in The Urban Design Reader 2007 (328), comments that regardless of the form sustainable cities might take, open space must remain an essential element.

Randolf Hester (1975) emphasises the importance of open space in the form of 'neighbourhood space'. He also emphasises the importance of community participation in the creation of neighbourhood space and provides user-need check lists and techniques for facilitating such participation.

Much research has been carried out on the nature and benefits of urban green space, and is summarised by Kazmierczak and James (2007) (see Chapter 4). James et al (2009) are developing an interdisciplinary framework for researching urban green space to gain a better understanding of how it contributes to people's wellbeing in cities and to the sustainability of urban environments.

Cooper Marcus and Sarkissian comment that their book "Housing As If People Mattered" ...

"... emphasises children's needs ... because they are the chief users of common outdoor space[s], and the most influenced by their design ..." (1986: 13)

This research challenges the notion that communal outdoor space is primarily for the use of children. It advocates imaginative fusion of many possible compatible uses of communal neighbourhood space to create multivalence. By doing so, not only children but all ages will be attracted to make use of communal spaces, indoors and outdoors, in medium-density housing environments.

Alexander et al (xii, xiii) give an example of a suggested 'pattern language' for an 'accessible green'. The authors explain how an accessible green might be designed in connection with larger and smaller patterns to which it relates. 
(C) 2012 Stephen Pattinson Chapter 5: Principles M. Arch Thesis

The key points of an 'accessible green' (see pattern \#60) according to Alexander et al are:-

- people will only walk to green open places if they are close by, therefore locate green open spaces within three minutes walk - about 750 feet (approximately 250 metres) - of every house and workplace

- Greens therefore should be uniformly scattered at 1,500 foot intervals (roughly 500 metres throughout the city

- Each green should be at least 150 feet (approximately 50 metres) across

- The total area of a green urban space needs to be at least 60,000 square feet (roughly 550 square meters)

Alexander et al. note this pattern for an accessible green could consist of various smaller patterns such as 'tree places' (\#171 - they suggest paying special attention to established trees, looking after them, and shaping the green to create 'positive outdoor space' (\#106 - the idea that outdoor spaces are not merely left over bits but purposeful and useful spaces that have sunshine, shade, shelter from the wind or rain, a pleasant view out, and that are good places for people). The authors suggest that other patterns which 'accessible green' could overlap with are such things as local sports (\#72), animals (\#74), and pools and streams (\#64).

The process of selecting, developing and integrating patterns allows ideas about spatial and physical concepts to be shared explicitly by a community of people. The more 'patterns' that a community can overlap within a space, the richer that place is likely to be in terms of shared use and meaning.

By integrating the above-mentioned pattern 'accessible green' with patterns for trees, walls, paths, streams, playgrounds, and sports fields etc., a community is able to create a richer place. Following this reasoning, if the intention is to create 
multivalent communal space then such a space is likely to be more well-resolved and meaningful if it is integrated at various levels with other meaningful patterns.

A very helpful text on how to create great outdoor open spaces for people is People Places by Cooper Marcus and Frances (1998). The authors introduce their text with the comment that users of the guidelines in this book are motivated by a care for people expressed through the quality of the built environment, the places that they make. The book provides guidelines for a full range of outdoor open spaces, including neighbourhood parks, mini-parks and pocket parks.

A final chapter in the book gives guidelines for carrying out post-occupancy evaluations (POE) of open spaces, that is, obtaining feedback from users on how well the spaces suit their needs and meet their expectations. 


\section{Principle \#4: Communal Space Fit for Children}

As housing intensifies in New Zealand's larger cities, communal space will become increasingly important for children. Children make up a significant portion of the population in New Zealand's urbanised areas.

According to Statistics New Zealand, 2006 census, just over half (52\%) of New Zealand's 1,168,000 children aged from 0 to 19 years live in New Zealand's four largest urbanised areas - Auckland (Super City), Wellington (includes Porirua and the Hutt Valley), Christchurch and Hamilton. This means there are approximately 608,000 children in these main urban areas. The breakdown is as follows:-

\begin{tabular}{|c|c|c|c|c|c|}
\hline \multicolumn{6}{|c|}{ Children in New Zealand's four largest urbanised areas (from Statistics NZ, 2006 census) } \\
\hline & $0-4$ years & $5-9$ years & $10-14$ years & $15-19$ years & Totals \\
\hline $\begin{array}{l}\text { Auckland } \\
\text { (North Shore } \\
\text { Waitakere, } \\
\text { Auckland, } \\
\text { Manukau \& } \\
\text { Papakura) }\end{array}$ & 90,684 & 91,728 & 95,874 & 96,327 & 374,613 \\
\hline $\begin{array}{l}\text { Wellington } \\
\text { (and Porirua, } \\
\text { Upper \& } \\
\text { Lower Hutt) }\end{array}$ & 25,323 & 24,657 & 25,371 & 27,483 & 102,834 \\
\hline Christchurch & 21,477 & 21,396 & 22,797 & 25,875 & 91,545 \\
\hline Hamilton & 9,564 & 9,138 & 9,555 & 11,148 & 39,405 \\
\hline Totals & 147,048 & 146,919 & 153,597 & 160,833 & 608,397 \\
\hline
\end{tabular}

Table 5.2 Children in New Zealand's four largest urbanised areas (from Statistics NZ, 2006 census)

Cooper Marcus and Sarkissian (1986) comment that much of the built environment tends to overlook the needs of children who, as the above figures for New Zealand show, make up a large user group of the urban environment. 
Evidence is emerging that as New Zealand's urban areas intensify, children are living in housing environments that do not meet their needs. Examples include toddlers living in small flats above ground level shared by more than one family, with minimal balcony space (writer's personal observation, Mt Eden, 27.3.10); of children playing on roads with more than the usual vehicle traffic flow because of the higher density of the residential environment (writer's observation, Albany, 14.10.09); of children in medium density housing having no play space other than car parking areas (writer's personal observation, Onehunga, 28.3.10); and of increasing numbers of families with children living in high rise apartments not designed for children (Witten 2010).

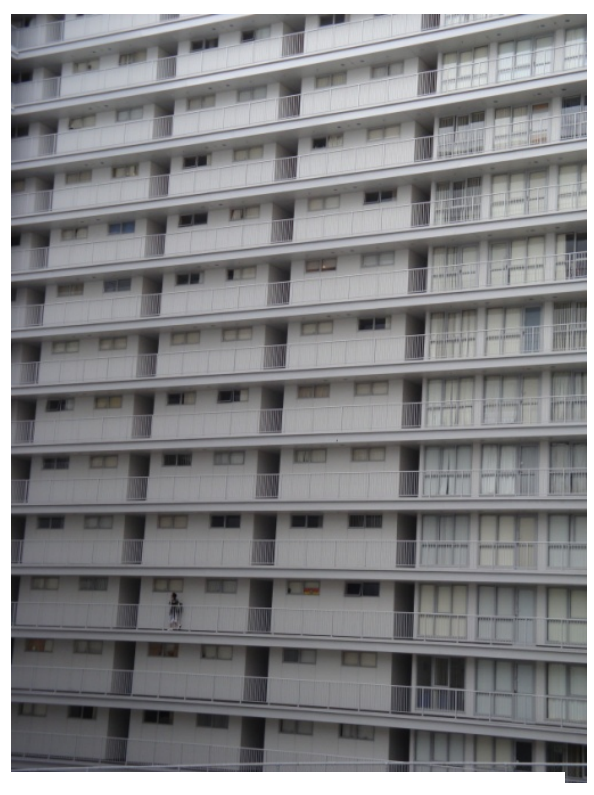

Fig. 5.6 Witten finds increasing numbers of families with children living in high rise apartments in Auckland's CBD.

(Photo: Karen Witten)

An example of the impacts on children of city environments that give little attention to children's needs can be found in a recent study by Synergia Ltd for Manukau (commissioned by Council and Ministry of Health). Dr Adrian Field, a director of Synergia Ltd, presented the findings of "Manukau Built Form and Spatial Structure Plan" Health Impact Assessment (HIA) Report (2008) (available on Ministry of Health website) at a seminar titled “Children's changing worlds: Do our cities work for children?" (organised by the New Zealand Centre for Sustainable Cities, Wellington, 16 June 2010). Synergia Ltd assessed Manukau's built form for accessibility, liveability, vitality, suitability for active transport (walking and cycling), and safety.

Although this study primarily focuses on children's use of a local shopping centre, and not housing, the study is still relevant to this research because it gives a 
contemporary picture of children's changing world's in New Zealand's cities. The comments in this study about public spaces in a shopping centre may also apply to open spaces around homes. Also, comments about connectivity and accessibility apply to the home in relation to the rest of children's urban environments.

The study focused on Year $7 \& 8$ children (approximately 11 and 12 year olds, mostly Maori and Pacific Island children) at Clendon Park Primary School. The researchers investigated the children's use of the Manukau shopping centre and the nearby Hayman Park. Dr. Field comments that one of the issues the study investigated is the impact on children of 'getting around the city by car' versus 'getting around the city by walking and cycling'. Field notes that the car is dislocating children from their neighbourhoods.

The study notes that children are typically taken from home to the shopping centre by car, and dropped off at the Mall or The Warehouse. It notes that there is not much for children to do at the shopping centre apart from visiting Rainbows End theme park. However, at a cost of $\$ 45$ per adult and $\$ 35$ per child, the theme park is not a regular option. There is nothing for children to do at the town square. Field comments that the civic spaces of the shopping centre seem designed to discourage children being there. The town centre is perceived by parents as being unsafe for children with respect to traffic, alcohol and strangers.

An apartment tower in the shopping centre, that accommodates some families with children, has no 'back yard' and no play area. Children are not free to come and go from the building for fear that, if they were given security swipe cards for access, they might loose the swipe cards.

The study notes that the civic centre needs to be better provided with green open spaces. There is no swimming pool in the area. Hayman Park, being distant from 
the shopping centre and landscaped with earth mounds and trees that screen it from view, is perceived by parents and children as an isolated place with no passive surveillance. It is considered an unsafe place for children. The study recommends that suitable places be provided for children at the shopping centre, spaces for unstructured meeting and play. A more pedestrian-oriented retail centre (not just walking from car park to Mall) would provide more eyes for watching over children playing around the shopping area. Also, places for unstructured play could encourage more inter-generational mixing.

One of the overall recommendations of the study, comments Dr Field, is a needed shift from car-centric to pedestrian-centric planning. The study notes that this is as much a social shift as a planning shift. For example, Manukau shopping centre lacks localised sporting facilities. Most sport for children is organised to occur on week nights or weekends, and venues are usually accessible only by car. Urban structure planning for the shopping centre needs to consider sports facilities for children and active transportation (walking and cycling) that connects easily with the facilities.

Claire Freeman (2010b), another presenter at the Sustainable Cities seminar “Children's Changing Worlds: Do Our Cities Work for Children?", comments that planning processes typically focus on recreation and education for children and young people but rarely consider the suitability of housing environments and shopping facilities for children and young people. As children grow up, their independent mobility range from the home gradually increases. Cities, however, are becoming increasingly fragmented. This makes it difficult for children to connect easily with other neighbourhoods except by being driven around by car. Freeman notes that some new suburbs even omit footpaths. Around the home and the school, Freeman notes a tendency towards insular living. Upmarket houses shut off the street with high fences, gates and bars. Some school grounds are enclosed 
with high wire-net fencing, topped with barbed wire, for protection against intrusion. Children become cut off from their neighbourhoods.

Because of the difficulty children experience in getting around the city (except by car), Freeman (2010a) comments that urban environments are becoming 'terra incognita' for children. Freeman notes that children need to connect with their peers in city centres - 'hanging out', 'meeting', 'joining in', 'colonising place', 'playing', 'challenging' and 'breaking silly rules' (like 'keep off the grass').

According to Claire Freeman, today's children are growing up in dynamic worlds that are diverse and multi-cultural. Through the use of communal play spaces, Freeman notes that children are able to build bridges where there are traditionally borders for example, Freeman showed a slide of Catholic and Protestant children in Ireland playing together on a neighbourhood green.

Kate Swanson (2010) explores a different type of border - "the borderlands of the mind" as Swanson puts it, "have disrupted young people's lives, particularly the lives of indigenous youth" in Canada, a country regarded as one of the best places in the world to live. Swanson explains that not only do indigenous young people straddle cultural borders but they also live in the borderlands of the adult/child world, "doubly stigmatised as both indigenous and young" (431).

Communal space is about overcoming borders, initiating bridges. Swanson notes that, despite the desperate circumstances of many indigenous youth, "young people have the capacity to engage with their revolutionary imaginations to overturn internal borders and work for positive change" (433).

One of the ways Swanson observes indigenous youth making positive changes is through new technologies including "online forums, texting, and social networking 
sites [virtual communal space], as these can be deeply meaningful venues for social interaction" (434). Swanson reports that, through their resilience and use of new technologies, "youth are playfully reworking borders to build bridges between communities" (429).

"There are so many borders that divide people, but for every border there is also a bridge."

- Gina Valdés (in Swanson 434)

Barbara Lambourn, NZ National Advocacy Manager for UNICEF (United Nation's International Children's Emergency Fund) commented at the above-mentioned seminar on "Children's Changing Worlds" that the concept of "child-friendly cities" was discussed and launched at the 1996 UN Conference on Human Settlements held in Istanbul (from which emerged the Habitat II Agenda). Lambourn comments that the very early years are most important in a child's development. Therefore the places where children live, play and relate with the environment are very important. The UNICEF motto is "Cities that are fit for children are fit for all".

This theme is taken up by Gleeson and Sipe in "Creating Child-friendly Cities: Reinstating Kids in the City" (2006). They mention child obesity and the disenfranchisement of children in the city. Children need safe, clean and healthy living environments where they can engage in free-play and recreation $(14,15)$.

With over 600,000 children living in New Zealand's four largest urbanised areas, the size of this user group warrants the best efforts of city place-makers.

As noted above by Adrian Field, meeting the needs of children in cities requires a social shift as much as a shift in planning focus to enable, for example, childrens' 
unstructured play in safe city environments. Cities that are fit for children are fit for all. Meeting the needs of children in the city, and in higher density housing environments, is a good start. But the task of building sustainable communities must involve the willing and active participation of the whole community.

\section{Principle \#5: Communal Space to Foster 'Sense of Community'}

What role might communal space play in fostering "a sense of community and strong local identity in neighbourhoods"? The literature shows that there are four or five key components to 'sense of community', all of which can be enhanced by neighbourhood communal space (see Chapter 4):

- Community identity

- Community attachment

- Pedestrianism

- Social interaction, and

- Local autonomy

Thorns comments that the word 'community' implies a degree of group cohesion. It could be based on territory, work, ideas, or skills (15).

Thorns notes that planners' intentions to create a sense of community in residential areas can differ from observed outcomes for residents. Designs focusing on physical neighbourhood features but lacking social content suggest "too much faith in social engineering via improvements in the physical environment" (63). Thorns notes that designers also tend to underestimate the extent to which individuals seek their own values and privacy ahead of community values. A common feature Thorns finds in all types of communities is a basic conflict between individual and community values (Thorns 134, 135, 147, 149). 
New urbanism is based on an underlying principle that "community planning and design must assert the importance of public over private values" (Bressi , cited by Grout and Wang 206). Robert Putnam (2000) attributes the decline in social capital over the last thirty years in the USA to a number of factors including individualism, high mobility and the isolating effect of the suburbs. Putnam considers that smart growth / new urbanist design principles have a positive role in facilitating a resurgence of social capital $(214,307)$.

Keller notes that often plans for the physical environment intended to foster social interaction and a sense of community are based mainly on professional idealism and assumptions that are un-researched (4). This is changing. An example is research by Joongsub Kim (2001) and his investigation of 'sense of community' in new urbanist designs.

Grout and Wang (215) refer to Joongsub Kim's study (2001) of a new urbanist neighbourhood design at Kentlands (206). From academic literature, Kim identified four essential components of 'sense of community' in the literature:

- community attachment

- pedestrianism

- social interaction, and

- community identity

Kim's research compares the 'sense of community' between Kentlands and a conventional residential development. Kim carried out extensive surveys and interviews of residents in the two neighbourhoods. He observed that in all four measures (attachment, identity, pedestrianism, and social interaction) residents of the new urbanist neighbourhood consistently rated their 'sense of community' higher than the residents of the conventional suburb. Kim concludes that new urbanist design contributes to a greater sense of neighbourhood identity, 
attachment, walkability, and social interaction - in short, a greater 'sense of community'.

Kim drew this conclusion from his investigation of specific physical design features in the two neighbourhoods. Kim was able to relate the stronger 'sense of community' to the physical design features of the new urbanist neighbourhood. These physical features include such things as:-

- $\quad$ street width (narrower in new urbanist)

- distance between footpaths and houses (shorter in new urbanist)

- residential density (higher in new urbanist)

- mixture of housing types (more types in new urbanist)

- $\quad$ street layout (more well-connected)

- $\quad$ street trees and landscaping

- $\quad$ public greens, tot lots, footpaths (open space, children's playgrounds more accessible in new urbanist)

A fifth characteristic can be added to Kim's components of 'sense of community' is local autonomy. Frey, in his table summarising criteria occupying 'common ground' in the sustainable city debate, notes that cities should have ...

"... a degree of local autonomy, the ability of individuals and the communities to shape their own environment according to their needs and aspirations; this would also support if not generate a sense of place and community, a sense of belonging."

Frey (1999) in The Urban Design Reader (2007: 340)

Park asserts that ...

"... under a system which makes residence the basis for participation in the government, the neighbourhood becomes the basis of political control." (580) 
Hester $(11,13)$ relates the scale of 'neighbourhood autonomy' to the scale of a political issue affecting it, as suggested by Milton Kotler:

"What is needed... is a practical definition [of 'neighbourhood']... that recognises the social, spatial, and political aspects of a neighbourhood ... Milton Kotler has proposed such a definition: 'The neighbourhood is a political settlement of small territory and familiar association, whose absolute property is its capacity for deliberative democracy.' ... Kotler's definition implies that the size of the small territory depends on the political issue. If the location of a thoroughfare will affect several thousand people, ... that is a small territory. If the location of a minipark will affect only a block, that is a small territory. ...[N]eighbourhood changes in the minds of the residents according to the nature of the political crisis or issue..."

Jane Jacobs describes her neighbourhood's battle in Greenwich, Manhattan, to prevent an expressway being built through their block in her book "The Death and Life of Great American Cities", 1961). Suzanne Keller summarises Jacob's views on neighbourhood autonomy and the street level and at the district level:

"Jane Jacobs ...considers self-management necessary for a successful city neighbourhood. It should operate at two levels - the street and the district. The street neighbourhoods are essentially those where the routine of daily life goes on - meetings, shopping, and just strolling. The vitality, concerns, needs and interests of residents are expressed there.

"On the district level, the more impersonal, organisational forces of the city become decisive. Jacobs sees these districts, represented by effective leaders or spokesmen, as intermediaries between the powerful city and the powerless street. She correctly observes that selfmanagement means different things at different levels.

"At the street level it involves resident participation, active concern for common local problems, and a network of interdependent human relationships ...

"At the district level, the ability to formulate broad policies, to relate to the city as a whole, and to deal with city hall directly to obtain necessary public improvements and services becomes crucial. Street and district thus have distinctive, yet equally essential, organisational objectives - one stresses the unique personal character of the local habitat, the other forms a common link to a wider external world on which the local unit depends."

("The Urban Neighbourhood ..." Keller 144 -145) 
Autonomy is therefore needed at both the street and district levels. Hamdi (xvii) anticipates changing roles for design professionals to support neighbourhood autonomy. He sees the expert's role as enabling local participation, helping communities envisage and shape their own 'best fit' solutions for the structures and content of their built environments ... incrementally. He sees design professionals enabling others to imagine their future and one that begins now, assisting them to cultivate and sustain change in a way that offers communities a 'shared sense of purpose'.

\section{Conclusion:}

Multivalent communal space is a component of the sustainable city. This research has identified five key aspects which if applied to communal space will (potentially) give the quality of multivalence to that space. The potential for multivalence will be realised when as many of these five aspects as possible are integrated into communal space in a way that:

- is imaginatively creative, putting the parts together in a new way,

- transforms the space by the fusion of as many parts as possible,

- links disparate parts together into a new whole,

- allows the parts to modify each other.

This multivalence will transform neutral time and space into memorable place and occasion, so that, rather than feeling anonymous and powerless in the city, people are more likely to feel connected, that they are somebody living somewhere ... ... AND ...

.... multivalance of communal space in clustered, medium density housing may provide an answer (at the neighbourhood level) to the question - "what is the form of the sustainable city?" 


\section{CHAPTER 6 - Case Study - Pinehill Park}

Part 1: The Case Study $\quad 152$

1.1. An explanation of the approach taken for this Case Study 153

1.2. Background information about Pinehill Park 158

1.3. Observations of the existing use of Pinehill Park 172

1.4. Interviews with residents of terrace housing 173

$\begin{array}{ll}\text { Part 2: Results of The Case Study } & 174\end{array}$

2.1. Observations of Pinehill Park 174

2.2. Comments relating to the observations of Pinehill Park 177

2.3. Interviews with residents of the terrace houses 181

2.4. Responses correlated with the Five Principles 182

2.4.1. Walkable Neighbourhoods 182

2.4.2. Clustered Housing 184

2.4.3. Green Open Space 187

2.4.4. Needs of Children and Teenagers 192

2.4.5. Sense of Community 196

$\begin{array}{ll}\text { Conclusion } & 200\end{array}$

Note: Ethics Approval No. 17632

See Appendix 5 for the Survey and Interview forms

See Appendix 6 for The Ethics Approval documents, including the Ethics Approval Information Sheet and Consent Form 
(C) 2012 Stephen Pattinson Chapter 6: Case Study-Pinehill Park M. Arch Thesis

\section{PART 1: The Case Study}

This Case Study on Pinehill Park explores the public / communal space of the park adjacent to the medium-density terrace housing at Pinehill Crescent, Upper Hutt. This public park, owned and maintained by Upper Hutt City Council, was created as part of a new 75-lot subdivision at Pinehill Crescent from 2002 to 2004 . The subdivision was developed as a joint venture between Harwood-Pacific (an Australian housing company) and Housing New Zealand Corporation. The terrace houses were built by Malcolm Gillies' company Golden Homes Limited in 2006/7. A playground was installed in the park by Upper Hutt City Council in 2007/8.

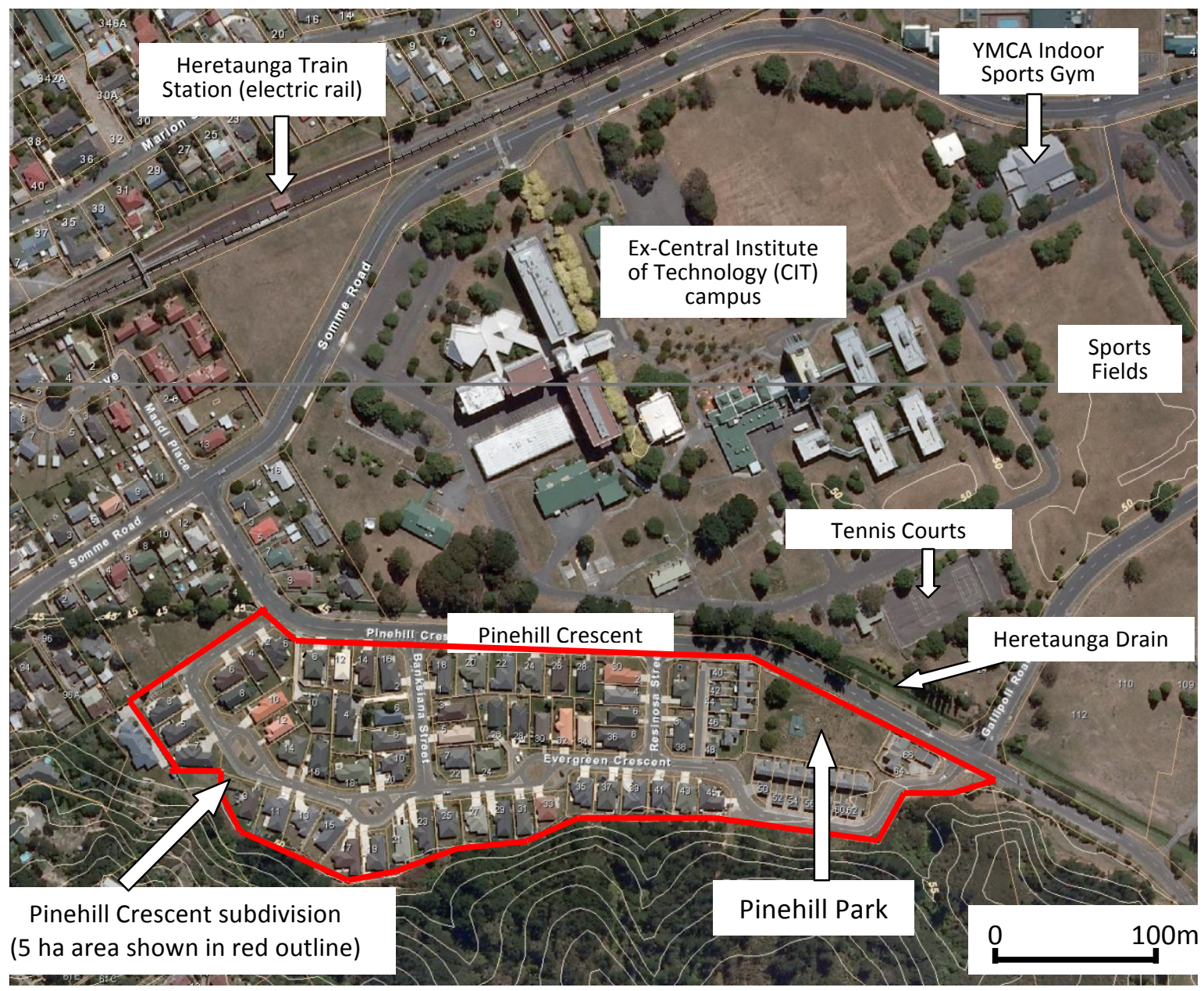

Figure 6.1 Aerial view of Pinehill Crescent subdivision (75-lots), Trentham, Upper Hutt. Pinehill Park is at the east end of the subdivision, with new terrace housing on three sides of the park. Pinehill Park was created for the 75 new lots as part of the land subdivision. Source: Upper Hutt City Council Xplorer aerial map - Approximate Scale 1:5,000 @ A4 size (North is up the page) 


\subsection{An explanation of the approach taken for this Case Study}

Initially, a suggested approach to this research was to carry out a literature search and, from the literature, to develop an analytical framework for communal space in medium-density housing. Analytical means a detailed analysis of the physical makeup of quality communal space. In other words, the objective of the literature review would be to create a conceptual framework of all the physical requirements for a common space to work successfully as shared space in a housing development. Armed with this conceptual framework, the next step would be to use the framework as a normative set of design guidelines against which to assess the quality of a number of existing case studies. The case studies, maybe $8-12$ in number, would be selected for their representative characteristics of particular types of communal space in medium-density housing developments.

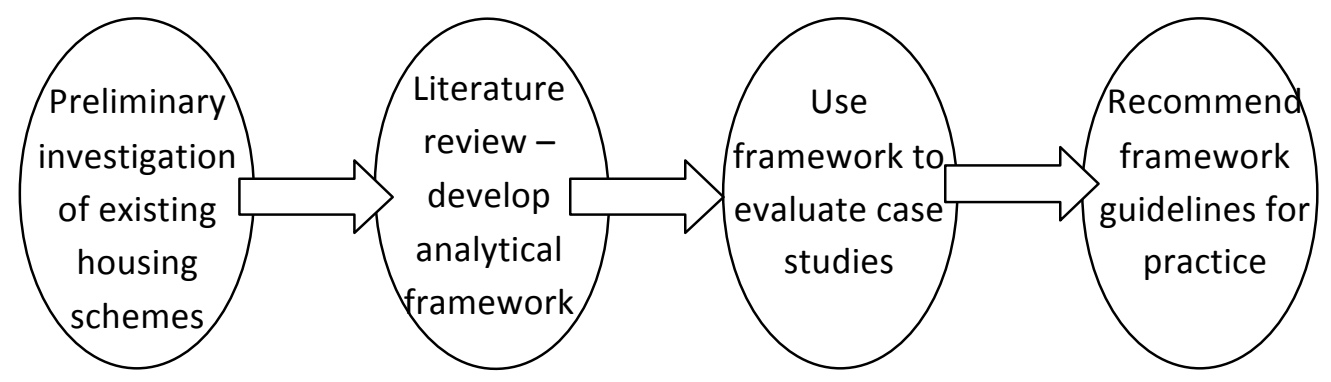

Figure 6.2 Linear Theory-to-Practice Approach

S.J. Pattinson

The writer discovered, however, that there is very little in the academic literature specifically addressing communal space in medium-density housing, and even less addressing multivalent communal space. The concept or principle of multivalent communal space has had to be constructed more or less 'from scratch', not by creating new knowledge, but by bringing various existing knowledge areas together and looking at them interactively in new ways. 
Consequently, the literature review has informed a concept of multivalent communal space, suggesting various uses and meanings that can overlap and enhance each other in common ground in medium-density housing. The outcome from the literature review was not an analytical framework for appraising existing developments, but rather a concept of multivalence in communal space.

This suggests a different approach is needed now for the Case Study work. Rather than evaluating a number of housing developments against a theoretical framework, a different purpose for the case study work emerges. The new purpose is to find out from people who actually live in medium-density developments what their point of view might be towards multivalent communal space.

What this approach to the case study work means is that the principle of multivalent communal space can be developed from three different perspectives or viewpoints - practitioners' (see Chapter 3), academic researchers' (see Chapters 4 \& 5), and the community's point of view, i.e. the perspective of residents (this chapter).

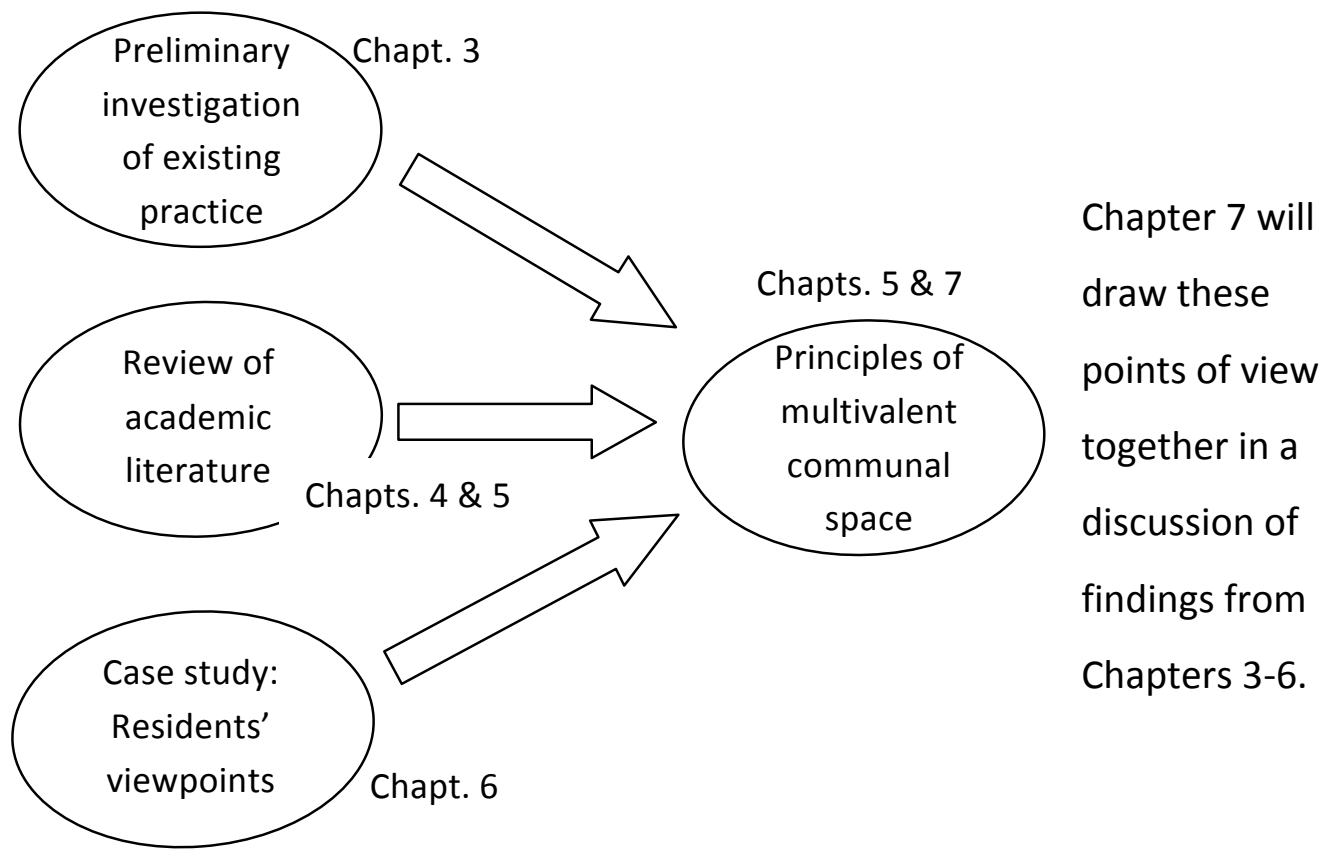

Figure 6.3 Participatory Approach: Dialectical Relationship Between Theory and Practice. S.J. Pattinson 
The approach outlined means that the case study work in this chapter, instead of being a summative appraisal, will be a participatory exploration, giving residents who use communal space in medium density housing an opportunity to contribute their experience, knowledge and creativity towards the development of the concept of multivalent communal space.

So far, there has been much breadth to this research - preliminary field visits to 200 existing medium-density housing developments and a wide scope to the literature review. What is needed now is some depth. Within the writer's ability, training and experience some depth has already been sought in each aspect of the literature review. Now this needs to be supplemented with some depth of enquiry into actual users' (residents') perspectives of communal space in medium density housing.

To achieve some depth from the residents' points of view, the writer has decided to focus on one Case Study rather than many, and to supplement this later (in the discussion in Chapter 7) with site observations and comments from dialogues with residents, urban designers, town planners, architects and developers that occurred during the preliminary field trips.

The Case Study approach outlined above has a reasonable academic basis. The idea of including ordinary people's viewpoints in the development of theory, rather than theory being developed exclusively from a distance by academics, seems to be supported by Pierre Bourdieu, an influential sociologist whose work over the last three decades of the $20^{\text {th }}$ Century is having a significant impact on concepts about 'sense of place' and social use of space.

In "Habitus: A Sense of Place" (2002: 3-6) the editors Jean Hillier and Emma Rooksby give an introduction to Bourdieu's work. According to Hillier and Rooksby, Bourdieu, in exploring the question 'what motivates human action?', proposes a theory which "connects structure and agency in a dialectical relationship between culture, structure and power". In other words, Bourdieu proposes that people 
develop and discover their 'habitus', that is their sense of place in their world through an embodied dialogue that takes place between their individual dispositions and the social power structures that are part of their living environment. Bourdieu makes room in his theory for both individual choice and social determinism. In other words, according to Bourdieu, people make their own choices but those choices are definitely influenced by social control and conditions that affect their everyday lives.

Hillier and Rooksby point out that Bourdieu vigorously challenges the traditional linear dichotomy that presumes that practice is the application of academic theory. Hillier and Rooksby point out that this traditional idea that practice follows theory is especially prevalent in professional views of the built environment. In this traditional view "theory becomes a totalising view-from-nowhere rather than being a dialectical relationship with practice" (Hillier and Rooksby 4, 5). In "Habitus: A Sense of Place", Hillier and Rooksby follow Bourdeiu's approach of 'building theory on practice and stories of everyday life worlds' which is what this Case Study will be doing by exploring the views of residents who daily use communal space in medium density housing.

Hillier and Rooksby further point out that Bourdeiu, in seeking to understand human behaviour, distinguishes between "synoptic and participatory views". While the synoptic view stands apart from the activity "describing what is taking or has taken place, participatory views regard action from what is avowedly a participant's standpoint" (Hillier and Rooksby 5). Although the editors point out that Bourdeiu deliberately avoids any clear definition of 'habitus' (Hillier and Rooksby 377 ) it has been described by others as involving dispositions which generate practices in the context of everyday social structures.

Up to this point in the research the synoptic views of the writer and authors of the academic literature have predominated. Now it is time to give voice to the practices 
of the everyday users of communal space in medium density housing. By doing this in this case study, the users' participatory voice can contribute in dialectical relationship to the development of theory on multivalent communal space.

Several housing developments could have qualified for selection for the Case Study. Pinehill Park in Trentham, Upper Hutt, was selected for the following reasons:-

- The site for the terrace house development at Pinehill Park has a combination of many ideal physical features, including the following facts:

- The site is flat, sheltered from southerly winds by the ridge to the south, and the long side of the site faces north (good for the sun);

- Good public transport exists nearby (3 minutes walk to a bus stop and 6 min walk to Heretaunga train station for rail journeys of 35 minutes to Wellington city and 7 minutes to Upper Hutt city centre);

- Good recreation facilities are nearby - grounds of ex-Central Institute of Technology campus (includes tennis courts and sports fields with exercise stations), rifle range 5 mins drive, golf course 5 minutes drive, YMCA gym less than 10 minutes walk;

- The development on this site is interesting in that there are two distinctly different housing typologies which offer opportunities for comparison;

- The park was created at the time of subdivision for the benefit of the residents of all 75 lots created in the subdivision, however the position of the park and the arrangement of terrace housing seems to suggest that the park will be used mainly by the residents of the terrace housing - this case study will investigate this perception;

- The 'community ownership' of the park seems ambiguous on several fronts does the park 'belong' to the wider community of Trentham in Upper Hutt, or does it 'belong' to this neighbourhood of 75 new allotments, or does it 
'belong' predominately to the residents of the terrace housing immediately adjacent to it?

- Because the park can be perceived as being possibly 'communal' (for the terrace house residents) more than 'public' (for anybody and everybody) it will be interesting to investigate its potential multivalence, in terms of uses and meanings, for the terrace house residents;

- This housing development is located in the Wellington region where the writer lives and therefore is accessible to the writer for observations of the site and interviews with the residents;

- The writer is familiar with this development from participation in public consultation during the design phase of the development from 2001-2003.

The following is a brief history and overview of the development of Pinehill Park.

\subsection{Background information about Pinehill Park}

\section{2a) History of the Pinehill site:}

The following is an historical timeline of the development of Pinehill Park as part of the new housing development in Pinehill Crescent, Trentham, Upper Hutt:

\begin{tabular}{|l|l|}
\hline \multicolumn{1}{|c|}{ Key dates } & \multicolumn{1}{c|}{ Items / events } \\
\hline Pre 1940's & $\begin{array}{l}\text { There seems to have been farming activity on this site and horse stables } \\
\text { for the Army (NZ Defence Force Army Camp at Trentham). }\end{array}$ \\
\hline $\begin{array}{l}\text { Early 1940's } \\
\text { (During WW2) }\end{array}$ & $\begin{array}{l}\text { The land was owned by the Army and the eastern end of the site on } \\
\text { Pinehill Crescent (where Pinehill Park now is) was developed by the } \\
\text { Public Works Department with a sewage plant for the Army Camp. }\end{array}$ \\
\hline (date unknown) & $\begin{array}{l}\text { The site is transferred from the Army to the Department of Justice and } \\
\text { about 40 single family houses are built on the site for prison staff. }\end{array}$ \\
\hline $\begin{array}{l}\text { (dates } \\
\text { unknown) }\end{array}$ & $\begin{array}{l}\text { The sewage plant catchment was later extended to include Wi Tako } \\
\text { Prison, Prison staff housing along Pinehill Crescent as far as Wier Grove } \\
\text { (next to the railway line), and the Central Institute of Technology (CIT). }\end{array}$ \\
\hline 1993 & Decommissioning and closure of the sewage plant. \\
\hline 1999 (?) & Prison staff and their families vacate the site. \\
\hline 2000 (?) & $\begin{array}{l}\text { Housing New Zealand Corporation (HNZC) acquires the site from the } \\
\text { Justice Department. }\end{array}$ \\
\hline
\end{tabular}




\begin{tabular}{|c|c|}
\hline \multicolumn{2}{|l|}{2001} \\
\hline $16 / 5 / 01$ & $\begin{array}{l}\text { The Upper Hutt Leader (Local newspaper) - Front page headline: } \\
\text { "Housing NZ seeks views on future of former prison houses" - this } \\
\text { newspaper article advises the local community about HNZC's intention } \\
\text { to provide new housing at Pinehill Crescent and gives an open invitation } \\
\text { to a public meeting to be held that evening. }\end{array}$ \\
\hline $16 / 5 / 01$ & Developer holds $1^{\text {st }}$ public consultation meeting (Silverstream School) \\
\hline $23 / 5 / 01$ & $2^{\text {nd }}$ public consultation meeting (Silverstream School) \\
\hline $31 / 5 / 01$ & $3^{\text {rd }}$ public consultation meeting (Brentwood School) \\
\hline $11 / 6 / 01$ & $\begin{array}{l}4^{\text {th }} \text { public consultation meeting (Silverstream School) - Appointment of } \\
\text { Pinehill Community Committee (PCC) to liaise with HNZC on its } \\
\text { commitments to the local community. }\end{array}$ \\
\hline Remainder 2001 & PCC has a number of meetings with HNZC \\
\hline \multicolumn{2}{|l|}{2002} \\
\hline $1 / 7 / 02$ & $\begin{array}{l}\text { The Government releases a press statement (see Appendix } 2 \text { ) } \\
\text { announcing the development of new housing in Pinehill Crescent in a } \\
\text { joint venture between the Australian company Harwood-Pacific and } \\
\text { HNZC, the first public-private partnership of its kind in NZ which may } \\
\text { become a model for future developments. (This press release was } \\
\text { reported in the Upper Hutt Leader the same week on 3/7/02). }\end{array}$ \\
\hline $26 / 11 / 02$ & $\begin{array}{l}\text { The developer lodges the Resource Consent application for the } \\
\text { subdivision at Upper Hutt City Council (UHCC). }\end{array}$ \\
\hline \multicolumn{2}{|r|}{ 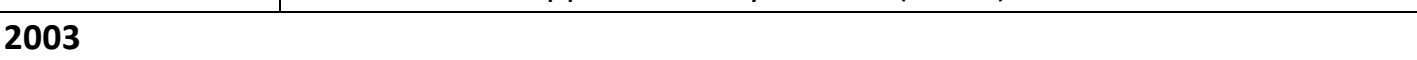 } \\
\hline $14 / 2 / 03$ & UHCC grant the Resource Consent. \\
\hline 2004/2005 & Construction of the subdivision. \\
\hline $2006 / 2007$ & Construction of the houses. \\
\hline 2007/2008 & UHCC installs a new children's playground in Pinehill Park. \\
\hline
\end{tabular}

Table 6.1 Chronology of Pinehill Park (S.J. Pattnson)

Regarding the development of the Park at Pinehill Crescent, according to the writer's recollection, the subdivision developer's public consultation process did not include any information or discussion about the location or nature of the Park. Similarly, a resident of one of the terrace houses who was occupying the terrace house at the time that the Council installed the children's playground in the park, advises the writer that to his knowledge local residents were not consulted about the development of the park or the location or nature of the playground equipment. 
For the purposes on this research on multivalent communal space in medium density house, this Case Study will seek from local residents of the terrace houses who have used the Park for the last 2 or 3 years how they find it, what it means to them and any suggestions or ideas they might have on its hypothetical potential.

1.2b) Physical layout of the terrace housing and the park:

1.2b(i) Description of the layout of the terrace housing and park

The subdivision of the site and issuing of property titles was completed in 2004. Malcolm Gillies bought the lots for all fourteen terrace houses from the subdivision developer, Pinehill Developments Joint Venture (between the Australian-based housing development company Harwood-Pacific and Housing New Zealand Corporation). The terrace housing (on Lots 78, 79 and 80 in the survey plan - Fig. 6.4 below) is situated on three sides (East, South and West) of a new park (Lot 77 - the proposed reserve vested in Upper Hutt City Council in lieu of the developer's subdivision reserve contribution).

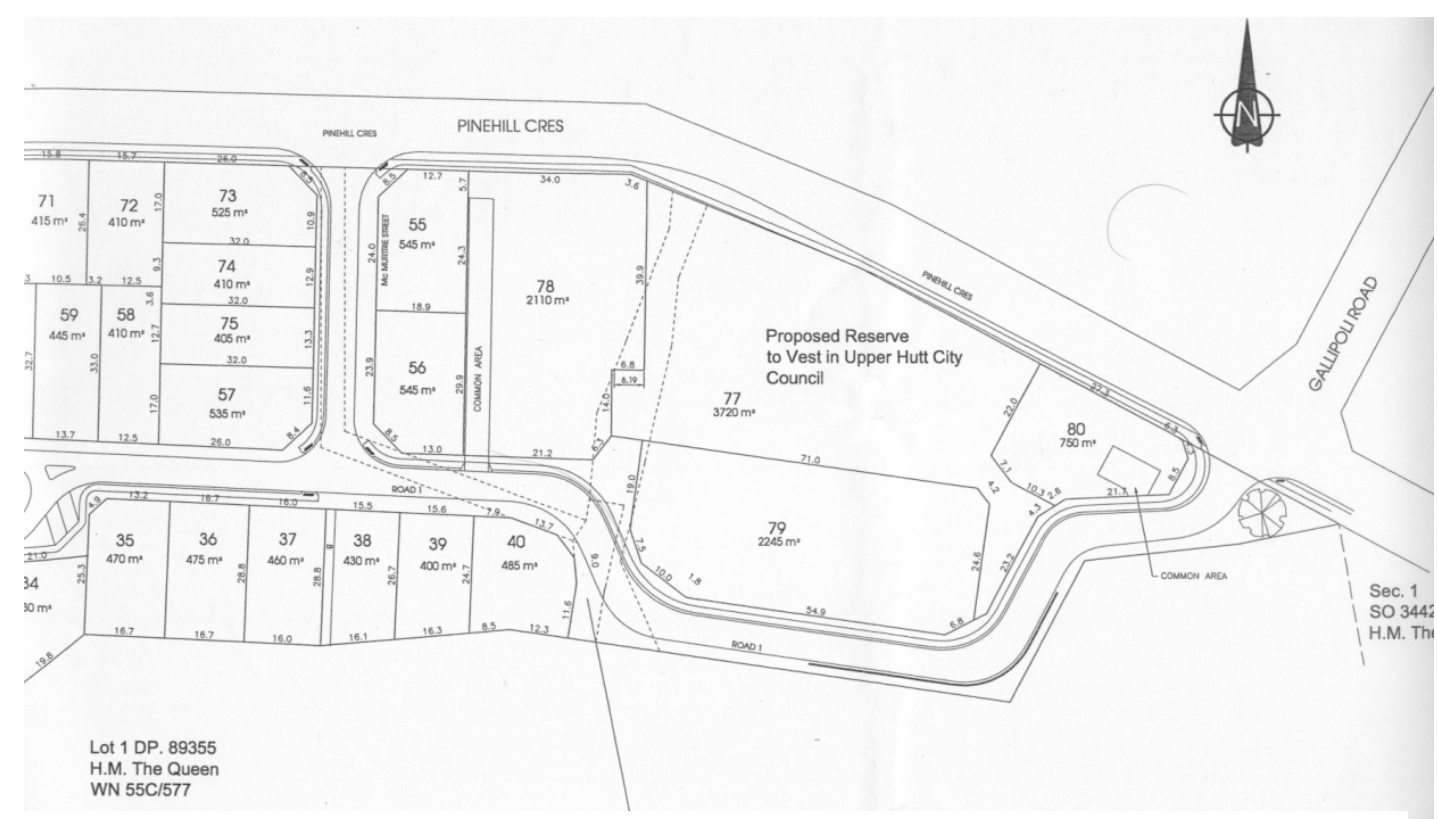

Figure 6.4 - Survey Plan of the proposed new Reserve (Lot 77), Pinehill Crescent flanked on the East, South and West by lots for new terrace housing (Lots 78, 79, 80).

Source: Upper Hutt City Council, from Resource Consent plans submitted by TCB (Truebridge Callender Beach Ltd - Land Surveyors) 


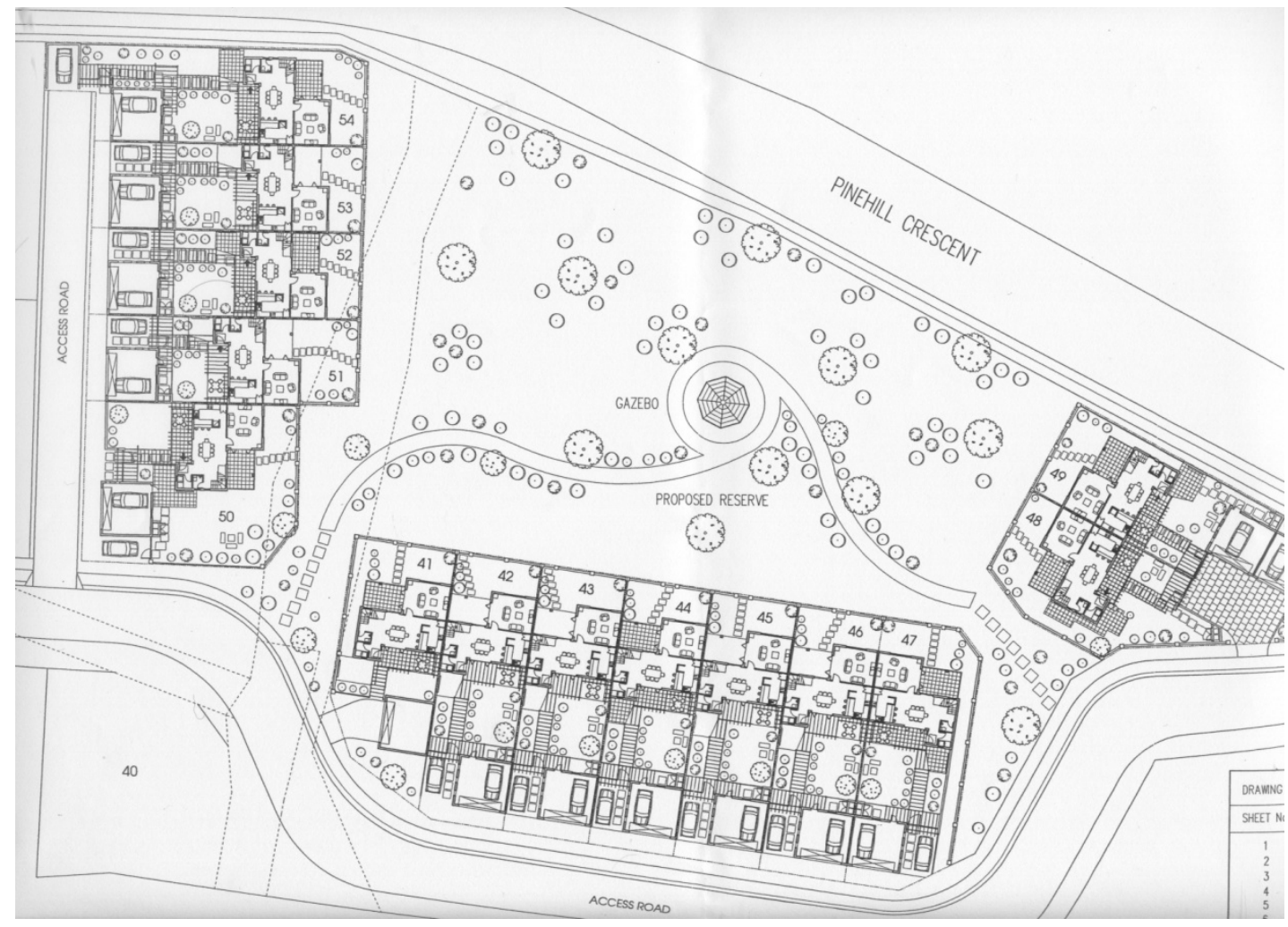

Figure 6.5 - Site Plan of the proposed 14 new terrace houses, 5 on the Western side of the park, 7 on the Southern side, and 2 on the Eastern side of the park.

Source: Upper Hutt City Council, from Resource Consent plans submitted by TCB (Truebridge Callender Beach Ltd - Land Surveyors). Note: the Nos. 41-54 are not the street address numbers, but the lot numbers in the overall 75-lot subdivision plan.

Upper Hutt Developments Ltd built the fourteen terrace houses and then onsold them. (The other 61 houses in the 75 -lot subdivision were built by Harwood-Pacific, 16 of which were taken over by Housing New Zealand Corporation for its tenants. None of the 14 terrace houses were taken over by Housing New Zealand Corporation. Malcolm Gillies verbally confirmed to the writer that all the terrace houses were built and sold by Upper Hutt Developments Ltd). He also informed the writer that the terrace houses were designed by Harwood-Pacific, and the designs approved by Upper Hutt City Council as part of the subdivision Resource Consent process. 
The proposed reserve shown on the above plan (Fig. 6.5) is as it was shown at the Resource Consent application stage (26.11.2002) and it is an indicative layout only. It is not the final layout of the park. The final layout of the park was determined later by Upper Hutt City Council after the Council concluded negotiations with the subdivision developer for the reserve in lieu of the reserve contribution. The final agreement included a cash contribution from the developer of approximately $\$ 35,000$ towards the development of the park. The Council's Annual Report 2007/8 shows that Council spent $\$ 34,000$ in that financial year installing a playground at Pinehill Park. The layout of the park as it is now built is shown in the aerial view below (Fig. 6.6).

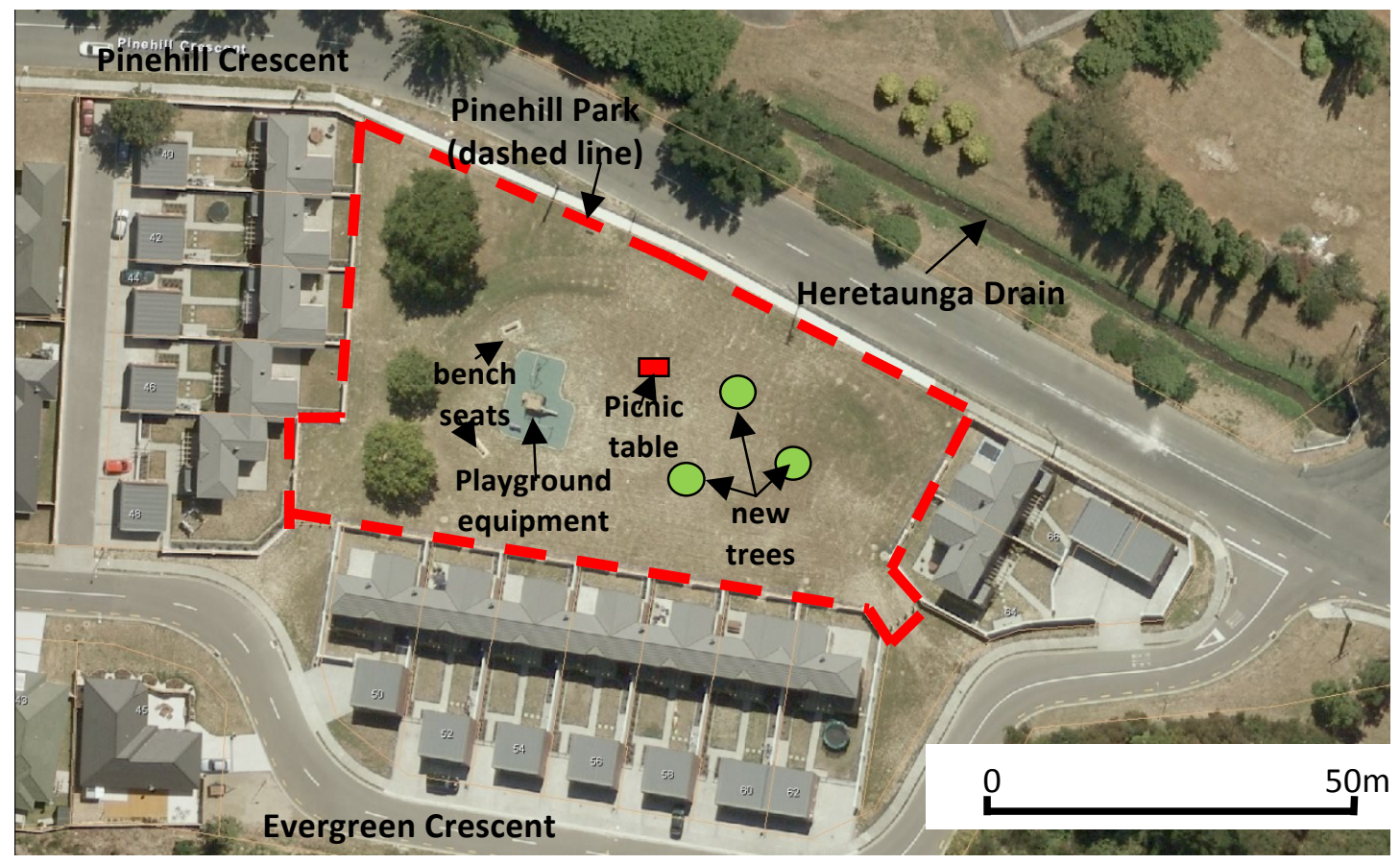

Figure 6.6- Aerial view of Pinehill Park, showing existing mature trees and playground equipment and two park benches installed by Council in 2007/8. More recently a picnic table and three new trees were also installed by Council. (North is up the page)

Note: the two slightly greener areas at the top-left and top-right ends of the park are where imported earth berms have been built up and grassed.

The road at the top of the park is Pinehill Crescent. The new road along the bottom of the photograph built for this subdivision is Evergreen Crescent. (Source: Google map) 


\section{$1.2 \mathrm{~b}$ (ii) Plans of the terrace houses}

The 14 terrace houses are two-storey and all have the same floor plan and are built with the same cladding materials - weatherboard to First Floor Level and cavity brick veneer over timber framing to Ground Floor level. Party walls between adjoining houses are solid-filled reinforced concrete masonry.

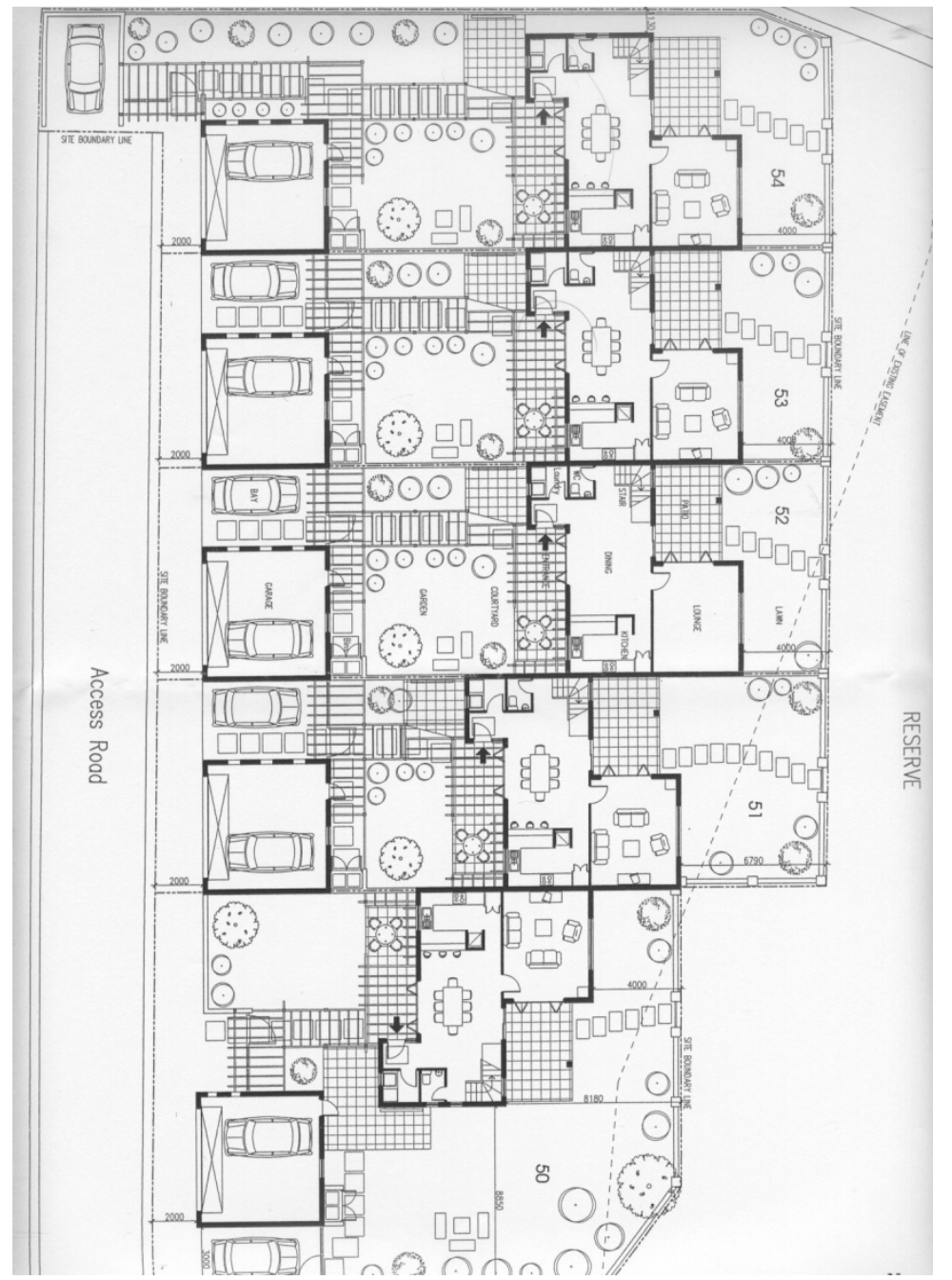

The three different orientations of the houses (some facing to the East, some West, and some North) provide some variation in terms of sun exposure to the houses and private yards, and to views of the park and beyond. Further minor variation is achieved by the mirror imaging of the floor plan for some of the houses, and, in a couple of instances (the southern two houses in the group of five on the West side of the park see Fig. 6.7 opposite), a step-back in the alignment of the fronts of the houses.

Figure 6.7 - Plan view of the group of five houses on the West side of Pinehill Park.

Note: the southern two houses in this group of five have a step-back in the alignment of the fronts of the houses, which adds some variety to the layout.

Source: Upper Hutt City Council, from Resource Consent plans submitted by TCB (Truebridge Callender Beach Ltd - Land Surveyors). $\quad$ (North is up the page) 


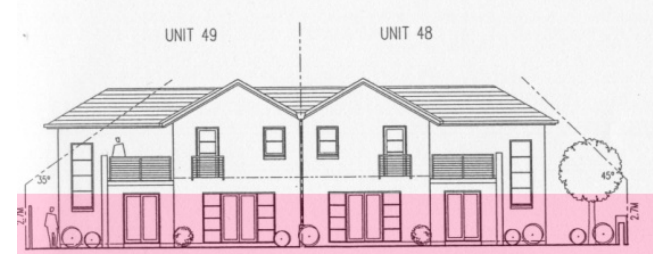

WEST ELEVATION (View from Reserve)

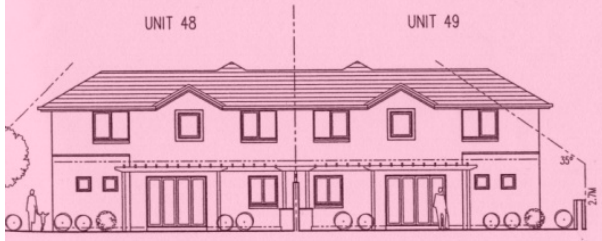

EAST ELEVATION (View from Courtyard)

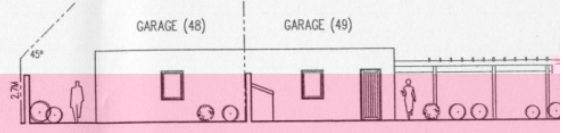

NORTH ELEVATION

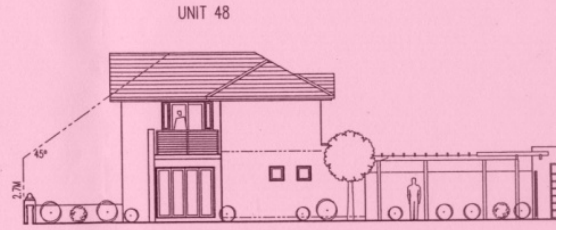

SOUTH ELEVATION

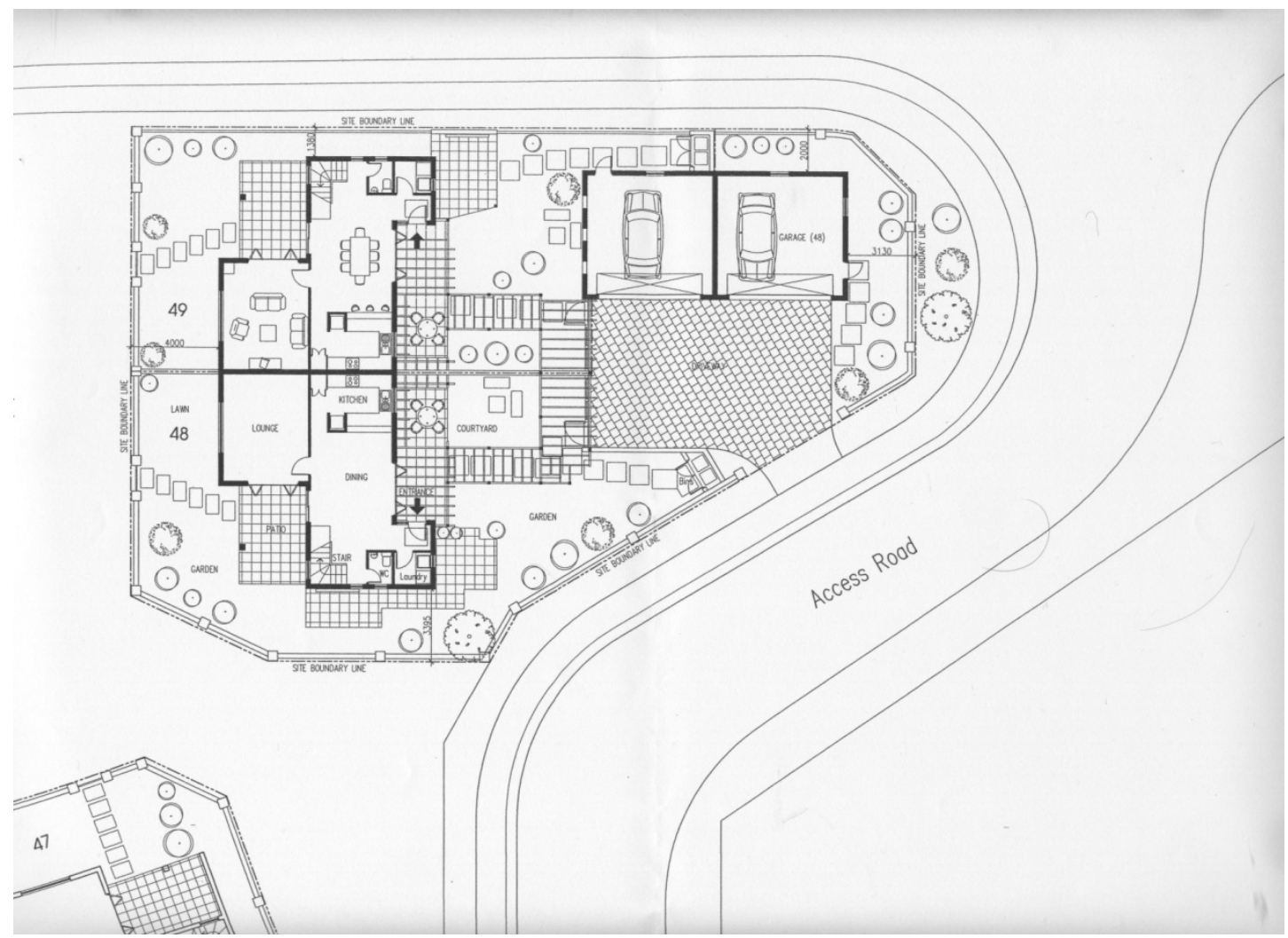

Figure 6.8a,b - Plan and Elevations of the two houses on the East side of Pinehill Park.

Source: Upper Hutt City Council, from Resource Consent plans submitted by TCB (Truebridge Callender Beach Ltd - Land Surveyors). (North is approximately up the page in the Plan view) 
(C) 2012 Stephen Pattinson Chapter 6: Case Study-Pinehill Park M. Arch Thesis

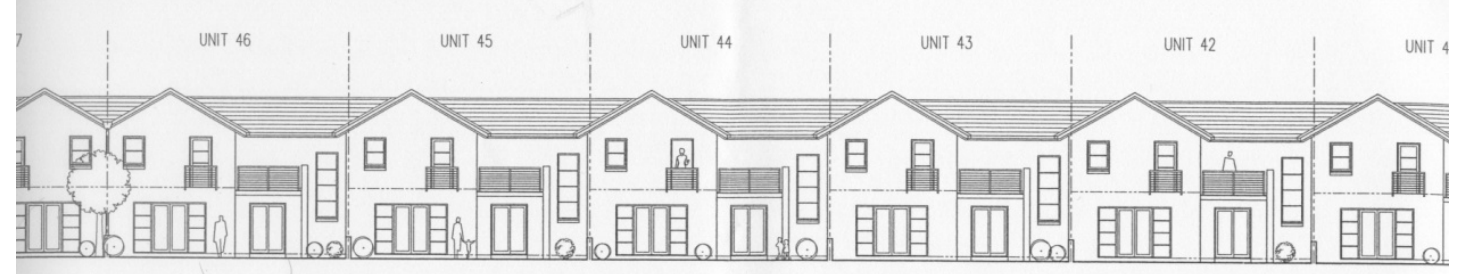

NORTH ELEVATION (View from Reserve)

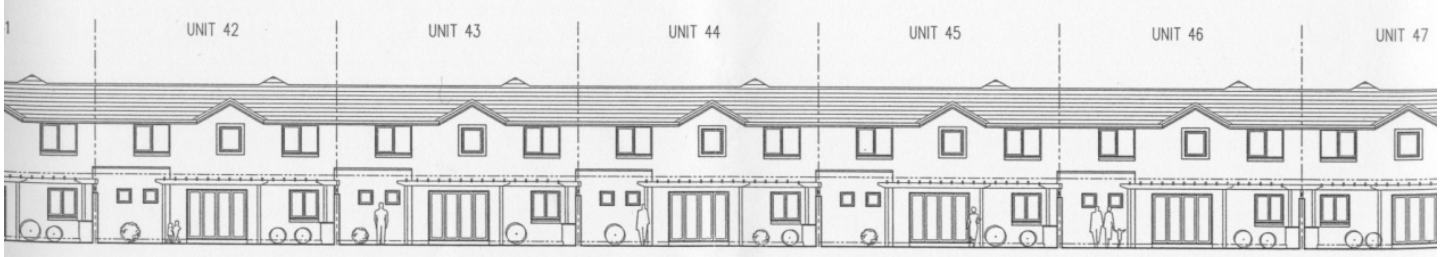

SOUTH ELEVATION (View from Courtyard)

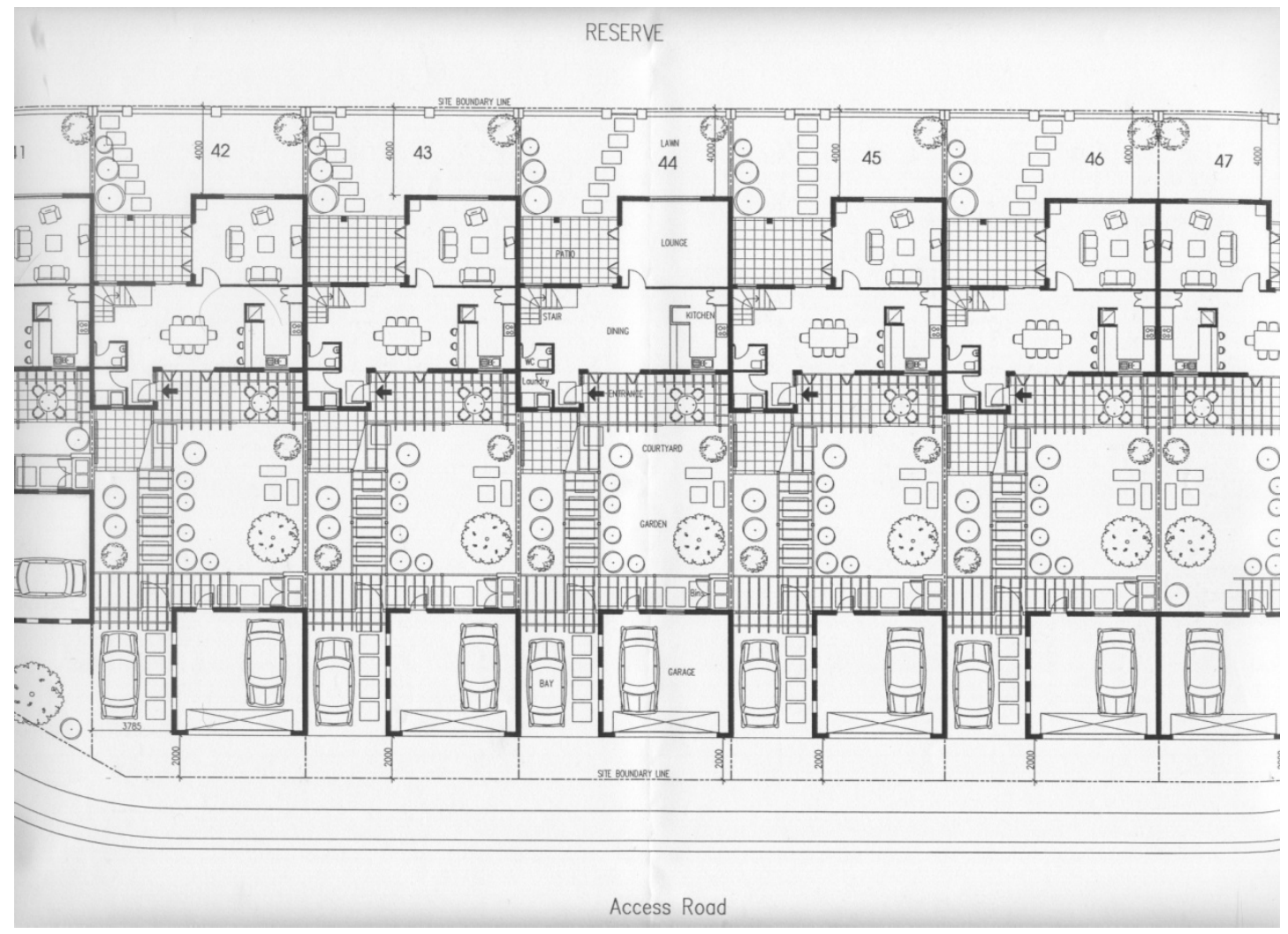

Figure 6.9a,b Plan and Elevations of the seven houses on the South side of Pinehill Park. Source: Upper Hutt City Council, from Resource Consent plans submitted by TCB (Truebridge Callender Beach Ltd - Land Surveyors). (North is approximately up the page in the Plan view) 
The houses are well designed, with a wide frontage onto Pinehill Park. The wide frontage allows for generous windows front and back (i.e. onto the park on one side and onto a private courtyard on the other side). This wide frontage also allows a shallow depth of plan, meaning good cross-ventilation through the house when both front and back windows are open.

The Ground Floor (see Figure 6.10c) consists of a separate Lounge and an open-plan kitchen-dining area which opens onto a private courtyard between the house and detached double garage. This private courtyard is well-proportioned, being approximately square (about $11 \mathrm{~m} \times 10 \mathrm{~m}$ ). These courtyards are well landscaped and provide an attractive outdoor entertainment area adjacent to the KitchenDining space. Solid garden walls (about $2 \mathrm{~m}$ high) provide visual privacy to these courtyards, however, because most of the houses are in a straight alignment there is some overlooking of these courtyards by the neighbour's upstairs windows.

The Ground Floor of the house is L-shaped in Plan view, forming two sides of a semiprivate patio beside the Lounge. The patio area is like an outdoor room, roughly square (about $4 \mathrm{~m} \times 4.5 \mathrm{~m}$ ). A third side of the patio is formed by the party wall of the adjoining house. This party wall has no windows, therefore the patio is not overlooked by neighbours. The drawings show a First Floor Level balcony over this patio, but these balconies were not built in the final project. The Lounge opens onto this patio on one side, and on another side it also opens onto a semi-private yard facing the Park. This yard is the full width of the lot (about $10 \mathrm{~m}$ ), and provides a $4 \mathrm{~m}$ setback to the Lounge from a low, solid garden wall on the Park boundary. The First Floor (see Fig. 6.10 below) consists of a double-bedroom and en-suite, another double-bedroom, a third, single bedroom, and a toilet/bathroom (which has a shower and a bath). As mentioned above, the main double-bedroom is shown on the drawings as having sliding doors opening onto an exterior balcony (no door off the hall, surprisingly) but these balconies were not built in the final project. 


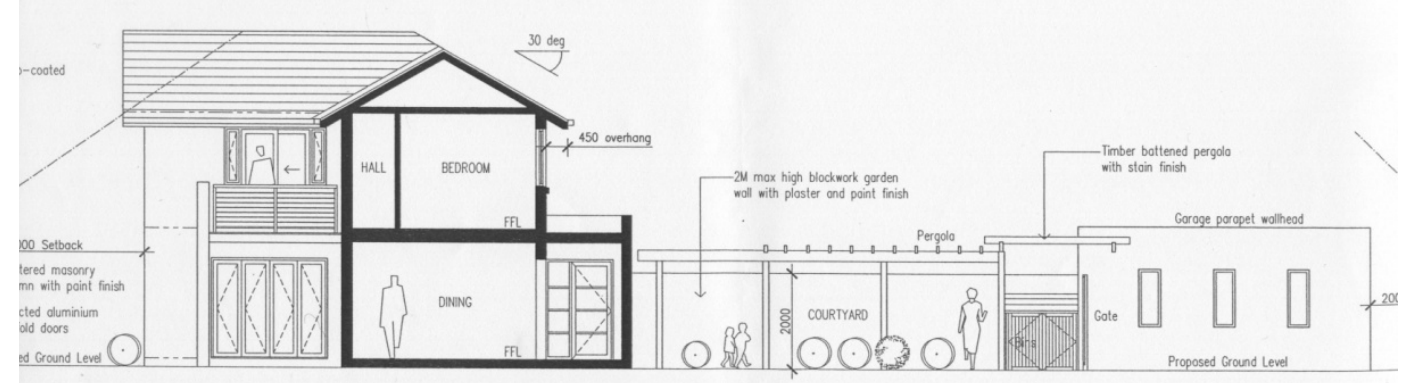

Figure 6.10a - Typical Cross-Section of a terrace house at Pinehill Park.

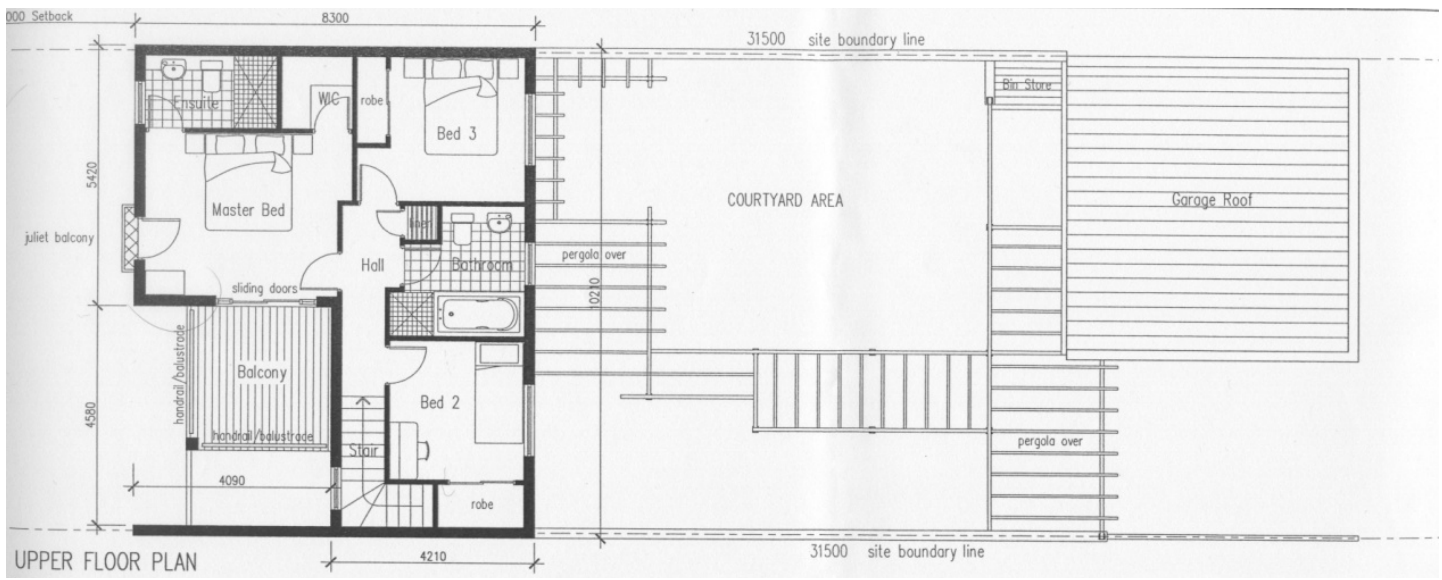

Figure 6.10b - Typical Upper Level Floor Plan of a terrace house at Pinehill Park.

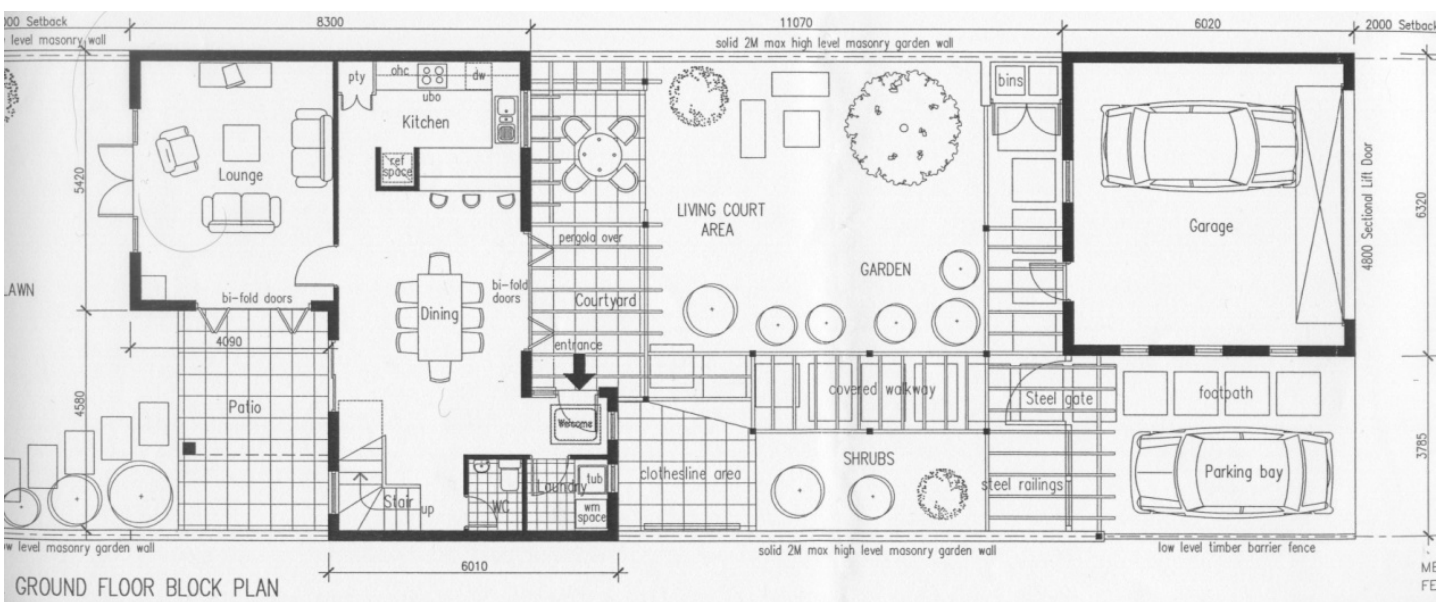

Figure 6.10c - Typical Ground Floor Plan of a terrace house at Pinehill Park.

Source: Upper Hutt City Council, from Resource Consent plans submitted by TCB (Truebridge Callender Beach Ltd - Land Surveyors).

Note: Copyright ownership of the Plans and Drawings belongs to Newport Homes Ltd c/Harwood-Pacific 
There is generous private vehicle parking space for all the terrace houses two cars in the double-garage and a third car park space beside the garage by the entrance gate to the private courtyard. For the houses on the South side of the Park there is further parking for three more (visitors) vehicles per house on a concrete apron between the garage and the footpath. The writer observes that this is a popular location for residents of these houses to wash their vehicles, and, coincidentally, to greet or chat with any passersby.

1.2b(iii) Photographs of Pinehill Park and the terrace houses:

The following are photographs of Pinehill Park and the surrounding terrace houses taken by the writer during November 2010.

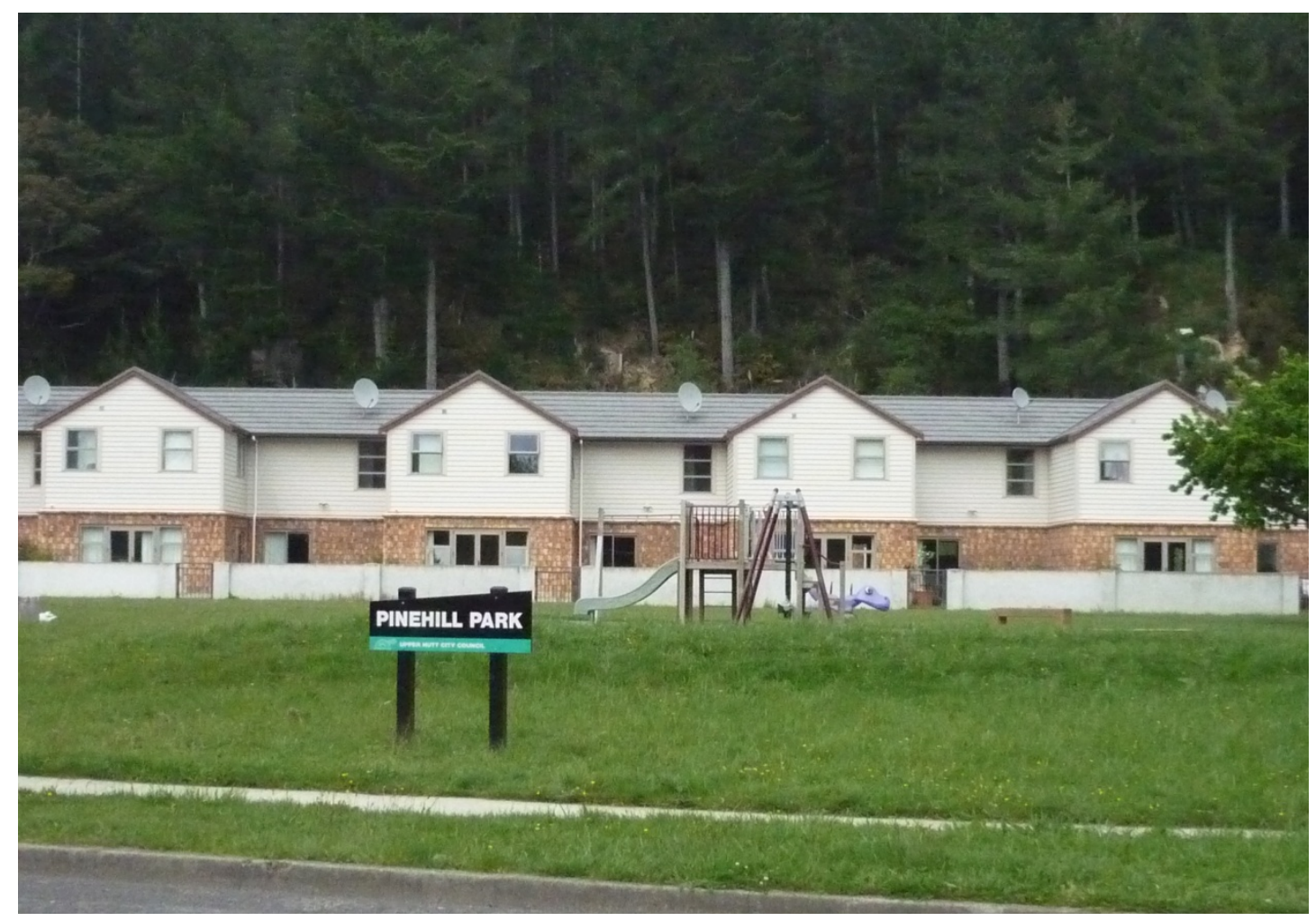

Figure 6.11 - Pinehill Park, viewed from roadside (Pinehill Crescent, Trentham, Upper Hutt) (Photo: S. J. Pattinson) 


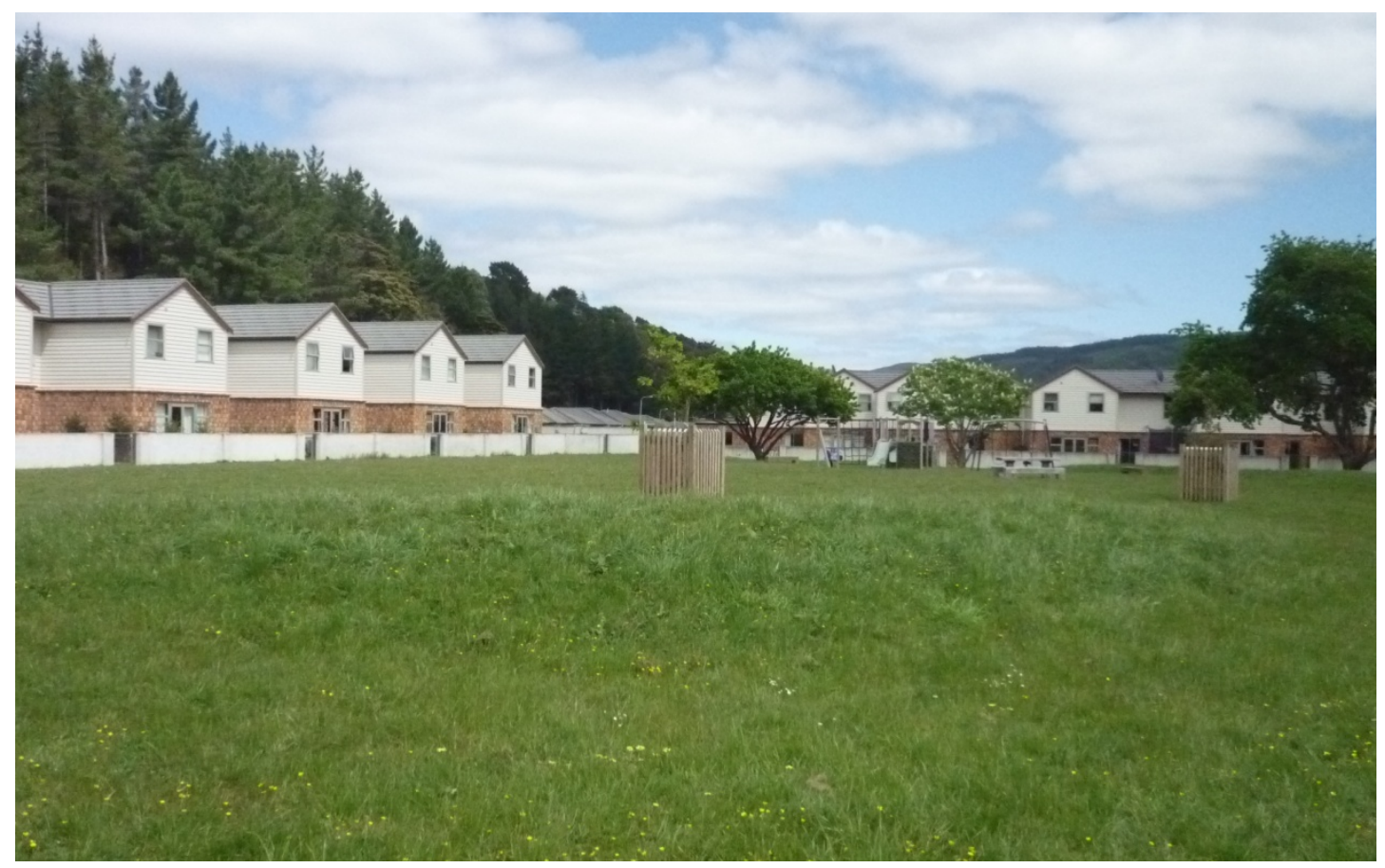

Figure 6.12 - Pinehill Park, viewed from the eastern end of the park (looking to the west). Note the raised grass berm in the foreground.

(Photo: S. J. Pattinson)

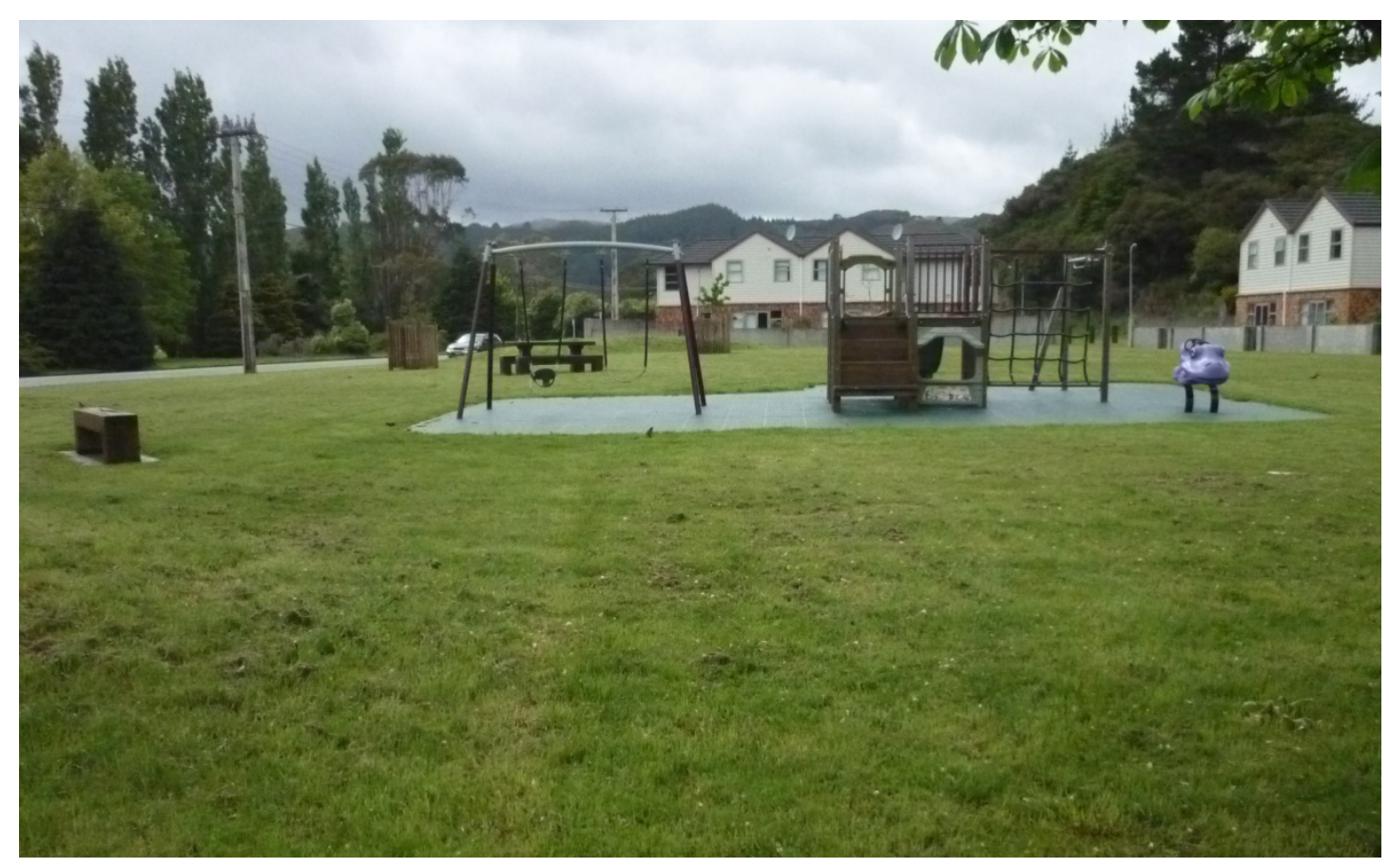

Figure 6.13 - Pinehill Park, viewed from the western end of the park (looking to the east). Note the bench seat on the left in the foreground, and the picnic table and tree saplings in the park beyond the playground.

(Photo: S. J. Pattinson) 


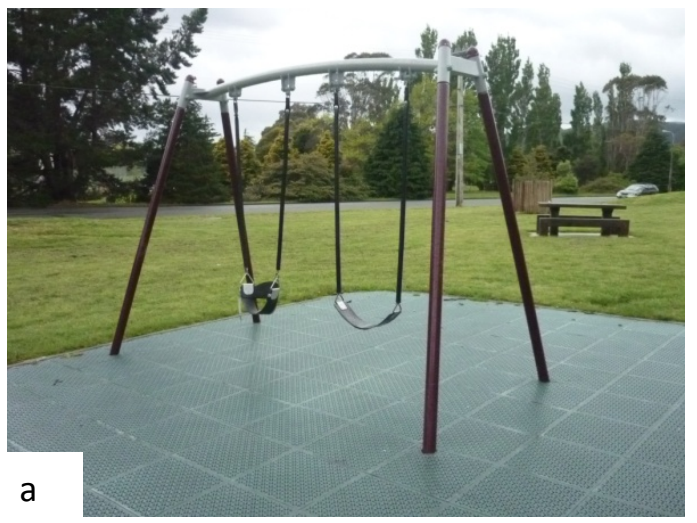

Fig. 6.14 a,b,c,d Pinehill Park playground

Young children's playground consists of swings, steps and platform, tobblers slide, climbing net, 'monkey-bar', 'rocking-croc', and safety matting. Children can also climb the trees by the playground.

(Photos: S. J. Pattinson)
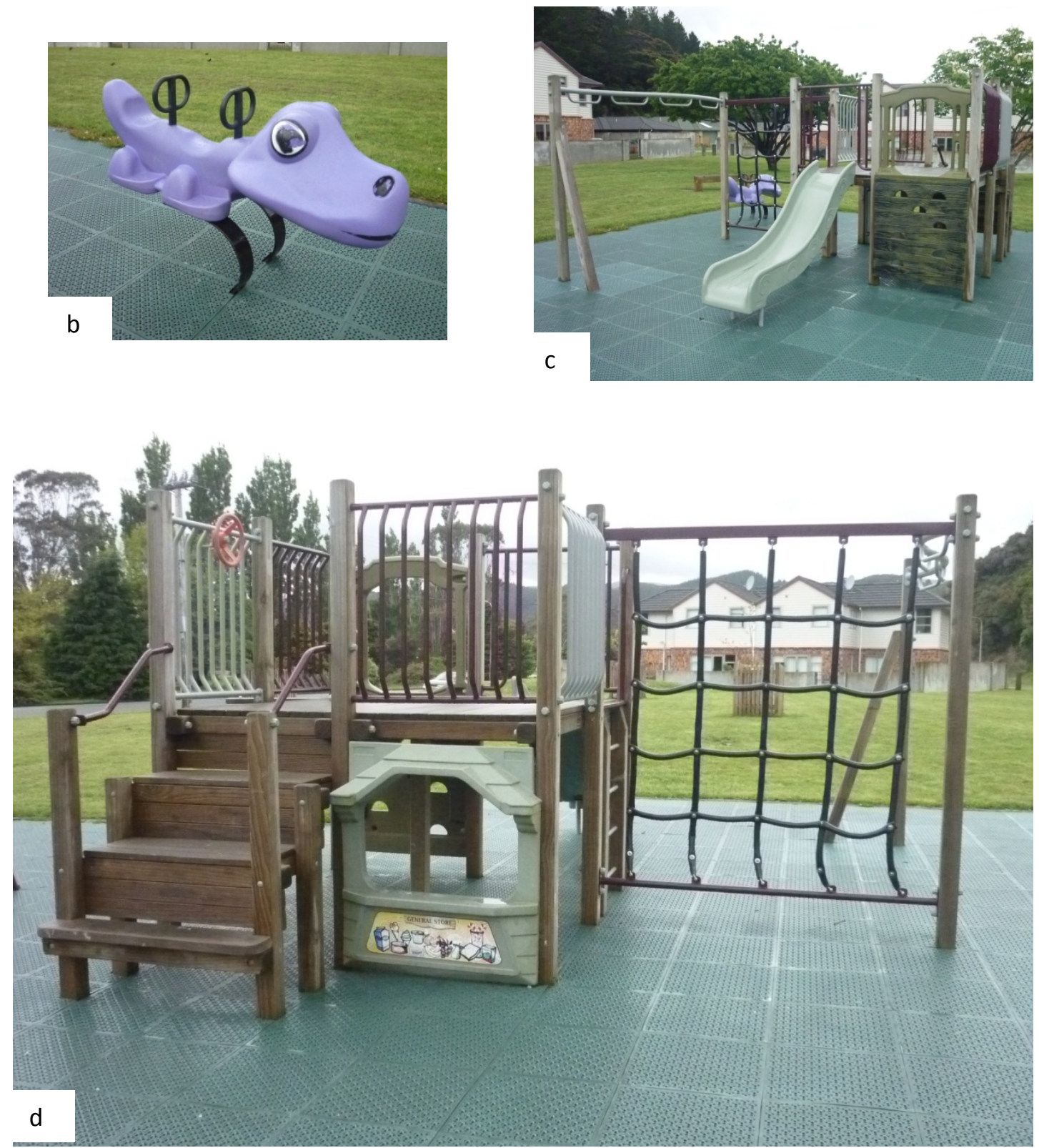
(C) 2012 Stephen Pattinson Chapter 6: Case Study-Pinehill Park M. Arch Thesis
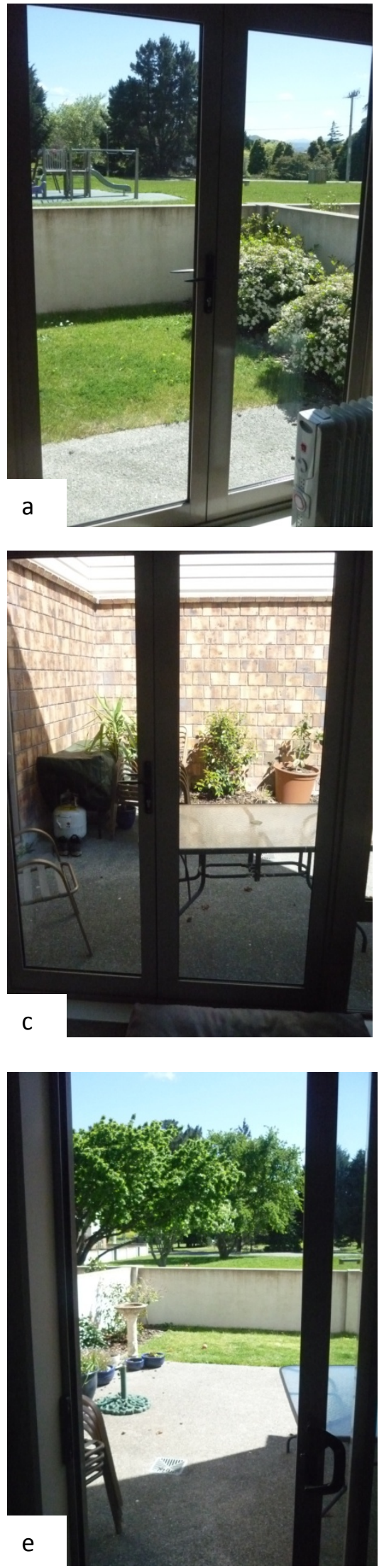
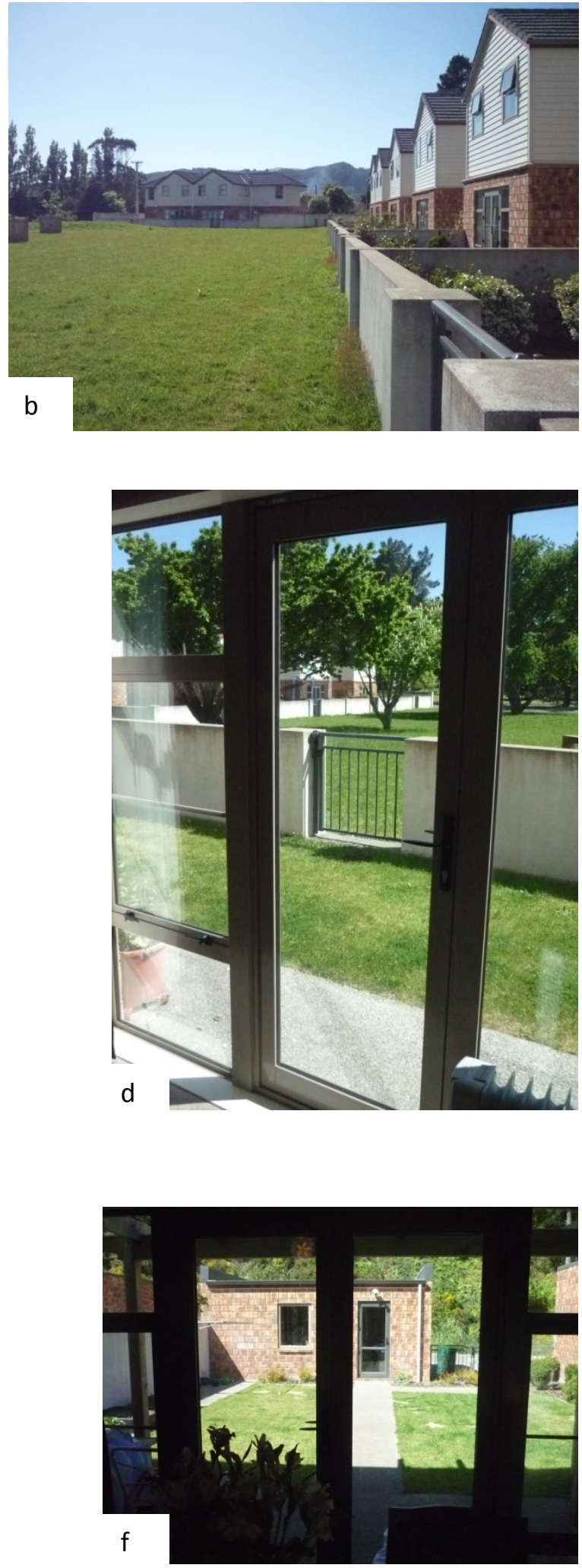

Fig. $6.15 \mathrm{a}-\mathrm{f}$ Views outdoors from inside one of the terrace houses at Pinehill Park. (Photos: S.J.Pattinson) 
1.2c) Comments about the assumed lack of use of the existing park:

The writer lives in Upper Hutt and has driven past the park (never stopping there) maybe once or twice every month since it was created about 3 years ago. In all that time the writer recalls only once ever seeing anyone using the park. This has given the writer the impression that this park hardly ever gets used, an impression reinforced by the comments of others including a senior planning officer of the Council. The writer (and the Council's senior planning officer) is therefore very interested to see what this case study reveals about the use of the Park.

\subsection{Observations of the existing use of Pinehill Park}

Guided by advice from Cooper Marcus and Francis (345-356) Chapter 8 "PostOccupancy Evaluation" of outdoor 'people-places', the writer carried out careful observations of Pinehill Park during the course of a week in November 2010. (see Appendix 3 for template of observation sheet). The park was observed in 1 to 5 hour time periods in mornings, afternoons and evenings of both weekdays and weekends to find out if and when the park is used, and by whom - in terms of gender and agegroup, immediate residents or from the wider community, and whether they arrive at the park on foot, by bike or by car. The park was observed for a total of 21 hours during this particular week in November, and the weather was fine throughout the observation periods.

The observations were made from several vantage points. The writer made these observations without engaging in any conversation with the users of the park and before the next stage of the case study (interviews with residents of the terrace houses) because the writer wanted to observe the park as objectively as possible from a distance in its 'natural' state, i.e. to observe the park as it is normally used, without interference from the observer. 
All traffic passing the park during the 21 hours of observation was also noted, including vehicles, cyclists, joggers and walkers. This observation was made to see how many people passing by stop to use the park. Part 2 of this chapter summarises the key findings from these observations.

\subsection{Interviews with residents of terrace houses adjoining Pinehill Park}

A survey and interviews of residents living in the terrace houses next to the park was carried out over a four-day period from Wednesday $15^{\text {th }}$ to Saturday $18^{\text {th }}$ December 2010. The purpose of the survey and interviews was to find out from the residents how they use the park and what they think of it. The survey/interviews consisted of two open-ended questions. The first question asked "What does it mean to you living beside the park? (How do you use the park, what do you like/dislike?)". The second question asked "How would you design the park? (What other layouts, uses, landscaping, furniture or equipment etc do you think the park could have? How could the park be designed to mean more to you?)".

The questions were accompanied with aerial view maps of the terrace housing and Pinehill Park for respondents to draw and make notes on if they wished to. The way the survey/interviews were conducted was by the writer and an assistant visiting each of the terrace houses. Residents were given the choice of taking the survey forms and completing them in their own time for collection by the writer at a later time. Alternatively residents could discuss the survey questions in an interview with the writer, while the writer's assistant took notes of respondents' comments.

Most respondents choose to do both, that is to take the survey forms and fill them out in their own time, and then discuss their responses in an interview with the writer and the assistant when they returned to collect the response forms. This seems to have the effect of giving the respondents time to provide considered comments. Interviews typically lasted for 30 to 60 minutes. 
(C) 2012 Stephen Pattinson Chapter 6: Case Study-Pinehill Park M. Arch Thesis

\section{PART 2: Results of the Case Study}

This chapter presents the results of observations of the existing use of Pinehill Park and of the survey and interviews with residents living in the terrace housing adjoining the park. Common themes in the responses from the terrace house residents are analysed for correlation with the principles for multivalent communal space derived from current practice and the literature review.

\subsection{Observations of Pinehill Park}

First is a summary of the key findings from the observations (see Appendix 4 for the complete set of observation sheets). 
The observations of Pinehill Park totalled a period of 21 hours 5 minutes.

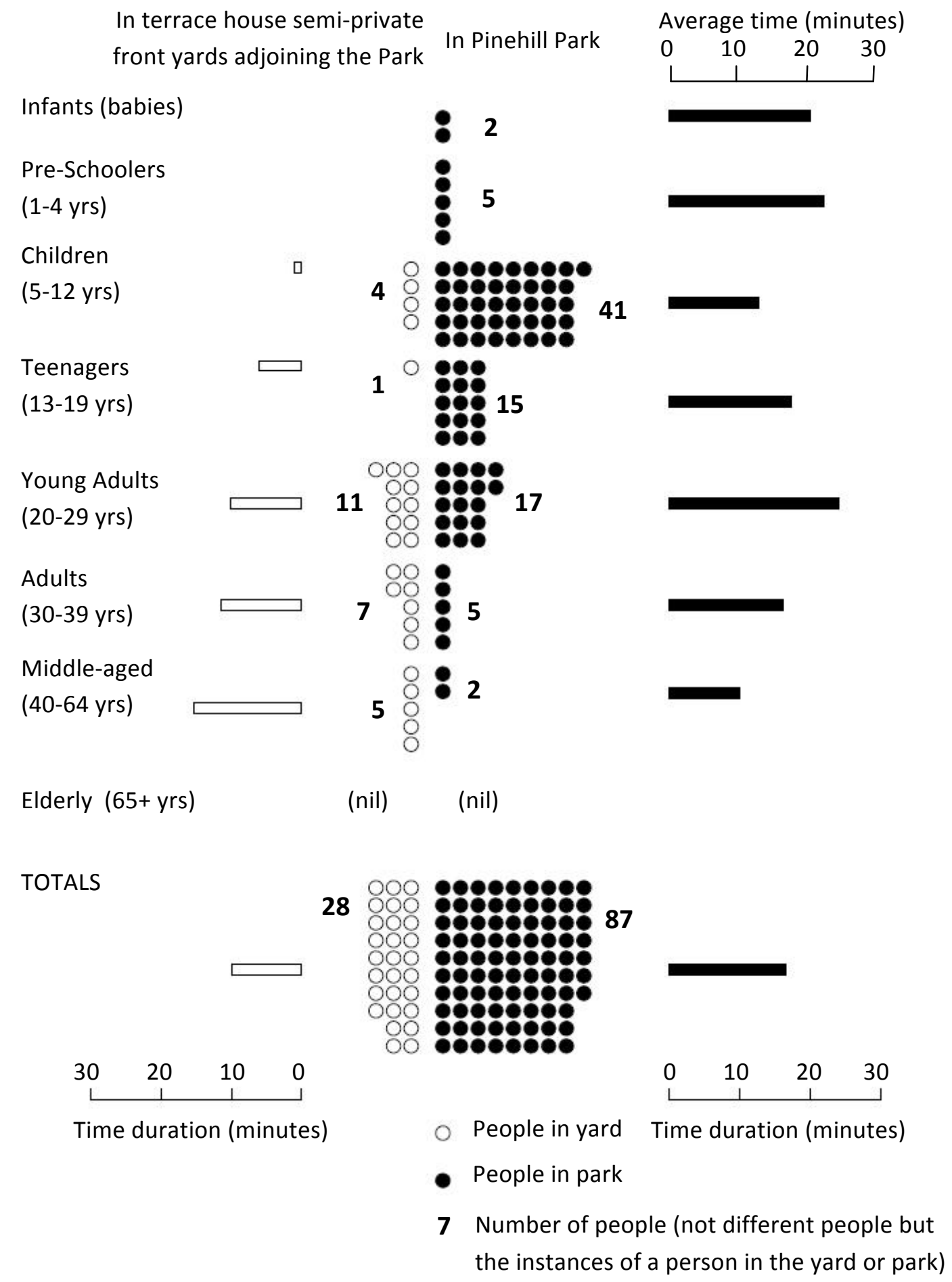

Figure 6.16 - Users of Pinehill Park during a 21-hours total observation. (See Appendix 4 for a summary of observation data)

S.J.Pattinson 
Having carried out these observations of the Park, the next step was to meet the residents of the terrace houses and interview those who were willing to participate to obtain their views of what it is like living next to the Park, and what the Park means to them. It is clear from these observations that the residents of the terrace houses are the major users of the Park and these users are predominately young adults, teenagers and young children.

The pattern of use by the terrace house residents is interesting. Once there are some children in the Park, other children from the terrace houses come out and join them. Their use of the Park is mainly for short periods of time, chatting or doing active things such as kicking a ball around, throwing a Frisbee, playing on the playground equipment (especially the swings), and climbing the trees.

Making the Park multivalent might prolong use of the Park for longer periods of time, and draw out adult users as well as children, possibly encouraging more intergenerational interaction. This would have the benefit of providing some older eyes in the Park to watch toddlers while young adults, teenagers and young children play. This would be a good safety strategy because, paradoxically, the more activity there is in the Park involving young adults and children, the more likely it can be that toddlers are momentarily unsupervised (as was observed on one occasion by the writer and the incident is related on p.179). In this situation it is risky for toddlers to be momentarily unsupervised, because the Park is beside Pinehill Crescent, which at times can be quite a busy road, with some traffic clearly exceeding the speed limit. Also, the ditch on the other side of the road (Heretaunga Drain, see Figs. 6.6 $\&$ 6.17) has very steep banks and is quite deep (about $2.5 \mathrm{~m}$ ), posing another potential hazard for toddlers and young children.

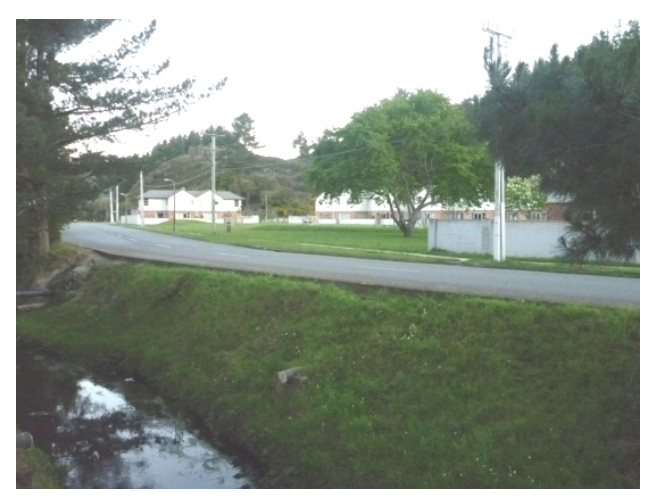

Figure 6.17 - Heretaunga Drain (Pinehill Park in background) Photo: S.J.Pattinson 


\subsection{Comments relating to the observations of Pinehill Park:}

It is clear from the observations, that contrary to prior impressions, Pinehill Park does get a reasonable amount of use. There are fairly clear patterns to the way the Park is currently used.

The most frequent users are school-aged children (5-12 years). They mostly come from the terrace houses, although there are several who walk up from the detached houses along the road. On average their visits to the park are fairly short but regular (that is after school and on weekend afternoons). They tend to run in and out from their houses for periods of play of about 10-15 minutes at a time, and they play mostly on the playground equipment, especially the swings (the boys like climbing on the swing frame), platform and rocker. They also like climbing the trees. They spend very little time in their yards that face the park, because they tend to use the park as their big back yard. The arrival of one or two children in the park would often bring others out of the terrace houses to play.

During the observations, no adults were in the park with school-aged children. The only adults in the park were those supervising preschoolers. The one exception was a middle-aged woman who came into the park for a few minutes to throw a frisbee with two school-aged children whom the writer later discovered (in an interview) were her grandchildren visiting for the afternoon. It seems that the park is regarded as a safe place for school-aged children to play without adult supervision. This is possibly because the 14 terrace houses (where most of the children using the park come from) directly overlook the park, and the children return to the houses regularly during their play time.

There does not seem to be much to attract teenage boys into the park. A few were seen walking across it and one teenage boy used the swing as a climbing frame. Some young teenage girls socialise around the swings, typically for $1 / 2$ an hour at a time. The writer later discovered in interviews that these girls live in the terrace 
houses and frequent the park with their friends who visit them. Like the younger children they are also in and out of the houses frequently. These girls joined in with the rugby ball play for approximately $1 / 4$ of an hour on Sunday afternoon. It was noticeable that the arrival of one or two young teenage girls would bring out others from the terraces houses into the park.

Young adults (early to mid 20s) use the park almost exclusively for rugby ball kicks and passes, usually for quite extended periods of time (up to 40-50 minutes). Teenagers and younger children were obviously welcome to join in with them, and did so most times that the young adults were out. There seemed to be quite a relaxed, neighbourly familiarity between most of the young people and children using the park. All the children and teenagers have their small groups with whom they usually play, but when the young adults come out with the rugby ball, this is the one activity that seems to bring them all together.

During the total 21 hours of observation only 2 infants (0-1 years) were brought to the park. Most of the preschoolers using the park are brought by mothers from along the road, and typically stay for about 20 minutes. The children use the playground equipment and the adults spend virtually their whole time in the park supervising the preschoolers. In the whole 21 hours of observation, only 1 car stopped at the park, and that was a couple bringing a preschool child to the playground, staying for a relatively short time (7 minutes).

The park is virtually unused by adults, except for those who bring preschoolers to the playground. There seems to be nothing in the park to invite its use by adults, the middle-aged or elderly people.

A number of times during the observations children crossed the road adjacent to the park (Pinehill Crescent). This was to retrieve a tennis ball, a golf ball, and the rugby ball (the latter retrieved by young adults). Usually these balls ended up in the ditch (Heretaunga Drain) on the other side of the road (see Figs. $6.6 \& 6.17$ ). 
On one occasion a toddler escaped the notice of his young adult supervisors during a Sunday afternoon when the park was at its busiest with young people and children all playing rugby kicks together. The toddler was on the playground equipment but suddenly ran from the playground out across the road to the edge of the ditch on the other side of the road. There was an oncoming car which fortunately was travelling fairly slowly and, on noticing the toddler, stopped in time to avoid any harm. Two young adults sprinted across the park and the road and retrieved the toddler from the edge of the ditch. This all happened in about 50 seconds.

The writer observed that the car was travelling from east to west along Pinehill Crescent (towards the railway line) and because this is a straight stretch of road along the side of the park, the toddler on the road was visible to the driver. This is not the case for vehicles coming in the opposite direction. A bend in the road just before the park means that the park is blind to drivers travelling from west to east. The writer observed that vehicles frequently speed along this stretch of road.

\begin{tabular}{|c|c|c|c|c|c|c|c|}
\hline \multicolumn{8}{|c|}{ Traffic on Pinehill Crescent, passing Pinehill Park (Observed Mon $1^{\text {st }}$ to Sun $7^{\text {th }}$ Nov 2010) } \\
\hline & $\begin{array}{c}\text { Mon } 1^{\text {st }} \\
11.30 \mathrm{a} \\
\text { m- } \\
4.30 \mathrm{pm}\end{array}$ & $\begin{array}{l}\text { Tue } 2^{\text {nd }} \\
3-8 p m\end{array}$ & $\begin{array}{l}\text { Wed 3 }^{\text {rd }} \\
6.30- \\
11.30 \mathrm{am}\end{array}$ & $\begin{array}{c}\text { Sat } 6^{\text {th }} \\
10.30- \\
11.30 \mathrm{am} \\
6.10-6.40 \mathrm{pm}\end{array}$ & $\begin{array}{c}\text { Sun } 7^{\text {th }} \\
\text { 9-9.30am } \\
12.05-2.05 p m \\
3.35-5.40 \mathrm{pm}\end{array}$ & TOTAL & $\begin{array}{c}\text { No. that } \\
\text { visit the } \\
\text { park }\end{array}$ \\
\hline $\begin{array}{l}\text { Vehicles } \\
\text { vehicles } \\
\text { per } \\
\text { minute } \\
\text { (vpm) }\end{array}$ & $\begin{array}{l}3- \\
4.30 \mathrm{pm} \\
199 / 90= \\
2.2 \mathrm{vpm}\end{array}$ & $\begin{array}{l}4.30- \\
6 \mathrm{pm} \\
234 / 90= \\
2.6 \mathrm{vpm}\end{array}$ & $\begin{array}{l}8-9.00 \mathrm{am} \\
141 / 60= \\
2.35 \mathrm{vpm} \\
11.15- \\
11.30 \mathrm{am} \\
13 / 15= \\
0.9 \mathrm{vpm}\end{array}$ & $\begin{array}{c}\text { No } \\
\text { count }\end{array}$ & $\begin{array}{c}\text { No } \\
\text { count }\end{array}$ & $\begin{array}{l}\text { Assuming } 2 \\
\text { vpm, about } \\
2,500 \text { vehicles } \\
\text { passed site } \\
\text { during } 21 \mathrm{hrs} \\
\text { observation }\end{array}$ & $\begin{array}{l}1 \times \text { car (2 } \\
\text { adults }+ \\
\text { toddler } \\
\text { visit for } 7 \\
\text { minutes) }\end{array}$ \\
\hline Cyclists & 5 & 18 & 16 & 1 & 4 & 44 & $\begin{array}{l}1 \text { boy } \\
\text { cycles } \\
\text { over } \\
\text { mound }\end{array}$ \\
\hline Joggers & 3 & 5 & 2 & 0 & 2 & 12 & nil \\
\hline Walkers & 11 & $\begin{array}{c}24 \\
\text { +skate- } \\
\text { border }\end{array}$ & 21 & 4 & 11 & 72 & $\begin{array}{c}2 \text { girls } \\
\text { walk over } \\
\text { mound }\end{array}$ \\
\hline $\begin{array}{l}\text { Soldiers } \\
\text { (Boot } \\
\text { Camp) }\end{array}$ & & & $\begin{array}{c}215 \text { in } \\
\text { uniform } \\
\text { with full kit }\end{array}$ & & & 215 & nil \\
\hline
\end{tabular}


The table above (Table 6.2) shows all the traffic passing the park during the 21 hours of observation, including vehicles, cyclists, joggers and walkers. This information is included to show that many people pass the park without stopping to use the park. The observed average rate was 2.3 vehicles passing the site every minute. The lowest observed rate was 0.9 vehicles per minute just before lunch on a week day. Assuming therefore an overall average of 1.5 vehicles per minute, approximately 2,000 vehicles would have passed the park during the 21 hour observation period. Of these only one stopped to use the park. Apart from three children taking a few seconds to traverse the grassed mounds, no cyclists, joggers or walkers (in total 128) stopped to use the park during the 21 hour observation period.

It is clear from the above traffic statistics that Pinehill Park is not a destination park. Very few people other than those living in the terrace houses around the park and some of the residents in the nearby detached housing use this park. There appears to be nothing about the park to draw in those who go past it. It should be noted that as a consequence a lot of potential neighbourly or social interaction is never realised. It would seem that the park could do more for this neighbourhood and district. It may be that parks are only perceived to be for the benefit of young children. Could parks such as this do more? 


\subsection{Interviews with residents of the terrace houses adjoining Pinehill Park}

Responses were received from 13 of the 14 terrace houses. From four houses, one response for each house was received. From five houses, two responses per house were received. From three houses, three responses each were received, and from one house, four respondents participated. This is a total of 27 respondents (or 55\%) from an overall terrace house population of 49 (assuming there are two adults only living at the 14th house where no one was home each time the writer and assistant called over the four day interview period). The following shows the breakdown of respondents according to broad age-groups (adults, teenagers and children):

ADULTS (20+ years)

- How many adults residing in terrace houses: 29

- How many adults responded:

19

- Percentage: $66 \%$

TEENAGERS $(13-19$ years $)$

- How many teenagers residing in terrace houses: 10

- How many teenagers responded: 7

- Percentage: $70 \%$

CHILDREN ( $1-12$ years)

- How many children residing in terrace houses: 10

- How many children responded: 1

- Percentage: $10 \%$

TOTAL 27 respondents from total terrace house population of $49=55 \%$

The terrace houses were built during 2006/7 and respondents have lived there for periods ranging from four months to $31 / 2$ years. There is a mixture of countries of origin amongst the respondents, including South Africa, Zimbabwe, Singapore, United Kingdom and New Zealand. 
Because the two questions in the survey/interview were open-ended, respondents were able to express their views to whatever extent they thought was relevant to the topic. Responses have been analysed with respect to the five aspects of multivalent communal space (sustainability, clustered housing, green open space, children in the city, and sense of community) developed from the literature review. (These five aspects of mulitivalence were not discussed with the respondents.)

(For a statistical analysis of the survey/interview responses see Appendix 5)

\subsection{Responses correlated with the Five Principles}

\subsubsection{Walkable Neighbourhoods}

The interview questions are focused on the open space of the park - how residents use it, what they think about it, how it might be made more meaningful to them. The questions did not raise the issues of walkability or environmental sustainability. However, because the questions are open-ended, some respondents have included in their responses comments about sustainability issues relating to their terrace houses and the park, and to the walkability of their neighbourhood.

Although located in 'drivable suburbia' where cars are probably the normal mode of transport, there are aspects of the neighbourhood that seem to invite walking:

- We looked at other properties in Petone and Wellington, two-storey townhouses. They were more expensive and didn't have what this has - it is close to the train, buses, a gym, and it has a double garage. (Couple, 50+)

- It is just 5 minutes walk to the bus or train, [the train] takes 7 minutes to Upper Hutt or 35 minutes to Wellington City (Couple, 50+)

- I walk to a gym on the other side of the CIT several times a week. The gym adds to the local environment (Adult Male 50+)

- Mums and children walk to the park from the nearby housing, sometimes in groups - usually about 3 or 4 parents but once we saw 8 adults and their children walk to the park (Couple, 50+) 
Some aspects of the park seem to deter walking, such as lack of paths and lighting (which makes it especially difficult for people with disabilities to use the park), and also lack of character or attractive features that might invite more use of the park:

- $\quad$ The park needs some paths (Adult Male 30-50)

- The park grounds need to be flat for $C$ to walk about (resident with disability) (Couple, 50+)

- I have never walked in the park the whole time I've been here [18 months]. It is characterless. There is nothing to draw you into it; it's more like a wide street berm. If the space was used to its optimum, then it would draw you into it. I don't want to go in there [the park], there is nothing to attract me to it. It needs fencing and landscaping to separate the park from the street. It needs some visual barriers to make the park ours, so that it is not just like a wide street berm as it is now (Adult Male 50+)

- There is enormous potential out there. At the moment the park is like having a house that's 100 square metres, but only using 10 square metres of it. It could have paved paths and walkways, little areas like outdoor rooms, little divisions, nooks and crannies, semi-circles, a water feature, a communal gas BBQ that is coin operated. (Adult Male 50+)

Some respondents comment on sustainability issues, such as vegetable gardening and planting fruit trees (i.e activities that provide physical exercise outdoors):

- I am planning on planting a cherry tree [to help with shading the windows] out in the left inside corner of the front yard. I also want to put in an organic garden (Adult Female 50+)

- We would like to see communal fruit trees, but not too many (Couple, 50+)

- Other neighbours have said about gardening, underneath is rocks and clay, difficult to garden (Couple, 50+) 


\subsubsection{Clustered Housing}

Several respondents comment on their terrace housing in relationship to its outdoor spaces and to the park. There are many comments about the sense of openness that the park gives to their home environment, for example:

- The park is an asset to the place, it gives a sense of more space and is an extension of the back yard (Adult Female 30-50)

- We had a property with large lawns and lots of space before moving here, but we get the same feeling here (Couple, 50+)

- The park is good, you don't have neighbours everywhere (you're not boxed in with houses) there are no big fences and it is open planned. You can see outside which is a nice view (Teenage Male 16-19)

One couple who immigrated to New Zealand 7 years ago commented, paradoxically, that they find their terrace house has more of a sense of openness than the closely spaced detached housing in the Wellington suburb where they lived previously:

- We don't like the way houses are built so close together in New Zealand, that is why we like living here, it gives us a feeling of personal space (Couple, 50+)

One respondent made some perceptive comments that although the terrace houses have private outdoor space he thinks the indoor/outdoor flow could be improved:

- Indoor/outdoor flow doesn't work. It looks like it has a lot of indoor-outdoor flow, but it is deceptive because the outdoor areas aren't sheltered. The patio has no cover [overhead shelter]. There needs to be shelter and pergolas to extend the house into the environment (Adult Male 50+)

The writer thought yards might be overlooked by neighbours first floor windows, but if this is an issue none of the respondents comment about it. Residents indicate by their responses that they feel their terrace houses are well designed for privacy:

- $\quad$ The settings of the house make it that you don't really see or hear the neighbours apart from in the park (Adult Female 30-50)

- The house has good party walls (good sound insulation). You don't hear neighbours next door - have only heard the smoke alarm when it has gone off. (Couple, 50+) 
One respondent, however, comments that he doesn't use his yard facing the park because he thinks it lacks privacy from the park:

- Our own backyard [facing the park] is not private enough. The patio needs roofing, and screening from the park for more privacy for a family BBQ. (Adult Male 50+)

One couple comment that the detached housing in the Wellington suburb of Johnsonville (where they lived prior to moving here) felt less private because the houses were close together and they could see into neighbours' windows on three sides from their kitchen:

- At our [previous] house in Johnsonville, [a detached single-family house close to its neighbours], we could see into the neighbours' places on both sides of the house, and down into the [third] neighbour's bathroom below where the man used to walk about naked - which wasn't a pretty sight! This [terrace house] is great. It has a feeling of openness. You are further away from your neighbours compared to our place in Johnsonville. (Couple, 50+)

[Regarding their comment about feeling 'further away from ... neighbours' in attached terrace housing than they did in detached suburban housing, this couple explained that although the suburban houses were physically more distant from each other than the terrace houses are, the terrace house patio and solid party wall (which has no windows in it that would otherwise overlook the patio and lounge) and the spaciousness of the park all create a greater sense of distance from their terrace house neighbours compared with their previous suburban neighbours.]

Some residents comment that there is a demand for these terrace houses:

- These [terrace houses] are sought after. We have had two land agents phone, one just this week, asking if we were wanting to sell. (Couple, 50+)

Some residents comment about issues of security, identity (the houses all look exactly the same), and functional planning (like lack of adequate storage space):

- I thought security would be a problem [being close to] the park, but that is not the case. (Adult Male 30-50)

- We call it Coronation Street [the houses all look the same]. (Adult Male 50+)

- The house is not our own, it lacks identity and we can't personalise it. We're both busy people so renting suits us. But the landlord doesn't let us put nails or picture 
hooks in the walls and the pictures fall off the 'no-nail' hangers we've tried, so we can't put our own stamp on the house. (Couple, 50+)

- The place needs storage. The only storage in the house is a small cupboard under the stairs, which is inefficient for storage. We all have double garages, but use the garage for storage - many of the cars are parked outside. (Adult Male 50+)

One couple commented that the windows of the houses are doubled glazed. Another mentioned that their house gets quite hot inside with the sun. The writer observed that there is no screening of the windows from direct sunlight (for example, roof overhangs do not shade the window, there is no shelter over glazed exterior doors and no pergolas or other shading devices to protect the windows from solar gain. Another couple commented that their house can get very cold in the middle of winter resulting in high power bills for electric space heating:

- Winter power bills can get up to $\$ 400$ per month; the three wall heaters, dining, lounge downstairs plus one upstairs for the bedrooms, are not enough. When it gets really cold we have to put extra bar heaters on, which really uses up the power. Why didn't they put in solar hot water heating like the Scandinavian system with the solar tubes on the roof? We had this back in the UK and it heated the water even on overcast days (Couple, 50+) 
(C) 2012 Stephen Pattinson Chapter 6: Case Study-Pinehill Park M. Arch Thesis

\subsubsection{Green Open Space}

Residents generally appreciate and enjoy the park. Residents comment on their thoughts, feelings and current use of the park as follows:

- We like the open space (Couple, 50+)

- It's a nice and quiet park (Teenage Male 16-19)

- The park provides uninterrupted, extra open space (Adult Male 50+)

- I feel the park extends across into the CIT [ex-Central Institute of Technology] grounds. The grass, the trees, it just keeps going, and then there is the backdrop of the [Rimutaka] hills (Adult Male 50+; Adult Female 50+)

The park is perceived as being primarily (if not solely) for the children:

- I really enjoy living beside the park, mainly for the kid's sake. It is great for them to be able to go out and expend some energy with their peers (Adult Male 30-50)

- The mother said she doesn't require anything of the park for herself, just for her children (Adult Female 30-50)

- It is a lovely playground for little children which is lovely to see, but nothing for us (Adult Male 50+)

There are some residents who don't use the Pinehill Park and they give their reasons why:

- I don't use the Park. I work full-time in Wellington. There are other parks that I go to like Harcourt Park and the park across the road [CIT]. It has a tennis court and I play tennis there with my friends (Adult female 30-50)

- Both my husband and I work full time. We don't use the park unless walking across it to have a wine with a neighbour (Adult Female 50+)

It seems from responses that residents generally perceive the park to be trouble free, although some express displeasure with rubbish:

- Been here $31 / 2$ years. Never had any trouble (Adult Female 50+)

- Its not a vandal area (Adult Male 50+)

- We like how the park is generally very clean (2 Teenage Females, both 16-19)

- I help keep the park clean by picking up papers and throwing them in our bin (Teenage female 16-19) 
(C) 2012 Stephen Pattinson Chapter 6: Case Study-Pinehill Park M. Arch Thesis

- There are no rubbish bins and quite often you will find rubbish from the park blowing into our yards (Adult Female 20-30)

Some residents use the park passively:

- It feels good to be living beside the park. I enjoy looking out on the park on sunny day. I like watching the ducks waddling around which gives the park a serene feeling (Adult male 30-50)

There are others who use the park incidentally or occasionally:

- We get fish and chips and eat them out in the park (Adult Female 30-50)

- People sometimes stop in their cars to use the park (Adult Female 50+)

Some residents use the park like an extension of their yard:

- It's like a big back yard. It's nice to go from the house out to the park (Couple with children)

- Younger children use it more. The teenagers sometimes go outside into the park and walk across it. It becomes extra space, spill over space for the teenagers when visitors come, while us adults talk inside (Adult Male 50+)

Some use the park for special activities:

- Nice to go out and see the stars (Couple, 50+)

- There is a local lady who walks a white Shetland pony up and down the street and into the park, which is lovely. We don't mind that at all. (Couple, 50+)

Terrace house residents are aware of some use of the park by others:

- Everyone uses the park not just the terrace house people (Adult Male 30-50)

- People stop in cars to use the park (Adult Female 50+)

- Seen young mums come and sit on a rug under the trees - they sat there while their children played; they walked from nearby [detached] housing. (Couple, 50+)

However, responses indicate that Pinehill Park is perceived as being under- utilised as shared open space for the terrace housing:

- $\quad$ This is the first [housing] of this set up we have seen with a 'common'. (Couple, 50+) 
(C) 2012 Stephen Pattinson Chapter 6: Case Study-Pinehill Park M. Arch Thesis

- New Zealander's don't know how to do housing around parks. We could learn from the English. They understand 'commons', for example East End, the gates and fences (Female, Couple 50+). [The writer commented about seeing a Park in the movie 'Notting Hill' - the couple climbing over the fence to get in]. Yes! I want a park that motivates me enough to want to climb the fence to get in! (Male, Couple 50+)

- As a ratepayer I would like to see more invested into this park - the park is an integral part of a good development, and the potential of the park needs to be thought through. (Adult Male 50+)

- I have seen the picnic table used only once in the last six months. The picnic table is exposed psychologically - out in the middle of the park, not near trees, and it's exposed physically to the wind (Adult Male 50+)

- We didn't choose the house because of the park, but it is a bonus. It is a great place to live. I'd like to see it [the park] maximised... like a square in England, where everyone goes. (Adult Male 50+)

The terrace house residents were asked, if given a say, how would they design the park that their houses face onto? There comments and suggestions include ...

... more park furniture, purposefully arranged for social use; several respondents commented that they would like to see a number of social areas developed in the park:

- When I think of sitting areas in the park there is only one of them, yet a lot of people want to sit (Adult Male 30-50)

- Make two or three areas - not just for the children (Adult Female 50+)

- Put chairs / seating / tables around the side so different families can use on special occasions, own area to use. Obviously children will socialise together. But do own thing with family while the children socialise together (Adult Male 30-50)

- Not a large number of families use the park. It needs more trees and benches - it would make more people use it (Adult Female 50+)

- Needs seating, shade, communal area, bbq tables, and outdoor furniture (Adult Male 50+).

... more equipment for additional activities, including more play equipment:

- Should have more equipment, eg slides, swings, more benches and a bin! (2 Teenage Females, both 16-19). Needs another bench (Teenage Male, 15 years). Have a BBQ added (Teenage Male 16-19)

- A basketball hoop, (Adult female 30-50) 
- A flying fox would be appreciated by the kids (Adult Male 30-50)

- Petanque (Adult Female 50+)

- Small pool would be nice- a big one would be expensive to maintain, about the size of our back yard would be good. It would be good to cool off. I think the adults would use it (Adult Male 30-50). A pool ... would be cool (Teenage Male 16-19)

... more appropriate landscaping:

- Have something instantaneously attractive (Adult Male 50+)

- Add a picnic area and trees at east end of the park. Put the picnic tables and benches under the trees, or nearer the trees, for shade. (Teenage Male 16-19; Teenage female 16-19)

- Trees are too big

- Have smaller trees in the park so it doesn't cut off the view from house (Teenage Male 16-19)

- The trees have been put in the wrong areas and get in the way of the playing area, e.g. rugby etc (Adult Female 20-30)

- Add a flower garden for older people to sit in or I would use it if I had a girlfriend. It would be nice to look at (Teenage Male 16-19)

- Add a garden maze - a mixture of bushes and flowers. At the centre of the maze, have a BBQ area with benches for friends and family to sit on (Adult male 30-50)

- a sculpture, a feature, something that says something meaningful that has a story (Adult Male 50+)

- Have a NZ rustic feel. Have designs on benches/tables (Adult Male 50+)

... add a water feature:

- I would love a water feature. There are risks with it but it can be designed to make it safe (Adult Male 50+)

- I would like to read a book in the park but I would like more trees and benches and a water feature. When we bought the house we were told there was going to be a fountain or water feature in the park (Adult Female 50+)

- Have floating walkways, like a Japanese peace garden (Adult Male 50+)

... add paths and lighting:

- $\quad$ The park is a bit dark at night time, a bit scary (Adult female 30-50)

- On one occasion at night time there was a threatening incident in the park, but we couldn't see what was happening because there was no lighting (Adult Female 50+)

- Don't want flood lighting. Security lighting would be good, (Adult Male 50+) 
(C) 2012 Stephen Pattinson Chapter 6: Case Study-Pinehill Park M. Arch Thesis

- Security lighting might attract drinkers at night. We don't want bottles left in the park (Adult Female 50+)

- Possibly some lights along front walls and central area. Not flood lights, but more intimate (Adult Male, 50+)

- It needs paths that light up here and there, not spotlights but intimate lighting. Clever in-ground lighting, light up gardens, footpaths, to gently follow at night (Adult Male 50+)

Several residents question the purpose of the earth mounds:

- I don't know what the berms [mounds] are for. Take the berms [grass mounds] away and level it out (Teenage Male 16-19)

- The little hills on the park can be quite annoying. It would be nice to see it flat (Adult Female 20-30)

The grass and ground conditions of the park are a significant issue for most residents:

- The ground is clay so in winter when it rains heavily the park becomes very marshy and boggy. Then in summer when it dries out the ground turns to rock and is very hard (Adult Female 50+)

- I don't play in the park. The ground is rough and uneven - me and my mates hurt our ankles if we play soccer on it, so we don't. Make it flat, then more people will come out and use the park (Teenage Male 11-15)

- The grass is only cut once a month. It needs to be cut more often, it gets long quickly. Sometimes it needs mowing once a week (Couple with children; Several couples 50+; Adult Male 50+; Adult Female 20-30)

- The grass is a park mix - heavy duty, stalky and uncomfortable on bare feet. The edges could be 'finished' properly with a mowing strip along the park side of our garden walls to get a clean edge around the park (Adult Male 50+)

- $\quad$ Treat the grass, there are black bugs that bite you (Adult Male 30-50)

- Be more like botanical gardens and a lot less like a rugby field (Adult Male 50+)

Many of the sentiments of the residents are summarised in the following responses:

- I would be delighted to see the park used differently. At the moment it lacks care, attention and character. It would be great to put more thought into it, like develop it into a series of outdoor rooms. That way it would get used $90 \%$ of the year apart from the $10 \%$ when it's really bad weather. Make it like a big backyard, like a private park. (Adult Male 50+; Couple with children; Teenage Male 16-19) 


\subsubsection{Needs of Children and Teenagers}

Although the survey/interview questions do not ask or prompt about children, many of the responses focus on children in the park and their safety with respect to the road along the side of the park. Several respondents express their appreciation of having the park for their children:

- The park means a lot because my 9 year old daughter plays there (Adult Male 30-50)

- The kids use the park a lot; whenever the weather is fine the children go out there (Couple with children)

Many of the responses mention the social benefits of the park for the children:

- It encourages socialising amongst the youngsters - kids playing, meeting each other (Adult Male 30-50)

- My son and his friends often use the park (Adult Female 30-50)

Some responses mention the physical benefits of the children being active in the park:

- Good to see the children outside playing (Adult Female 50+)

- If the kids are stuck inside playing computers and they see someone in the park they will go out to play with them, the park draws them outside (Adult Male 30-50)

- Used heaps in the evening especially by kids playing sport (Adult Female 30-50)

The park is appreciated by those who take care of children as it gives them somewhere close to home where the children can engage in supervised play:

- I play in the park with the kids I look after here during the day (Teenage female 1619)

- Our grandchildren all go out and play in park when they are staying or visiting, and have teamed up with other neighbours grandchildren. They are in and out of house ... mixing together (Couple, 50+)

- I use the park when I visit my grandad and grandma. I like the swings, boat and shop. I would like a bigger climbing frame, a tree house and a crocodile horse (Child, Male, 5 years)

Several respondents comment about the range of playground equipment being limited. The equipment that is there is appreciated but more of it is needed for the number of children who use it, and for a wider age range: 
- The playground area is designed for small children (Couple with children)

- They have to put in more swings because two swings is not enough for the kids to play (Teenage female 16-19)

- We would like to have a playhouse for the children in one of the trees in the park. There was one, but it was taken down because it was thought to be too dangerous. It was very popular with the children (Couple 50+)

- The park needs a playground at both ends. Sometimes 12 children are all wanting to go on the swings. The playgrounds could be different but swings are the main attraction (Couple with children)

Several respondents point out that there are aspects of the park which present safety issues for the children:

- $\quad$ The park needs to be more child friendly (Adult Male 50+)

- Young children can't go out into the park unsupervised (Adult Male 50+)

- When we have a BBQ at home with visitors in our yard, the children all want to go on the swings, at the other end of the park [which is about 50 metres away]. If park was fenced along the road, then we could let them go out and play by themselves while we have our $B B Q$ with our visitors (Couple with children)

- The new trees planted in the middle of the Park could prevent us from seeing the children in the play area at the far end of the park (Couple with children)

- To play here in the park, young children have to be supervised; you cannot just send them out. I'm not into fences, but If you could block off from the road with a fence then it would make the park a relatively safe enclosure for the children, like a big playpen, with all the houses looking down on the park (Adult Male 50+)

Comments about the park needing to be safer for children in relation to the road were made by most of the respondents. These are discussed later, after the following comments about the needs of teenagers in relation to the park. Some teenagers expressed how they currently use the park:

- We use the park to hang out with friends. We use the benches to sit and eat. Maybe we could have some separate parts so that when there are kids in the park, we can still hang out there (2 Teenage Females, both 16-19)

- We use the park often to eat fish and chips with mates and to kick a ball with friends- (Teenage Male 16-19) 
(C) 2012 Stephen Pattinson Chapter 6: Case Study-Pinehill Park M. Arch Thesis

- I generally use the park with a couple of mates, sitting on the swings with friends, talking, maybe playing a few games such as tag. I am happy with the park layout currently (Teenage Male, 15 years)

There are comments however about how the current design of the park reduces the teenagers' use of it, and how activities impact some of the residents:

- Don't put trees or tables or benches in the open play area in the middle of the park. The new trees in the middle of the park are in the way. The trees should be on the border of the park, and the picnic tables too (Adult Male 30-50)

- The new trees in the middle of the park have cut out a lot of the area for kicking balls etc (Adult Male 30-50).

- The new trees push the ball playing to side of the park close to our house, which means the ball comes too close to my house (Adult Female 30-50; Adult Female 50+)

- The ground needs to be levelled out so that its not so rough in the playing field (Adult Female 30-50)

- The whole park needs to be very flat for playing soccer; get rid of both of the raised grass mounds (Teenage Male 11-15)

A few suggestions are made about activities in the park suitable for teenagers:

- Set out a field/playing area to play soccer or rugby. Move the playground to one side to free up some more space for the field (Teenage Male 16-19)

- A basketball hoop would draw older kids out (Couple with children)

- I don't want the noise of basketballs bouncing on the concrete (Adult Male 50+)

- I don't like it when teenage kids run around kicking a football because it generates a lot of noise - difficult to relax in a situation like that (Adult Male 30s)

- Don't put a skate park here. It would attract the wrong people to the park (Teenage Male 11-15; Adult Male 50+; Couple with children)

One of the respondents, a teenage boy, has been involved in efforts to get a local skate park installed in the area for younger teenagers (around 13 years old) where they would feel safe. This is because they find they get bullied by older teenagers at the main skate park in Upper Hutt. He commented that he doesn't want a skate park here at Pinehill Park. (His disappointment over being unable to establish a local skate park, plus the fact that he is not a skater himself, means he is no longer pursing this venture). He comments: 
- Young teenagers are the "forgotten" age. Parks have things for 0-5 year olds and over 18's can go into the city, but there is nothing for the in-between age.

The road is a concern for the majority of the terrace house residents. This majority concern is reflected in the fact that 16 of the 26 respondents mention their concern about the safety of children in the park with regard to the road:

- In the mornings it is busy - a lot of people travel down that road. Cars don't go 50 kilometres, they speed past (Couple with children)

- It is not safe for children. Children always have to be supervised. I can't relax there, as have to watch the children. They can run out on road (Adult Male 30-50)

- If 2-3 year olds [children] run in that direction [pointed to road], there is nothing to stop them before the road (Another couple with children)

- We have noticed motorbikes sometimes use the park as a shortcut. This could be extremely dangerous if/when there are young children playing (Couple 50+)

- People do burn outs on the road at the 4-way crossing [the intersection of Pinehill Crescent, Evergreen Crescent and Gallipoli Road at the eastern end of the terrace housing] ... The noise draws the children out to see what is going on, and if one car got out of control it could hit a child (Couple with children)

Several suggestions are made by respondents to improve safety for children:

- Speed bumps would deter people from using this road, and make drivers detour to other roads instead of using this one (Couple with children: Adult Male 30-50)

- There aren't any road signs to show there is a park. Road signs about a park would make drivers aware that there could be children in the area (Adult Male 30-50)

- I want the park to be fenced so that I will feel comfortable when my sister's kids are playing. I know that they will be safe (Teenage Female 16-19)

- It needs to be fenced along the road side of the park to keep children from running out on the road (Adult Male 30-50)

- It doesn't have to be a high fence ... just a border. The other entrances to the park need to be fenced and gated as well (Couples with children).

- A fence along the road could have a couple of gates so that the public know they can still use the park (Adult Female 30-50) 


\subsubsection{Sense of community}

Several respondents mention the ambiguous nature of the park. The suggestion noted above to install a fence along the Pinehill Crescent side of the park may make the park feel more clearly communal (i.e. for the use of the terrace house residents only) rather than public (i.e. open to everybody). Some respondents mention that they would like the park to be private - just for the terrace house residents. Other respondents say they would like the park fenced along the road and other entrances but in a way that clearly conveys that it is still a public park.

This issue of sense of ownership affects not just whether it is a public or communal park, but also within the development some respondents indicate that they feel unsure about using parts of the park which they feel belong more to the residents closest to them. Because of the terrace houses looking in from three sides of the park, the public may feel unsure about using the park. One of the respondents comments that they have observed cars stop for a while but then drive off again without entering the park and he wonders if this has something to do with the ambiguous nature of the park which he considers to be an ill-defined space:

- It's an ill-defined space, which makes people ill at ease, or appear to be. Maybe they are thinking, "Am I allowed to enter in here or aren't I?". There is no sense of being invited in. There is no sense of 'welcome to our park', no sense of the sharing of the space, and no psychological indicators to say that it is for them, maybe more a feeling they should move on! (Adult Male 50+)

- Who does the Park belong to? I don't know. There is no real declaration as to what the park space is. What space is it? Is it ours? Is it a public park? It doesn't have a name - or does it? (Adult Male 50+)

Some respondents clearly have a 'sense of ownership' of the park:

- We see this as our park (Couple with children)

- The park seems like it's the people who live in the terraced housing- "its our park" and "my back yard" (Teenage Male 16-19) 
- When I see other people not from the terrace housing walk through the park, I feel like they are on my backyard (Adult Male 50+)

Some residents would like to see more of the parks potential as a communal asset for the terrace house residents to be developed:

- I wouldn't want the park to be sectarian, keep it communal for all residents but you might have responsibility for taking care of certain parts of it, but others could use it all (Adult Male 50+)

Some clearly envisage the park as being for the terrace house residents, the people along the road, and the wider public:

- The park should be for everyone to enjoy [not just for the terrace house residents] (Adult female 30-50)

- Having no park fencing means it doesn't close you off to the rest of the community (Adult Female 30-50)

- If fenced, the park would be more convenient and more people would come and use it (Couple with children)

- Fencing would not change the park. It all depends on the background you are raised in. Certain people see fenced as 'keep out', but have two signs saying that it is a public park, "please take care of the park", "use but please keep it tidy". Fencing won't affect the park as more relaxed New Zealand people won't see it as a closed area. A McDonald's playground is closed in, but you can still go in and out. Have something solid parts to the fence, but also make it see through, and with gates (Adult Male 30-50)

\section{Pedestrianism / walkability}

See 'Walkable Neighbourhoods' (p. 182)

\section{Social Interaction}

Many of the respondents spoke of the potential of the park for facilitating social interaction and a sense of community: 
- As part of a community you can sit and watch people enjoy the park. You meet people out in the park and it is safe (Adult Female 30-50)

- People chat over front fence to others in the park. It expands the boundaries of a small section and helps community relations. There is an openness to the 'common' (Couple, 50+)

- The park allows the kids to play together, so the kids in the area become good friends (Adult Female 20-30)

- I like the fact that the park is open because it encourages interaction between kids and adults (Adult Female 30-50)

- Children wave as they go past. A child walked along the fence and waved (Couple, $50+)$

- I see different age groups all playing alongside others successfully with out any problems (Adult Female 30-50)

- People will respect the park if the facilities are good. We could try to make something more of community - maybe a coffee shop / café (Adult Male 50+)

Residents of the terrace housing community have held a few shared events in the park. These have included a New Year's Eve party where they brought out tents, tables, BBQs, food, drink, karaoke, and a bouncy castle for the children (Adult Male 30-50). They are talking about a celebration in the park for Waitangi Day, 2011.

At the beginning of December 2010, a resident in the detached housing down the road held a street party as a Christmas celebration for the local neighbourhood and invited the residents of the terrace housing. One of the terrace housing families attended and said there were about 20 adults at the street party plus some children. The party was held on one of the traffic islands in Evergreen Crescent. Because the terrace house residents received invitations, some commented about the street party in their responses, wondering why this neighbourhood Christmas celebration hadn't been held in Pinehill Park. They commented that maybe the residents of the detached housing feel that the park belongs to the terrace house residents. These events demonstrate that efforts are being made to bring the neighbourhood 
together and encourage a sense of local community. Respondents comment that they see the park as having a role in facilitating this.

One couple mentioned a previous environment they lived in with the Defence Force which gave them an experience of sharing some communal facilities. The comments of this experience were positive. They commented that they believe communities need to be made up of a cross-section of society. Therefore they liked the heterogeneous nature of this community. The facilities included a tennis court and a huge grass area where their children could fly a kite and play - they never had to worry about them playing outdoors and never feared for their wellbeing because it was a village environment that was gated and safe.

This couple also commented about their experience of the close at Salisbury Cathedral in England - houses grouped around a communal open space which had a commanding vista inviting you into the space. They think that housing schemes in New Zealand that share communal open spaces could learn from examples like this.

Two other couples made very similar comments, one mentioning about the example of English commons and gardens, and the other mentioning about a relative living at Hamstead Heath, a garden suburb with a variety of people, apartments and gardens.

These respondents all indicated that whilst there may not be many precedents for housing around 'commons' in New Zealand, there is much that can be learnt from overseas examples. They like living here at Pinehill Park and would like to see the full potential of this development realised. 


\section{Conclusion}

The key finding of the observations is that the Park is mainly used by children, specifically, mainly the children living in the terrace houses adjoining the park. Teeangers sometimes hang out at the park but there is little to attract them to it. Young adults use the park for rugby ball play, which others join in on. Adults seldom use the park at all.

The key points from the survey and interviews are that respondents generally like living beside the park; it provides a sense of spaciousness to their home environment; it is generally regarded as belonging to them but they also see it as belonging to the wider public although they acknowledge that ownership of the park is ambiguous; the park is seen as under-utilised, it has been 'done to them' yet all respondents were interested in and even enthusiastic about its design and have clear and detailed ideas about how the park could be better developed for all age groups, and especially so for the safety of young children with respect to the road. All survey and interview respondents were willing to share their ideas about how to address their concerns about the park, and develop its potential to make it more attractive and meaningful to them - they had just never been asked before.

This concludes the Case Study. It now remains in Chapter 7 to discuss the findings from all facets of the research, that is, from the preliminary investigations of current practice in the design of existing medium density developments in Auckland, Wellington, Christchurch (Chapter 3), the literature review (Chapters 4 and 5), and this case study of Pinehill Park (Chapter 6). The purpose of the discussion in Chapter 7 will be to draw together the results from these various sources to construct a framework of principles for achieving multivalent communal space in medium density housing, and to consider if the application of these principles will make more efficient use of land and enhance the living environment for residents. 


\section{CHAPTER 7 - Discussion: Multivalent communal space}

\section{Introduction}

Question 4: Will the application of the five principles for multivalent communal space achieve more efficient use of urban land and enhance medium density living environments for residents?

This research finds communal space is potentially rich with multi-faceted meanings. By applying the five principles of multivalent communal space in an integrated way, these principles will facilitate more efficient use of land and enhance living environments for residents.

He tangata, he tangata, he tangata - yes, it is all about people, but as the bell bird needs the flax bush, so communities need supportive living environments. People and environment: the one shapes the other.

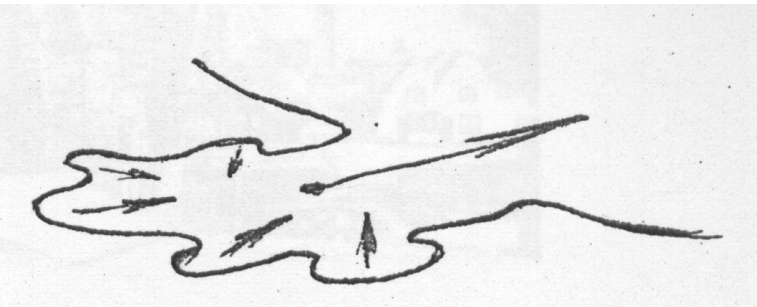

Fig. 7.1 - The 'bay' structure (Dr Richard Toy)
Richard Toy describes the 'bay' as the archetypal form giving choice for connectedness:

"...the bay offers the opportunity and choice for haven or to venture out into a wider community..."

With housing design, people shape the 'bay', and the bay shapes the community. Communal open space is at the heart of the bay - the centre of the bay structure. Practitioners, academics and residents agree that the 'bay' and 'courtyard' work as appropriate urban forms for expressing and supporting 'a sense of community'.

A brief recap follows of the chief task of this thesis - to make the 'bay' multivalent. 
(C) 2012 Stephen Pattinson Chapter 7: Discussion M. Arch Thesis

\title{
THE RESEARCH PROBLEM:
}

Individualism generally results in more floor space per person; urban intensification supplies less site space per dwelling. The outcome is less urban open space. The problem is how to deliver more amenity for residents within less open space.

\section{THE RESEARCH AIM:}

To develop principles of multivalent communal space that foster more efficient use of urban land and enhance the living environment for residents in medium density housing developments.

\author{
PROBLEM \\ AIM \\ individualism: demanding \\ multivalent communal space \\ more floor space \\ less site space available

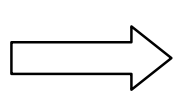 \\ enhance living environment by \\ delivering more amenity in \\ multivalent communal space \\ losing open space

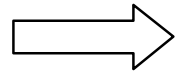 \\ preserve open space by making \\ more efficient use of land

\section{THE RESEARCH DESIGN:} \\ The author's research paradigm is that people and their living environments both \\ matter - as mentioned above, the one shapes the other (Hall 4, 106; Sommer 7). To \\ develop principles by using a participatory approach (i.e. including the viewpoints of \\ practitioners, academic researchers and the community) for multivalent communal \\ space in medium density housing the intention is to make more efficient use of \\ urban land and enhance the living environment for residents.
}




\section{THE RESEARCH QUESTIONS:}

1. What is the nature of communal space in existing medium density housing in New Zealand?

2. What is the potential multivalence of communal space in medium density housing?

3. What are the principles for achieving multivalent communal space in medium density housing?

4. Will the application of these principles achieve more efficient use of urban land and enhance the living environment for residents?

According to Hillier and Rooksby, Bourdieu challenges the traditional assumption of practice being applied theory (3-6). The following diagram expresses a participatory approach, inclusive of the views of practitioners (current practice), academics (from the literature of various disciplines), and the community (represented by residents of terrace housing in a case study of Pinehill Park, Upper Hutt, Wellington).

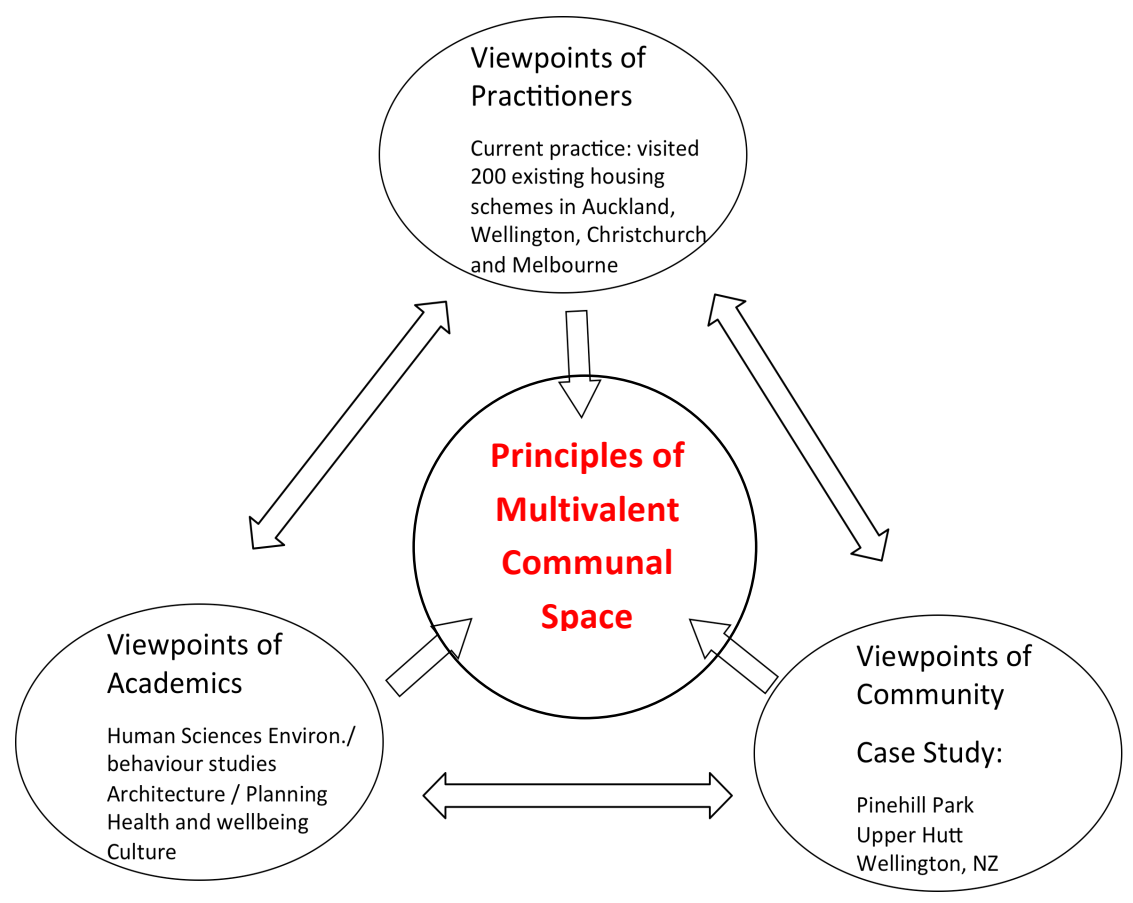

Fig. 7.2 A participatory approach to developing theory and practice, inclusive of the viewpoints of practitioners, researchers, and the community (adapted from Hamdi, 2010). 
Five Principles for Multivalent Communal Space in Medium Density Housing: From these three viewpoints (Fig. 7.2), five interactive principles have emerged for multivalent communal space in medium density housing within Hildebrand Frey's framework for "Sustainable Cities". Frey notes there is considerable consensus among academics on criteria for sustainable city form (339-340). Five agreed criteria for sustainable cities identified by Frey correlate with findings from this research for multivalent communal space in medium density housing (Fig. 7.3).

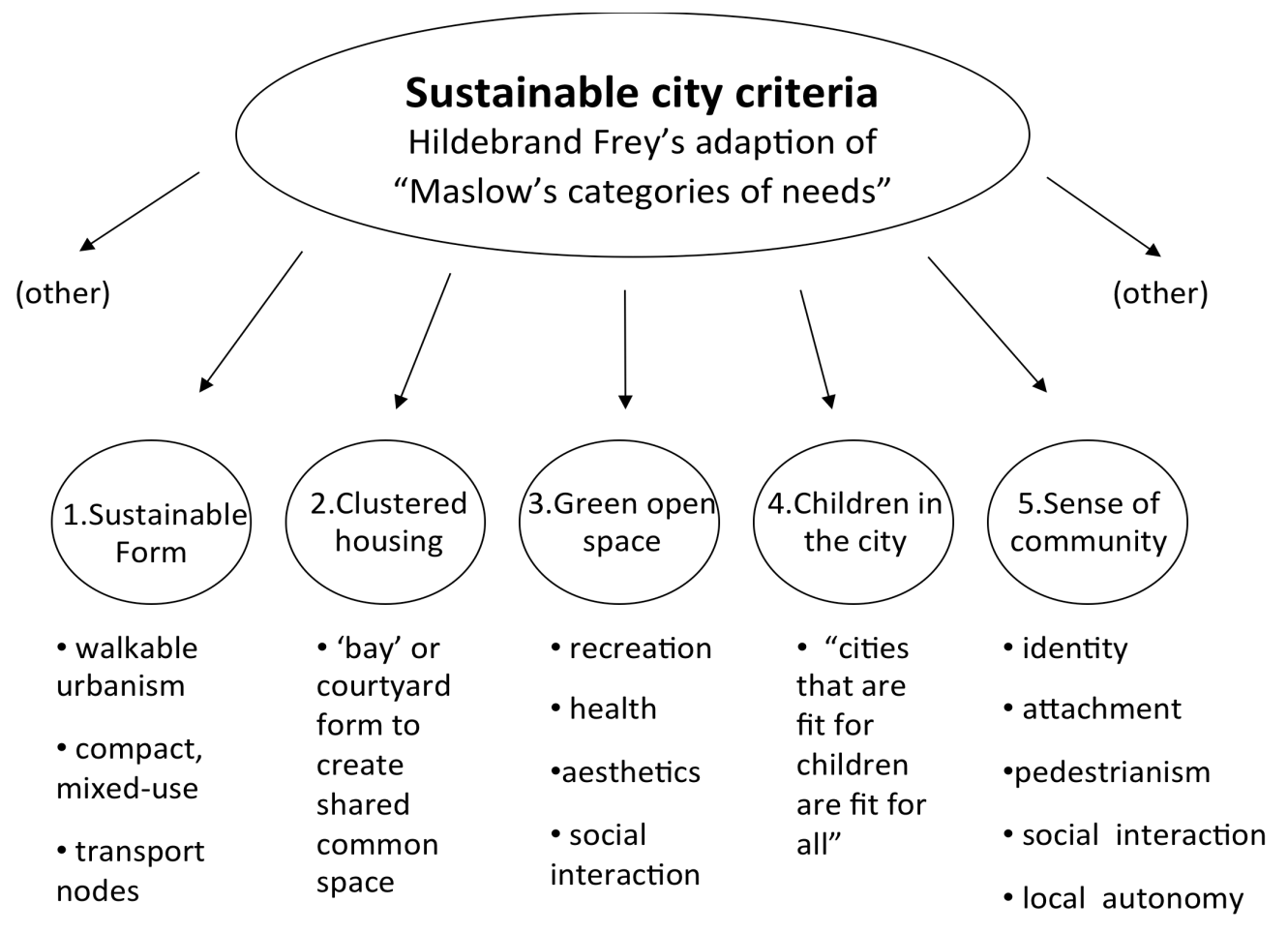

Fig. 7.3 Five principles for multivalent communal space in medium density housing within the framework of agreed criteria for sustainable city form.

(S.J. Pattinson, adapted from Frey in The Urban Design Reader 2007, 339-340)

These five proposed principles (viz., sustainable neighbourhoods; clustered housing; green open space; the needs of children; and 'sense of community'), when applied in an integrated way, will achieve multivalent communal space in medium density housing. Tables $7.1-7.5$ show how these five interactive principles emerge from the viewpoints of practitioners (current practice), academic researchers (current 
theory) and residents (in Case Study research). Briefly, the five interactive principles for multivalent communal space are:

1. Walkable neighbourhoods - Compact urban form of a certain density and diversity of land use supporting transit-oriented development (TOD) is required for walkable urban environments (Lozano 165-166; Leinberger 114; Calthorpe 91)

2. Clustered housing - perimeter housing up to four storeys arranged around central open space makes the most efficient use of land (Martin and March 20, 21, 45; Scoffham and Vale 67-69; Cooper Marcus and Sarkissian 7-9, 189).

3. Green open space - The central open spaces created by the clustering of houses provide a natural focus for a wide variety of overlapping uses, providing residents with many benefits, including recreation, physical health and mental well-being, aesthetic enjoyment, sharing in voluntary work, and social interaction (Lozano 149, 179; Alexander et al. 304; Kazmierczak and James 354).

4. Children and Teenagers - a wide body of literature addresses the needs of children in cities. Cooper Marcus and Sarkissian provide comprehensive design guidelines, noting that well-designed communal spaces within housing developments can be particularly beneficial to children of all age groups.

5. Sense of community - the design of the physical features of a neighbourhood can foster a sense of local community. Four components of 'sense of community' have been identified, viz. neighbourhood identity, neighbourhood attachment, pedestrianism and social interaction. Another important factor for a 'sense of community' is local political autonomy (Joongsub Kim 2001; Jan Gehl 21-24; Milton Kotler, in Hester 11; Jane Jacobs 117). 
(C) 2012 Stephen Pattinson Chapter 7: Discussion M. Arch Thesis

\section{A Correlation of Best Practice, Theory and Community Input:}

In the following Tables $7.1-7.5$ the viewpoints of practitioners, academic researchers and residents are correlated for each of the five principles of multivalent communal space. The viewpoints of practitioners are from current practice (see Chapter 3). The viewpoints of academic researchers are from current theory in literature in various disciplines (see Chapter 4). Community viewpoints are represented by the responses of residents of the terrace housing at Pinehill Park, Trentham, Upper Hutt to the survey and interviews in the Case Study in Chapter 6.

In the 'Practitioners' column of Tables 7.1 - 7.5 some New Zealand medium density housing projects are shown that illustrate key aspects of each of the five principles of multivalent communal space. In the 'Academics' column of Tables $7.1-7.5$ the key points of each of the five principles are shown along with the main literature references for these key points.

The five principles for multivalent communal space emerged from Chapters 3 and 4 (investigations of current practice and review of current theory). These principles are discussed in Chapter 5. In the Case Study of Pinehill Park in Chapter 6, residents of the terrace housing were not informed about the proposed five principles for multivalent communal space. The writer did not want to influence or lead the respondents in the survey/interview questions. The two survey/interview questions were deliberately kept simple and open-ended to allow respondents to express their own viewpoints about Pinehill Park.

The 'Community' column of Tables 7.1 - 7.5 shows some of the responses from residents in the terrace housing at Pinehill Park. Responses do not necessarily correlate with all the key points of the five principles because these were never discussed with the residents. The responses show, however, that most aspects of the five principles are addressed in the respondents' comments. 


\begin{tabular}{|c|c|c|c|}
\hline \#1 & Walkable Neighbourhoods: & TABLE 7.1 & (S.J. Pattinson) \\
\hline & Pract & Academics (Current Theory) & Community (Pinehill Park Residents) \\
\hline & $\begin{array}{l}\text { Merivale Mews, Christchurch, NZ. } \\
\text { Offices, shops, tavern, restaurant, } \\
\text { village green and housing clustered } \\
\text { around a central landscaped pond. } \\
\text { Architect: Peter Beaven (Google Maps) } \\
\text { Ropata Village: } \\
\text { retirement care, } \\
\text { medical / dental } \\
\text { and pharmacy. } \\
\text { Lower Hutt, NZ } \\
\text { Architect: Roger } \\
\text { Walker (Photo: } \\
\text { S.J.Pattinson) }\end{array}$ & $\begin{array}{l}\text { Public transport / Transit Oriented } \\
\text { Development Calthorpe } 1993 \\
\text { Compact, mixed-use development } \\
\text { Frey, in The Urban Design Reader } 2007 \\
\text { Walkable urbanism Leinberger } 2009 \\
\text { Shared streets Southworth \& Ben- } \\
\text { Joseph 2003: 6-7, 125 } \\
\text { Safety, security / Crime Prevention } \\
\text { Through Environmental Design } \\
\text { Newman 1972: 9,10; AMCORD PND17; } \\
\text { NZMinistry of Justice - CPTED 2005 } \\
\text { Optional, recreational and social } \\
\text { activities outdoors (eg walking) are } \\
\text { influenced by and very sensitive to } \\
\text { the quality of outdoor space, so } \\
\text { attention to environmental detail } \\
\text { is important. Gehl 1996: 131, 135ff }\end{array}$ & $\begin{array}{l}\text { "It is just five minutes walk to the bus or train } \\
\text { which takes } 7 \text { minutes to Upper Hutt or } 35 \\
\text { minutes to Wellington City " (Couple, 50+) } \\
\text { "I walk to a [local] gym ... several times a week } \\
\text { (Male 50+) } \\
\text { "The park needs some paths" (Male 30-50) } \\
\text { "The park needs [paths] for C to walk } \\
\text { about" (disabled resident) (Couple, 50+) } \\
\text { "The park is dark at night, a bit scary" (Female } \\
30-50) \text { "... there was a threatening incident in } \\
\text { the park, we couldn't see what was happening" } \\
\text { (Female } 50+\text { ) "Security lighting would be } \\
\text { good" (Male } 50+\text { ) "Needs lights for gardens, } \\
\text { footpaths, not floodlights - intimate, in-ground } \\
\text { lighting to gently follow at night" (Male 50+) } \\
\text { "There is enormous potential out there. I'd like } \\
\text { to see it maximised, have something attractive } \\
\text {...that would draw you into the Park"(Male } 50^{+} \text {) } \\
\text { "I don't use the Park. There are other parks I go } \\
\text { to and play tennis with friends." (Female 30-50) } \\
\text { "We don't use the park unless crossing it to } \\
\text { have a wine with a neighbour. I would like to } \\
\text { read a book in the park but I would like more } \\
\text { trees, benches, a waterfeature." (Female 50+) }\end{array}$ \\
\hline
\end{tabular}




\begin{tabular}{|c|c|c|c|}
\hline \multirow[t]{2}{*}{$\# 2$} & \multicolumn{3}{|l|}{ Clustered Housing: } \\
\hline & Practitioners (Best Practice) & Academics (Current Theory) & Community (Pinehill Park Residents) \\
\hline & $\begin{array}{l}\text { Perimeter } \\
\text { terrace housing } \\
\text { around shared } \\
\text { open space with } \\
\text { swimming pool. } \\
\text { Takapuna, } \\
\text { Auckland, NZ. } \\
\text { Architect: Jensen, } \\
\text { Chambers, Young }\end{array}$ & $\begin{array}{l}\text { Attached housing clustered around } \\
\text { central communal open space. } \\
\text { Martin \& March 20, 41, 45; Scoffham } \\
\text { and Vale 67-69; Carmona et al. 183; } \\
\text { Cooper Marcus and Sarkissian 7-9,189 } \\
\text { Levitt } 110 \\
\text { 2-and 3-storey attached housing, } \\
\text { of timber-framed construction, } \\
\text { arranged around perimeter of } \\
\text { planted courtyard social space. } \\
\text { Cameron McClean c.1977 }\end{array}$ & $\begin{array}{l}\text { "The settings of the [attached] house make it } \\
\text { so that you don't really see or hear neighbours } \\
\text { apart from in the Park." (Female } 30-50 \text { ) "At } \\
\text { our previous [detached] house we could see in } \\
\text { the neighbours' houses on } 3 \text { sides. You can't } \\
\text { see into your neighbour's place here ... it gives } \\
\text { us a feeling of personal space." (Couple 50+) } \\
\text { "You're not boxed in with houses ... it is open" } \\
\text { (Male 16-19) } \\
\text { "People chat over the front fence to others in } \\
\text { the Park. It expands the boundaries of a small } \\
\text { lot and helps community relations."(Couple 50+) } \\
\text { "As part of a community you can sit and watch } \\
\text { people enjoy the Park. You meet people out in } \\
\text { the Park and it is safe." (Female 30-50) } \\
\text { "Why didn't they put in solar heating [i.e. solar } \\
\text { water and solar space heating]? When it gets } \\
\text { really cold we have to put extra bar heaters on, } \\
\text { which really uses up the power. ... We would } \\
\text { also like communal fruit trees." (Couple, 50+) } \\
\text { "I am planting a cherry tree [to shade the } \\
\text { windows] and an organic garden" (Female 50+) } \\
\text { [requires outdoor space and physical activity] }\end{array}$ \\
\hline
\end{tabular}




\begin{tabular}{|c|c|c|c|}
\hline \#3 & \multicolumn{3}{|c|}{ Green Open Space: $\quad$ TABLE 7.3} \\
\hline & (Best Practice) & Academics (Current Theory) & Community (Pinehill Park Residents) \\
\hline & 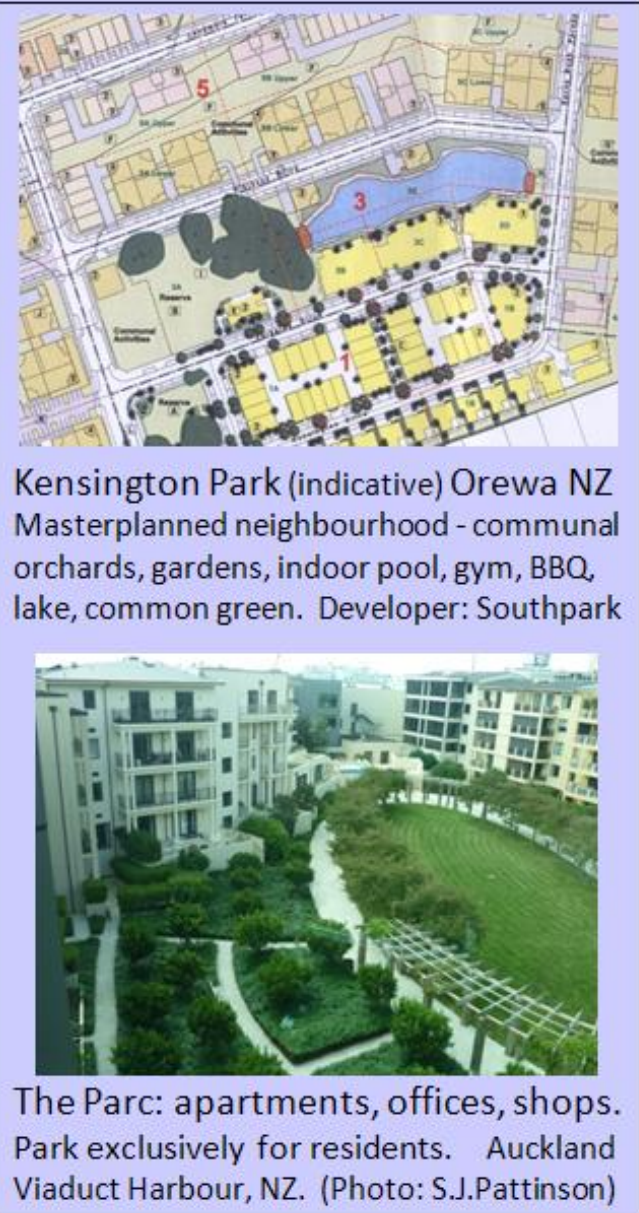 & $\begin{array}{l}\text { Benefits of urban green space: } \\
\text { - Recreation } \\
\text { Kazmierczak and James } 2007 \\
\text { Alexander et al. } 304-309 \\
\text { Calthorpe } 91 \\
\text { - Health - physical and mental } \\
\text { Kazmierczak and James } 2007 \\
\text { - Aesthetics / status / value } \\
\text { Kazmierczak and James } 2007 \\
\text { - Social inclusion and interaction } \\
\text { Kazmierczak and James } 2007 \\
\text { Jacobs } 89-111 \\
\text { - Local autonomy } \\
\text { Kazmierczak and James } 2007 \text {; Frey, } \\
\text { in The Urban Design Reader } 2007\end{array}$ & $\begin{array}{l}\text { "The Park is an asset to the place, it gives a } \\
\text { sense of more space and is an extension of the } \\
\text { back yard." (Female } 30-50 \text { ) "The Park seems } \\
\text { like it's for the people who live in the terrace } \\
\text { housing - its 'our Park' and 'my back yard"" } \\
\text { (Male 16-19) "We see this as our Park" (Couple } \\
\text { with children) "The Park encourages socialising } \\
\text { amongst youngsters - kids playing, meeting } \\
\text { each other" (Male 30-50) } \\
\text { "I really enjoy living beside the Park, mainly for } \\
\text { the kid's sake... to be able to go out and expend } \\
\text { some energy with their peers" (Male } 30-50 \text { ) } \\
\text { "It's used heaps in the evening, especially by } \\
\text { kids playing sport." (Females 30-50) } \\
\text { "I don't play in the Park. The ground is rough } \\
\text { and uneven - me and my mates hurt our ankles } \\
\text { if we play soccer..." (Teenage Male 11-15) } \\
\text { "The grass is stalky and uncomfortable on bare } \\
\text { feet. The ground is clay so in winter ... its boggy } \\
\text {... in summer it ... is very hard." (Female 50+) } \\
\text { "Nobody asked us about the playground, picnic } \\
\text { table or trees before they were put in; don't put } \\
\text { trees, tables or benches in the open play area } \\
\text { in the middle of the Park." (Male } 30-50 \text { ) "Put } \\
\text { them round the edges of the Park." (Male 50+) }\end{array}$ \\
\hline
\end{tabular}




\begin{tabular}{|c|c|c|c|}
\hline \#4A & Places for Children: & TABLE 7.4A & (S.J. Pattinson) \\
\hline & Practitioners $(\mathrm{B} \epsilon$ & Academics (Current Theory) & Community (Pinehill Park Residents) \\
\hline & $\begin{array}{l}\text { Talbot Park, Glen Innes, Auckland NZ } \\
\text { (Housing New Zealand Corporation } \\
\text { social housing). Standard play } \\
\text { equipment for very young children. } \\
\text { (Photos: S.J. Pattinson) }\end{array}$ & $\begin{array}{l}\text { Pre-school Children - } \\
\text { Cooper Marcus and Sarkissian provide } \\
\text { comprehensive advice on facilities to } \\
\text { meet pre-schoolers' needs, including } \\
\text { design guidelines for doorstep play, } \\
\text { balconies, yards, tot lots, sand play, } \\
\text { water play, etc. (138-150) } \\
\text { Five to Twelve Year Olds - } \\
\text { Cooper Marcus and Sarkissian provide } \\
\text { guidelines for young children's needs } \\
\text { for mobility, biking, ball games, flow } \\
\text { of play, equipment variety, lookouts, } \\
\text { adventure playgrounds, huts and self- } \\
\text { made enclosures etc. (151-179) } \\
\text { Children as Placemakers - } \\
\text { Include children in the process of } \\
\text { planning the areas they will use. In } \\
\text { addition to standard play equipment } \\
\text { provide children with the opportunity } \\
\text { to create, deconstruct and recreate } \\
\text { their own play environments. (Cooper } \\
\text { Marcus and Sarkissian 153-4) }\end{array}$ & $\begin{array}{l}\text { "The new trees in the middle of the park can } \\
\text { prevent us from seeing the children at the far } \\
\text { end of the park. If 2-3 year olds run [from the } \\
\text { playground out] to the road, there is nothing } \\
\text { to stop them. Drivers do burn outs. The noise } \\
\text { draws the children to the road to see what is } \\
\text { going on" (Couple with children) "The park } \\
\text { needs a fence along the roadside and speed } \\
\text { bumps on the road." (All parents) } \\
\text { "The kids use the park a lot." "The park allows } \\
\text { the kids to play together, so the kids in the area } \\
\text { become good friends." (Couple with children; } \\
\text { Female 20-30) "If the kids are stuck inside } \\
\text { playing computers and they see someone in the } \\
\text { park they will go out to play with them, the } \\
\text { park draws them outside." (Male } 30-50 \text { ) } \\
\text { "Our grandchildren play in the park... with } \\
\text { neighbours' grandchildren. There was a tree- } \\
\text { house [in the park] but it was taken down. It } \\
\text { was very popular with the children" (Couple } \\
50^{+} \text {"I would like a bigger climbing frame and } \\
\text { tree-house." (Grandson, } 5 \text { yrs) (This suggests } \\
\text { the boy is outgrowing the standard playground } \\
\text { equipment intended for very young children) } \\
\text { "The park needs a playground at both ends ... } \\
\text { they could be different" (Couple with children) }\end{array}$ \\
\hline
\end{tabular}




\begin{tabular}{|c|c|c|c|}
\hline \#4B & \multicolumn{3}{|c|}{ Places for Teenagers: $\quad$ TABLE 7.4B } \\
\hline & Practitioners (Best Practice) & Academics (Current Theory) & Community (Pinehill Park Residents) \\
\hline & $\begin{array}{l}\text { During visits by the author to over } 200 \\
\text { medium-density housing developments, } \\
\text { outdoor facilities for teenagers were } \\
\text { observed at only two developments, } \\
\text { viz. Council flats, Arlington Street, } \\
\text { Wellington (where about } 20 \text { young } \\
\text { people were playing volley ball on a } \\
\text { basketball half-court); and Pinehill Park, } \\
\text { Upper Hutt (where } 5 \text { teenagers were } \\
\text { kicking a rugby ball, using the swings, } \\
\text { and climbing over the swing frame). }\end{array}$ & $\begin{array}{l}\text { Cooper Marcus and Sarkissian (180- } \\
\text { 184) suggest a range of facilities for } \\
\text { teenagers in housing developments, } \\
\text { including space for ball games, } \\
\text { hanging out (informal gathering } \\
\text { places), teen entertainment, a teen } \\
\text { centre, and access (e.g. convenient } \\
\text { public transport) to off site facilities. } \\
\text { Cooper Marcus and Sarkissian } \\
\text { comment that "many teenage needs } \\
\text { will be met at school, in the } \\
\text { community, and in local parks, not } \\
\text { within the housing site" (180). They } \\
\text { advise, however, that "provision for } \\
\text { adolescent leisure should be part of } \\
\text { any housing program" (184). } \\
\text { Planning processes typically focus on } \\
\text { parks and schools for meeting the } \\
\text { needs of young people but rarely } \\
\text { consider facilities for them in housing } \\
\text { environments (Freeman 2010). } \\
\text { Communal facilities for teenagers e.g. } \\
\text { workshops, photography dark room, } \\
\text { music room (McCamant \& Durrett 27) }\end{array}$ & $\begin{array}{l}\text { "Young teenagers are the 'forgotten age'. } \\
\text { Parks have things for } 0-5 \text { year olds, and over } \\
18 \text { 's can go into the city, but there is nothing } \\
\text { for the in-between age" (Teenage boy, 13-15) } \\
\text { "We use the park to hang out with friends. We } \\
\text { use the benches to sit and eat. Maybe we } \\
\text { could have some separate parts so that when } \\
\text { there are kids in the park, we can still hang out } \\
\text { there" (Teenage Girls, both 16-19) } \\
\text { "Should have more equipment, e.g. slides, } \\
\text { swings, more benches and a bin!" (Teenage } \\
\text { Females both 16-19) "Add a BBQ" (Male 16-19) } \\
\text { "The new trees in the middle of the park ... } \\
\text { push the ball playing to the side of the park } \\
\text { close to our house" (Female 50+) "The ground } \\
\text { needs to be levelled out ... for playing soccer" } \\
\text { (Female } 30-50 \text { ) (Boy 11-15) "Move the } \\
\text { playground to one side to free up some more } \\
\text { space for the soccer/rugby area" (Male 16-19) } \\
\text { "The teenagers sometimes go outside into the } \\
\text { park ... It becomes extra ... spill over space for } \\
\text { the teenagers when visitors come, while us } \\
\text { adults talk inside." (Male } 50+\text { ) "A [swimming] } \\
\text { pool ... would be cool." (Male 16-19) }\end{array}$ \\
\hline
\end{tabular}




\begin{tabular}{|c|c|c|c|}
\hline \#5 & \multicolumn{3}{|c|}{ Sense of Community: $\quad$ TABLE 7.5} \\
\hline & Practitioners & Academics (Current Theory) & Community (Pinehill Park Residents) \\
\hline & $\begin{array}{l}\text { Salisbury Garden } \\
\text { Court, Wadestown } \\
\text { Houses sited round shared tennis court. } \\
\text { "A Place to Stay" film by Marie Russell. } \\
\text { (Aerial view from Wellington City Council) }\end{array}$ & $\begin{array}{l}\text { Neighbourhood identity } \\
\text { Kim } 2001 \\
\text { Neighbourhood attachment } \\
\text { Kim 2001; Morgan } 2010 \\
\text { Pedestrianism } \\
\text { Leinberger 2009; Kim 2001 } \\
\text { Social interaction } \\
\text { Kim 2001; Gehl 1996, 1977 } \\
\text { Local autonomy } \\
\text { Kotler, in Hester (11); Jacobs (117); } \\
\text { AMCORD 2005 PND5; Wood, in Bell \& } \\
\text { Tyrwhitt (327); Newman (1972); Frey, } \\
\text { in The Urban Design Reader, 339-340 }\end{array}$ & $\begin{array}{l}\text { "Terrace house residents have held shared } \\
\text { events in the park, including a New Year's Eve } \\
\text { party where they brought out tents, tables, } \\
B B Q \text { 's, food, drink, karaoke, and a bouncy } \\
\text { castle for the children. They are talking about a } \\
\text { celebration in the Park for Waitangi Day [on } \\
\text { February 6, 2011]" (Male 30-50) } \\
\text { "Mums and children walk to the Park from the } \\
\text { nearby housing, sometimes in groups - usually } \\
\text { about } 3 \text { or } 4 \text { parents, but once we saw } 8 \text { adults } \\
\text { and their children walk to the Park"(Couple50+) } \\
\text { "Not a large number of families use the park. It } \\
\text { needs more trees and benches" (Female 50+) } \\
\text { "Only one sitting area ... yet a lot of people } \\
\text { want to sit" (Male } 30-50 \text { ). "Make } 2 \text { or } 3 \text { areas } \\
\text { - and not just for the children" (Female 50+) } \\
\text { "Put chairs / seating / tables around the side so } \\
\text { different families can use on special occasions, } \\
\text { have their own area to use..." (Male } 30-50 \text { ) } \\
\text { "Keep the park communal for all residents but } \\
\text { different families might have seating / tables } \\
\text { around the edge of park to use on special } \\
\text { occasions and have responsibility for taking } \\
\text { care of their own area ... We could try to make } \\
\text { something more of community" (Male 50+) }\end{array}$ \\
\hline
\end{tabular}




\section{The five principles for multivalence are inter-dependent}

The purpose of the following brief discussion is to reflect on Tables 7.1 -7.5 and to point out the inter-relatedness of things. Twelve potential uses for communal space in medium density housing were indentified in Chapter 4. None of the twelve are mutually exclusive: they all complement or reinforce each other in some way. For example, doing voluntary gardening to help maintain the landscaping of a communal area can simultaneously relieve stress and restore mental vitality, provide some physical exercise, give occasion for some social interaction, while the upkeep of the grounds contributes to neighbourhood identity and attachment. Similarly, daily brief exchanges of greetings with neighbours while traversing a pleasant common green can gradually build neighbourly relations, foster intergenerational exchange and support, provide opportunities for tutoring or mentoring young ones, and generally build the social fabric essential to the self-policing of a community.

All twelve meanings of communal space are embodied in one way or another in the five principles. It is not surprising therefore that the five principles are inter-related: they need each other. For example, 'a sense of community' (the fifth principle) requires a pedestrian-oriented rather than car-oriented environment, hence the need for 'walkable urbanism' (the first principle). And the reverse is also true - the first principle ('walkable neighbourhoods') needs the fifth principle ('sense of community') if a housing environment is to be more than just a showcase for Environmentally Sustainable Design (ESD) features.

The point is that the five principles of multivalent communal space are interactive and inter-dependent. They are not optional. All are necessary to achieve multivalence of the 'bay'. 
(C) 2012 Stephen Pattinson Chapter 7: Discussion M. Arch Thesis

\section{Resolving 'incompatible' mixes}

A valuable lesson from the development of 'shared streets' is that apparently conflicting uses, e.g. moving vehicles and playing children, should not automatically be regarded as 'incompatible'. In the case of shared streets, integrated solutions developed by the combined insights of several disciplines has resulted in the mixing of cars and children in environments that actually turn out to be safer than conventional streets.

At Pinehill Park, some respondents (teenagers) said they would like a basketball hoop and playing area and some respondents (adults) said they did not want this. Likewise, some said they wanted a tennis court and others said they did not because, like basketballs, tennis balls bouncing on hard surfaces can be noisy. Maybe a solution can be developed whereby a tennis court and basketball area can be located along the road boundary of the park (i.e. in the most distant location from the houses but so that surveillance is still possible) where noise will be less of an issue. A high wire netting fence along the road boundary for the tennis/basketball area can be part of the border strategy to protect children from road traffic. The tennis/basketball activities, while being available for all residents and all ages, also provide a point of interest to invite use of the park by passersby.

The point is to look at supposedly 'incompatible' uses holistically and collectively to recognise the 'relatedness' of things, and how mutual resolution of apparently conflicting uses can actually result in a more multivalent outcome for communal spaces. 


\section{Application of the Five Principles for Multivalent Communal Space}

\section{'Re-Developing' Pinehill Crescent Subdivision, Trentham, Upper Hutt}

Pinehill Park was selected for the Case Study because its current arrangement seems to have potential for multivalence. Tables $7.1-7.5$ indicate from the responses of residents that this is a special site and the community of residents there could, if they wanted to, transform 'their' park from univalence (being meaningful only to children) to multivalence (being meaningful for the whole community). For reasons similar to the selection of the Case Study site, the whole subdivision in which this site is located is now selected for an exercise in applying the five principles for multivalent communal space. The purpose of this exercise is to investigate research question $4-$ will the application of these principles result in more efficient use of land and enhance residents' living environment?

This is a broad-brush exercise - it is conceptual and indicative only. The reader is asked to imagine much that goes unsaid in the following diagrams but which is implied already or would be developed if this were a real project. For example, little is said about the location of the subdivision, but it is known from Chapter 6 that it is well served by public transport (bus and electric rail), and is close to many recreational facilities within walking distance, including tennis courts, sports fields, a YMCA fitness centre, a rifle range, two golf courses, Trentham Memorial Park, horse racing at Trentham Racecourse and a Trentham wetland ecological restoration project. This all relates to Principle No. 1 - 'Walkable Neighbourhoods'. This subdivision, if re-developed, has the potential to be a genuinely 'walkable' environment (i.e. pedestrian-oriented rather than car-oriented). 
But to achieve 'walkability' a higher density threshold needs to be achieved, meaning more concentrated development. Upper Hutt City Council is currently processing a District Plan change which is intended to allow higher density residential close to the Central City and suburban neighbourhood shopping centres. The intention is to reduce the current minimum lot size from $400 \mathrm{~m}^{2}$ down to $300 \mathrm{~m}^{2}$ per dwelling. The writer has therefore hypothetically 're-developed' the 5 hectare Pinehill Crescent site assuming $300 \mathrm{~m}^{2}$ minimum lot sizes according to two different development patterns - firstly using a conventional approach (detached housing on $300 \mathrm{~m}^{2}$ lots) and secondly by applying the five proposed principles for multivalent communal space - and comparing the difference. Will the application of the five principles result in more efficient use of the 5 hectare site, and enhance the living environment for its residents?

The exercise is conceptual - it has to be imagined that if this were a real exercise the details could be successfully addressed later. At this stage the reader is asked to imagine that this exercise is being carried out by a team consisting of practitioners, researchers and the community - that it is Tables $7.1-7.5$ being put into effect by collaborative effort. In a real situation, detailed issues - such as identifying private, communal and public land so there is no ambiguous space, or the detailed layout and design of 'shared streets' etc. - would all be addressed by the team at the proper time. But at this conceptual stage the objective is to investigate the potential application of all five principles in broad terms only, and then make a comparison of the two hypothetical and approximate outcomes.

The following diagrams show the two alternative developments: Fig. 7.4 - the subdivision as existing; Fig. 7.5 - three terrace houses are added to Pinehill Park to illustrate a concept about density; Fig. 7.6 - the subdivision re-developed with detached houses on minimum $300 \mathrm{~m}^{2}$ lots; and Figs. $7.7-7.10$ show the subdivision re-developed with multivalent communal spaces. 


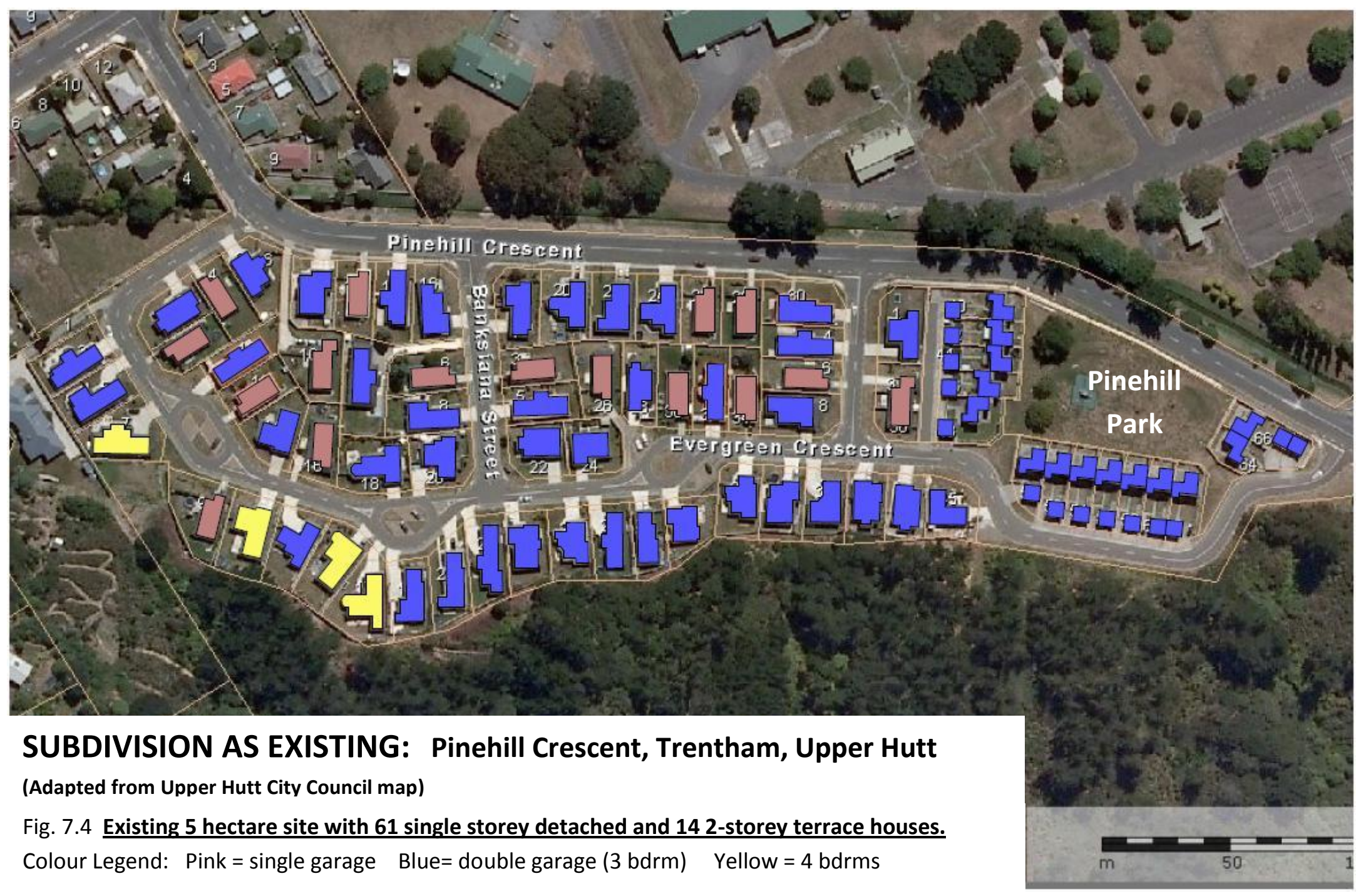




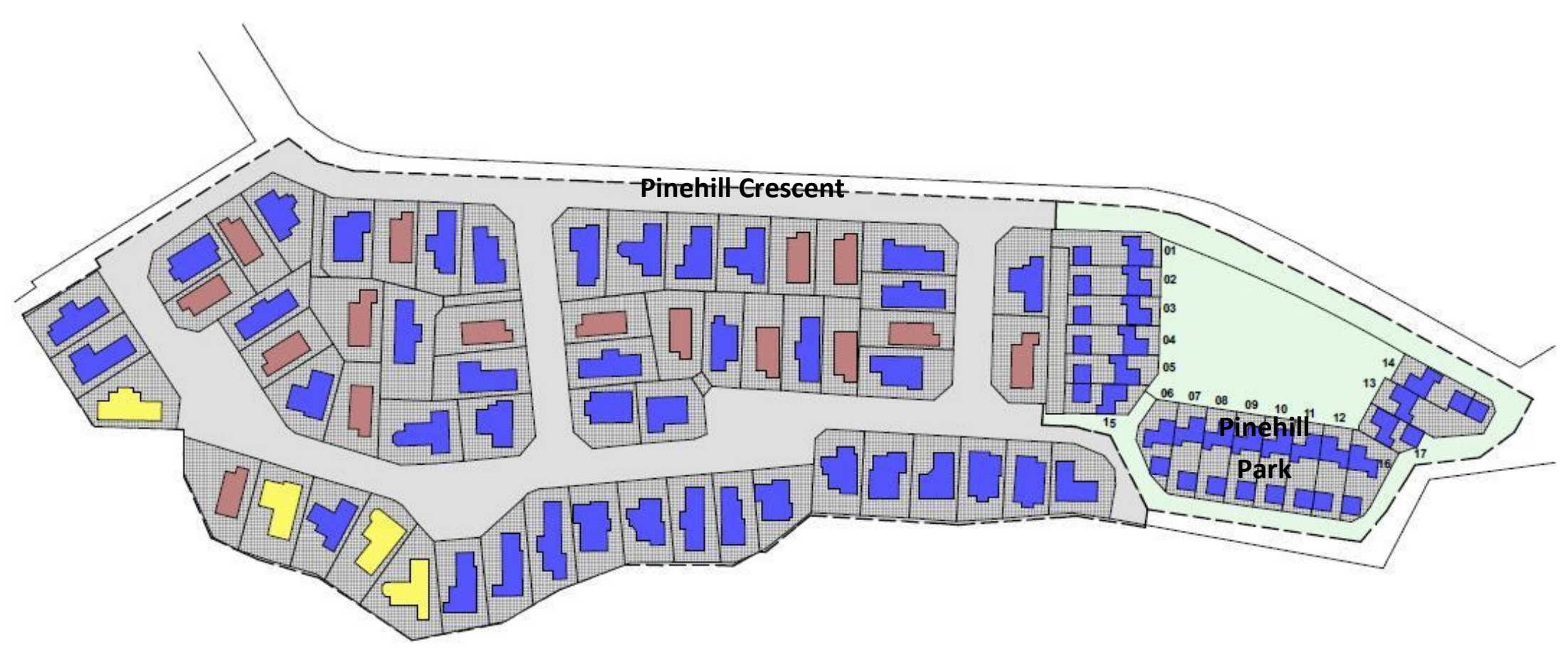

Fig. 7.5 Three terrace houses $(15,16,17)$ added to the cluster housing at Pinehill Park. Currently the density of the terrace housing is $12 \mathrm{dph}$ gross (including Pinehill Park and half the width of perimeter roads) - a little less than the density of the detached houses ( $15 \mathrm{dph}$ gross). By adding three terraces, the densities are the same (both $15 \mathrm{dph}$ gross ). The advantage of cluster housing is the creation of shared open space, in this case, Pinehill Park which is about $3,500 \mathrm{~m}^{2}$ of open space.

\section{SAME DENSITY: Two types of development (detached, and cluster around park)}

Pinehill Crescent, Trentham, Upper Hutt (hypothetical illustration)

(Source: S. J. Pattinson) 


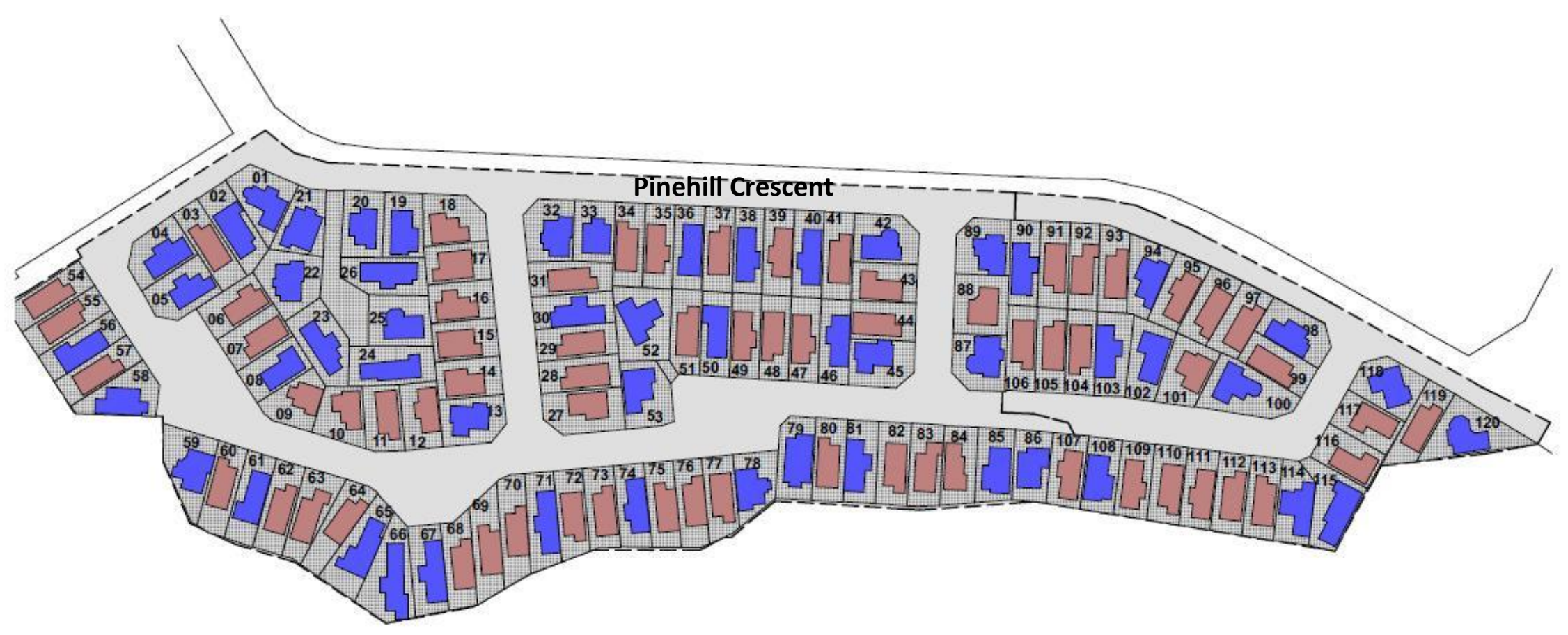

Fig. 7.6 Hypothetical development of a $\mathbf{5}$ hectare site with $\mathbf{1 2 0}$ detached single storey houses Colour Legend: Blue $=3 \mathrm{bdrm}$ (double garages ) Pink = smaller houses with single gargaes

120 DETACHED HOUSES: Minimum lot $300 \mathrm{~m}^{2}$ (Average size $320 \mathrm{~m}^{2}$. Density 22dph gross)

Pinehill Crescent, Trentham, Upper Hutt (hypothetical illustration) (Source: S. J. Pattinson) 


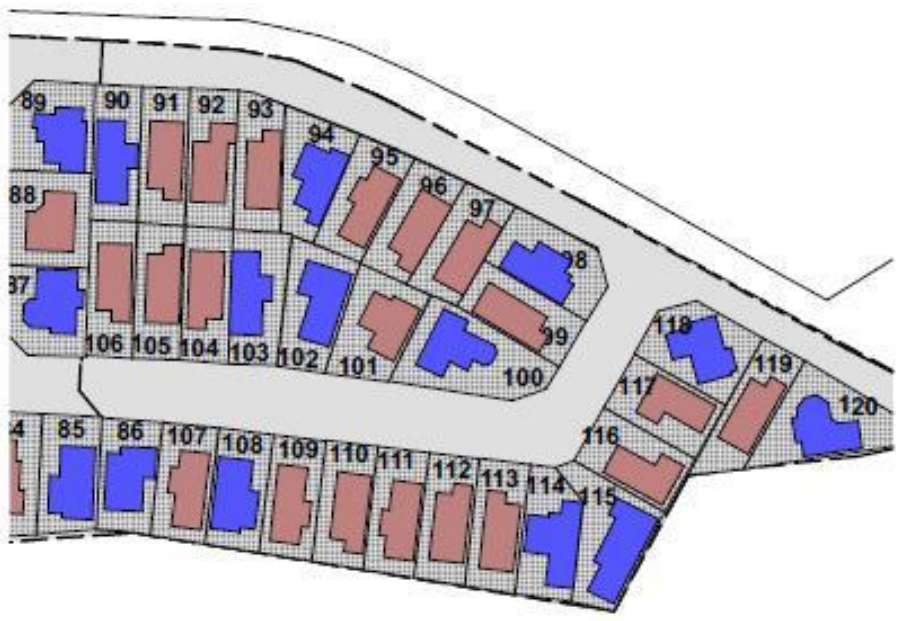

32 detached houses

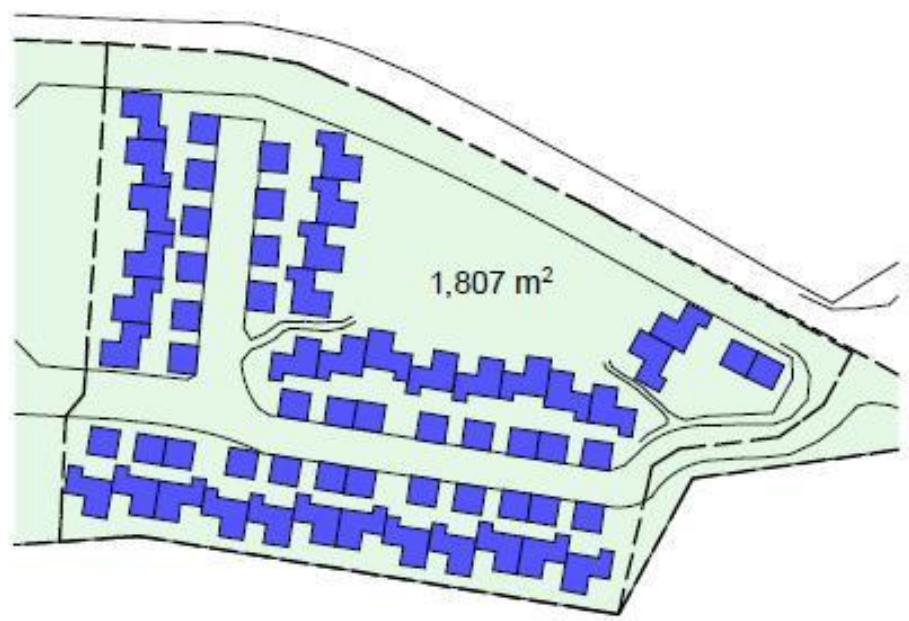

32 clustered terrace houses

Fig. 7.7 Hypothetical re-development of Pinehill Park to accommodate 32 houses - detached (left); clustered (right). Both approaches intensify the land to the same density ( $22 \mathrm{dph}$ ), using the same house types as existing, i.e. the same single storey detached housing as existing but with smaller yards and fewer $3 \mathrm{bdrm} / \mathrm{dble}$ garage types; the clustered development uses the same terrace house model as existing (all 2-storey with double garages) and has $1,800 \mathrm{~m}^{2}$ park.

DETACHED vs CLUSTER HOUSING WITH OPEN SPACE (Density 22dph gross for both)

Pinehill Crescent, Trentham, Upper Hutt (hypothetical illustration)

(Source: S. J. Pattinson) 


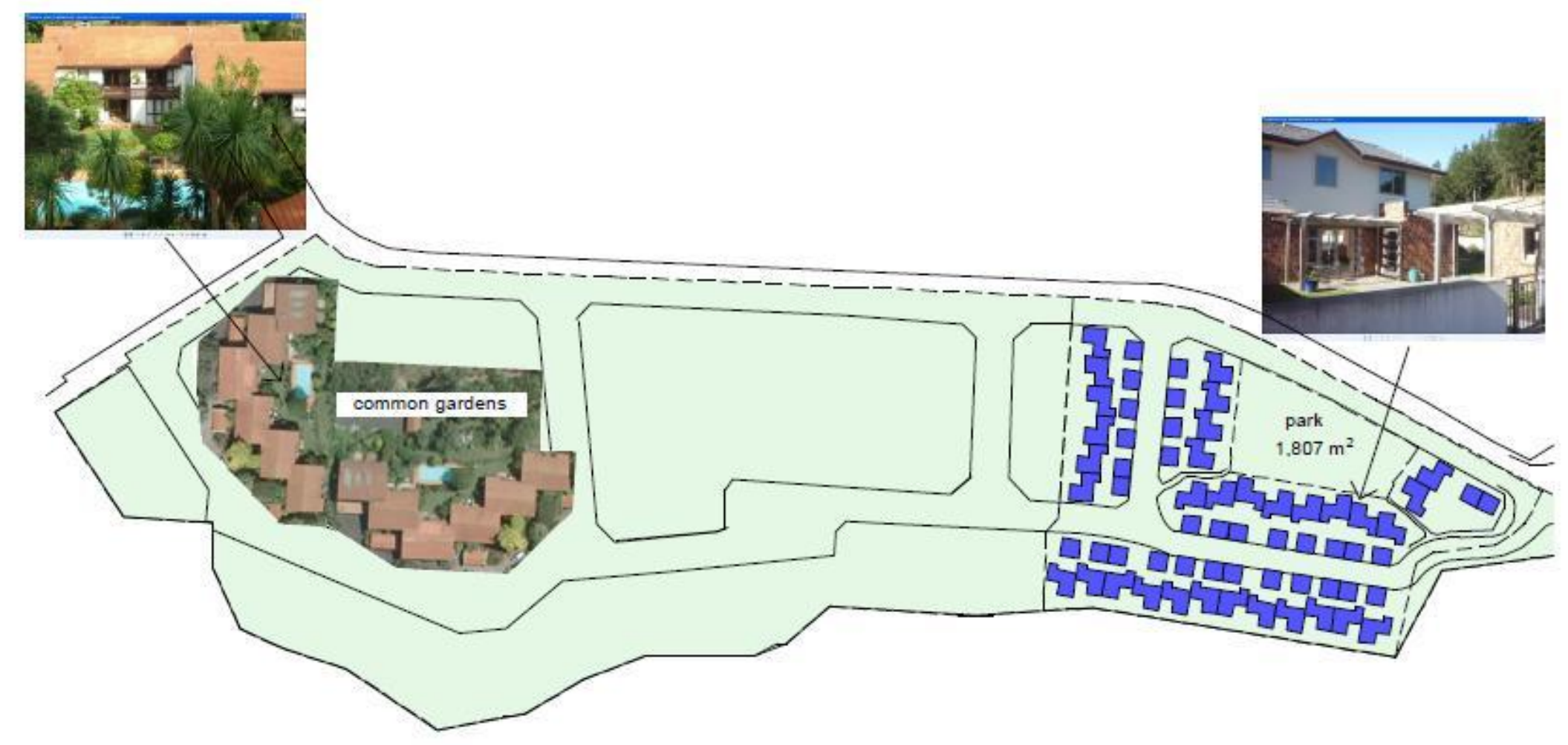

Fig. 7.8 Retaining the intensified 32 terrace houses at Pinehill Park (on the right of diagram), 48 units are added borrowing the concept of 'best practice' clusters (from Owens Street, Epsom, Auckland) at the left end of the subdivision. These 'concept clusters' include communal swimming pools and gardens, as shown in the aerial view and photo.

CLUSTER HOUSING WITH OPEN SPACE - Concept Stage 1 (right) \& Stage 2 (left)

Pinehill Crescent, Trentham, Upper Hutt (hypothetical illustration)

(Source: S. J. Pattinson) 


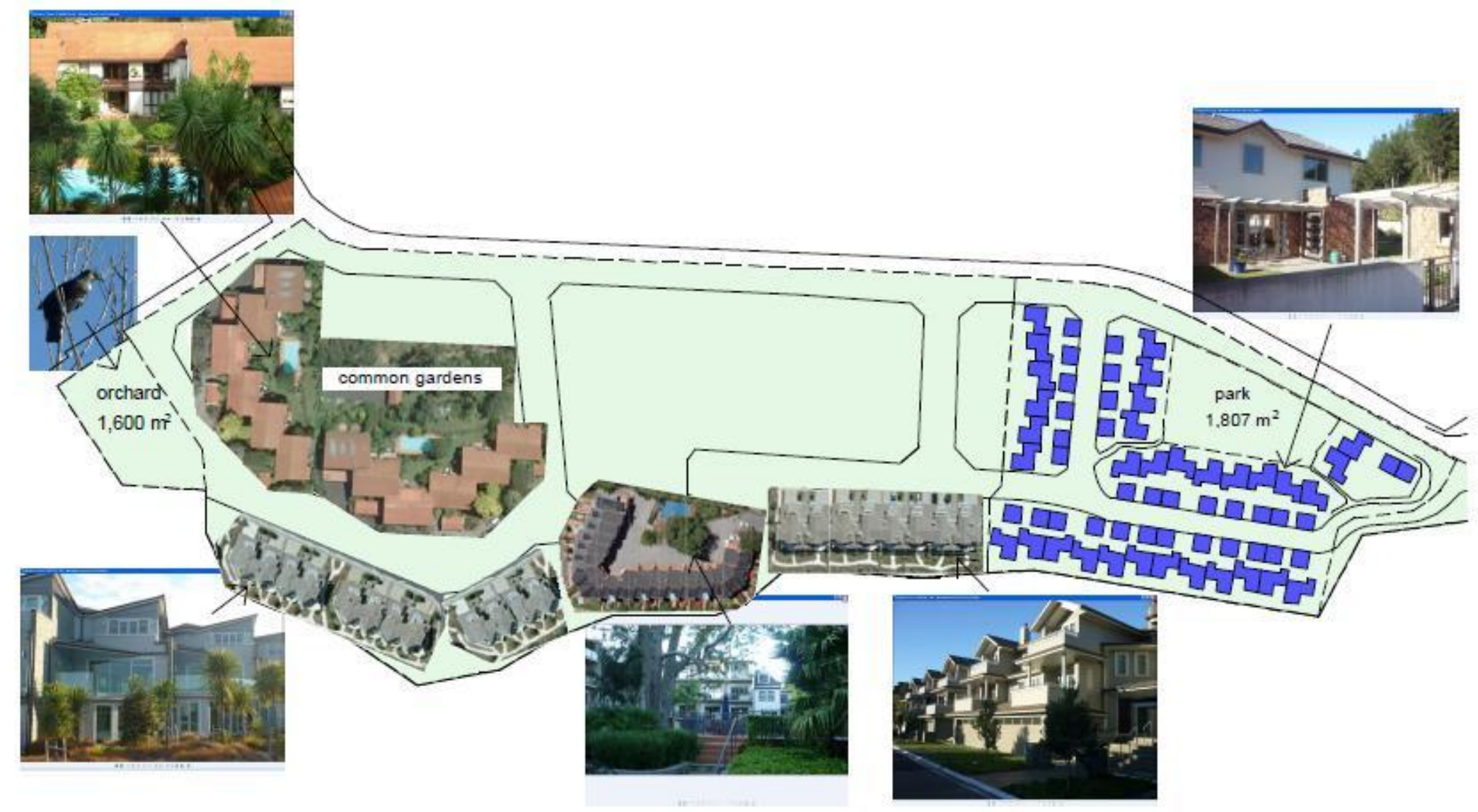

Fig. 7.9 Terraces added to south boundary. Borrowing more 'best practice' clusters, 18 terrace houses (from Takapuna) are added at the middle of the south boundary, and 20 from Whisper Cove ( 8 to the right and 12 to the left of the Takapuna cluster)

CLUSTER HOUSING WITH OPEN SPACE - Concept Stage 3 (south boundary terraces and orchard)

Pinehill Crescent, Trentham, Upper Hutt (hypothetical illustration) (Source: S. J. Pattinson) 


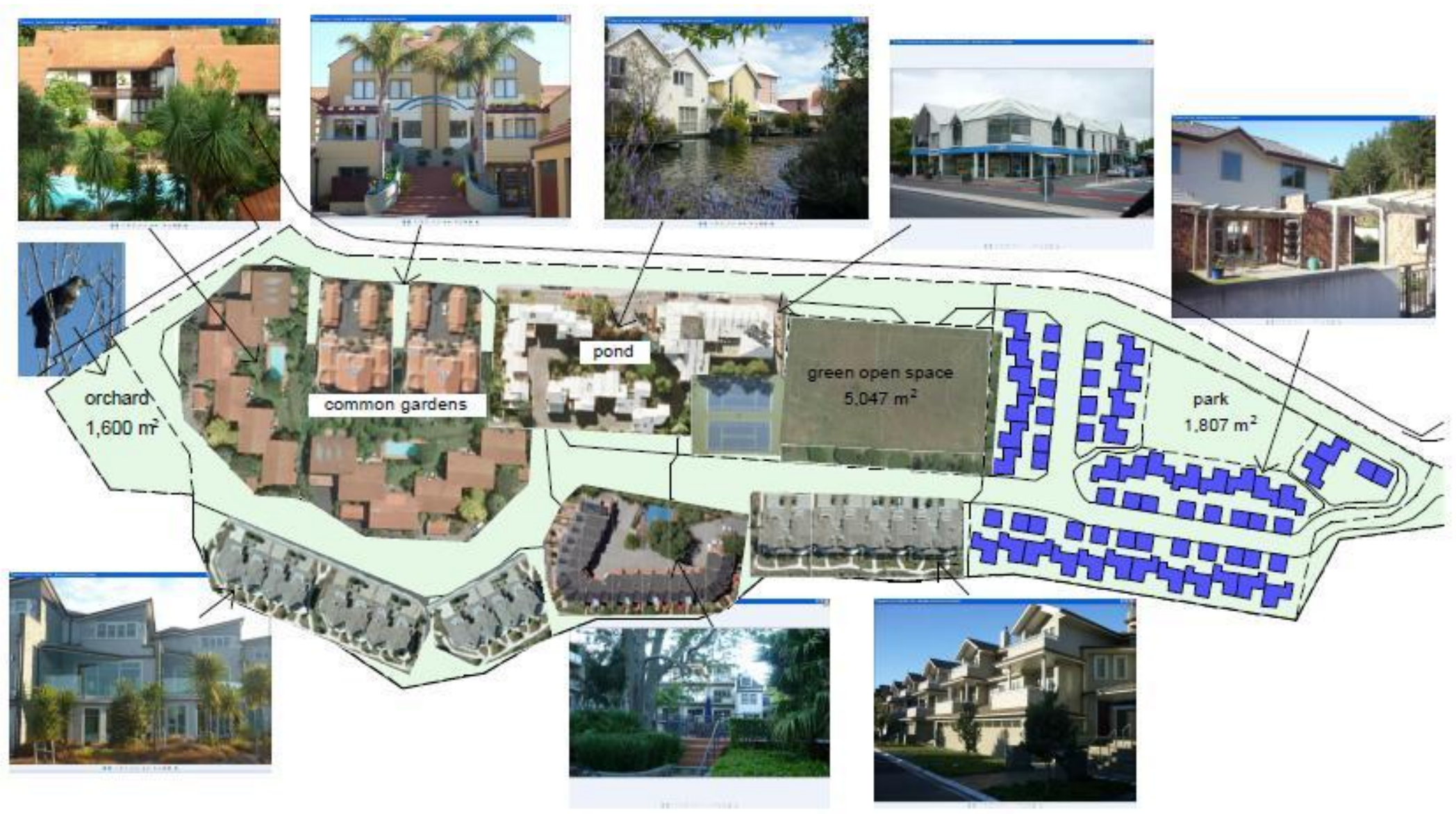

Fig. 7.10 Completing the overall concept, more 'best practice' clusters are added, 10 units from Gulf Harbour (Whangaparaoa) and 12 units with a water feature and retail centre (from Merivale, Christchurch). Also added are two tennis courts and soccer playing field.

CLUSTER HOUSING WITH OPEN SPACES - Concept Stage 4 (centre)

Pinehill Crescent, Trentham, Upper Hutt (hypothetical illustration)

\section{TOTAL: 140 DWELLINGS}

(Source and Photos: S.J. Pattinson) 
The clustered housing concept (Fig. 7.10) is built up from 'best practice', 'theory' (Martin \& March; Lozano; Leinberger; Cooper Marcus and Sarkissian, etc.), and community participation (e.g. Case Study respondents' suggestions for a café and social arenas for adults; more variety for children; facilities for teenagers; a water feature, etc.) It can be seen from a comparison of the two alternative developments (Figs. $7.6 \& 7.10$ ) that the development employing the principles of multivalent communal space (Fig. 7.10) has significant advantages over the conventional development (Fig. 7.6), summarised in the Table below (Table 7.6):

\begin{tabular}{|c|c|c|}
\hline & $\begin{array}{l}\text { Scheme A: Detached houses } \\
\text { on } 300 \mathrm{~m}^{2} \text { lots } \\
\text { (See Fig. 7.6) }\end{array}$ & $\begin{array}{l}\text { Scheme B: Alternative } \\
\text { development applying five } \\
\text { principles of multivalent } \\
\text { communal space (Fig. } 7.10 \text { ) }\end{array}$ \\
\hline $\begin{array}{l}\text { Overall area of } \\
\text { development: }\end{array}$ & 5 hectares & 5 hectares \\
\hline Total houses: & 120 & 140 \\
\hline House types / sizes: & Less variety and choice & More variety and choice \\
\hline Garaging: & Mostly single & Mostly double \\
\hline Number of storeys: & 1 & $2-3$ \\
\hline Average lot size: & $320 m^{2}$ & $\begin{array}{l}140 \mathrm{~m}^{2} \text { (estimate only) plus } \\
\text { share in communal open } \\
\text { spaces and facilities }\end{array}$ \\
\hline $\begin{array}{l}\text { Communal Open } \\
\text { Spaces: } \\
\text { NB: a Case Study } \\
\text { respondent suggested } \\
\text { a coffee spot/ café for } \\
\text { the neighbourhood. } \\
\text { The retail centre in } \\
\text { Fig. } 7.10 \text { borrowed } \\
\text { from Merivale, Chch, } \\
\text { could be imagined as } \\
\text { a community centre } \\
\text { with café, youth and } \\
\text { child care facilities etc }\end{array}$ & $\begin{array}{c}\text { Nil } \\
\text { (children play on streets) }\end{array}$ & $\begin{array}{l}\text { - } \text { orchard }\left(1600 \mathrm{~m}^{2}\right) \\
\text { - } \text { comm. gardens } \\
\text { - } 2 \text { tennis courts } \\
\text { - } \text { park }\left(1800 \mathrm{~m}^{2}\right) \\
\text { - landscaped pond } \\
\text { - } \text { trees, biodiversity } \\
\text { - } \text { community centre, } \\
\text { café, youth centre, } \\
\text { child care, etc. as } \\
\text { focal point for } \\
\text { neighbourhood } \\
\text { identity/attachmt/ } \\
\text { social interaction/ } \\
\text { local autonomy }\end{array}$ \\
\hline
\end{tabular}

Table 7.6 Comparison of conventional vs. cluster housing development (S.J. Pattinson) 
(C) 2012 Stephen Pattinson Chapter 7: Discussion M. Arch Thesis

It was noted earlier (p. 182) that the terrace housing at Pinehill Park is located in 'drivable suburbia' where cars are probably the normal mode of transport. And, in fact, one of the features which appealed to a couple who responded to the Case Study survey and interviews was that their terrace house 'has a double garage'. This contradiction of having double garages in a neighbourhood that is meant to be 'walkable' reflects the 'neverland' character of this housing development (and many others in New Zealand like it). As mentioned earlier (p. 105), Leinberger comments that 'neverland' developments may be a mistake or a transitional phase, but either way, in his opinion they are not sustainable, market-viable developments.

In the comparison of the two hypothetical developments at Pinehill Crescent (Table 7.6) it is clear that if, as in the current market, people want double garaging, then the terrace housing (Fig. 7.10) rather than the compact detached housing (Fig. 7.6) is more likely to satisfy this demand. But if the price of petrol continues to rise and people in 'walkable' localities such as Pinehill Crescent start choosing more active transport modes (cycling and walking to shops and local amenities) then the second garage might be converted to alternative uses, for example, a workshop, recreation or storage space, or an additional bedroom or guestroom.

As can be seen in the comparison of the alternative developments in Table 7.6, the hypothetical application of the proposed five principles for multivalent communal space at Pinehill Crescent has resulted in more efficient use of the land and an enhanced living environment for residents. Not only are there more dwellings in the clustered housing scheme, but also (unlike the conventional development) there are several generous communal spaces (indoors and outdoors) for residents to develop and enjoy. These communal spaces serve a variety of uses - gardens and orchards, playing fields, volley-ball courts, swimming pools and a range of community facilities - for a full range of age groups. It suggests a living environment which potentially could be rich and meaningful for the whole community. 


\section{CHAPTER 8 - Conclusion}

People and the environment both matter: one shapes the other. Shaping the environment for 'community' is best done collaboratively, with the community participating in the process of place-making. Communal space in medium density housing can enrich the life of a community by being multivalent. This thesis proposes principles that will make communal spaces multivalent.

All five principles (walkability, clustered housing, green open space, places for children and teenagers, and a sense of community) are necessary and interdependent for shaping mulitvalent communal space in medium density housing. The hypothetical application of these five principles at Pinehill Crescent resulted in more efficient use of land while also enhancing the living environment for residents, as can be seen by a comparison of the alternative developments in Figs. 7.6 \& 7.10 and Table 7.6. Not only are there more dwellings in the clustered housing scheme, there are also a wide variety of generous spaces (indoors and outdoors) available for residents to develop and enjoy.

It is considered these five principles for multivalent communal space are generic, and will achieve similar results in other medium density housing developments. The Case Study investigated the viewpoints of residents of only one development - the terrace housing at Pinehill Park, Upper Hutt. The five principles for multivalent communal space were applied hypothetically in Chapter 7 to just one site - the subdivision at Pinehill Crescent, Upper Hutt (Figs. 7.7 - 7.10). However, the principles are considered to be relevant to medium density housing generally. It was found that the application of these five principles made a significant positive difference to the efficiency of land use and to the quality of the living environment in the hypothetical re-development of the Pinehill Crescent subdivision. The five principles are applicable generally to other developments for the following reasons: 
1. Walkability - Pinehill Crescent is a typical subdivision. The hypothetical redevelopment in this chapter demonstrates how a subdivision in a typical location could be intensified (in this case from scratch) to make existing public transportation more viable by providing a higher population density to support it, take advantage of existing recreational amenities, and provide a pedestrian-oriented living environment more conducive to fostering a sense of community. Other localities could do the same;

2. Clustered housing - The 'concept cluster housing' in the re-development of Pinehill Crescent is 'borrowed' from 'best-practice' examples located throughout New Zealand (Snell's Beach, Gulf Harbour, Takapuna, Epsom, and Christchurch). The concept indicates variety and choice in the range of house types, style and layout which can be achieved in any locality in a way that is appropriate to the local context and local community aspirations;

3. Green open space - The open space made possible by the clustering of houses offers a variety of uses. At Pinehill Crescent, opportunities are indicated for a communal orchard, vegetable gardens, ornamental pond/water feature, café, a community building for local meetings and/or offices and/or some small retail ventures, youth facilities, outdoor playing fields, tennis courts, communal swimming pools, plus several outdoor spaces for recreation and relaxation. Similar opportunities could be created in any location;

4. Places for children and teenagers - the Pinehill Crescent concept responds to children's and teenagers' generic needs. It suggests a rich variety of opportunities that can be created for meeting these needs in a clustered housing environment with multivalent communal spaces. These include spaces for younger children (doorstep play, 'tot lots' etc.) and older children (open space for ball play, biking etc) all with appropriate levels of passive surveillance, and a variety of places for teenagers to hang out. These needs 
(C) 2012 Stephen Pattinson Chapter 8: Conclusion M. Arch Thesis

have been addressed using principles and patterns generally relevant to children and teenagers wherever they live;

5. Sense of community - the academic literature identifies the components of 'sense of community' to be neighbourhood identity, neighbourhood attachment, pedestrianism and social interaction. The conceptual Pinehill Crescent re-development shows how the layout of a residential environment can foster pedestrianism (by creating a more interesting environment to walk in) and social interaction (by providing a wide range of communal spaces that are meaningful to all age groups in the community). These physical layouts and uses of communal spaces are indicative of a wide variety of possible design solutions. This is the beauty of the bay-structure as Richard Toy notes:

"latent with .. great complexity ... the bay structure has a potential to accommodate an almost infinite variety of conditions and combinations of human belonging ..."

It is up to individual communities to create their own 'best-fit' design solutions. The five principles for multivalent communal space, and their application at Pinehill Crescent (Fig. 7.10), were developed by corroborating the viewpoints of practitioners, researchers and residents. The result is indicative of a potential which any community could realise. Each community will imbue its living environment with its own special 'place identity', and its residents will develop their own special 'place attachment'.

The academic literature also identifies 'local autonomy' as a vital component of 'sense of community'. The residents of the Pinehill Park terrace housing had not been asked before how they would like 'their' park to be - it had been done for them. They demonstrated in their responses that, collectively, they are well able to direct the shaping of their own environment to suit their own needs and aspirations. Their example may inspire other communities to say - 'yes, we also can do that'. 
M. Arch Thesis 


\section{Bibliography}

A Place to Stay: A Film about Salisbury Garden Court. By Marie Russell, with Shane Loader. DVD. Community Media Trust, 2009.

Addison: The Gardens Stage 3. Promotional Brochure by McConnell Property. Obtained from on-site sales office, 18 August 2009.

Alexander, Christopher. "A City is Not a Tree." Human Identity in the Urban Environment. Eds Gwen Bell and Jacqueline Tyrwhitt. Hammondsworth; Penguin Books, 1972. 401-428.

Alexander, Christopher, et al. A Pattern Language. New York: Oxford UP, 1977.

Alexander, Ernest. "Density Measures: A Review and Analysis." Journal of Architectural and Planning Research 10.3 (1993): 181-202.

Altman, Irwin. The Environment and Social Behaviour: Privacy, Personal Space, Territory, Crowding. Monterey: Brooks Cole, 1975.

AMCORD: Australian Model Code for Residential Development. Canberra: Commonwealth of Australia, 1997.

Auckland Council. Good Solutions Guide for Apartments. Auckland: Auckland Council, (no date).

---. Good Solutions Guide for Medium Density Housing. Auckland: Auckland Council, 2007.

---. Good Solutions Guide for Mixed Use Town Centres. Auckland: Auckland Council, 2005.

---. Monitor Auckland: Our Community, Households and Families, Household Size. 14 October 2009. 06 Nov 2010. <http: monitorauckland.arc.govt.nz/ourcommunity/households-and-families/household-size.cfm>.

---. "Practice Note: Minimum Apartment Size”. BLD-125-PN. 2010.

---. "Section 16: Residential." North Shore City District Plan June 2002 (Updated Jan. 2011) http://www.northshorecity.govt.nz/YourCouncil/PublicDocuments/DistrictPlan/D ocuments/section16-residential.pdf Retrieved 28 February 2011

---. "Section 6: Managing Growth." North Shore City District Plan June 2002 (Updated June 2009) 
http://www.northshorecity.govt.nz/YourCouncil/PublicDocuments/DistrictPlan/D ocuments/section6-managing-growth.pdf Retrieved 28 February 2011

---. Regional Growth Strategy. http://www.arc.govt.nz/auckland/aucklandsgrowth/residential-intensification.cfm Retrieved 20 Nov. 2009.

Bamford, Gregory. Understanding Housing Density. 2nd Australasian Housing Researchers' Conference, 20th - 22nd June 2007, U of Queensland, Brisbane, Australia. http://www.uq.edu.au/housingconference2007/docs/Bamford_2ndAHRC2 007.pdf Retrieved 02.8.2010

Bently, lan, et al. Responsive Environments: A manual for designers. Oxford: Architectural Press, 2007.

Berghauser Pont, Meta, and Per Haupt. "The Relation between Urban Form and Density." Journal of Urban Morphology 11.1 (2007) Viewpoints. <http://urbanmorphology.orgjournal/viewpoints0107.html>.

Biddulph, Mike. Introduction to Residential Layout. Oxford: Elsevier, 2007.

---. “Villages Don't Make a City" Journal of Urban Design 5.1 (2000): 65-82.

Bird, Clinton. Minimum Standards for Residential Apartments. Auckland: U. of Auckland, Nov. 2005.

Borsi, Katharina. "Drawing and Dispute: The Strategies of the Berlin Block." Intimate Metropolis. Eds. Di Palma, Vittoria, Diana Periton and Marina Lathouri. Abingdon, Oxon: Routledge, 2009.

Brand, Diane. "Non-Western Urbanism." Victoria U. of Wellington, Wellington, 25 May 2009.

Burchell, Robert et al. TCRP Report 74: The Costs of Sprawl 2000. Transit Cooperative Research Programme. Washington DC: National Academy Press, 2002.

Burton, Elizbeth, and Lynne Mitchel. Inclusive Urban Design: Streets for Life. Oxford: Architectural Press, 2006.

Calthorpe, Peter. The Next American Metropolis: Ecology, Community and the American Dream. New York: Princeton Architectural Press, 1993.

Campoli, Julie, and Alex MacLean. Visualizing Density. Cambridge, Massachusetts: Lincoln Institute of Land Policy, 2007. 
Carmona, Matthew, Tim Heath, Taner Oc and Steven Tiesdell. Public Places - Urban Spaces: The Dimensions of Urban Design. Oxford: Architectural Press. 2003

CCCB, Centre of Contemporary Culture of Barcelona. In Favour of Public Space: Ten Years of the European Prize for Urban Public Space. Barcelona: CCCB, 2010.

Chermayeff, Serge, and Christopher Alexander. Community and Privacy. New York: Anchor, 1965.

Churchman, Arza. "Disentangling the Concept of Density." Journal of Planning Literature 13.4 (1999): 389-411.

Colquhoun, Ian, and Peter Fauset. Housing Design in Practice. Harlow UK: Longman, $1991 a$.

---. Housing Design: An International Perspective. London: Batsford, 1991b.

Conzen, Michael. "The elusive common denominator in understanding urban form." Journal of Urban Morphology 14.1 (2010) 55-58

Cooper Marcus, Clare, and Wendy Sarkissian. Housing as If People Mattered: Site Design Guidelines for Medium-Density Family Housing. Berkeley: $U$ of California Press, 1986.

Cooper Marcus, Clare, and Carolyn Francis. People Places. Canada: John Wiley \& Sons, 1998.

Cotton, Peter. "Density Fact Sheet." Your Development (2008). 18.8.2010 <http: yourdevelopment.org/factsheet/pdf/id/58 >.

Crosbie, Michael. Multi-Family Housing: The Art of Sharing. Mulgrave: Images Publishing, 2003.

---. Living Together: Mulit-Family Housing Today. Mulgrave: Images Publishing, 2007.

Davis, Sam. The Architecture of Affordable Housing. Berkeley: University of California Press, 1995.

Diamond, Jack. "Residential Density and Housing Form." Journal of Aesthetic Education 29.3 (1976): 15-17.

Duany, Andres, Elizabeth Plater-Zyberk, and Jeff Speck. Suburban Nation: The Rise of Sprawl and the Decline of the American Dream. New York: North Point Press, 2000.

Essex County Council. The Essex Design Guide. Essex County Council, 2005. 
---. The Urban Place Supplement to the Essex Design Guide. Essex County Council. http://www.the-edi.co.uk/whatistheedi.php. Retrieved 3 Oct. 2010

Evans, David, and Paul Gruba. How to Write a Better Thesis. Melbourne: Melbourne University Press, 2009.

Evans, Paul. "Housing Layout and Density." Land Use and Built Form Studies: Working Paper No. 75. Cambridge, England: U. of Cambridge, 1973.

Faull, Richard. "The Marvels and Challenges of the Human Brain." Talking Heads Lecture Series. Radio New Zealand, 31 October 2010.

Field, Adrian. "Children's Changing Worlds: Do our cities work for children?" New Zealand Centre for Sustainable Cities. U. of Otago, Wellington. 16 June 2010.

Forsyth, Ann. Measuring Density: Working Definitions for Residential Density and Building Intensity. Design Center for American Urban Landscape, 2003.

Freeman, Claire. "Children's neighbourhoods: social centres to 'terra incognita'” Children's Geographies 8.2 (2010a): 157-176.

---."Children's Changing Worlds: Do our cities work for children?" New Zealand Centre for Sustainable Cities. U. of Otago, Wellington. 16 June 2010.

Frey, Hildebrand. "Compact, Decentralised or What? The Sustainable City Debate, from Designing the City: Towards a More Sustainable Urban Form 1999." The Urban Design Reader. Eds. Larice, Michael, and Elizabeth Macdonald. London: Routledge, 2007. 328-343

Galloway, Anne and Robin Skinner. "Research-led Design; Design-led Research". Colloquium on research design. Victoria U. of Wellington, Wellington. 19 October, 2010.

Gehl, Jan. The Interface between Public and Private Territories in Residential Areas. Melbourne: Melbourne University, 1977.

---. Livet Mellem Husene. (The Life Between The Houses: Outdoor Activities Outdoor Environment). Arkitektens Forlag. $2^{\text {nd }}$ Ed. Architectural Press, Copenhagen. 1980. (Held at School of Architecture Library, U. of Auckland)

---. Life between Buildings: Using Public Space. Copenhagen: Arkitektens Forlag, 1996.

Gleeson, Brendan, and Neil Sipe. Creating Child Friendly Cities: Reinstating kids in the city. London: Routledge, 2006. 
Goode, Justine. "Remembering a Real Mensch." Architecture New Zealand 5 (2007): 92.

Gray, John. "Suburban Intensification and Indigenous Settlement Patterns." Tāone Tupu Ora: Indigenous knowledge and sustainable urban design. Eds. Stuart, Keriata, and Michelle Thompson-Fawcett, for NZ Centre for Sustainable Cities at U. of Otago, Wellington. Wellington: Steele Roberts Aotearoa, 2010. $60-81$

Greater London Council. The Planning of a New Town. London: Greater London Council, 1965.

Groat, Linda, and David Wang. Architectural Research Methods. New York: John Wiley \& Sons, 2002.

Hall, Edward T. The Hidden Dimension. 1966. New York: Anchor, 1990.

Hall, Peter, and Colin Ward. Sociable Cities: The Legacy of Ebenezer Howard. New York: John Wiley \& Sons, 1998.

Hamdi, Nabeel. The Placemaker's Guide to Building Community. London: Earthscan, 2010.

Hermanuz, G., M. Gutman, and R. Plunz. Reweaving the Urban Fabric: Approaches to Infill Housing. New York State Council on the Arts. New York: Princeton Architectural Press, 1988.

Hester, Randolph. Neighbourhood Space. Stroudsberg, Pennsylvannia: Dowden, Hutchinson \& Ross, 1975.

Hillier, J., and E. Rooksby. Habitus: A Sense of Place. Ed. E. Rooksby and J Hillier. Hampshire: Ashgate, 2002.

Hinshaw, Mark. True Urbanism: Living In and Near the Center. Chicago: Planners Press, 2007.

Howard, E. Garden Cities of To-Morrow. 1902. London: Faber \& Faber, 1946.

Jacobs, Jane. The Death and Life of Great American Cities. 1961. New York: Random House Vintage Books, 1992.

James, J. R. "Residential Densities and Housing Layouts." Town and Country Planning. December 1967: 552-61.

James, P. et al. "Towards an integrated understanding of green space in the European built environment." Urban Forestry \& Urban Greening 8 (2009): 65-75. 
Janz, Wes, and Bob Beckley. "Multivalence, Context and Synthesis in Urban Design." JAE 36.3 (1983): 7-13.

Jencks, Charles. Modern Movements in Architecture. 2nd ed. Harmondsworth: Penguin, 1986.

Jenkins, Douglas Lloyd. At Home: A Century of New Zealand Design. Auckland: Random House, 2005.

Judd, Bruce. Designed for Urban Living: Recent Medium-Density Group Housing in Australia. Red Hill ACT: RAIA, 1993.

Judd, Bruce, and John Dean. Medium Density Housing in Australia. Red Hill ACT: RAIA, 1983.

Kazmierczak, A. and P. James. "The Role of Urban Green Spaces in Improving Social Inclusion." Proceedings in the 7th International Post-Graduate Conference in the Built and Human Environment. Salford. Ed. D. Amaratungas. 27-29 March 2007.

Kyttä, Marketta. "The Extent of Children's Independent Mobility and the number of actualized affordances as criteria for child-friendly environments." Journal of Environmental Psychology 24.2 (2004): 179-198.

Keller, Suzanne. The Urban Neighborhood: A Sociological Perspective. New York: Random House, 1968.

Kim, Joongsub. "Sense of Community in Neo-Traditional and Conventional Suburban Developments." Thesis, University of Michigan, 2001.

Lambourn, Barbara. “Children's Changing Worlds: Do our cities work for children?” New Zealand Centre for Sustainable Cities. U. of Otago. Wellington. 16 June 2010.

Lefaivre, Liane and Ingeborg de Roode, Eds. Aldo van Eyck: The Playgrounds and The City. Stedelijk Museum Amsterdam. Rotterdam: NAi Publishers, 2002.

Leinberger, Christopher. The Option of Urbanism: Investing in a New American Dream. Washington DC: Island, 2009.

Levitt, David. The Housing Design Handbook: A Guide to Good Practice. London: Routledge, 2010.

London, Geoffrey, and Simon Anderson. Take 7 - Housing Australia: How Architects Can Make a Difference. Manuka ACT: RAIA, 2008. 
Lozano, Eduardo. Community Design and the Culture of Cities: The Crossroad and the Wall. Cambridge: Cambridge University Press, 1990.

Lynch, Kevin. Good City Form. 1981. Cambridge: MIT Press, 1990.

Lynch, Kevin, and Gary Hack. Site Planning. Cambridge: MIT Press, 1984.

Madanipour, Ali. Public and Private Spaces of the City. New York: Routledge, 2003.

Martin, Leslie, and Lionel March. Urban Space and Structures. Cambridge: Cambridge University Press, 1972.

McCamant, Kathryn, and Charles Durret. Cohousing: A Contemporary Approach to Housing Ourselves. Berkeley: Ten Speed Press, 1994.

---. Creating Cohousing: Building Sustainable Communities. Gabriola Island, BC Canada: New Society, 2011.

McClean, Cameron. "Renewable Energy and Human Settlements." Unpublished staff paper - Building Economics; held at School of Architecture Library, U. of Auckland, New Zealand. c.1977

McIndoe, Graeme. "Modernity and Modernism - The Modernist City: Skyscrapers in the Park". SARC 351 - Urban Design Theory and Practice, Victoria U. of Wellington, Wellington, 12 March 2010.

McInnes, Gabrielle, Stephen J. Pattinson and Mark de Lisle. "After The Goldrush". Unpublished undegraduate Thesis on the viability of a pedestrian scale, densely populated, low energy settlement. For Bachelor of Architecture degree. School of Architecture, U. of Auckland. New Zealand. 1980.

McKay, Bill. "What Is a Developer?" Houses New Zealand Issue 05 September 2007

McKnight, John, and Peter Block. The Abundant Community: Awakening the Power of Families and Neighborhoods. San Francisco: Berrett-Koehler, 2010.

Mead, David, and Allan McGregor. "Regional Intensification: Intensive Housing Demand and Supply Issues". Report. Auckland: Hill Young Cooper Ltd, 2007.

Morgan, Paul. "Towards a Development Theory of Place Attachment." Journal of Environmental Psychology 30 (2010): 11-22.

Moudon, Anne. "Urban Morphology as an Emerging Interdisciplinary Field." Urban Morphology 1 (1997): 3-10. 
Mumford, Lewis. "The Garden City Idea and Modern Planning from Introduction to Garden Cities of To Morrow 1946." The Urban Design Reader. Eds. Larice, Michael and Elizabeth Macdonald. London: Routledge, 2007. 43-53.

Murray, Shane, Diego Ramirez-Lovering and Simon Whibley. Eds. reHousing: 24 housing projects. Melbourne: RMIT Publishing, 2008.

Nelson, Arthur C. "Household Size Could Reduce Demand for Housing and Slow Recovery." 7 May 2010. Impact Lab. http://www.impactlab.net/2010/05/07/increase-in-household-size-could-reducedemand-fo-housing-and-slow-recovery/ Retrieved 06 Nov.2010

Newman, Oscar. Defensible Space: Crime Prevention through Urban Design. New York: MACMILLAN, 1972.

New Zealand Ministry for the Environment. Urban Design Case Studies. NZ Urban Design Protocol. Wellington: Ministry for the Environment, 2005.

---. Urban Design Case Studies: Local Government. NZ Urban Design Protocol. Wellington: Ministry for the Environment, 2008.

---. Medium Density Housing. DRAFT Case Studies, Prepared by Boffa Miskell Ltd. Wellington: Ministry for the Environment, (publishing pending 2011).

New Zealand Ministry of Health. HIA (Health Impact Assessment) Report commissioned by Manukau the Healthy City. "Manukau Built Form and Spatial Structure Plan" by Synergia Ltd and Hapai te Hauora Tapui Ltd. 2008. http://www.moh.govt.nz/moh.nsf/indexmh/hiasupportunitcompleted\#manukauhia Retrieved 29.11.10

New Zealand Ministry of Justice. "National Guidelines for Crime Prevention through Environmental Design in New Zealand - Part 1: Seven Qualities of Safer Places". NZ Urban Design Protocol. Wellington: Ministry of Justice, 2005.

---. "National Guidelines for Crime Prevention through Environmental Design in New Zealand - Part 2: Implementation Guide". NZ Urban Design Protocol. Wellington: Ministry of Justice, 2005.

Norberg-Schultz, Christian. Existence, Space and Architecture. New York: Praeger, 1971.

Office of National Statistics. "Focus on People and Migration 2005". London, 2005. Office of National Statistics (UK). 29 Dec 2010. <http://www.statistics.gov.uk/statbase Product.asp?vlnk=12899>.

---. "Social Trends No. 37." 2007. 
Oldenburg, Ray. The Great Good Place: Cafes, Coffee Shops, Community Centres, Beauty Parlours, General Stores, Bars, Hangouts, and How They Get You Through the Day. New York: Paragon House, 1989.

Park, Robert E. "The City: Suggestions for the Investigation of Human Behaviour in the City Environment." The American Journal of Sociology 20.5 (1915): 580.

Perry, Clarence. "The Neighbourhood Unit from Regional Plan of New York and its Environs 1929." The Urban Design Reader. Eds. Larice, Michael, and Elizabeth Macdonald. London: Routledge, 2007. 54-65.

Polyzoides, S., R. Sherwood, and J. Tice. Courtyard Housing in Los Angeles. New York: Princeton Architectural Press, 1992.

Project for Public Spaces, Inc. How to Turn a Place Around: A Handbook for Creating Successful Public Spaces. New York: Project for Public Spaces, 2008.

Putnam, Robert. Bowling Alone: The Collapse and Revival of American Community. New York: Simon \& Schuster, 2000.

Rapoport, Amos. "Toward a Redefinition of Density." Environment and Behaviour 7.2 (1975): 133-58.

Sax, John, Executive Director, Southpark Corporation. Personal interview. 20 Oct. 2009.

Scoffham, Ernie, and Brenda Vale. "How Compact Is Sustainable - How Sustainable Is Compact?" The Compact City - a Sustainable Urban Form? Ed. M Jenks. London: Spon, 1996.

Sommer, Robert. Personal Space: The Behavioural Basis of Design. 1969. Bristol: Bosko, 2007.

Southworth, Michael, and Eran Ben-Joseph. Streets and the Shaping of Towns and Cities. Washington D.C.: Island Press, 2003.

Statistics New Zealand. "National Family And Household Projections: 2006 (base) 2031 update." July 2010.

http://www.stats.govt.nz/browse_for_stats/population/estimates_and_pr ojections/NationalFamilyAndHouseholdProjections_HOTP20062031update/Commentary.aspx Retrieved 01.11.2010

---. 2006 Census: Territorial Authority by Sex and Age Group for the Census - Usually Resident Population Count. 2010. 
Swanson, Kate. "'For every border, there is also a bridge': overturning borders in young Aboriginal peoples' lives." Children's Geographies 8.4(2010):429-436

Thorns, David. The Quest for Community: Social Aspects of Residential Growth. London: George Allen \& Unwin, 1976.

Tibbalds, Francis. Making People-Friendly Towns: Improving the public environment in towns and cities. London: Spon Press, 2001.

Tindall Foundation Annual Report, 2010 (2011 Calendar).

Toy, Richard. "Auckland: Water-City of the South Pacific" Auckland at Full Stretch Issues of the Seventies. Eds. Graham Bush and Claudia Scott. Auckland City Council and University of Auckland Board of Urban Studies, 1977

Trancik, Roger. "What is Lost Space?" (1986). The Urban Design Reader. Eds. Larice, Michael and Elizabeth Macdonald. London: Routledge, 2007. 63-69.

Turner, D., J. Hewitt, C. Wagner, B. Su, and K. Davies. "Best Practice in Medium Density Housing Design". Report for Housing New Zealand Corporation. Auckland: UNITEC, 2004.

Turner, David, and Bin Su. "A Classification Methodology Based on Site Layout Analysis for Medium Density Housing". Report. Auckland: UNITEC, 2005.

Untermann, R, and R. Small. Site Planning for Cluster Housing. New York: Van Nostrand, 1977.

Upper Hutt City Council. District Plan 2004. Section 18 Residential Zone Rules. Amended May 2010. http://www.upperhuttcity.com/store/doc/DP-18Residential-Zone-Rules.pdf Retrieved 28 February 2011

US Census Bureau. "No. Hs-12. Households by Type and Size: 1900 to 2002." 2003.

Vale, Robert, and Brenda Vale. Time to Eat the Dog: The Real Guide to Sustainable Living. London: Thames and Hudson, 2009.

Walljasper, Jay. The Great Neighborhood Book: A Do-it-Yourself Guide to Placemaking. A Project for Public Spaces Book. Gabriola Island BC: New Society Publishers, 2007.

Walters, David. Designing Community: Charrettes, Masterplans and Form-based Codes. Oxform: Architectural Press, 2007.

Webster, Helena. Bourdieu for Architects. London: Routledge, 2011. 
Wellington City Council. District Plan. Chapter 4 Residential Areas: Introduction, Objectives and Policies. Operative 27/07/00. Last Amended 09 March 2010 http://www.wellington.govt.nz/plans/district/volume1/pdfs/v1chap04.pdf Retrieved 28 February 2011

---. Residential Design Guide. Operative 27/07/00. Last Amended 10 July 2009. http://www.wellington.govt.nz/plans/district/volume2/pdfs/v2resdesguide.pdf Retrieved 28 February 2011

Witten, Karen. "Intensification, Housing Affordability and Families: learning from the Auckland CBD." Growth Misconduct? Can we do better on urban intensification? NZ Centre for Sustainable Cities and U. of Otago - The $14^{\text {th }}$ Public Health Summer School. Wellington. 16 February 2010.

Whyte, William. The Social Life of Small Urban Spaces. New York: Project for Public Spaces, 2004.

Wood, Elizabeth. "Housing Design: A Social Theory." Human Identity In the Urban Environment. Eds Bell, Gwen, Tyrwhitt, Jacqueline. Hammondsworth: Penguin Books, 1972. 327-351.

Woodward, Alistair. "Put me back on my bike." Seminar, New Zealand Centre for Sustainable Cities. U. of Otago, Wellington. 26 May 2010.

Zacharias, John, and Arthur Stamps. "Perceived Building Density as a Function of Layout." Perceptual and Motor Skills 98 (2004): 777-784

Zhou, Jingman. Urban Housing Forms. Oxford: Elsevier, 2005. 


\section{Appendices}

Appendix 1: Floor space and household size

(12 pages)

Appendix 2: Government Press Release - Pinehill Crescent

(1 page)

Appendix 3: Template of observation sheet

(1 page)

Appendix 4: Observations of Pinehill Park

(14 pages)

Appendix 5.1: Survey / Interview Forms

(5 pages)

Appendix 5.2: Analysis - Survey / Interview Responses

(23 pages)

Appendix 6: Ethics Approval Information Sheet \& Consent Form

(2 pages)

Appendix 7.1: List of Sites Visited for thesis research

(14 pages)

Appendix 7.2: Field Notes - Melbourne

(41 pages)

Appendix 7.3: Preliminary Investigations

(47 pages)

Appendix 8: Analytical Framework (Wellington schemes) 
Appendix 1: Floor space and household size 


\section{APPENDIX 1: Floor Space and Household Size}

A significant driver in the demand for housing (and hence urban space) is the falling average household size. This appendix explains the method of data collection and analysis carried out by the writer to explore the topic of floor space per person in relation to household size. The findings (see Chapter 1 ) are summarised here:

The minimum floor space required per individual in a one- or two-person dwelling is more than the minimum floor space required to house an individual in a five- or six-person dwelling - two or three times more, in fact ... With household sizes falling, this effect, multiplied several thousand times in the population of a city, results in a dramatic increase in the built floor space required to house a population, without any increase in population size.

This has nothing to do with the trend towards bigger houses, although that trend is a related issue exacerbating the underlying issue of falling household size being studied here. Evans notes that net floor space per dwelling corresponds in a linear function to the number of bedspaces per dwelling. Based on UK Parker Morris standards for minimum dwelling sizes, Evans finds that one bedspace requires a theoretical minimum floorspace of $34.0 \mathrm{~m}^{2}$; two bedspaces requires $47.5 \mathrm{~m}^{2}$; three bedspaces, $61.0 \mathrm{~m}^{2}$; four bedspaces, $74.5 \mathrm{~m}^{2}$; five bedspaces, $88.0 \mathrm{~m}^{2}$; and six bedspaces require a theoretical minimum floorspace of $101.5 \mathrm{~m}^{2}(14,15)$. Evans $(16)$ comments: "[as] bedspaces per dwelling increase the floorspace per person falls ...".

Based on these minimum dwelling sizes, for a given population (Evans uses the example of a density of 400 persons per hectare) a certain total minimum area of floorspace is required to house this population in six-person dwellings (Evans provides the figure of $6,766 \mathrm{sq} . \mathrm{m} / \mathrm{ha}$ ); to house the same population in "two bedspace dwellings the amount of floor space rises to 9,500 sq.m/ha; in the case of one bedspace dwellings the ... floorspace ... is 13,600 sq.m/ha" (19), i.e. double the floorspace requirement for housing this population in six-person dwellings. 
Evans identifies five major land-uses in a housing development - "buildings, roads, garages and parking spaces, private open space, and public open space" (21) - and notes the decline of both private and public open space with increases in building floorspace and carspace (22-27). Having described how minimum floorspace increases (without any change in population size) as bedspaces per dwelling decrease, Evans notes that dwelling size and bedspaces per dwelling, and their effect on open space, need to be considered in housing design $(27,28)$.

For a clearer understanding of the impact of household size on open space, the writer analysed six medium density (attached) house plans ranging from one- to sixperson (i.e. one- to six-bedspace) dwellings. The six house plans were selected by the writer from Colquhoun \& Fauset (1991a) to correlate approximately with Parker Morris standards of minimum net dwelling sizes (based on bedspaces per dwelling), i.e. a one-person (bedspace) house-plan of $33 \mathrm{~m}^{2}$ (341); a two-person house-plan of $46 \mathrm{~m}^{2}$ (289); a three-person house-plan of $58 \mathrm{~m}^{2}$ (272); a four-person house-plan of $76 \mathrm{~m}^{2}$ (273); a five-person (or compact six-person) house-plan of $88 \mathrm{~m}^{2}$ (274); and a six-person (bedspace) house-plan of $95 \mathrm{~m}^{2}$ (275, modified).

The Parker Morris standards were used because there is national standard for minimum floor space for New Zealand housing. The New Zealand Building Code does not regulate minimum areas for residential floor space (other than for 'Old Peoples Homes' - see NZBC G5 Interior Environment). In the United Kingdom, Parker Morris Standards recommended in 1961 are still referred to as a guide for minimum floor space in dwellings (for example, Essex Design Guide, 192).

Some New Zealand local authorities have determined their own minimum floor space standards for residential apartments. For example, Appendix 12 in Auckland Council's District Plan sets minimum space standards for apartments based on recommendations by Clinton Bird (2005). As explained below, these are similar to 
the Parker Morris standards. Auckland adopted minimum floor space standards because of public furore over small apartment sizes, many being around $18 \mathrm{~m}^{2}$ total area (NZ Herald 26.5.2005), some as small as $10 \mathrm{~m}^{2}$ (Auckland Council 2010).

The Parker Morris Report (1961) titled "Homes for Today and Tomorrow" concluded that "internal space standards should be related to the number of occupants of a dwelling, rather than a minimum room size which had been the previous criterion" in the 1950's in the UK. The minimum space requirements set by Parker Morris became mandatory for public housing in the UK in 1969 but were never imposed on private sector housing (Colquhoun \& Fauset, 1991a: 290, 291).

\begin{tabular}{|l|c|c|c|c|c|c|}
\hline & \multicolumn{6}{|c|}{ No. of people (i.e. bedspaces) per dwelling } \\
\hline & 1 & 2 & 3 & 4 & 5 & 6 \\
\hline $\begin{array}{l}\text { Flats } \\
\left(\mathrm{m}^{2} \text { net }\right)\end{array}$ & 30 & 44.5 & 57 & 70 & 79 & 86.5 \\
\hline $\begin{array}{l}\text { Storage } \\
\left(\mathrm{m}^{2} \text { net }\right)\end{array}$ & 2.5 & 3 & 3 & 3.5 & 3.5 & 3.5 \\
\hline
\end{tabular}

Table A1.1 Parker Morris minimum space standards for flats (Colquhoun \& Fausett, 1991a: 290)

The District Plan for Auckland Central Area Appendix 12 “Minimum Residential Apartment Standards" sets minimum gross floor areas of $35 \mathrm{~m}^{2}$ for studio apartments, $45 \mathrm{~m}^{2}$ for one-bedroom, $70 \mathrm{~m}^{2}$ for two-bedroom and $90 \mathrm{~m}^{2}$ for threebedroom apartments, as shown in Table A1.2:

\begin{tabular}{|c|c|c|c|c|c|c|c|c|c|c|c|c|}
\hline & $\underline{\text { Living }}$ & $\frac{\underline{\text { Kitchen/ }}}{\underline{\text { Eat }}}$ & $\frac{\text { Bedrm }}{\underline{1}}$ & $\frac{\text { Bedrm }}{\underline{2}}$ & $\frac{\text { Bedrm }}{\underline{3}}$ & $\frac{\text { Bathrm }}{\underline{1}}$ & $\frac{\text { Bathrm }}{\underline{2}}$ & $\underline{\text { Laundry }}$ & $\underline{\text { WR+Entry }}$ & Balcony & TOTAL & ROUNDED \\
\hline$\underline{\text { Studio }}$ & $\underline{11.00}$ & $\underline{5.10}$ & $\underline{9.00}$ & $\underline{0.00}$ & $\underline{0.00}$ & $\underline{3.00}$ & $\underline{0.00}$ & $\underline{0.84}$ & $\underline{0.72+0.36}$ & $\underline{5.00}$ & $\underline{35.02}$ & $35.00 \mathrm{~m}^{2}$ \\
\hline 1 bed & $\underline{15.00}$ & $\underline{10.80}$ & $\underline{9.00}$ & $\underline{0.00}$ & $\underline{0.00}$ & $\underline{3.00}$ & $\underline{0.00}$ & $\underline{0.84}$ & $\underline{1.08+0.36}$ & $\underline{5.00}$ & $\underline{45.08}$ & $\underline{45.00 \mathrm{~m}^{2}}$ \\
\hline 2 bed 11 & $\underline{24.00}$ & $\underline{13.20}$ & $\underline{9.00}$ & $\underline{9.00}$ & $\underline{0.00}$ & $\underline{3.00}$ & $\underline{0.00}$ & $\underline{1.26}$ & $\underline{2.16+0.36}$ & $\underline{8.00}$ & $\underline{69.98}$ & $70.00 \mathrm{~m}^{2}-$ \\
\hline $3+$ bed $^{2}$ & $\underline{28.00}$ & $\underline{16.20}$ & $\underline{9.00}$ & $\underline{9.00}$ & $\underline{9.00}$ & $\underline{3.00}$ & $\underline{3.00}$ & $\underline{1.26}$ & $3.24+0.36$ & $\underline{8.00}$ & $\underline{90.06}$ & $90.00 \mathrm{~m}^{2}$ \\
\hline
\end{tabular}

Table A1.2 Residential Apartment Standards (Auckland Council District Plan: Central Area Appendix 12)

It will be seen that the Auckland Central apartment space standards are similar to the Parker Morris standards. For example, an Auckland 3 bedroom apartment is 
required to have a minimum gross area of $90 \mathrm{~m}^{2}$. Assuming two bedspaces per bedroom, this apartment could accommodate 6 people in $90 \mathrm{~m}^{2}$ gross area. This compares with the Parker Morris minimum standard of $86.5 \mathrm{~m}^{2}$ net area for 6 bedspaces. Similarly, a two-bedroom Auckland apartment with a minimum gross area of $70 \mathrm{~m}^{2}$, assuming 2 bedspaces per bedroom, compares with the Parker Morris minimum $70 \mathrm{~m}^{2}$ net for a 4-person flat; a one-bedroom Auckland apartment with a minimum gross area of $45 \mathrm{~m}^{2}$, assuming two bedspaces, compares with the Parker Morris minimum area $44.5 \mathrm{~m}^{2}$ net for a 2-person flat; and an Auckland studio apartment of $35 \mathrm{~m}^{2}$ minimum gross area compares with a Parker Morris standard one-person flat of $30 \mathrm{~m}^{2}$ minimum net area.

Colquhoun \& Fauset (1991a) comment on the importance of the interior layout of individual dwellings to the overall layout of any residential area:

"Although the layout of a [multi-unit] residential area is concerned with the integration of the dwellings with the landscape, the roads, and ... other factors ... [t] he occupants of each dwelling make up the basic social unit and it is just as important to obtain the right kind of internal environment for the life of the occupants, be they single people or a large family. A housing scheme cannot be successful unless it embraces the design of the individual dwellings with their spatial grouping on the site." (270)

Therefore, some consideration is being given here to the planning of individual dwellings. The purpose is to explore the impact on open space of acceptable minimum floor space requirements for a range of household sizes, from a singleperson to, say, a six-person dwelling. This will give an indication of the effect on open space of falling average household sizes, quite apart from the trend toward bigger houses or any increase in population size. 


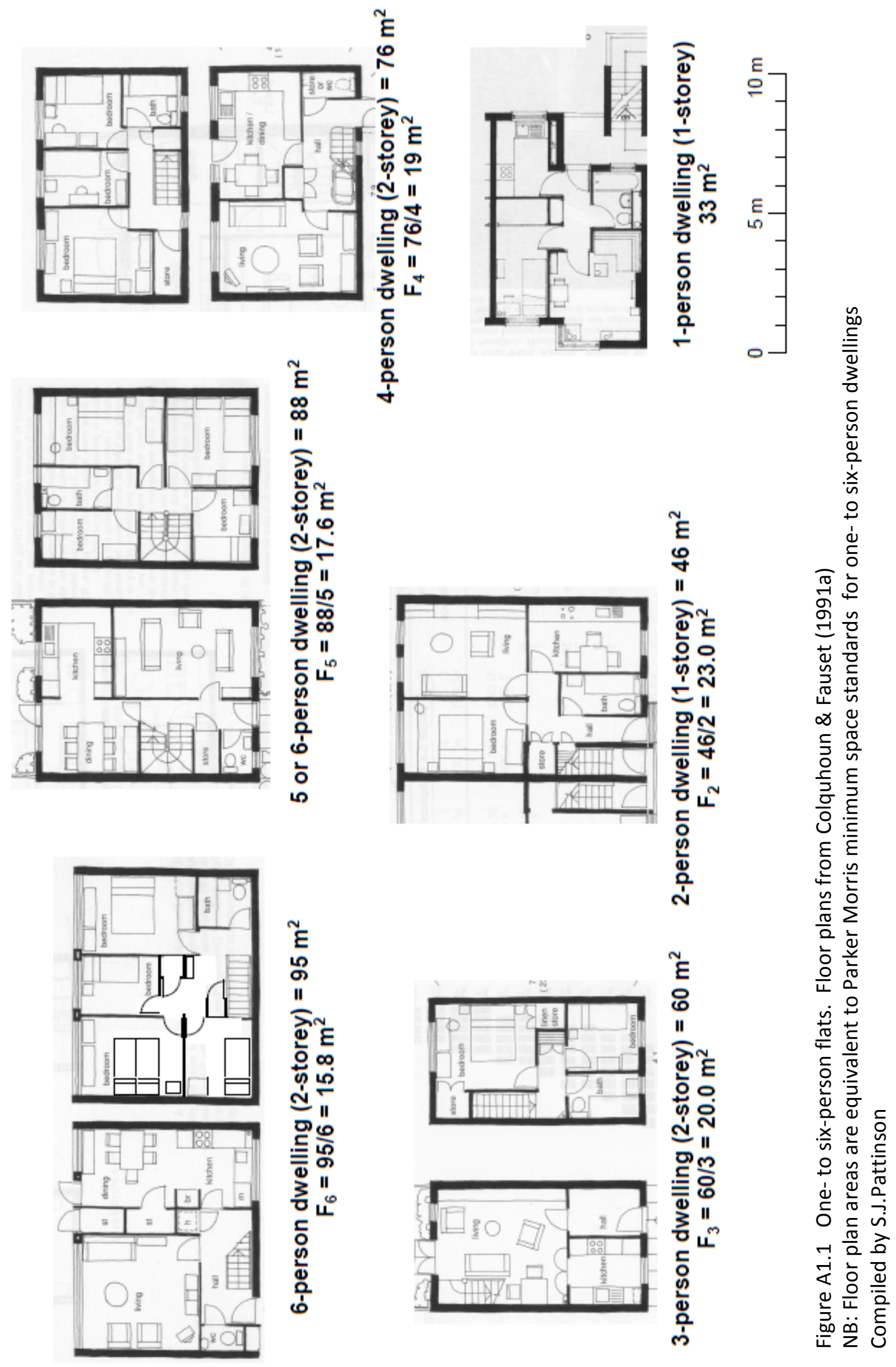



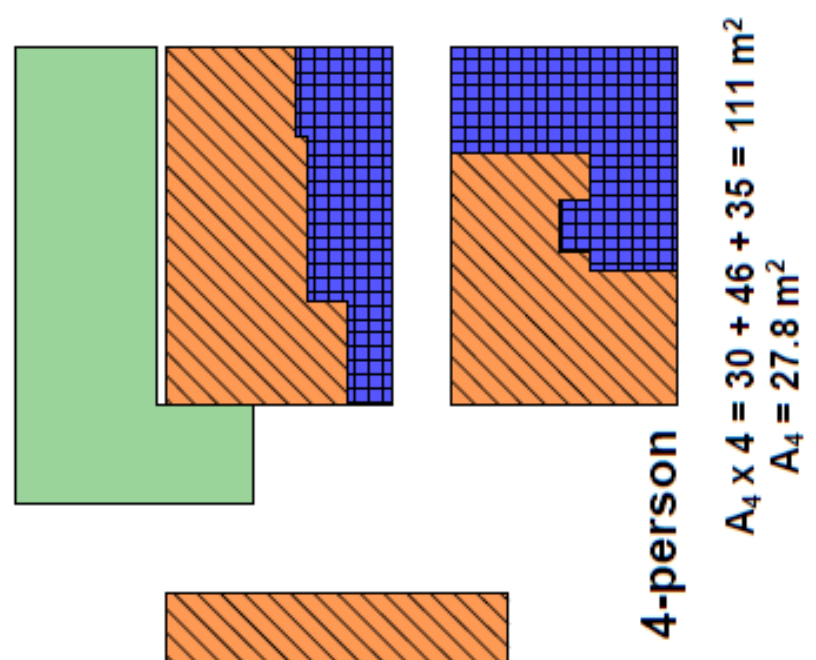
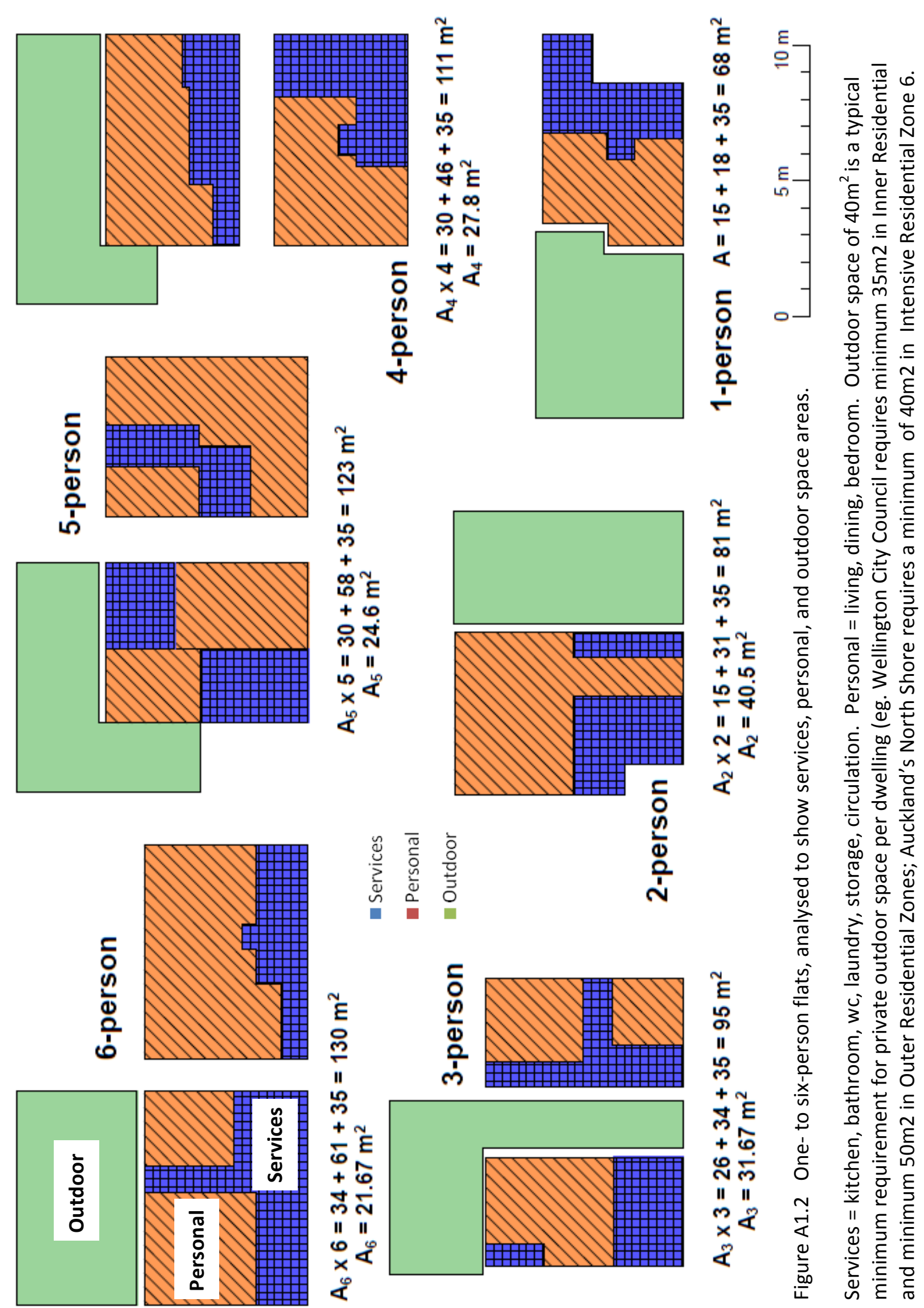


\section{Dwelling size ( $\mathrm{m} 2)$ based on no. of bedspaces}

(excludes exterior and inter-tenancy walls, but includes interior partitions)

\begin{tabular}{lrrrrrrr} 
& 1 & 2 & 3 & 4 & 5 & \multicolumn{1}{c}{} \\
Services* & 15 & 15 & 26 & 30 & 30 & 34 \\
Personal* & 18 & 31 & 34 & 46 & 58 & 61 \\
Outdoor** & 35 & 35 & 35 & 35 & 35 & 35 \\
& & & & & & \\
Tot.(Serv.+Pers.) & 33 & 46 & 60 & 76 & 88 & 95 \\
Tot.+outdoors & 68 & 81 & 95 & 111 & 123 & 130
\end{tabular}

Services = kitchen, bathroom, laundry, storage, circulation

Personal = living, dining, bedroom

* based on dwelling floor plans equivalent to Parker Morris minimum space standards (dwelling floor plans selected from Colquhoun \& Fauset, 1991a:

$341,289,272,273,274,275)$

** minimum outdoor space based on Wellington rules for Inner Residential Zone

Table A1.3 Analysis of dwellings equivalent to Parker Morris minimum standards for one- to sixperson dwellings (based on the number of bedspaces per dwelling)

From Table A1.3, the floor space per per son for each size dwelling can be derived as shown in Table A1.4 below:

\section{Floorspace per person (m2)}

\begin{tabular}{lrrrrrr} 
& \multicolumn{1}{c}{1} & \multicolumn{1}{c}{2} & \multicolumn{1}{c}{3} & \multicolumn{1}{c}{4} & \multicolumn{1}{c}{5} \\
Services & 15.0 & 7.5 & 8.7 & 7.5 & 6.0 & 5.7 \\
Personal & 18.0 & 15.5 & 11.3 & 11.5 & 11.6 & 10.2 \\
Outdoor & 35.0 & 17.5 & 11.7 & 8.8 & 7.0 & 5.8 \\
& & & & & & \\
Tot.(Serv.+Pers.) & 33.0 & 23.0 & 20.0 & 19.0 & 17.6 & 15.8 \\
Tot.+outdoors & 68.0 & 40.5 & 31.7 & 27.8 & 24.6 & 21.7
\end{tabular}

Table A1.4 Floor space per person for one- to six-person dwellings (based on dwelling sizes equivalent to Parker Morris minimum space standards) 
The trend toward smaller household size gives the impression that personal living space is getting smaller: the reality is that individual space is actually getting larger. The bar graphs below show that as household size reduces from six to one person per dwelling, the dwelling size decreases but the amount of space required to house each individual increases. An individual in a six-member household needs $20 \mathrm{~m}^{2}$ of space: to live alone requires $40 \mathrm{~m}^{2}\left(80 \mathrm{~m}^{2}\right.$ if allowing for minimum outdoor space).

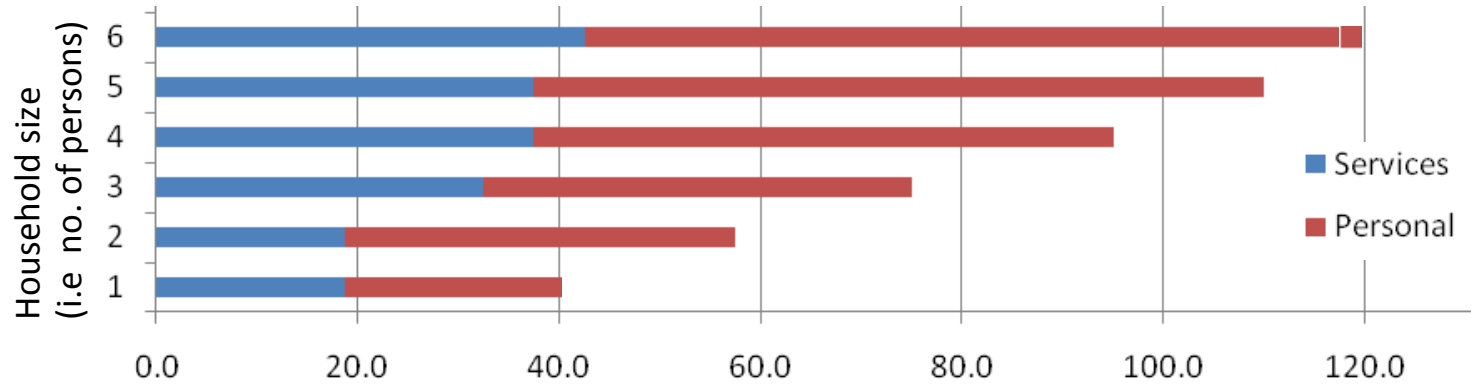

Figure A1.3 Dwelling area reducing with household size (S.J.Pattinson) Dwelling size $\left(\mathrm{m}^{2}\right)$

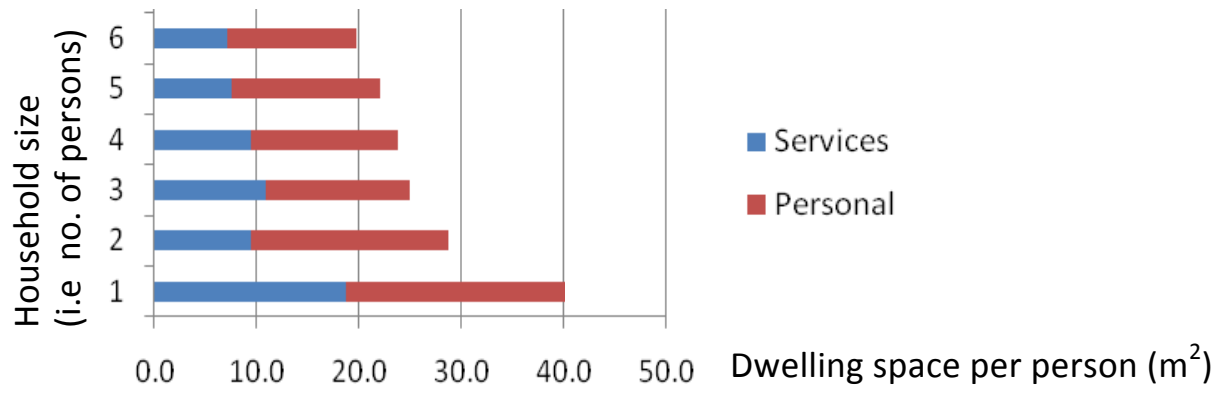

Figure A1.4 Individual space increasing with reducing household size

S.J.Pattinson

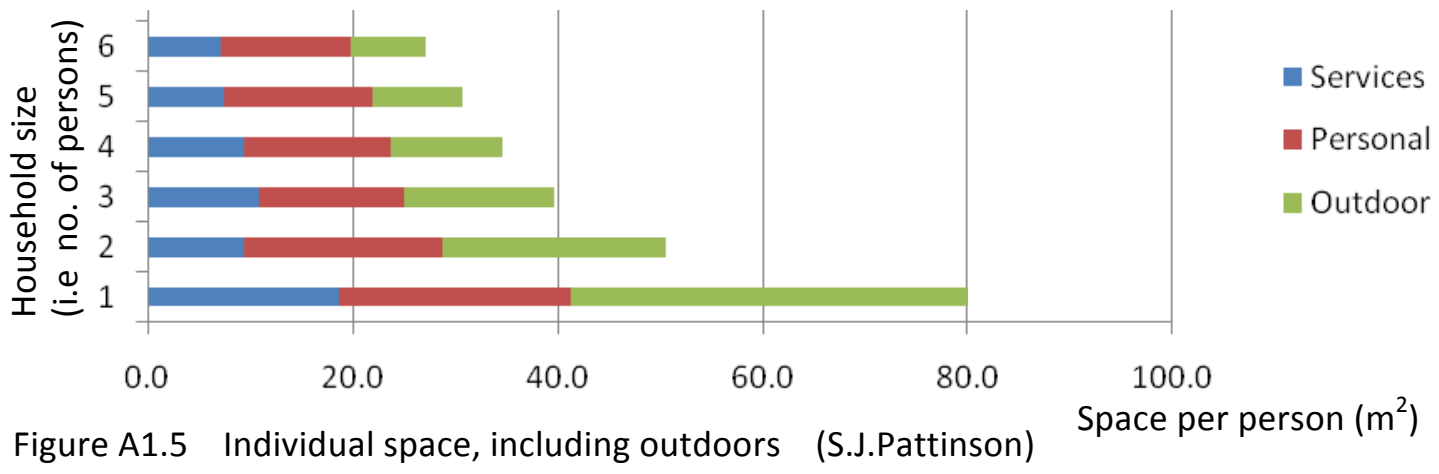

Services $=$ kitchen, bathroom, wc, laundry, storage, circulation. Personal = living, dining, bedroom 

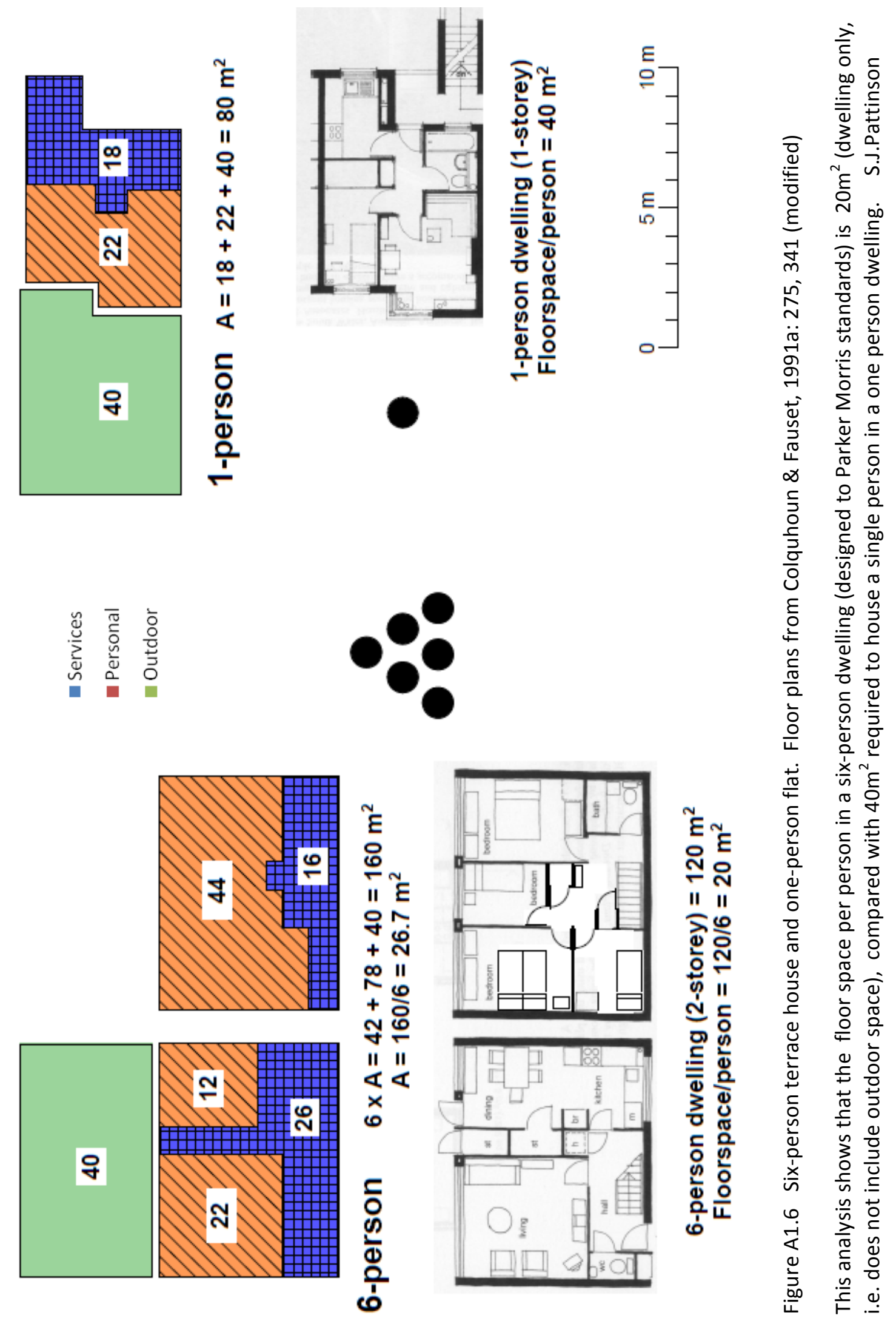


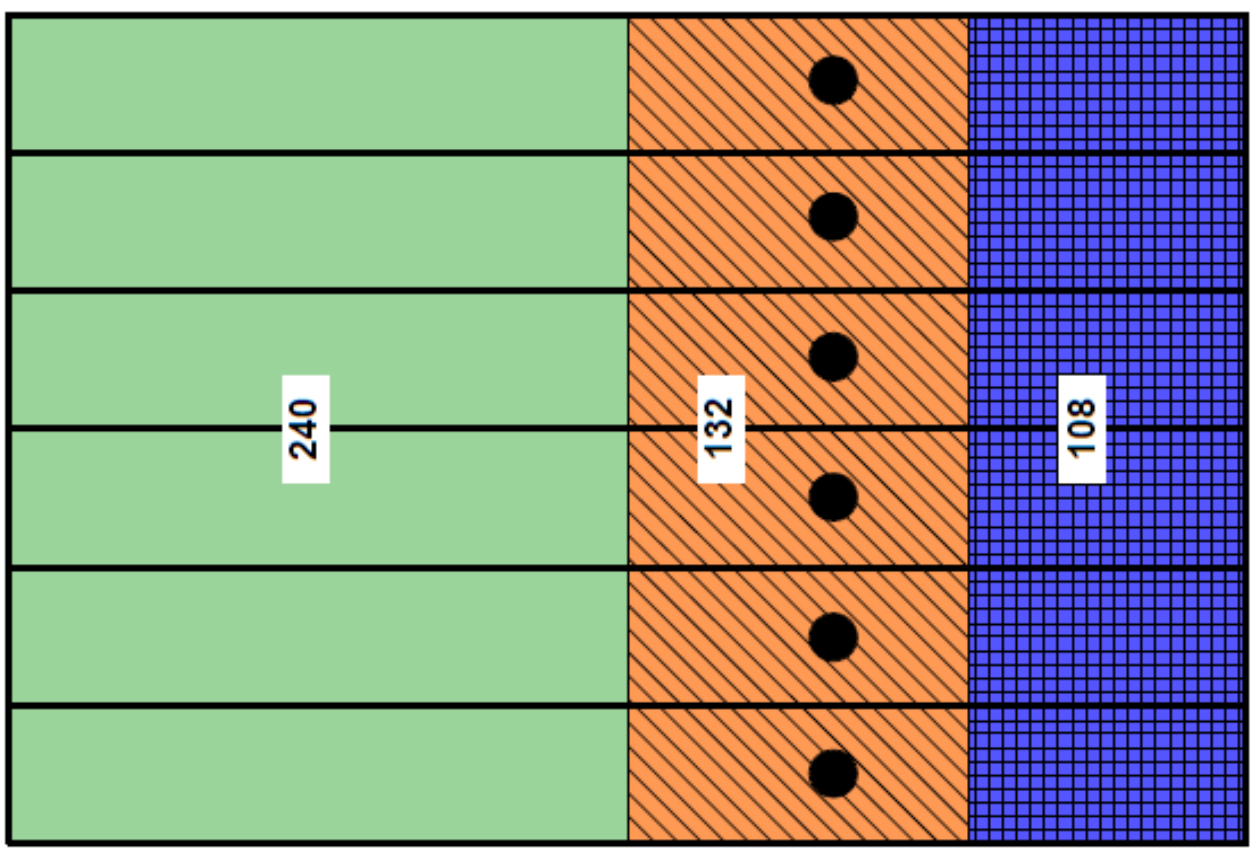

겅

훈 के

ज号 vi

先

ㅎํㄴ

은

ธิ

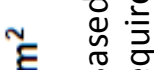

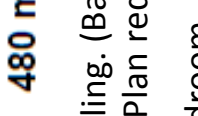

奉㐫

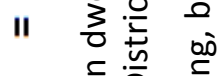

-

$\times \quad \frac{2}{2}$

$\times \quad$ 辛高

$\infty \quad \stackrel{0}{0} \frac{\bar{c}}{0} \frac{\pi}{11}$

$\infty \quad \frac{1}{2} \frac{11}{\frac{\pi}{2}}$

吾

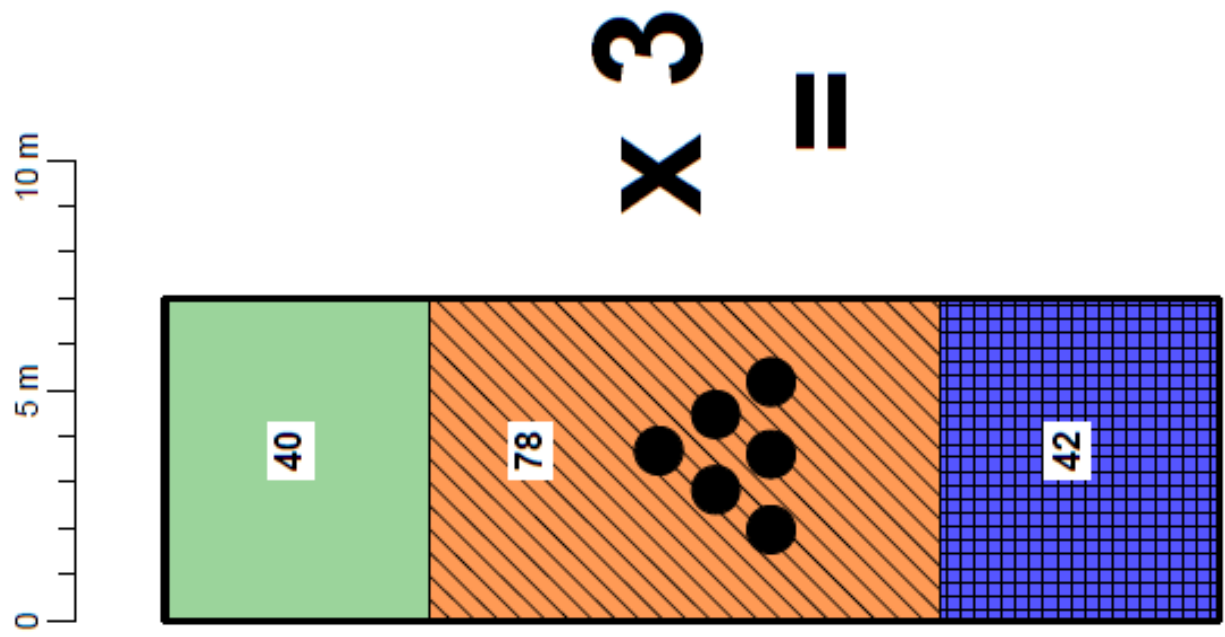

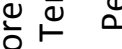

है

$\times \frac{2}{\pi}$

$m$.

品 齐

들

㐫

ㄷํㅇำ

ะ

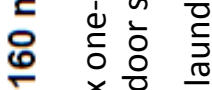

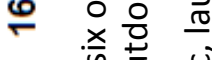

产苛

을 हो

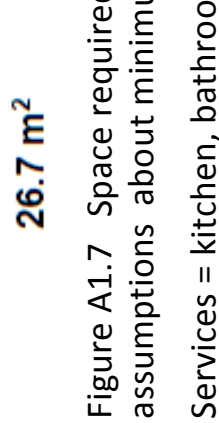


Through the process of individualism, households are getting smaller. However, the area of individual space is significantly increasing, without taking into account the trend towards bigger houses (the dwelling sizes used in the above analyses are considered to be acceptable minimum sizes) and without accounting for population increase. Figures A1.1 to A1.7 and Tables A1.1 to A1.4 show how changing one sixperson household to six one-person households doubles the minimum building floor space and triples the site space required per individual.

Meanwhile, the available site space per dwelling is significantly decreasing, with some Council's encouraging amalgamation of adjacent $1000 \mathrm{~m}^{2}$ residential sites into one larger site allowing for Sydney-style terrace housing accessed by rear lanes to dwellings on $137 \mathrm{~m} 2$ sites, or thereabouts (personal interview, Matt Riley, Senior Urban Designer, Auckland Council, 17 Aug. 2009). The following figures illustrate this trend:
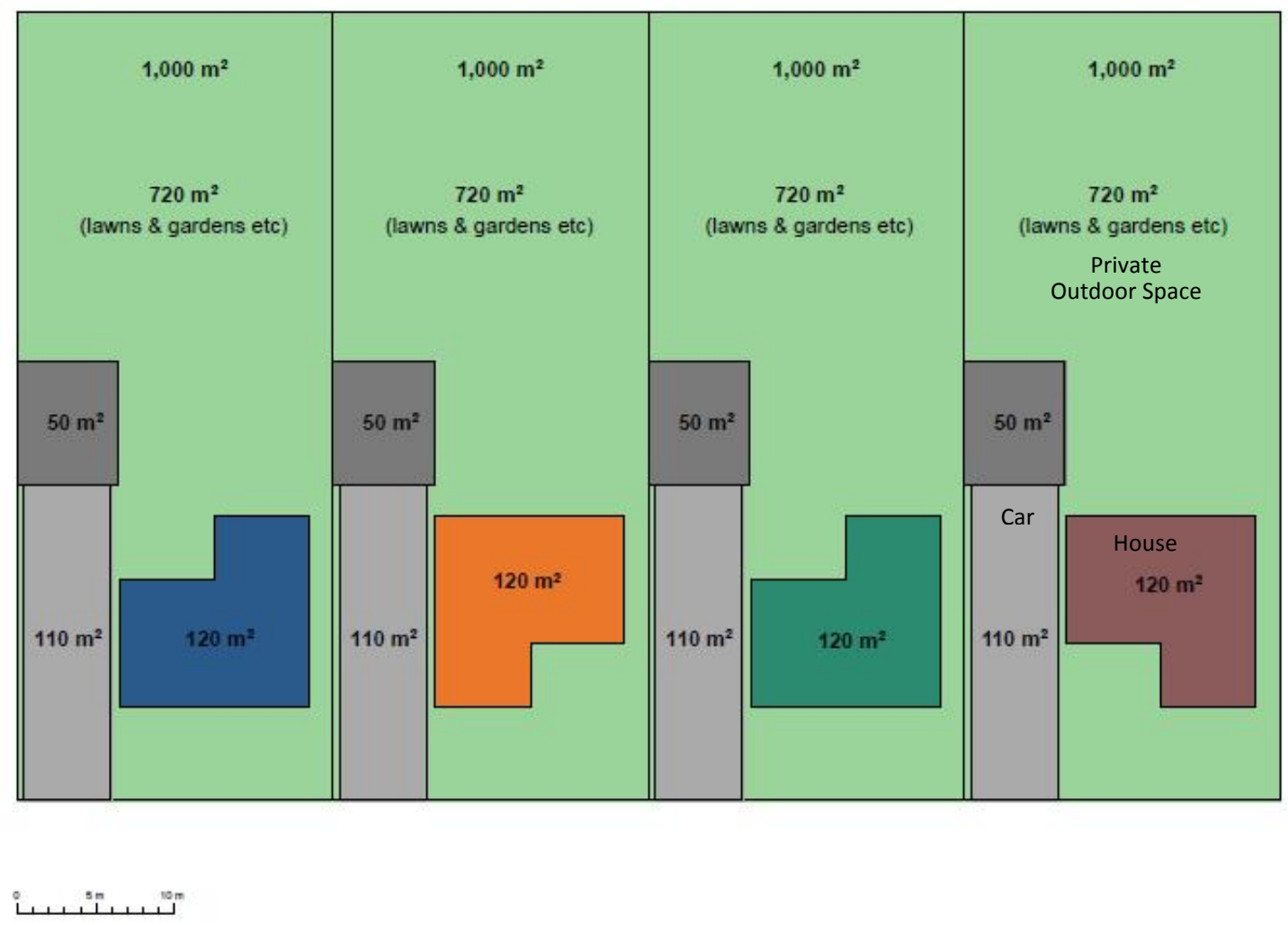

See Fig. 1.5 (Thesis Chapter 1) $4 \times 1000 \mathrm{~m}^{2}$ lots, $120 \mathrm{~m}^{2}$ dwelling on each. (Total 24 residents, assuming average 6 persons per household) (S.J. Pattinson) 


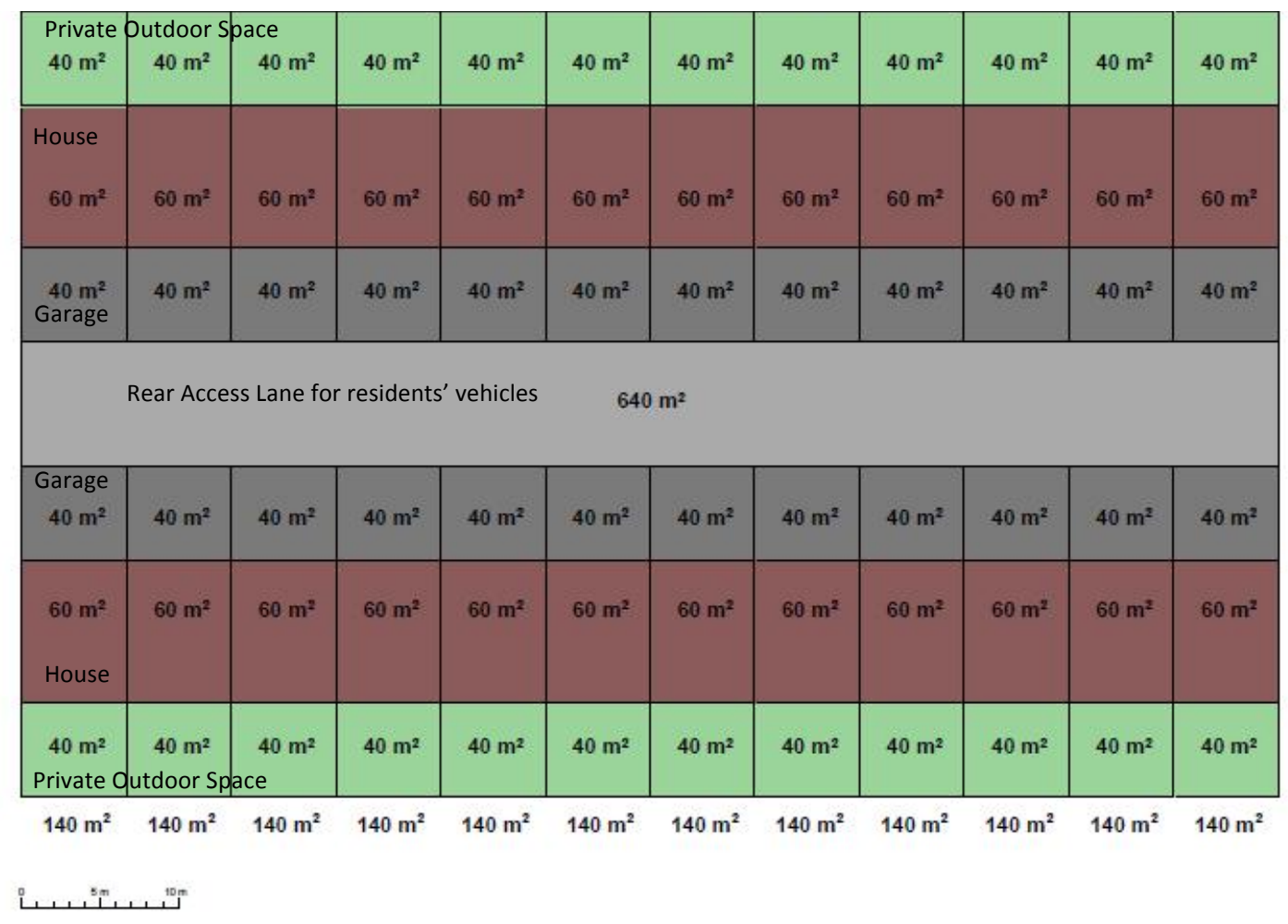

See Fig. 1.6 (Thesis Chapter 1) $24 \times 140 \mathrm{~m}^{2}$ lots plus rear access lane (total area 4,000 $\mathrm{m}^{2}$ ). (Total 24 residents assuming one person per house) (S.J. Pattinson)

The terrace houses may be one- or two- storey. Table 1.2 in Thesis Chapter 1 shows that the conversion in Figs. 1.6 and 1.7 from larger detached dwellings to smaller attached terrace houses tends to result in greater individual floor space per person. Figs. 1.6 and 1.7 illustrate the resulting loss of open space.

\section{References:}

Evans, Paul. "Housing Layout and Density" Land Use and Built Form Studies: Working Paper No. 75. Cambridge, England: U. of Cambridge, 1973.

Bird, Clinton. Minimum Standards for Residential Apartments. Auckland: U. of Auckland, Nov. 2005.

Auckland Council. Practice Note: Minimum Apartment Size. BLD-125-PN. 2010. 
Appendix 2: Government Press Release - Pinehill Crescent 


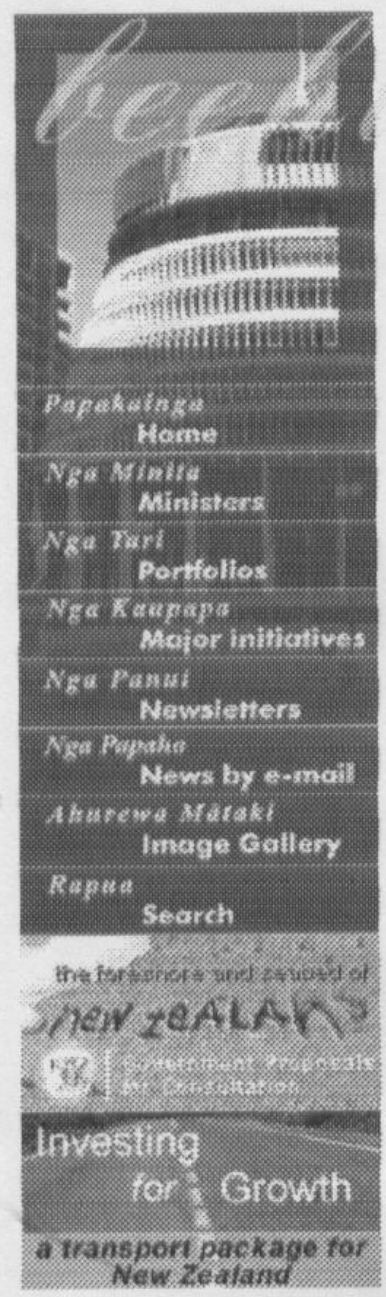

\section{beehive.govt.nz}

\section{Hon Tariana Turia}

01 July 2002

\section{New State Houses for Pinehill}

Acting Housing Minister Tariana Turia and Rimutaka Labour MP Paul Swain today announced that 70 new houses, including 20 new state houses, are to be built on Housing New Zealand land at Pinehill in Upper Hutt.

Mrs Turia said a proposal from a private developer has been accepted for 70 new houses to be built on the five-hectare site.

The proposal, which is subject to final contract negotiations, would see the area subdivided, and then Harwood Pacific Limited contracted to buy the sites and build the houses.

Housing New Zealand would buy about 20 of the homes within a pre-agreed price, and Harwood Pacific would sell the remaining 50 homes on the open market.

"This arrangement between Housing New Zealand and a private developer resulted from extensive community consultation," said Tariana Turia. "It could become a model for other state housing areas," she said.

Local MP Paul Swain said he was delighted with the announcement.

"This announcement is excellent news and will add to the very positive future outlook for Upper Hutt. The area is really moving ahead. Recently South Pacific Tyres announced that they are taking on more staff. The International School on the old CIT site is another exciting development," he said

Housing NZ bought the former Rimutaka Prison staff housing village site in December 2000 . The houses had mostly been empty for at least two years prior to purchase and were in a state of disrepair.

Mrs Turia said the Pinehill redevelopment project had involved extensive community consultation in the past year. It also required careful consideration by Government due to the complex nature of the commercial arrangements.

"The community consultation has included four public meetings and planning workshops. The community expressed their desire to avoid any concentration of state housing in the redevelopment.

http://www.beehive.govt.nz/ViewDocument.cfm?DocumentID=14439 


\section{Appendix 3: Template of Observation Sheet}




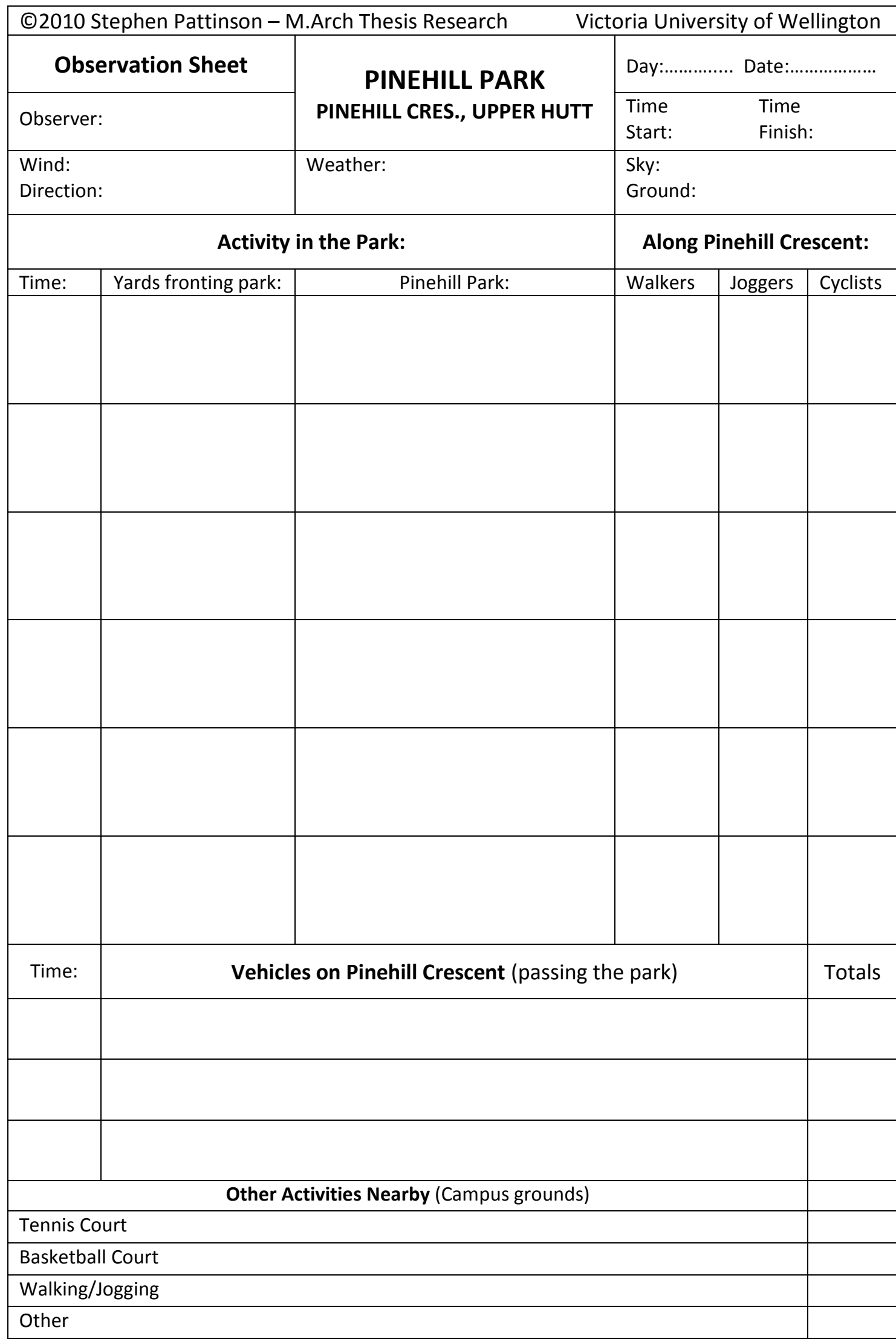




\section{Appendix 4: Observations of Pinehill Park}




\section{Appendix 4-Observation of Pinehill Park: Notes and data}

Regarding the observations of Pinehill Park carried out by the writer in the Case Study (see Chapter 6, 172), the writer's approach to these observations was guided by advice from Cooper Marcus and Francis on how to carry out Post-Occupancy Evaluations (POEs) of neighbourhood parks and similar outdoor spaces. Cooper Marcus and Francis advise to:

"... observe actual activities in the park for at least four separate half hour periods, preferably on different days at different times of the day, perhaps a weekday and a weekend morning; and a weekday and a weekend afternoon..." (People Places, 351)

Elsewhere Cooper Marcus and Francis advise "at least one hour ... at each visit" (346) and "For each observation period, keep a complete record of all that is happening in the space - by age, sex, ethnicity, type of activity, and location ..." (351). They describe this approach as "systematic" (348) and comment that "this approach ... can achieve considerable insight into actual use of designed places" (346).

Following their approach, here is a summary of the writer's notes and data from observations of Pinehill Park.

Observation Monday, 1 November 2010, 11.30 am to 4.30 pm (5 hours continuous):

- During the first 4 hours of observation, nobody used the park. From $3.30 \mathrm{pm}$ to 4.00 pm 3 guys (who appear to be in their late teens/early 20 's) were kicking a rugby ball around the park. They were the only Park users during this 5 hour observation period. 
- Also during this 5 hour period, nobody appeared in any of the front yards (bordering the Park) of the terrace houses apart from 2 guys sitting on the boundary garden wall (who later came out and kicked the rugby ball around the Park).

- An average of about 2 vehicles per minute passed the site throughout most of the observation period but none of them stopped at the Park.

- Meanwhile, 8 young people in 3 groups were using the tennis courts across the road at the CIT throughout most of the 5 hour observation period.

Observation Tuesday, 2 November 2010, 3.00 pm to 8.00 pm (5 hours continuous):

- No one was observed in the Park or the front yards until $3.34 \mathrm{pm}$ when a young girl entered the Park from the south-west corner of the Park (at the Evergreen Crescent entry) spending 1 minute on the swing and a couple of minutes climbing a tree before leaving, staying for 5 minutes total in the Park.

- From 3.40 pm to 4.00 pm (20 minutes total) a lady with a toddler in a pram and a young boy walking (looked to be about 3 years) arrived at the Park on foot from the street. They used the swing, climbing frame, the 'rocker-croc' and the shade of the trees. They left the Park on foot along Gallipoli Road.

- From 4.00 pm onwards there was some activity beginning to happen in the Park. There was a group of 3 young girls mostly using the swings from 4.00 pm to $4.30 \mathrm{pm}$. A lady with a toddler arrived at $4.45 \mathrm{pm}$ on foot from the south-west corner of the Park (at the Evergreen Crescent entry) - the toddler used the swing, the climbing frame, the 'rocking-croc' and the slide. They left the park 25 minutes later at $5.10 \mathrm{pm}$ out the south-west corner where they had entered. A young couple walked diagonally across the Park 
from the same entry and left along Gallipoli Road. At $5.35 \mathrm{pm}$ an elderly man with a toddler used the 'rocking-croc', climbing frame and the slide and left through the south-west corner after staying for 13 minutes. At about the same time 3 young girls came back to the park and mostly used the swings where they sat chatting either at the swings or sitting on the grass.

- At 6.00 pm 3 young boys came out and joined the girls bringing a frisbie. These children were using the swing, the climbing frame, the slide and the monkey bar. They all stayed in the Park playing games, doing somersaults, playing on the playground equipment until $6.37 \mathrm{pm}$. The Park is now empty.

- The group of children who just left appear to live in the terrace houses around the Park. Being early evening, the playground equipment and swings are now in the shadow cast by the low evening sun of the trees at the western end of the Park. (The sun however did not set until after observation of the Park finished at 8.00 pm.)

- The Park remained empty for almost an hour until 7.34 pm when a young teenage boy entered the Park, did some gymnastics on the swing frame for 2 minutes and then left the Park. There was no further activity in the Park for the remainder of the observation period which finished at $8.00 \mathrm{pm}$.

- During this 5 hour period there was no activity in the front yards of the terrace houses apart from the above mentioned children entering their houses through their yard gates and one male resident who was outside in the evening for 35 minutes watering his garden. 
Observation Wednesday, 3 November 2010, 6.30 am to 11.30 am (5 hours continuous):

- Nobody used the Park during this whole 5 hour period. At 9.30 am a resident of one of the terrace houses came out briefly into their front yard, and that was the only instance of the front yards being used during this time.

Observation Saturday, 6 November 2010, 10.30 am to 11.30 am (1 hour continuous):

- The writer was curious to see if there was any evidence in the Park this morning of Guy Fawkes celebrations the night before - there was none. It had started raining last evening at about $8.00 \mathrm{pm}$ but this morning the rain had cleared.

- At 10.55 am a lady comes out into her front yard for 4 minutes using a cordless telephone.

- At 10.43 am, 2 teenage girls came out of one of the terrace houses and used the swings until 11.05 am when it started raining lightly, and they went back inside.

- There are some puddles in the grass by the playground equipment. The ground is very clayey and the water just lies on the ground surface without soaking away.

- At 11.09 am a lady from another terrace house throws some bread over her front yard garden wall into the Park to feed several ducks which frequent the park.

- The light rain continued intermittently until 11.30 am until this observation finished, there having been no further activity in the Park for the last 20 minutes. 
Observation Saturday, 6 November 2010, 6.10 pm to 6.40 pm (30 minutes continuous):

- It has been raining off and on during the day and there have been some strong cold winds from the south-west, but by $6.10 \mathrm{pm}$ the weather was fine and overcast with some sunny patches in the weather.

- $6.16 \mathrm{pm}, 2$ young boys came out of the terrace houses carrying golf irons and hit a golf ball around the Park until they went back inside at $6.40 \mathrm{pm}$. During their play the golf ball went across the road on one occasion and into the stream that runs along the other side of the road in the CIT grounds. The ball was retrieved by the boys and they carried on playing in the park.

- Two young girls from the terrace houses also came out to the Park at 6.32 $\mathrm{pm}$ and use the swings. The girls talk with the young boys briefly when the golf ball comes close to the playground equipment.

Observation Sunday, 7 November 2010, 9.00 am to 9.30 am (30 minutes continuous):

- The weather is fine

- Again there was no evidence in the Park this morning of any significant Guy Fawkes celebrations the night before (Saturday night) apart from 3 or 4 crackers on the grass by the playground equipment.

- There was no activity either in the Park or in the front yards of the terrace houses during this $1 / 2$ hour observation. 
Observation Sunday, 7 November 2010, 12.05 pm to 2.05 pm (2 hours continuous):

- This observation begins with a man and a toddler 2 or 3 years old in the Park using the 'rocking-croc', the swing, the climbing platform and the slide. They also play hide-and-seek in the hut-like shelter beneath the climbing platform. The man and the toddler take turns hiding in this shelter. The man holds the boy while he attempts to climb the vertical rope netting. The man also lifts the toddler into the lower branches of the largest tree for 1 minute. They leave the park walking along the footpath at $12.24 \mathrm{pm}$. The park is now empty. (The weather is fine, but the wind is steady and cool from the southwest).

- At $12.37 \mathrm{pm}$ a boy and girl come into the Park from one of the terrace houses and use the playground equipment for 2 minutes, then return into their terrace house.

- At $12.44 \mathrm{pm}$ a women and boy come out form one of the terraces houses bringing a Frisbee ring which they throw around the Park to each other for 3 minutes. The boy runs up the slide, across the net, swings along the monkey bar, leans on the 'rocking-croc' and then climbs up the tree all in the space of 1 minutes, at which the lady returns to the terrace house (at $12.49 \mathrm{pm}$ ) leaving the boy up the tree, but the boy immediately comes down and goes back into the terrace house.

- At $12.51 \mathrm{pm}$ a girl comes out of a terrace house and climbs a tree for 3 minutes and then returns to the terrace house.

- The sky is blue, but the wind is still steady.

- $\quad 1.16$ pm, 2 young adult males from one of the terrace houses kick a rugby ball around the Park and soon afterwards a young lady from the same terrace house throws some bread over the garden wall into the Park for the birds. 2 minutes later, 2 young boys from the adjacent terrace house come 
out and join them for 2 minutes kicking the rugby ball. At 1.26 pm (after 10 minutes of being in the Park), the 2 young adult males leave the Park).

- At $1.38 \mathrm{pm}$ the young woman who had been feeding the birds brings the rugby ball out into the Park by herself. She kicks the ball across to the swings, and then gets on a swing, climbs on the rope net and then throws the rugby ball into the air several times catching it on her way back to the terrace house at $1.43 \mathrm{pm}$ (she has been in the Park for 5 minutes).

- At 1.43 pm a young girl from another terrace house, followed by a young boy from the same house, come out into the Park and use the rocker and the climbing frame. They run around the grass and then return to their house after being in the Park for 8 minutes.

- $1.52 \mathrm{pm}$ at teenage girl comes into the Park and uses the swing. At the same time, 2 young adults (a guy and girl) enter the Park with a rugby ball and kick it around the Park. Joined 2 minutes later by a third young person from the same terrace house. The teenage girl leaves the swing and the Park at 1.58 pm after being there for 6 minutes and returns to her terrace. But she comes back again 3 minutes later at $2.01 \mathrm{pm}$ to the swing, being joined by a second teenage girl at $2.04 \mathrm{pm}$. Meanwhile a fourth young person (a guy) joins the group kicking and throwing the rugby ball. It goes into the stream in the ditch on the other side of Pinehill Crescent Road, and two guys go across the road to retrieve it.

- The observation finished at $2.05 \mathrm{pm}$ with the 4 young people playing with the rugby ball, and the 2 young girls on the swings. 
Observation Sunday, 7 November 2010, 3.35 pm to 5.40 pm (just over 2 hours continuous):

- During this observation period the weather is fine, sunny and calm, there being just a slight breeze from the south-west. The sky is blue.

- No one is in the Park. No one is in the front yard of the terrace houses apart from 2 adult males and a woman at one house who are having drinks and appear to be unpacking a new barbeque for their patio. There is no activity in the Park until $3.52 \mathrm{pm}$ until a girl runs out of one of the terrace houses, chases a pair of paradise ducks and plays on the playground equipment. She returns to her house after being in the Park for less than 5 minutes.

- At 4.06 pm quite a bit of activity begins to happen in the Park. 2 young adult males come out from a terrace house kicking the rugby ball; a teenage girl comes out from another terrace house to the swing. 2 minutes later a couple arrive by car and bring their toddler to the swing, then 1 minute later a young boy comes out of another terrace house and joins the rugby, followed a minute later by 3 other young boys (about 10-12 years) who run into the park from the south-west corner (the entry to the Park off Evergreen Crescent) and they go to the playground equipment. 2 minutes later (now $4.12 \mathrm{pm}$ ), 2 teenage girls come into the Park from one of the terrace houses and play on the rocker. At $4.16 \mathrm{pm}$, the man and woman put the toddler back in the car and drive off after having been in the Park for 7 minutes mostly using the swing and the slide. At 4.15 pm, 2 girls come out of a terrace house to the swings, 1 with a guitar, 1 of the girls sits on the swing while the other sits on the grass playing the guitar. A boy comes to the swings along with 2 other boys who alternate between the climbing platform and the swing frame (these are the 3 boys who entered the Park from the south-west corner). They leave the Park through the same southwest entry at $4.23 \mathrm{pm}$. ( 1 of these 3 boys is on a bike, and another is 
carrying a skateboard. They had left these on the grass by the trees when they entered the Park.)

- At 4.40 pm, 2 young boys come out of one of the terrace houses and go to the playground equipment. 1 minute later, a boy bikes into the Park from the street, across to the girls on the swings, where they talk briefly.

- The writer recorded a lot of detail concerning users and activities in the Park over the remaining hour of this observation which is summarised briefly here by saying that there is now the most people in the Park that the writer has observed during this whole week. There are 3 groups in the Park all being young adults and children. 1 group consists of 3 young adults kicking the rugby ball around the Park, watched by 3 more young adults in the yard of one of the terrace houses leaning on the garden wall on the edge of the Park. A second group consists of 3 young boys from the adjacent terrace house joining in the rugby kicks and passes with their neighbours (the young adults in the first group). The third group consists of 2 young teenage girls on the playground equipment, mostly on the swings. Belonging to the first group is a young toddler sitting on the rocker watching the activity in the Park. At $5.37 \mathrm{pm}$, the writer noted that in total there are 12 young adults and children using the Park. At this point the toddler suddenly left the play equipment and ran across the road. A car was approaching from the east and seeing the toddler slowed to a stop. Two of the young adults dashed across the park and the road and reached the toddler just as the toddler reached the edge of the ditch (Heretaunga Drain) on the other side of the road. Fortunately there were no cars coming from the other direction (from the west) which, because of the bend in the road, is a blind approach to the Park for drivers.

- At $5.40 \mathrm{pm}$ the toddler was taken inside while some of the young people and children remained playing in the Park. 
PINEHILL PARK, Trentham, Upper Hutt - How it is used and by which age groups SUMMARY

\begin{tabular}{r|l|l} 
Mon 1st - Sun 7th Nov 2010 & 1,265 mins (21 hrs 5 mins) total observation time
\end{tabular}

\begin{tabular}{|c|c|c|c|c|c|c|c|c|c|c|c|c|c|c|c|c|c|c|c|c|c|c|c|c|c|}
\hline & Car & Bike & Walk & Terr/H Sex & Time & F/yard & Park & Time & Analy & is: & Mat or & & & & & & & & & Climb & & & & & \\
\hline & & & & (average stay in & mins) & no. & no. & (ave) & Table & Bench & grass & Swing & Platfm & Shop & Slide & $\mathrm{Ne}$ & & ar & Rocker & Tree & Mound & Ball & Frisbee & Golf & Games \\
\hline nfants & & & & & & 0 & 2 & 20 & & & & & & & & & & & & & & & & & \\
\hline Pre-schoolers & & & & & & 0 & 5 & 21.8 & & & & & & & & & & & & & & & & & \\
\hline Children & & & & & 1.1 & 4 & 41 & 12.7 & & & & & & & & & & & & & & & & & \\
\hline Teenagers & & & & & 6 & 1 & 15 & 17.3 & & & & & & & & & & & & & & & & & \\
\hline Young adults & & & & & 10.2 & 11 & 17 & 24 & & & & & & & & & & & & & & 39 & & & \\
\hline Adults & & & & & 11.4 & 7 & 5 & 16 & & & & & & & & & & & & & & & & & \\
\hline Middle-aged & & & & & 15.2 & 5 & 2 & 10 & & & & & & & & & & & & & & & & & \\
\hline Elderly & & & & & & 0 & 0 & & & & & & & & & & & & & & & & & & \\
\hline & & & & TOTALS: & $\begin{array}{l}9.9 \\
\text { ave }\end{array}$ & 28 & 87 & $\begin{array}{c}16.5 \\
\text { ave }\end{array}$ & & & & & & & & & & & & & & & & & \\
\hline
\end{tabular}

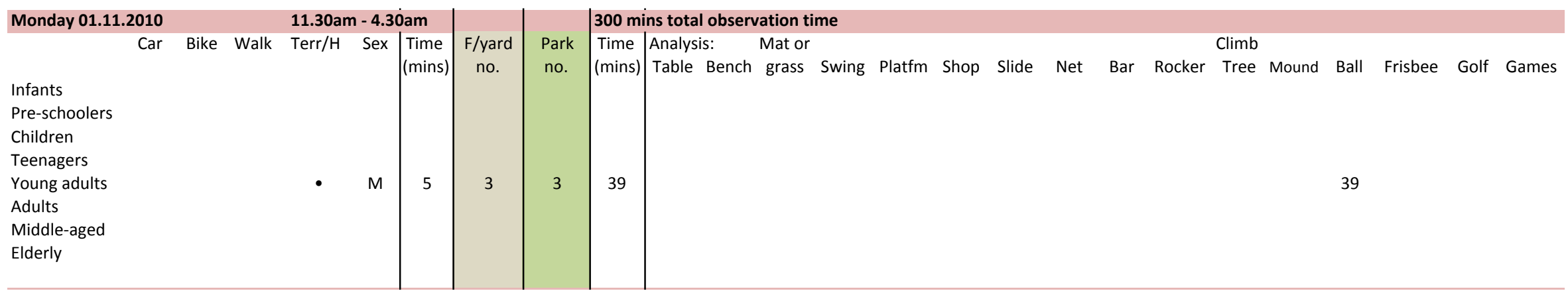




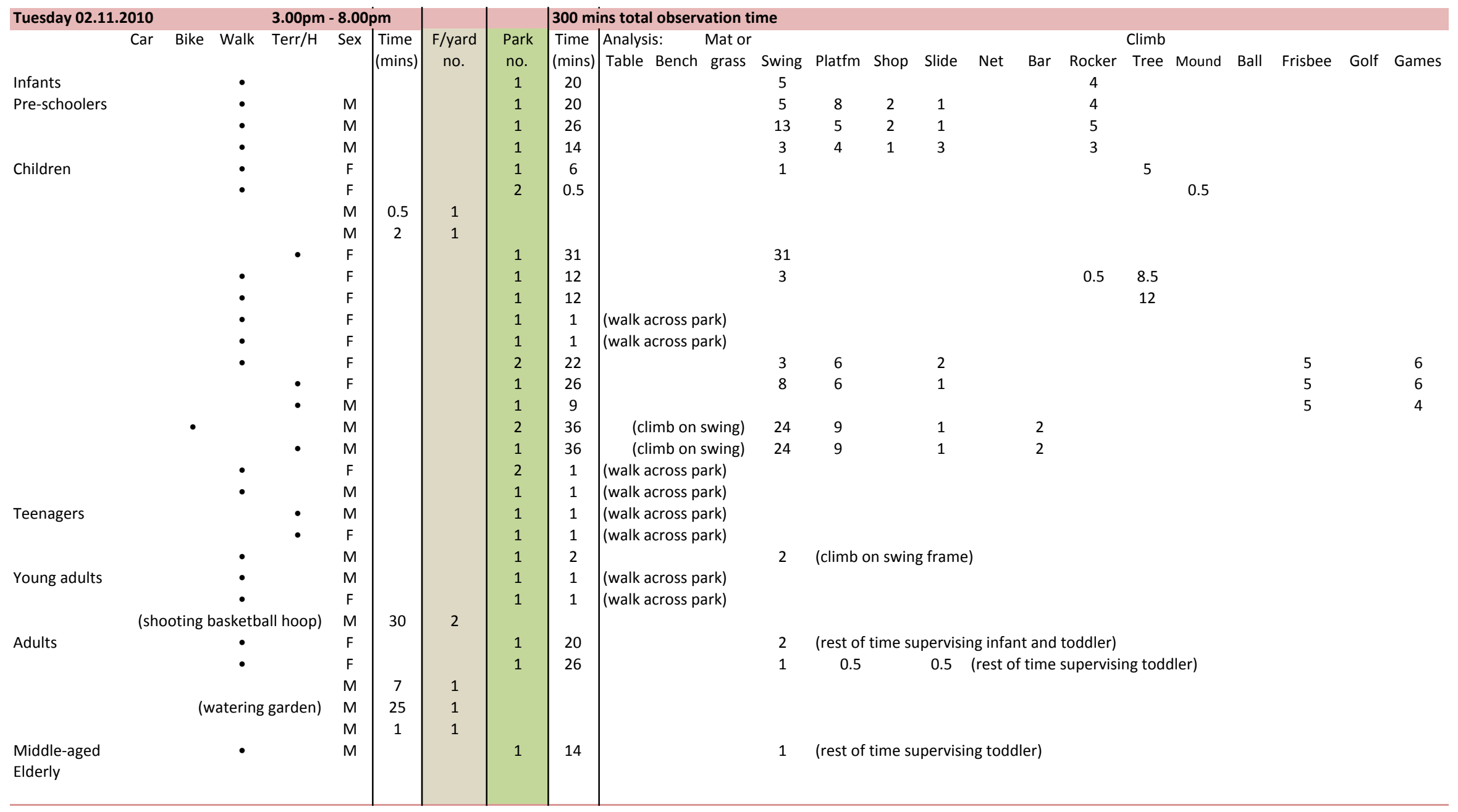




\section{(C) 2012 Stephen Pattinson Appendix 4-Observation notes and data M. Arch Thesis}

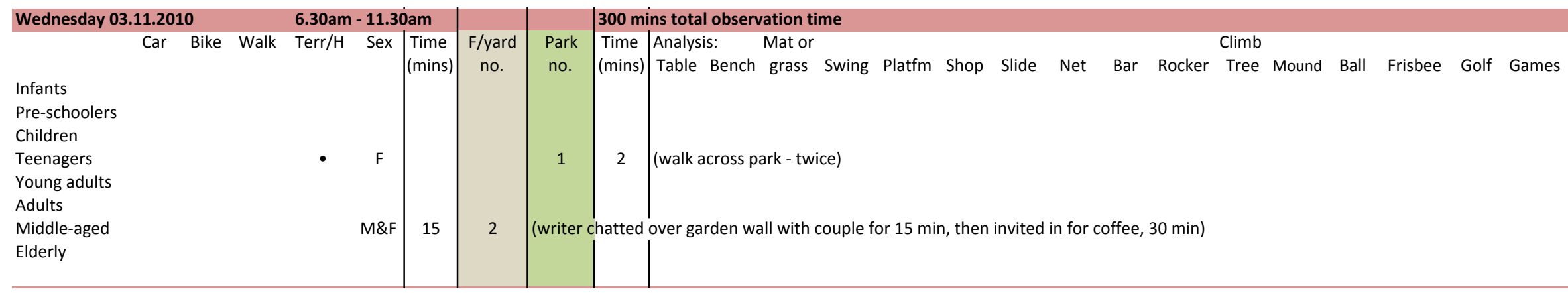

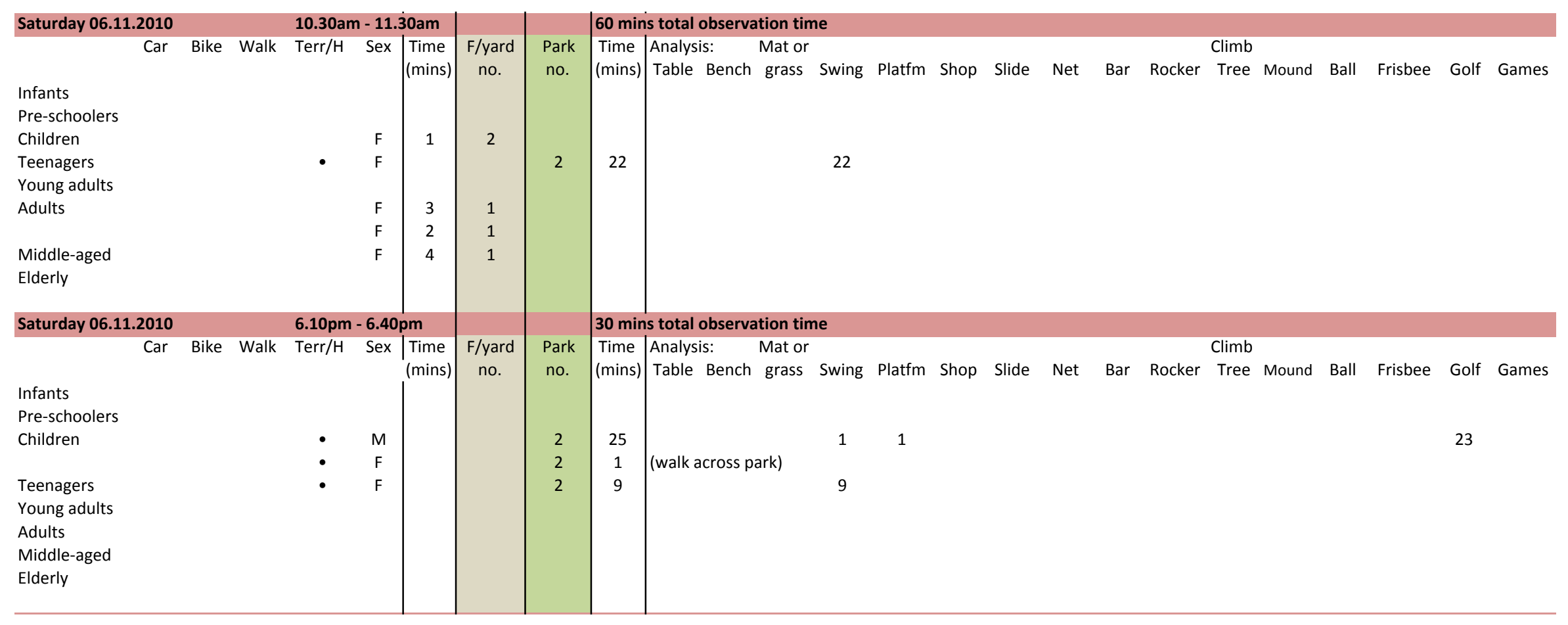




\section{(C) 2012 Stephen Pattinson Appendix 4-Observation notes and data M. Arch Thesis}

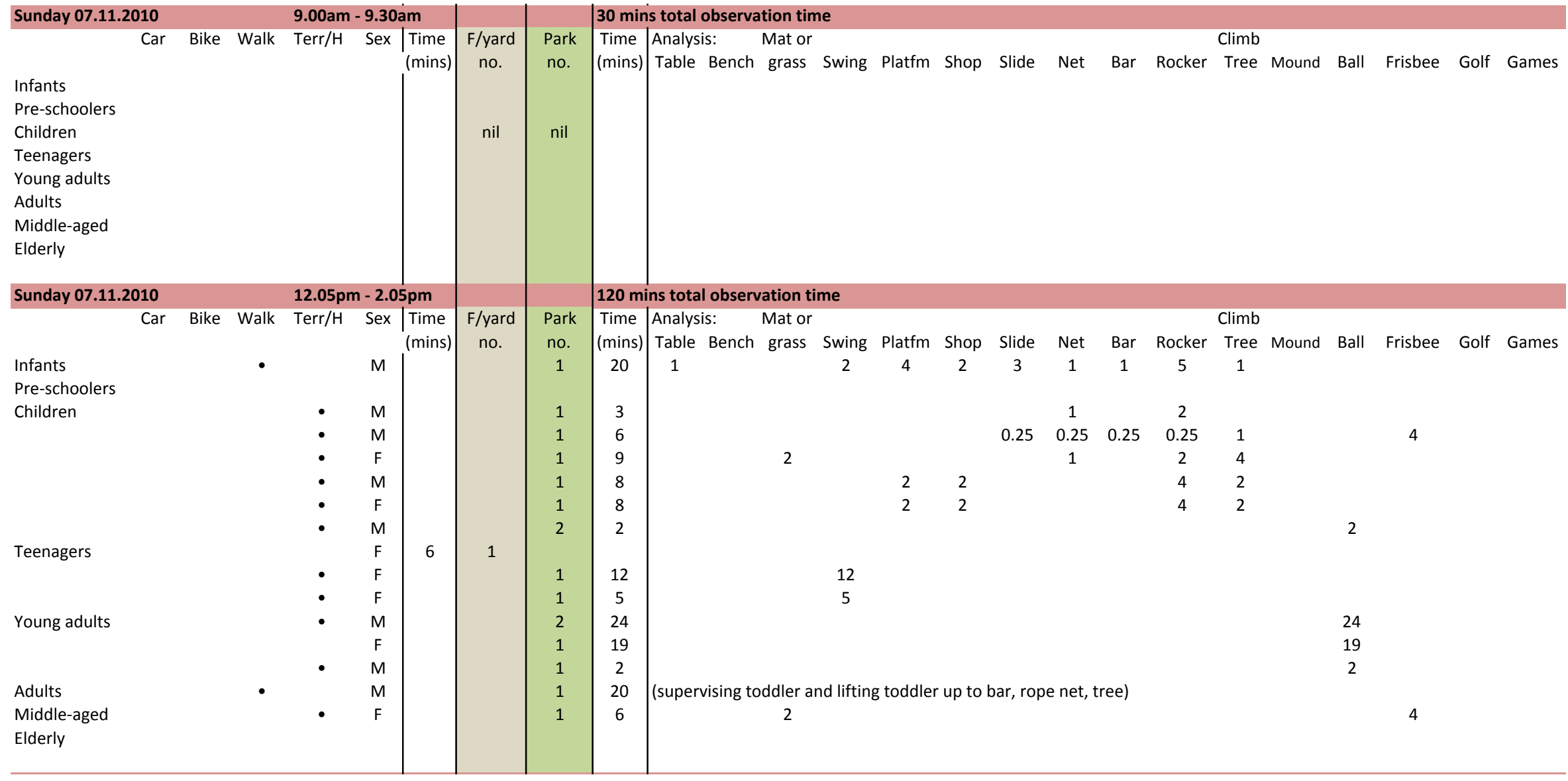




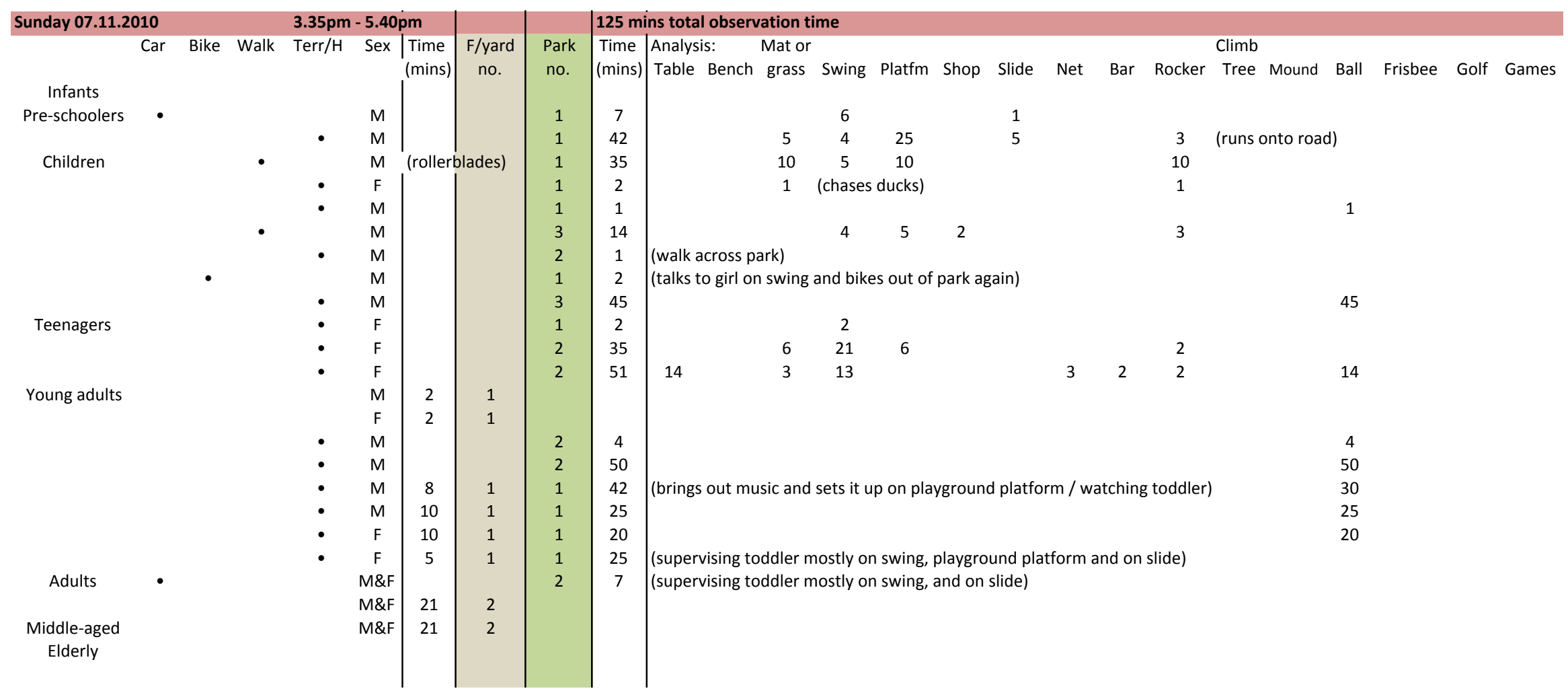




\section{Appendix 5.1: Survey / Interview Forms}




\section{PINEHILL PARK RESEARCH}

\section{Survey Information}

This survey is part of a Master's thesis on 'Common Ground' in medium-density housing. Demand for housing means more use needs to be made of less urban land. This research looks at how the use of common land in medium-density housing might improve the quality of life for residents. A Housing New Zealand Design Guide says that community ownership of open spaces means "a community feels as though the space is there for them".

As the nearest residents to Pinehill Park, this survey asks the occupants of the 14 terrace houses around the park for comments on the uses and potential of the Park.

\section{Questions asked in this survey:}

1: What does it mean to you living beside the park?

(How do you use the park? What do you like / dislike?)

2: How would you design the park?

(What other layouts, uses, landscaping, furniture or equipment etc do you think the park could have? How could the park be designed to mean more to you? Please note that this is a hypothetical design exercise only, and not intended to suggest that this park will be redeveloped in any way.)

\section{Optional questions:}

\begin{tabular}{|c|c|c|c|c|c|c|}
\hline Age range & $1-5$ & $6-10$ & $11-15$ & $16-19$ & $20-30$ & $30-50$ \\
\hline
\end{tabular}

Country of Origin

How long have you lived next to the park? years months How many living in your household? adults pre-school children school children teenagers

\section{Thank you for your assistance.}

If you have any queries about this survey, or if there is anything else you would like to say about your thoughts on the design of Pinehill Park you may contact the writer ....

Stephen Pattinson B.Arch., ANZIA, Registered Architect Ph. (04) 9384555 sipattinson@paradise.net.nz_Ｄecember 2010

Thesis research is by Stephen Pattinson and is toward a Master of Architecture Degree, Victoria University of Wellington, School of Architecture, Ethics Approval Number 17632.

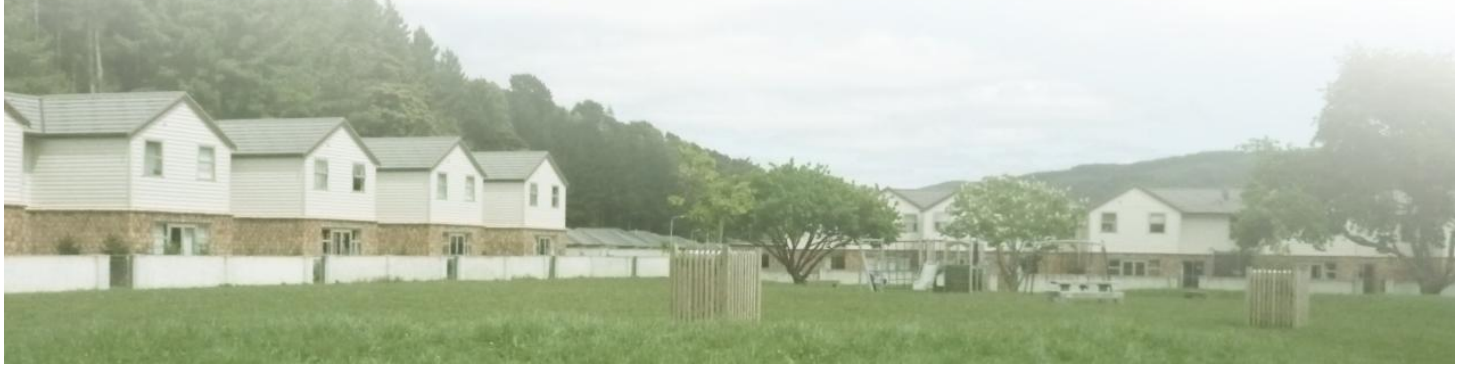




\section{PINEHILL PARK RESEARCH}

\section{Response Form}

You may write / sketch your responses on the accompanying map of Pinehill Park.

Question 1: What does it mean to you living beside the park?

(How do you use the park? What do you like / dislike?)

Question 2: How would you design the park?

(What other layouts, uses, landscaping, furniture or equipment etc do you think the park could have? How could the park be designed to mean more to you?)

Please note that this is a hypothetical design exercise only, and not intended to suggest that this park will be redeveloped in any way.

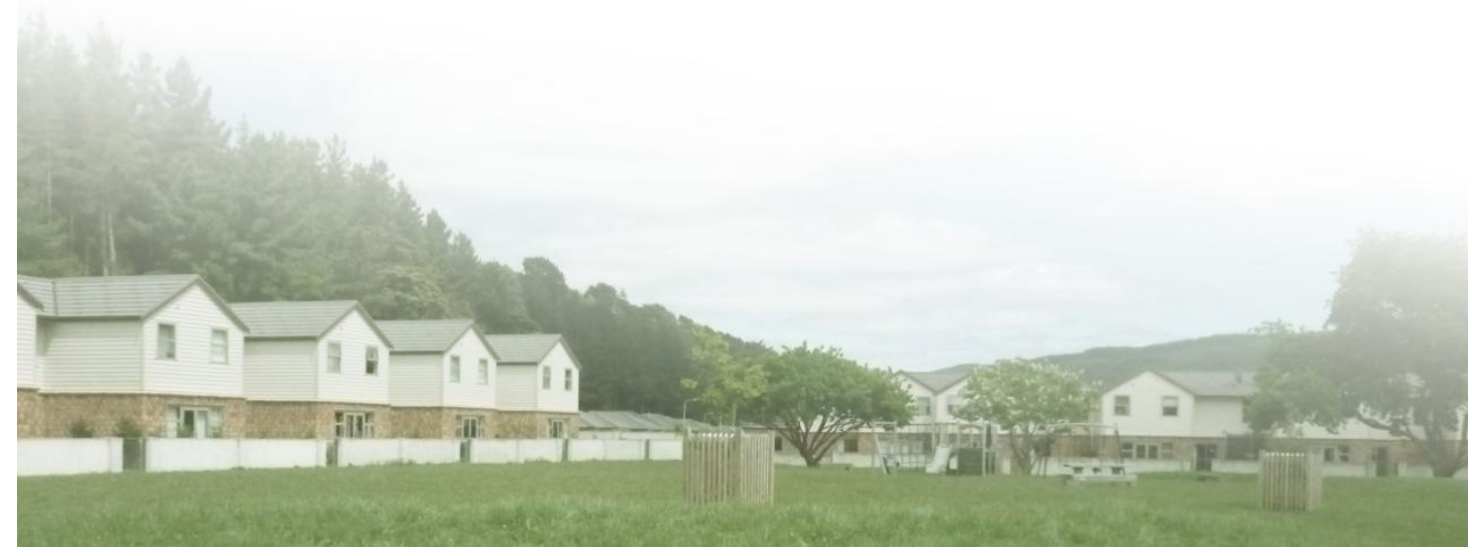




\section{OPTIONAL:}

To assist with this research could you please provide the following information

Age range $1-5$

6-10

$11-15$

16-19

20-30

$30-50$

$50+$

Sex

$M / F$

Country of Origin

How long have you lived next to the park?

years

.months

How many live in your household?

adults

pre-school children

school children teenagers

Thank you for your assistance.

December 2010

If you have any queries about this survey, or if there is anything else you would like to say about your thoughts on the design of Pinehill Park you may contact the writer ....

Stephen Pattinson B.Arch., ANZIA, Registered Architect, Ph. (04) 9384555 sipattinson@paradise.net.nz

Thesis research is by Stephen Pattinson and is toward a Master of Architecture Degree, Victoria University of Wellington, School of Architecture, Ethics Approval Number 17632.

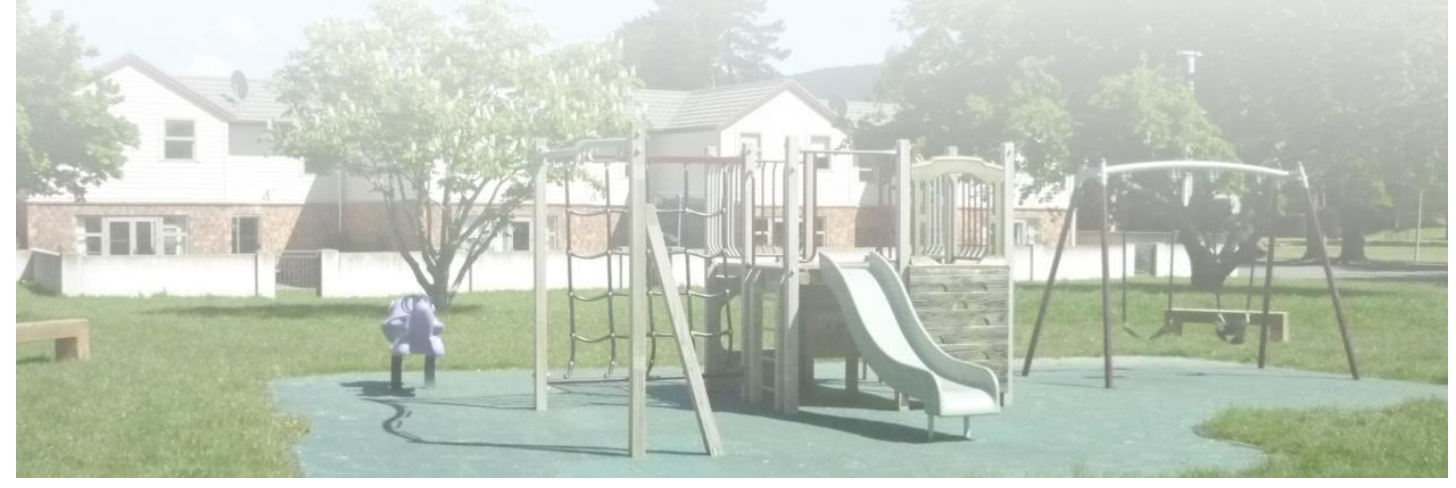




\section{PINEHILL PARK RESEARCH}

\section{Response Form}

You may write / sketch your responses on the accompanying map of Pinehill Park.

Question 1: What does it mean to you living beside the park?

(How do you use the park? What do you like / dislike?)

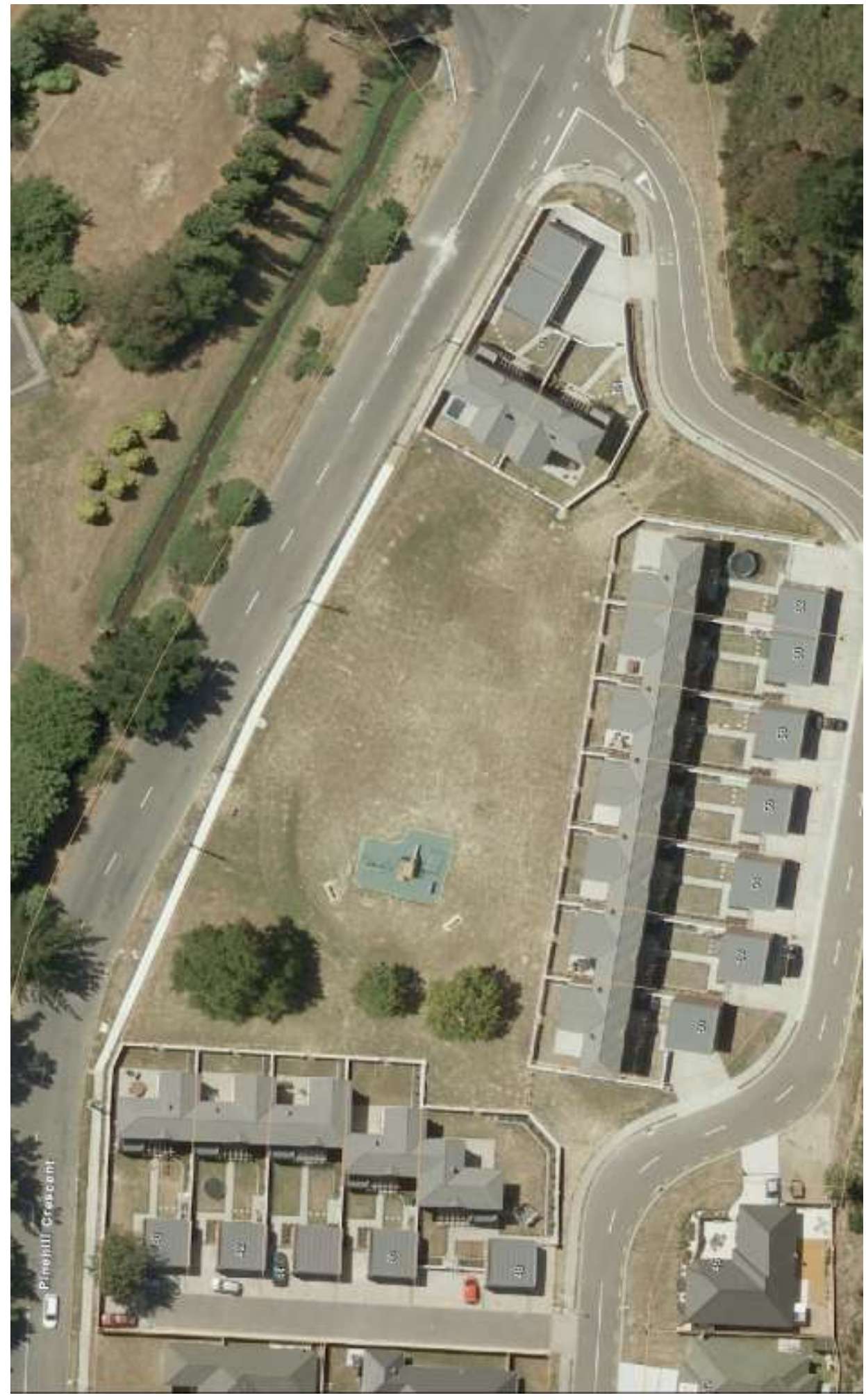




\section{Question 2: How would you design the park?}

(What other layouts, uses, landscaping, furniture or equipment etc do you think the park could have? How could the park be designed to mean more to you?)

Please note that this is a hypothetical design exercise only, and not intended to suggest that this park will be redeveloped in any way.

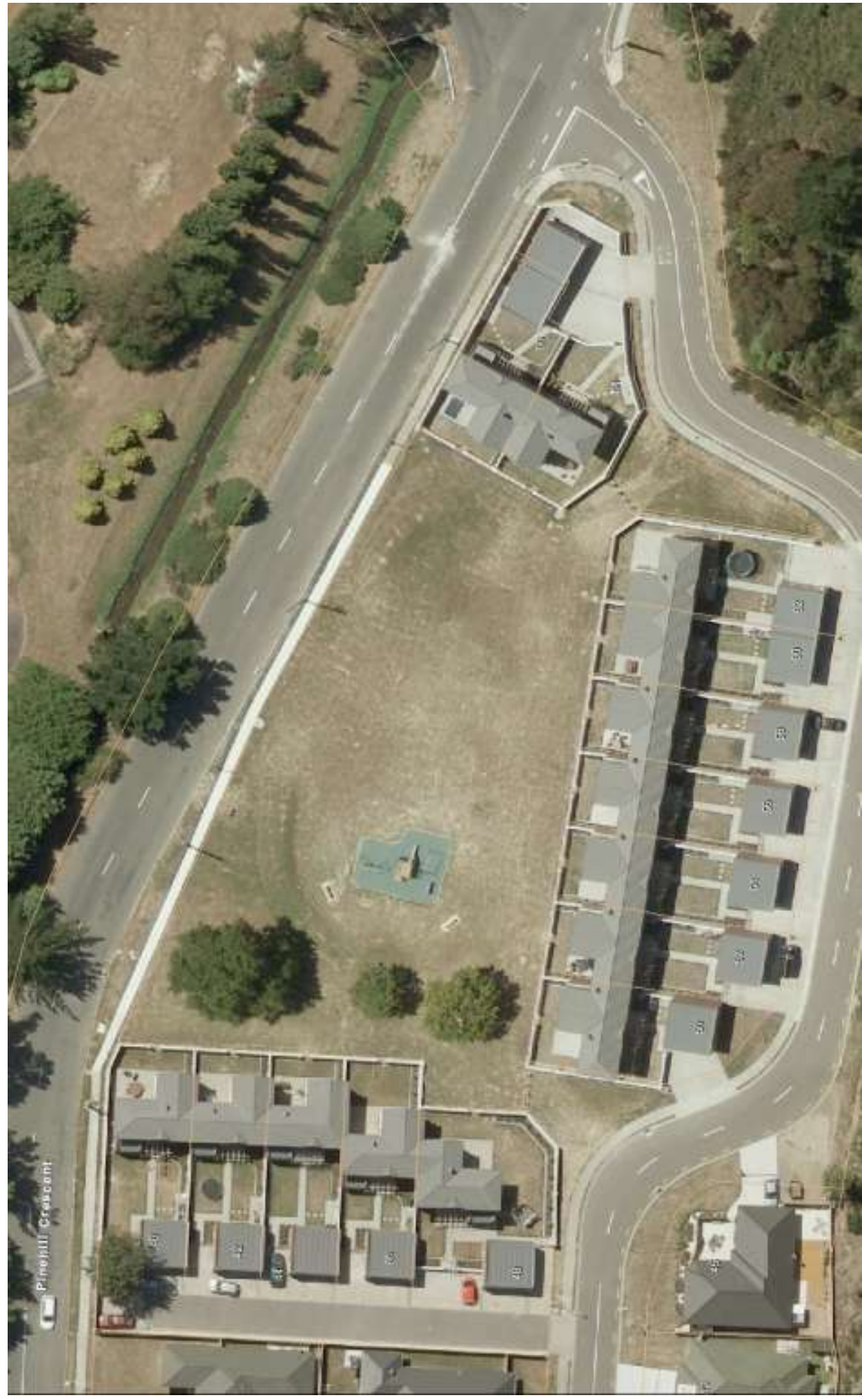




\section{Appendix 5.2: Analysis - Survey / Interview Responses}




\section{APPENDIX 5.2: Analysis - Survey / Interview Responses Pinehill Park Case Study}

This part of Appendix 5 presents the data from respondents of the survey and interviews carried out with residents of the terrace housing at Pinehill Park (see Chapter 6, p. 181f).

Respondents comments have been categorised according to the five proposed principles of communal space in medium density housing:-

1. Walkable neighbourhoods

2. Clustered Housing

3. Open Green Space

4. Children and Teenagers

5. Sense of Community

The first table, Table A5.1 presents an overview of the data. The subsequent tables analyse the data further into various aspects of each of the five principles. These principles were never mentioned to participants in the survey and interviews. Respondents have given their views and comments regarding two open-ended questions:

1. What does it mean to you living beside the Park?

2. How would you design the Park?

Respondents were free to mentioned anything they felt was relevant to these two questions. 


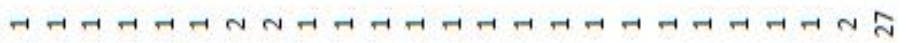

๓

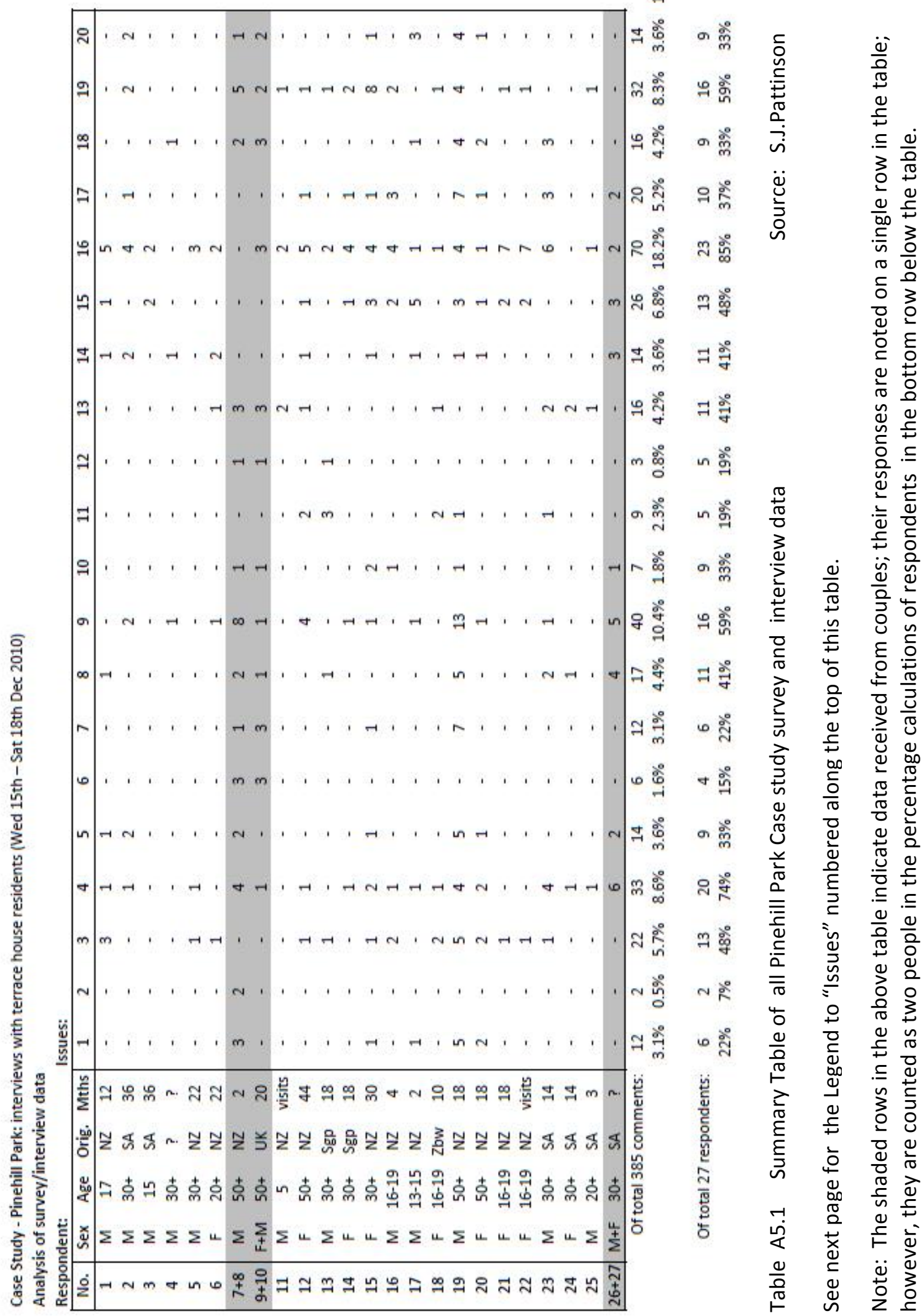



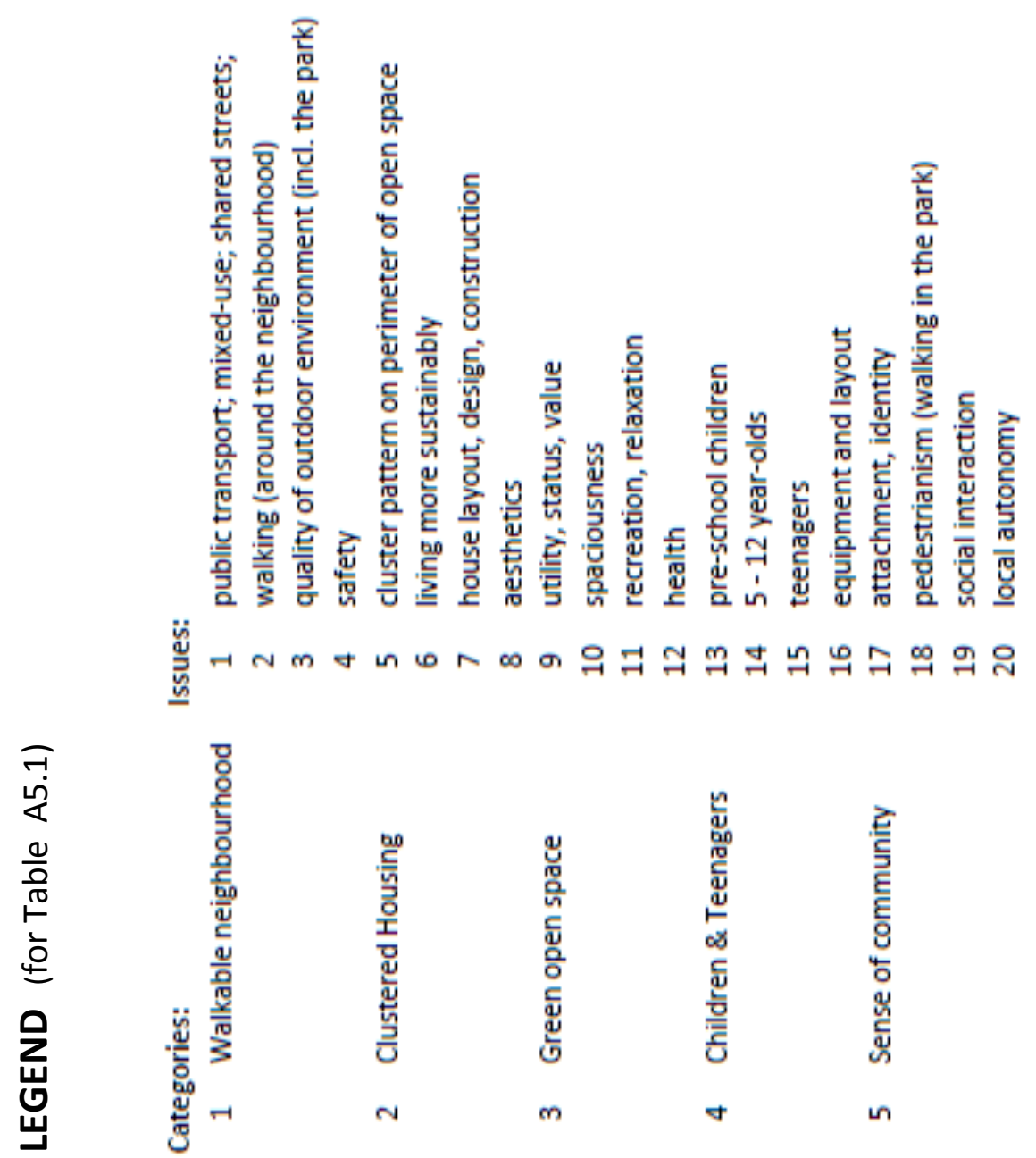

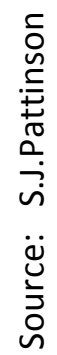




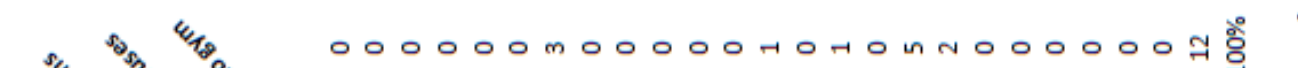

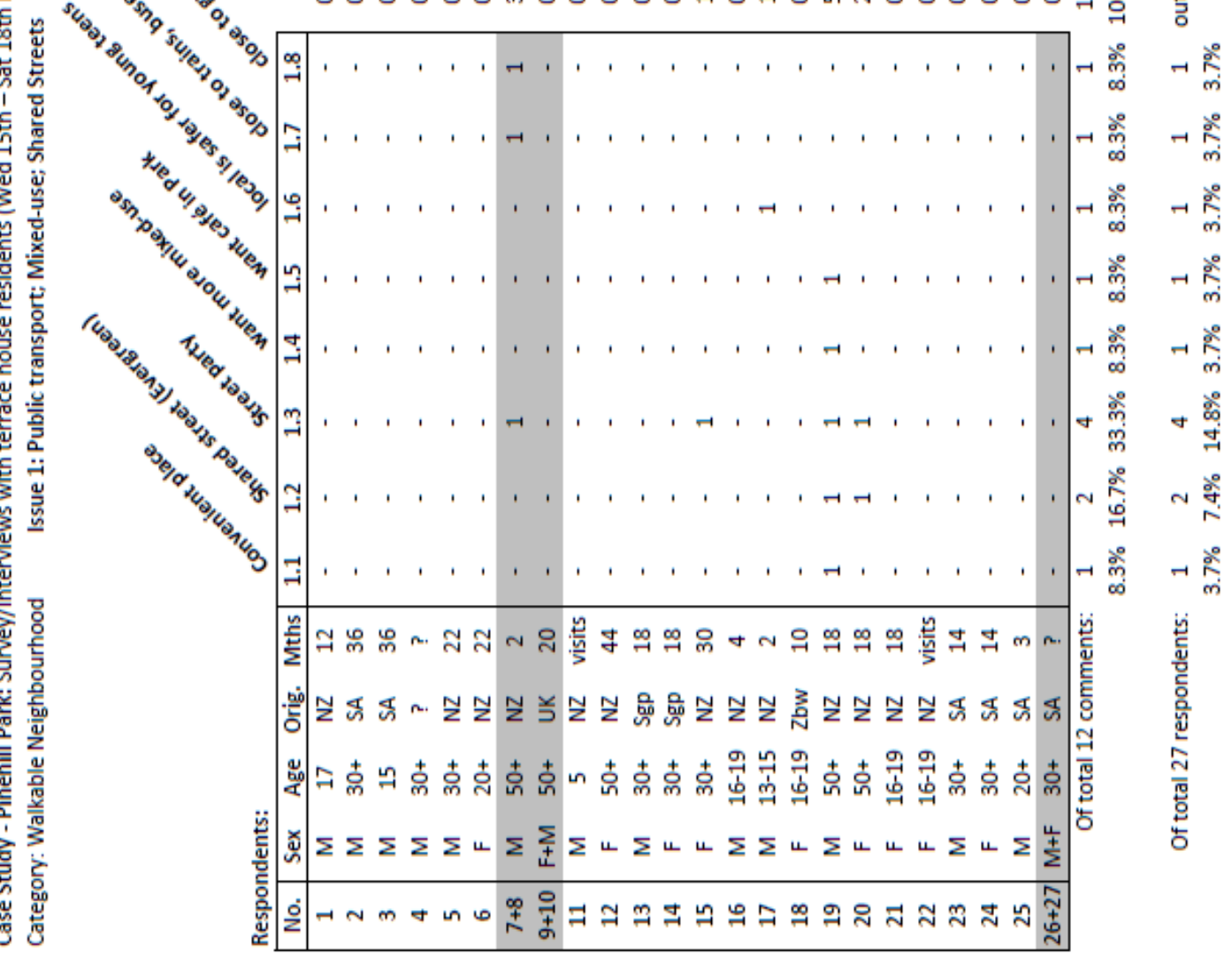




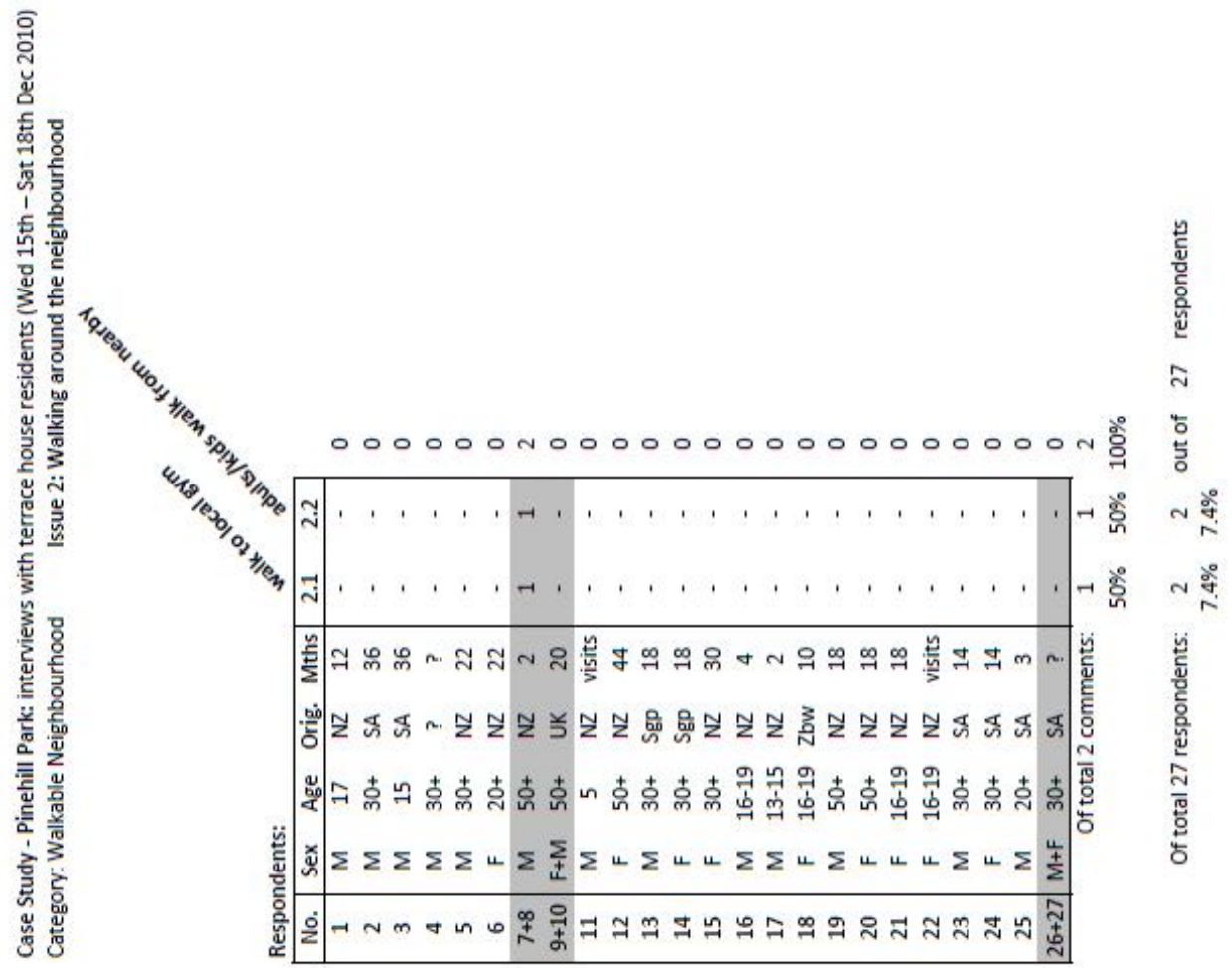




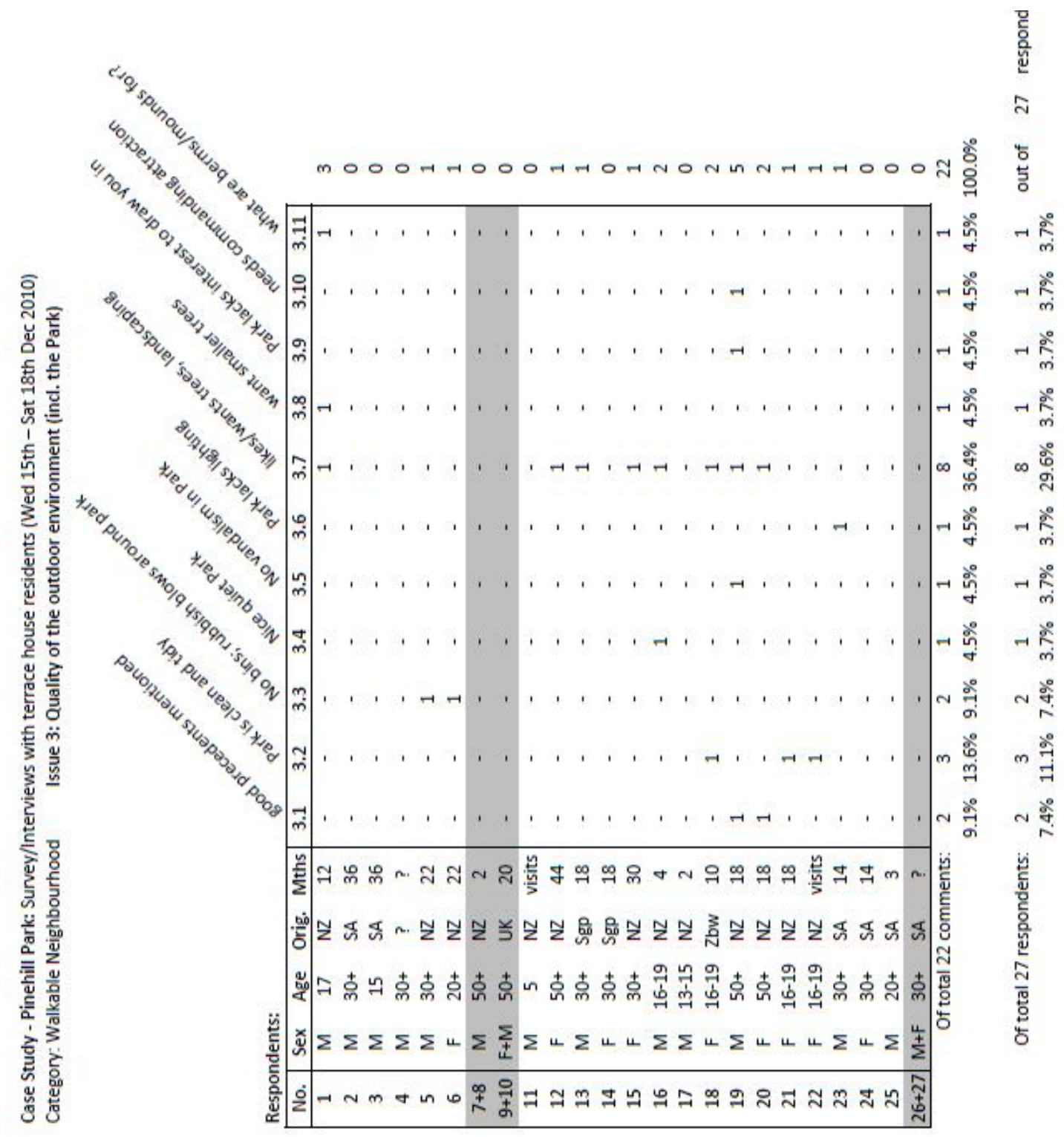




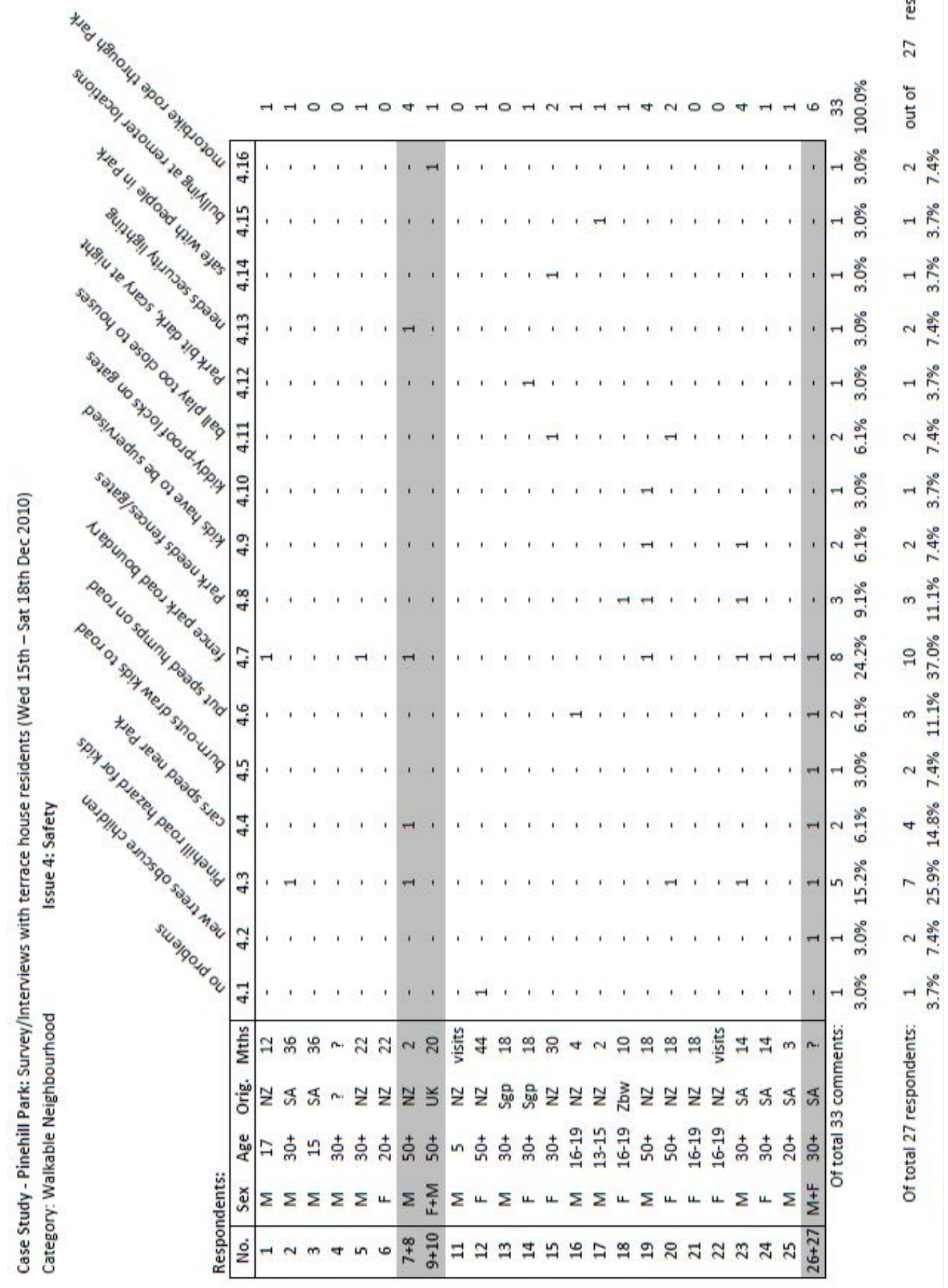




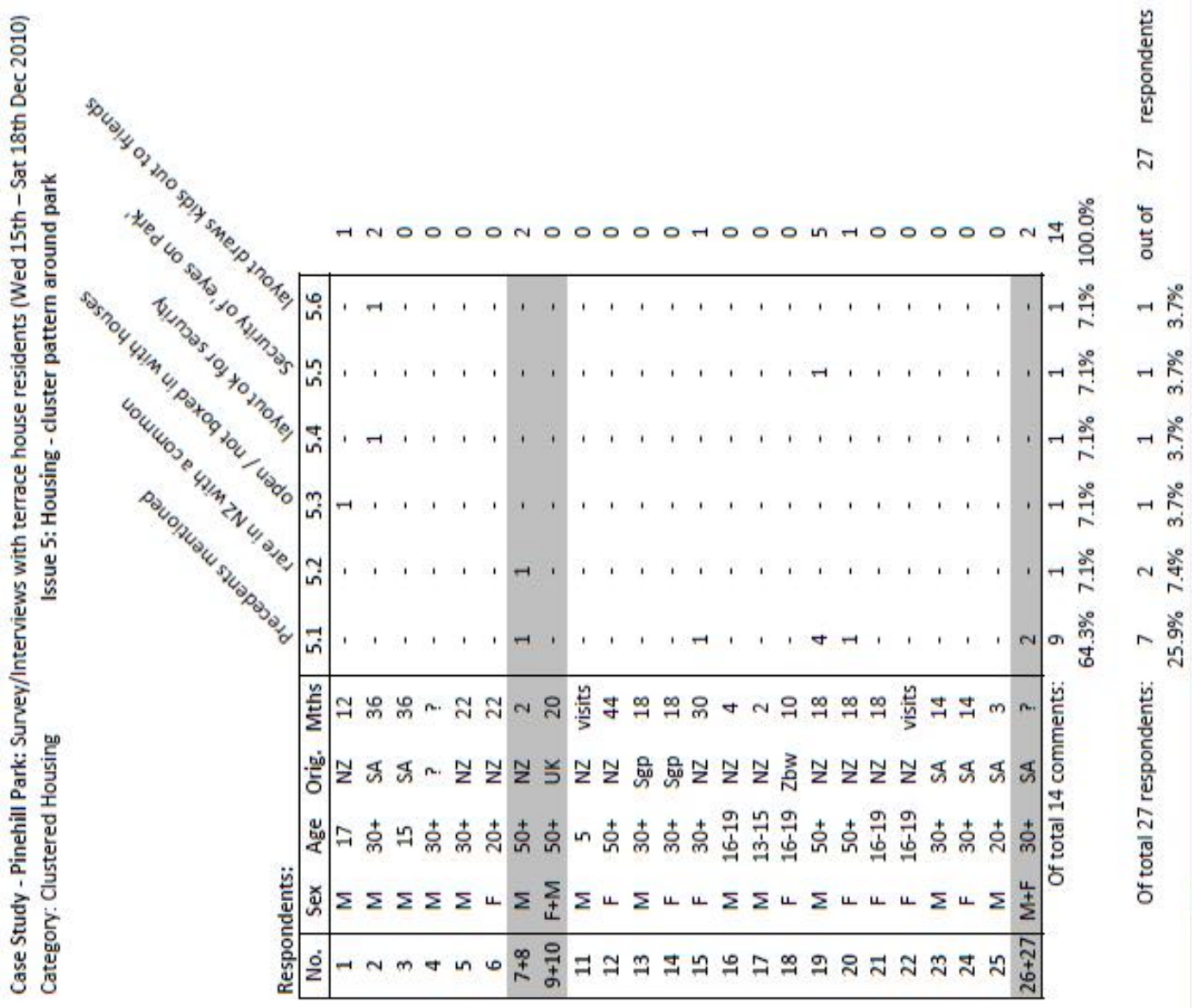




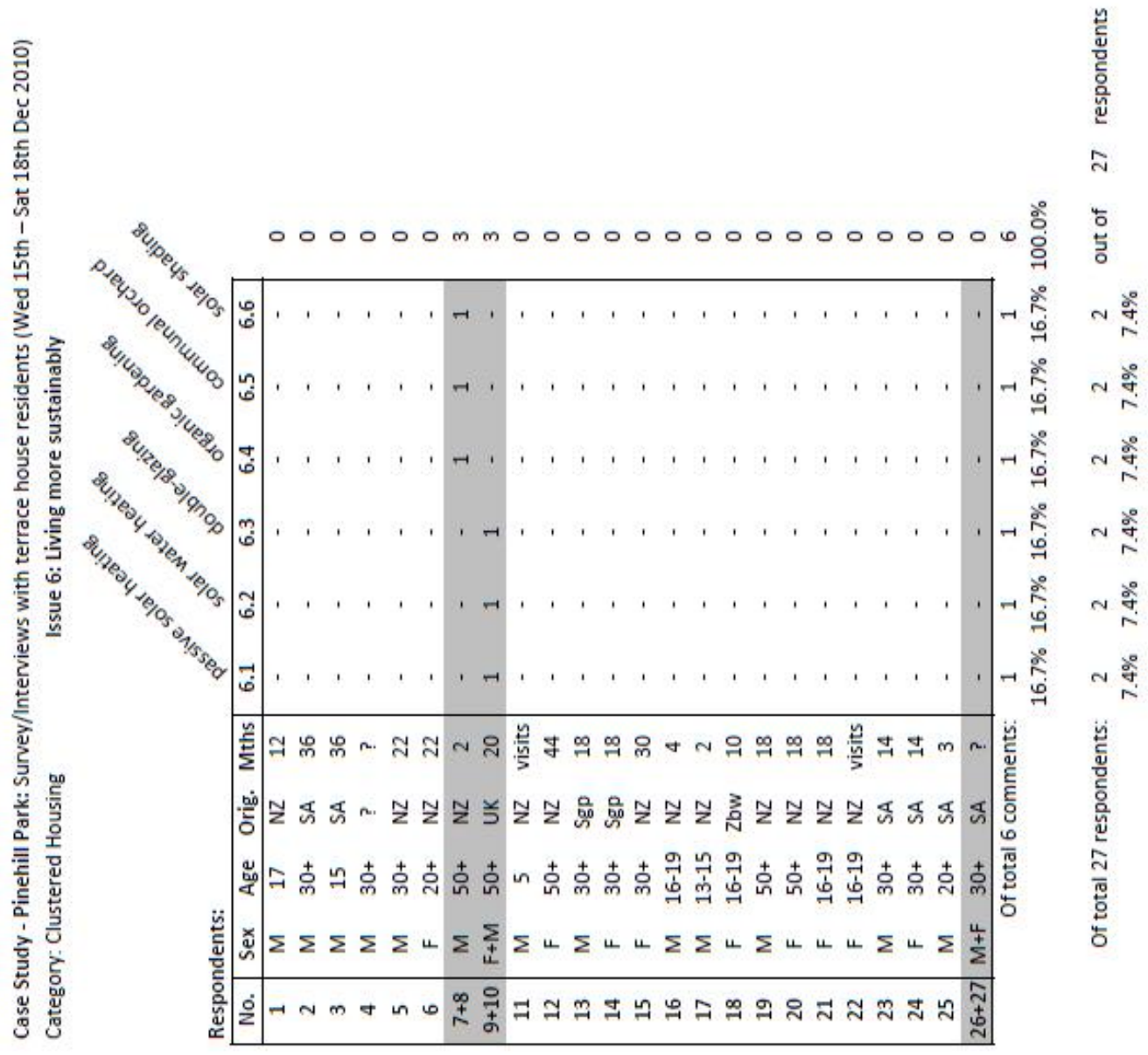




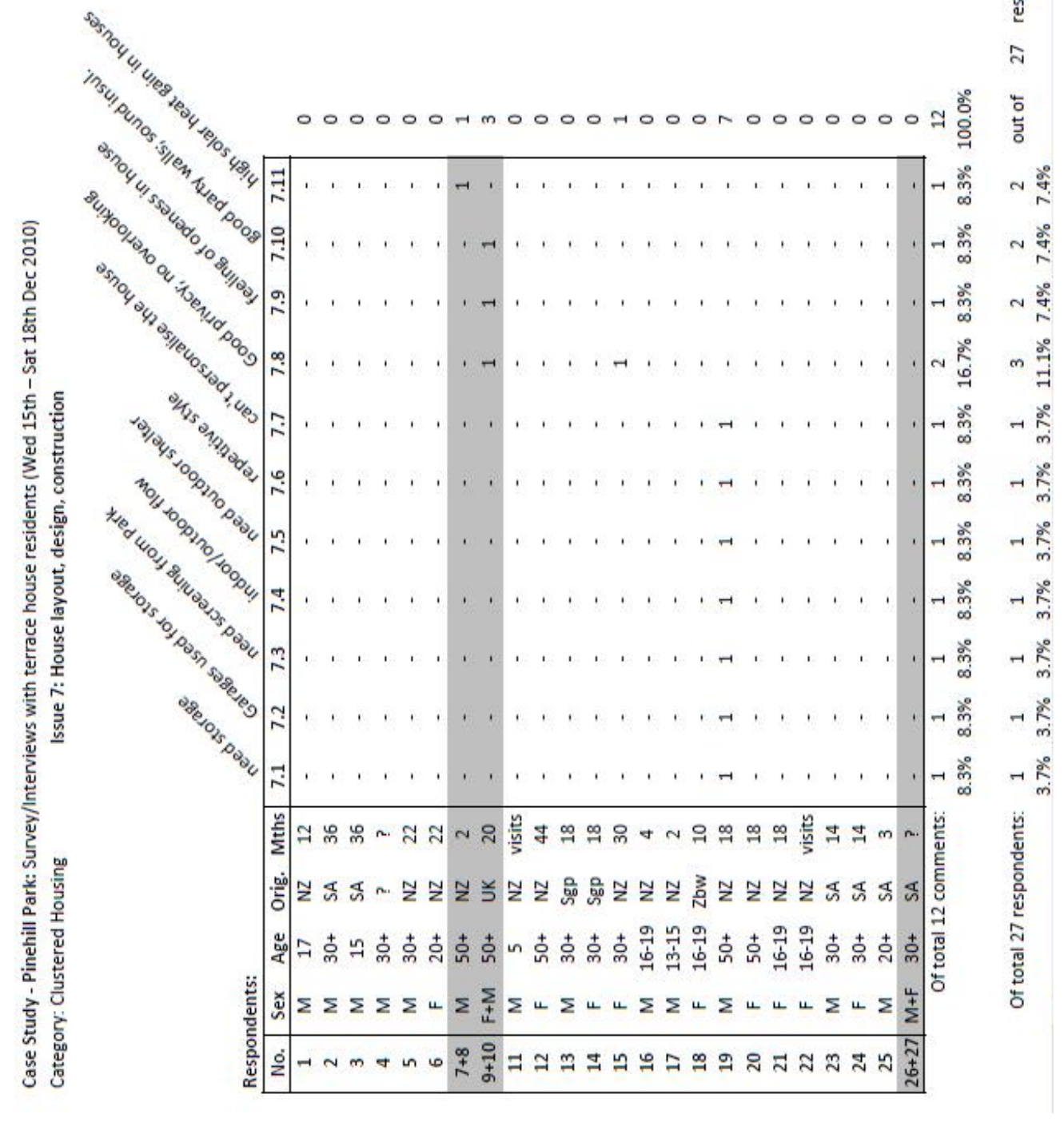




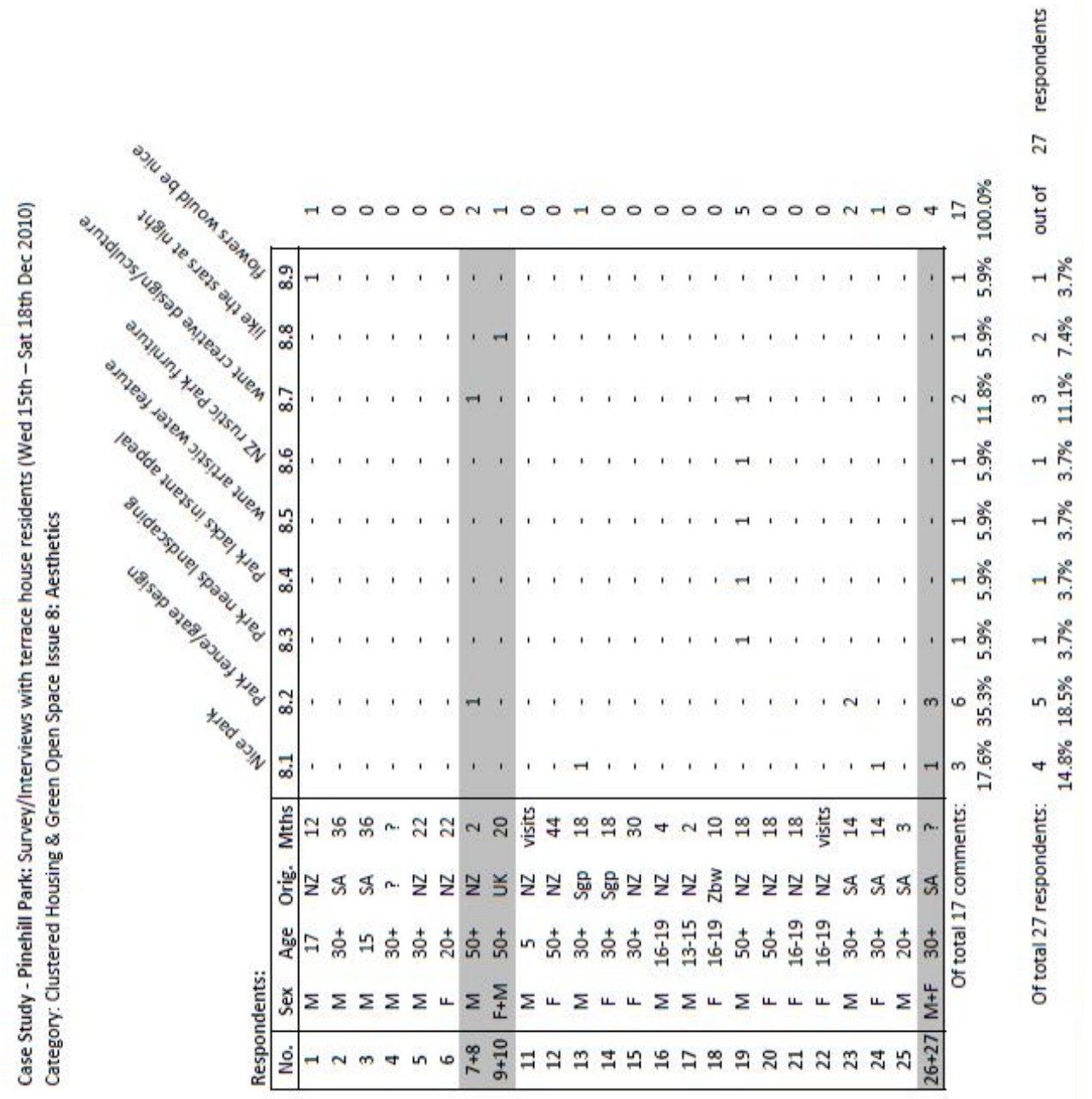




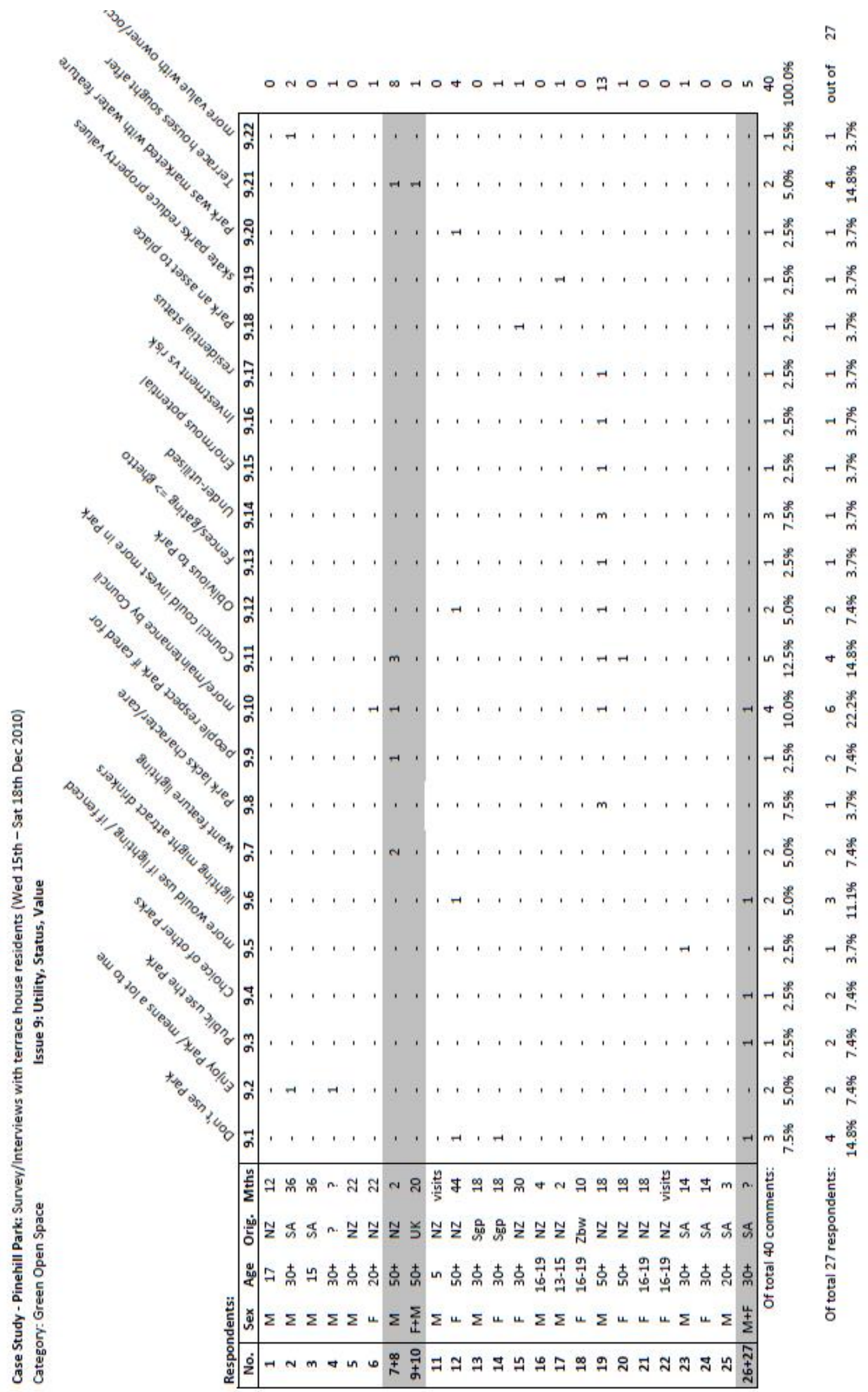




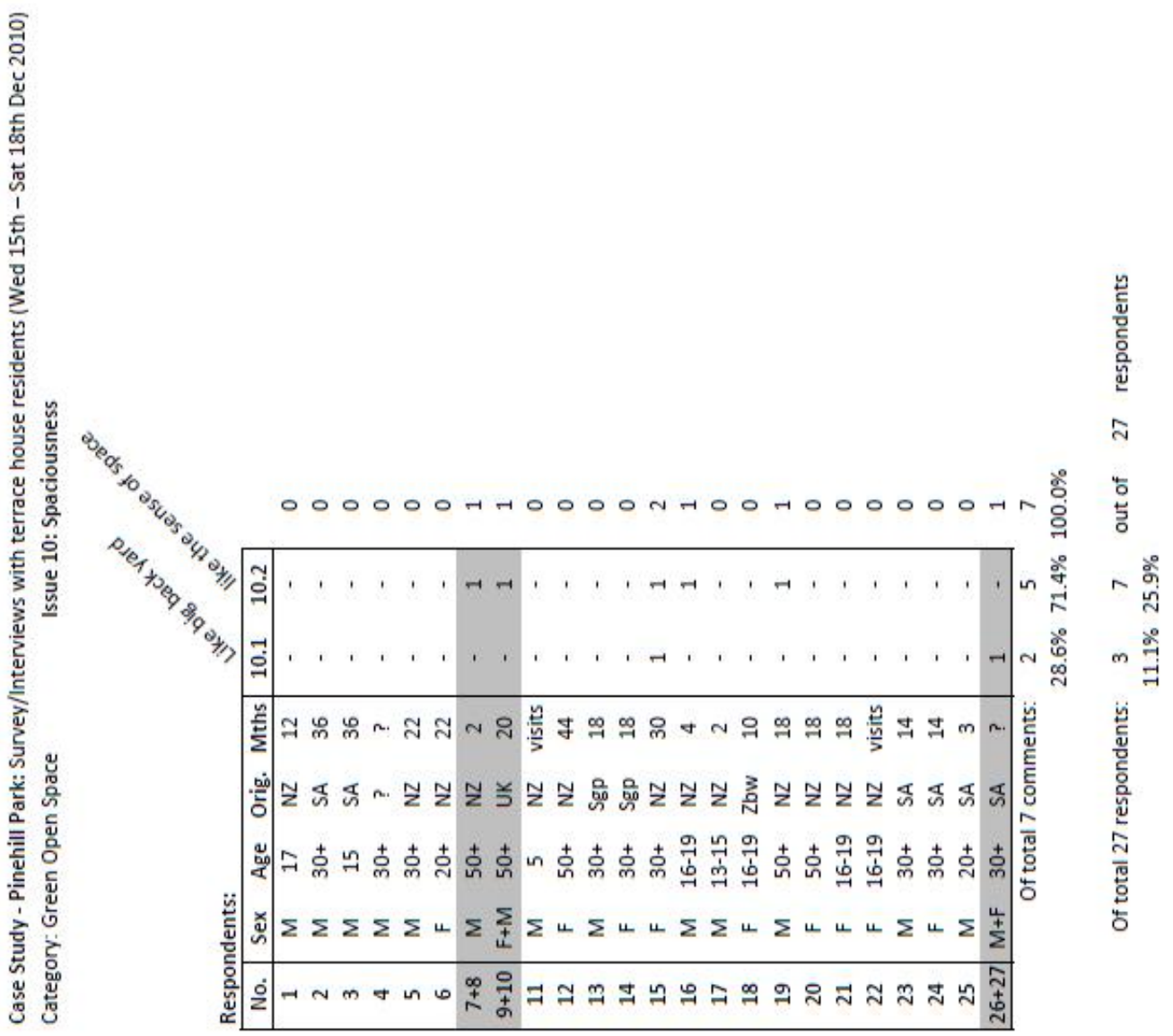




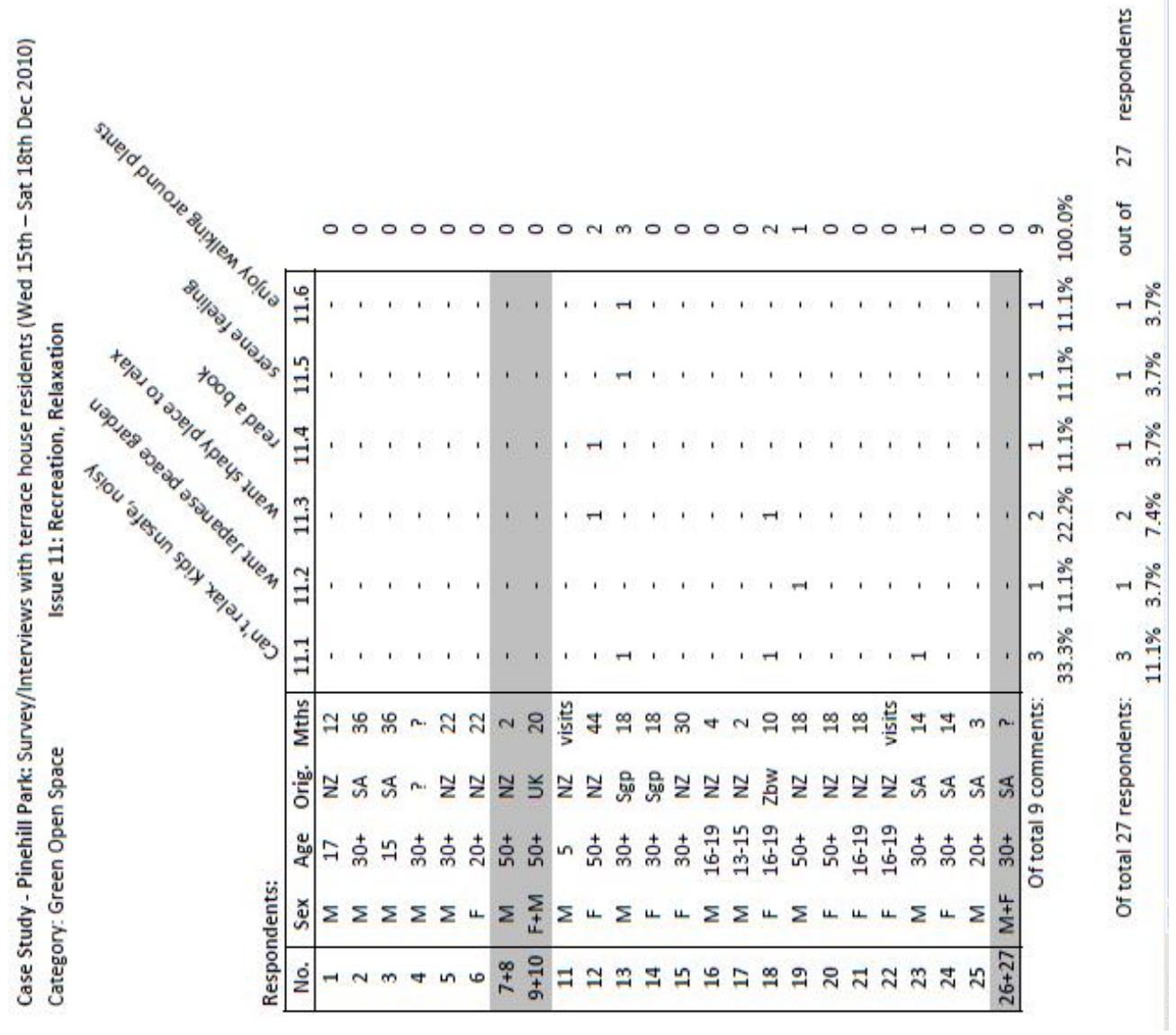




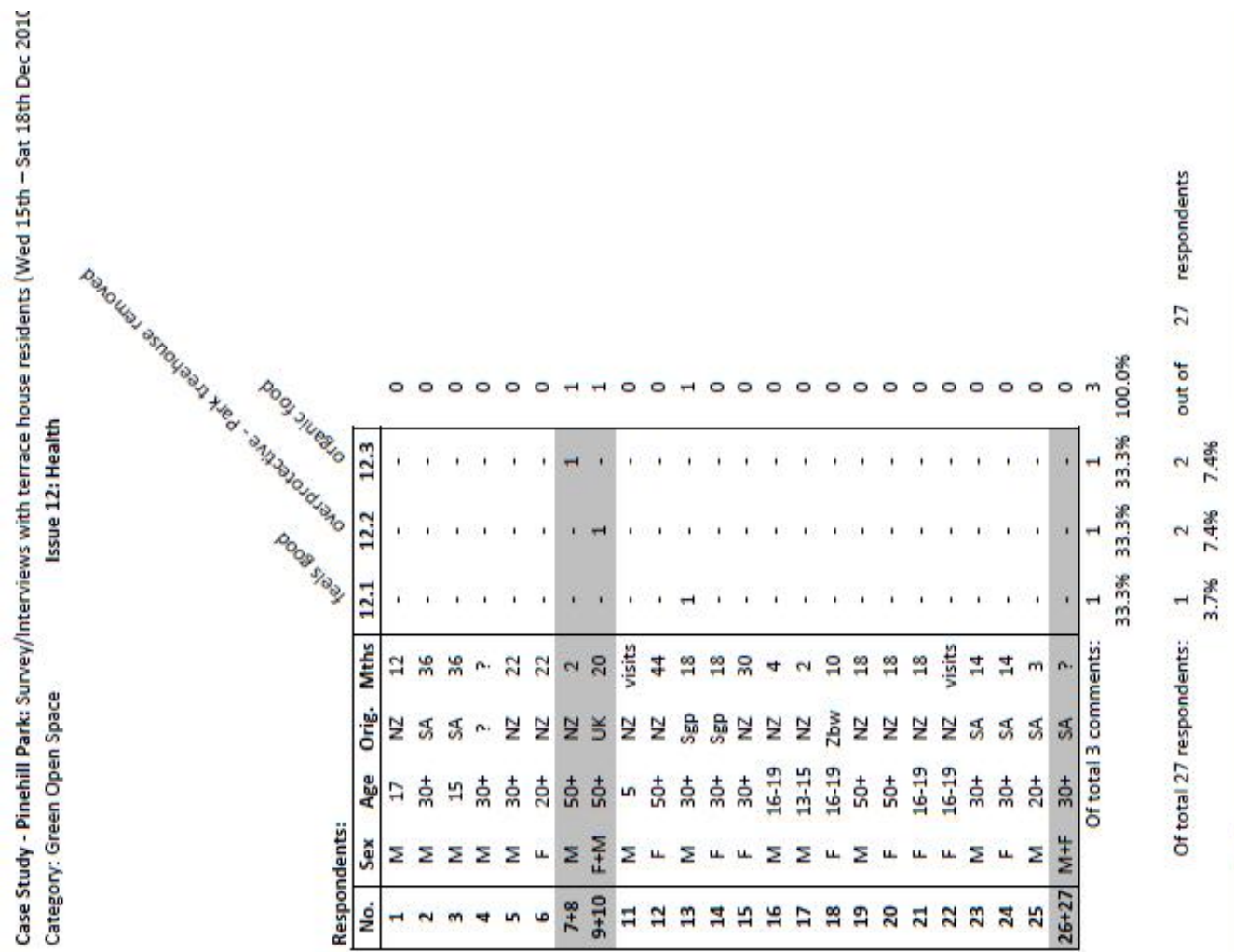




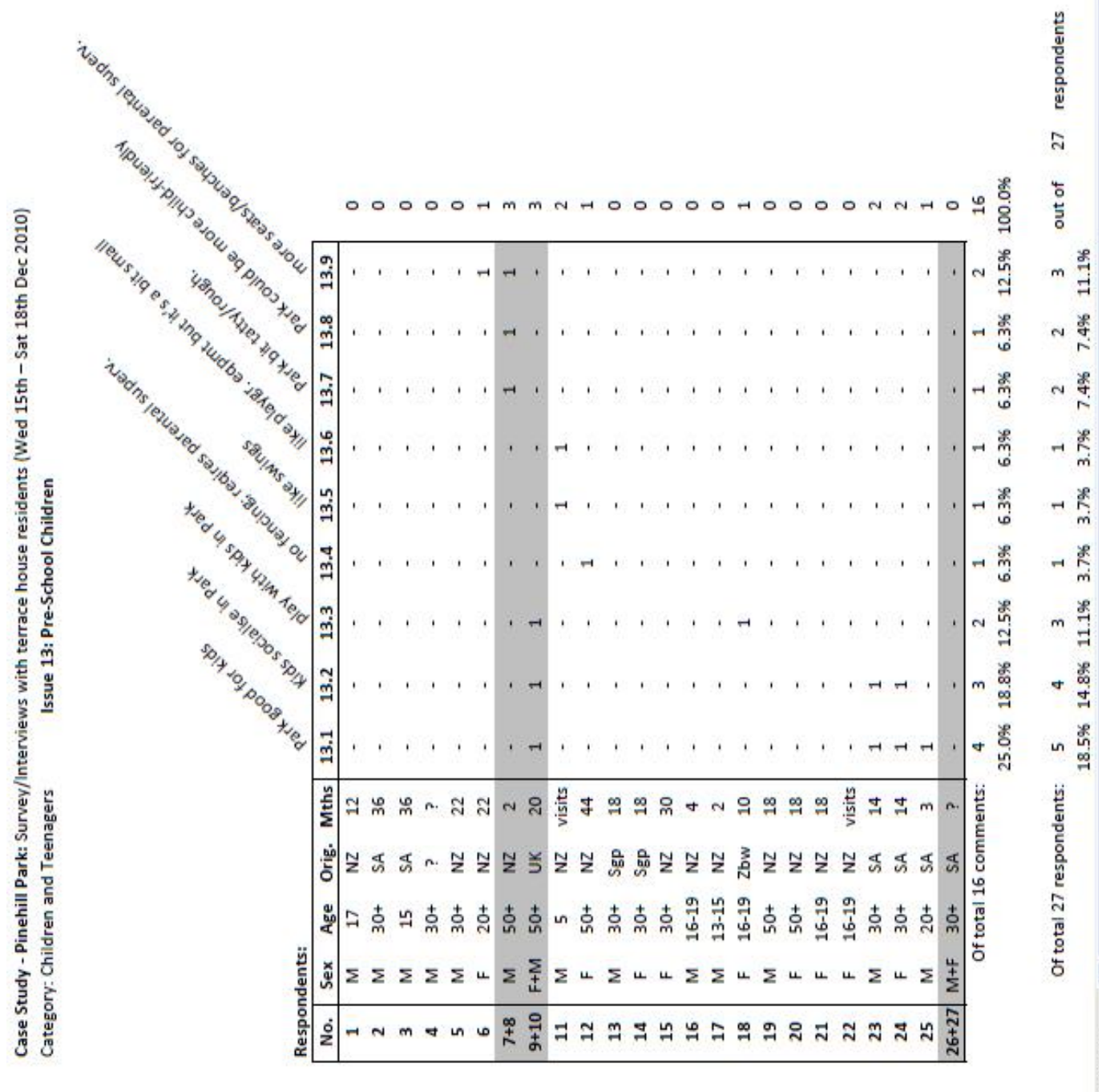




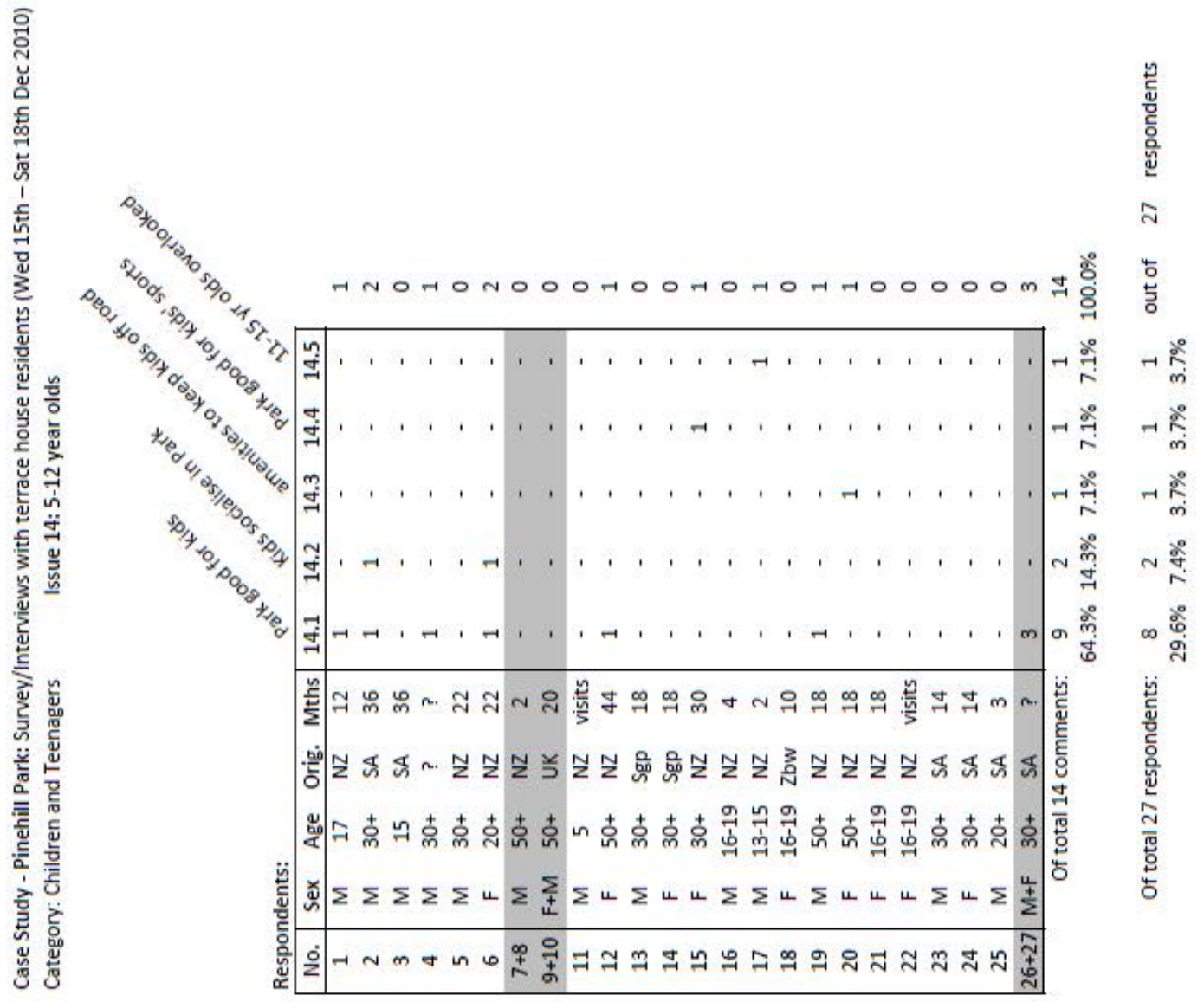




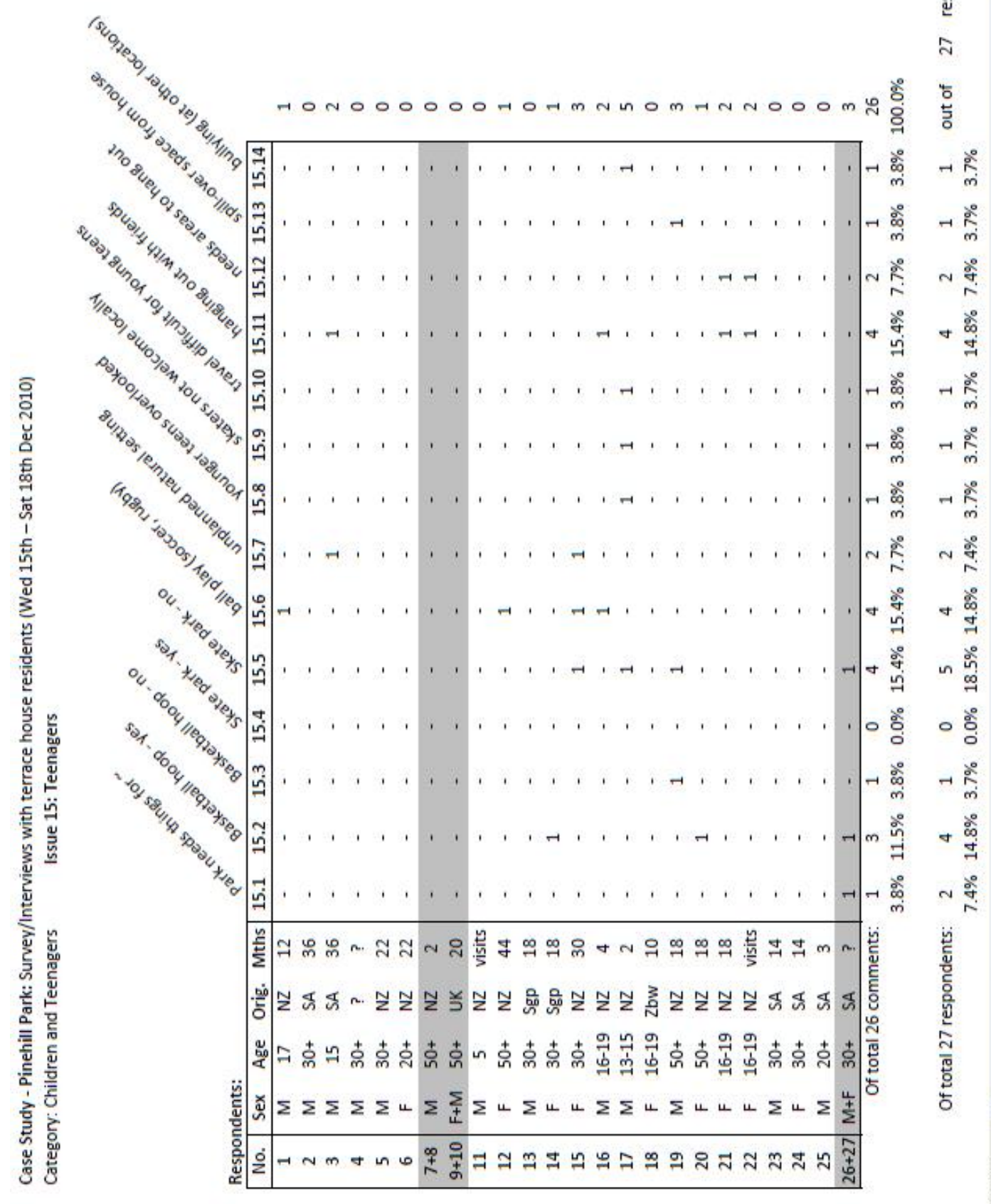




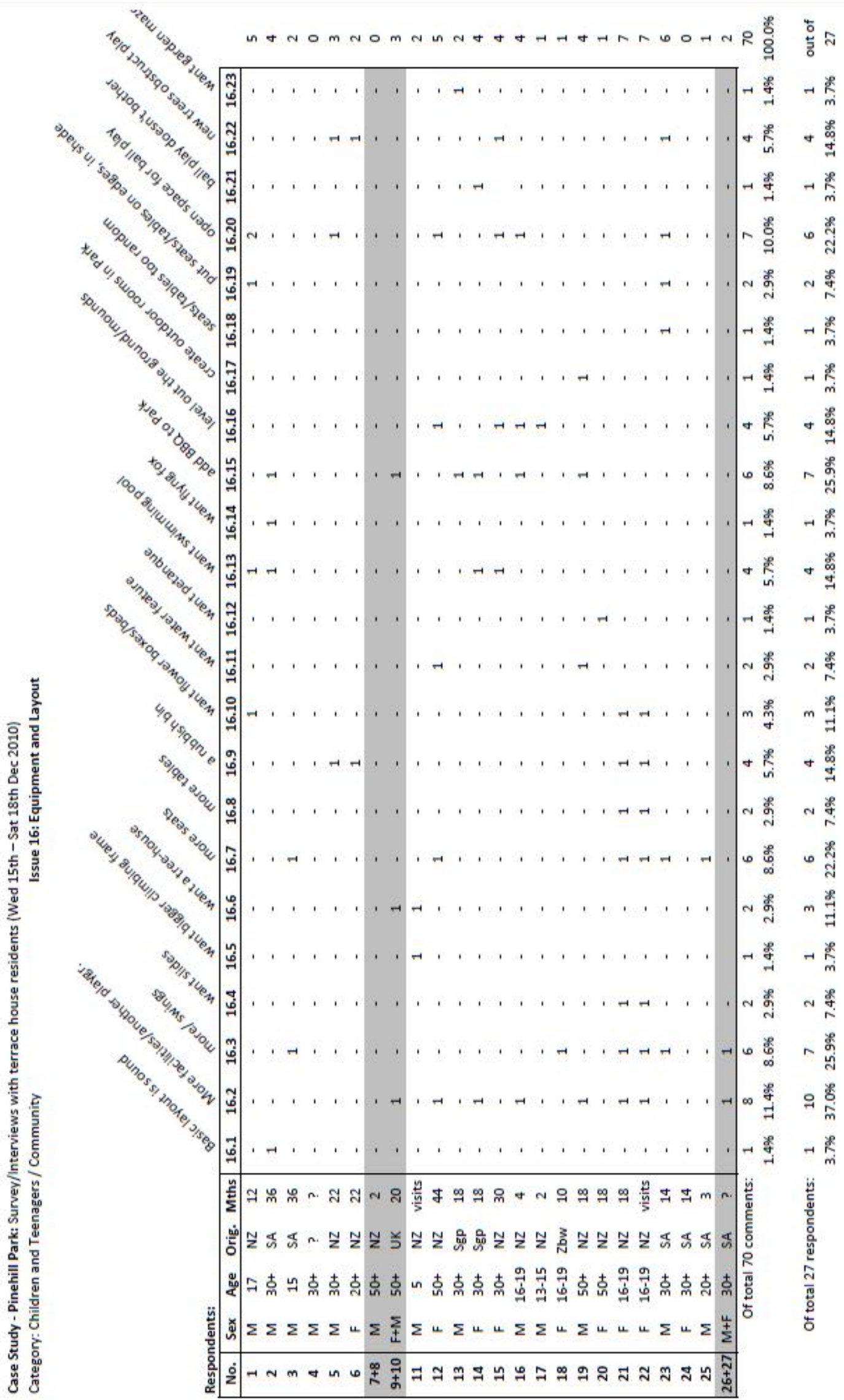




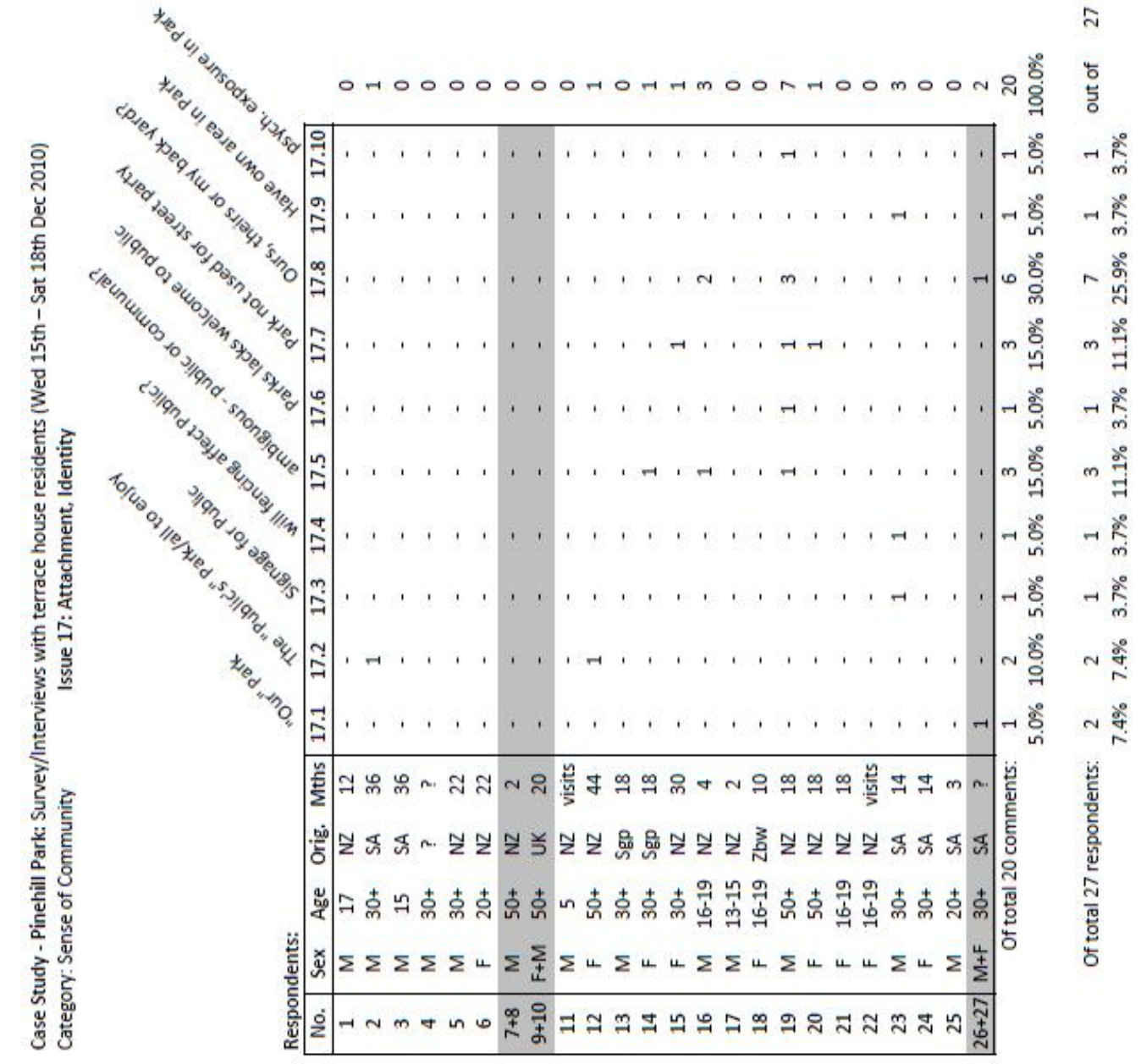




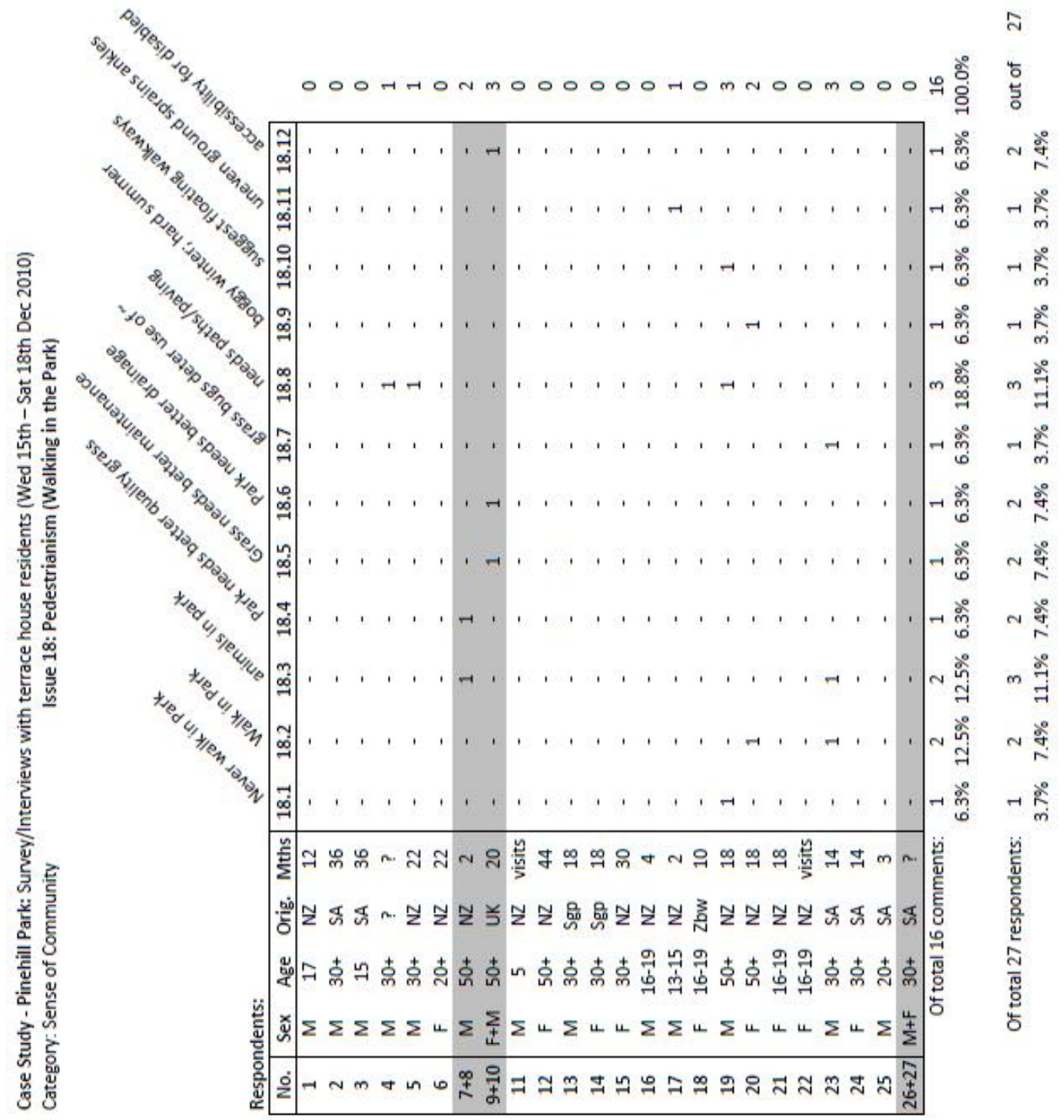




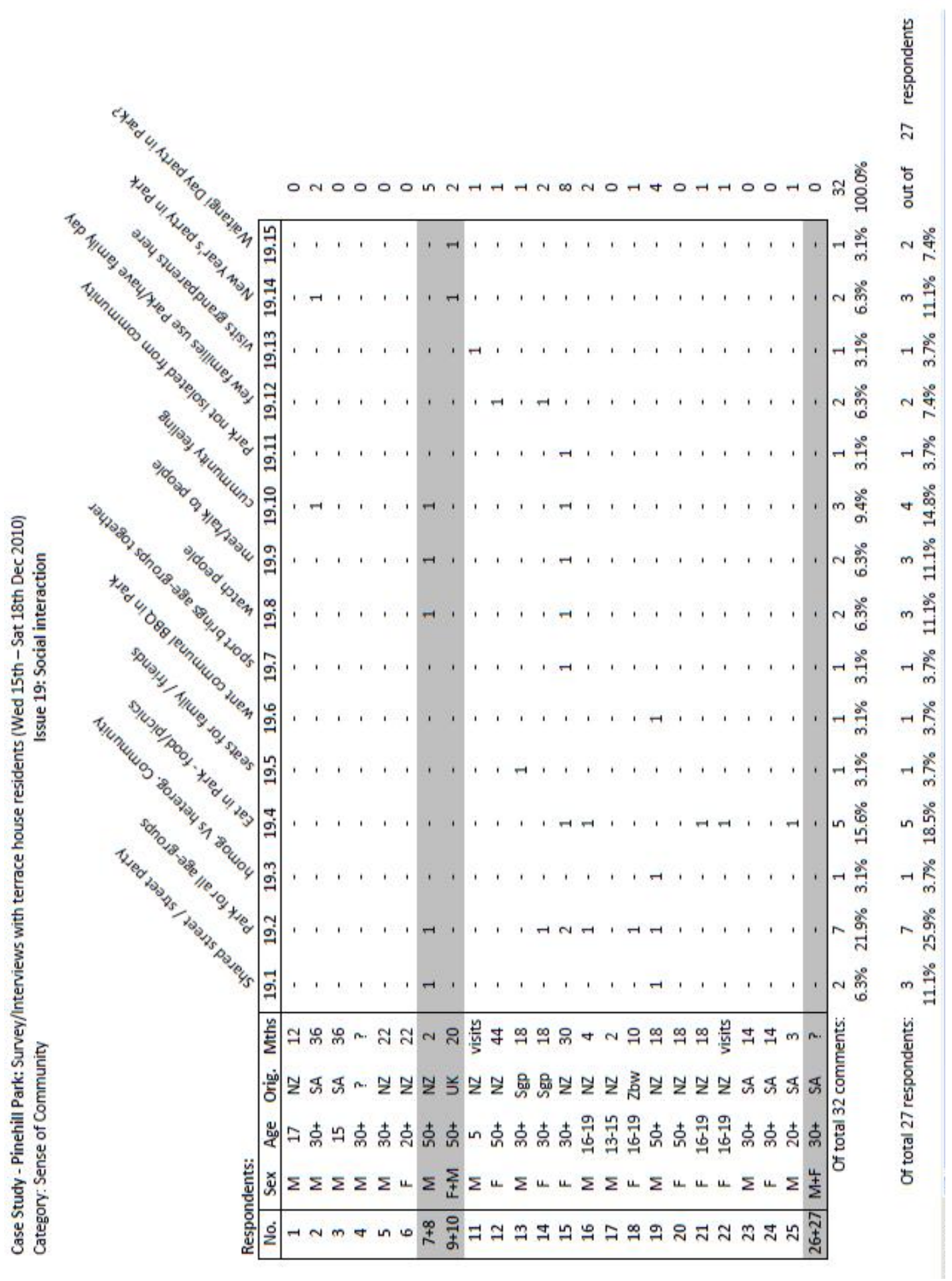




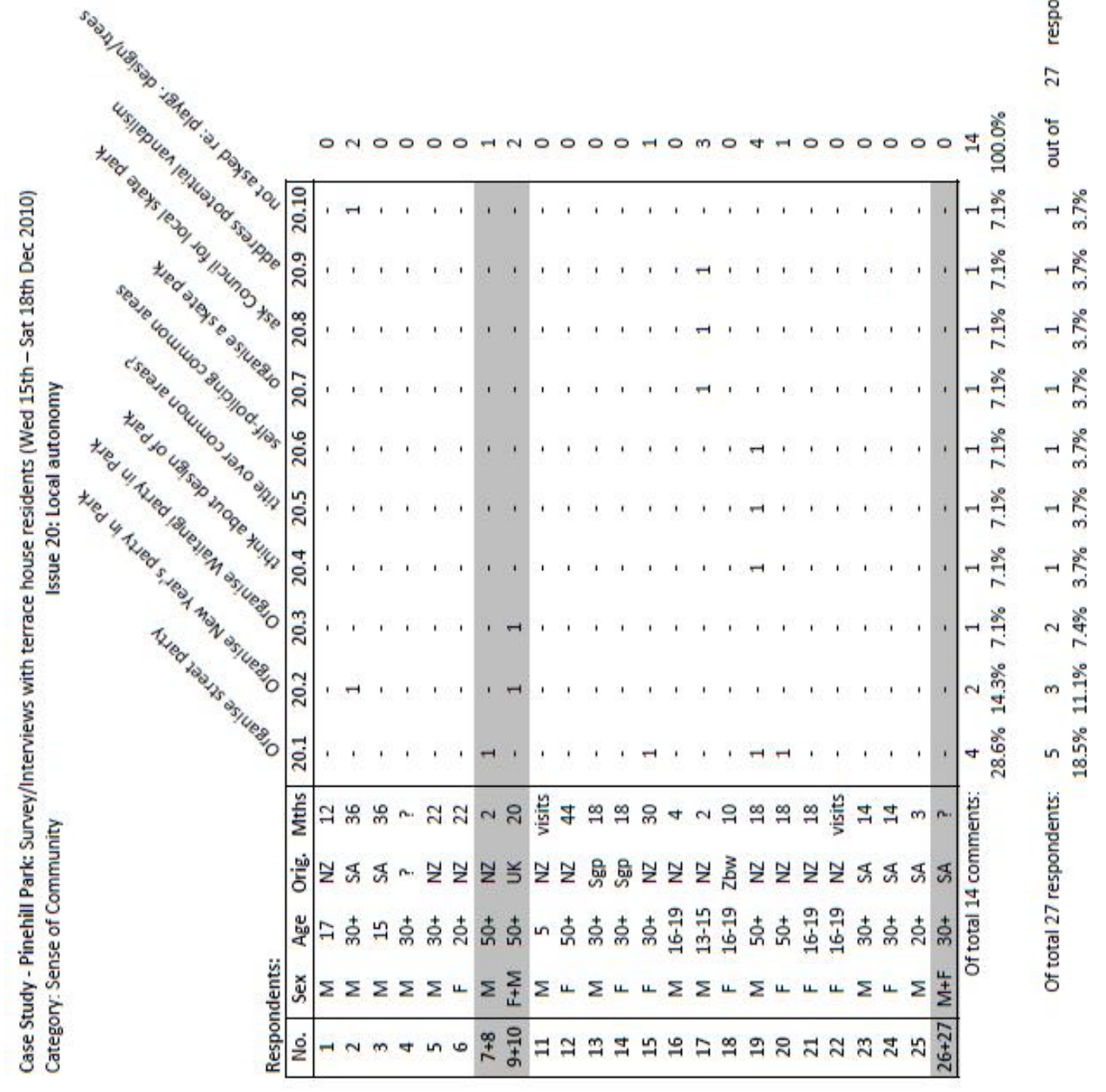




\section{Appendix 6: Ethics Approval Information Sheet \& \\ Consent Form}




\section{Participant Information Sheet for a Study of Communal Space in Medium Density Housing}

Researcher: Stephen Pattinson: School of Architecture, Victoria University of Wellington

This ethics statement has been approved by the Victoria University of Wellington Human Ethics Committee.

I am a Masters student carrying out research for a thesis for a Master of Architecture degree at Victoria University of Wellington on the topic of communal space in medium density housing in New Zealand:

- How important is communal space in medium density housing?

- What does quality communal space look like?

- How is it best delivered - by market mechanisms, by regulation, or some other way?

The University requires that ethics approval be obtained for research involving human participants. I am inviting people to participate in this study. Participants will be asked to respond to some questions related to medium density housing in general, and communal space in particular. The questions are mainly related to issues of quality and design, cost and implementation.

It is envisaged that responding to questions may take anywhere from 15 minutes to 90 minutes to complete, depending on the level of detail you may wish to provide.

Responses collected from interviews and discussions with participants will help form the basis of my research project and will be put into a written report, mostly on an anonymous basis. The default position is that data will be anonymous, unless the right is specifically waived by the participant. Information in the thesis will only be attributed to sources by name where participants give their prior informed consent.

All material collected will be kept confidential. No other person besides my supervisor, Morten Gjerde, and myself will see the information collected. The thesis will be submitted for marking to the School of Architecture and deposited in the University Library. A copy of the thesis will also be given to The New Zealand Centre for Sustainable Cities (Otago University, Wellington) which has provided some funding for this research.

It is intended that one or more articles will be submitted for publication in scholarly and professional journals, and may also be presented at seminars or conferences.

If you have any questions or would like to receive further information about the project, please contact

me at 04938455 or 0272263374 or my supervisor, Morten Gjerde, DDI ph. 04463 6233, at the School of Architecture at Victoria University, P O Box 600, Wellington.

Signed:

Stephen Pattinson 
VICTORIA UNIVERSITY OF WELLINGTON

CONSENT TO PARTICIPATE IN RESEARCH

\section{Common Ground: Communal Space in Medium Density Housing}

\section{Informed consent}

I have been given and have understood an explanation of this research project. I have had an opportunity to ask questions and have them answered to my satisfaction. I understand that I may withdraw myself (or any information I have provided) from this project at any time within 4 weeks after the interview is completed without having to give reasons or without penalty of any sort.

\section{Anonymity}

The information gathered in this research will be used anonymously in the thesis and in any publications in scholarly and professional journals, and any presentations at seminars or conferences (unless agreed otherwise with the participant beforehand in writing - see below). I understand that any information I provide will be kept confidential to the researcher, the supervisor and the person who transcribes the recordings of our interview. The published results will not use my name, and no opinions will be attributed to me in any way that will identify me. I understand that the recording of interviews will be electronically wiped at the end of the project unless I indicate that I would like them returned to me.

\section{Name attribution}

i) Where agreed with the participant beforehand in writing, information gathered in this research may be attributed to sources. I consent to information or opinions which I have provided being attributed to me in any reports of this research? Yes No

ii) Regarding any information that I have agreed to have attributed to me, I would like an opportunity to review it before the thesis is submitted? Yes No

iii) What I want done with the information I have provided after this thesis is submitted: The researcher keep it in secure file storage for future reference? Yes No Return it to me? Destroy it?

I understand that the data I provide will not be used for any other purpose or released to others without my written consent.

I agree to take part in this research

[or I agree that , who is under my guardianship, may take part in this research]

Signed:

Name of participant

(Please print clearly)

Date: 


\section{Appendix 7.1: List of Sites Visited for Thesis Research}


Stephen Pattinson - VUW M.Arch thesis research

APPENDIX 7.1 - List of medium- to high- density housing sites visited for thesis research on communal space:

\begin{tabular}{|c|c|c|c|c|}
\hline No. & Housing Scheme & Address & Communal space(s) & When built \\
\hline & AUCKLAND REGION & NEW ZEALAND & & \\
\hline & RODNEY & & & \\
\hline A01 & Whisper Cove & Arabella Lane, Snells Beach & Landscaped wetland & $2008 ?$ \\
\hline A02 & Gulf Harbour Village & Gulf Harbour Drive, Whangaparoa Peninsula & Marina, restaurant, retail square & \\
\hline A03 & Ocean Park & Pinecresr Drive, Gulf Harbour & Golf course & \\
\hline A04 & Cape Cod & Cape Cod Drive, Gulf Harbour & Golf course & \\
\hline A05 & Greenway & Greenway Rise, Gulf Harbour & Golf course, small lake & \\
\hline A06 & Alverna Heights & Alverna Heights View, Gulf Harbour & Golf course & \\
\hline \multirow[t]{2}{*}{ A07 } & Kensington Park & Off Puriri Boulevard, Orewa & $\begin{array}{l}\text { Natural stream, native bush, lawns, } \\
\text { gardens, indoor swimming pool }\end{array}$ & $2008 ?$ \\
\hline & NORTH SHORE & & & \\
\hline A08 & $\begin{array}{l}\text { Primary school } \\
\text { (of interest because of its } \\
\text { bay-form layout) }\end{array}$ & 126 Oteha Valley Road & $\begin{array}{l}\text { Classrooms linked in crescent form } \\
\text { around } 2 \times \text { basketball courts on } \\
\text { edge of school playground }\end{array}$ & \\
\hline A09 & Retirement Village & Mayfair Drive, Northcross & Bowling green, petanque & \\
\hline A10 & Town housing (linked) & Cnr Fields Parade and Gleanor Avenue, Oteha & $\begin{array}{l}\text { None (adjacent to green open space } \\
\text { and children's small playground) }\end{array}$ & \\
\hline A11 & Town housing (linked) & 29 Fields Parade,Oteha & None & \\
\hline A12 & Town housing (linked) & 1- 5 John Jennings Drive, Oteha & Lawn & \\
\hline A13 & Town housing (close) & 2-26 John Jennings Drive, Oteha & None & \\
\hline A14 & Town housing (linked) & 28 - 30 John Jennings Drive, Oteha & Swimming pool and $\mathrm{BBQ}$ & \\
\hline A15 & Town housing (linked) & 36 - 40 John Jennings Drive, Oteha & None & \\
\hline A16 & Town housing (linked) & 7 - 15 John Jennings Drive, Oteha & None & \\
\hline A17 & Town housing (linked) & 15 Andersons Road, Oteha & Lawn & \\
\hline A18 & Town housing (linked) & Cnr Fields Parade and Nimstedt Avenue, Oteha & Swimming pool & \\
\hline A19 & $\begin{array}{l}\text { Town housing (close) and } \\
\text { town housing (linked) }\end{array}$ & Fields Parade and Sunnydale Place,Oteha & $\begin{array}{l}\text { None, adjacent to Bays City Park } \\
\text { (sports fields) and a primary school }\end{array}$ & \\
\hline A20 & $\begin{array}{l}\text { Town housing (close) and } \\
\text { terrace housing (linked) }\end{array}$ & 22 Northcross Drive, Oteha & $\begin{array}{l}\text { Swimming pool on site. Adjacent to } \\
\text { sports fields }\end{array}$ & \\
\hline
\end{tabular}




\begin{tabular}{|c|c|c|c|c|}
\hline A21 & Fairview retirement village & Off Fairview Avenue,Oteha & $\begin{array}{l}\text { Outdoors: Lawns, gardens, bowling } \\
\text { greens, petanque, vegetable } \\
\text { gardens and nursery } \\
\text { Indoors: dining, lounge, dance floor, } \\
\text { stage/music, cinema, bar, billiards, } \\
\text { library, cards/games, gym/weights, } \\
\text { sauna, indoor swimming pool and } \\
\text { spa, workshop }\end{array}$ & \\
\hline A22 & Town housing (close) & $\begin{array}{l}\text { Cnr Medallion Drive and Fernhill Way, Oteha } \\
\text { (i.e. Candlestick Place off Fields Parade) }\end{array}$ & $\begin{array}{l}\text { Wetland pond, children's } \\
\text { playground }\end{array}$ & \\
\hline A23 & Town housing (linked) & $70-76$ Fernhill Way, Oteha & none & \\
\hline A24 & Town housing (linked) & 78 \& 86 Fernhill Way, Oteha & none & \\
\hline A25 & Town housing (linked) & 71 - 77 Fernhill Way, Oteha & none & \\
\hline A26 & Town housing (linked) & 8 \& 12 Pannill Place, Oteha & none & \\
\hline A27 & Town housing (linked) & 16 \& 26 Pannill Place, Oteha & none & \\
\hline A28 & Town housing (linked) & 25 Pannill Place, Oteha & none & \\
\hline A29 & Town housing (linked) & 7-17 Pannill Place, Oteha & none & \\
\hline A30 & Town housing (linked) & 1 Pannill Place, Oteha & none & \\
\hline A31 & Terrace housing & 60 Masons Road, Oteha & Open green space & \\
\hline A32 & Terrace housing & 71 Spencer Road, Oteha & Open green space & \\
\hline A33 & Takapuna Motor Lodge & 52 Taharoto Drive, Takapuna & Swimming pool, lawn, playground & \\
\hline A34 & Terrace housing & 177 Hurstmere Road, Takapuna & $\begin{array}{l}\text { Housing arranged in C-shape around } \\
\text { mature tree and swimming pool }\end{array}$ & \\
\hline A35 & Mon Desir Apartments & $\begin{array}{l}\text { Cnr 171-175 Hurstmere Rd \& } 5 \text { The Promenade, } \\
\text { Takapuna }\end{array}$ & $\begin{array}{l}\text { Swimming pool, lawns and mature } \\
\text { trees, beach access }\end{array}$ & \\
\hline A36 & Terrace blocks on hillside & 20 Ian Marwick Place, Birkenhead & $\begin{array}{l}\text { Common paths. Lower blocks face } \\
\text { stream hidden in native bush } \\
\text { reserve }\end{array}$ & \\
\hline A37 & Blocks of linear units & 5 Waipa Street, Birkenhead & none & 1960s? \\
\hline A38 & $\begin{array}{l}\text { Blocks around central loop } \\
\text { access drive }\end{array}$ & 37 Waipa Street, Birkenhead & Central lawns, swimming pool & \\
\hline A39 & Townhousing (linked) & 41 Waipa Street, Birkenhead & $\begin{array}{l}\text { None. Adjacent to extensive native } \\
\text { bush reserve }\end{array}$ & \\
\hline A40 & HillsideTownhouse Blocks & 55 Verbena Road, Birkenhead & None & \\
\hline
\end{tabular}




\begin{tabular}{|c|c|c|c|c|}
\hline A41 & The Castle Glade & 8 Flynn Street, Birkenhead & $\begin{array}{l}\text { Blocks of terrace houses on hillside } \\
\text { surrounded by native bush reserve. } \\
\text { Swimming pool. }\end{array}$ & \\
\hline A42 & Retirement detached units & John Bracken Way, Beach Haven & $\begin{array}{l}\text { None. Cul-de-sac. Adjacent to } \\
\text { extensive sports fields, park, with } \\
\text { outdoor bowls, tennis club nearby }\end{array}$ & \\
\hline A43 & $\begin{array}{l}\text { Terrace housing around } \\
\text { triangular vehicle court }\end{array}$ & 118 - 122 Beach Haven Road, Beach Haven & Swimming pool & \\
\hline A44 & Town housing (linked) & 92 Bush Road, Albany & $\begin{array}{l}\text { Tennis court, lawn, trees, swimming } \\
\text { pool }\end{array}$ & \\
\hline A45 & Terrace housing & 5 -53 Carolina Place, Albany & Swimming pool, communal building & \\
\hline A46 & Terrace housing & $\begin{array}{l}\text { Cnr Point Ridge Avenue } \& 35-49 \text { Genoa Way, } \\
\text { Albany Heights }\end{array}$ & $\begin{array}{l}\text { Clustered in crescent form around } \\
\text { common lawn, swimming pool, BBQ } \\
\text { and communal building }\end{array}$ & \\
\hline A47 & Terrace housing & 11 The Avenue, Albany Heights & $\begin{array}{l}\text { Swimming and lap pools, B-B-Q, } \\
\text { gazebo, tennis court, lawn }\end{array}$ & \\
\hline A48 & Town housing (close) & $\begin{array}{l}34-74 \& 35-75 \text { Lucas Point Road, } \\
\text { Albany Village }\end{array}$ & Swimming pool & \\
\hline A49 & Town housing (linked) & 427 Albany Highway, Albany & $\begin{array}{l}\text { Tennis court, swimming pool, BBQ, } \\
\text { gazebo, lawn, playground, petanque }\end{array}$ & \\
\hline \multirow[t]{2}{*}{ A50 } & Town housing (linked) & 437 Albany Highway, Albany & Tennis court, lawn & \\
\hline & WAITAKERE CITY & & & \\
\hline A51 & Hobsonville Landing & Hobsonville & Wharves, Landing, Coastal area & $2009 / 10$ \\
\hline A52 & Hobsonville Point & Hobsonville & $\begin{array}{l}\text { Green open spaces in medium- } \\
\text { density housing developments }\end{array}$ & \\
\hline A53 & Terrace housing & 15-17 Harbour View Rd, Te Atatu Peninsula & $\begin{array}{l}\text { None (adjacent to green open } \\
\text { space) }\end{array}$ & \\
\hline A54 & $\begin{array}{l}\text { Various medium density } \\
\text { developments (terrace } \\
\text { housing etc) }\end{array}$ & Gunner Drive, Te Atatu Peninsula & $\begin{array}{l}\text { Various green open spaces } \\
\text { throughout subdivision plus coastal } \\
\text { mangrove wetland }\end{array}$ & \\
\hline A55 & Earthsong & 457 Swanson Road, Ranui & Various green open spaces & \\
\hline A56 & $\begin{array}{l}\text { Waitakere Gardens } \\
\text { Retirement Village }\end{array}$ & Sel Peacock Drive, Henderson & $\begin{array}{l}\text { Wide range of indoor and outdoor } \\
\text { communal spaces }\end{array}$ & \\
\hline A57 & Tuscany Towers & 1 Ambrico Place, New Lynn & Green open space and tennis courts & \\
\hline
\end{tabular}




\begin{tabular}{|c|c|c|c|c|}
\hline A58 & Town housing & 3 Ambrico Place, New Lynn & None other than central car court & \\
\hline A59 & Town housing & $\begin{array}{l}4 \& 6 \text { Ambrico Place, New Lynn (adjacent to old } \\
\text { brickworks relic and to green open space and } \\
\text { children's playground) }\end{array}$ & None other than central car court & \\
\hline A60 & Town housing & 12 \& 14 Ambrico Place, New Lynn & $\begin{array}{l}\text { None. (Adjacent to green open } \\
\text { space and children's playground) }\end{array}$ & \\
\hline A61 & Town housing & 1C Rankin Ave, New Lynn & None. (Adjacent to railway lines) & \\
\hline A62 & Town housing & 10-17 Crown Lyn Place, New Lynn & $\begin{array}{l}\text { Green open space, swimming pool } \\
\text { and tennis court }\end{array}$ & \\
\hline A63 & Town housing & 56 Rata Street, New Lynn & $\begin{array}{l}\text { Some green open space (on edge of } \\
\text { mangrove tidal creek }\end{array}$ & \\
\hline A64 & Town housing & 2 Arawa Street, New Lynn & None (along edge of bush section) & \\
\hline A65 & Town housing & 1 Melview Place, New Lynn & None (Adjacent to tidal wetland) & \\
\hline \multirow[t]{2}{*}{ A66 } & Town housing & 8 Margan Avenue, New Lynn & $\begin{array}{l}\text { None (Adjacent to tidal wetland and } \\
\text { green open space) }\end{array}$ & \\
\hline & AUCKLAND CENTRAL & & & \\
\hline A67 & Beaumont Quarter & 18 - 20 Beaumont Street, Auckland Central & $\begin{array}{l}\text { Gym, indoor swimming pool, several } \\
\text { green open spaces }\end{array}$ & \\
\hline A68 & Apartments & 10 Beaumont Street, Auckland Central & $\begin{array}{l}\text { Swimming pool, landscaped } \\
\text { courtyard }\end{array}$ & \\
\hline A69 & Auckland Viaduct Harbour & Waterfront, Auckland Central & Waterfront boulevard & \\
\hline A70 & Princes Wharf apartments & Princes Wharf, Auckland Central & & \\
\hline A71 & $\begin{array}{l}\text { The Quays \& Sebel } \\
\text { apartments }\end{array}$ & Auckland Viaduct Harbour, Auckland Central & Outdoor cafe & \\
\hline A72 & The Parc apartments & Auckland Viaduct Harbour, Auckland Central & Swimming pool, landscaped park & \\
\hline A73 & The Point apartments & Auckland Viaduct Harbour, Auckland Central & Landscaped courtyard & \\
\hline A74 & Latitude 37 apartments & Auckland Viaduct Harbour, Auckland Central & Landscaped courtyard and fountains & \\
\hline A75 & $\begin{array}{l}\text { Maritime Square Business } \\
\text { Park }\end{array}$ & Auckland Viaduct Harbour, Auckland Central & Gardens, paths and raised lawns & \\
\hline A76 & $\begin{array}{l}\text { Westin Hotel \& Lighter } \\
\text { Quay, Halsey Street }\end{array}$ & Auckland Viaduct Harbour, Auckland Central & Internal boat mooring & \\
\hline A77 & Stratis, Halsey Street & Auckland Viaduct Harbour, Auckland Central & Internal boat mooring & \\
\hline A78 & North Apart., Halsey Street & Auckland Viaduct Harbour, Auckland Central & Internal boat mooring & \\
\hline
\end{tabular}




\begin{tabular}{|c|c|c|c|c|}
\hline A79 & Tank Farm development & Wynyard Wharf, Auckland Central & $\begin{array}{l}\text { Fish markets; open public spaces; } \\
\text { tank structure features }\end{array}$ & \\
\hline A80 & Apartments & cnr Pitt \& Hopetoun Streets, Auckland Central & Car parking courtyard & \\
\hline A81 & Greys Avenue Flats & Greys Ave, Auckland Central & Lawns and gardens & \\
\hline A82 & Hebrew Synagogue & Greys Ave, Auckland Central & $\begin{array}{l}\text { Internal courtyard; community } \\
\text { rooms; adjacent to Myers Park }\end{array}$ & \\
\hline A83 & Apartments & Dockside Lane, Auckland Central & Formal internal courtyards & \\
\hline A84 & Apartments & Mahuhu Cres, Auckland Central & Adjacent to small public park & \\
\hline A85 & Terrace housing & Ronayne Place, Auckland Central & Small internal green & \\
\hline A86 & High-rise apartments & 86-88 The Strand, Auckland Central & Fun pool, tennis court & \\
\hline A87 & Terrace housing & Cotesmore Way on The Strand, Auckland Central & none & \\
\hline A88 & Apartments & 220 Victoria Street West & $\begin{array}{l}\text { Overlooks Victoria Park; } \\
\text { Lap pool in rear yard }\end{array}$ & \\
\hline A89 & 2-storey townhouses & Sheridan Lane_Freemans Bay & Cul-de-sac and common greens & \\
\hline A90 & Townhouses & 115 Franklin Road, Freemans Bay & none & \\
\hline A91 & $\begin{array}{l}\text { Townhouses } \\
\text { (Design by Marshall Cook) }\end{array}$ & 41A - 51B Napier Street, Freemans Bay & none & \\
\hline A92 & Luna Park apartment tower & Burton Street, Grafton & Adjacent to small Glenside Reserve & \\
\hline A93 & $\begin{array}{l}\text { Greenwich Park terrace } \\
\text { housing }\end{array}$ & Burton St, Grafton & Adjacent to small Glenside Reserve & \\
\hline A94 & Mixed-use terrace houses & 150 Symonds Street & none & \\
\hline A95 & Madison Apartment tower & 160 Symonds St & Adjacent to small Glenside Reserve & \\
\hline A96 & Terrace housing & Edwin Street, Mt Eden & $\begin{array}{l}\text { Central landscaped court with water } \\
\text { features }\end{array}$ & \\
\hline A97 & Terrace housing & Harold \& Mary Streets, Mt Eden & Central landscaped gardens & \\
\hline A98 & Terrace housing & $66 \mathrm{Mt}$ Eden Road, Mt Eden & none & \\
\hline A99 & Marino Gardens & 145-147 Mt Eden Rd, Mt Eden & Swimming pool, landscaped courts & 1936 \\
\hline A100 & $\begin{array}{l}\text { Zone } 23 \text { - Mixed Use: } \\
\text { Retail, offices, apartments }\end{array}$ & $21-23$ Edwin Street, Mt Eden & Café in retail courtyard & \\
\hline A101 & $\begin{array}{l}\text { Terrace housing and } \\
\text { mixed-use with apartments }\end{array}$ & 1 George St, Newmarket & $\begin{array}{l}\text { Landscaped triangular garden court. } \\
\text { Complex is adj. to Auckland domain }\end{array}$ & \\
\hline A102 & Terrace housing & 1 Marston St, Parnell & none & \\
\hline A103 & Linked townhouses & 123 Owens Rd, Epsom -Adj. One Tree Hill domain & Swimming pool; landscaped gardens & \\
\hline
\end{tabular}




\begin{tabular}{|c|c|c|c|}
\hline A104 & Terrace Housing & 93 - 103 Mays Rd, Onehunga & none \\
\hline A105 & $\begin{array}{l}\text { Edmund Hillary Retirement } \\
\text { Village }\end{array}$ & 221 Abbots Way, Remuera & $\begin{array}{l}\text { Various green open spaces, pond, } \\
\text { fountain }\end{array}$ \\
\hline A106 & Stonefields & Mt Wellington quarry & $\begin{array}{l}\text { New subdivision with some green } \\
\text { open spaces }\end{array}$ \\
\hline A107 & Talbot Park (HNZC) & Glen Innes & Green open spaces and play grounds \\
\hline A108 & Social housing & $\begin{array}{l}\text { Maybury Street, Glen Innes (near Ruapotaka } \\
\text { Marae and green open spaces) }\end{array}$ & Linear blocks on common land \\
\hline A109 & Social housing & 3-17 Rowena Crescent, Glen Innes & none \\
\hline A110 & $\begin{array}{l}\text { Various medium-density } \\
\text { developments }\end{array}$ & Jellicoe Road, Panmure & Generally none \\
\hline A111 & Terrace housing & 37-51 Ireland Road, Panmure & None. By rail station and soccer field \\
\hline A112 & Town housing & 50 Stanhope Road, Mt Wellington & none \\
\hline A113 & typical subdivisions & Sunnyhills \& Farm Cove, Pakuranga & Scattered green open spaces \\
\hline A114 & Terrace housing & Tiger Drive, Golflands, Botany Downs & Green open spaces \\
\hline A115 & Terrace housing & Haven Drive, Botany Downs & $\begin{array}{l}\text { Green open space, tennis court, } \\
\text { swimming pool }\end{array}$ \\
\hline A116 & Terrace housing & 7 Kelvin Hart Drive, Botany Downs & swimming pool \\
\hline A117 & $\begin{array}{l}\text { Vision Dannemora } \\
\text { Retirement Village }\end{array}$ & 30 Matarangi Road, Botany Downs & $\begin{array}{l}\text { Various landscaped outdoor spaces } \\
\text { bowling green }\end{array}$ \\
\hline A118 & Academy Botany Motor Inn & 4 Leixlep Lane, Botany Downs & Swimming pool in car court \\
\hline A119 & Terrace housing & 21 Armoy Drive, Botany Downs & Central outdoor swimming pool \\
\hline A120 & Terrace housing & 2 Bandon Close, Botany Downs & swimming pool and tennis court \\
\hline A121 & $\begin{array}{l}\text { Clustered Townhousing } \\
\text { with separate garaging }\end{array}$ & 49 Aberfeldy Avenue, Highland Park & $\begin{array}{l}\text { Landscaped central garden } \\
\text { courtyard }\end{array}$ \\
\hline A122 & Duplex and Triplex units & Tagata Way, Mangere & Vehicle access way \\
\hline A123 & Addison & Takanini & $\begin{array}{l}\text { Various green open spaces including } \\
\text { wetland and extensive sports fields }\end{array}$ \\
\hline A124 & Oaklands & Lime Crescent, Papakura & $\begin{array}{l}\text { Crescent shape terrace housing } \\
\text { looking onto green open space }\end{array}$ \\
\hline$\sim$ & $\begin{array}{l}\text { Indicates sites not visited } \\
\text { (information obtained } \\
\text { from internet) }\end{array}$ & & \\
\hline
\end{tabular}




\begin{tabular}{|c|c|c|c|c|}
\hline & Housing Scheme & Address & Communal space(s) & When built \\
\hline & CHRISTCHURCH & NEW ZEALAND & & \\
\hline $\mathrm{CO1}$ & Apartments & 1099 Ferry Rd, Ferrymead & $\begin{array}{l}\text { Overlooks estuary; } \\
\text { Lap pool; tennis courts }\end{array}$ & $2008 ?$ \\
\hline $\mathrm{CO} 2$ & Detached close & York Tong Place & Green open space & \\
\hline $\mathrm{CO3}$ & Norman Kirk Courts & 183 Strickland Street (cnr Brougham Street) & Some green open space / gardens & \\
\hline $\mathrm{CO4}$ & $\begin{array}{l}\text { Univ of Canterbury } \\
\text { (buildings enclosing shared } \\
\text { outdoor spaces) }\end{array}$ & Ilam Road, Ilam & $\begin{array}{l}\text { Various green open space, stream } \\
\text { and water features }\end{array}$ & \\
\hline $\mathrm{CO5}$ & $\begin{array}{l}\text { Aidanfield Retirement } \\
\text { Village }\end{array}$ & Aidanfield Drive, Halswell & $\begin{array}{l}\text { Some courtyards and open green } \\
\text { spaces; indoor swimming pool }\end{array}$ & \\
\hline C06 & Bishops Green & Te Pihopa Way, Halswell & Green open space & \\
\hline $\mathrm{CO7}$ & College House & 100 Waimairi Road, Ilam & $\begin{array}{l}\text { Green central courtyard; tennis } \\
\text { courts; green field; various indoor } \\
\text { communal spaces }\end{array}$ & \\
\hline C08 & Bishop Julius Hall & Waimairi Road, Ilam & $\begin{array}{l}\text { Landscaped quad fully, including } \\
\text { water feature and NZ flag pole, } \\
\text { enclosed by building form }\end{array}$ & \\
\hline C09 & Ilam Apartments (students) & Homestead Lane, Ilam & apartment blocks in open space & \\
\hline C10 & IHC home & $313-315$ Hendersons Road, Halswell & $\begin{array}{l}\text { Small garden courtyard; various } \\
\text { interior communal spaces }\end{array}$ & \\
\hline C11 & Lifestyle lots & Hawthorne Park off Sparks Road, Hoon Hay & N/A & \\
\hline C12 & Detached in cul-de-sac & Brookford Place, Westmorland & Stream; green open space & \\
\hline C13 & $\begin{array}{l}\text { Francis Reserve - } \\
\text { a "drive-to" park on the } \\
\text { suburban / rural fringe }\end{array}$ & Cashmere Road, Westmorland & $\begin{array}{l}\text { tennis courts, playground, green } \\
\text { field, picnic tables, wetland reserve }\end{array}$ & \\
\hline C14 & Townhouses & 143 Salisbury Street, central city & none & \\
\hline C15 & Townhouses & $152 \& 160$ Salisbury St, central city & none & \\
\hline C16 & $\begin{array}{l}\text { ABC Childcare (buildings } \\
\text { flanking communal } \\
\text { outdoor space) }\end{array}$ & 150 Salisbury St, central city & Outdoor green spaces / playgrounds & \\
\hline C17 & Cranleigh Mews & 10 Cranmer Square, central city & None. Opposite Cranmer Park & \\
\hline C18 & Townhouses & 42 Chester Street West, central city & None. Near Cranmer Park & \\
\hline C19 & Chester Mews & 48 Chester Street West, central city & None. Near Cranmer Park & \\
\hline
\end{tabular}




\begin{tabular}{|c|c|c|c|}
\hline $\mathrm{C} 20$ & Townhouses & 45 Chester Street West, central city & None. Near Cranmer Park \\
\hline C21* & St Mary's Apartments & 868 Colombo Street, central city & Landscaped green quads \\
\hline $\mathrm{C} 22^{*}$ & Cornwall Gardens & 27 Cornwall Street, St Albans & Stream and bush walking trail \\
\hline C23* & Tonbridge Mews & 17 Tonbridge \& 12 Shrewsbury Streets, Merivale & Paved courtyards \\
\hline C24* & Apartments & 8 Carlton Mill Road, Merivale & On Avon river side \\
\hline $\mathrm{C} 25^{*}$ & Apartments & Cnr Rossall Street \& Carlton Mill Road, Merivale & $\begin{array}{l}\text { By pedestrian bridge over Avon } \\
\text { River to north end of North Hagley } \\
\text { Park }\end{array}$ \\
\hline C26* & Merivale Mews & 140 Office Road, Merivale & $\begin{array}{l}\text { Pond, village green, retail shops on } \\
\text { corner of Office \& Papanui Roads }\end{array}$ \\
\hline $\mathrm{C} 27^{*}$ & Apartments & Cnr Fitzgerald \& Worcester, central city & $\begin{array}{l}\text { Paved and landscaped internal } \\
\text { courtyard; and common lawn / BBQ }\end{array}$ \\
\hline C28* & K-Block Apartments & 2 The Esplanade, Sumner & $\begin{array}{l}\text { Paved and landscaped internal } \\
\text { courtyard; overlooks Sumner Beach }\end{array}$ \\
\hline C29* & $\begin{array}{l}\text { HNZC competition entry } \\
\text { townhouses }\end{array}$ & $\begin{array}{l}19 \text { Sheppard Avenue to } 31 \text { Aurora Avenue, } \\
\text { Mt Roskill, Auckland }\end{array}$ & Central square \\
\hline C30 & $\begin{array}{l}\text { Provincial Chambers - } \\
\text { historic office building }\end{array}$ & 200 Durahm Street North, central city & Open green central courtyard \\
\hline C31 & $\begin{array}{l}\text { Maryville Courts } \\
\text { Retirement Village }\end{array}$ & Cnr Salisbury \& Manchester Streets, central city & $\begin{array}{l}\text { Various open green spaces and } \\
\text { gardens; internal communal spaces }\end{array}$ \\
\hline C32 & Social townhousing & 278 Glouchester Street (by John Chaplin) & Letterboxes in car court \\
\hline C33 & Townhouses & 37 Cornwall Street, St Albans & None. (Stream goes under driveway) \\
\hline C34 & Merivale Gardens & $16-18$ St Albans Street, St Albans & None \\
\hline C35 & St Lukes Apartments & $\begin{array}{l}170 \text { Peterborough Street, central city } \\
\text { (cnr Manchester Street) }\end{array}$ & Small landscaped common green \\
\hline C36 & Cramner Court & $\begin{array}{l}44 \text { Peterborough Street, central city } \\
\text { (cnr Montreal Street) }\end{array}$ & Swimming pool \\
\hline C37 & Cambridge Apartments & 41 Cambridge Terrace & $\begin{array}{l}\text { Central landscaped planter and } \\
\text { seating in paved courtyard; } \\
\text { opposite Avon River }\end{array}$ \\
\hline C38 & Oxford Terrace apartments & 66 Oxford Terrace & None. Near Avon River \\
\hline C39 & Terrace housing & 15-17 Warwick Street, Richmond & Letterboxes at gated entry \\
\hline C40 & Redcliffs Rest Home & Raekura Place, Redcliffs & $\begin{array}{l}\text { Landscaped courtyard; gardens; } \\
\text { small vegetable plot; indoor pool; }\end{array}$ \\
\hline
\end{tabular}




\begin{tabular}{|c|c|c|c|c|}
\hline & & & indoor communal dining \& lounges & \\
\hline C41 & Council Flats & $\begin{array}{l}14-30 \text { Conference Street, Airdale Place \& } \\
51-63 \text { Salisbury Street, central city }\end{array}$ & Green open spaces & \\
\hline C42 & Council Flats (Art Deco) & 56 Salisbury Street, central city & Raised green bays / gardens & \\
\hline $\mathrm{C} 43$ & Council Flats & $\begin{array}{l}\text { 40-59 Gowerton Place, Richmond (new flats) } \\
3-31 \text { Gowerton Place, Richmond (old flats) }\end{array}$ & $\begin{array}{l}\text { New flats - award winning for low- } \\
\text { impact sustainable environmental } \\
\text { design / landscaping / wetland }\end{array}$ & 2009 \\
\hline C44 & Council Flats? & Waltham Road, Sydenham & $\begin{array}{l}\text { Network of paths through common } \\
\text { ground }\end{array}$ & \\
\hline C45 & $\begin{array}{l}\text { Council Flats, Brougham } \\
\text { Village }\end{array}$ & $356-402$ Brougham Street, Sydenham & $\begin{array}{l}\text { Two green fields, one with small } \\
\text { playground; paved courtyards; } \\
\text { gardens; communal kitchen, lounge } \\
\text { and laundry facilities }\end{array}$ & \\
\hline C46 & Council Flats & 16 Cecil Place \& 33 Vienna Street, Waltham & $\begin{array}{l}\text { Small triangular courtyards; cul-de- } \\
\text { sacs that terminate inside the } \\
\text { housing development; communal } \\
\text { laundry and clothesline area }\end{array}$ & \\
\hline C47 & Tommy Taylor Court & 7-15 Cecil Place, Waltham & Landscaping, lawn and BBQ area & \\
\hline C48 & Terrace housing & 2-62 Waterford Avenue, Belfast & $\begin{array}{l}\text { Crescent-shaped housing form } \\
\text { enclosing western end of small lake } \\
\text { and wetland reserve on Northwood } \\
\text { Boulevard }\end{array}$ & \\
\hline C49 & Terrace housing & 18-46 Beechwood Drive, Belfast & $\begin{array}{l}\text { Linear housing form facing north } \\
\text { directly onto lake and wetland } \\
\text { reserve on Northwood Boulevard }\end{array}$ & \\
\hline $\mathrm{C50}$ & $\begin{array}{l}\text { Recreational corner site in } \\
\text { suburban context }\end{array}$ & Cnr Beechwood Drive \& Mistral Road, Belfast & $\begin{array}{l}\text { BBQ, seating and children's } \\
\text { playground }\end{array}$ & \\
\hline C51 & $\begin{array}{l}\text { Applefield Court } \\
\text { (detached housing in gated } \\
\text { community) }\end{array}$ & Applefield Court (entrance off Riverbank Road), & $\begin{array}{l}\text { None. Membership (compulsory) of } \\
\text { Styx Mill Country Club - tennis } \\
\text { courts, BBQ courtyard, adventure } \\
\text { playground, indoor swimming pool, } \\
\text { lappool, sauna, spa, gym, beauty } \\
\text { therapist, massage therapy, meeting } \\
\text { room, café and restaraunt }\end{array}$ & \\
\hline
\end{tabular}




\begin{tabular}{|c|c|c|c|}
\hline $\mathrm{C52}$ & Terrace housing & Hazelwood Terrace, Belfast & $\begin{array}{l}\text { Green open spaces. Membership } \\
\text { (compulsory) of Styx Mill Country } \\
\text { Club as above. }\end{array}$ \\
\hline C53 & $\begin{array}{l}\text { Pegasus (new planned } \\
\text { community) }\end{array}$ & Pegasus town, North Canterbury & $\begin{array}{l}\text { Green open spaces, lakes, kids zone } \\
\text { adventure playground, sportsfields, } \\
\text { golf course, short drive to surf club } \\
\text { and beach, mixed-use central town } \\
\text { square, urban lakefront (cafes, } \\
\text { restaurant, yacht club, swimming) }\end{array}$ \\
\hline C54 & $\begin{array}{l}\text { Pegasus medium-density } \\
\text { lakefront apartments; } \\
\text { apartments around urban } \\
\text { square; terrace housing }\end{array}$ & Pegasus town, North Canterbury & $\begin{array}{l}\text { (medium-density housing is in future } \\
\text { stages - yet to be constructed. } \\
\text { Promotional information obtained } \\
\text { from site office) }\end{array}$ \\
\hline $\mathrm{C55}$ & $\begin{array}{l}\text { Waitikiri Village - } \\
\text { townhousing }\end{array}$ & Waitikiri Drive, Marshland & $\begin{array}{l}\text { Common green open spaces, lake. } \\
\text { Near Waitikiri Golf Course }\end{array}$ \\
\hline & & & \\
\hline & & & \\
\hline$*$ & Designed by Peter Beaven & & \\
\hline$\sim$ & $\begin{array}{l}\text { Indicates sites not visited } \\
\text { (information obtained } \\
\text { from internet) }\end{array}$ & & \\
\hline & & & \\
\hline & & & \\
\hline & & & \\
\hline & & & \\
\hline & & & \\
\hline & & & \\
\hline & & & \\
\hline & & & \\
\hline & & & \\
\hline & & & \\
\hline
\end{tabular}




\begin{tabular}{|c|c|c|c|c|}
\hline No. & Housing Scheme & Address & Communal space(s) & When built \\
\hline & WELLINGTON REGION & NEW ZEALAND & & \\
\hline & KAPITI COAST & & & \\
\hline W01 & $\begin{array}{l}\text { Brookvale Village } \\
\text { (detached close) }\end{array}$ & 17 Redwood Close, Paraparaumu & Small open space & \\
\hline W02 & $\begin{array}{l}\text { Metlifecare Coastal Villas } \\
\text { Retirement Village }\end{array}$ & Spencer Russell Drive, Paraparaumu & & \\
\hline W03 & $\begin{array}{l}\text { Waterstone (a greenfield } \\
\text { site near rail line (possible } \\
\text { future train station) }\end{array}$ & Waterstone Avenue, Paraparaumu & $\begin{array}{l}\text { Outdoor space around landscaped } \\
\text { natural ponds }\end{array}$ & $\begin{array}{l}\text { Construction } \\
\text { in progress }\end{array}$ \\
\hline \multirow[t]{2}{*}{ W04 } & Proposed ecovillage & On main highway north of Paraparaumu & $\begin{array}{l}\text { Outdoor spaces around landscaped } \\
\text { natural ponds (wetlands) }\end{array}$ & $\begin{array}{l}\text { Design only } \\
\text { (project now } \\
\text { on hold) }\end{array}$ \\
\hline & PORIRUA CITY & & & \\
\hline W05 & Townhouses & $\begin{array}{l}101 \& 103 \text { Mana Esplanade, Mana } \\
\text { (off Service Lane behind shops) }\end{array}$ & $\begin{array}{l}\text { None. Near train station and shops } \\
\& \text { Ngatitoa Domain on harbor edge }\end{array}$ & \\
\hline W06 & Townhouses & $\begin{array}{l}\text { 2-28 Marina View, Mana } \\
\text { (typical of various townhouse developments } \\
\text { along Mana Esplanade) }\end{array}$ & $\begin{array}{l}\text { None. Overlooking boat marina and } \\
\text { near train station and shops \& } \\
\text { Ngatitoa Domain on harbor edge }\end{array}$ & \\
\hline W07 & Townhouses & Lakeside Villas, Whitby Village & None. On lake edge Whitby Village & \\
\hline W08 & Townhouses & $\begin{array}{l}\text { Tweed Road, Papakowhai (near Aotea lagoon } \\
\text { and public gardens) }\end{array}$ & $\begin{array}{l}\text { Small green courtyards defined by } \\
\text { cluster housing forms. }\end{array}$ & \\
\hline W09 & Townhouses & $\begin{array}{l}\text { Halladale Road, Papakowhai. (On hillside } \\
\text { overlooking NZ Police College and Aotea Lagoon) }\end{array}$ & $\begin{array}{l}\text { Small green open spaces. Adjacent } \\
\text { to bush, lake and wetland. }\end{array}$ & \\
\hline W10 & $\begin{array}{l}\text { Belmont Motor Lodge } \\
\text { (typical of motor } \\
\text { accomodation) }\end{array}$ & 1 Mungavin Avenue, Porirua East & $\begin{array}{l}\text { Square car courtyard flanked by } \\
\text { attached motel units on all sides }\end{array}$ & \\
\hline W11 & $\begin{array}{l}\text { Typical "greenfinger" } \\
\text { subdivision (detached) }\end{array}$ & Porirua East & $\begin{array}{l}\text { Green open spaces following bush- } \\
\text { lined creeks }\end{array}$ & \\
\hline W12 & $\begin{array}{l}\text { Social housing - long linear } \\
\text { blocks }\end{array}$ & Bounded by Dimock \& Owhiti Streets, Titahi Bay & $\begin{array}{l}\text { Large central common (subdivided } \\
\text { by fencing) }\end{array}$ & \\
\hline W13 & $\begin{array}{l}\text { Backpackers Oban Villa } \\
\text { (10 minutes walk from } \\
\text { Porirua Railway Station) }\end{array}$ & $\begin{array}{l}10 \text { Farm Road, Porirua Hospital Grounds, Porirua } \\
\text { [Individual rooms (singles and doubles) and } \\
\text { dormitory-type accommodation.] }\end{array}$ & Green open spaces & \\
\hline
\end{tabular}




\begin{tabular}{|c|c|c|c|c|}
\hline W14 & Redwood Village & 42 Main Road, Tawa & None & \\
\hline W15 & Townhouses & 230-234 Middleton Road, Churton Park & $\begin{array}{l}\text { Tennis court, swimming pool, green } \\
\text { open space }\end{array}$ & \\
\hline W16 & $\begin{array}{l}\text { Townhousing around cul- } \\
\text { de-sac }\end{array}$ & 46 Wingfield Place, Churton Park & None & \\
\hline W17 & $\begin{array}{l}\text { Melvina Major Retirement } \\
\text { Village }\end{array}$ & Burma Rd, Johnsonville & Bowling green & \\
\hline W18 & Athfield House & 105 Amritsar Street, Khandallah_ & Courtyards, communal work spaces & \\
\hline W19 & Townhouses & 4-30 Cottleville Terrace, Thorndon & none & \\
\hline W20 & Semi-circular form & 4 Grosvenor Terrace, Thorndon & Green space inside circular drive & \\
\hline W21 & Apartments & 42 Murrayfield Drive,Thorndon & $\begin{array}{l}\text { 3-sided apartment block around } \\
\text { garden courtyard }\end{array}$ & \\
\hline W22 & $\begin{array}{l}19^{\text {th }} \text { Century med-density } \\
\text { block of Victorian villas }\end{array}$ & Goring Street, Thorndon & $\begin{array}{l}\text { None } \\
\text { Between motorway and greenbelt }\end{array}$ & \\
\hline W23 & Townhouses & 11 Harriett Street, Thorndon & none & \\
\hline W24 & Habitat Pitarua Court & 9-11 Pitarua Street, Thorndon & courtyards & \\
\hline W25 & Thorndon Mews & 10 Pitarua Street, Thorndon & courtyards & \\
\hline W26 & Salisbury Garden Court & Between 131 \& 133 Cecil Road, Wadestown & Tennis court, bush, town greenbelt & \\
\hline W27 & Townhouses & 12 Agra Crescent, Khandallah & none & \\
\hline W28 & The Boundary & Hazlewood Avenue, Karori_ & Tennis court, gazebos, playground & \\
\hline W29 & Townhousing & Futuna Close, 100 Friend Street, Karori & lawns & \\
\hline W30 & Townhouses & 219 Karori Road, Karori & Car courts & \\
\hline W31 & Townhouses (4) & 67 Old Karori Road, Karori & none & \\
\hline W32 & Terrace houses & 27 The Rigi, Northland & Tennis courts, bush & \\
\hline W33 & Bishop Park & Eastbourne & $\begin{array}{l}\text { Public swimming pool, adventure } \\
\text { playground, and green open space } \\
\text { on beach frontage }\end{array}$ & \\
\hline W34 & Williams Park & Days Bay & $\begin{array}{l}\text { Bush, pond, pavilion café and green } \\
\text { open space on beach frontage }\end{array}$ & \\
\hline W35 & "Four-Pack" townhouses & 71 Randwick Crescent, Randwick & none & \\
\hline W36 & Woburn Court & 65 White Lines Road, Woburn & External entrance stairs & $1928 ?$ \\
\hline W37 & Ropata Village & 577 High Street, Lower Hutt & $\begin{array}{l}\text { Interior courtyard; communal dining } \\
\text { and lounge areas; medical facilities }\end{array}$ & $1986 ?$ \\
\hline
\end{tabular}




\begin{tabular}{|c|c|c|c|c|}
\hline W38 & Townhouses & 10 Park Avenue, Lower Hutt & none & $2008 ?$ \\
\hline W39 & $\begin{array}{l}\text { Shona McFarlane } \\
\text { Retirement Village }\end{array}$ & 66 Maybe Rd, Avalon & $\begin{array}{l}\text { Bowling green, landscaped interior } \\
\text { courtyard, near Fraser Park }\end{array}$ & \\
\hline W40 & $\begin{array}{l}\text { The Links \& The Glade } \\
\text { Social housing }\end{array}$ & Farmer Crescent, Pomare & $\begin{array}{l}\text { Green open space. Near Pomare } \\
\text { Train Station. Opposite Hutt River. }\end{array}$ & \\
\hline W41 & The Square & Manor Park & Green open space & \\
\hline W42 & Moera Community Library & 171 Randwick Road, Moera & Green open space, playgrounds & \\
\hline W43 & Siverstream Retreat & Reynolds Bach Rd & Courtyards, green open spaces & \\
\hline W44 & Retirement houses & Millwood Place, Silverstream & none & \\
\hline W45 & Townhouses & 3 Gard Street, Silverstream & none & \\
\hline W46 & Terrace houses & $\begin{array}{l}\text { 40-66 Evergreen Crescent } \\
\text { (off Pinehill Crescent), Heretaunga }\end{array}$ & Green open space & \\
\hline W47 & Cluster housing & Silverstream / Pinehaven, Upper Hutt & $\begin{array}{l}\text { Small 'village' clusters set } \\
\text { unobtrusively into pine forest }\end{array}$ & \\
\hline W48 & $\begin{array}{l}\text { Detached houses on } \\
\text { smaller lots }\end{array}$ & Messines Avenue, Trentham & $\begin{array}{l}\text { None. Adjacent to expansive green } \\
\text { open spaces (eg ex-CIT campus) }\end{array}$ & $2005 ?$ \\
\hline W49 & $\begin{array}{l}\text { Detached houses on } \\
\text { smaller lots }\end{array}$ & $\begin{array}{l}\text { Brentwood Street, Trentham } \\
\text { (ex- Brentwood Primary School site) }\end{array}$ & $\begin{array}{l}\text { None. Adjoins expansive open } \\
\text { space of Trentham Memorial park }\end{array}$ & $2006 ?$ \\
\hline & & & & \\
\hline$\sim$ & $\begin{array}{l}\text { Indicates sites not visited } \\
\text { (information obtained } \\
\text { from internet) }\end{array}$ & & & \\
\hline & & & & \\
\hline & & & & \\
\hline & & & & \\
\hline & & & & \\
\hline & & & & \\
\hline & & & & \\
\hline & & & & \\
\hline & & & & \\
\hline
\end{tabular}




\begin{tabular}{|c|c|c|c|c|}
\hline No. & Housing Scheme & Address & Communal space(s) & When built \\
\hline & MELBOURNE, VICTORIA & AUSTRALIA & & \\
\hline M01 & $\begin{array}{l}\text { Quest Royal Gardens } \\
\text { Apartments (Motel) }\end{array}$ & $\begin{array}{l}8 \text { Royal Lane } \\
\text { Fitzroy 3065, Melbourne }\end{array}$ & $\begin{array}{l}\text { Lawns, gardens, swimming pool, } \\
\text { picnic table, B-B-Q }\end{array}$ & $1970 ?$ \\
\hline M02 & Fleet Street Social Housing & $\begin{array}{l}21 \text { Palmer Street \& 3, 4, } 9 \& 10 \text { Fleet Street } \\
\text { Fitzroy 3065, Melbourne }\end{array}$ & Lawns, children's small play area & 1960's? \\
\hline M03 & Royal Terrace & $\begin{array}{l}50-68 \text { Nicholson Street } \\
\text { Fitzroy 3065, Melbourne }\end{array}$ & $\begin{array}{l}\text { Adjacent to Carlton Gardens (public } \\
\text { park) and Melbourne Museum }\end{array}$ & 1856 \\
\hline M04 & $\begin{array}{l}\text { Cairo Apartments } \\
\text { (opp. Melbourne Museum) }\end{array}$ & $\begin{array}{l}98 \text { Nicholson Street } \\
\text { Fitzroy 3065, Melbourne }\end{array}$ & $\begin{array}{l}\text { Central lawn/gardens. Adjacent to } \\
\text { Carlton Gardens (public park) }\end{array}$ & $\begin{array}{l}1930 ? \\
\text { Art Deco style }\end{array}$ \\
\hline M05 & $\begin{array}{l}\text { Hanover Street - range of } \\
\text { terraces from various eras }\end{array}$ & $\begin{array}{l}18-38 \text { Hanover Street } \\
\text { Fitzroy 3065, Melbourne }\end{array}$ & none & $\begin{array}{l}1860 ? 1960 ? \\
1970 ? 2000 ?\end{array}$ \\
\hline M06 & "Six-Pack" Flats & $\begin{array}{l}\text { 2-32, 34-50 King William St \& 3-25, 27-31, 41-47 } \\
\text { Hanover St, Fitzroy 3065, Melbourne }\end{array}$ & Garden paths and letterboxes & 1960 's ? \\
\hline M07 & Fitzroy Block - Historic & $\begin{array}{l}\text { Moor / Nicholson / Bell and Cremorne Streets } \\
\text { Fitzroy 3065, Melbourne }\end{array}$ & none & $19^{\text {th }}$ century \\
\hline M08 & Apartments & $\begin{array}{l}2-10 \text { Carlton Street } \\
\text { Carlton } 3053, \text { Melbourne }\end{array}$ & $\begin{array}{l}\text { Adjacent to Carlton Gardens (public } \\
\text { park) and Melbourne Museum }\end{array}$ & 1990's? \\
\hline M09 & $\begin{array}{l}\text { Atherton Gardens Estate } \\
4 \mathrm{x} \text { high-rise towers in park }\end{array}$ & $\begin{array}{l}\text { Social Housing - Brunswick, Gertrude \& Napier } \\
\text { Streets, Fitzroy } 3065\end{array}$ & $\begin{array}{l}\text { Park, trees, sports fields, } \\
\text { playground, common rooms }\end{array}$ & $\begin{array}{l}\text { Late } 1950 \text { 's or } \\
\text { early } 1960 \text { 's }\end{array}$ \\
\hline M10 & Apartments & $\begin{array}{l}26 \text { Victoria Street \& 212(?) Nicholson Street \& } \\
\text { 23(?) Bell Street, Fitzroy } 3065\end{array}$ & Swimming pool, BBQ, gardens & 1990's? \\
\hline M11 & $\begin{array}{l}\text { "K2" Apartments } \\
\text { (Social Housing) }\end{array}$ & $\begin{array}{l}\text { 2(?) Raleigh Street } \\
\text { Windsor } 3181 \text {, Melbourne }\end{array}$ & $\begin{array}{l}\text { Landscaping, seating, community } \\
\text { room (locked) and courtyard; } \\
\text { "Urban Park" interface with street }\end{array}$ & 2007 \\
\hline $\mathrm{M} 12^{\sim}$ & $\begin{array}{l}\text { D'LUX Apartments } \\
\text { formerly “Inkerman Oasis" }\end{array}$ & $\begin{array}{l}\text { cnr Inkerman \& Greeves Sts } \\
\text { St. Kilda, City of Port Philip, Melbourne }\end{array}$ & & $2003-2005$ \\
\hline M13 & First Point Apartments & $\begin{array}{l}\text { First Point } \\
\text { Port Melbourne, Victoria, } 3207\end{array}$ & Beachfront landscaping & $2003 ?$ \\
\hline M14 & $\begin{array}{l}\text { Port Melbourne } \\
\text { Waterfront Apartments }\end{array}$ & $\begin{array}{l}\text { Beacon Vista \& Pier Street (at waterfront) } \\
\text { Port Melbourne, Victoria } 3207\end{array}$ & & Mid-2000's? \\
\hline M15 & $\begin{array}{l}\text { Port Melbourne } \\
\text { New subdivision }\end{array}$ & $\begin{array}{l}\text { Port Melbourne } \\
\text { Victoria } 3207\end{array}$ & Landscaped green open space & Mid-2000's? \\
\hline
\end{tabular}




\section{Appendix 7.2: Field Notes - Melbourne}




\section{APPENDIX 7.2 - Field Notes (Melbourne)}

Following are Field Notes of medium density housing developments in Melbourne, Australia, visited by the writer in September 2009 as part of research for this thesis.

The writer took photographs and made notes while on field trips in Melbourne, then later prepared these formal Field Notes which also include material such as Google Maps and Google street views obtained from the internet. The Field Notes record basic information about each development such as the name of the development, its location, the date it was visited, the name of the architect/designer where known, contact details of the owner where known, type of housing, density (dwelling units per hectare) and context. The main purpose of the Field Notes was to record the layout and nature of any communal space and its relationship with the housing in each development visited.

The writer found quite a wide range of medium density housing typologies in Melbourne, some with fine examples of communal space, such as the first Field Note M01 - Quest Motel. Although designed for temporary motel accommodation, this is a particularly interesting example because of the generous provision of communal space in a relatively high density development (90 units/ha) consisting of a range of unit sizes, from one to four bedrooms. Ground level units have small semi-private yards bordering the central communal space. The location is central, about 5 minutes walk from Melbourne Museum and Carlton Gardens, and about 10 minutes walk from the heart of the CBD. 
() 2009 Stephen J. Pattinson

M. Arch. Thesis Research

Victoria University of Wellington, NZ
FIELD NOTES

Site Visits: Tuesday 08.09 .09 (page 1 of 4 )
Sheet No.

MO1 A

Updated: 14.09 .09

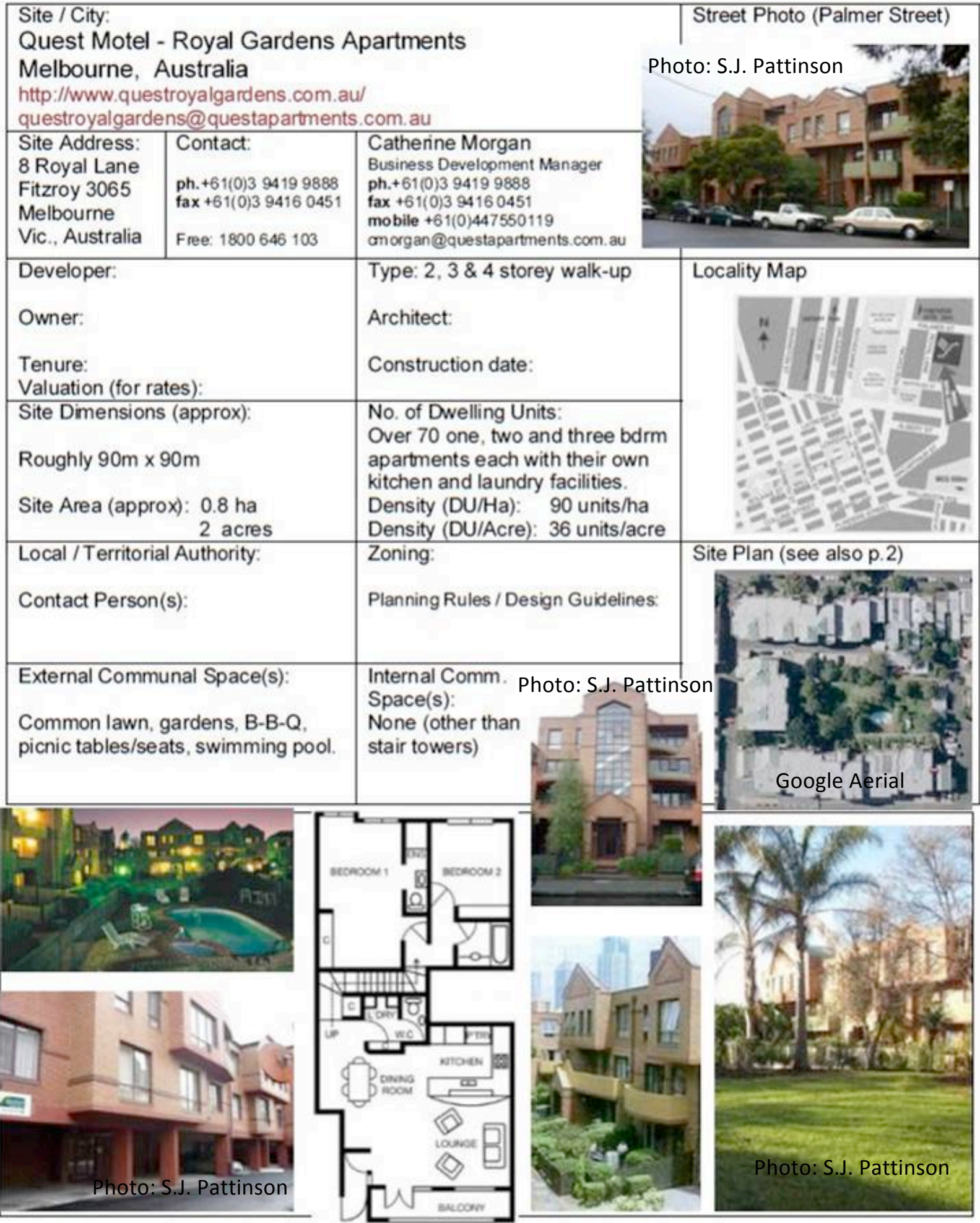




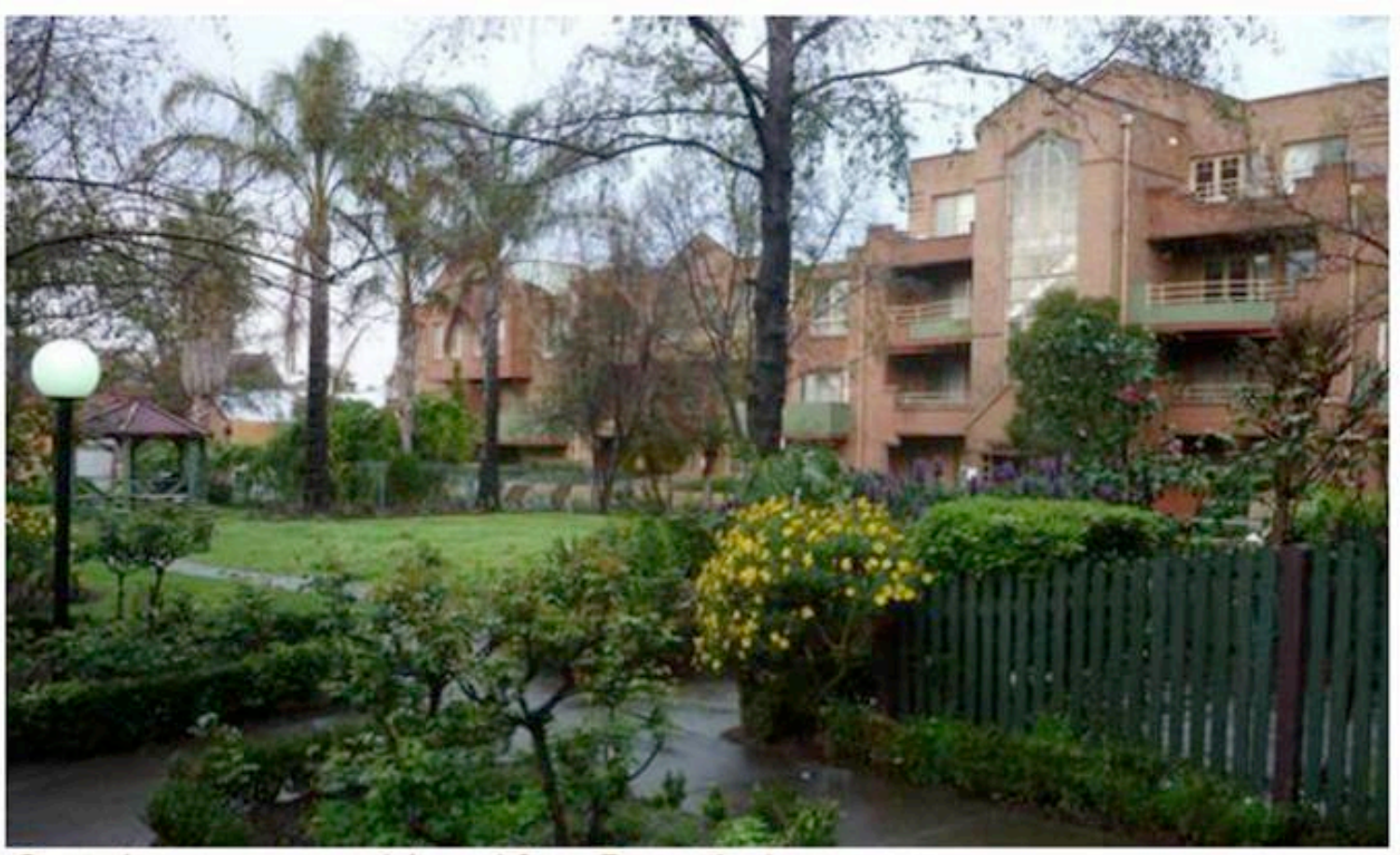

Central common area (viewed from Reception)

Photo: S.J. Pattinson

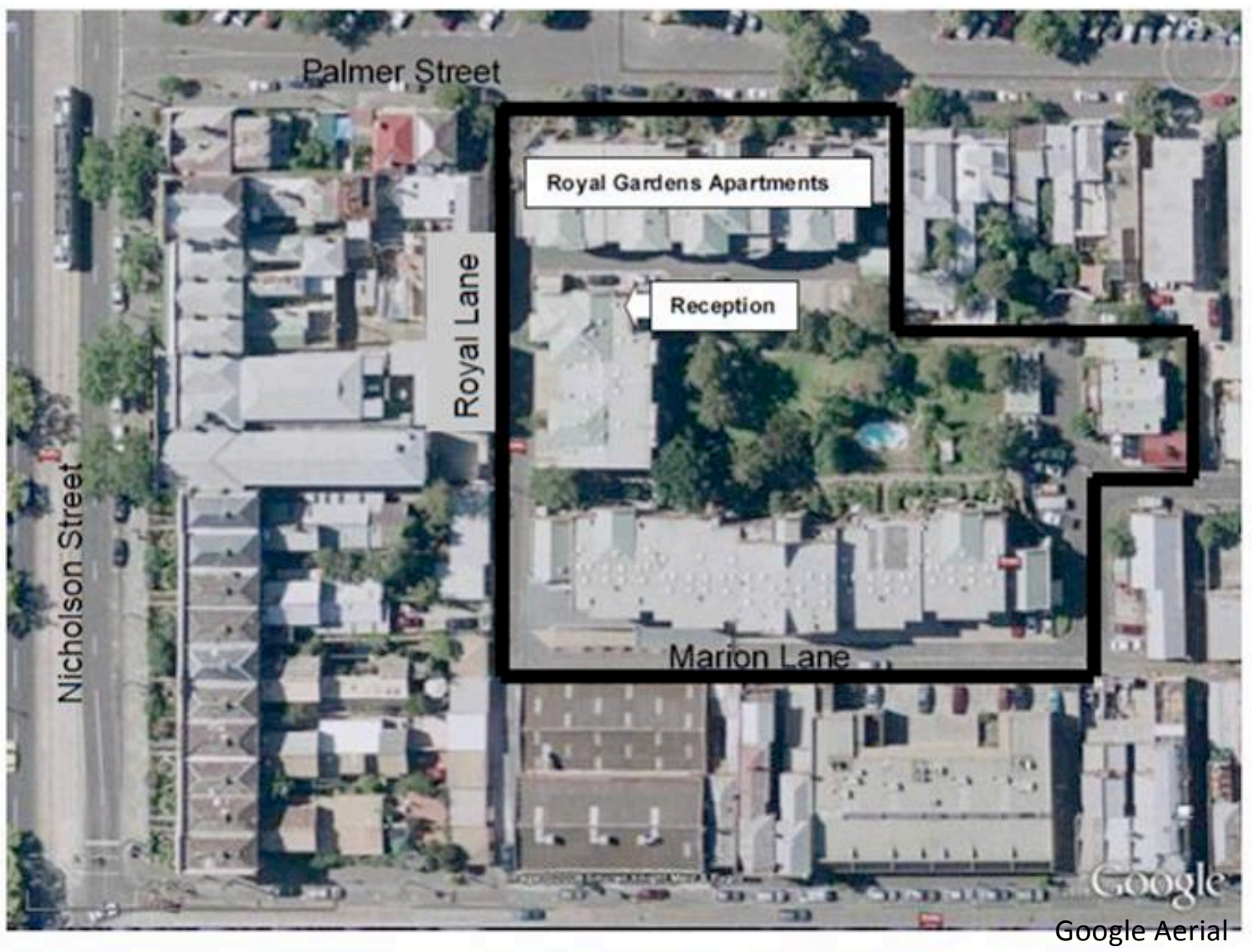

Aerial View of Site (indicative only) - Royal Gardens Apartments

(page 2 of 4 ) 


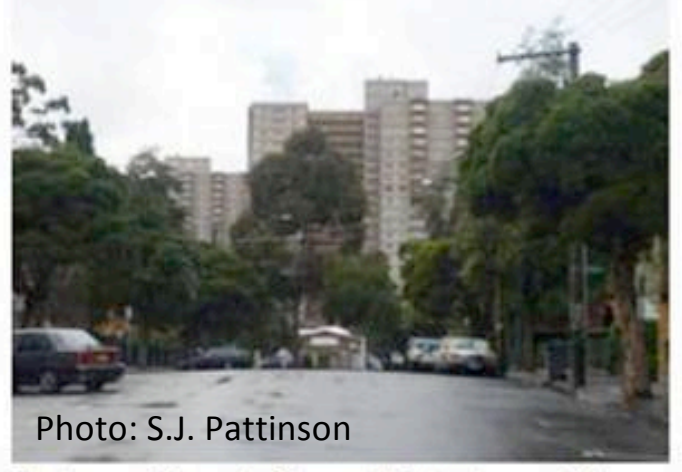

Palmer Street (from Nicholson St)

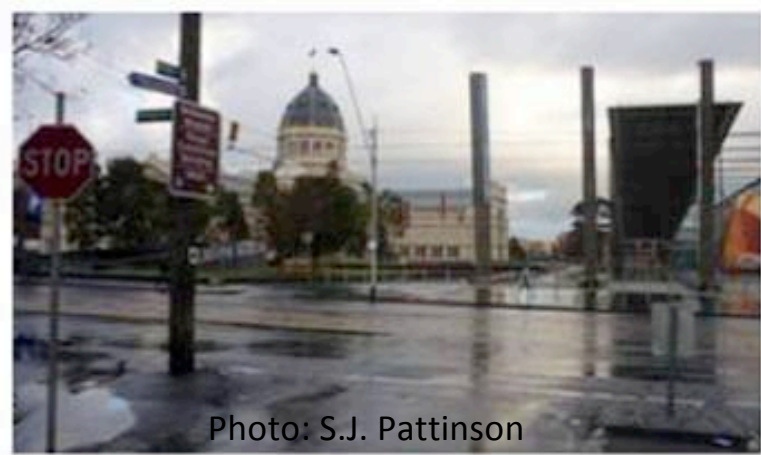

Royal Exhibition Bldg \& Melbourne Museum (from cnr Palmer Street \& Nicholson Street)

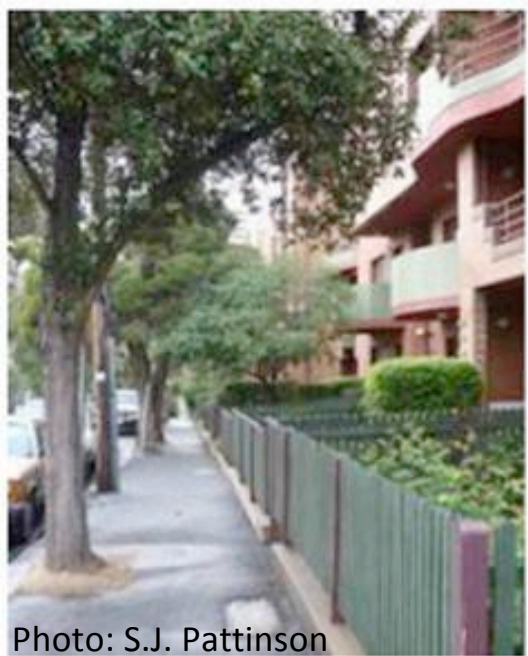

Palmer Street frontages

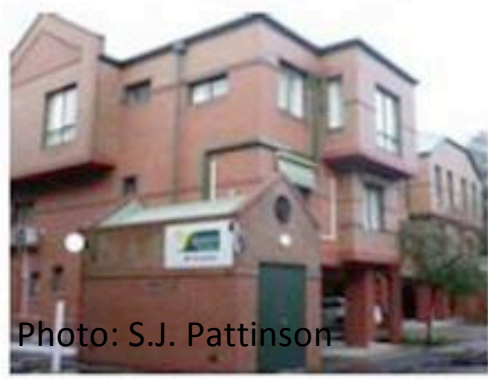

Royal Lane (secondary lane on left to Reception)

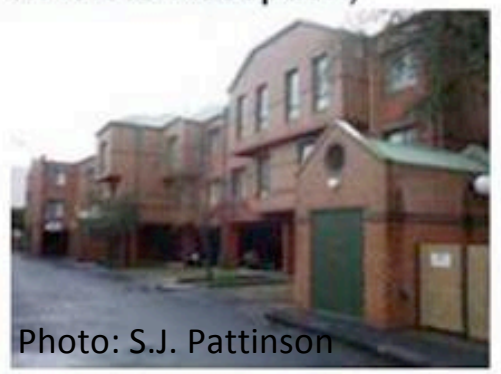

Royal Lane (from South)

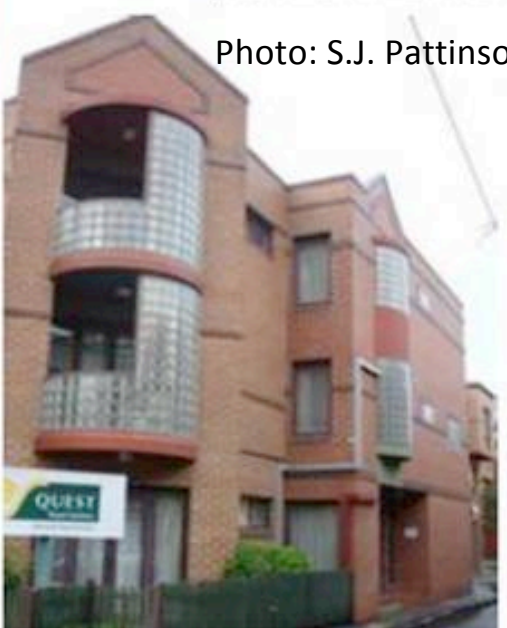

Cnr Palmer St \& Royal Lane

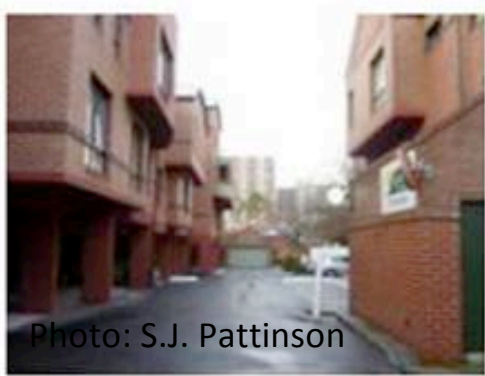

Secondary lane (to Reception on right)

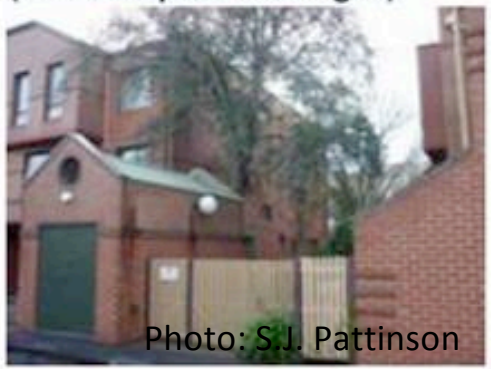

Restricted pedestrian access

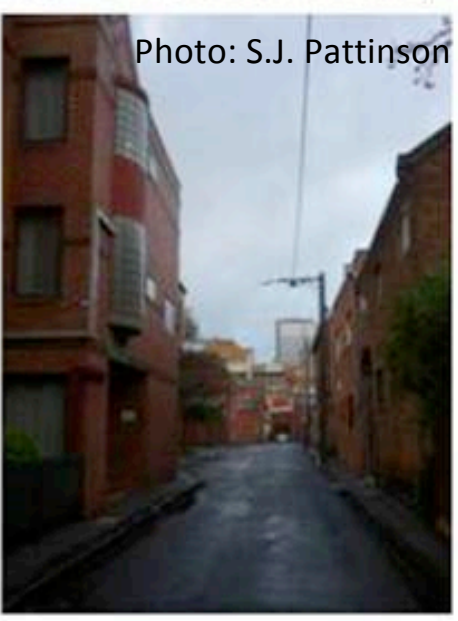

Royal Lane from Palmer

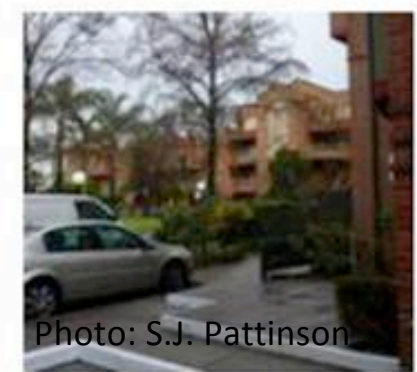

First glimpse of œntral outdoor common area

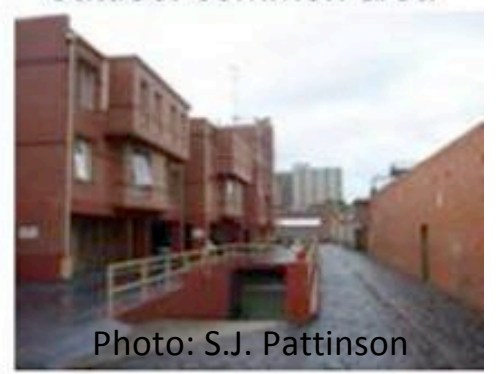

Marion Lane

(page 3 of 4 ) 


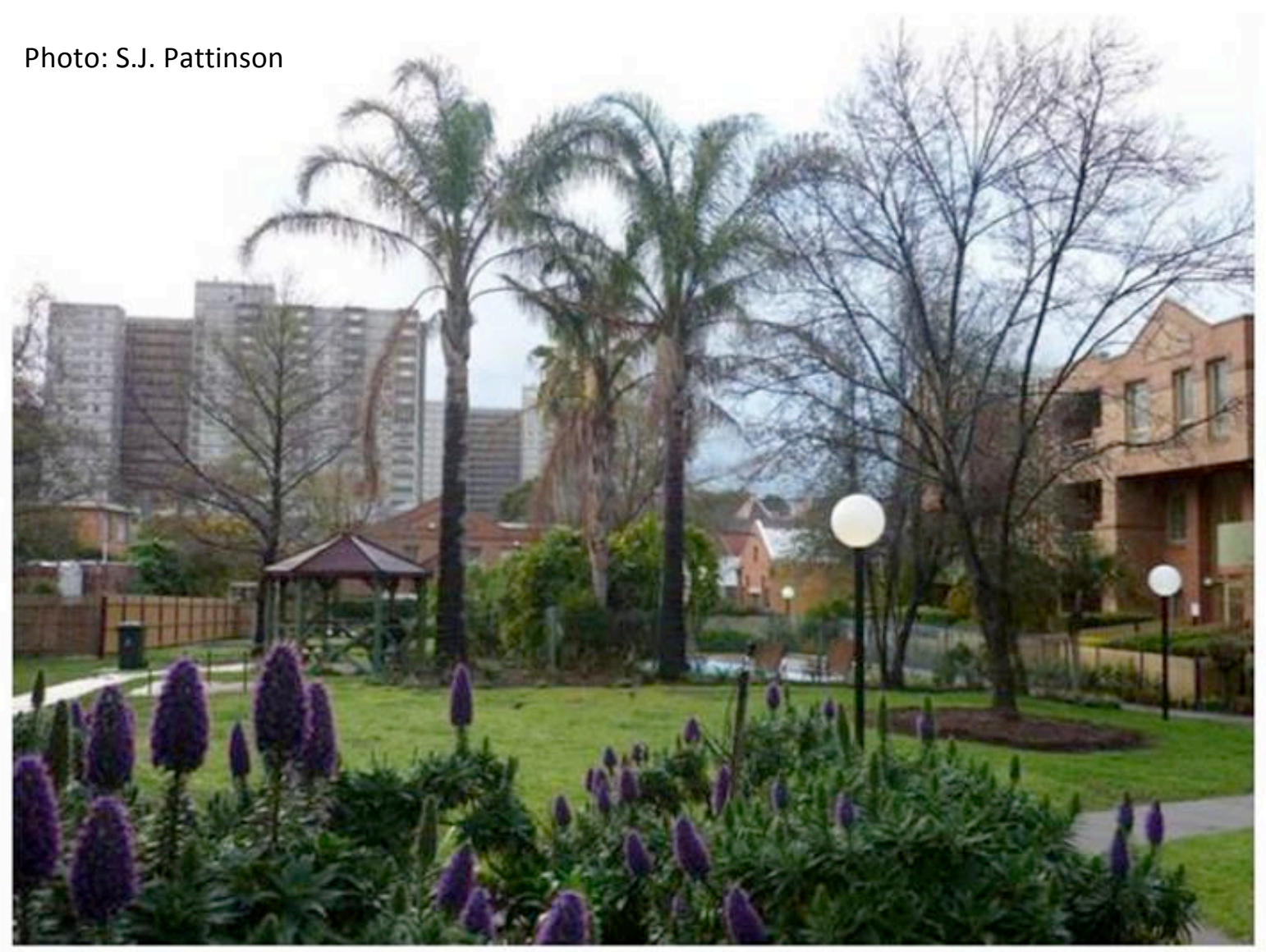

Central outdoor common area (lawn and gardens; BBQ to left; swimming pool to right)

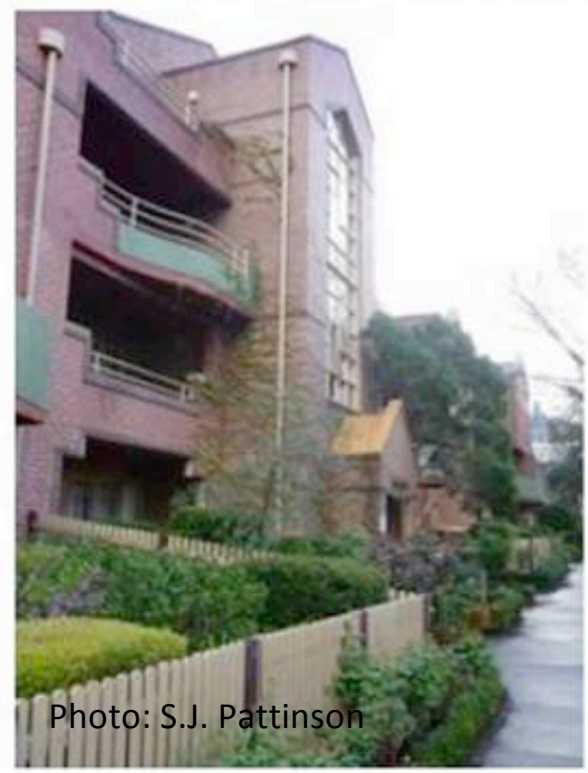

Apartments have either a private balcony or a fenced cottage garden. Guest parking and pedestrian paths flank the outdoor common area. (page 4 of 4 )
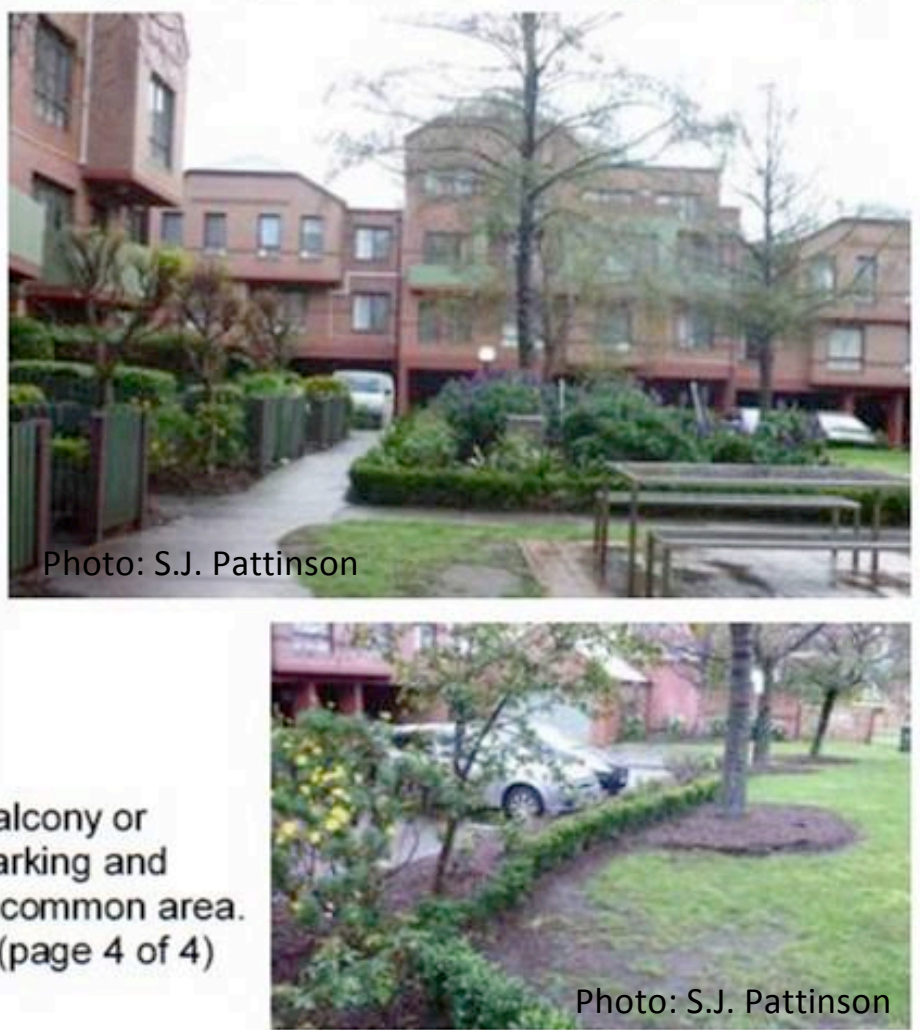


\begin{tabular}{|c|c|c|}
\hline $\begin{array}{l}\text { C } 2009 \text { Stephen J. Pattinson } \\
\text { M. Arch. Thesis Research } \\
\text { Victoria University of Wellington, NZ }\end{array}$ & $\begin{array}{c}\text { FIELD NOTES } \\
\text { Site Visits: Tuesday } 08.09 .09 \quad \text { (page } 1 \text { of } 2 \text { ) }\end{array}$ & Sheet No. \\
\hline
\end{tabular}

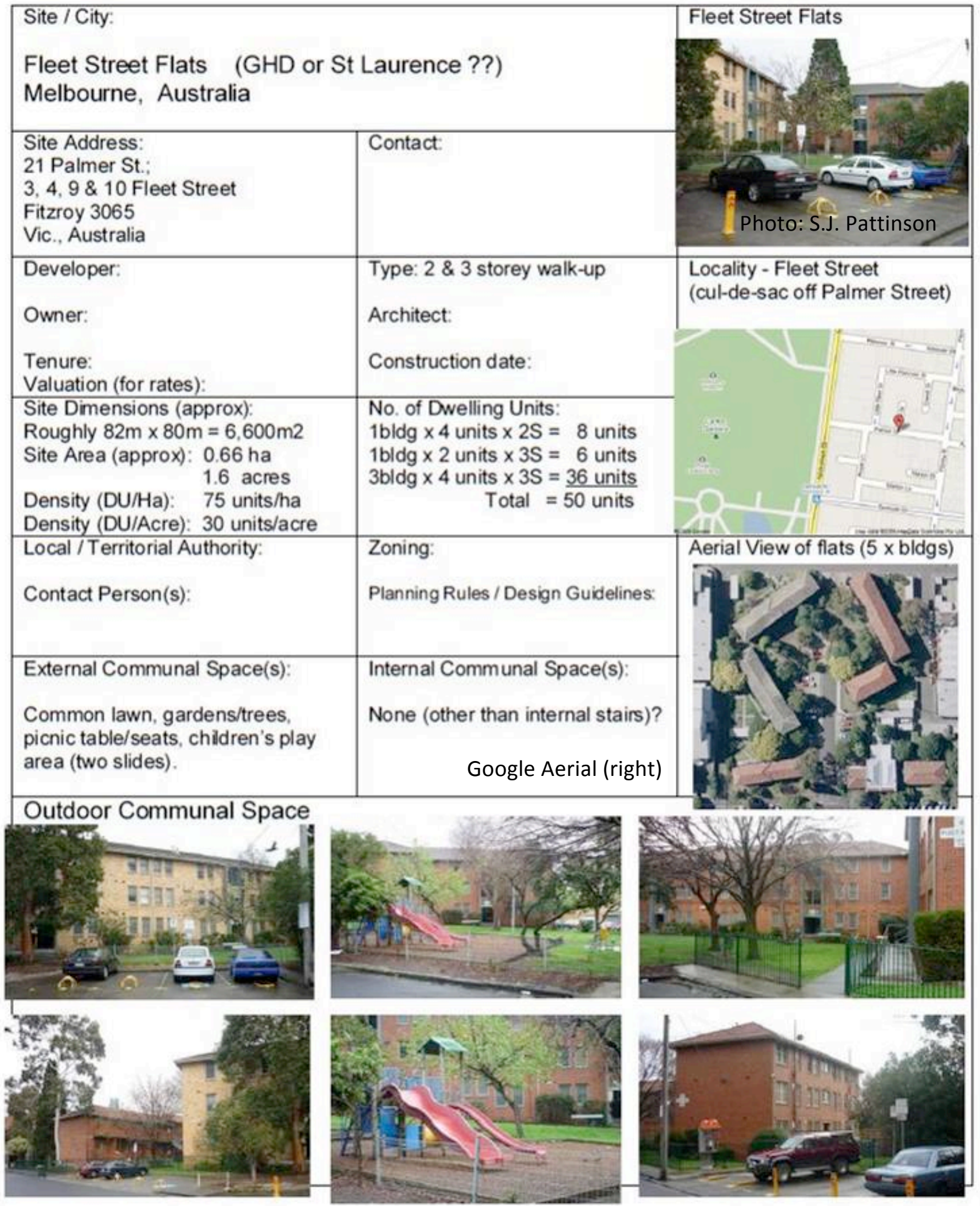

All Photos: S.J. Pattinson 

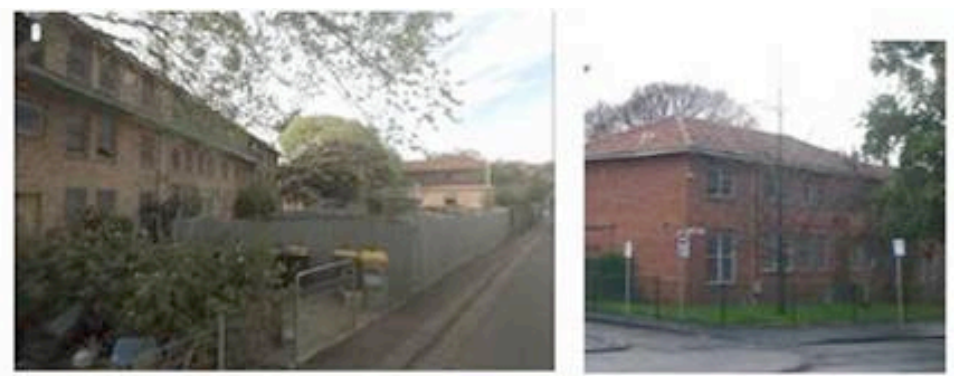

Little Fleet St

Palmer Street

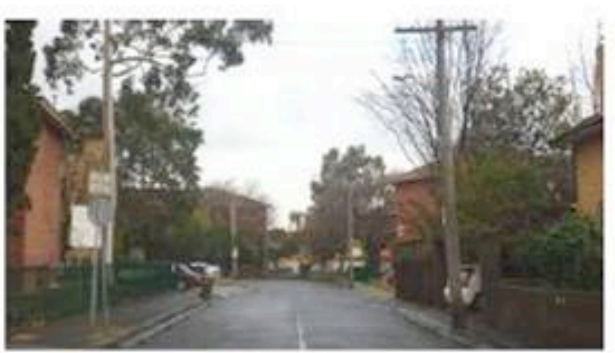

Fleet Street

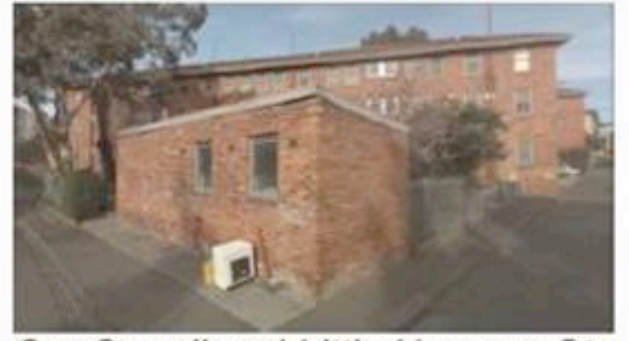

Cnr Cowell and Little Hanover Sts

Streetscapes All Google Street Views

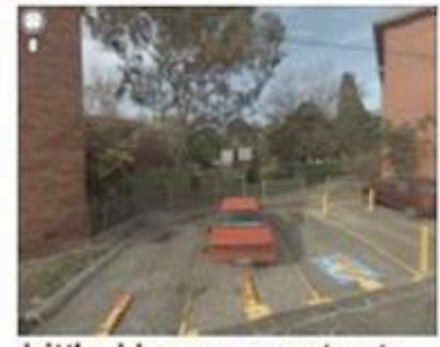

Little Hanover entry to Fleet St site

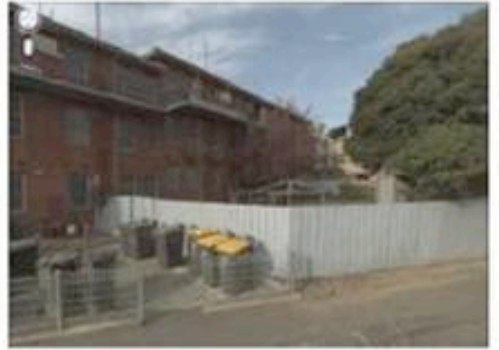

Little Hanover (west end)

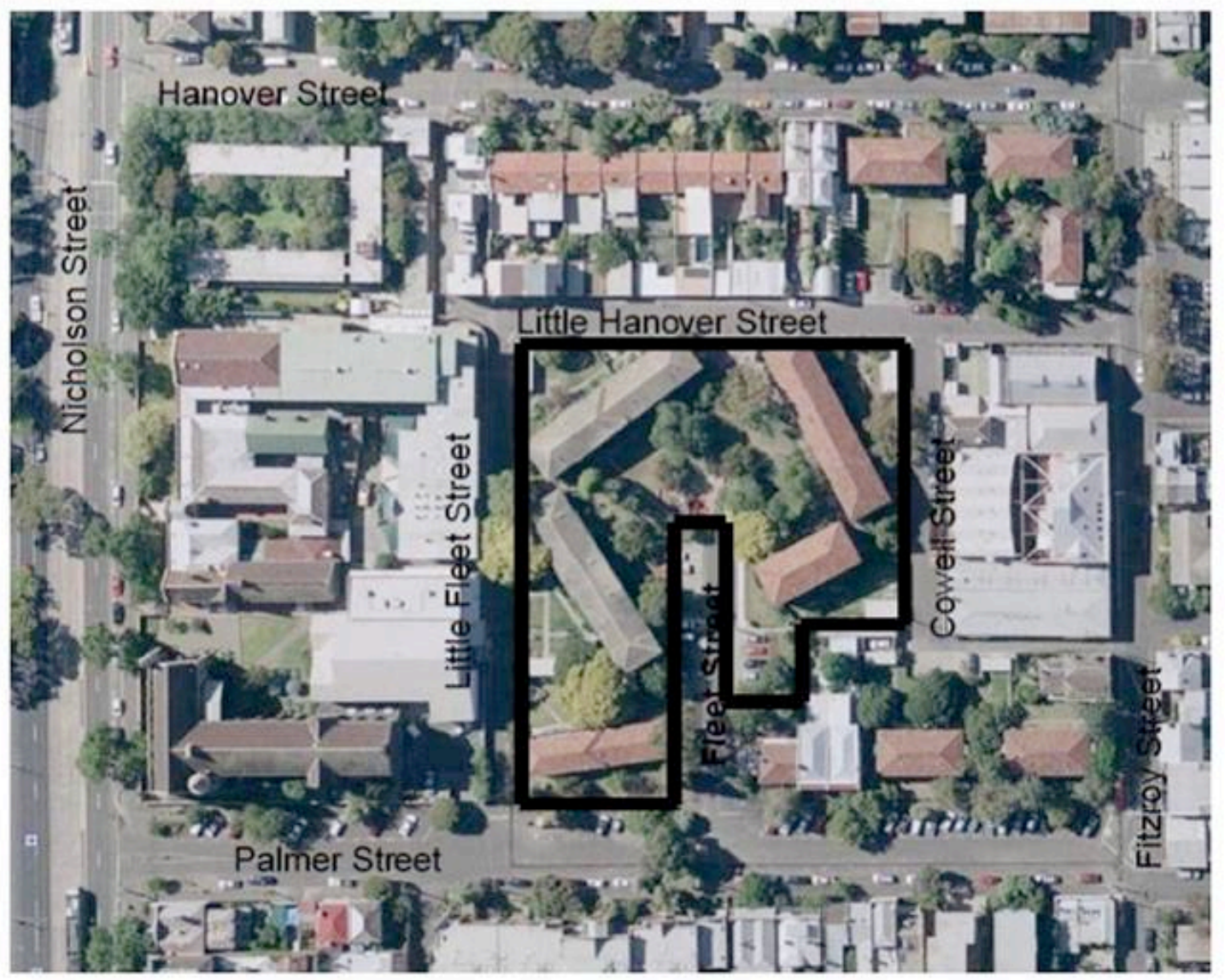

Aerial View of Site (indicative only)

Google Aerial Vieçw

(page 2 of 2) 


\begin{tabular}{|l|c|l|}
\hline $\begin{array}{l}\text { (C) 2009 Stephen J. Pattinson } \\
\text { M. Arch. Thesis Research } \\
\text { Victoria University of Wellington, NZ }\end{array}$ & FIELD NOTES & $\begin{array}{l}\text { Sheet No. } \\
\text { Site Visits: Tuesday 08.09.09 (page 1 of 2) }\end{array}$ \\
\hline
\end{tabular}

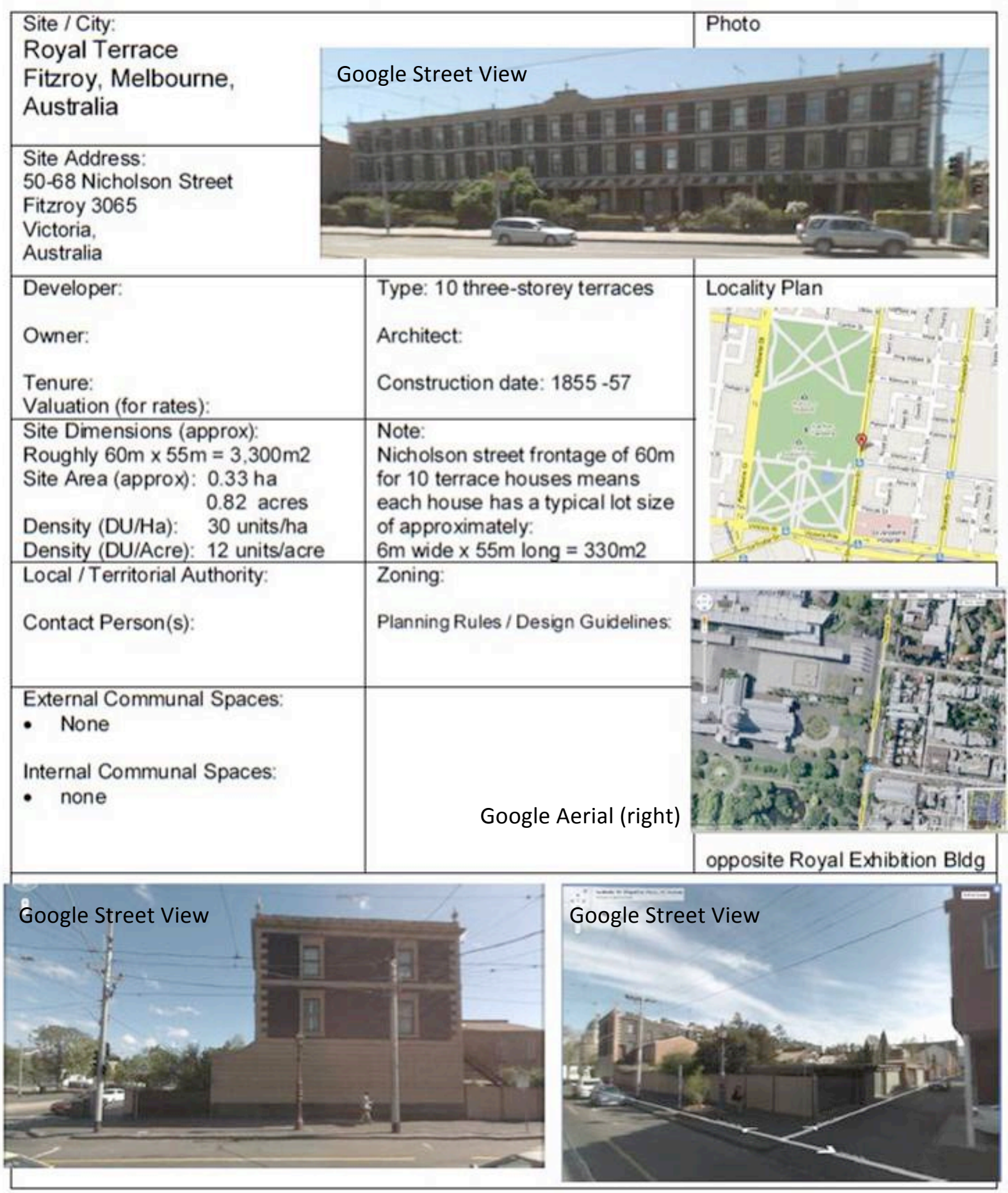



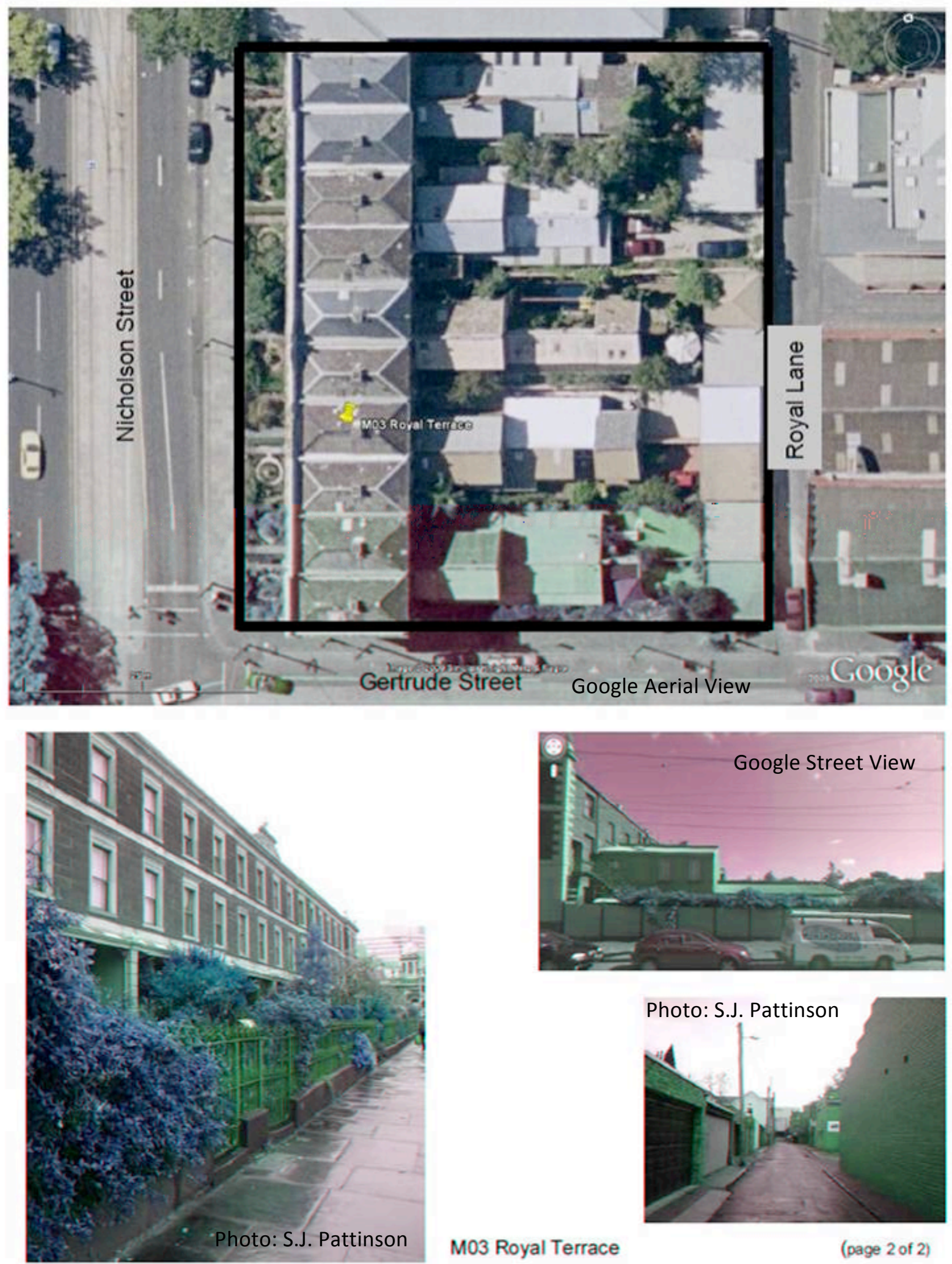

(page 2 of 2) 


\begin{tabular}{l|c|l|}
\hline $\begin{array}{l}\text { C. 2009 Stephen J. Pattinson } \\
\text { M. Arch. Thesis Research } \\
\text { Victoria University of Wellington, NZ }\end{array}$ & FIELD NOTES & $\begin{array}{l}\text { Sheet No. } \\
\text { Site Visits: Tuesday 08.09.09 }\end{array}$ \\
\hline
\end{tabular}

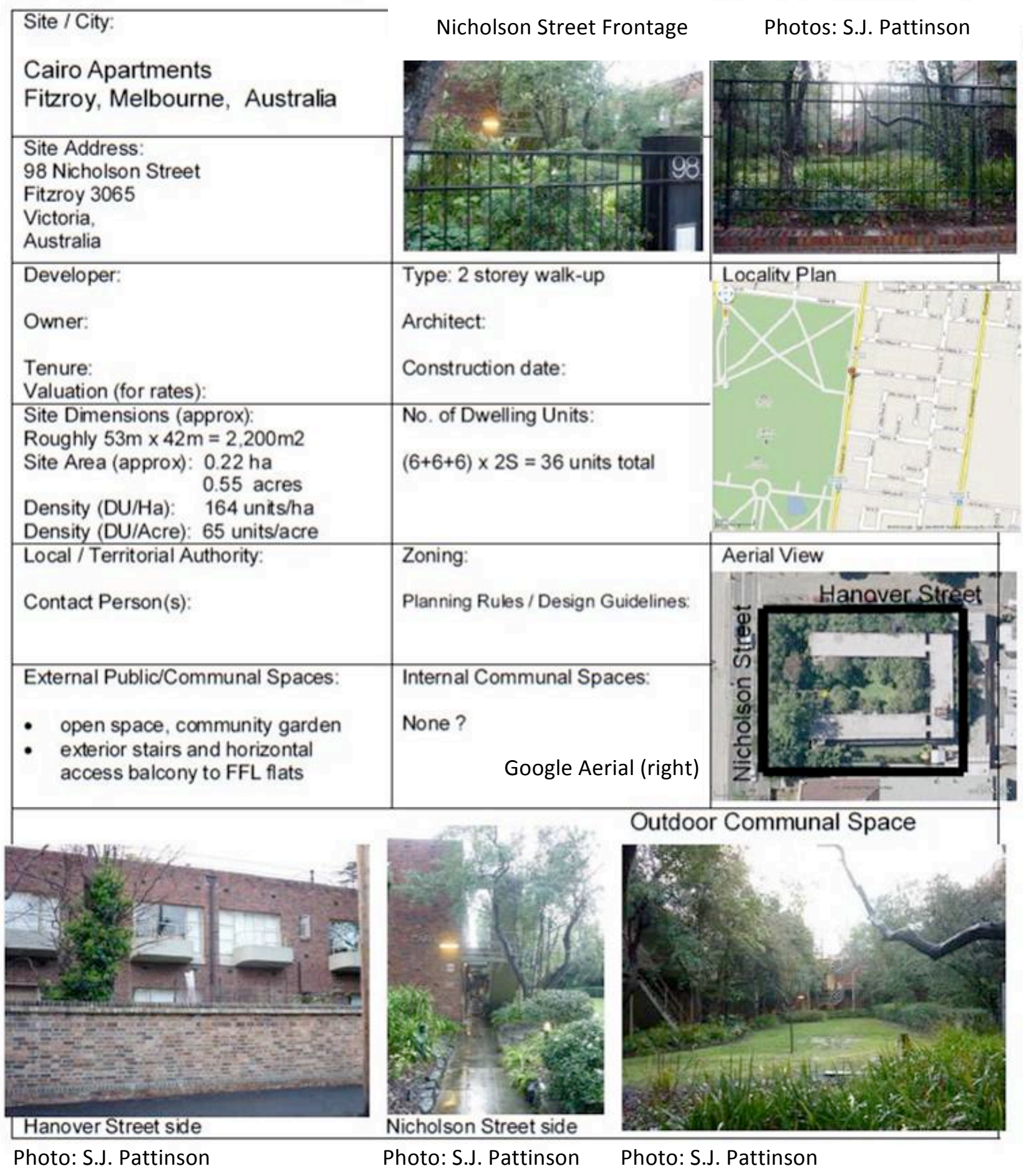



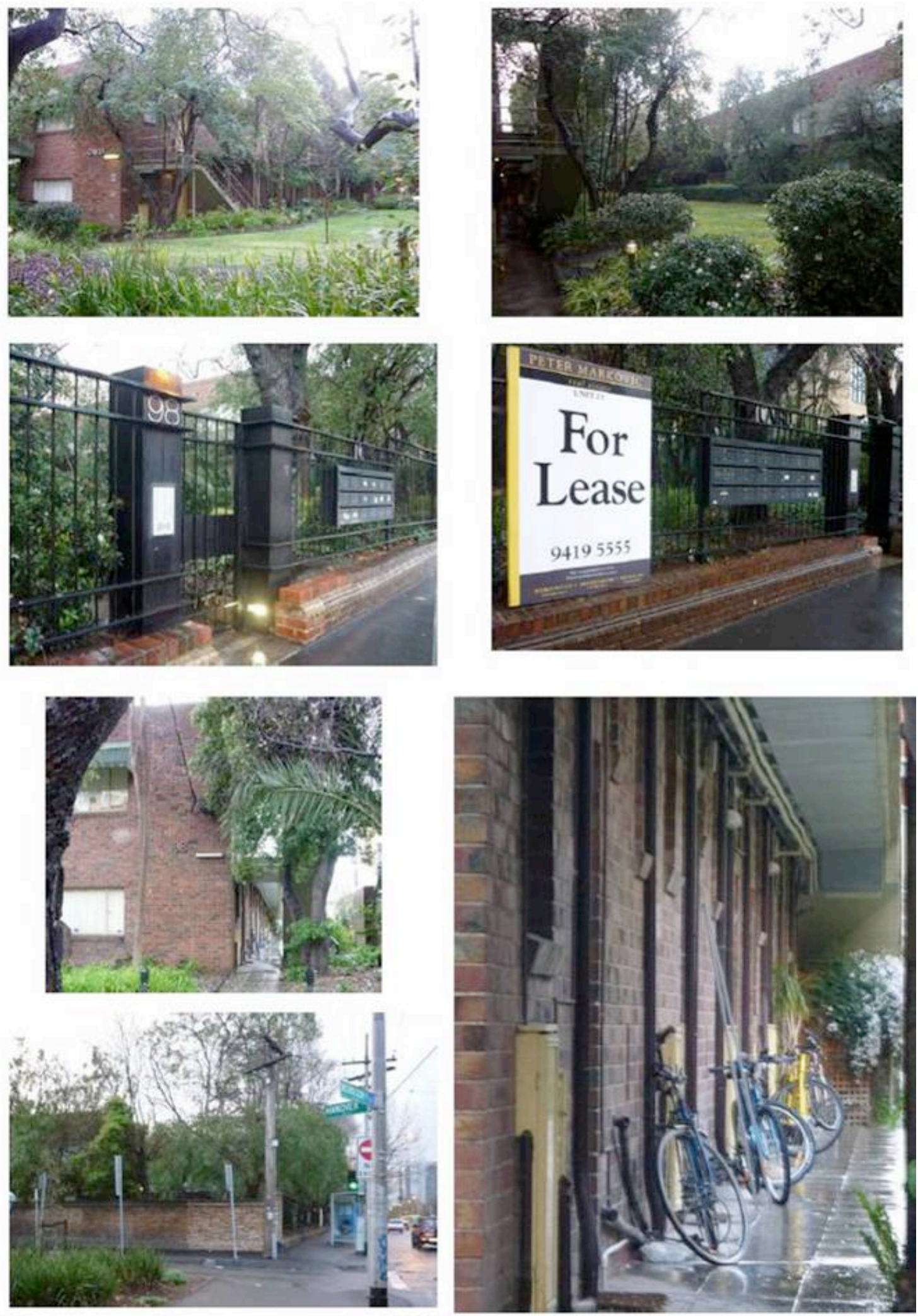

All Photos: S.J. Pattinson

M04 - Cairo Apartments

(page 2 of 2) 


\begin{tabular}{|l|c|l|}
\hline $\begin{array}{l}\text { () 2009 Stephen J. Pattinson } \\
\text { M. Arch. Thesis Research } \\
\text { Victoria University of Wellington, NZ }\end{array}$ & FIELD NOTES & Sheet No. \\
Site Visits: Tuesday 08.09.09 & M05 A \\
\hline
\end{tabular}

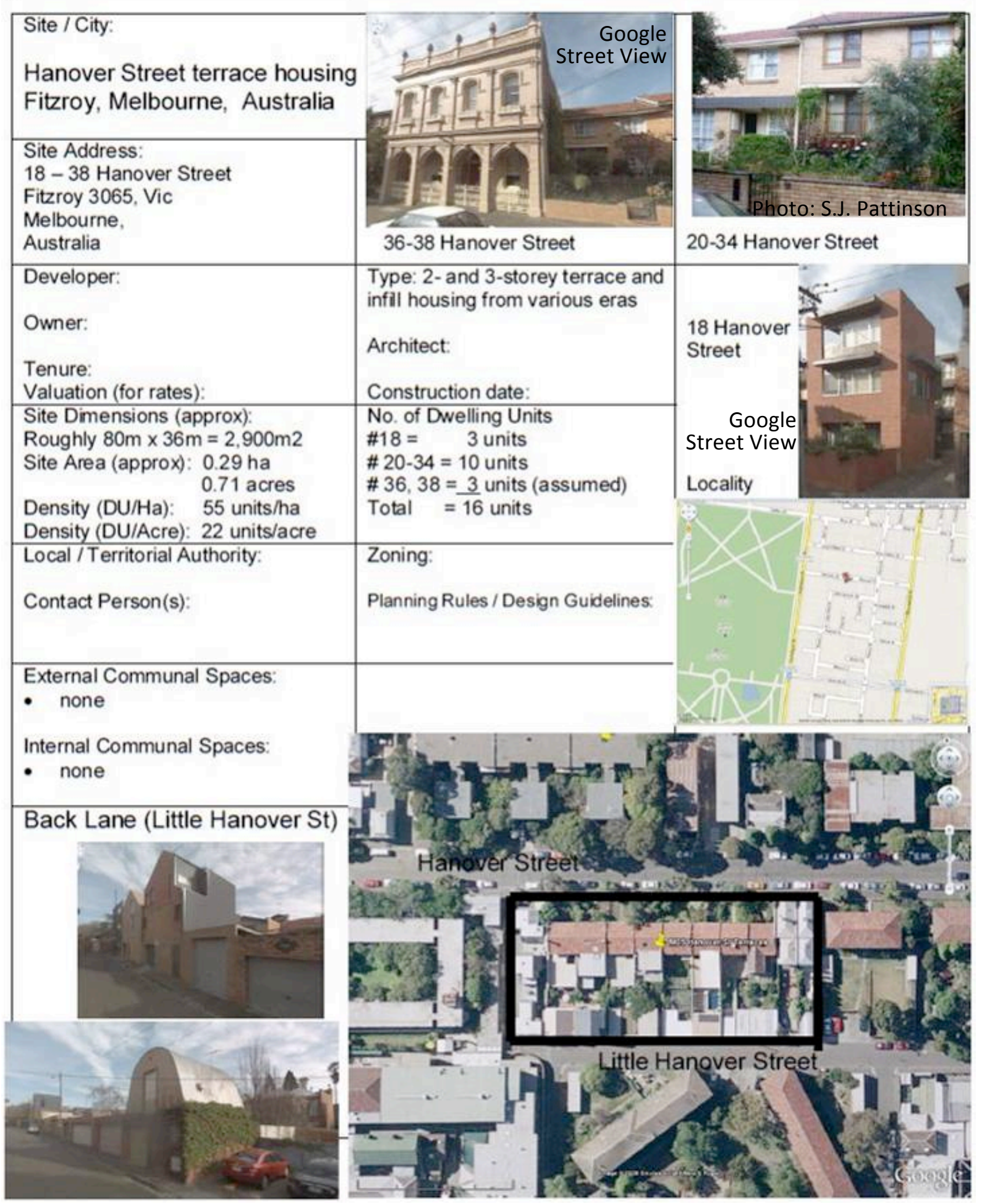

Google Street Views

Google Aerial View 
(C) 2009 Stephen J. Pattinson M. Arch. Thesis Research Victoria University of Wellington, NZ

\section{FIELD NOTES}

Site Visits: Tuesday 08.09.09

(page 1 of 2)

Sheet No.

MOG

Uodand. 15.09 .09

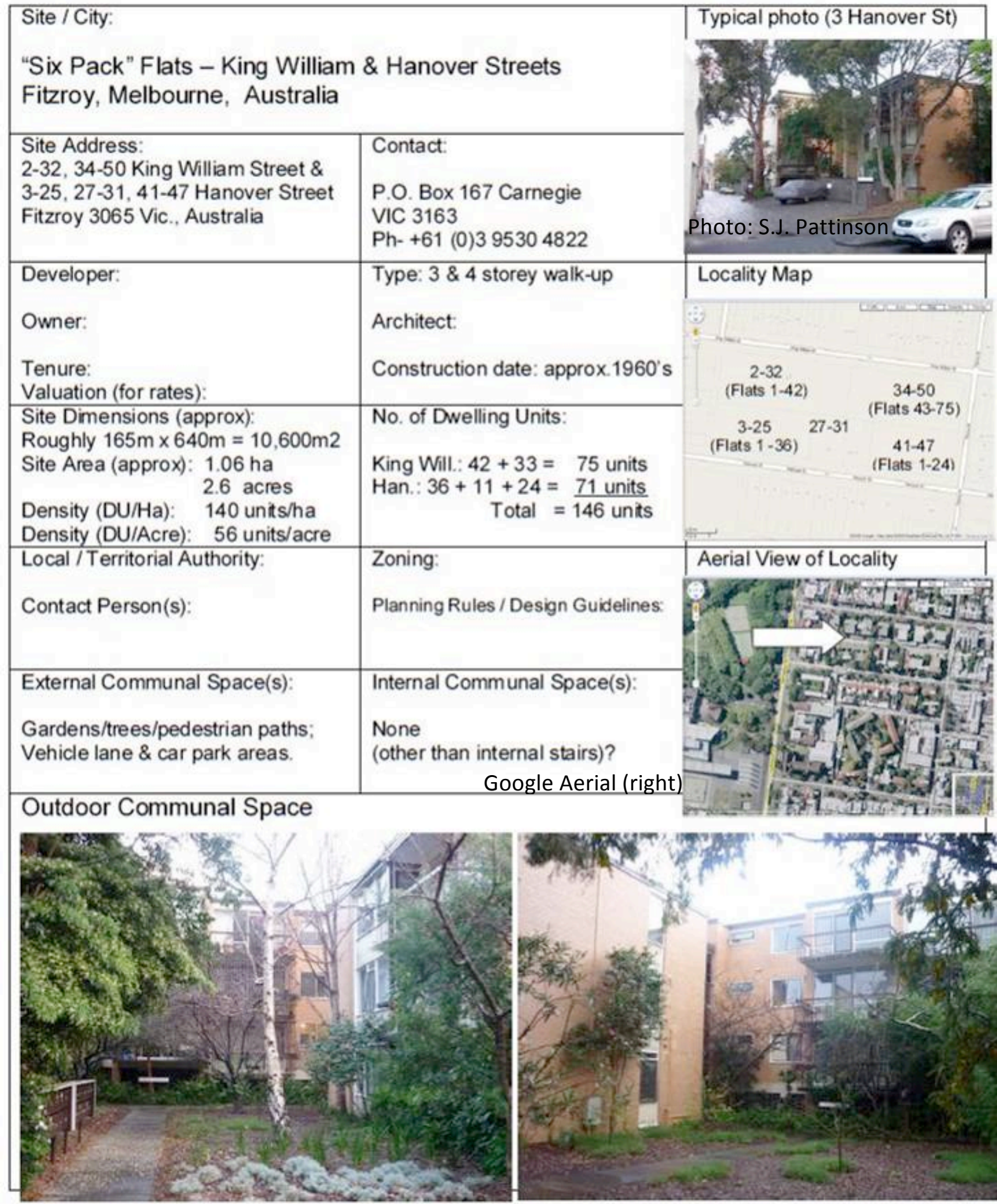

Photo: S.J. Pattinson

Photo: S.J. Pattinson 


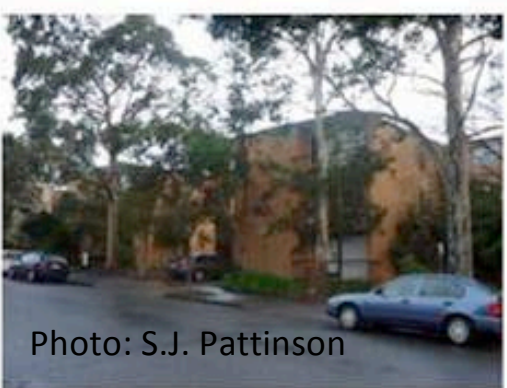

3-25 Hanover Street

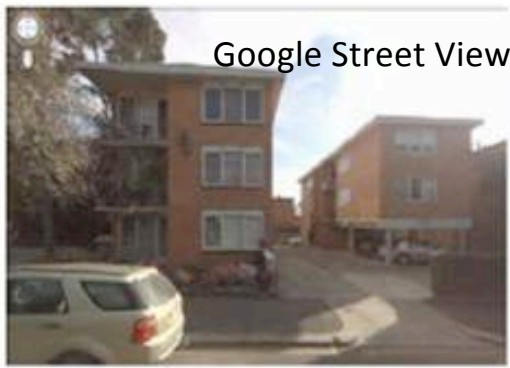

27 Hanover Street

Streetscapes

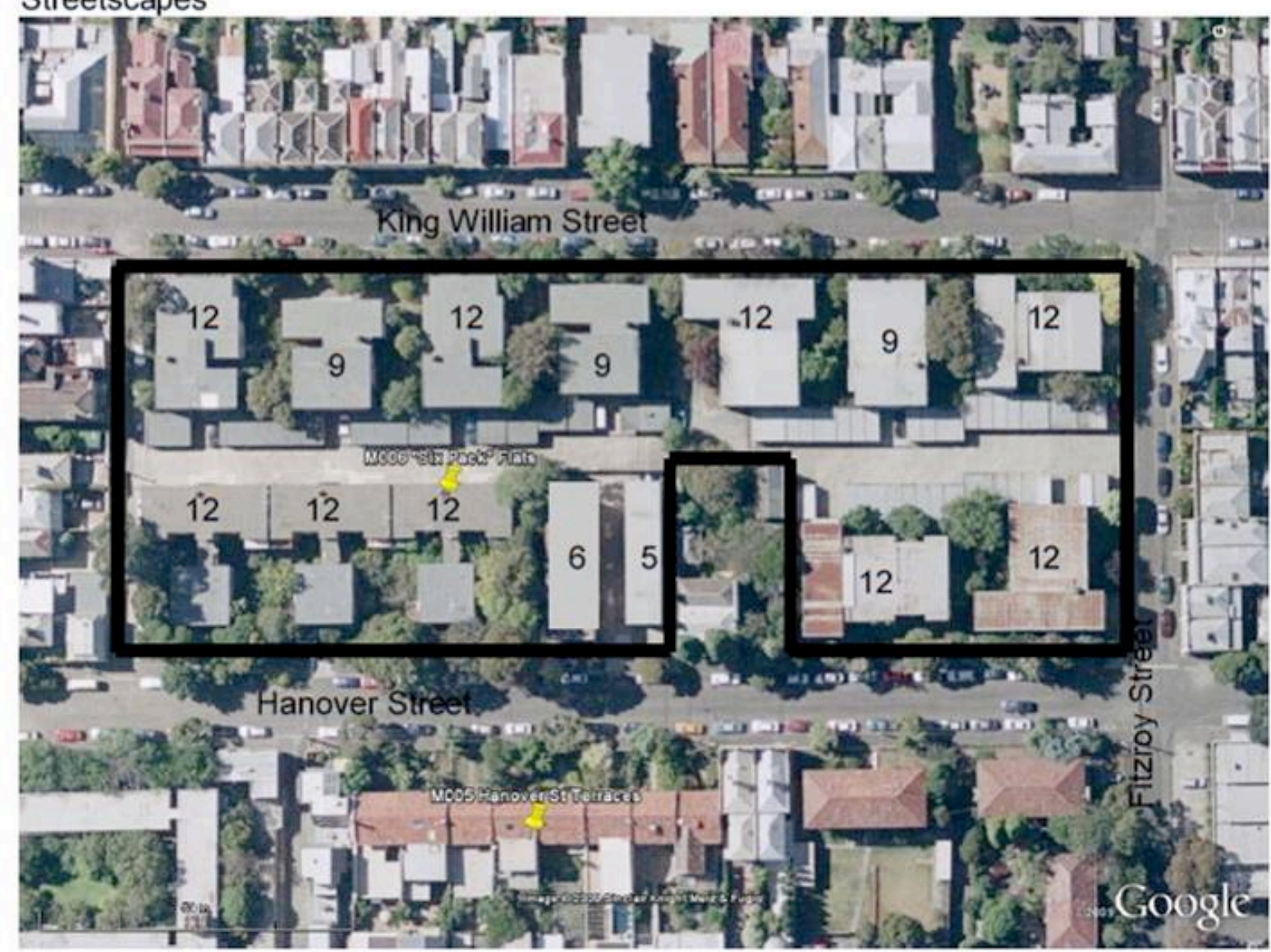

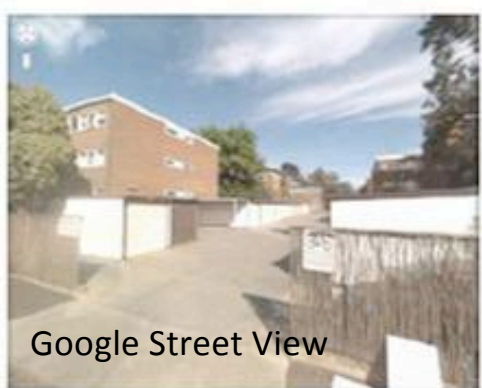

Fitzroy Street

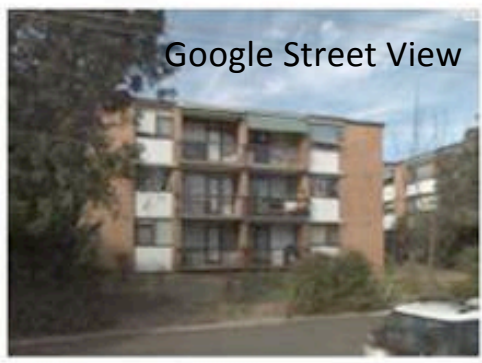

40(?) King William Street

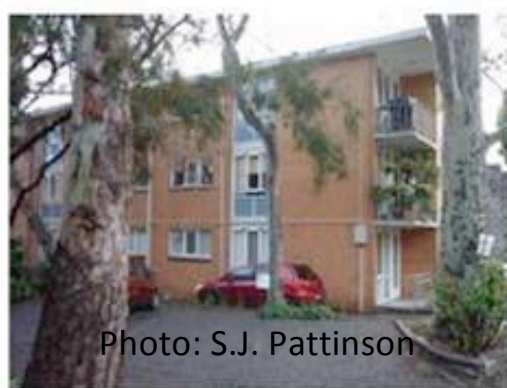

27 Hanover Street

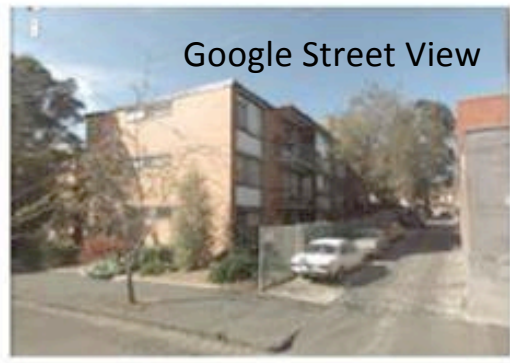

2 -32 King William Street

Aerial View of Site (indicative only)

Google Aerial View

(page 2 of 2) 


\begin{tabular}{|c|c|c|}
\hline $\begin{array}{l}\text { (c) } 2009 \text { Stephen J. Pattinson } \\
\text { M. Arch. Thesis Research } \\
\text { Victoria University of Wellington, NZ }\end{array}$ & $\begin{array}{c}\text { FIELD NOTES } \\
\text { Site Visits: Tuesday } 08.09 .09 \\
\text { (page } 1 \text { of } 2 \text { ) }\end{array}$ & $\begin{array}{l}\text { Sheet No. } \\
\text { MO }\end{array}$ \\
\hline
\end{tabular}

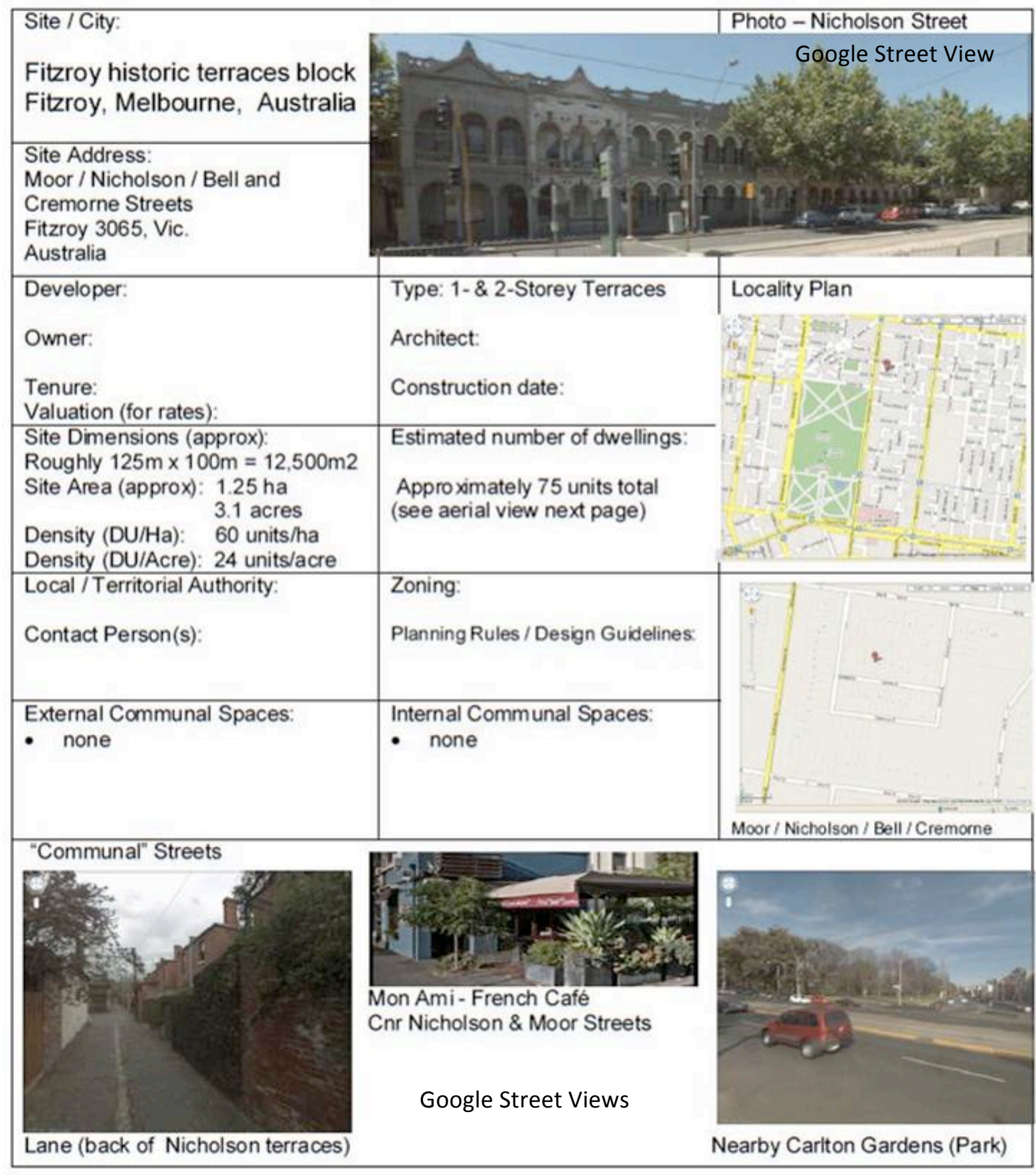


Aerial View of Neighbourhood Google Aerial View

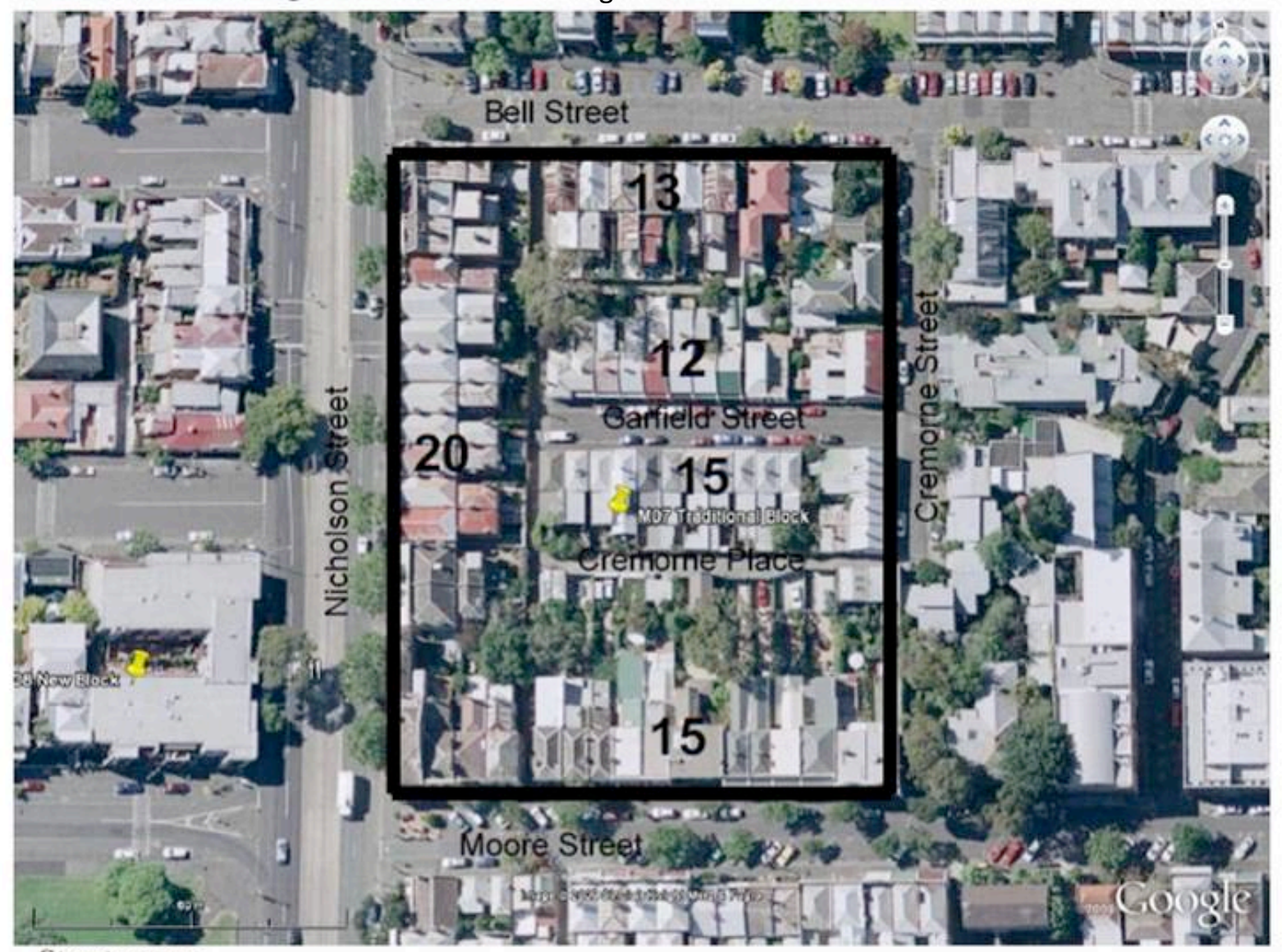

Streetscapes:

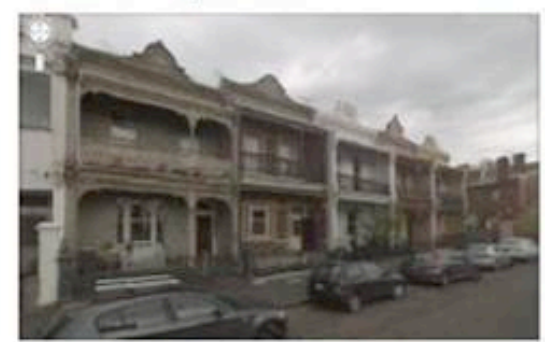

Bell Street

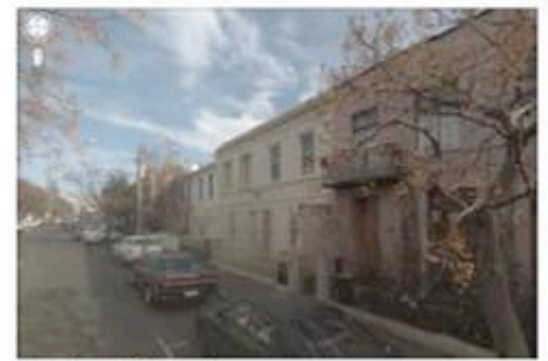

Moor Street

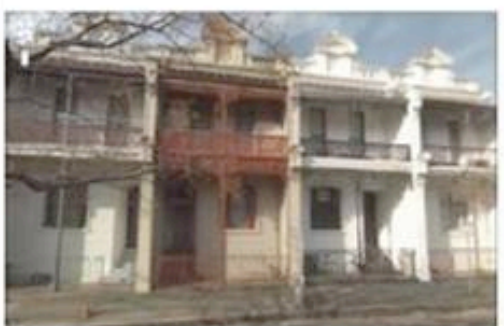

Moor Street

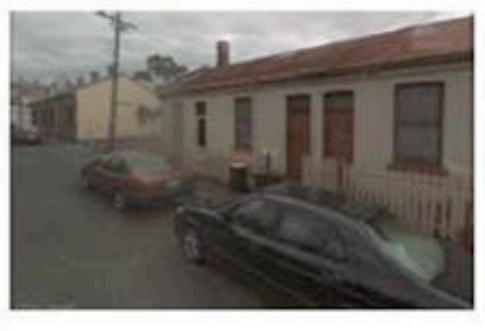

Cremorne Street

Google Street Views

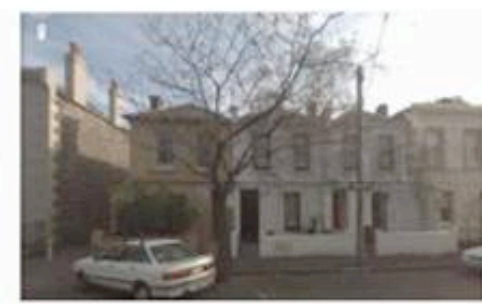

Moor Street

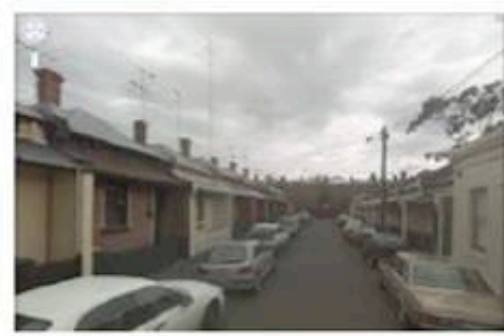

Garfield Street 


\begin{tabular}{|l|c|l|}
\hline $\begin{array}{l}\text { ㄷ 2009 Stephen J. Pattinson } \\
\text { M. Arch. Thesis Research } \\
\text { Victoria University of Wellington, NZ }\end{array}$ & FIELD NOTES & Sheet No. \\
\hline
\end{tabular}

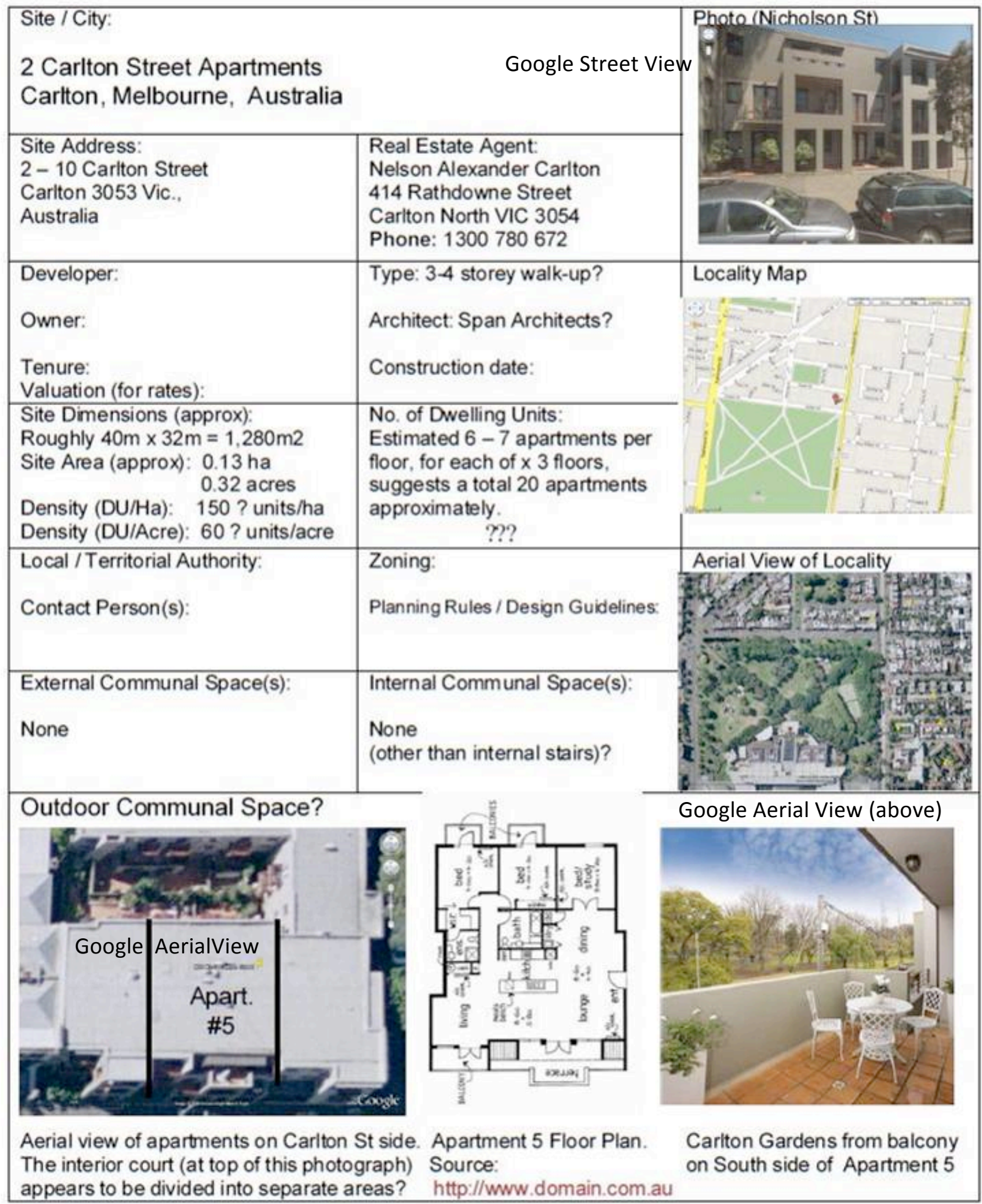



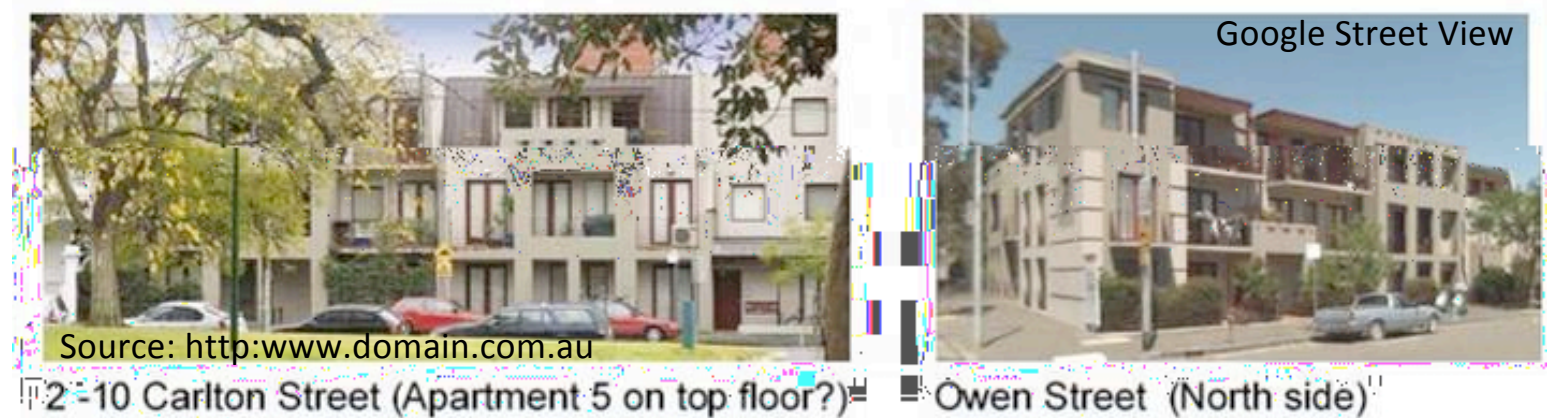

$2-10$ Carlton Street (Apartment 5 on top floor?) $=$

Owen Street (North side)"

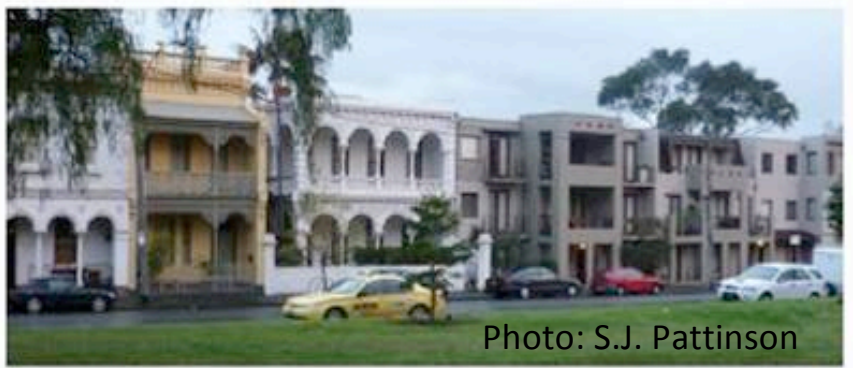

Carlton St (Apart. face south to Carlton Gardens) Streetscapes

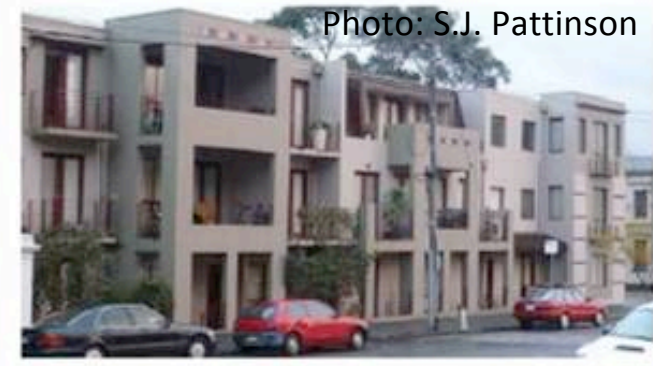

2 -10 Carlton Street (South side)

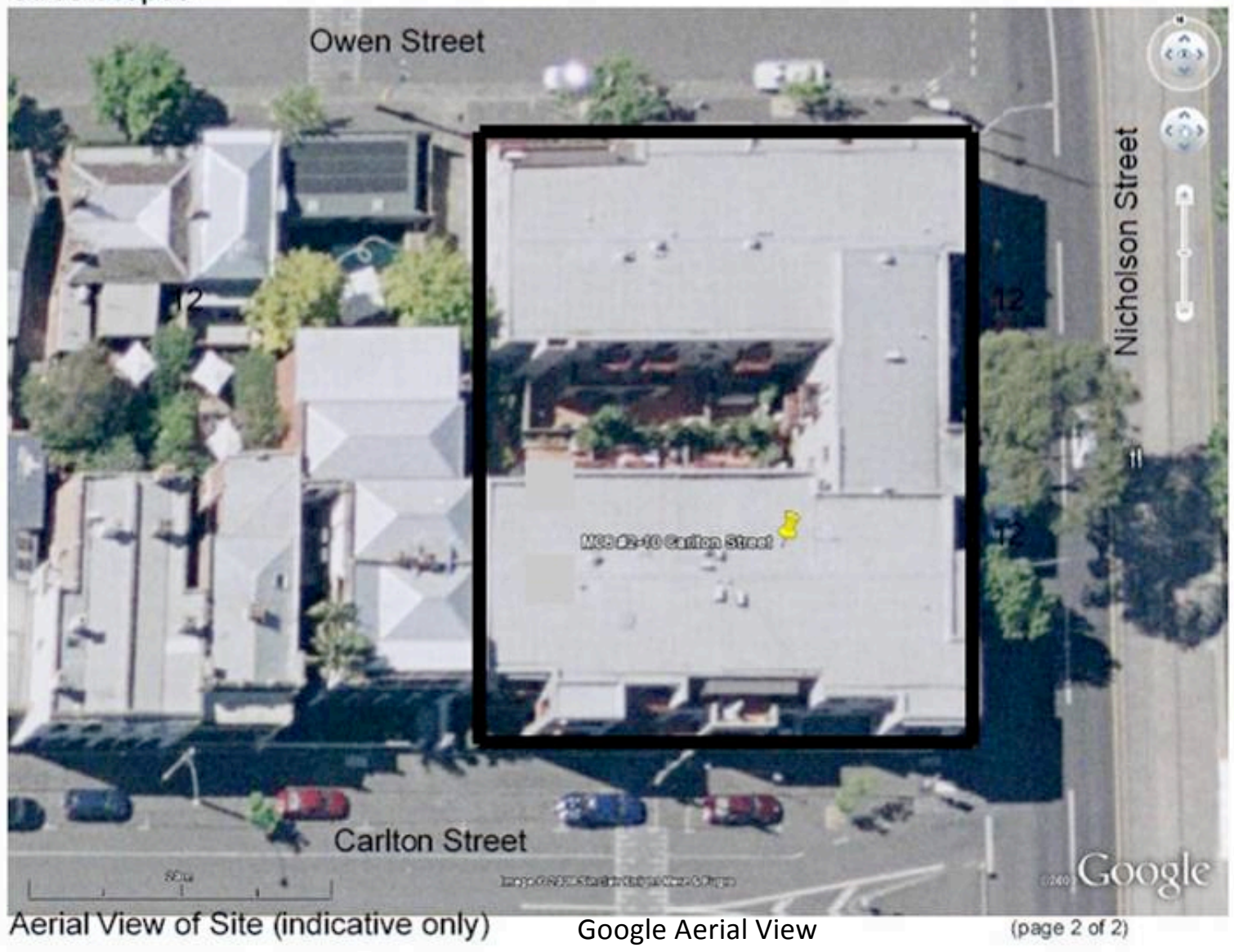




\begin{tabular}{|l|c|l|}
\hline $\begin{array}{l}\text { (9) 2009 Stephen J. Pattinson } \\
\text { M. Arch. Thesis Research } \\
\text { Victoria University of Wellington, NZ }\end{array}$ & FIELD NOTES & $\begin{array}{l}\text { Sheet No. } \\
\text { Site Visits: Tuesday 08.09.09 }\end{array}$ \\
\hline
\end{tabular}

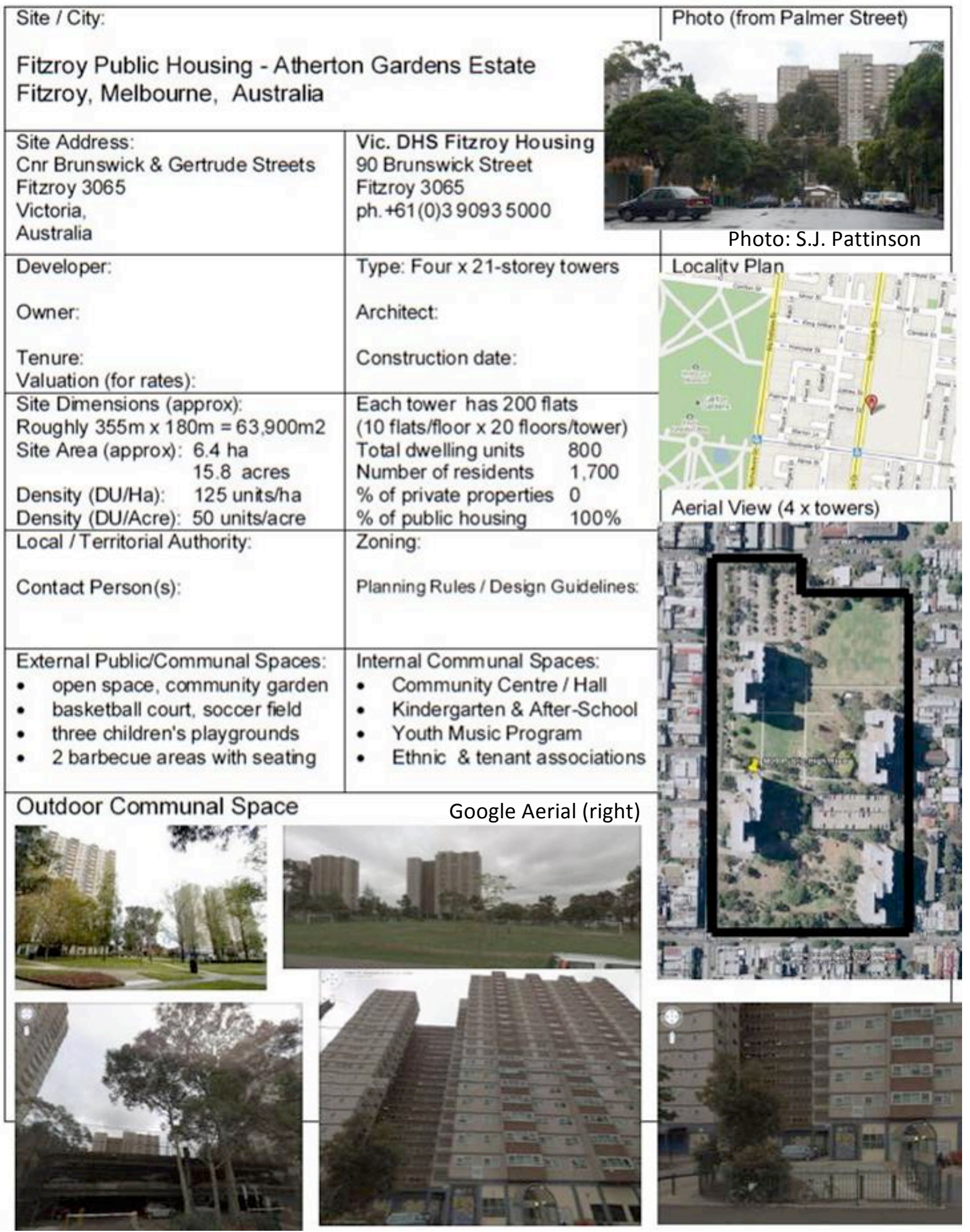

All Google Street views 
(C) 2009 Stephen J. Pattinson M. Arch. Thesis Research Victoria University of Wellington, NZ

\section{FIELD NOTES}

Site Visits:

(page 1 of 2)

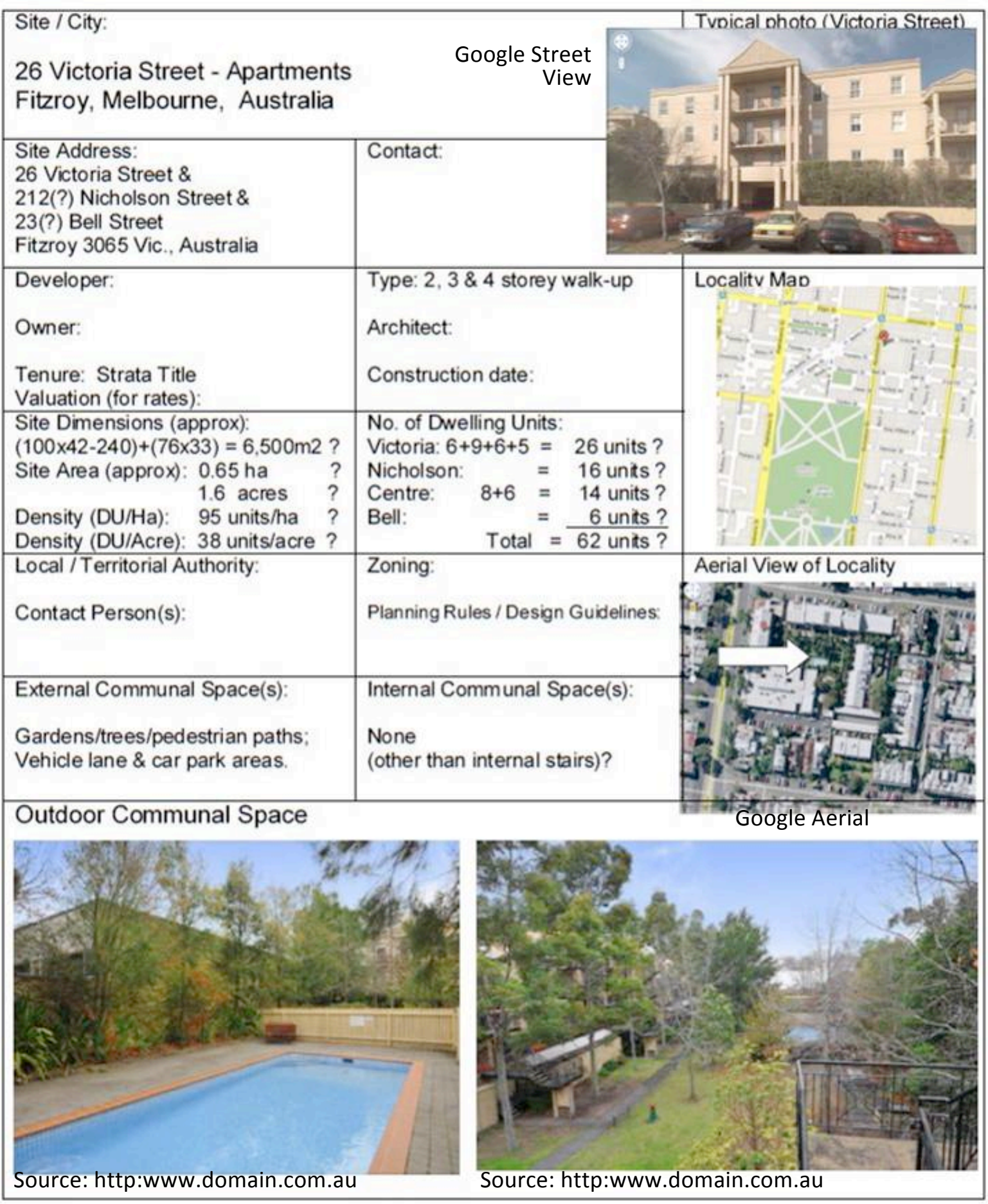



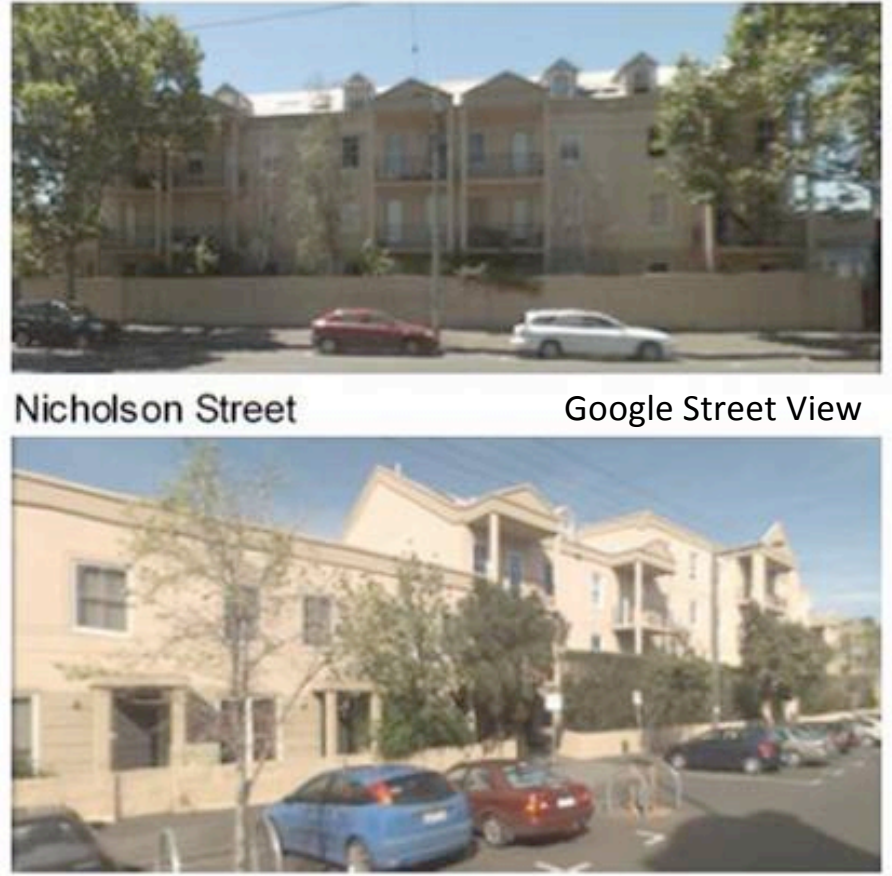

Victoria Street

Google Street View

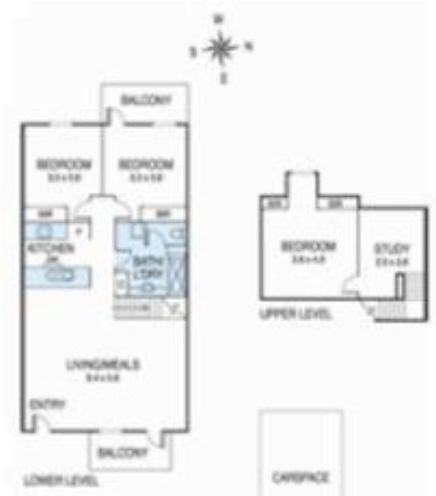

Source: http:www.domain.com.au

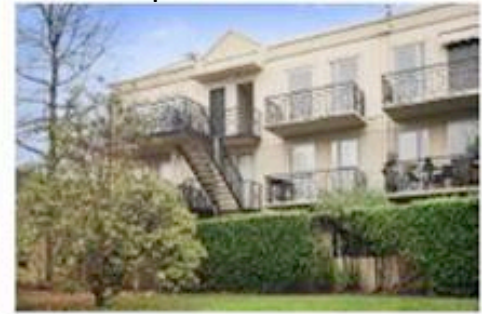

Apartment \#38

Source: http:www.domain.com.au

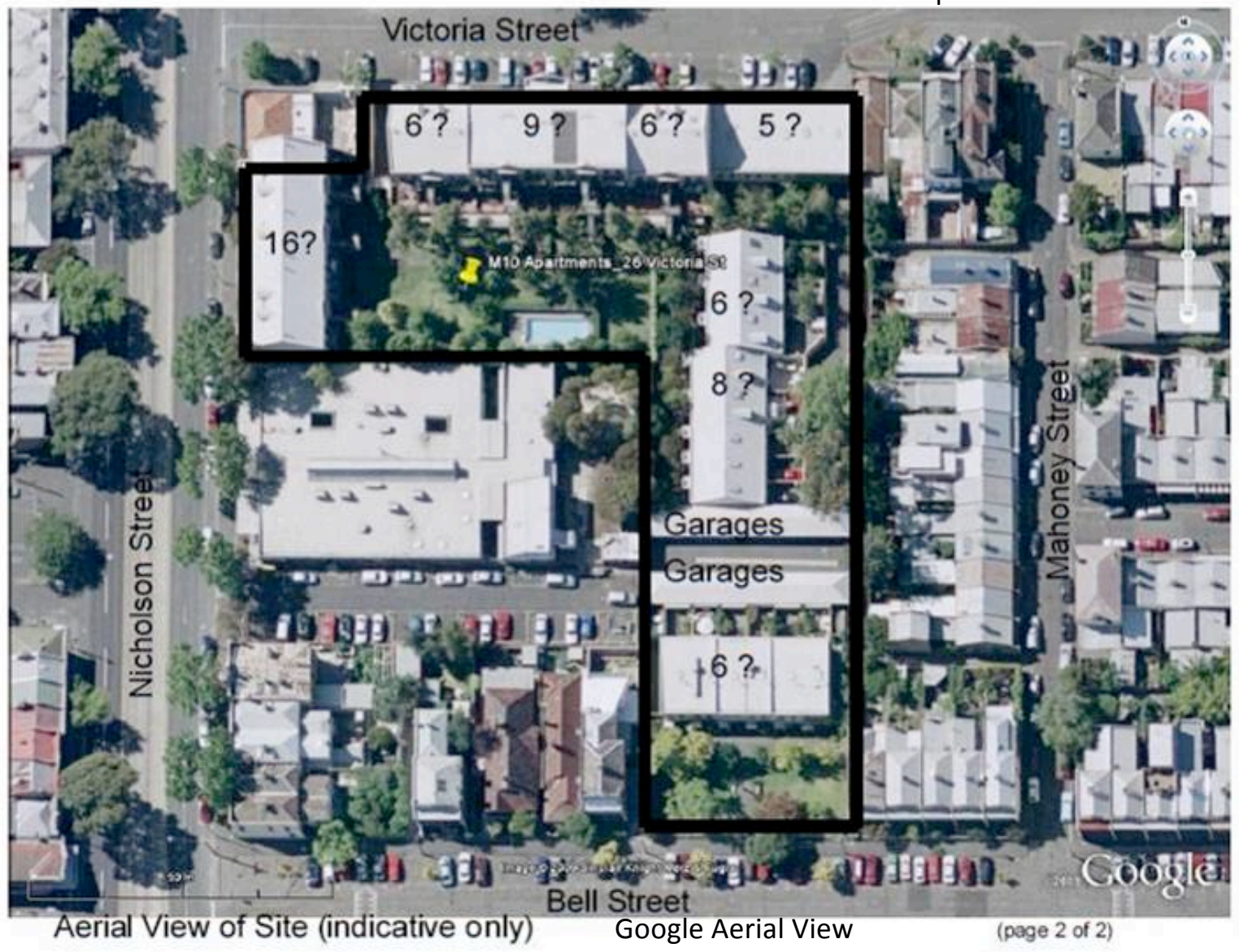




\begin{tabular}{|c|c|c|}
\hline $\begin{array}{l}\text { C } 2009 \text { Stephen J. Pattinson } \\
\text { M. Arch. Thesis Research } \\
\text { Victoria University of Wellington, NZ }\end{array}$ & $\begin{array}{l}\text { FIELD NOTES } \\
\text { Site Visits: Tuesday } 08.09 .09\end{array}$ & Sheet No. \\
\hline
\end{tabular}

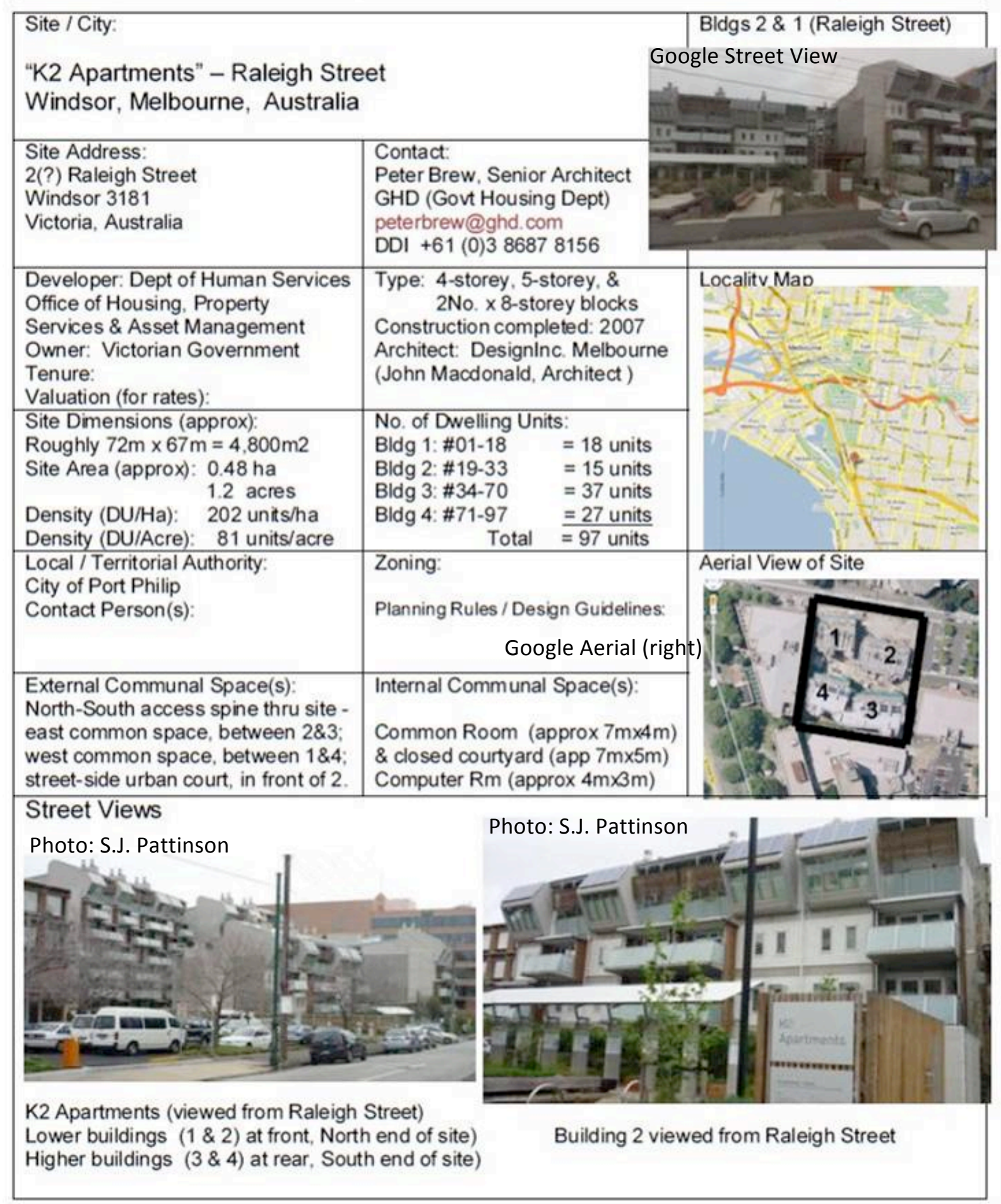


Photo: S.J. Pattinson

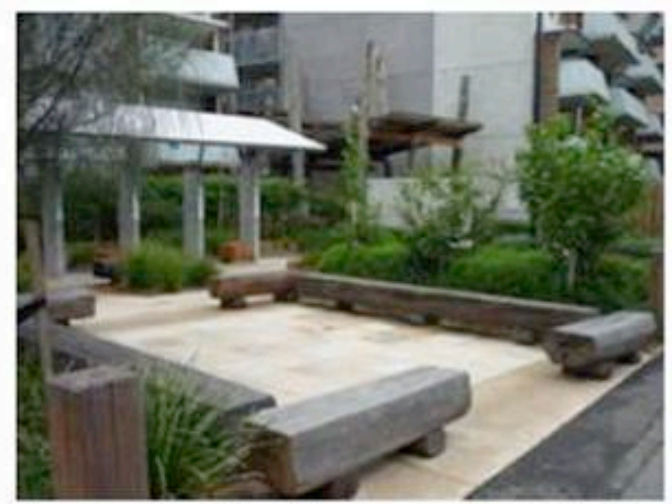

Urban Courtvard (public space off Raleiah Street)
Photo: S.J. Pattinson

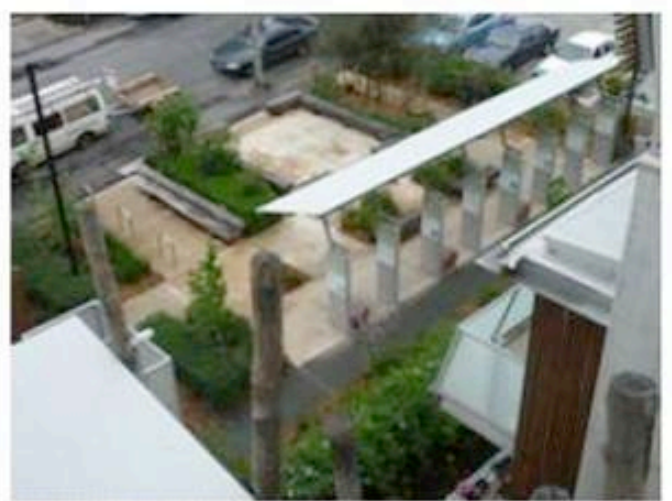

Urban Courtyard (viewed from Building 1)

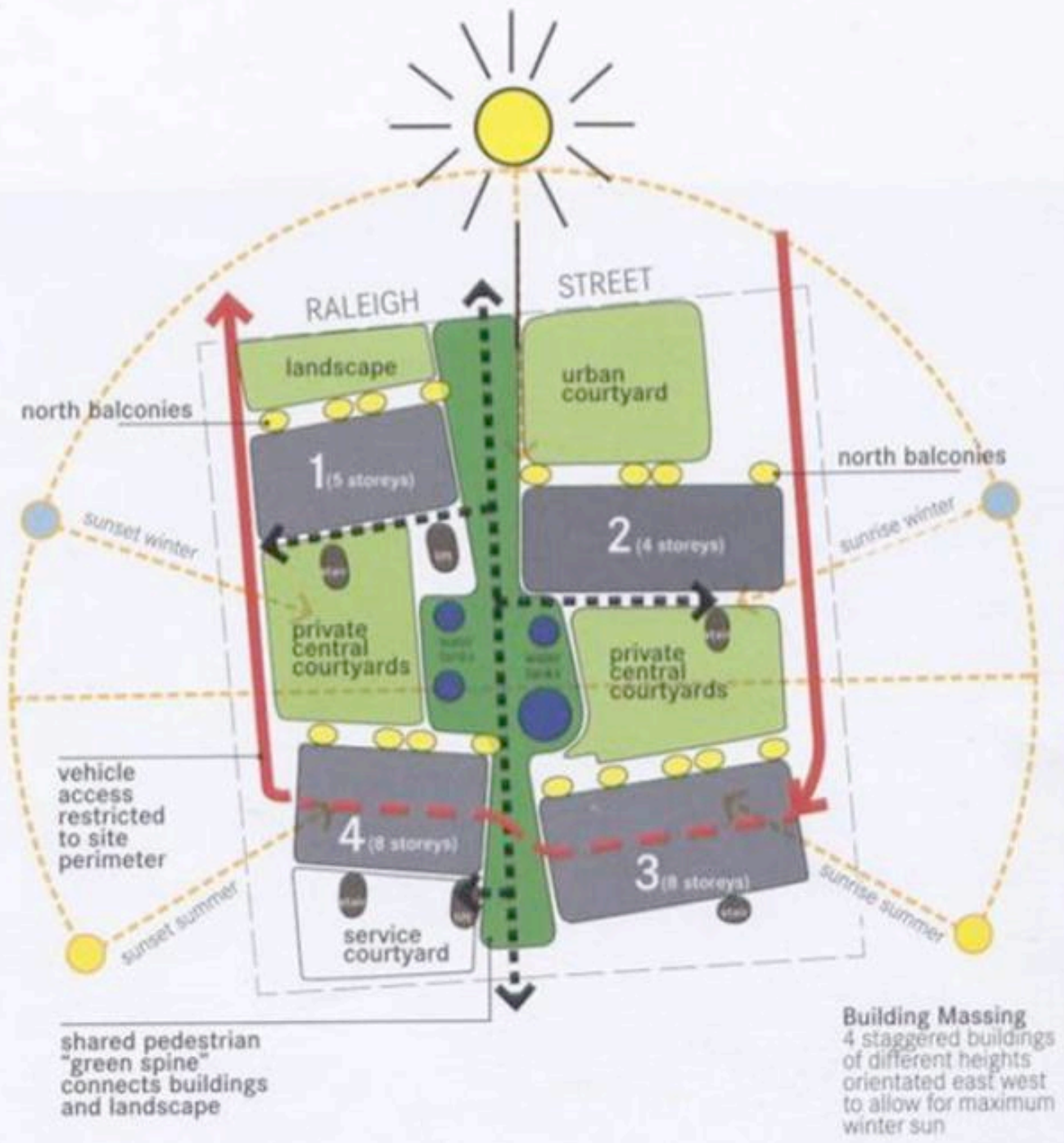

Site Planning Strategy

Source: Victorian Government Housing Department -

(page 2 of 4)

K2 Apartments brochure p. 3 


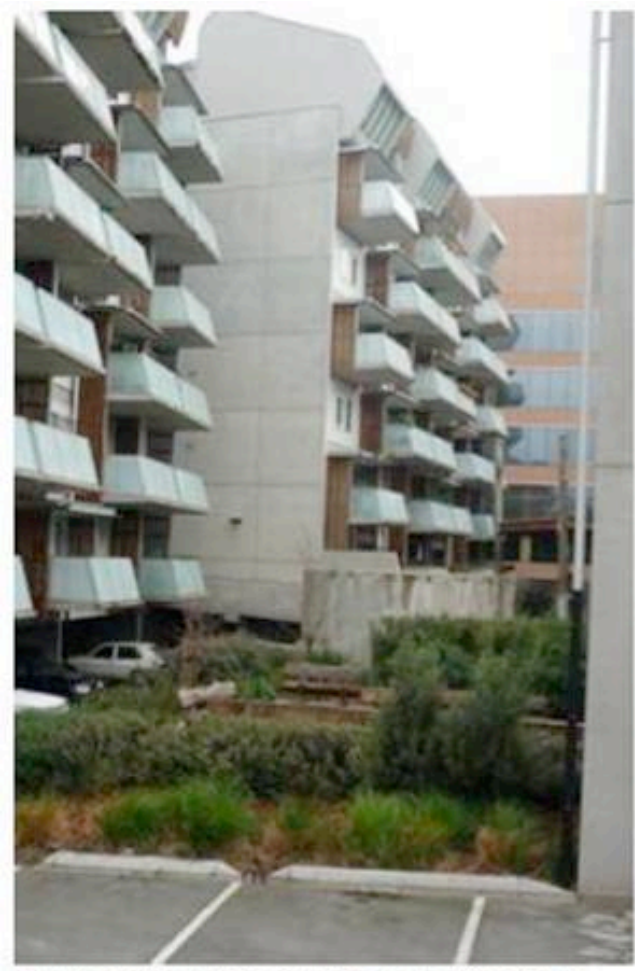

East courtyard viewed from parking bay

West "private" central courtyard

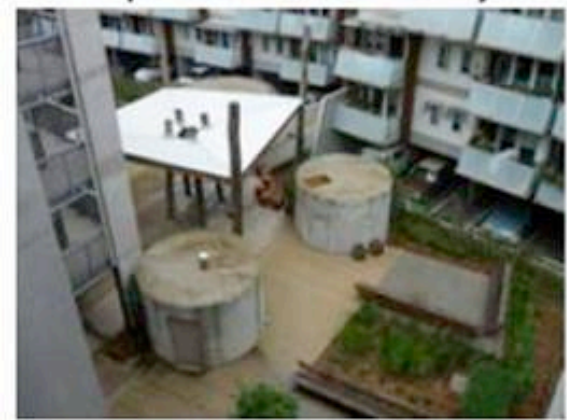

West courtyard has two seating areas

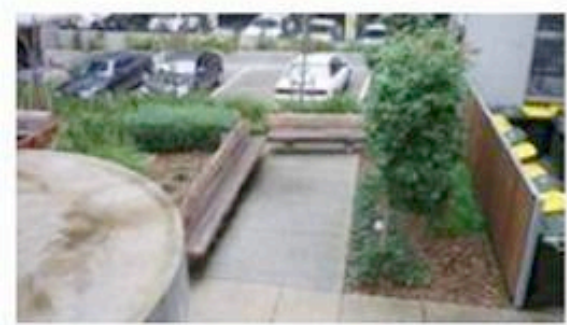

Part of West courtyard

All photos this page: S.J. Pattinson

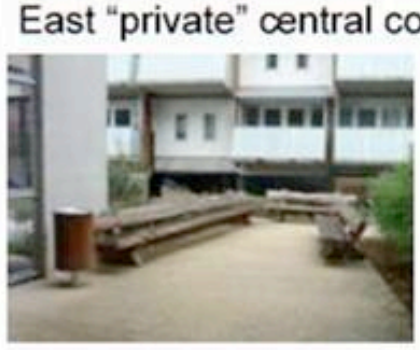

East courtyard

has two

seating areas
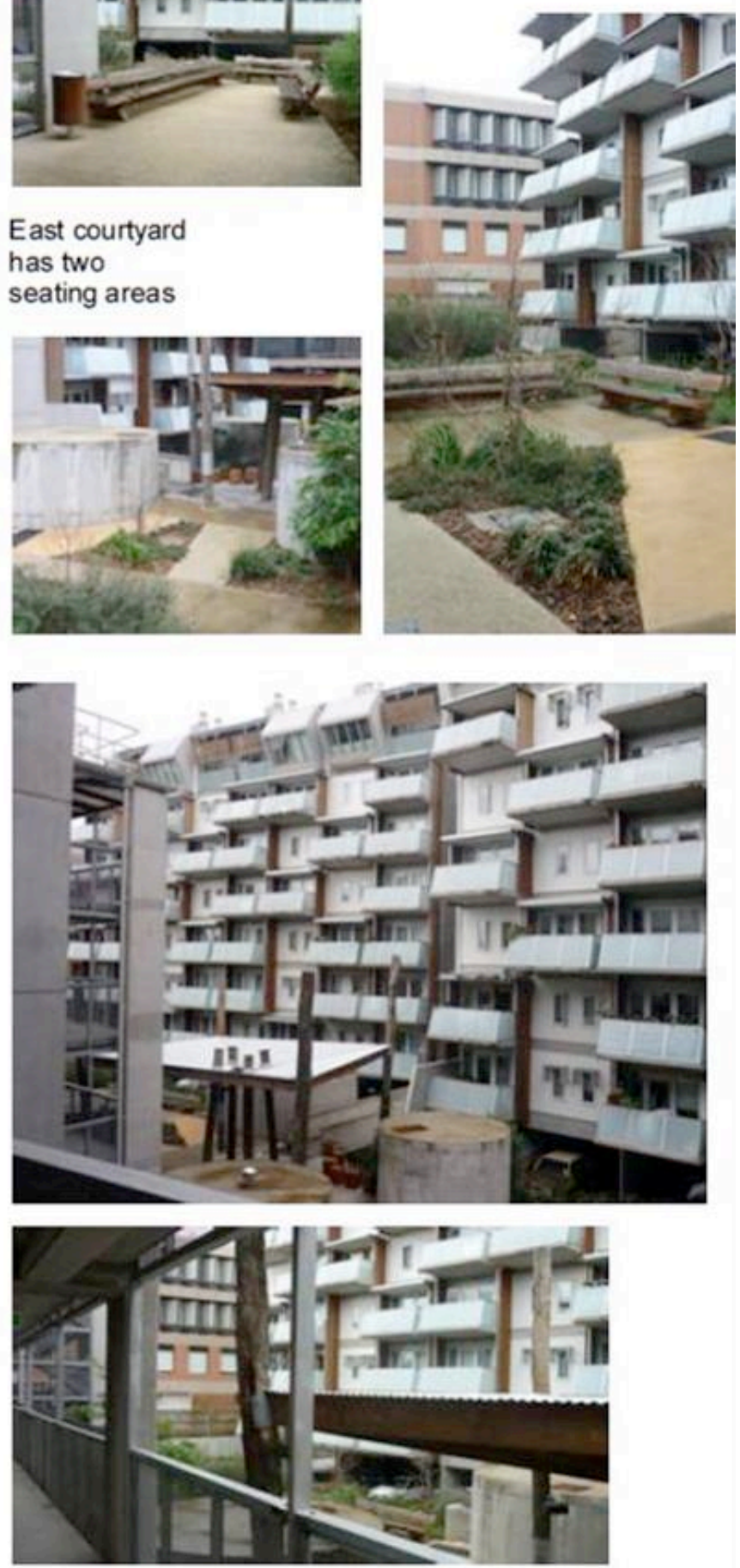

All four buildings (and the neighbouring buildings) overlook the East and West central courtyards

(page 3 of 4 ) 
Source of map (below) - Victorian Government Housing Department: Photographed on site by S.J. Pattinson - signage in "urban park"

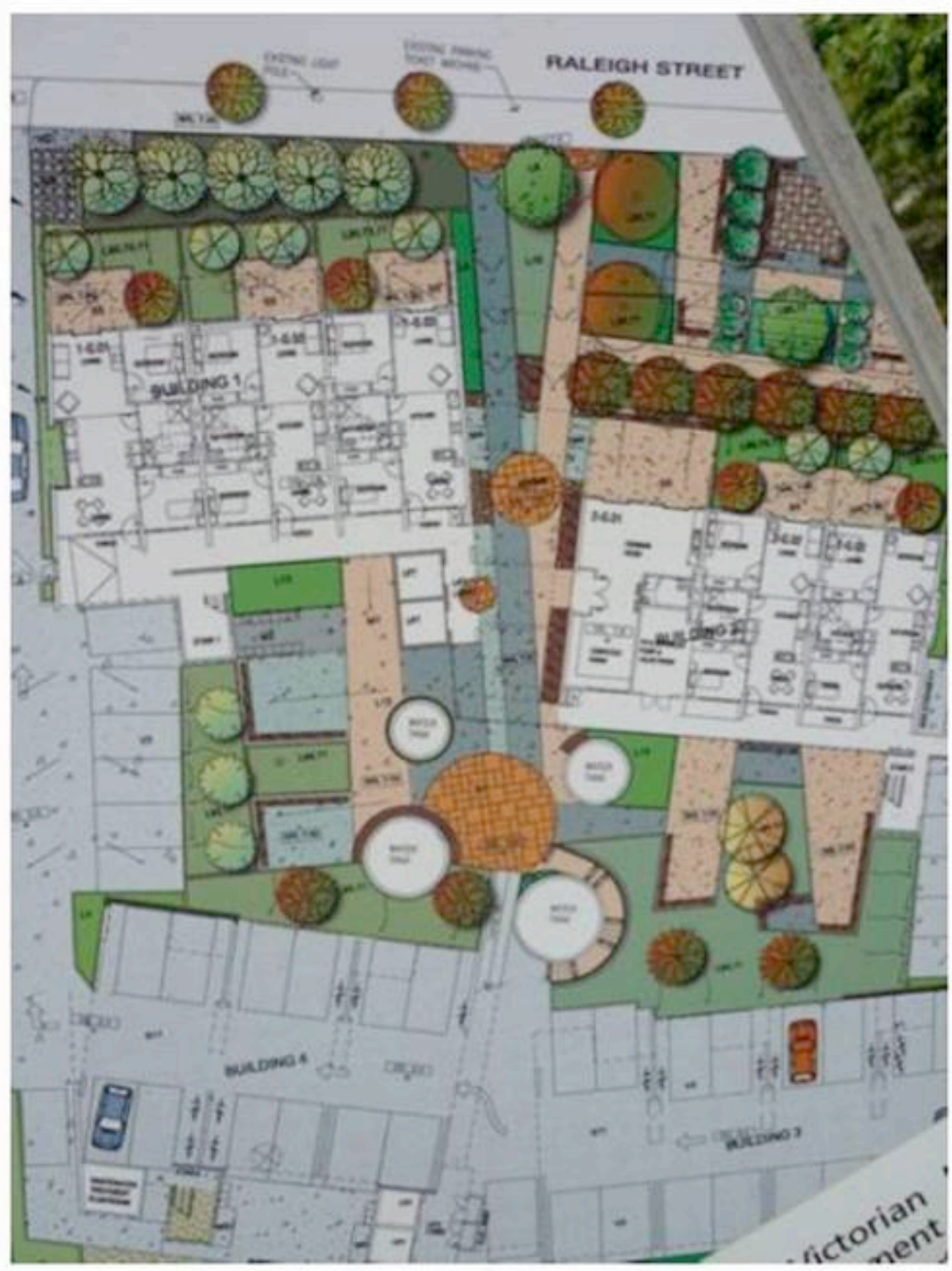

Entry (from Raleigh Street):
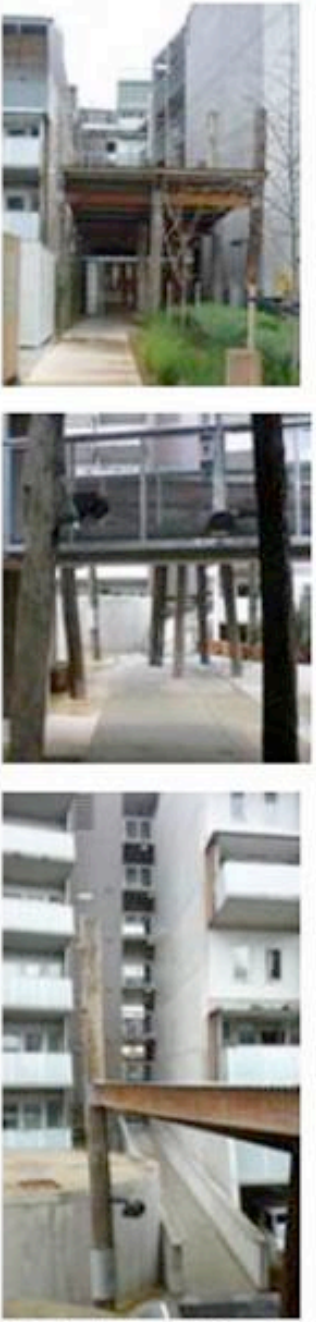

Common Room and Closed Courtyard access by appointment
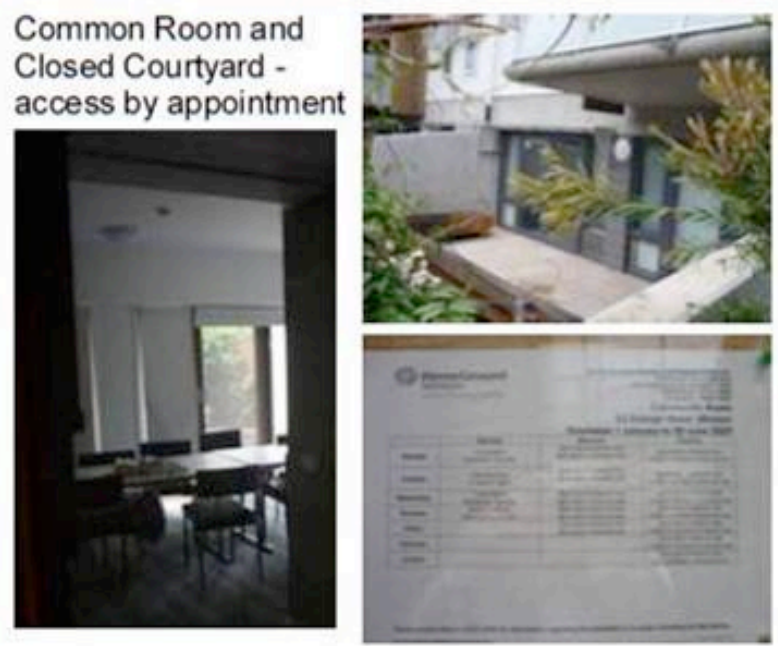

All photos this page: S.J. Pattinson
Typical access to units via lifts and external balconies:

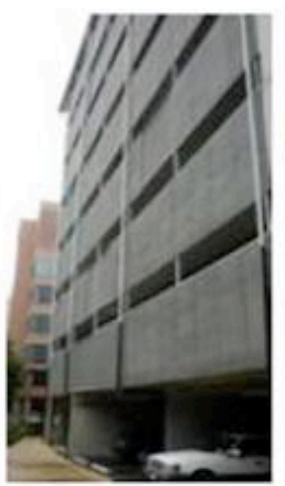

Central North-South spine
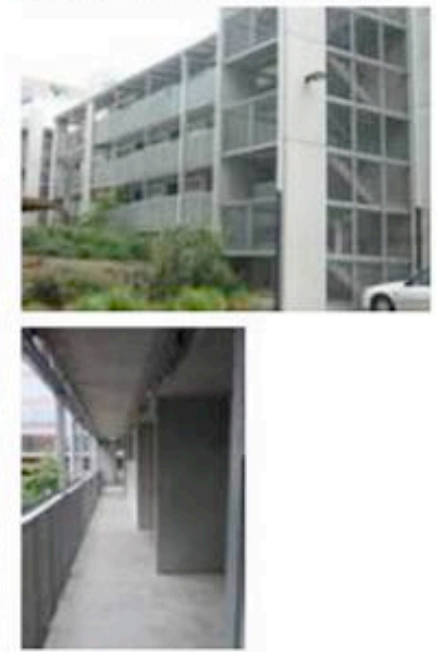

(page 4 of 4 ) 


\begin{tabular}{|c|c|c|}
\hline $\begin{array}{l}\text { (C) } 2009 \text { Stephen J. Pattinson } \\
\text { M. Arch. Thesis Research } \\
\text { Victoria University of Wellington, NZ }\end{array}$ & $\underset{\text { Site Visits: }}{\text { FIELD NOTES }}$ & $\mathrm{M}_{\text {Updatod. }}{ }^{\text {Sheet }}{ }^{\mathrm{A}} \mathrm{A}$ \\
\hline
\end{tabular}

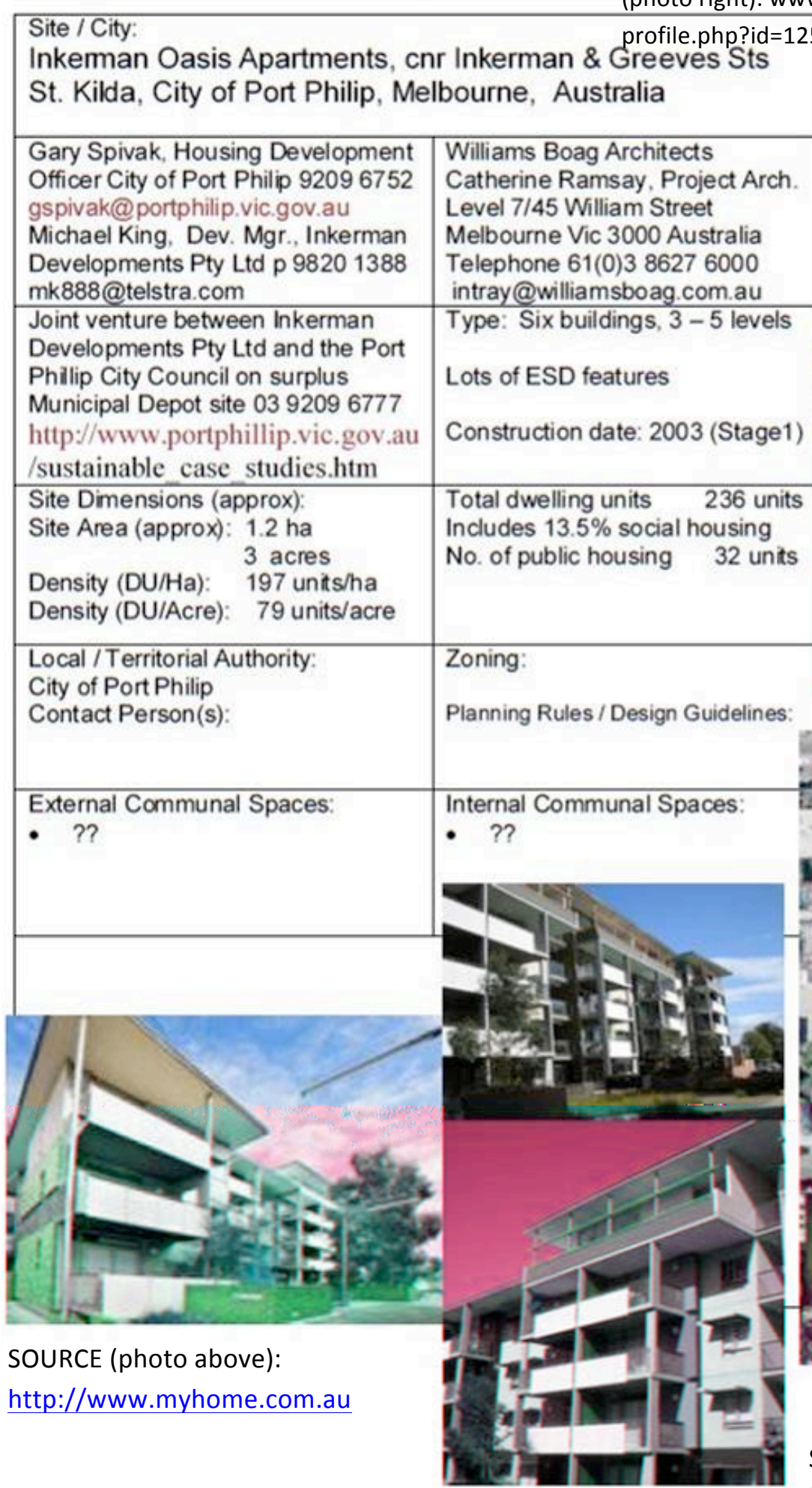

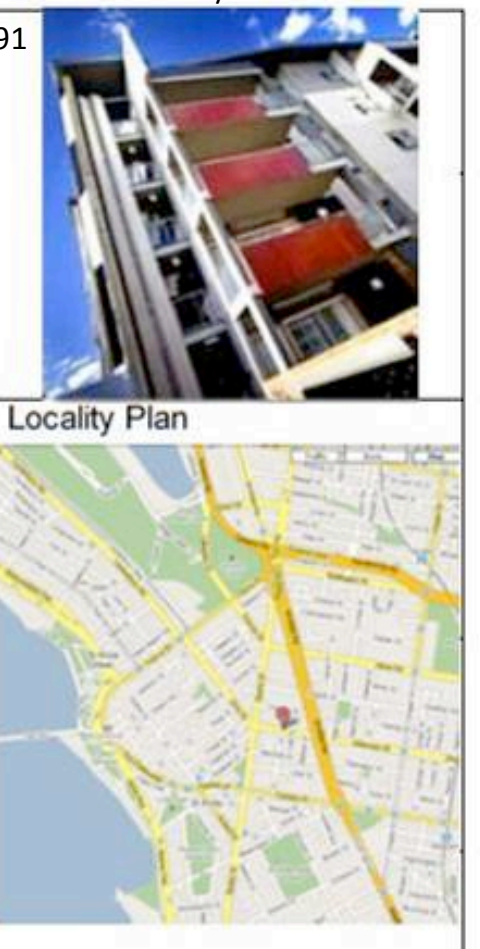

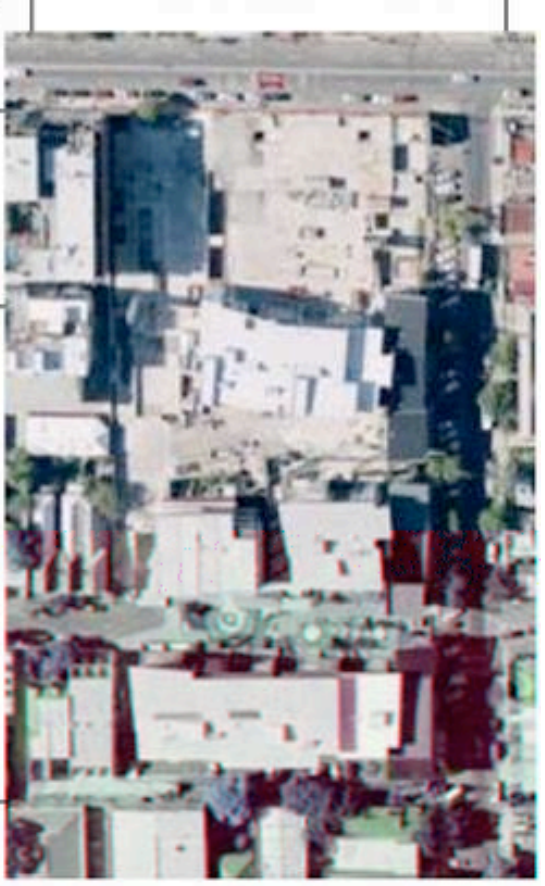

Google Aerial View

SOURCE (photo left and above left): http://www.urbanprop.com.au/past Projects.shtml 


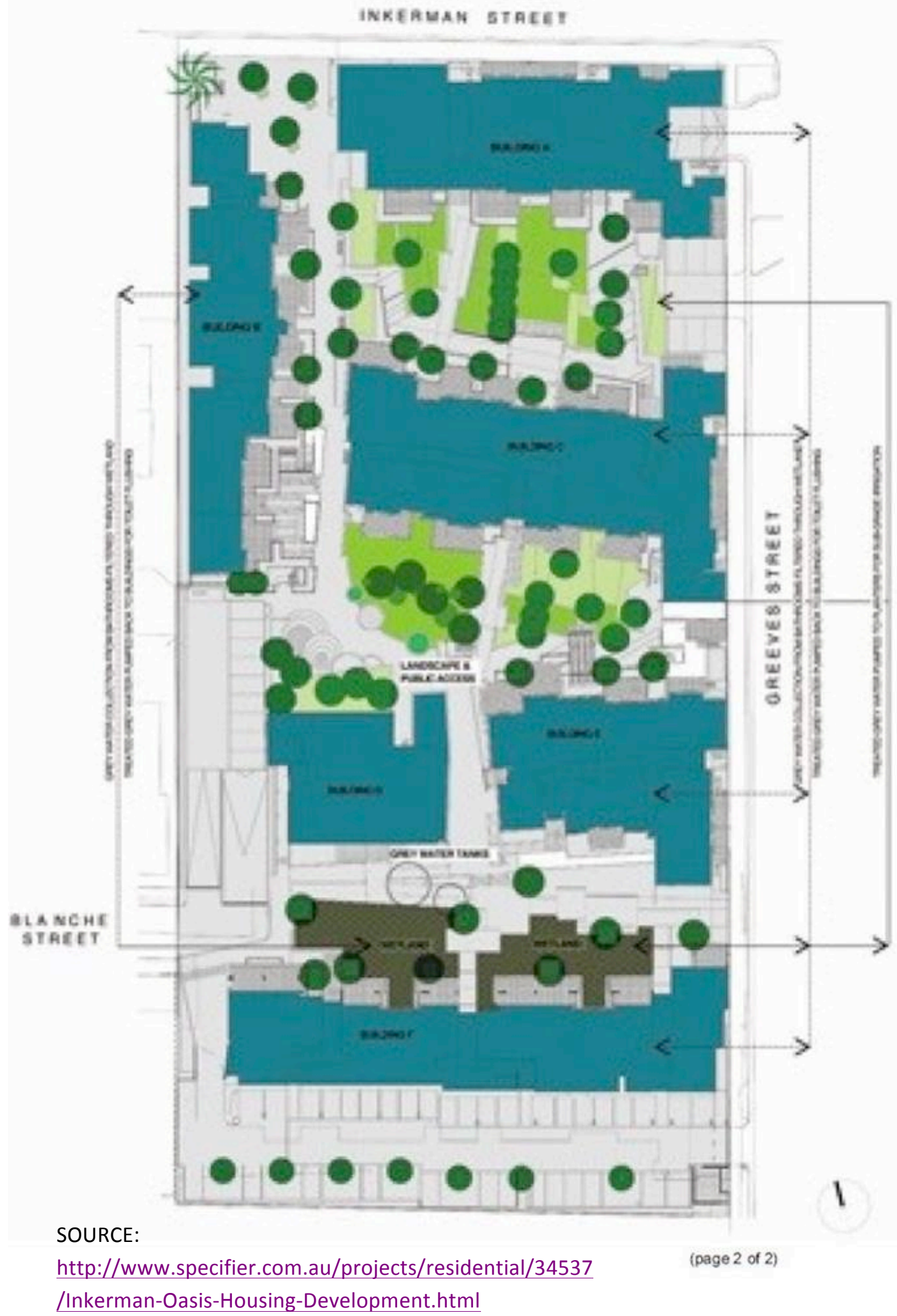




\begin{tabular}{|c|c|c|}
\hline $\begin{array}{l}\text { (c) } 2009 \text { Stephen J. Pattinson } \\
\text { M. Arch. Thesis Research } \\
\text { Victoria University of Wellington, NZ }\end{array}$ & $\begin{array}{l}\text { FIELD NOTES } \\
\text { Site Visits: Tuesday } 08.09 .09 \quad \text { (page } 1 \text { of } 2)\end{array}$ & Sheet No. \\
\hline
\end{tabular}

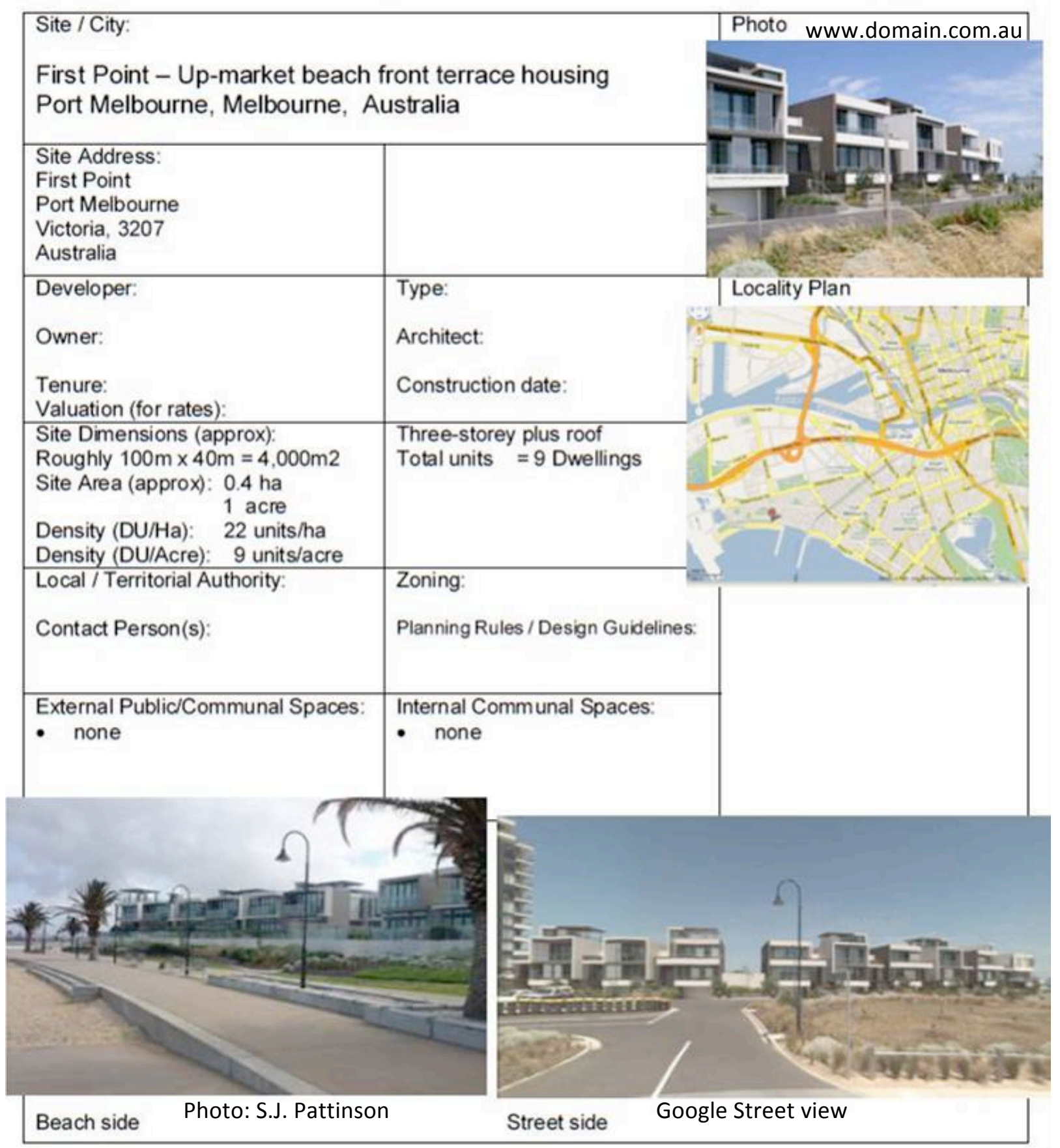



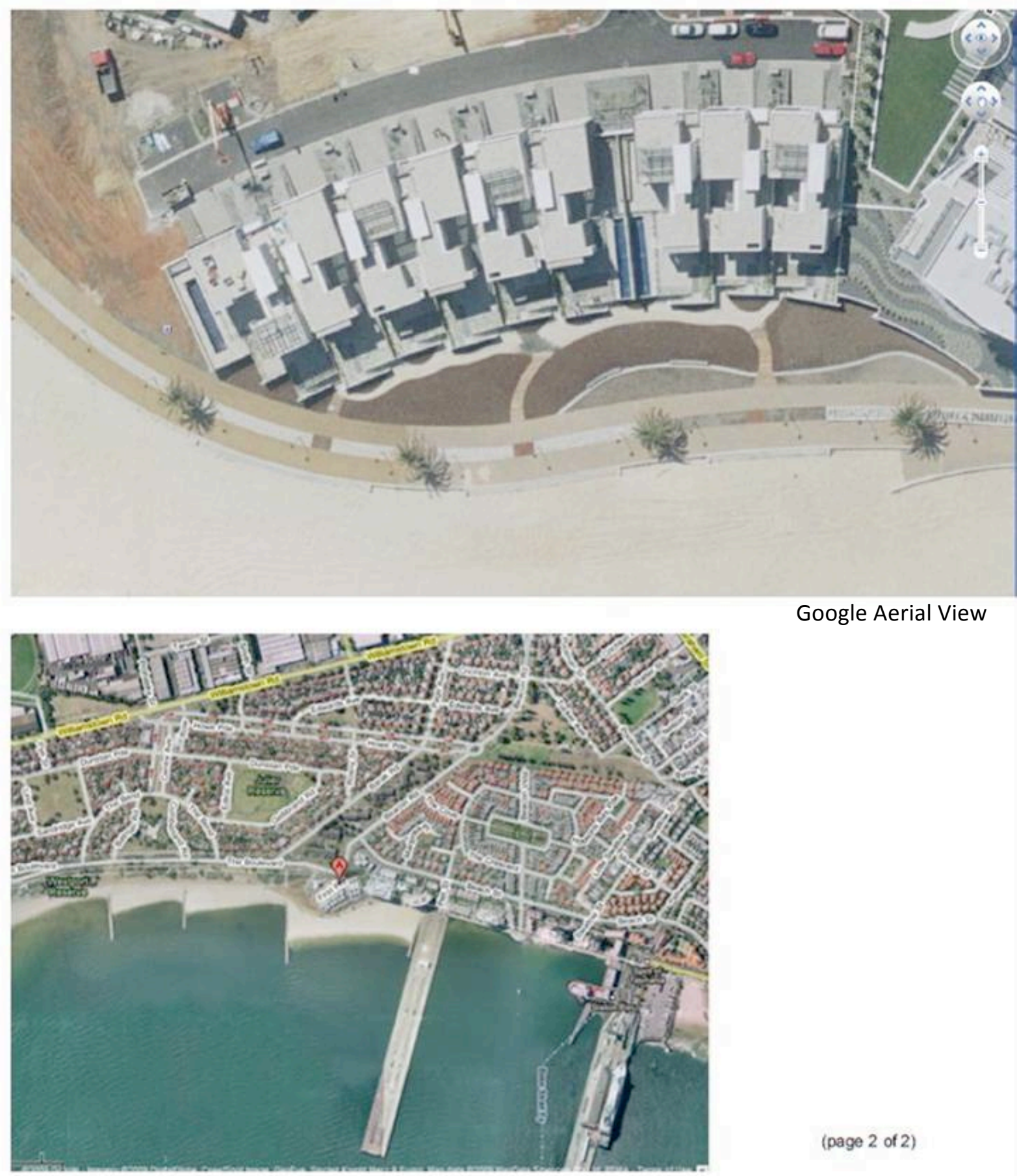

Google Aerial View

Google Aerial View

(page 2 of 2 ) 


\begin{tabular}{|l|c|l|}
\hline $\begin{array}{l}\text { (c) 2009 Stephen J. Pattinson } \\
\text { M. Arch. Thesis Research } \\
\text { Victoria University of Wellington, NZ }\end{array}$ & $\begin{array}{c}\text { FIELD NOTES } \\
\text { Site Visits: } 08.9 .09 \quad \text { (page 1 of 3) }\end{array}$ & $\begin{array}{l}\text { M14 A A } \\
\text { Updated 1210.09 }\end{array}$ \\
\hline
\end{tabular}

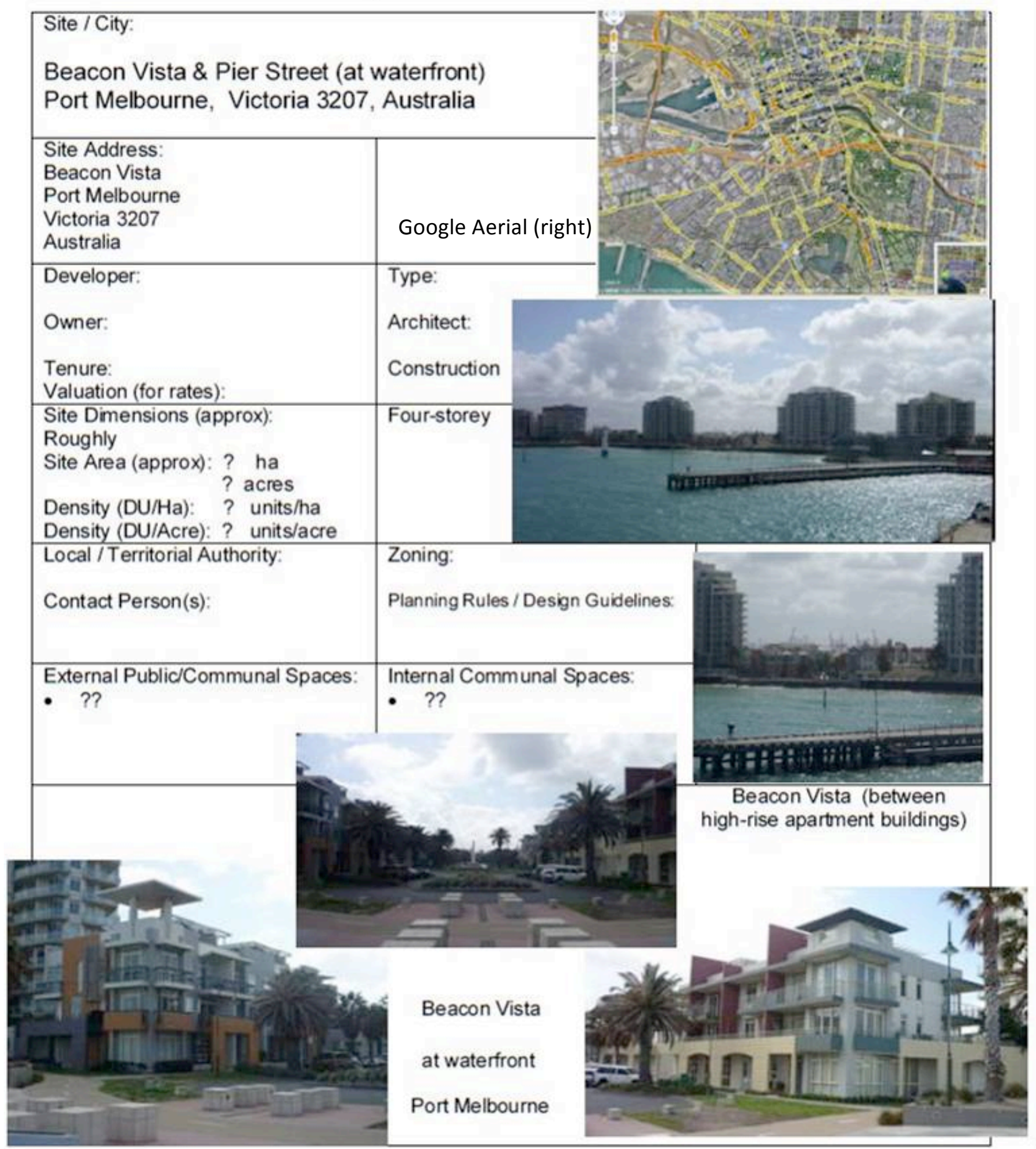

All photos this page: S.J. Pattinson 


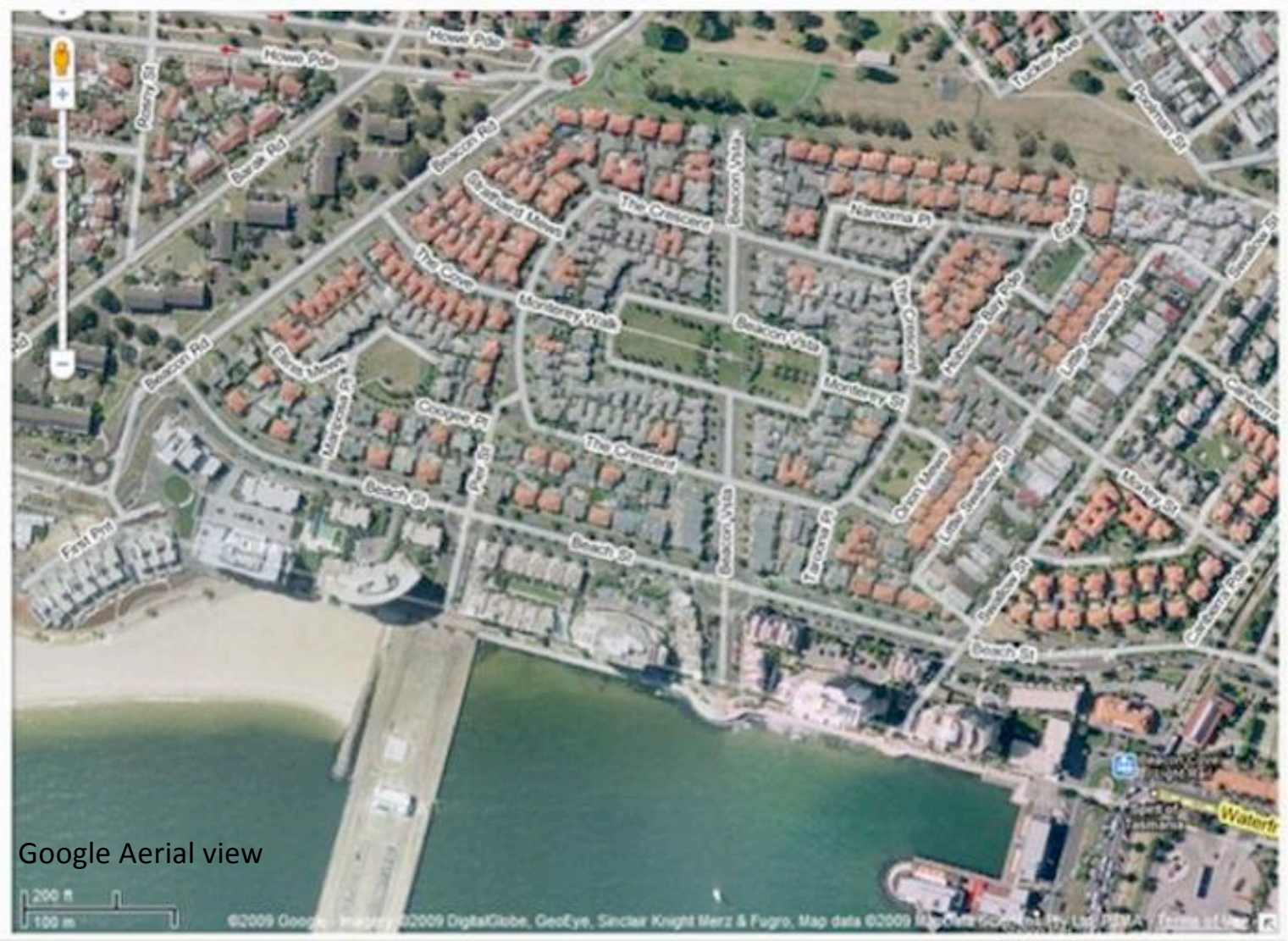

Part of Port Melbourne - Aerial view (First Point terraces at left, Pier St \& Beacon Vista centre)

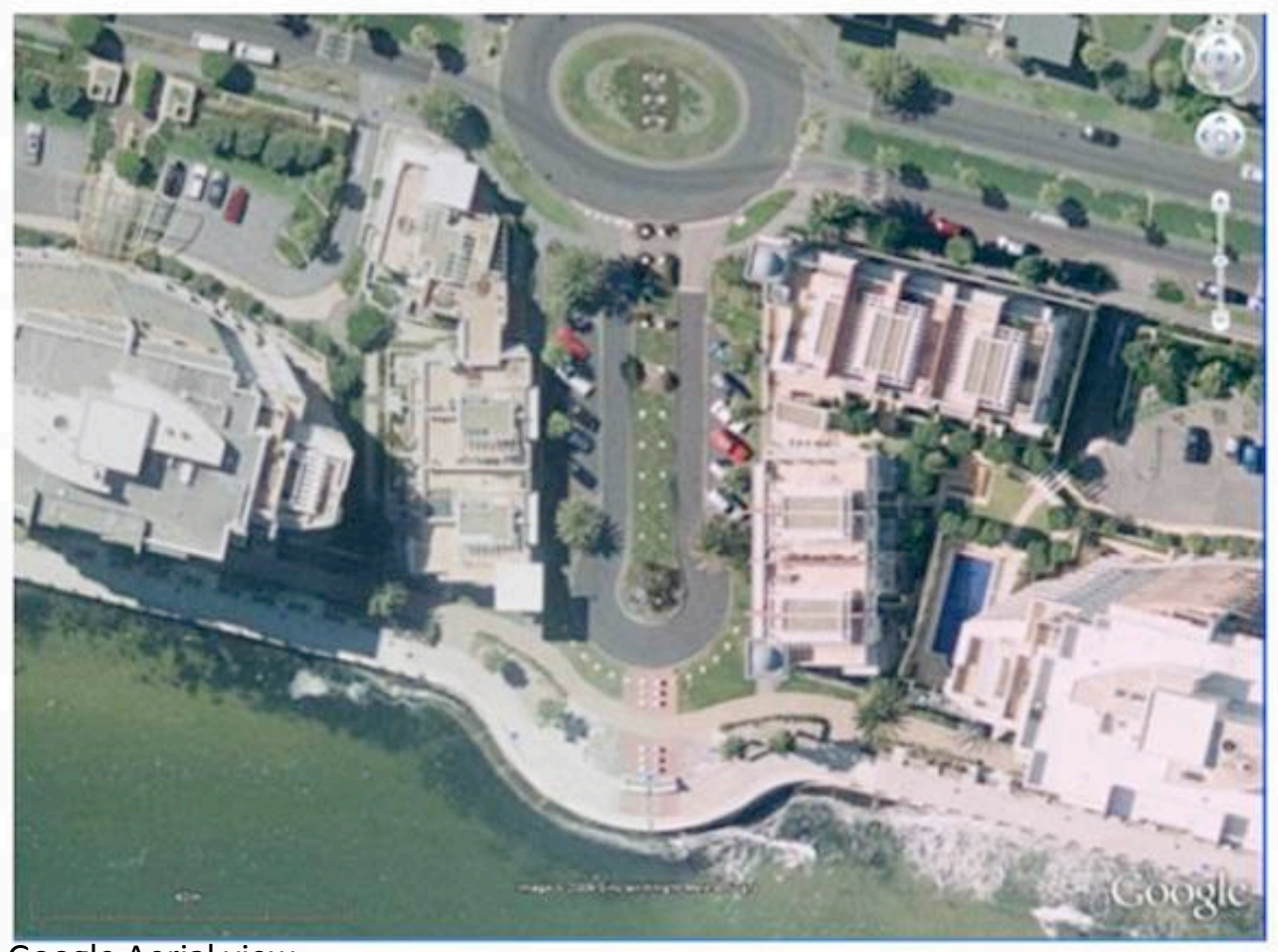

Google Aerial view Beacon Vista at waterfront, Port Melbourne

(page 2 of 3 ) 


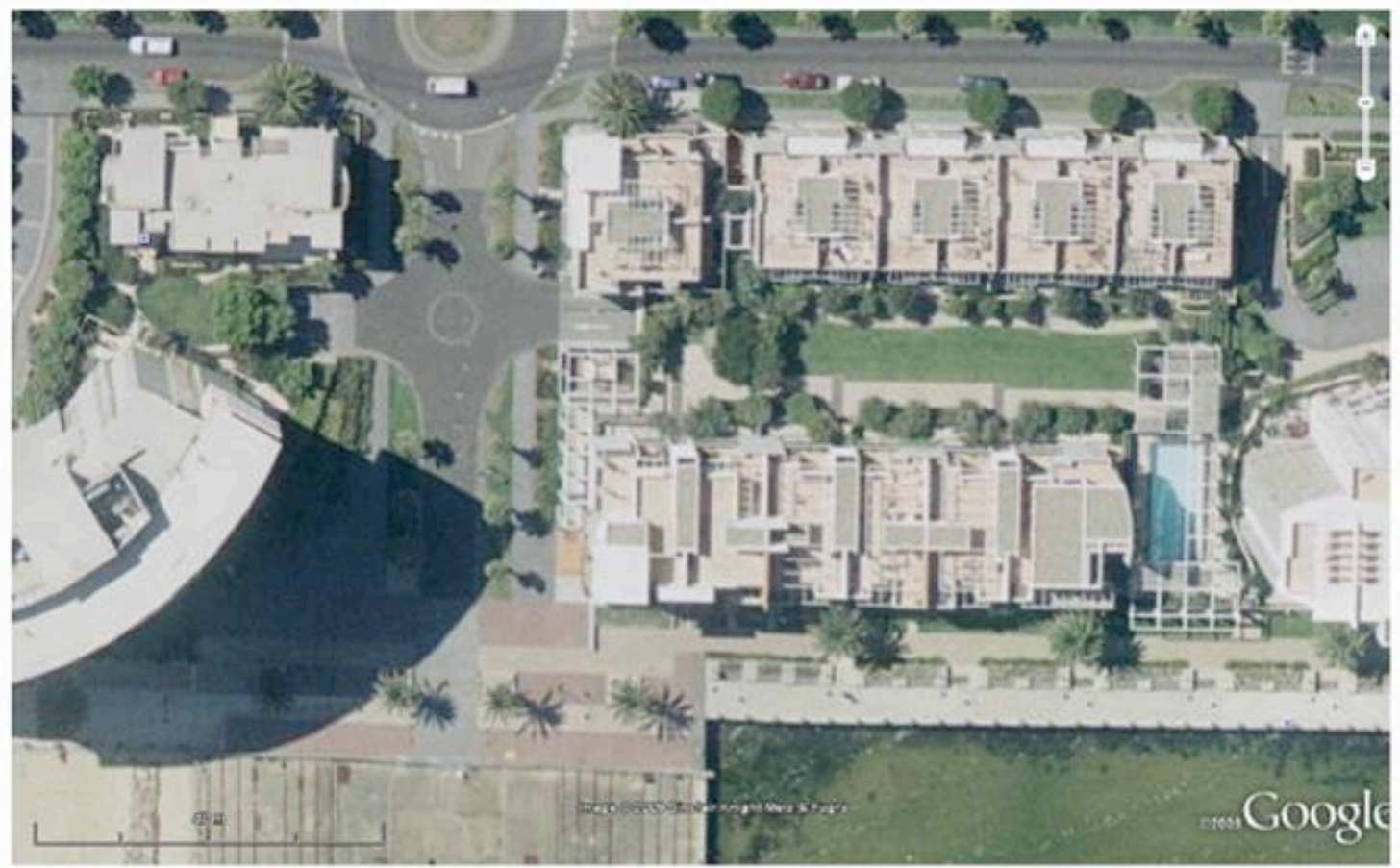

Pier Street at waterfront, Port Melbourne Google Aerial View

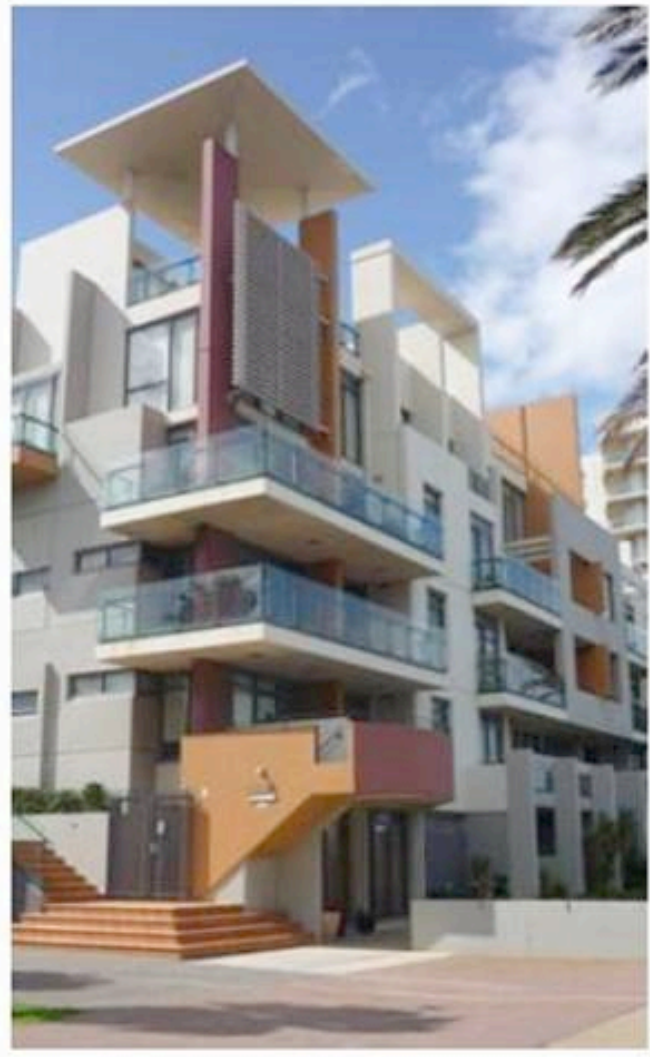

Photo: S.J. Pattinson

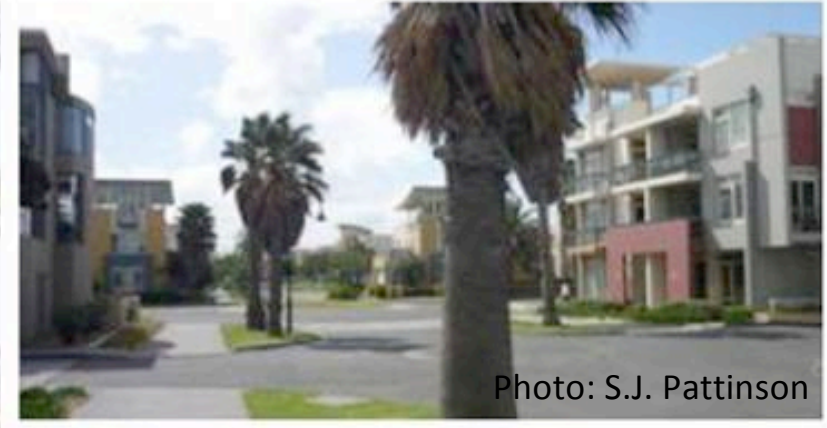

Pier Street (looking North from the waterfront)

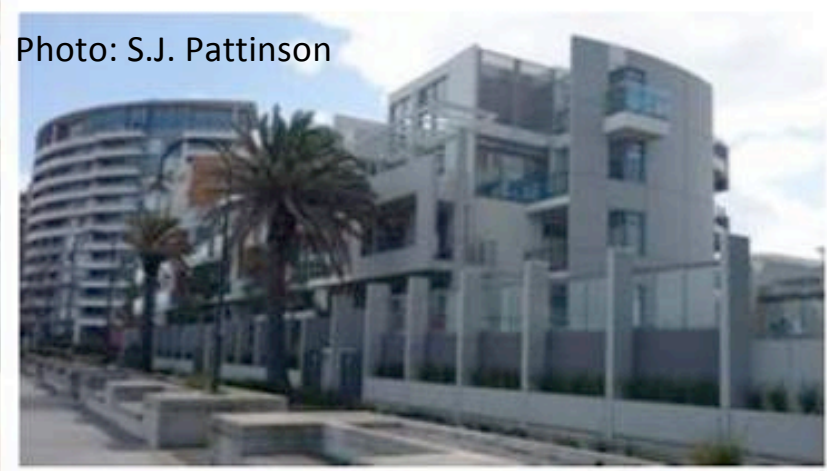

Medium-rise waterfront apartments at Pier Street 


\begin{tabular}{|l|c|l|}
\hline $\begin{array}{l}\text { () 2009 Stephen J. Pattinson } \\
\text { M. Arch. Thesis Research } \\
\text { Victoria University of Wellington, NZ }\end{array}$ & $\begin{array}{c}\text { FIELD NOTES } \\
\text { Site Visits: }\end{array}$ & $\begin{array}{l}\text { Sheet No. } \\
\text { (page 1 of 4) }\end{array}$
\end{tabular}

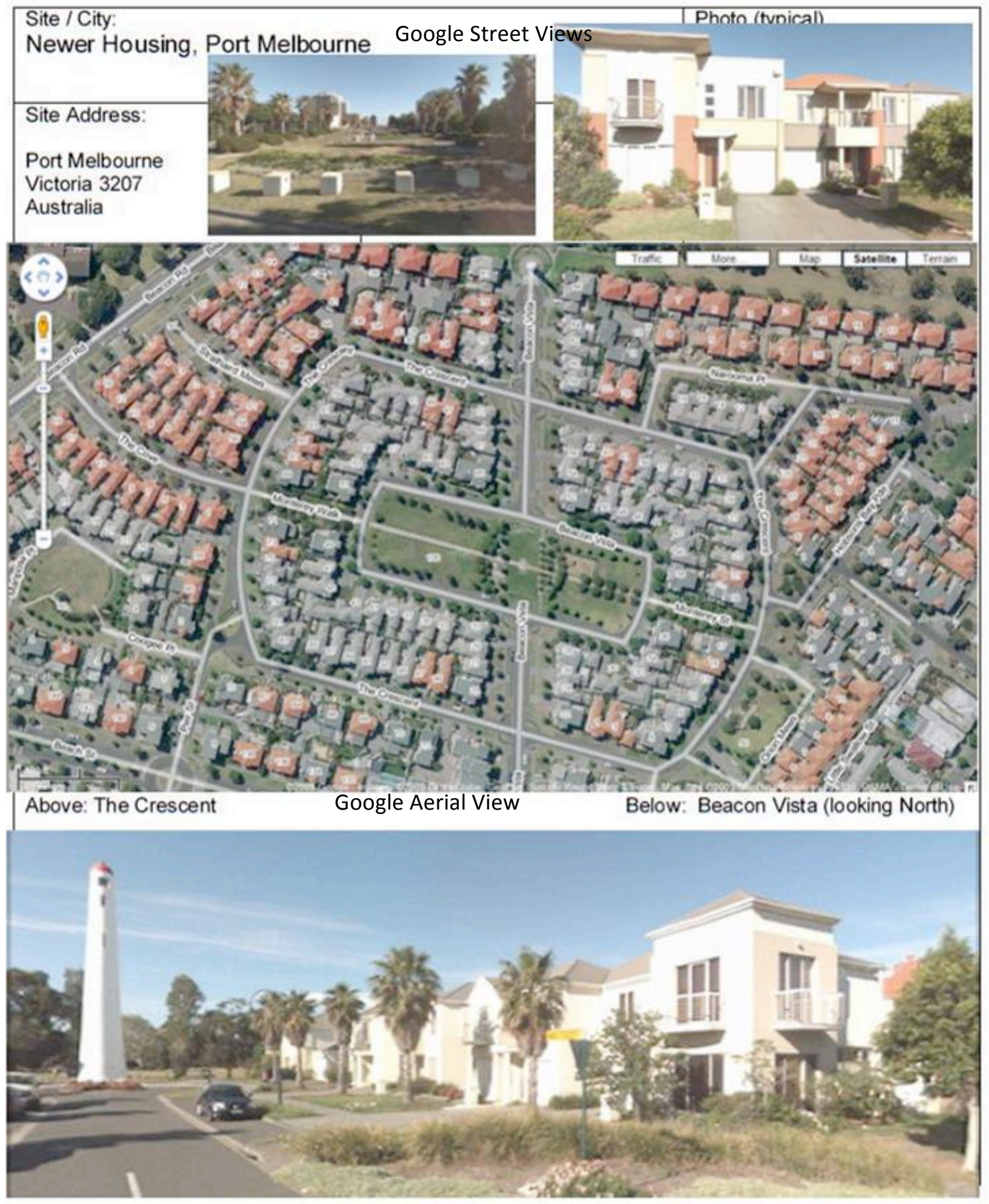

Google Street View 
Google Aerial View

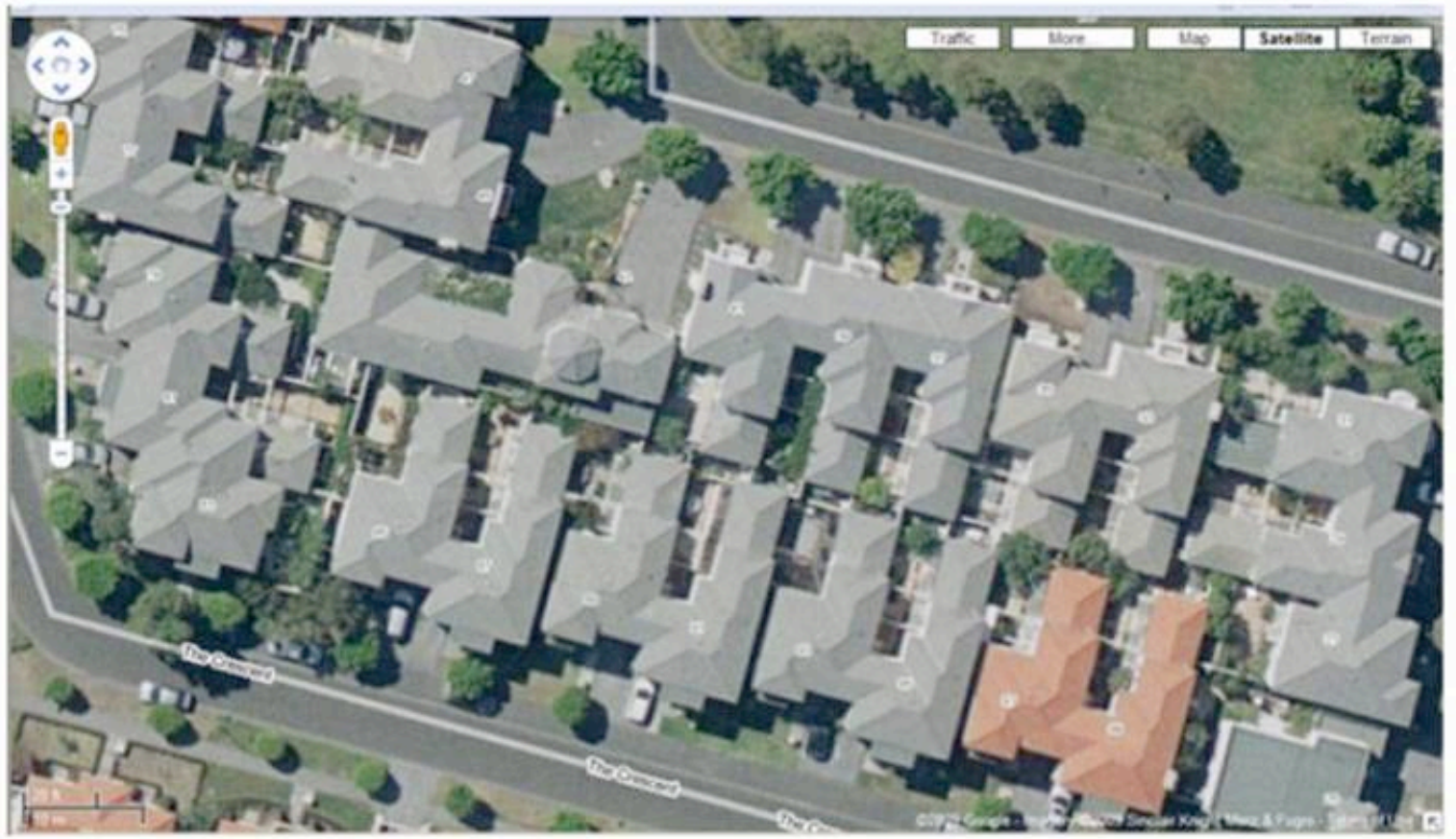

The Crescent, Port Melbourne - Typical (mostly 2-storey duplex and triplex)

Shown here total 25 units on approx. $120 \mathrm{~m} \times 50 \mathrm{~m}=6.000 \mathrm{~m} 2$. Density 42 units / ha ( 17 units / acre)

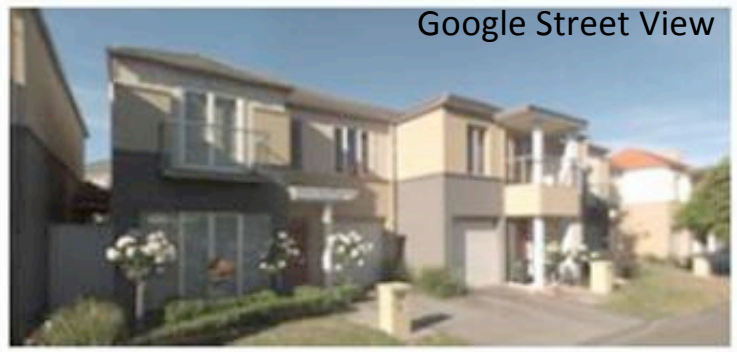

Nos. 93 \& 95 The Crescent (duplex), Port Melbourne

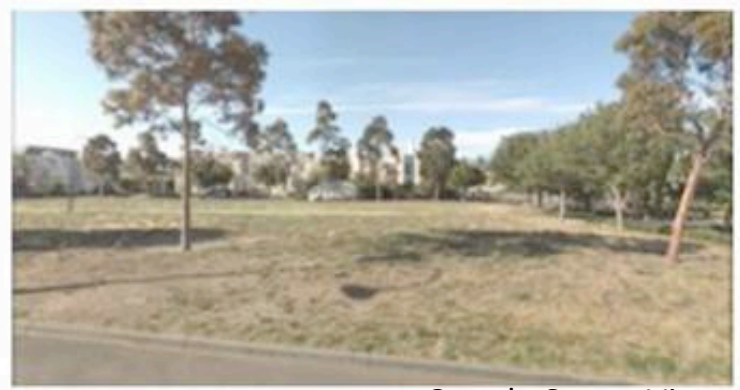

Central open space (public) Google Street View

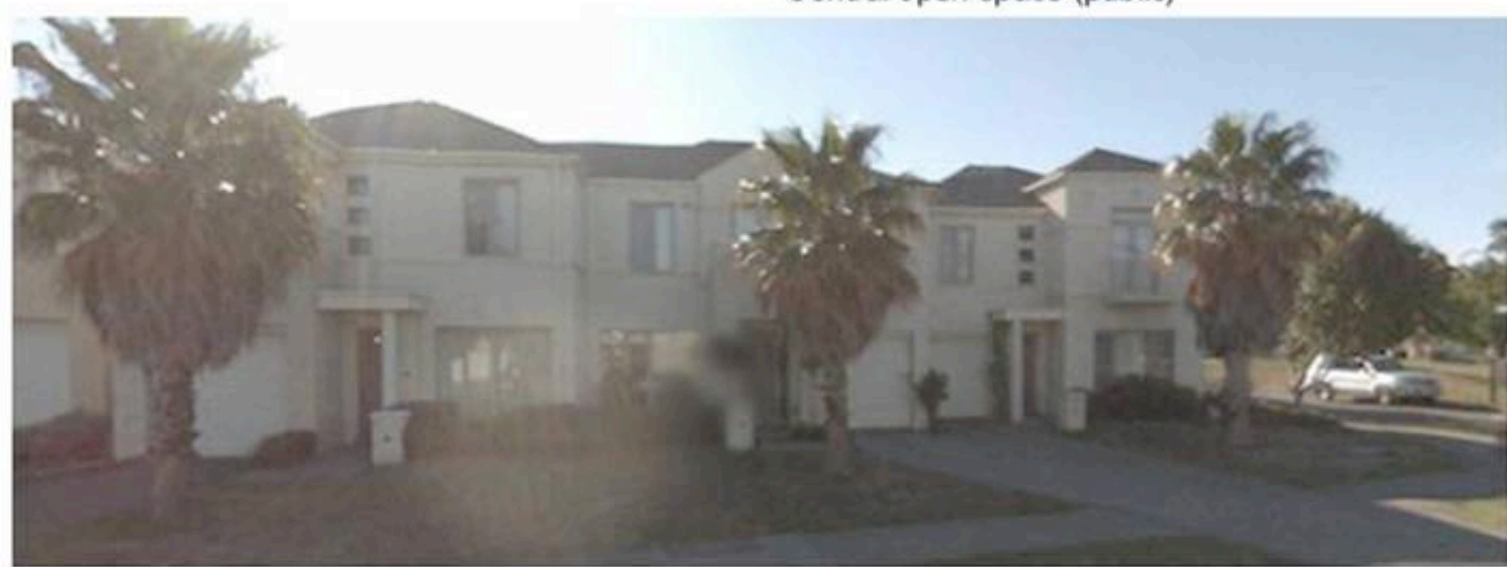

Nos. 27, 29 \& 31 Beacon Vista (triplex), Port Melbourne 


\section{Google Aerial View}

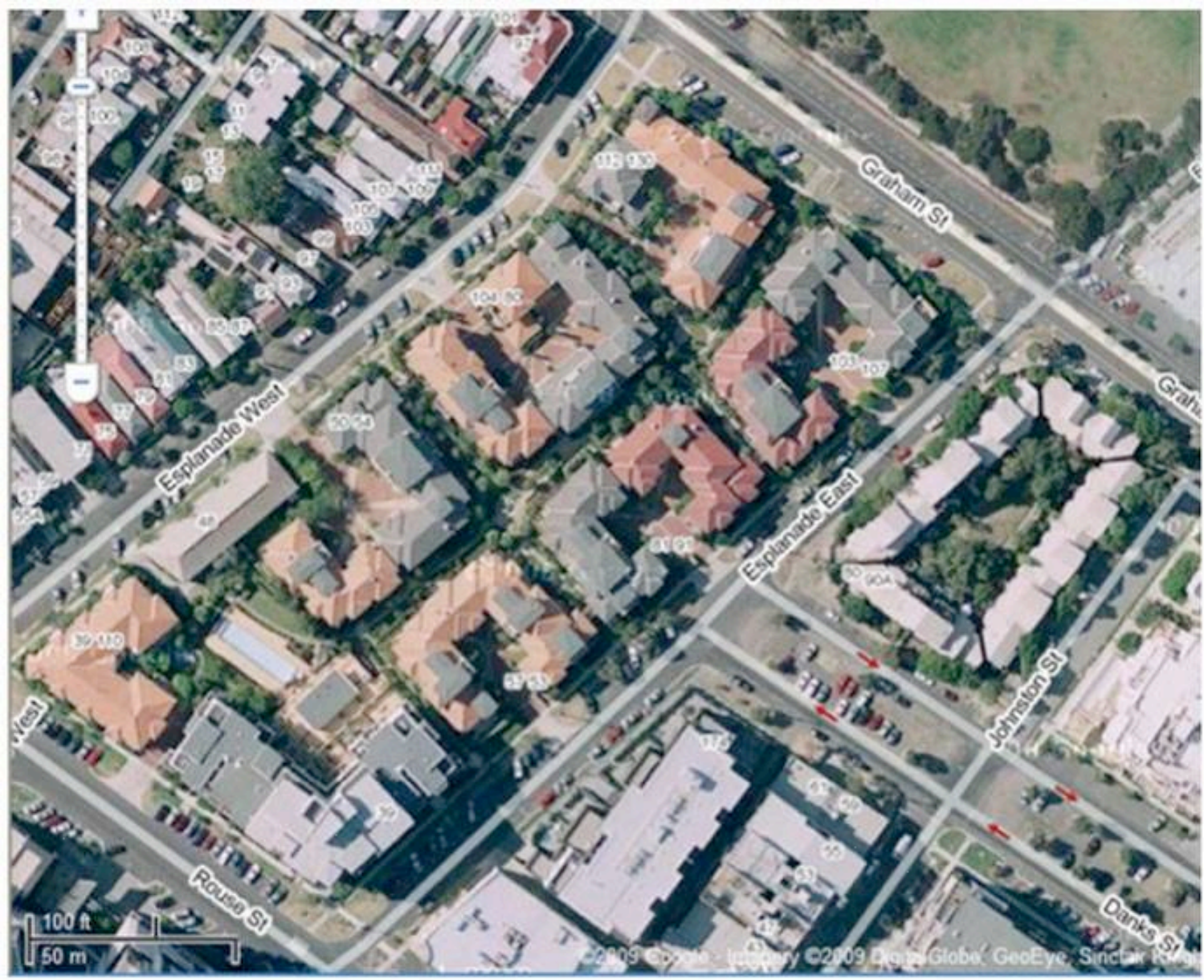

Esplanades East \& West. xxunits (?) on approx $18,600 \mathrm{~m} 2=$ approx $x \times$ units / ha (xx units /acre)

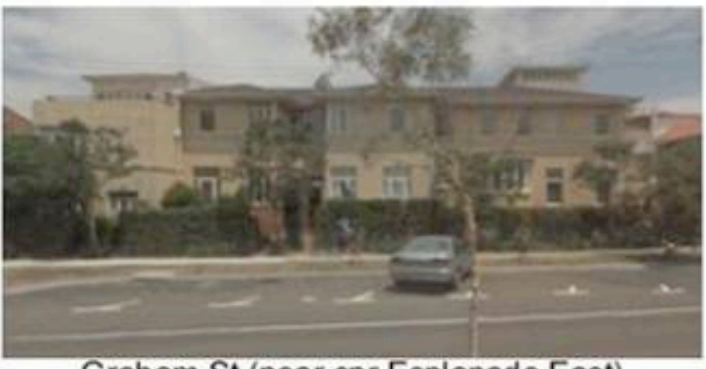

Graham St (near cnr Esplanade East)

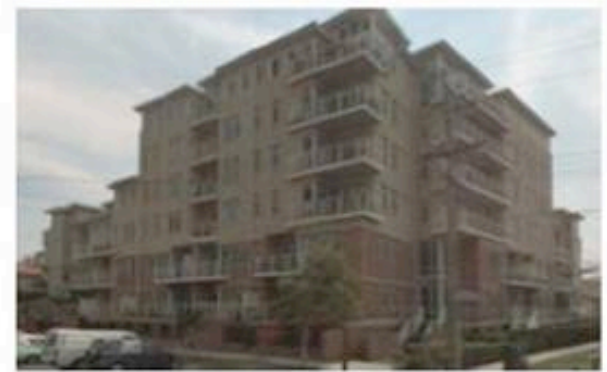

Cnr Rouse St \& Esplanade East

All photos this page: Google Street Views

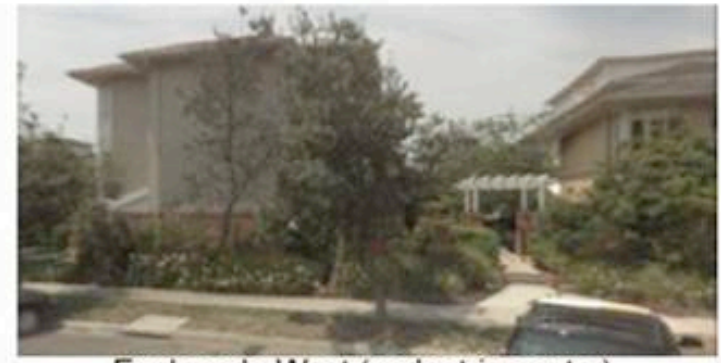

Esplanade West (pedestrian entry)

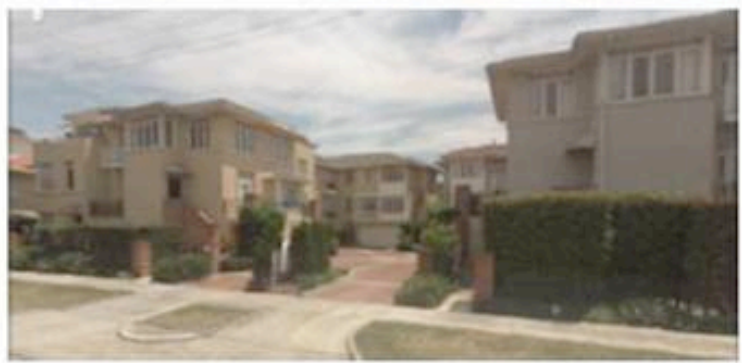

81 - 91 Esplanade East (by Danks St intersection)

(page 3 of 4 ) 
Google Aerial View

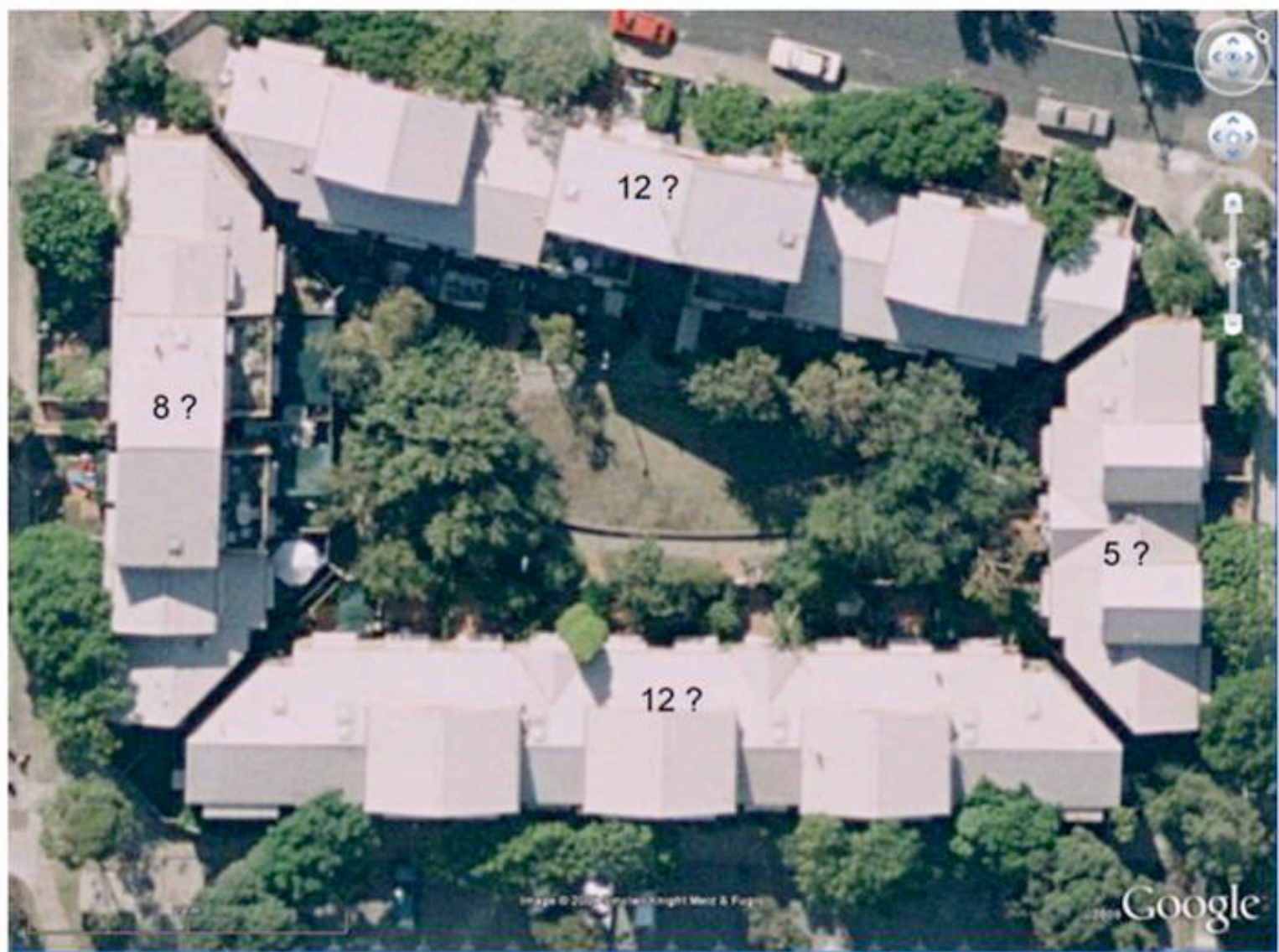

Plan view of brick cluster. 25 units (?) on approx $3,300 \mathrm{~m} 2=$ approx. 75 units / ha ( 30 units /acre)

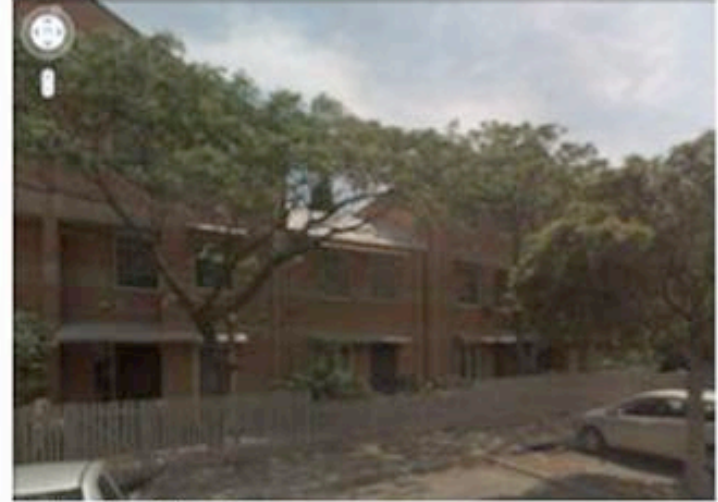

Johnston Street

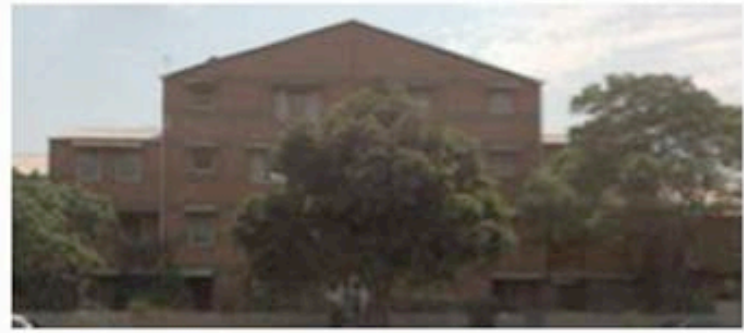

Danks Street

All photos this page: Google Street Views

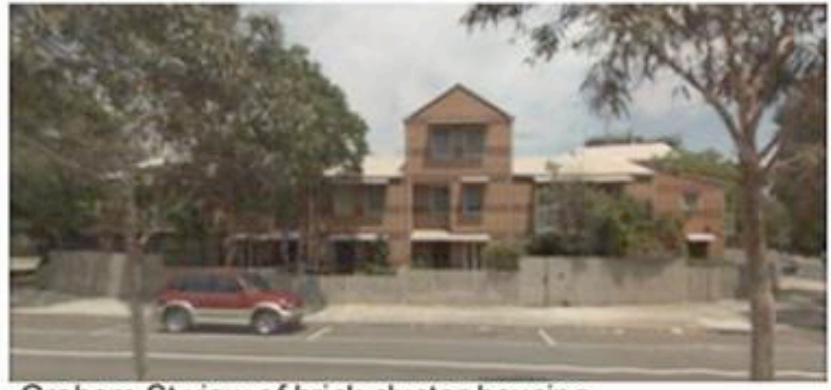

Graham St view of brick cluster housing

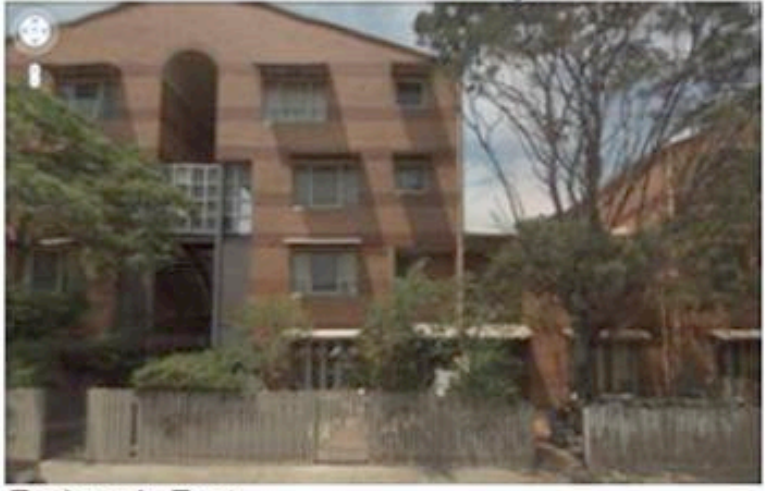

Esplanade East

(page 4 of 4 ) 


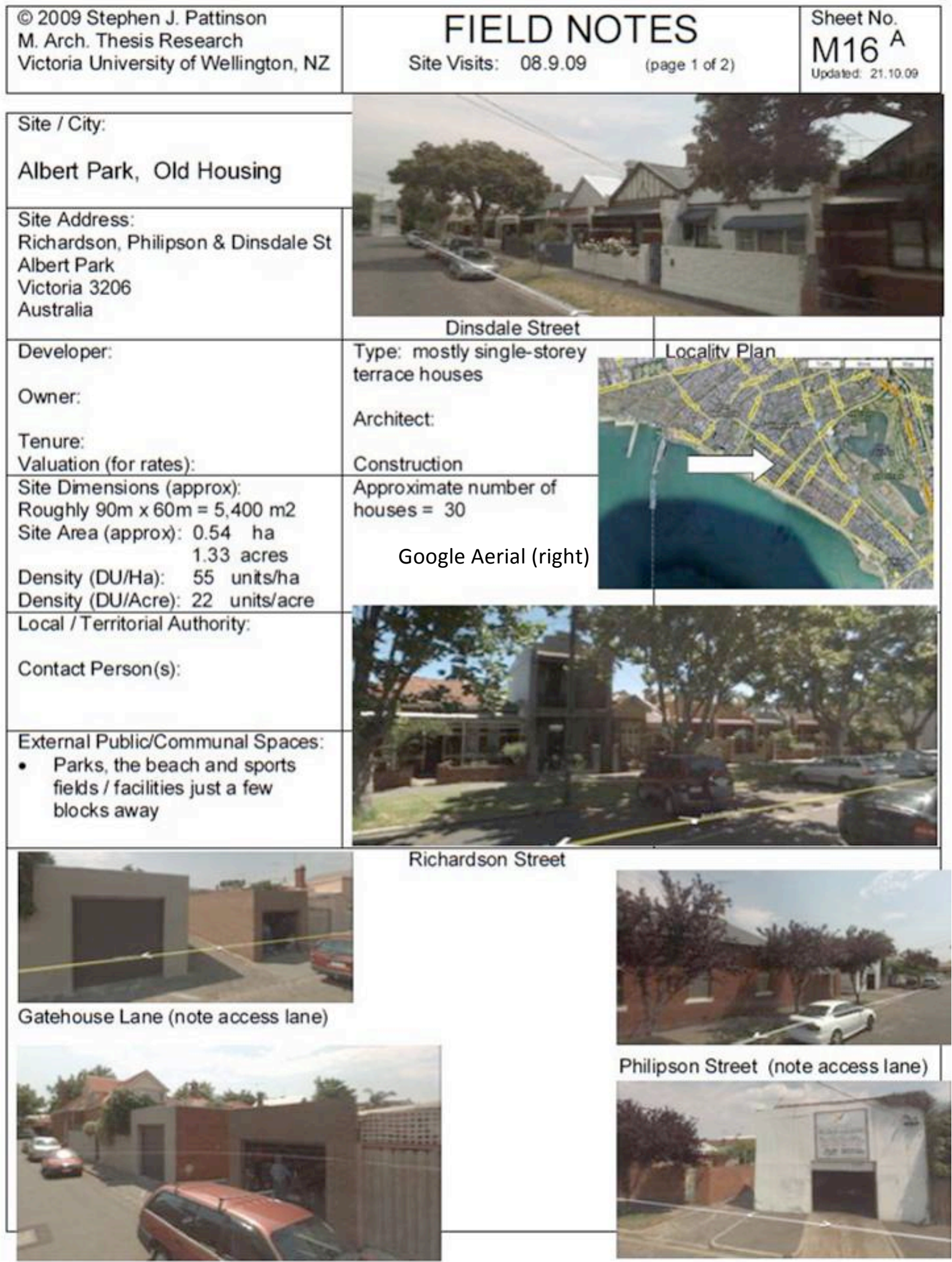

All photos this page: Google Street Views 
Google Aerial View

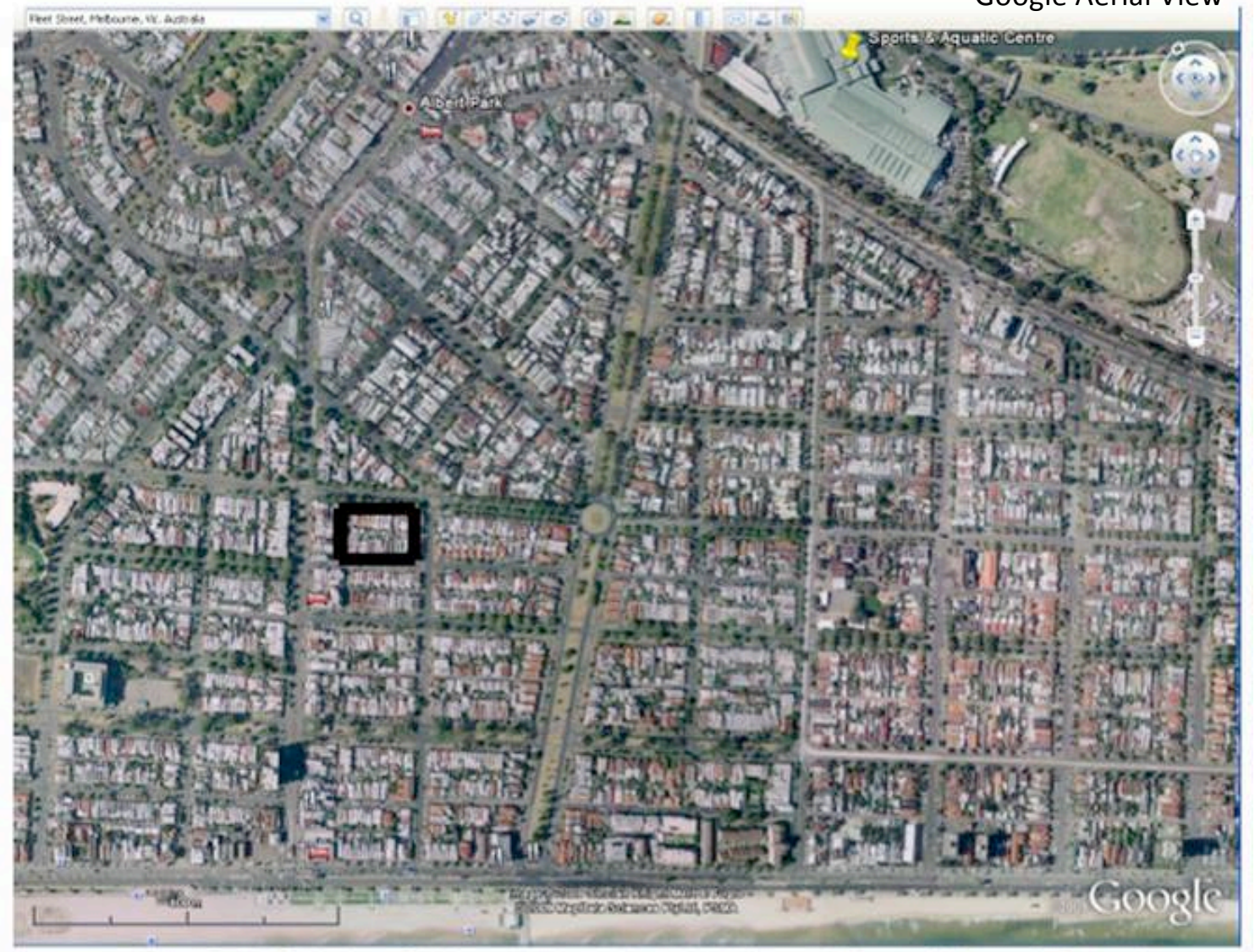

Albert Park Suburb - Old housing (near beach, parks and Sports \& Aquatic Centre)

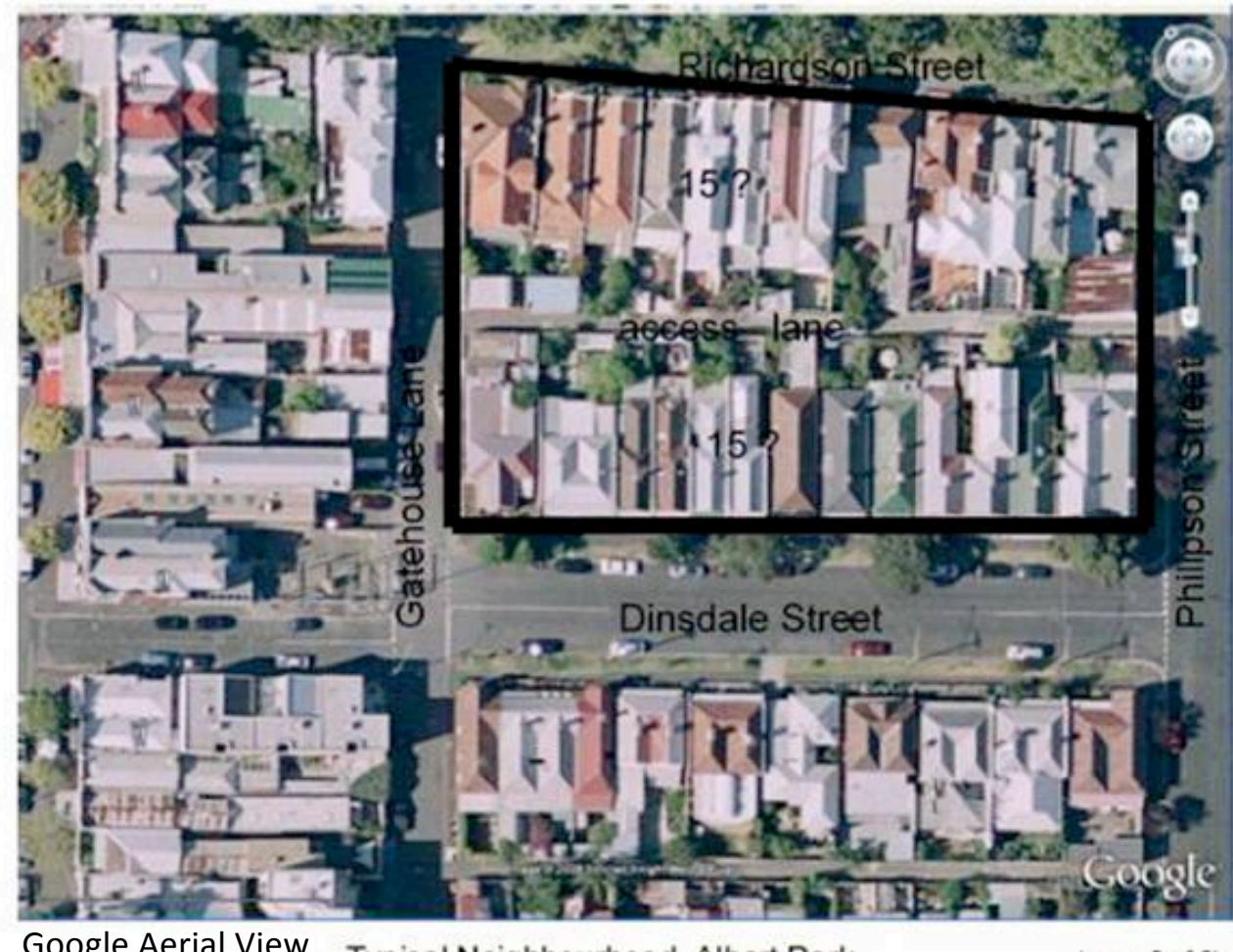

Google Aerial View Typical Neighbourhood, Albert Park

(page 2 of 2) 


\section{Appendix 7.3: Preliminary Investigations}




\section{APPENDIX 7.3 - Preliminary Investigations}

Following is a record of the writer's first impressions of communal space from preliminary investigations at medium density housing developments in the three main cities in New Zealand and in Melbourne, Australia. It includes observations and initial thoughts from field visits to 227 existing housing developments between August 2009 and July 2010 to view communal space first hand:
- Auckland (region), New Zealand
114 sites
- Wellington, New Zealand
47 sites
- Christchurch, New Zealand
54 sites
- Melbourne, Australia
12 sites

The writer's perspective on the relationship between people and the environment and on communal space is declared in the thesis (e.g. pages 13-16). This thesis has been an attempt at 'reflexive' research, i.e.

"investigation in which the researcher is aware of their contribution to the construction of meanings throughout the research process", a process in which "researchers ... explicitly recognise (or declare) ... their perspectives on the objects of their research" (Webster 6, 102)

The writer's views may change or be modified as the research progresses, but the following provides a record of the writer's perspective at the outset of the research. A one page summary of the main points from these preliminary investigations appears on page 50 of the thesis. 


\section{Field visits to existing medium-density housing to investigate communal space:}

"Well, you won't find much of that!" was a prominent New Zealand architect's response to the writer's comment that this research is about communal space in medium-density housing. If there is any communal space in New Zealand's housing developments, then the writer wanted to know what it looks like. Therefore, the research begins with the writer's preliminary investigations in Auckland, Wellington, Christchurch and Melbourne. The writer found some excellent examples of mediumdensity housing in these cities - some with especially high-quality landscaped outdoor spaces. What are the uses of the communal spaces in these examples? What do they mean? Are lap pools, tennis courts, landscaped gardens and manicured lawns primarily status symbols, for marketing and passive outlook? Do they help residents, an aggregate of strangers, become 'good neighbours'? Field visits ranged from 30 minutes to three hours - not long enough to answer these questions. The following is simply a record of observations and first impressions.

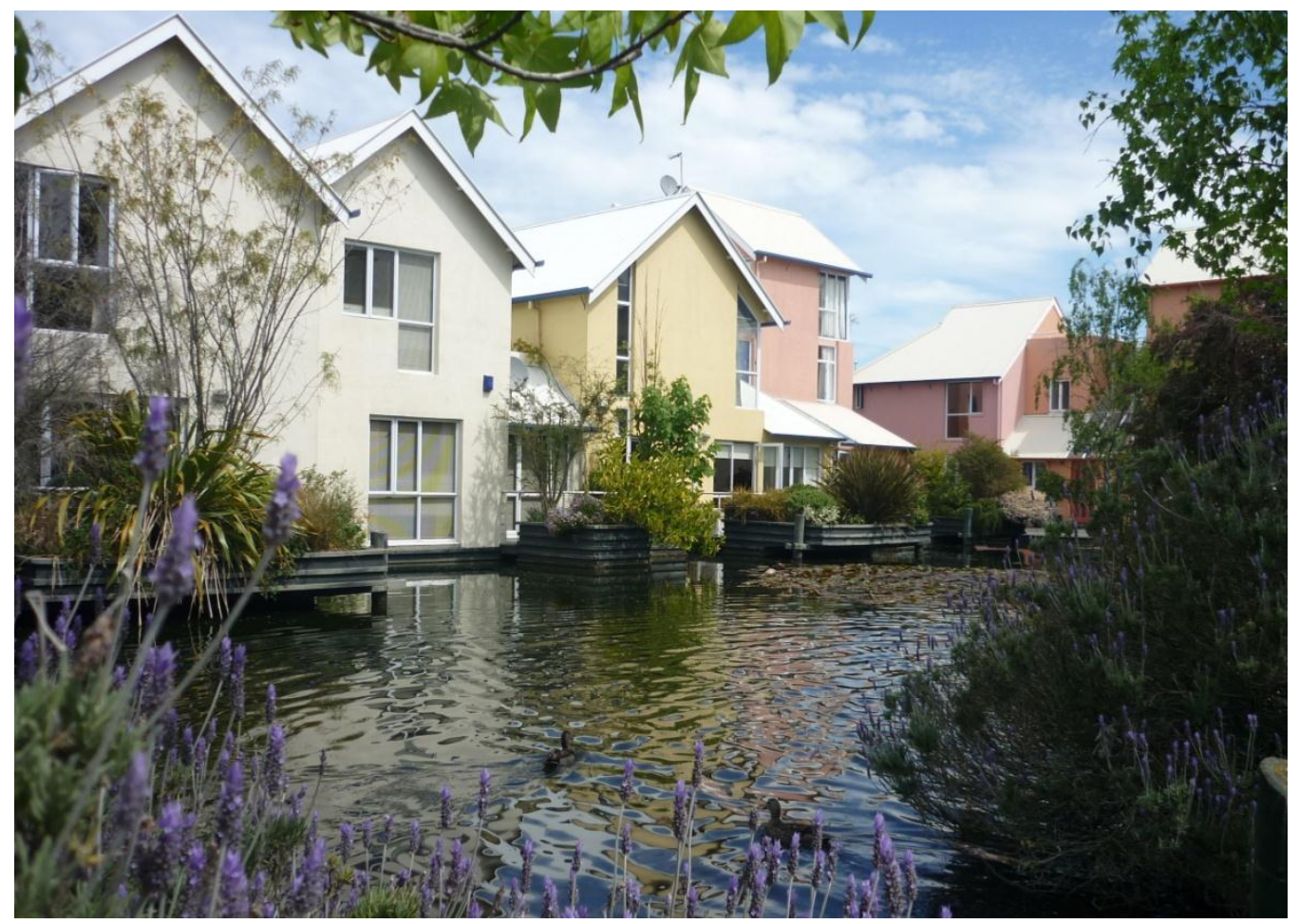

Fig.A7.3.1 Merivale Mews, Office Road, Merivale, Christchurch. Architect: Peter Beaven Beautiful outdoor space ... primarily for passive outlook?

(Photo: S. J. Pattinson) 


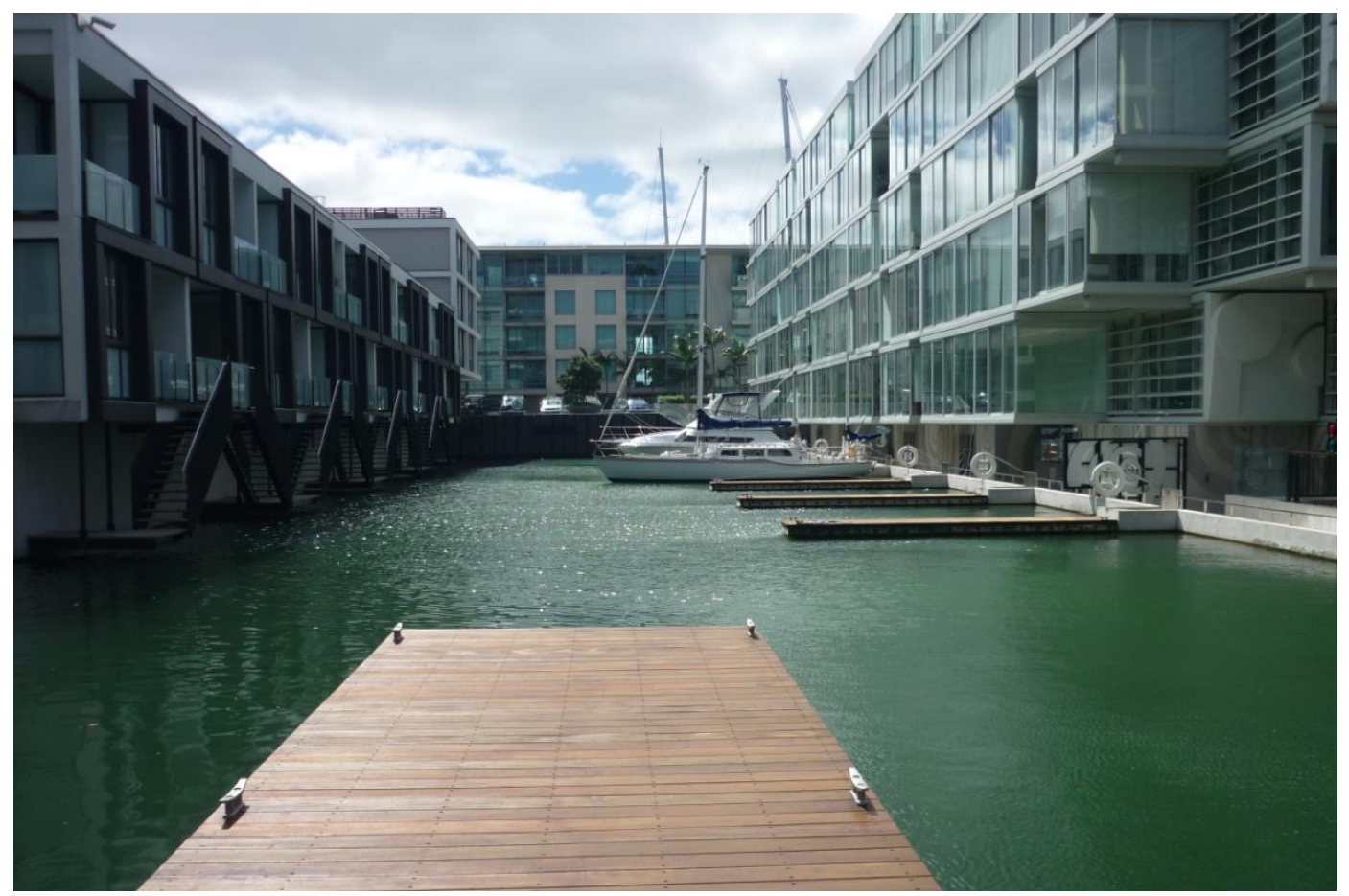

Fig.A7.3.2 Halsey, North and Stratis Apartments - Viaduct Harbour, Auckland.

Exclusive 'club' community.

(Photo: S. J. Pattinson)

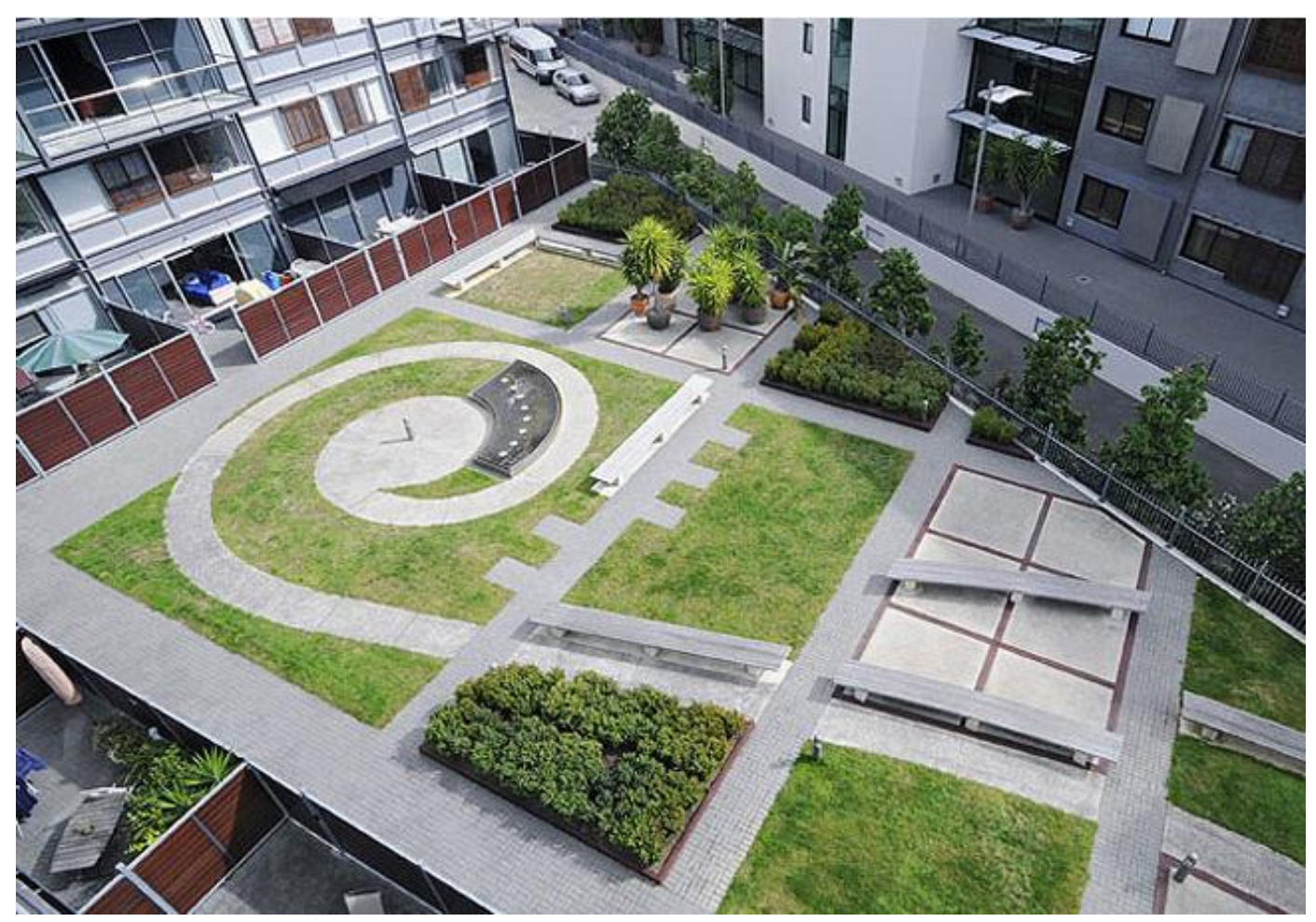

Fig.A7.3.3 Viaduct Point Apartments, Auckland. Gated outdoor space ... for landscaped outlook?
Architect: Craig, Craig, Moller (Photo: www.crockers.co.nz) 


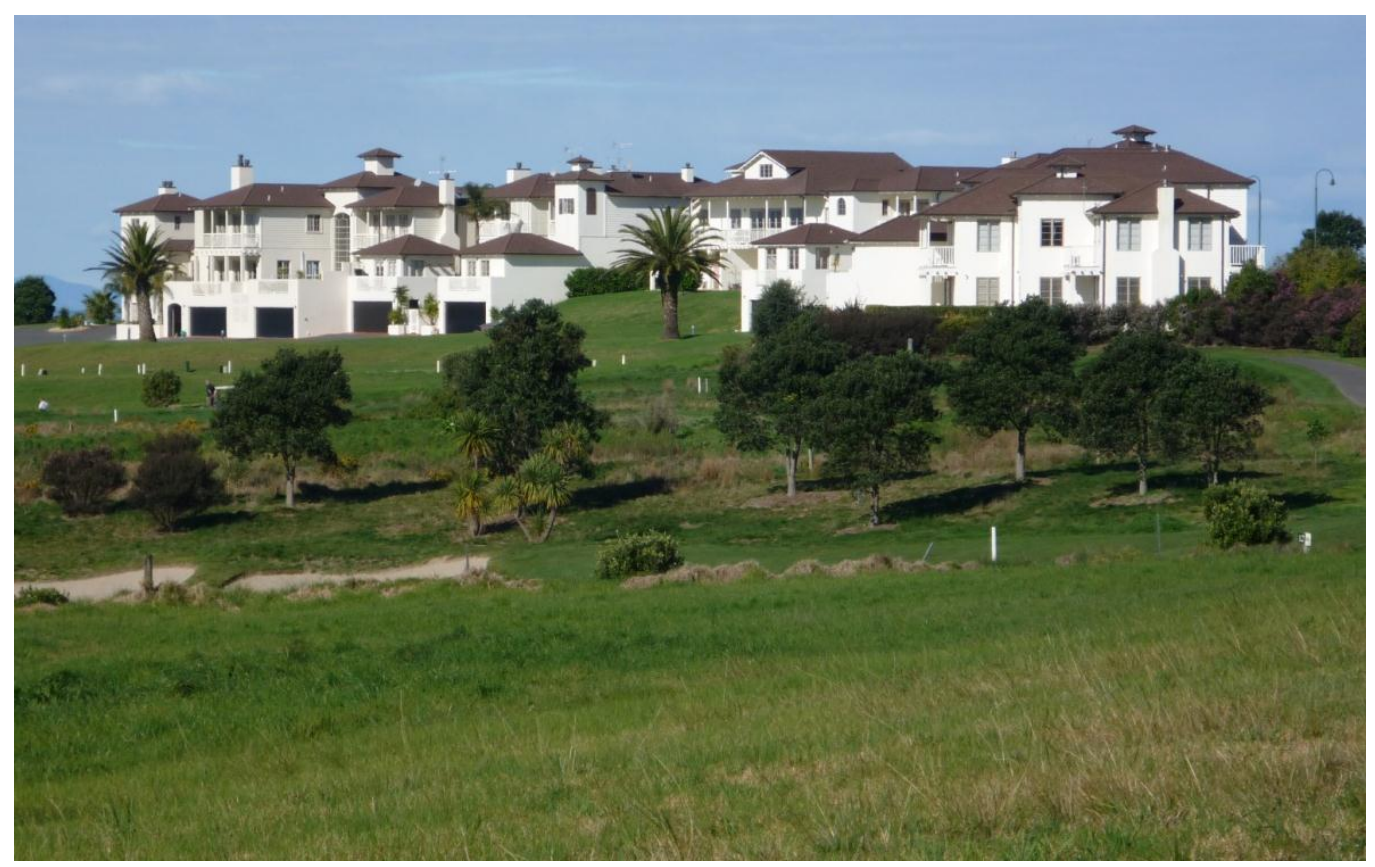

Fig.A7.3.4 Ocean Park, Pinecrest Drive, Gulf Harbour, Whagaparaora, Auckland.

(Photo: S. J. Pattinson)

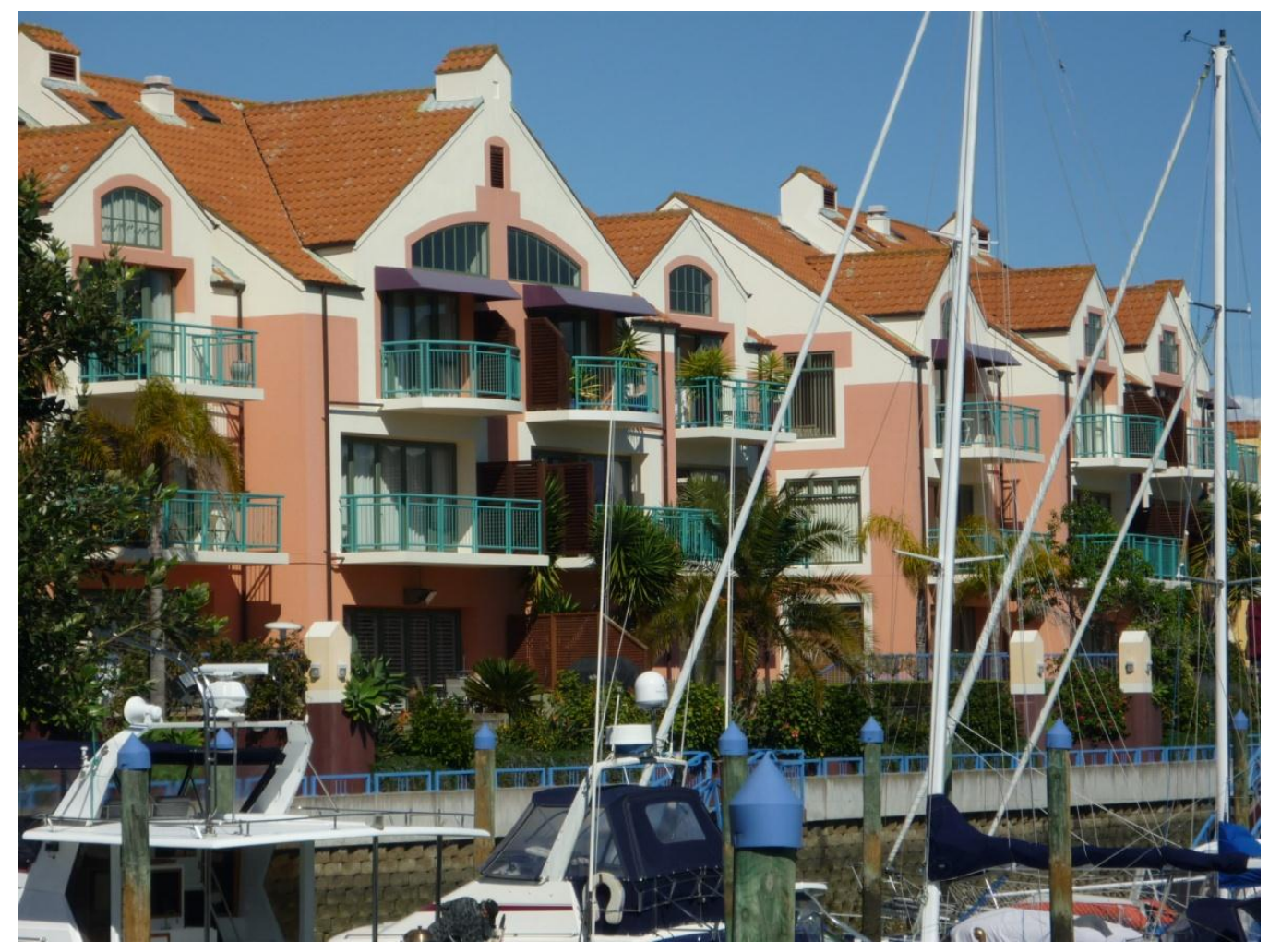

Fig.A7.3.5 Gulf Harbour Marina, Whangaparaora, Auckland.

Gated 'club' community. Outdoor space = status + recreation? (Photo: S. J. Pattinson) 

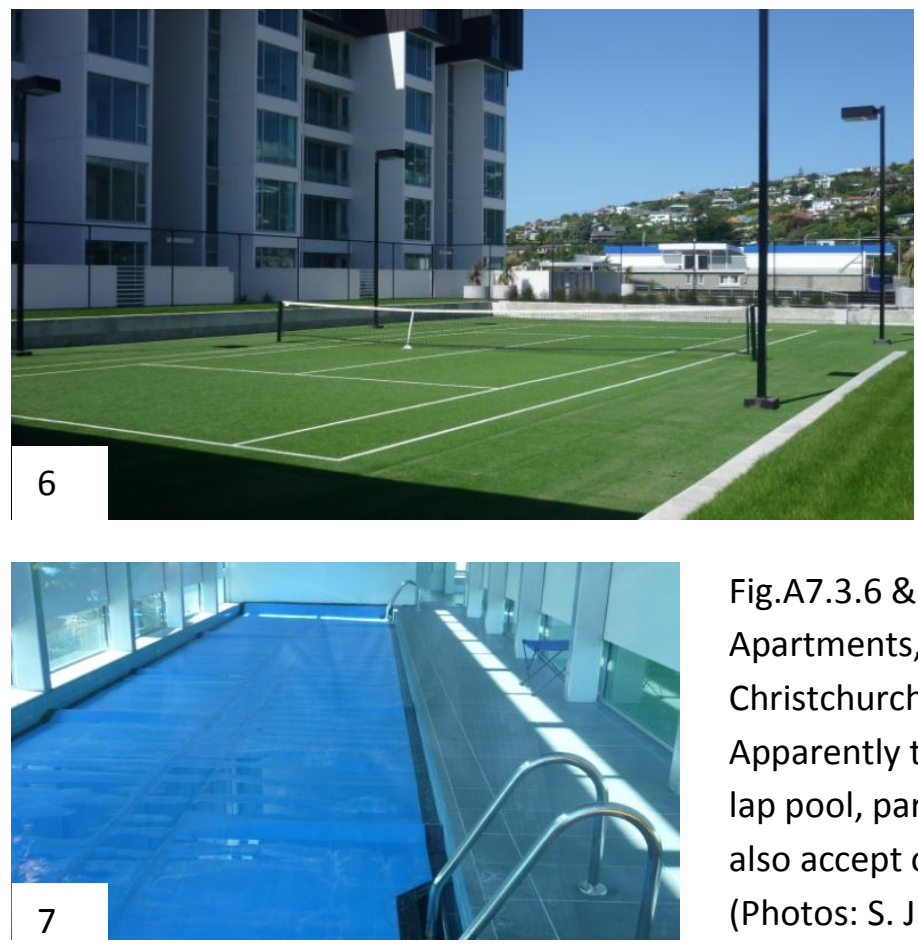

Fig.A7.3.6 \& 7 Tennis court and lap pool. Apartments, 1099 Ferry Road, Ferrymead, Christchurch. Architect: Andrew Barclay Apparently this tennis court and indoor lap pool, part of the apartment complex, also accept outside user membership. (Photos: S. J. Pattinson)

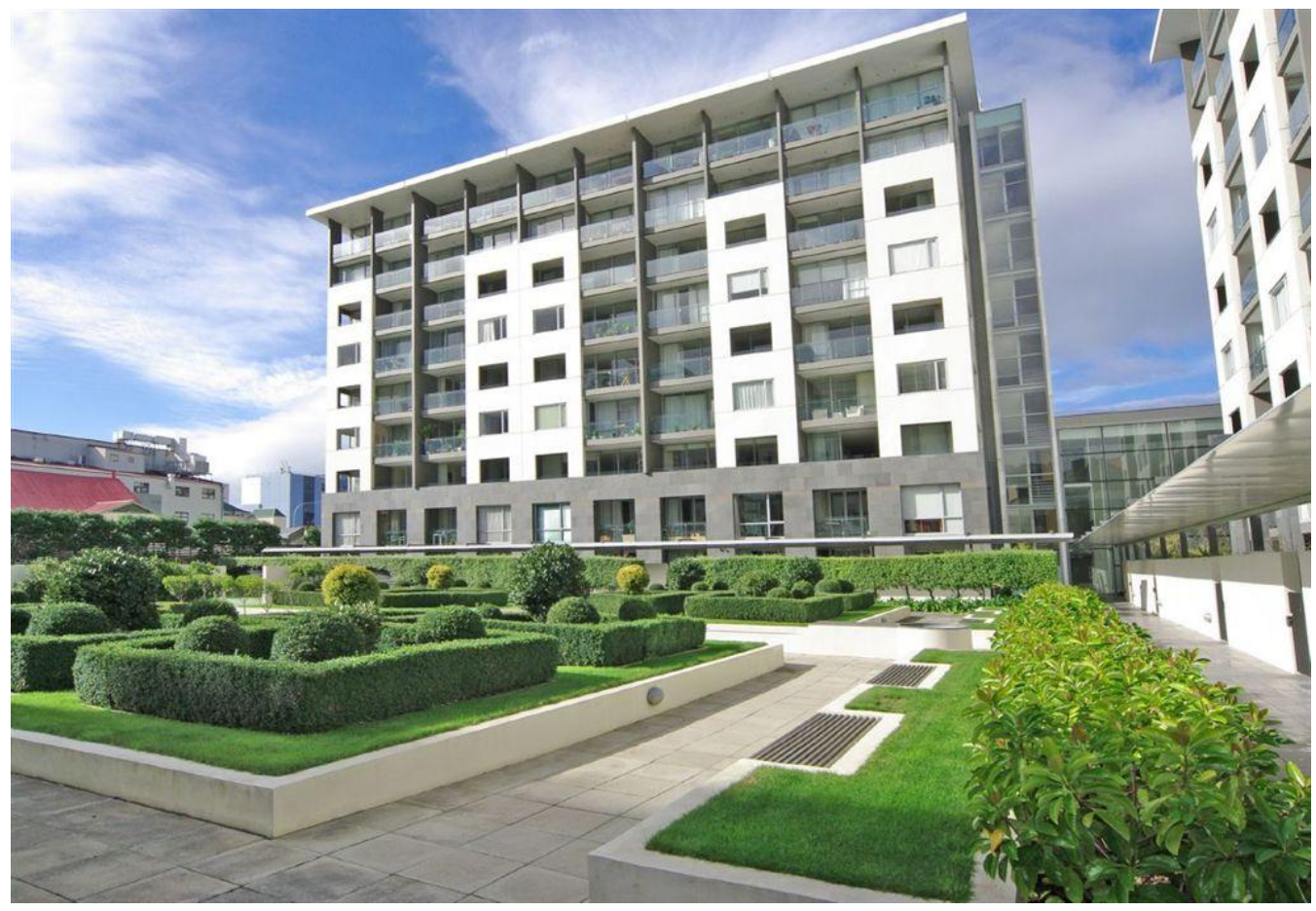

Fig.A7.3.8 Sanctum Apartments, Ebor Street, Wellington. Architect: Warren \& Mahoney Also has a tennis court and indoor lap pool. Are these raised lawns and topiary primarily for landscaped outlook?

(Photo: www.sanctum.co.nz) 

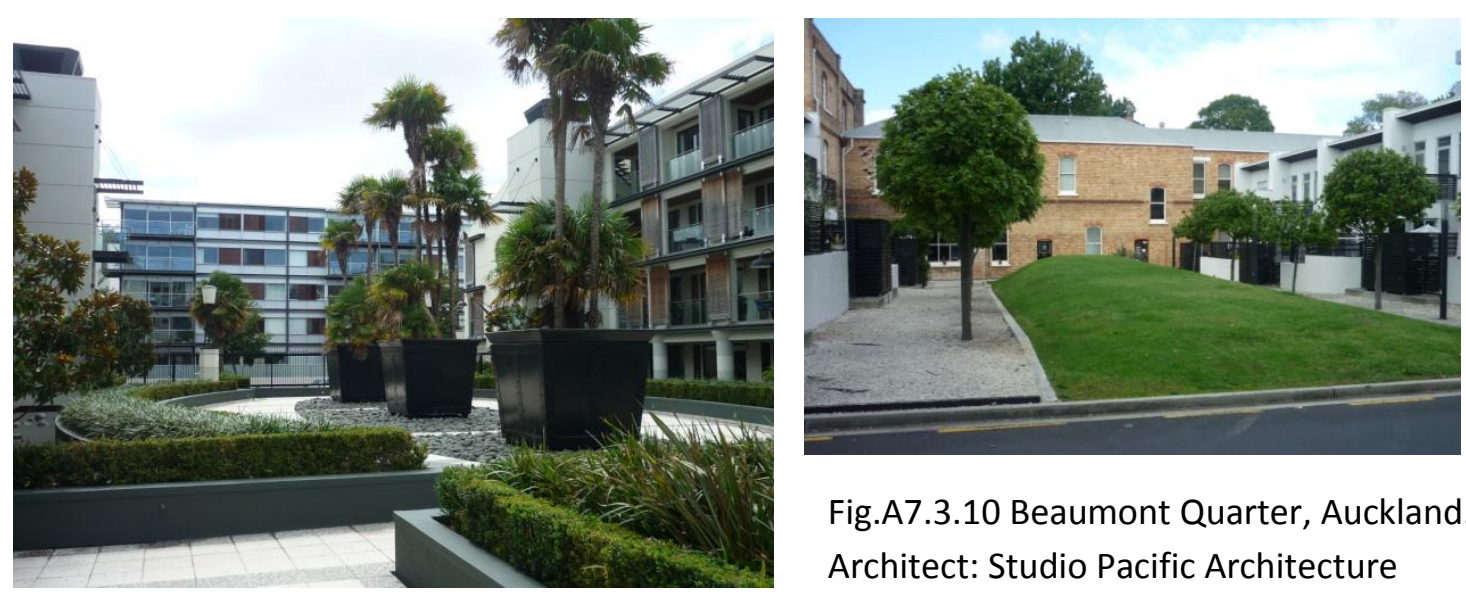

Fig.A7.3.10 Beaumont Quarter, Auckland. Architect: Studio Pacific Architecture

Fig.A7.3.9 Latitude 37 Apartments, Viaduct Harbour, Auckland. (Photo: S. J. Pattinson)

\section{Landscaped outlook?}

Fig.A7.3.11 Beaumont Quarter, Auckland. Architect: Ian Moore Architects (Photo: S. J. Pattinson) (Photo: S. J. Pattinson)
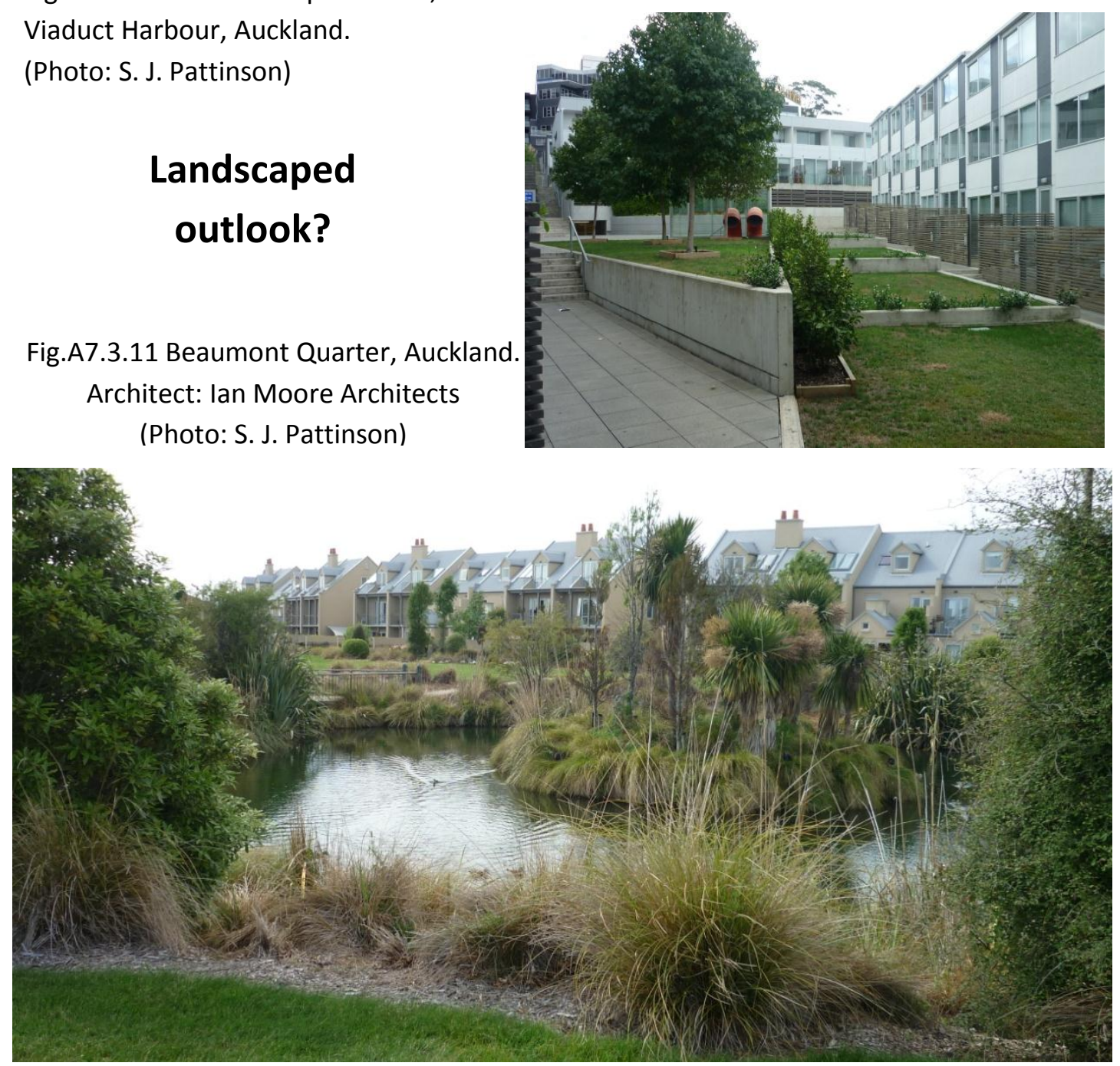

Fig.A7.3.12 Beechwood Drive, Christchurch. Natural wetland developed for environmental biodiversity and outdoor enjoyment? (Photo: S. J. Pattinson) 


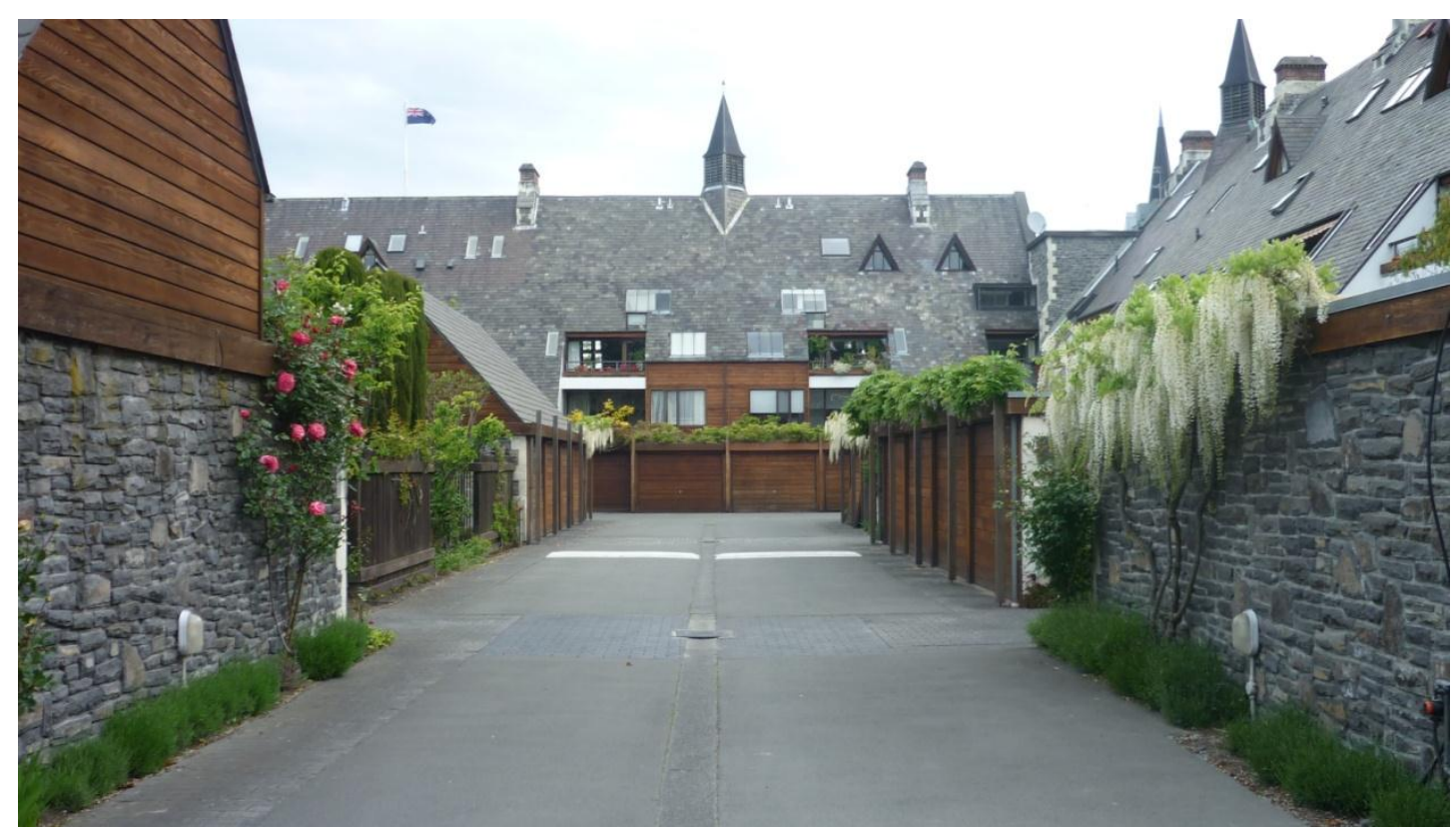

Fig.A7.3.13 Cranmer Court Apartments, 44 Peterborough Street, Christchurch. Internal garaging. Fenced communal swimming pool (left in photo) (Photo: S. J. Pattinson)

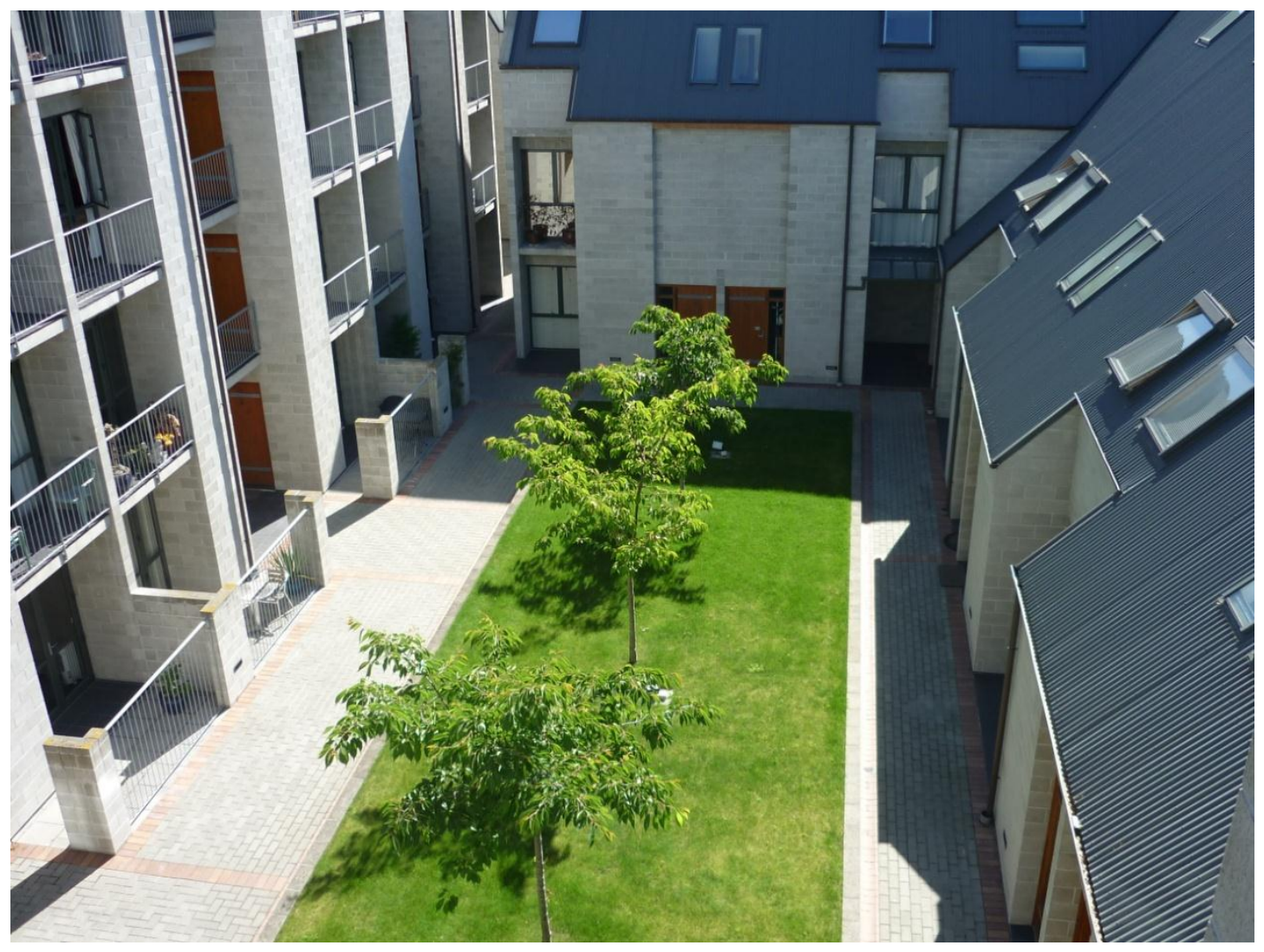

Fig.A7.3.14 St Mary Apartments, 868 Colombo Street, Christchurch. Architect: Peter Beaven Semi-private edge spaces in communal quad with raised central lawn. (Photo: S. J. 


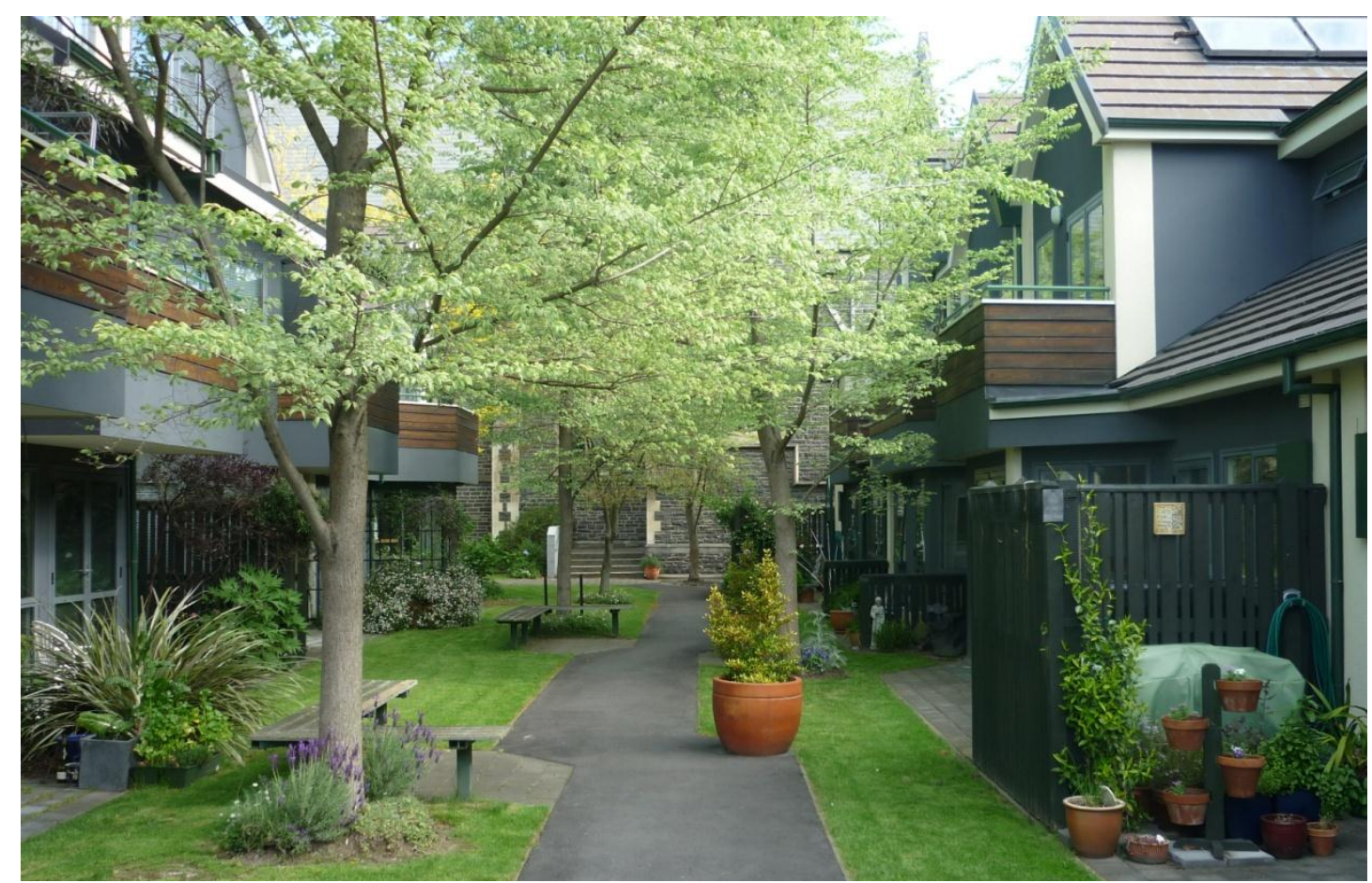

Fig.A7.3.15 St Luke's Apartments, 170 Peterborough Street, Christchurch.

Communal circulation space between dwellings.

(Photo: S. J. Pattinson)

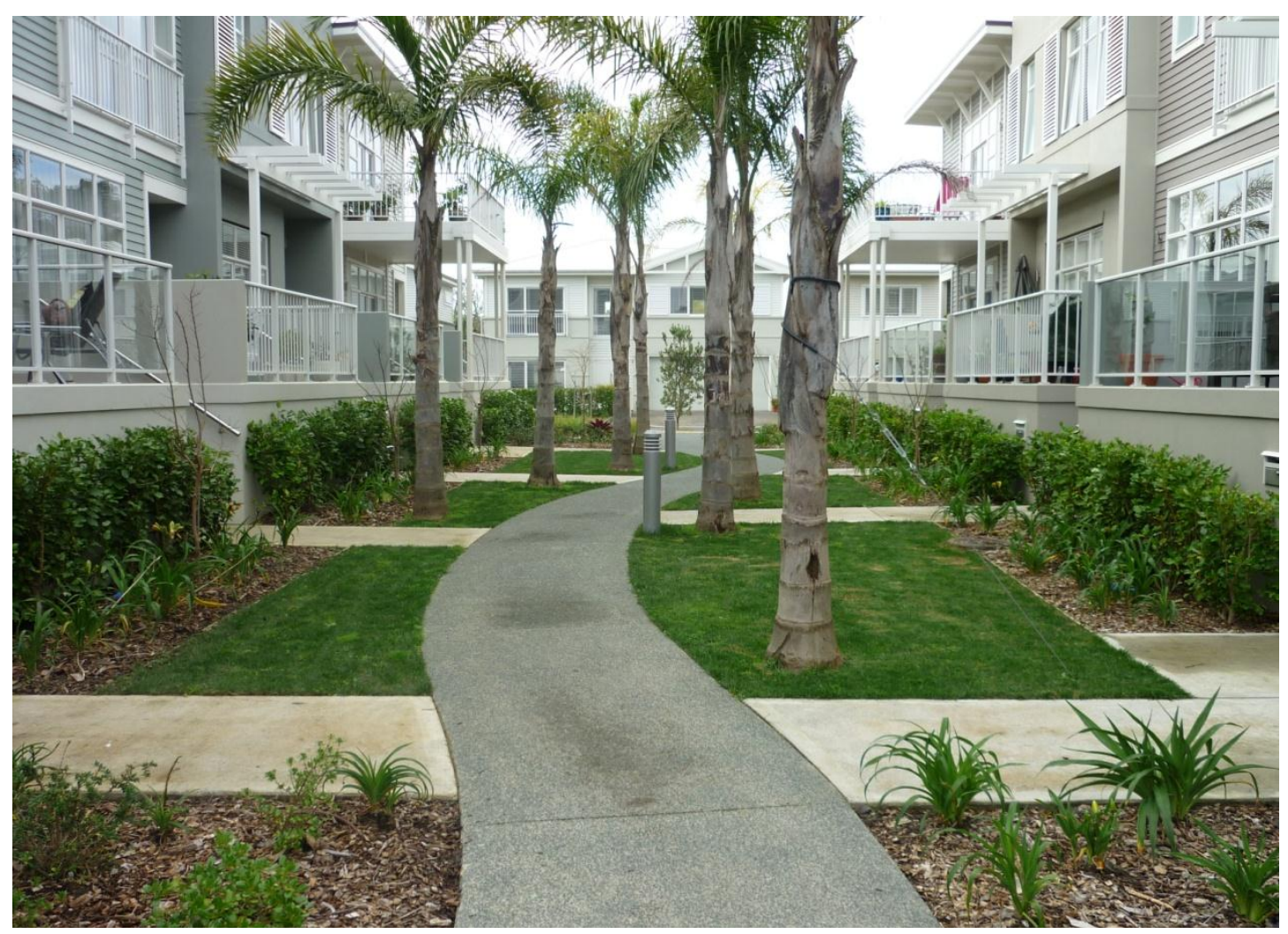

Fig.A7.3.16 Kensington Gardens, Puriri Ave, Orewa, Auckland.

Communal circulation space between dwellings.

(Photo: S. J. Pattinson) 

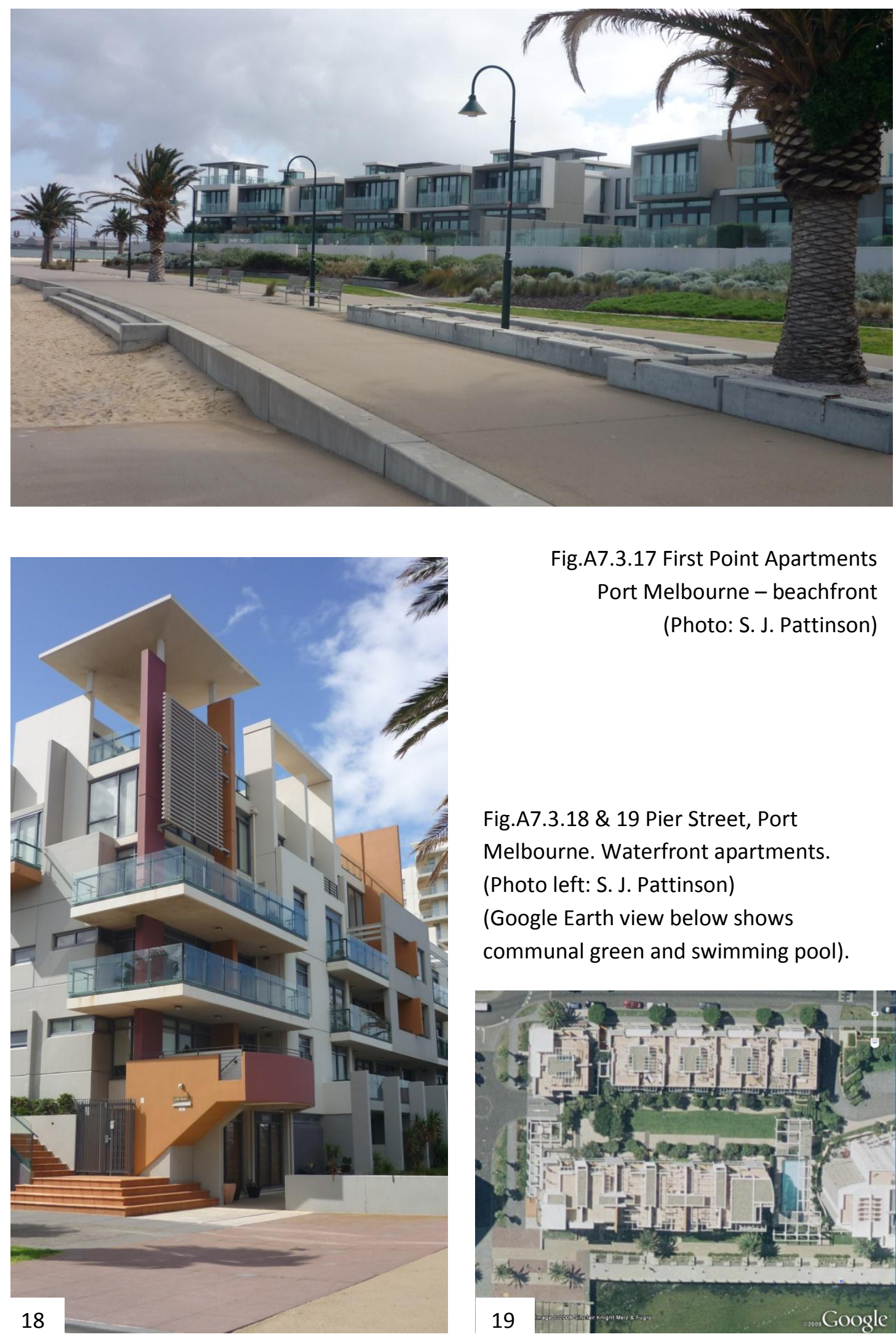

Fig.A7.3.17 First Point Apartments Port Melbourne - beachfront (Photo: S. J. Pattinson)

Fig.A7.3.18 \& 19 Pier Street, Port Melbourne. Waterfront apartments.

(Photo left: S. J. Pattinson)

(Google Earth view below shows communal green and swimming pool).

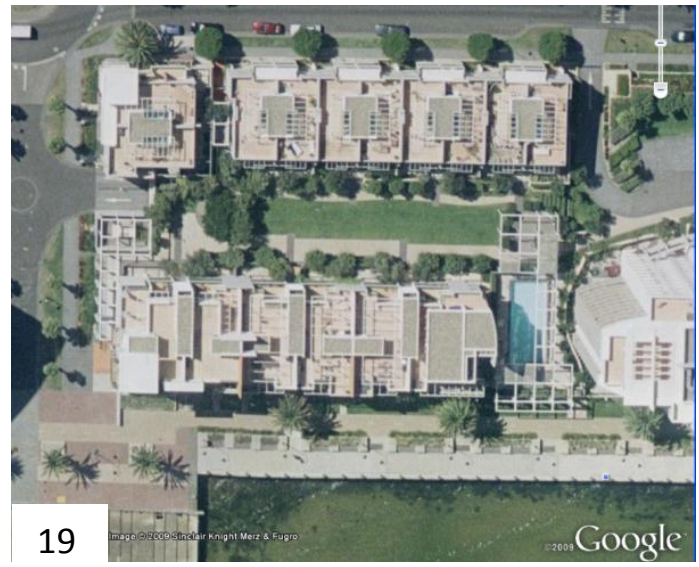




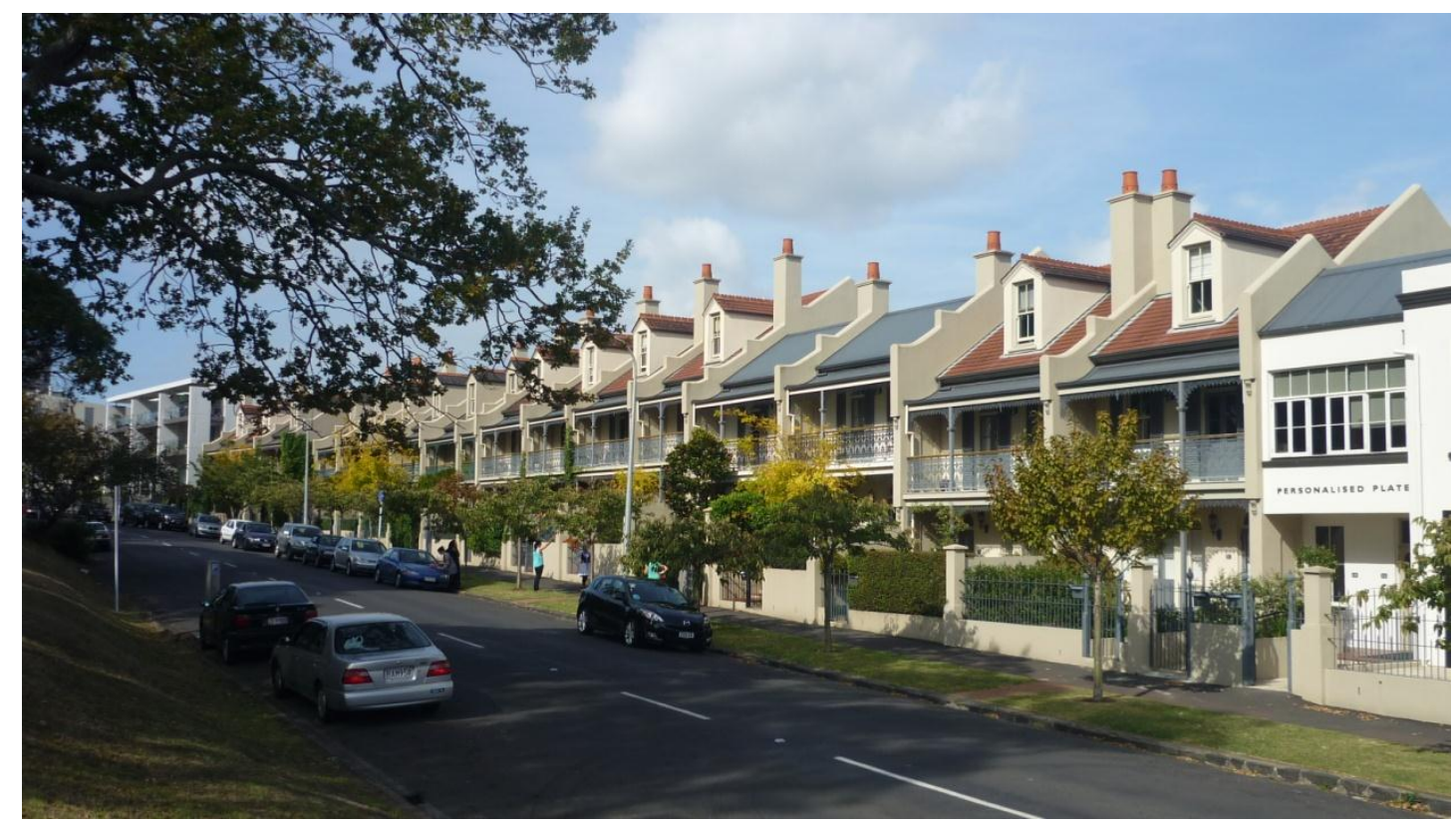

Fig.A7.3.20 Domain Terraces, Newmarket, Auckland.

Adjacent to vast green open spaces of Auckland Domain.

(Photo: S. J. Pattinson)

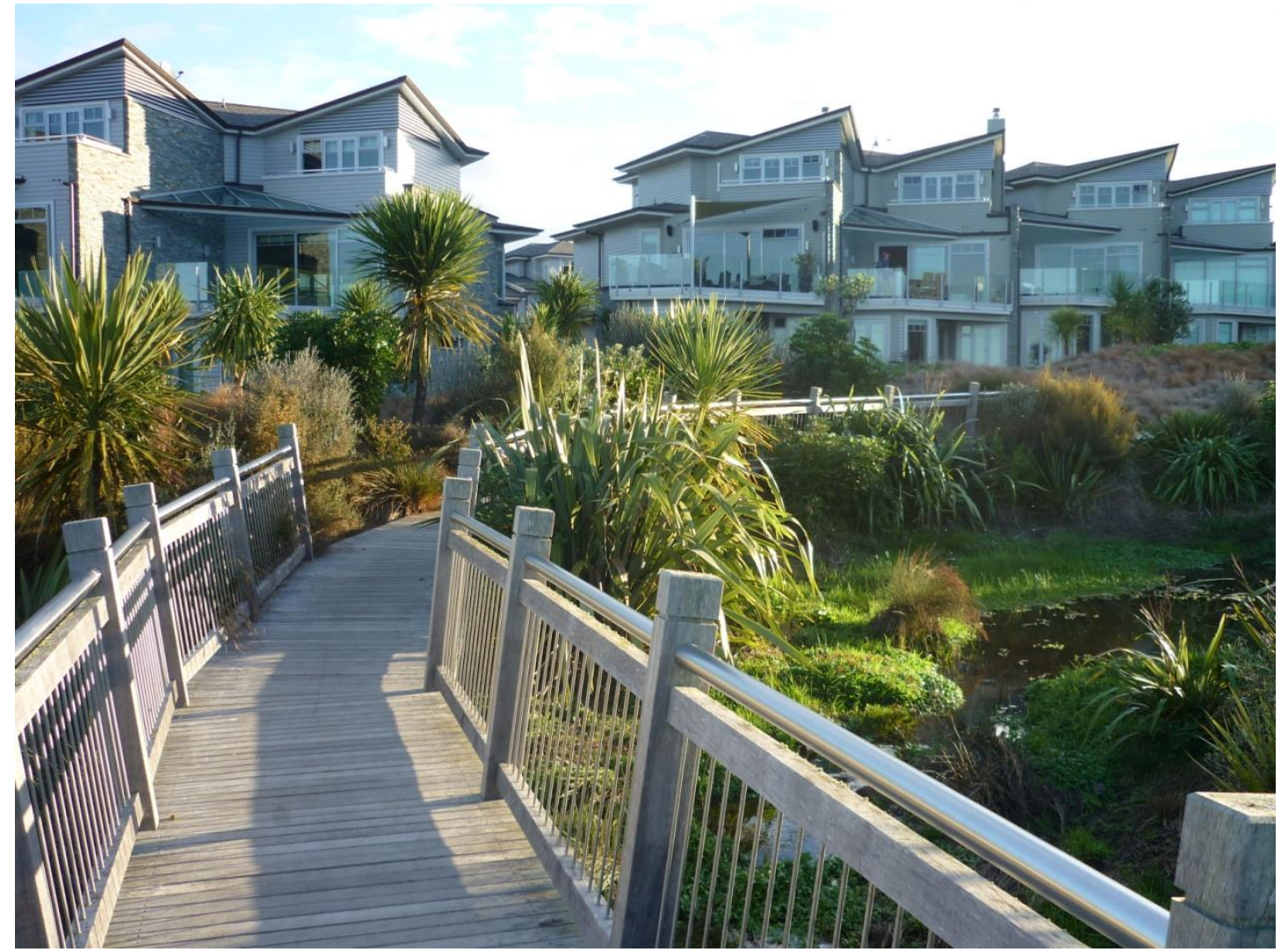

Fig.A7.3.21 Whisper Cove, Snells Beach, Rodney, Auckland.

Wetland bridge and path to beachfront.

(Photo: S. J. Pattinson) 


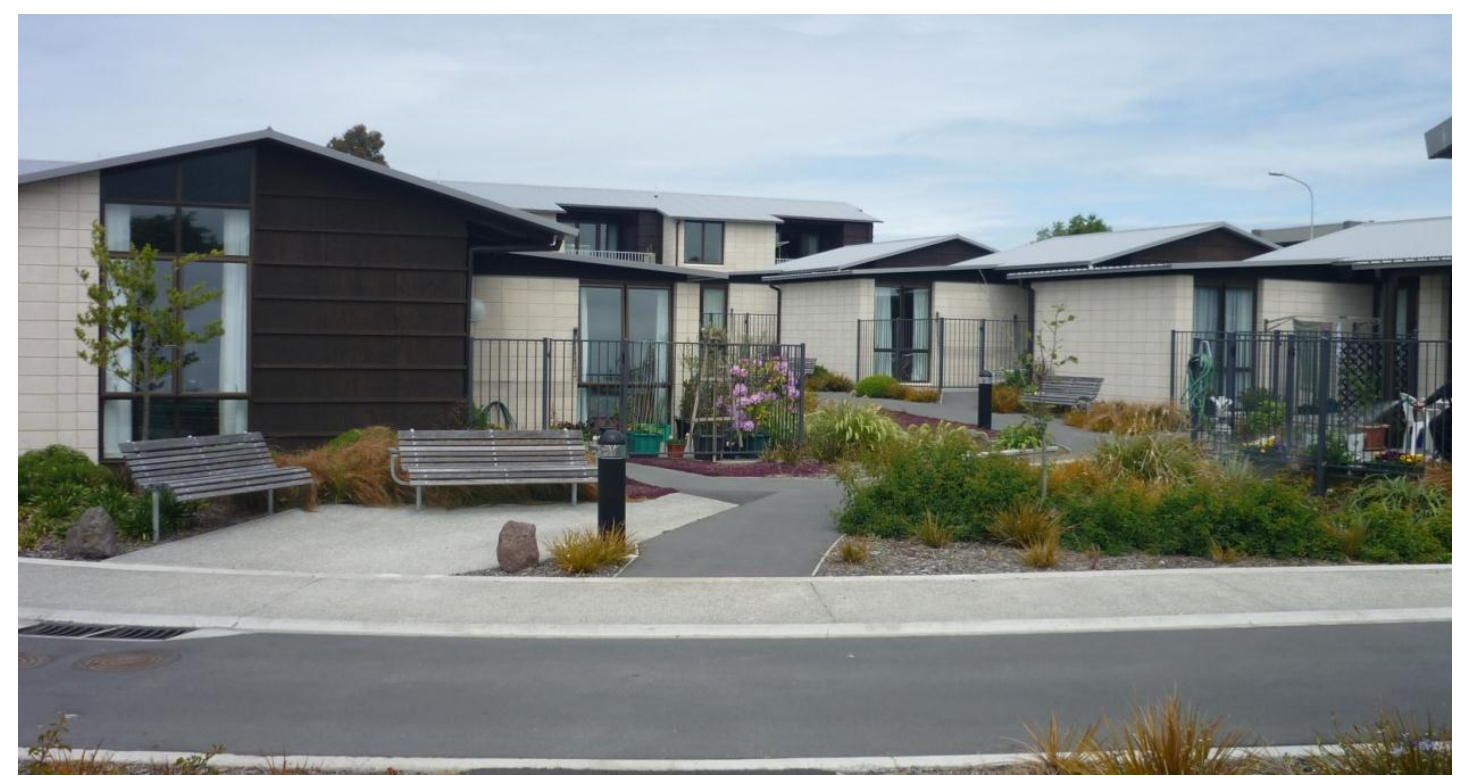

Fig.A7.3.22 40-59 Gowerton Place, Richmond, Christchurch. Council pensioner housing, 2009. Landscaped communal circulation space between dwellings. (Photo: S. J. Pattinson)

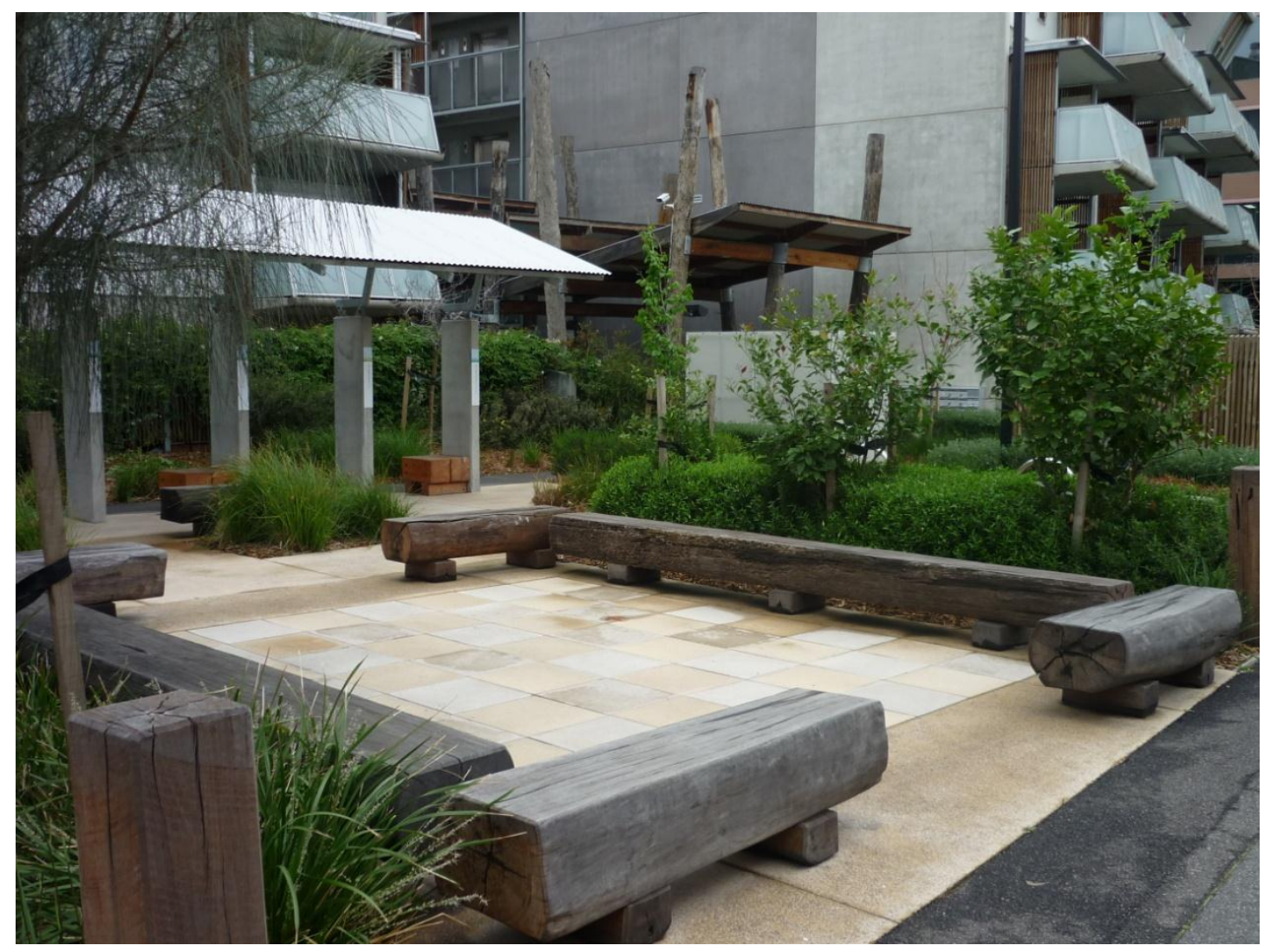

Fig.A7.3.23 Urban Park, K2 Apartments (Social Housing, Government Housing Dept) Melbourne. Located on street edge at Entry (used by 
Extensive playground and sports field areas, and lots of outdoor social spaces at Housing New Zealand Corporation's Talbot Park housing in Glen Innes, Auckland:
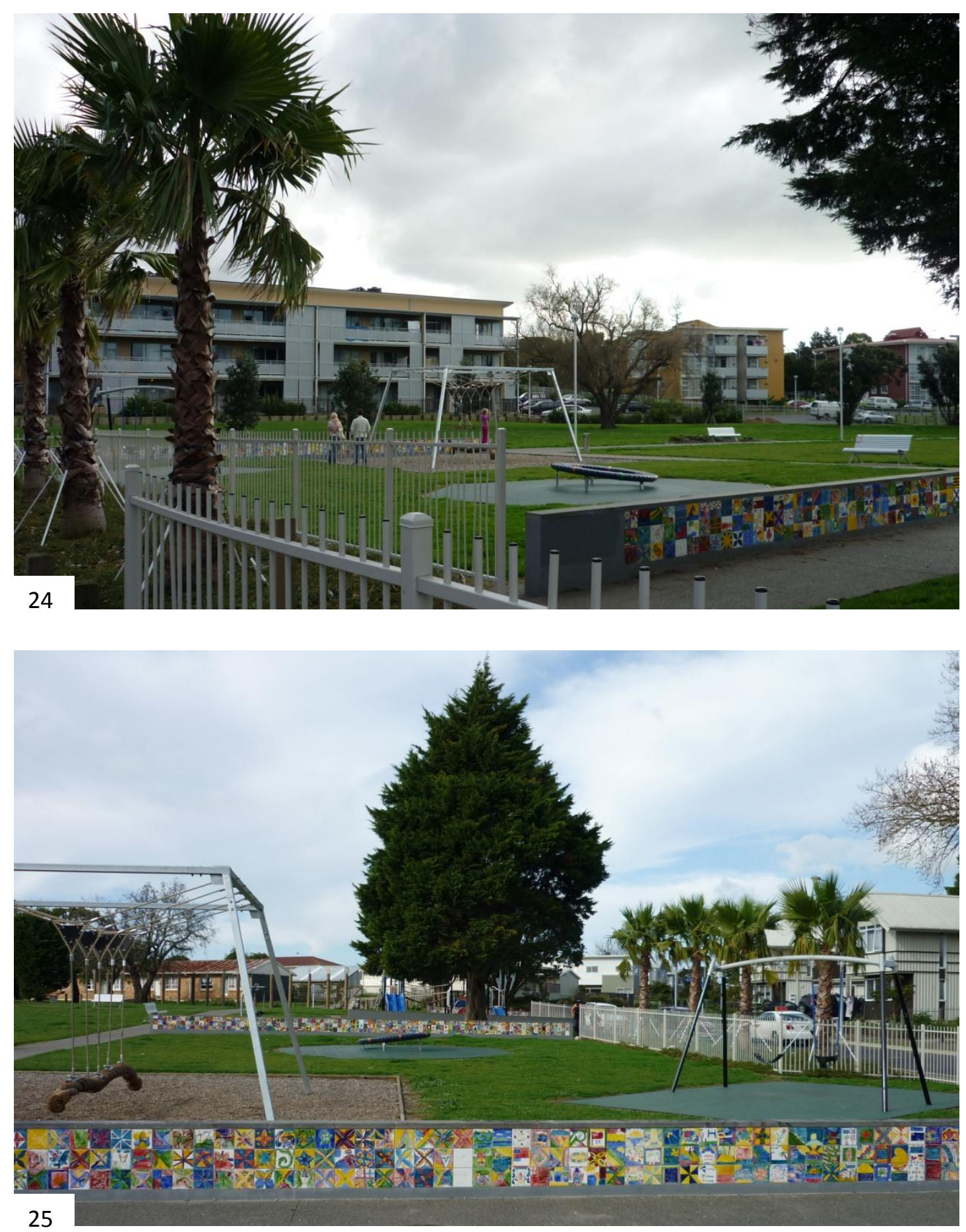

Fig.A7.3.24 \& 25 Children's playground area at Talbot Park, Glen Innes, Auckland. Hand-painted wall tiles were created by local primary school children. (Photos: S. J. Pattinson) 

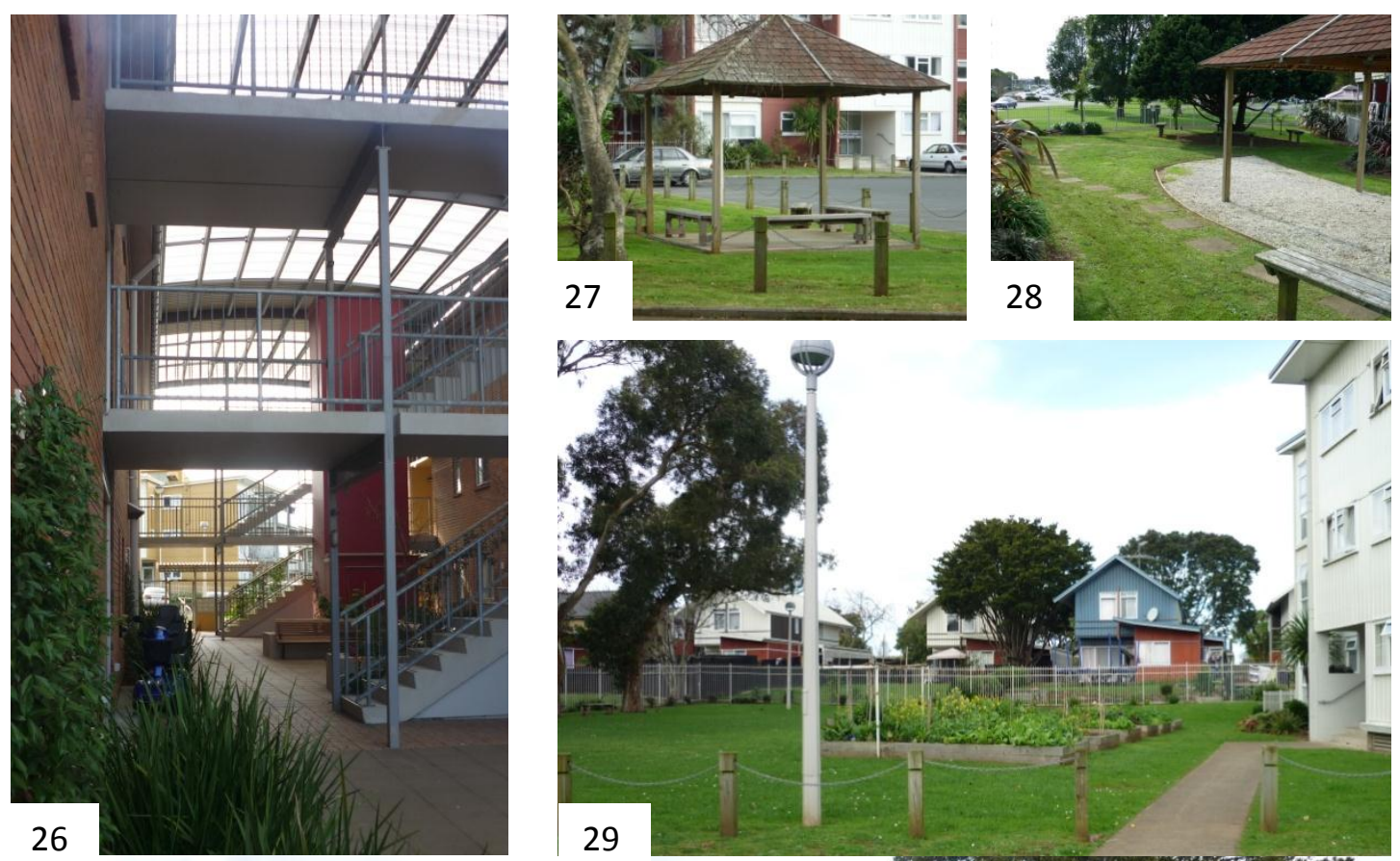

29
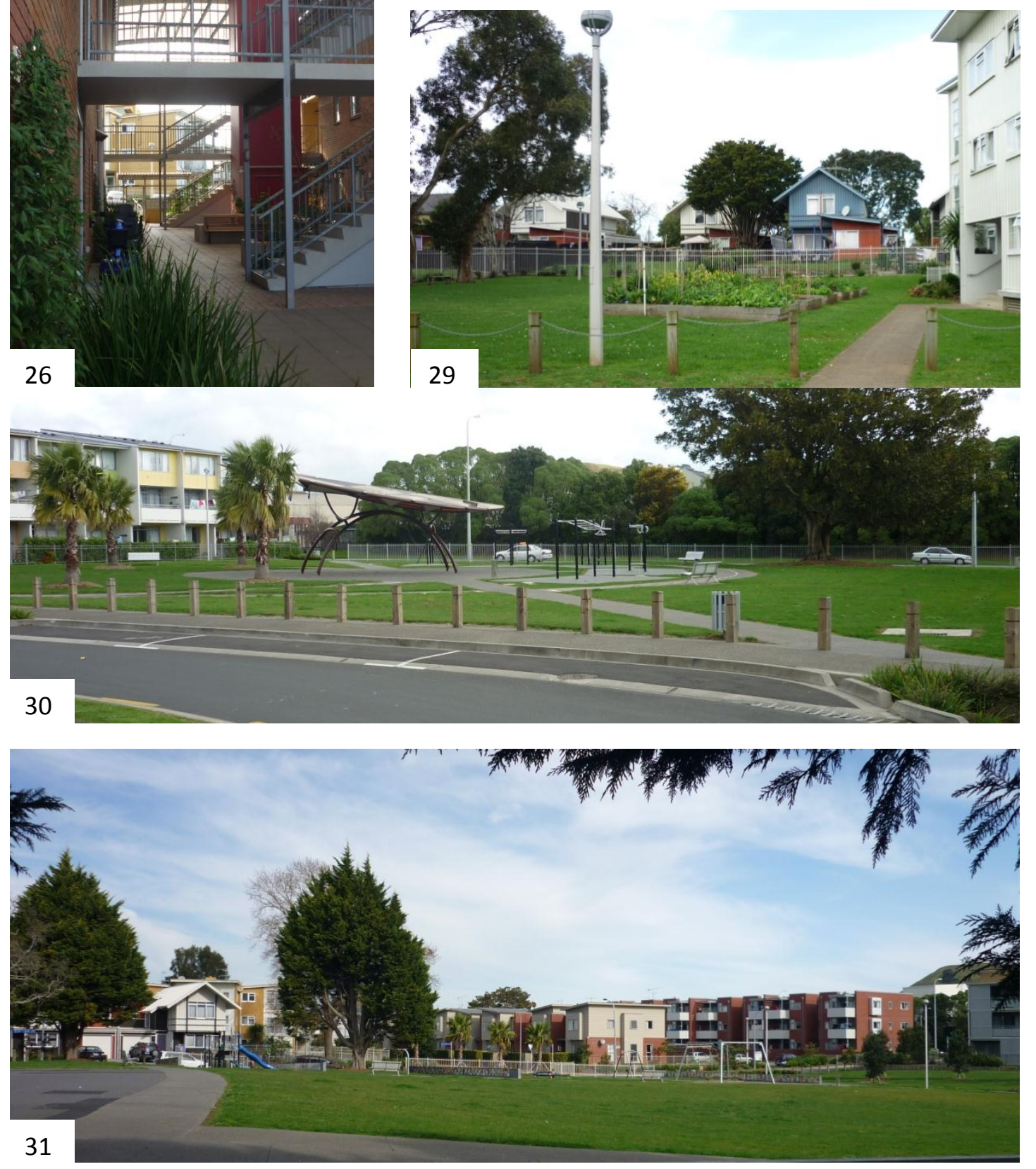

Fig.A7.3.26 - 31 Lots of sports fields and outdoor social areas at Talbot Park, Glen Innes, Auckland, including a communal vegetable garden.

(Photos: S. J. Pattinson) 


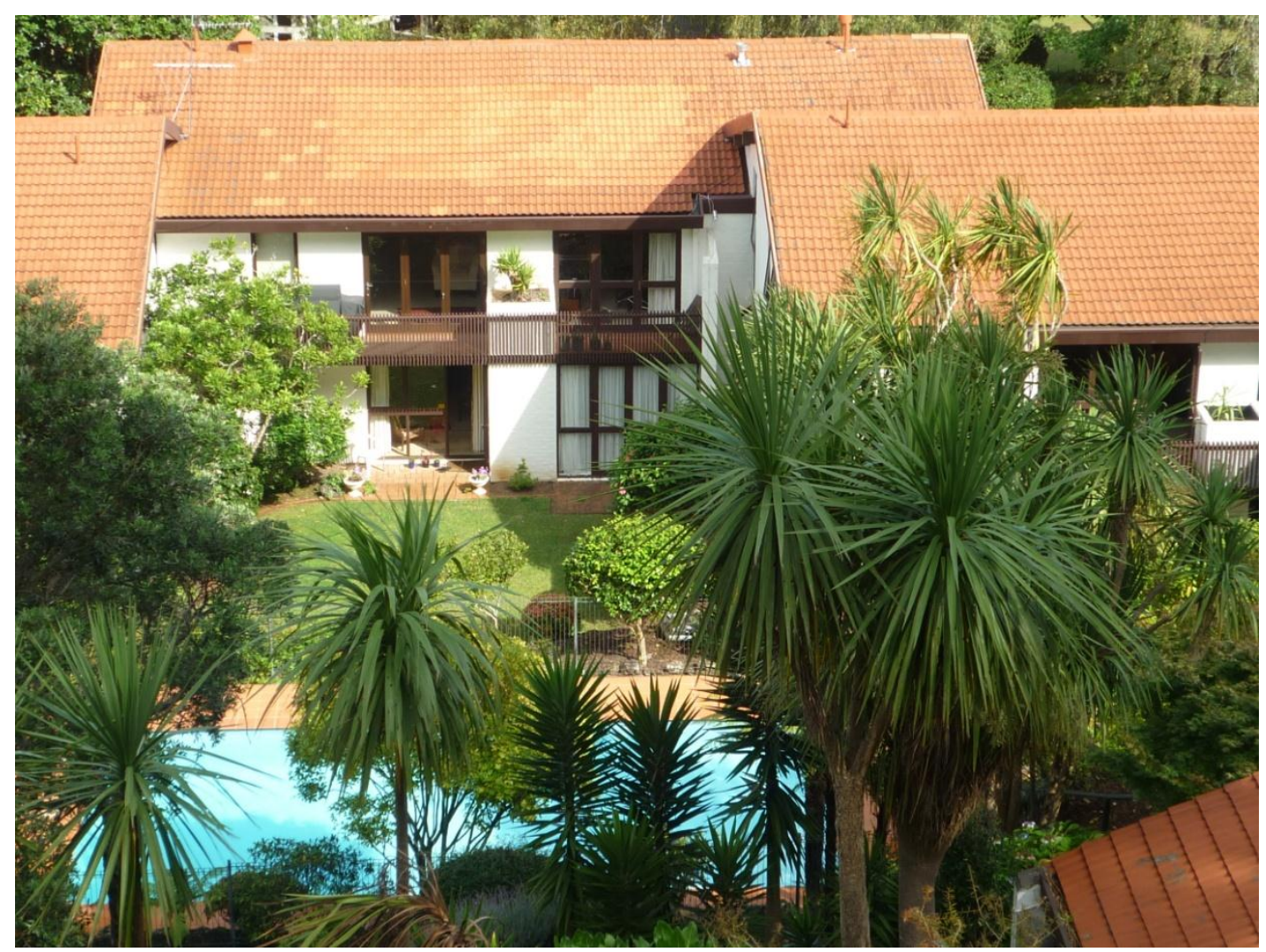

Fig.A7.3.32 123 Owen Street, Epsom, Auckland.

Cluster of units arranged around a shared pool and landscaped yard. (Photo: S.J.Pattinson)

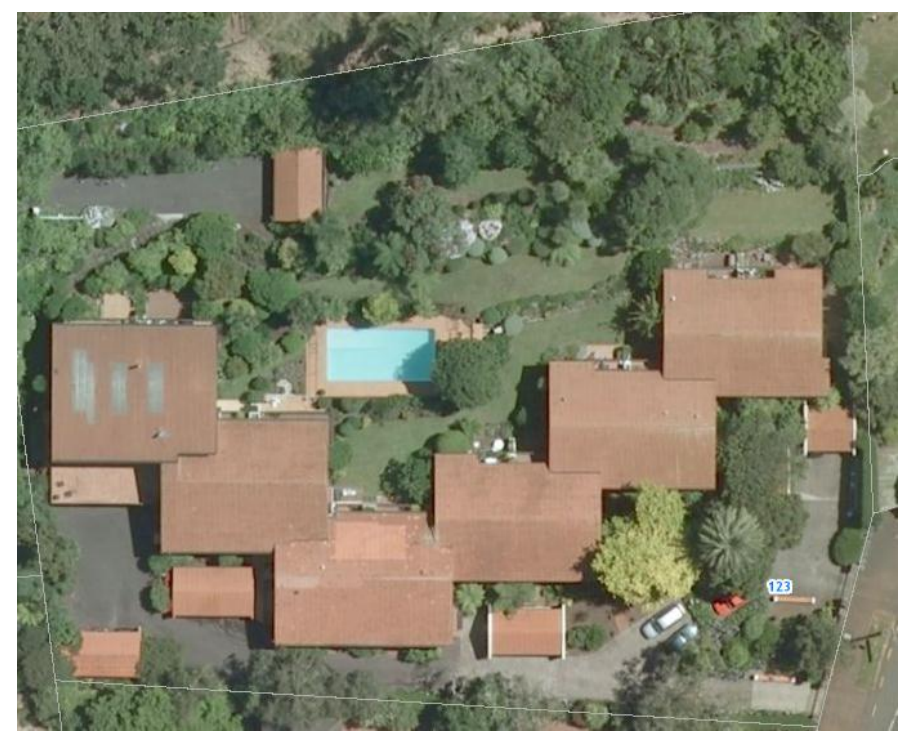

Fig.A7.3.33 123 Owen Street, Epsom, Auckland. Cluster of 18 units arranged around a shared pool and landscaped yard. (Alggi Aerial Map)
This housing development at Owens Street, Epsom is a good example of bay-shaped communal space in clustered housing. From Council records it appears to have been built in the 1970s (designer unknown). When talking about communal space, some people have said to the writer - "We don't understand it. What is it?". This example might help explain the concept for them.

This is one of the few communal spaces the writer observed being actively used by residents - two groups swimming, people gardening, and others outdoors. 


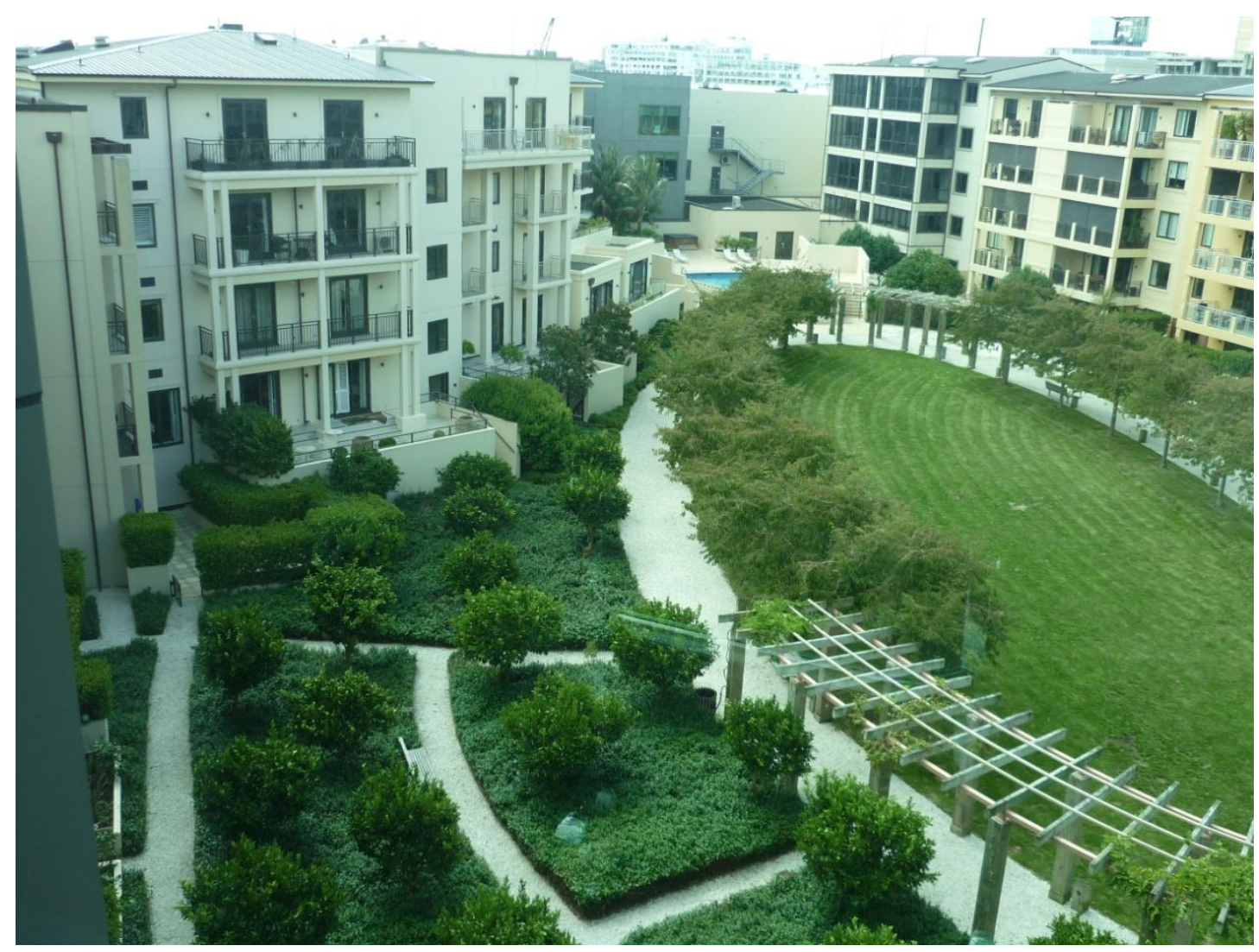

Fig.A7.3.34 The Parc, Viaduct Harbour, Auckland: Central communal green and swimming pool - primarily landscaped outlook?

(Photo: S. J.

The foregoing examples illustrate high-quality, medium-density housing developments. They also show some communal facilities that are spacious and well maintained. It is difficult to say what percentage of New Zealand's medium-density housing is of this high quality - it is not the purpose of this thesis to make such an assessment. The above examples might represent $10 \%$ to $15 \%$ of the developments the writer has visited.

More typically, communal space in medium-density housing is not of such high quality, and the public generally do not seem to want it in their back yard. It is therefore not unusual to find medium-density developments occupying less expensive land next to motorways, railway lines and industry. 
The following pages set out the writer's first impressions of communal spaces typical of the vast majority of such spaces in existing medium-density housing developments. Specifically, the writer observed that:

a) residents appear to spend little time outdoors in medium-density housing;

b) "location, location, location" can mean "motorway, railway and industry";

c) provision for cars can dominate a housing development;

d) outdoor recreational areas are limited to 'left-over' space;

e) cramming can squeeze out open space;

f) averaging does not work with landscaping, i.e. high quality landscaping in one small part of a housing development does not make barren areas in the rest of the development more liveable;

g) picnic areas and playgrounds may be just token gestures;

h) pools and playgrounds can be inaccessible to residents;

i) playgrounds can be unimaginative;

j) communal space can be ambiguous;

k) communal space may just be 'landscaped outlook' rather than social space;

I) communal facilities can be inadequate;

$\mathrm{m}$ ) the needs of children and teenagers are often overlooked;

n) the needs of the elderly may be overlooked;

o) neighbourhoods are fragmented;

p) fragmentation of neighbourhoods may be a reflection of 'silo' thinking;

q) holistic, integrated, multivalent thinking is needed.

The following pages provide examples, illustrations and explanations of the above observations. 


\section{a) Residents appear to spend little time outdoors}

One would expect to see a few people outdoors in a housing development like the one shown in Fig.A7.3.35 below. There are 115 houses in this development.

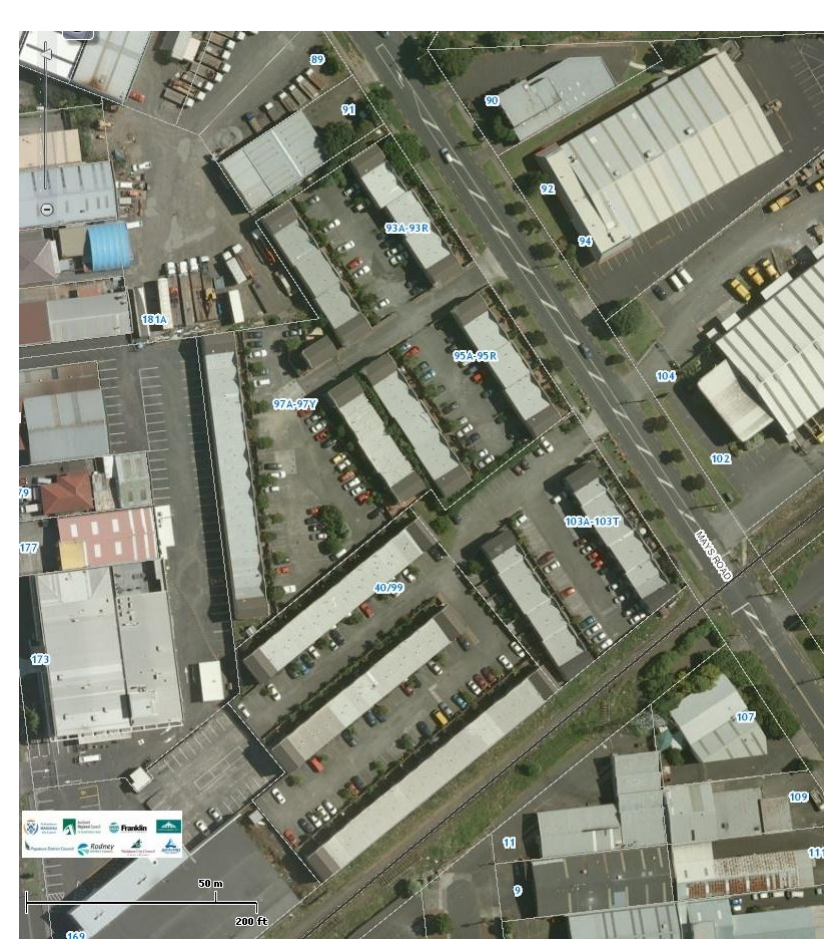

Fig.A7.3.35 95-103 Mays Road, Onehunga, Auckland, NZ. An industrial area.

There is no outdoor communal space in this development, other than vehicle areas.
Assuming a full occupancy rate of, say, $2.0-2.5$ people per household, there may be 250 people living here. The Sunday afternoon on which the writer made a 40 minute visit - a fine day in March -a total of 7 people were seen. One was putting out some rubbish, one was checking the mail and the other five (travelling alone) were in their cars, either arriving or leaving.

There were 96 parked cars.

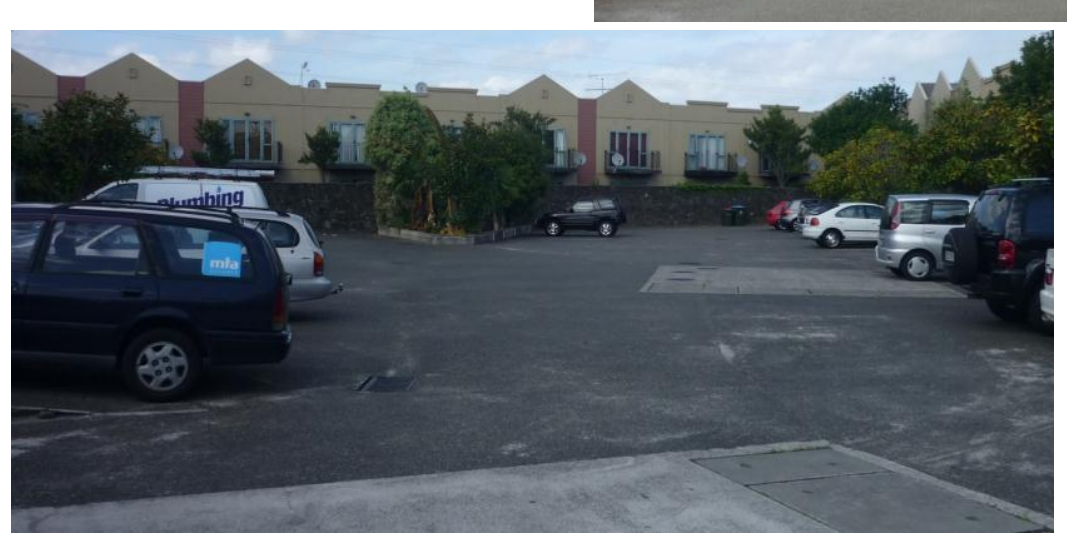

Fig.A7.3.36 \& 37 95-103 Mays Rd, Onehunga. All the residents appear to be indoors. 
If the residents come outside - what is there to see and hear?

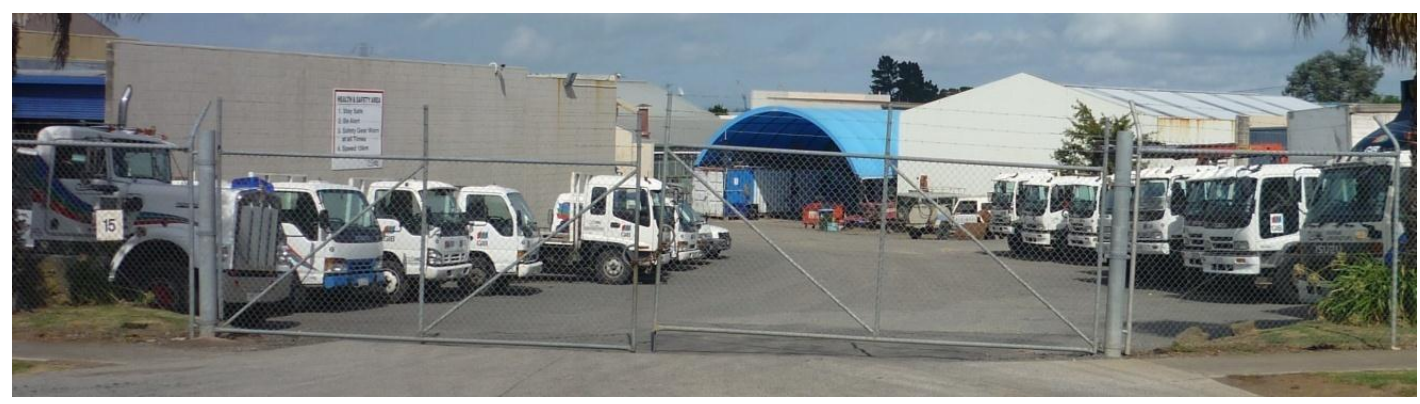

Fig.A7.3.38 North neighbour

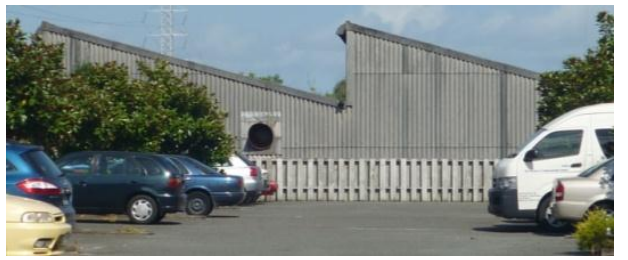

Fig.A7.3.39 West neighbour

Fig.A7.3.40 East neighbour
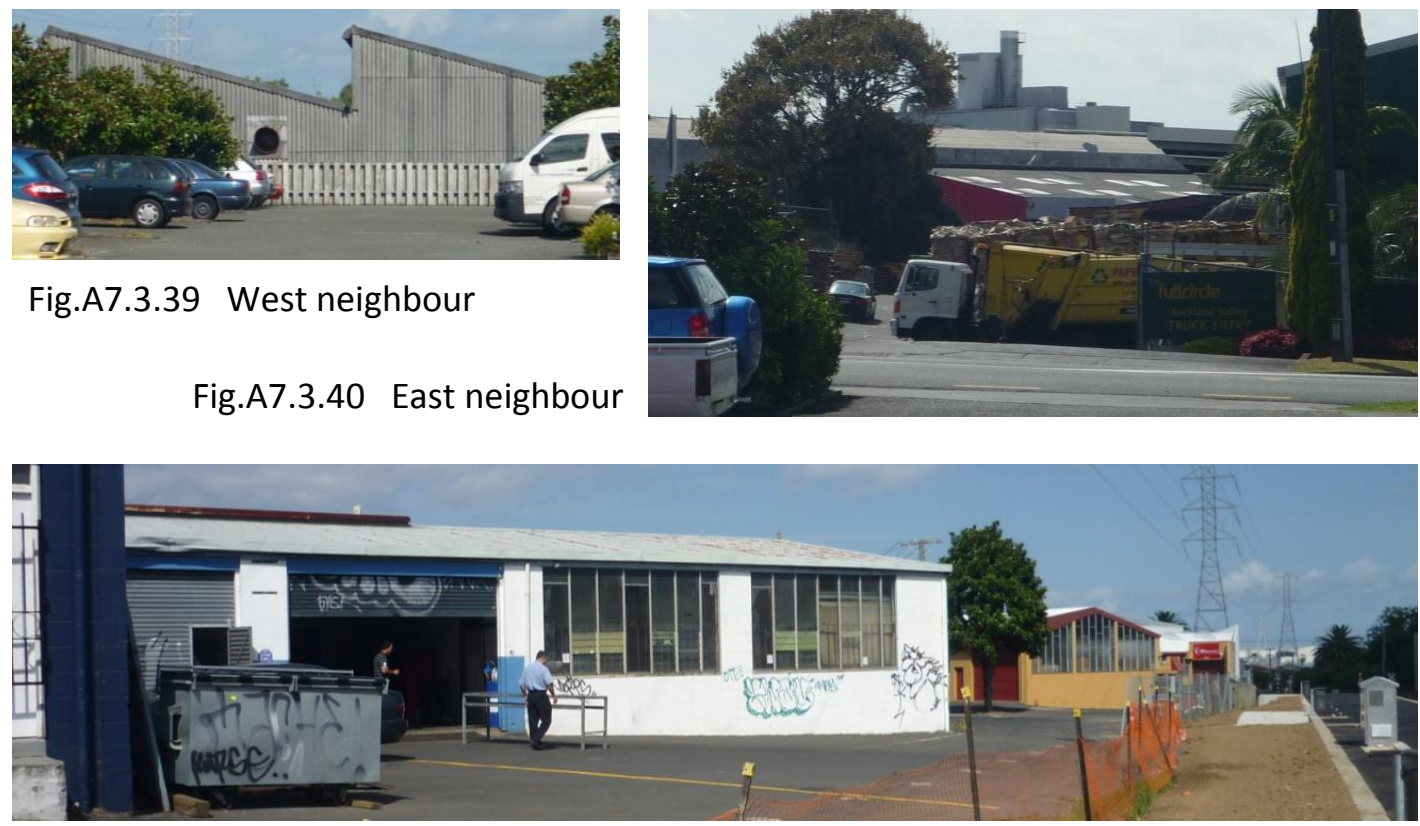

Fig.A7.3.41 South neighbour

(All photos: S.J. Pattinson)

Do they have yards...?

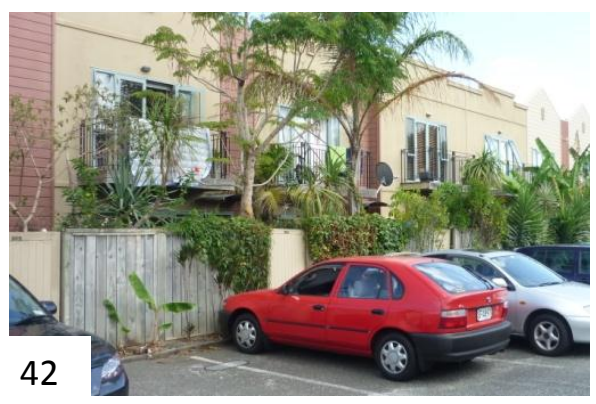

... front private yards ...

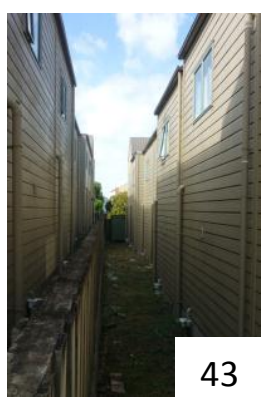

... rear yards ...

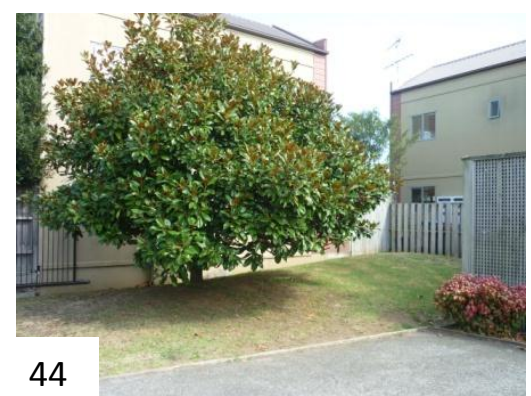

... and a lawn for 250 people.

Fig.A7.3.42 - 44 Yards at 95-103 Mays Road, Onehunga, Auckland. (All photos: S.J. Pattinson) 


\section{b) "Location, Location, Location" can mean "Motorway, Railway and Industry":}

Medium-density

housing is often

located on cheaper

urban land, adjacent

to motorways,

railway lines and

industrial activities.

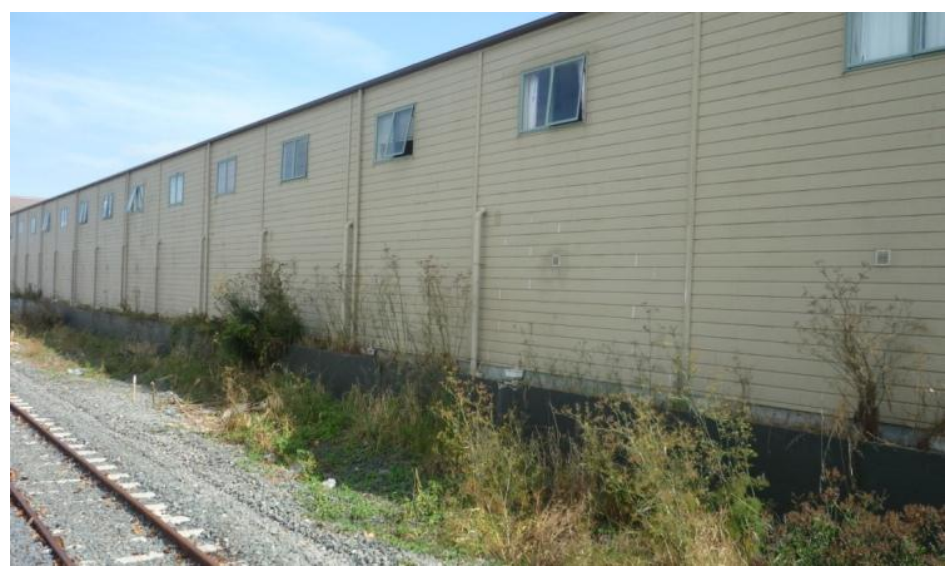

Fig.A7.3.45 95-103 Mays Road, Onehunga, Auckland. (Photo: S.J. Pattinson)

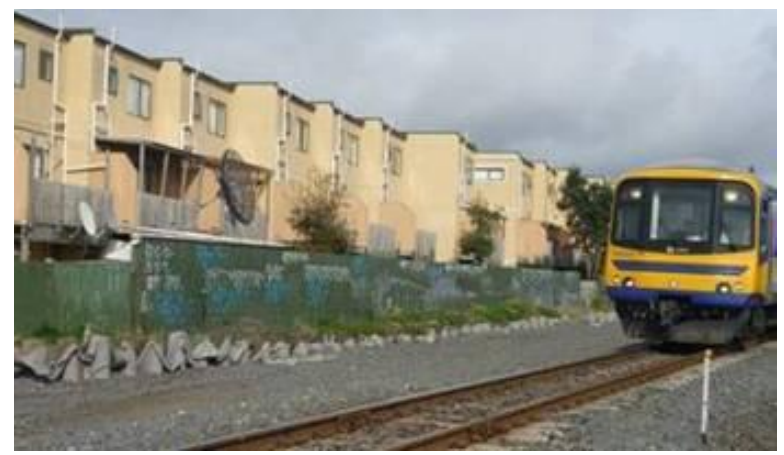

Fig.A7.3.46 New Lynn, Waitakere City, Auckland. 'Not-so-private' private back yards.

(Photo: S.J.Pattinson)

Fig.A7.3.47 Tawa, Wellington Squeezed between road, rail and open drain channel. (Google Map)

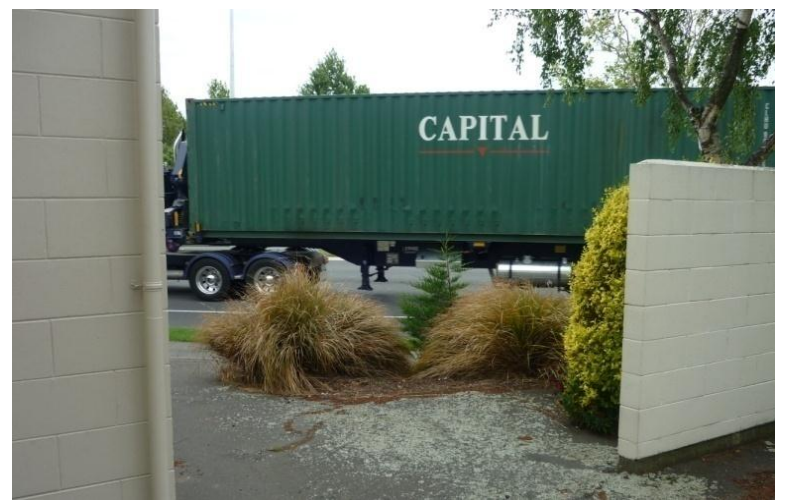

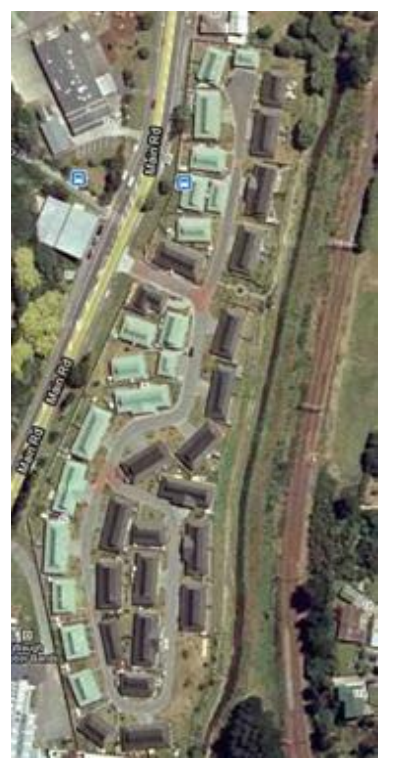

Fig.A7.3.48 Brougham Street, Christchurch, New Zealand There are young children living in this development, which has no protection from Brougham Street, a major arterial road. (Photo: S.J.Pattinson) 


\section{c) Provision for cars can dominate a housing development:}

Many housing developments visited are memorable for the dominance of cars, lack of people outdoors (even when the cars are there), and lack of useful open space:

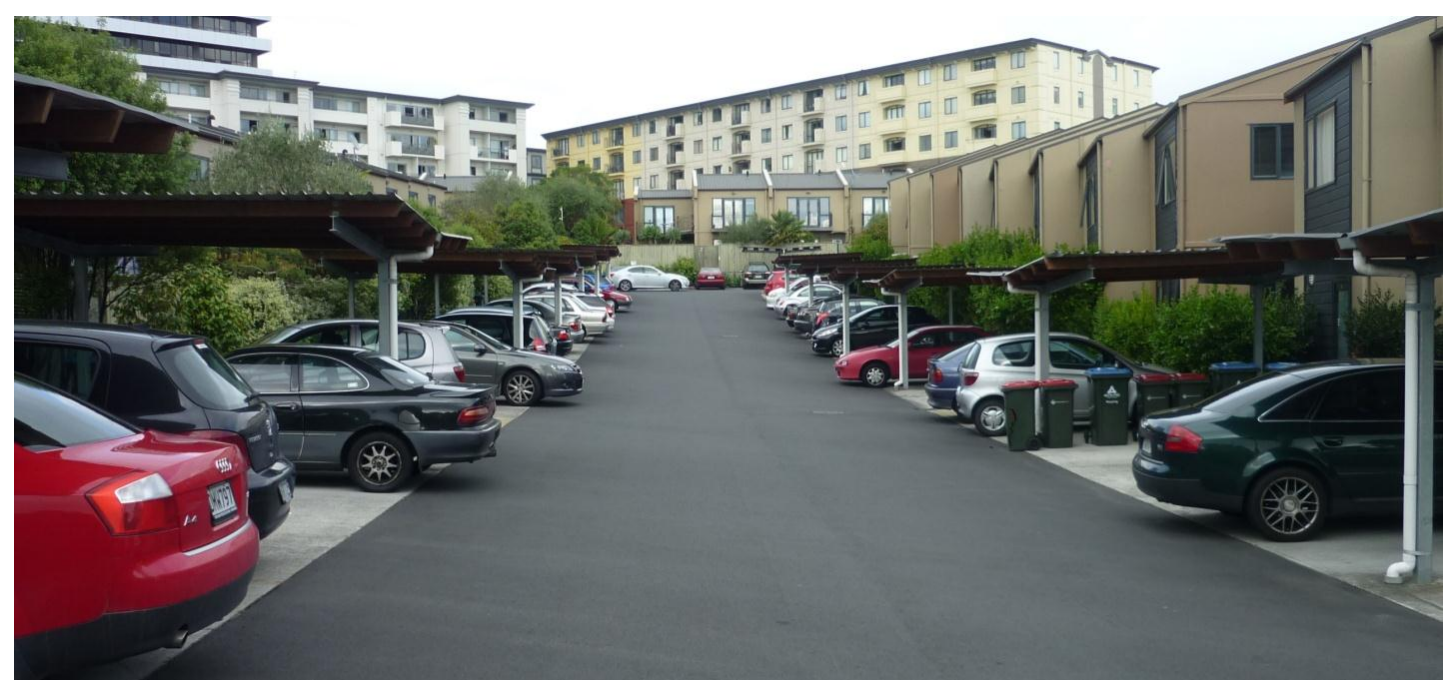

Fig.A7.3.49 Greenwich Park, 3 Burton Street, Grafton, Auckland, NZ.

Front yards are small, overgrown and dominated by car parking. (Photo: S.J. Pattinson)

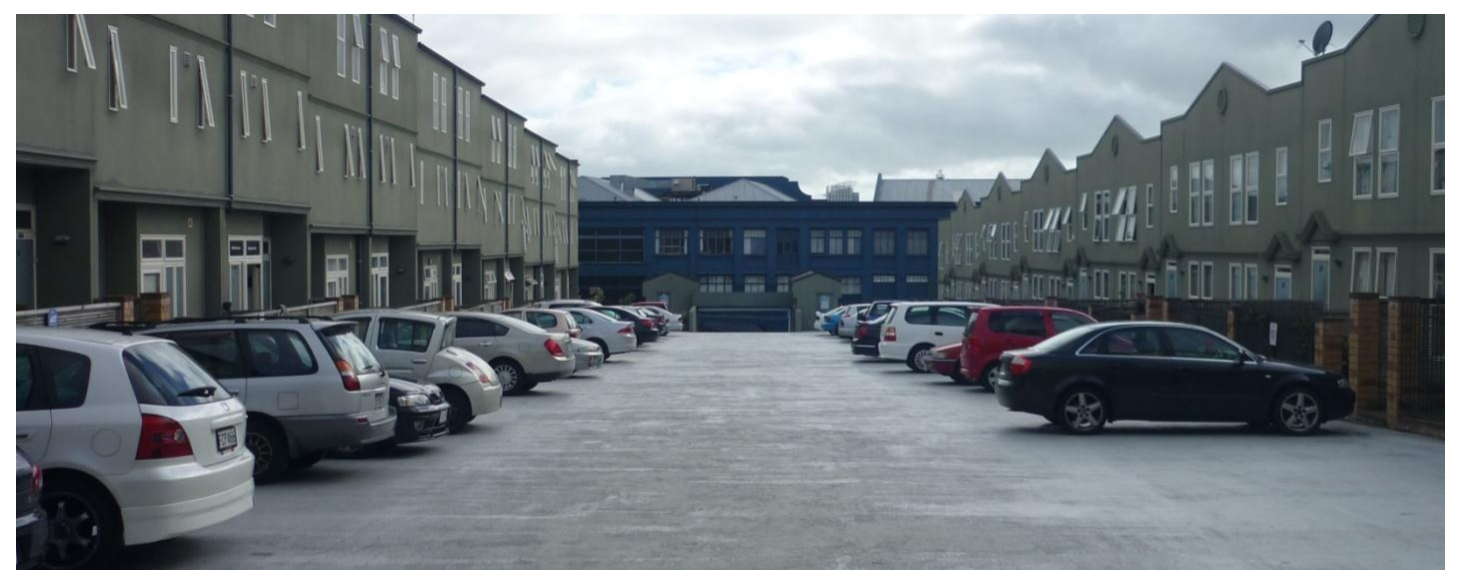

Fig.A7.3.50 150 Symonds Street, Grafton, Auckland, NZ.

(Photo: S.J. Pattinson)

No outdoor space except for semi-private yards and carpark.

\section{d) Outdoor recreational areas are limited to 'left-over' space}

The two projects illustrated above give priority to houses, cars, and minimal private yards. Any 'left-over' space is recreation. The 150 Symonds Street scheme has none. 
The 'left-over' space at 3 Burton Street (called Greenwich Park) is in the corners of the site. Next door are two apartment buildings - Luna Apartments (16 Burton Street) and Madison Apartments (160 Symonds Street). In total, there are about 246 dwellings in this block - Greenwich Park has 85 units, Luna Apartments 72 units, Madison Apartments 56 units, and 33 units at 150 Symonds Street. Assuming an average $2.0-2.5$ persons per dwelling unit, there are $500-600$ people living here. Their only outdoor space (apart from tiny private yards and balconies) is triangular Glenside Reserve - two picnic tables and seats, two park benches, and a bit of grass. The block is an island isolated by traffic. Glenside Reserve is the only outdoor recreational space conveniently accessible by walking for these $500-600$ residents.

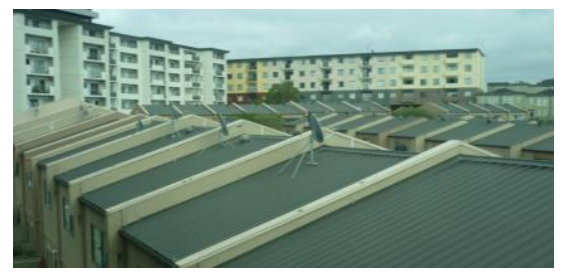

Fig.A7.3.51 \& 52 Glenside Reserve, Grafton -2 picnic tables, 2 park benches and a bit of grass for 600

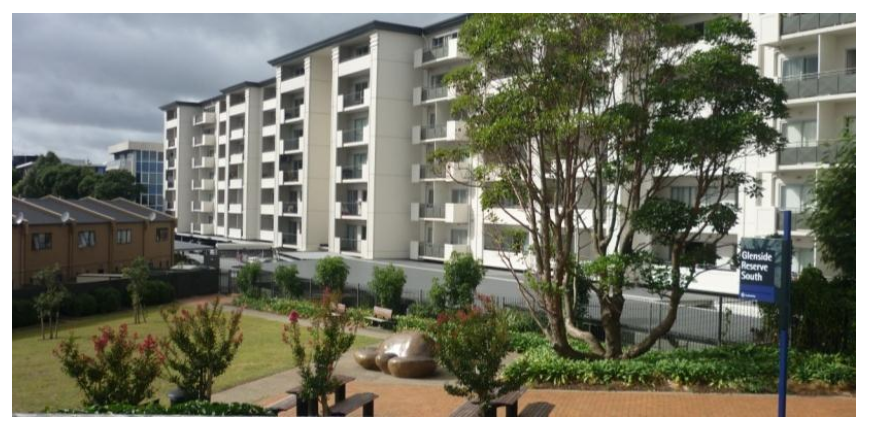
residents (Photos: S.J.Pattinson)

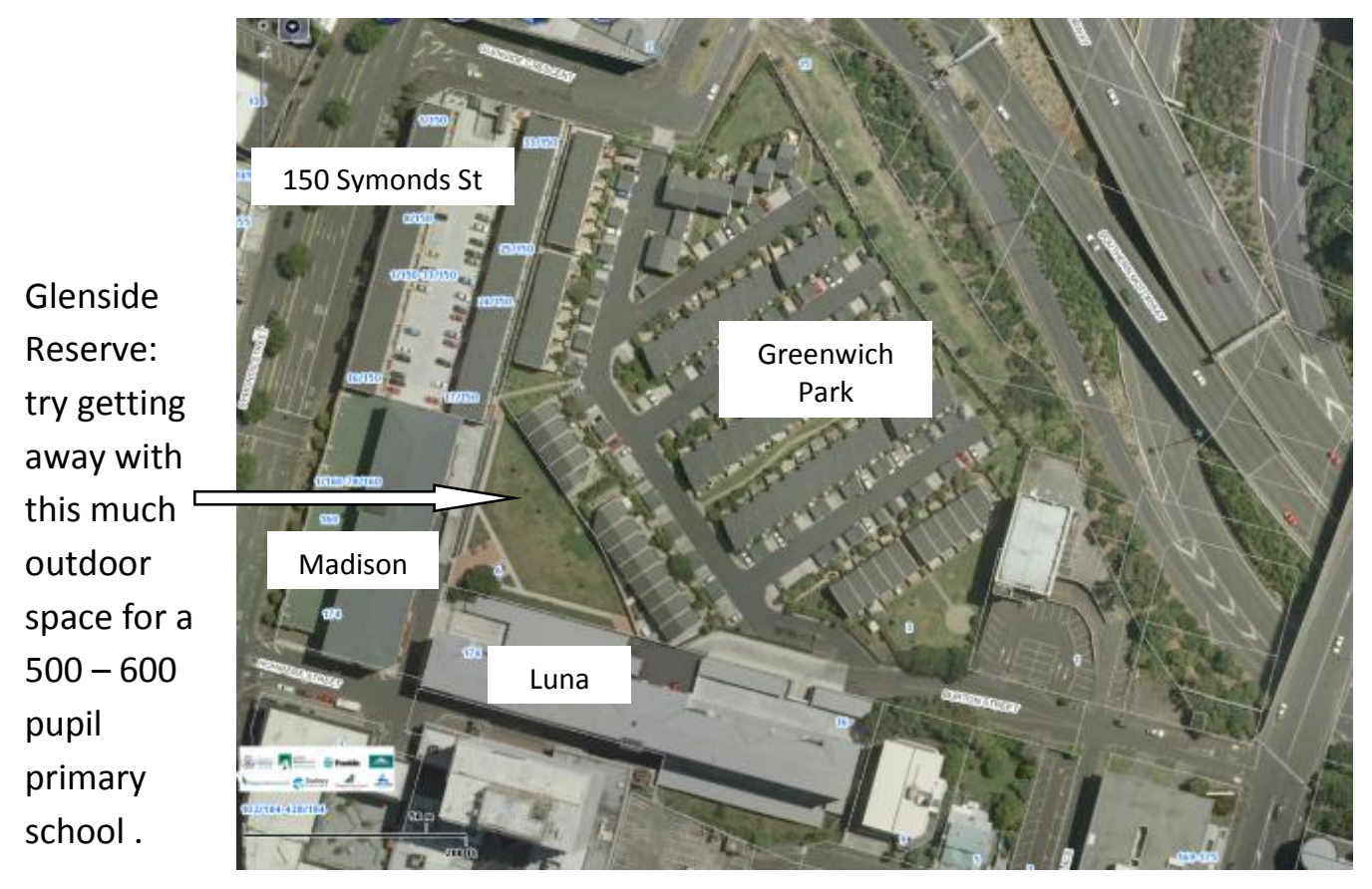

Fig.A7.3.53 Housing developments around Glenside Reserve (ALGGI Map) 


\section{e) Cramming can squeeze out open space:}

In "What is a developer?” ('Houses NZ' Issue 5, p.70), Bill McKay comments, “...most developers seem to be in it for the quick buck ... Houses... are ... built cheap and sold fast ... the role of the architect is to rack 'em, stack 'em, and pack 'em in." Like so ...

\section{Rack 'em ...}

Fig.A7.3.54 $66 \mathrm{Mt}$ Eden Rd, Mt Eden, Auckland. Built over an old carpark building, this is all the outdoor space for 42 double-stacked units. The units are $32 \mathrm{~m} 2$ each and there are families with young children living here.

(Photo: S.J.Pattinson)
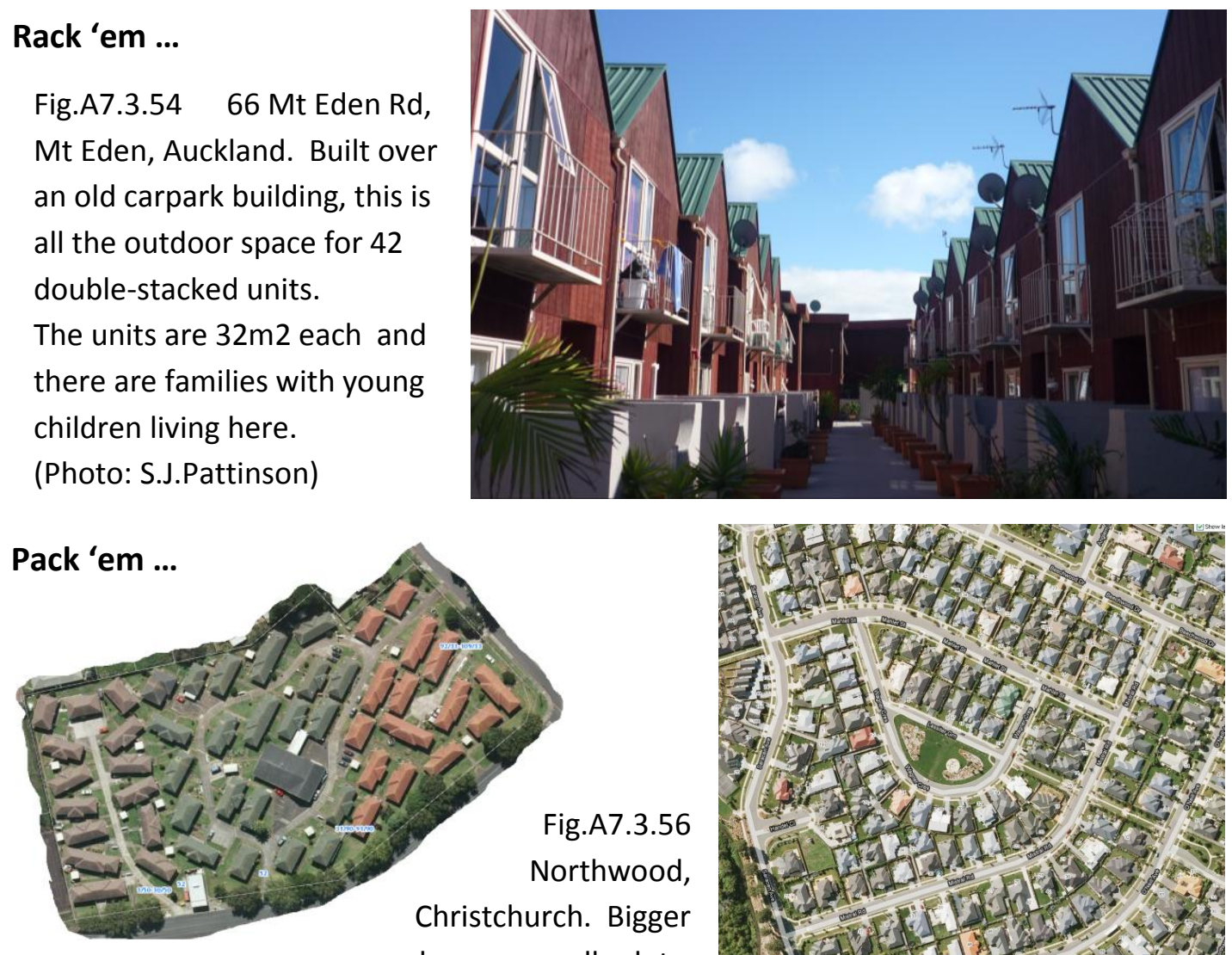

Fig.A7.3.55 Otahuhu houses, smaller lots.

(Alggi Map)

\section{Stack'em ...}

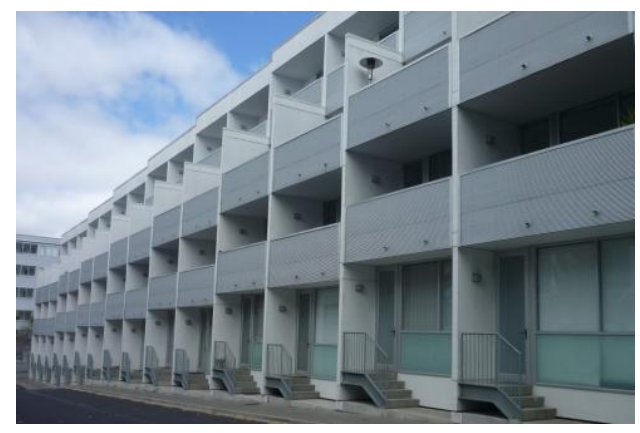

Fig.A7.3.57 Beaumont Quarter, Auckland. (Photo: S.J.Pattinson)
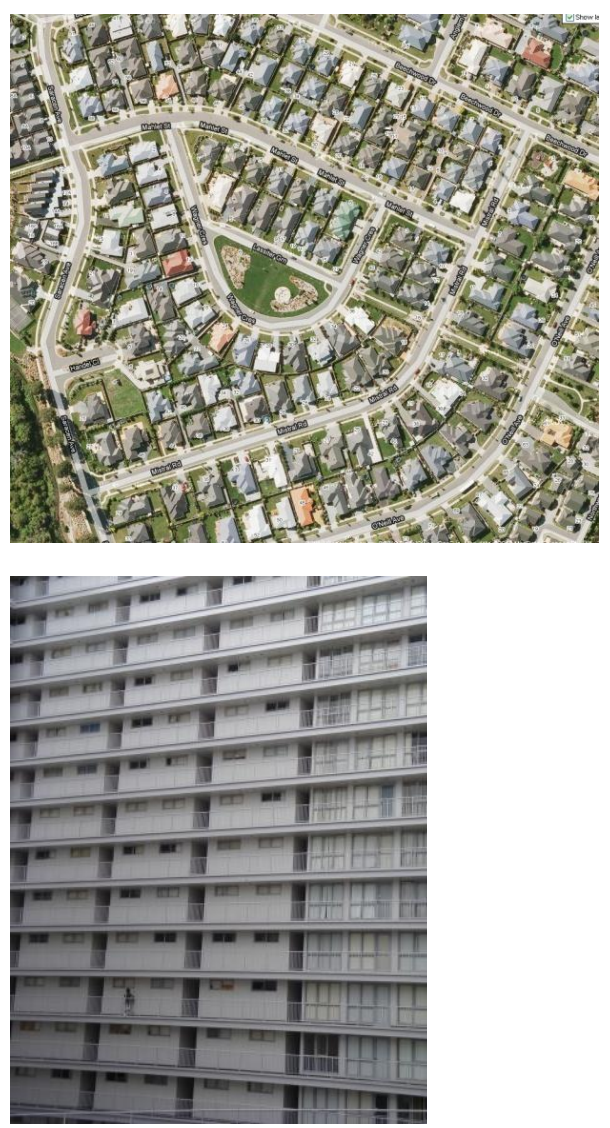

Fig.A7.3.58 Hobson Street, Auckland. Karen Witten reports of families with children in apartments like this one. (Photo: K.Witten) 


\section{f) Averaging does not work with landscaping:}

Something is wrong here. Can exotic landscaping in one small part of a housing development compensate for barren internal streetscapes elsewhere:
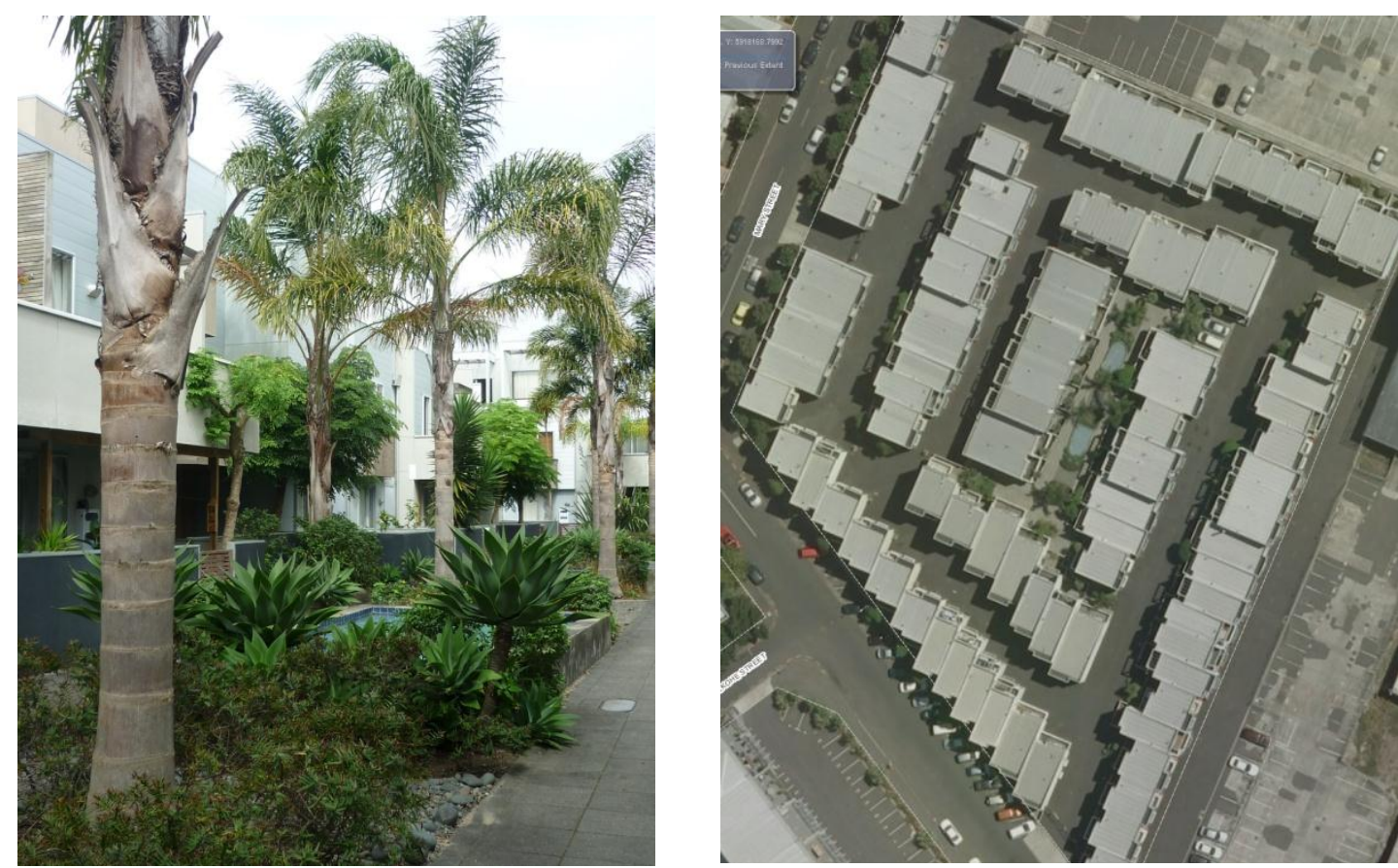

Fig.A7.3.59 Edwin Street, Mt Eden, Auckland. Fig.A7.3.60 Edwin Street, Mt Eden, Auckland Central landscaping. (Photo: S.J. Pattinson)

Green space - central units only (Alggi map)

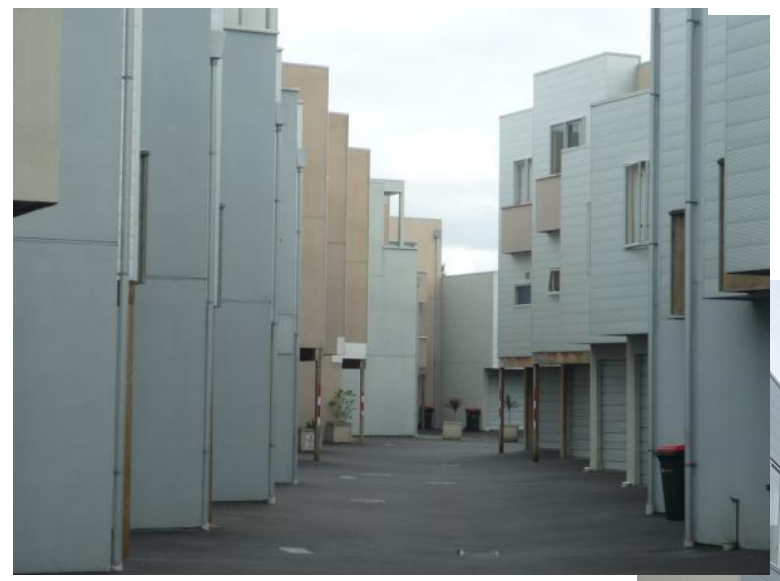

Fig.A7.3.61 typical internal streetscape Edwin Street, Mt Eden, Auckland.

(Photo: S.J. Pattinson)

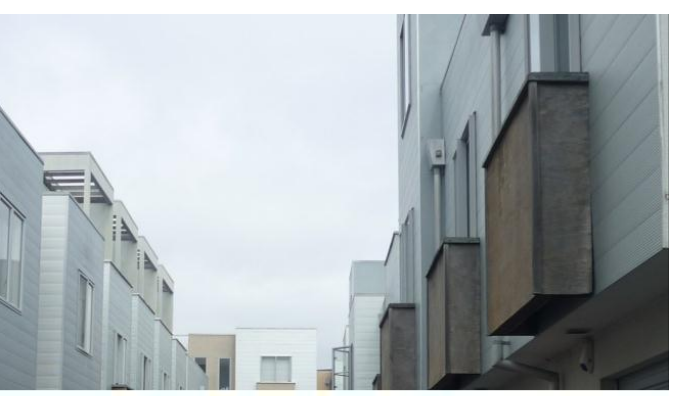

Fig.A7.3.62 typical internal streetscape Edwin Street, Mt Eden, Auckland.

(Photo: S.J. Pattinson) 
Across the road, another housing development also has applied landscaping to the central area of the project, and nothing to the internal streetscapes. What benefit does the small central landscaped open space provide for the majority of residents?

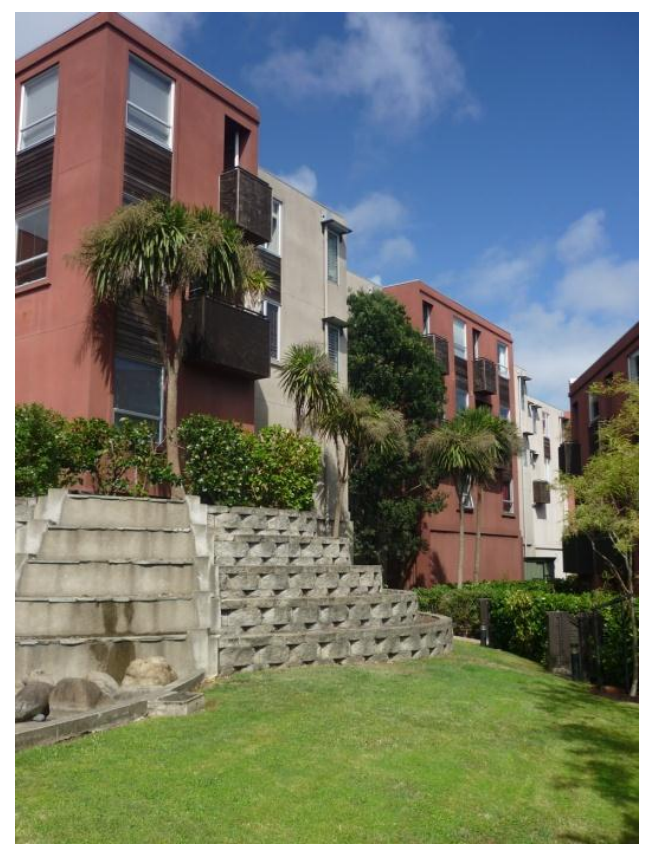

Fig.A7.3.63 Harold \& Mary Sts, Mt Eden. Central landscaping. (Photo: S.J.Pattinson)

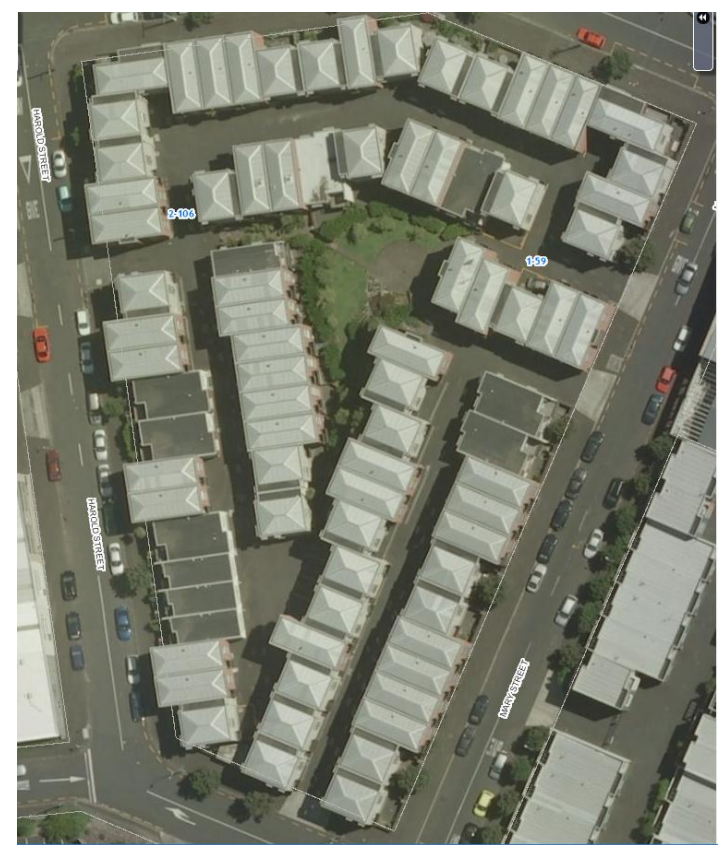

Fig.A7.3.64 Harold \& Mary Streets, Mt Eden, Auckland. (Alggi map)

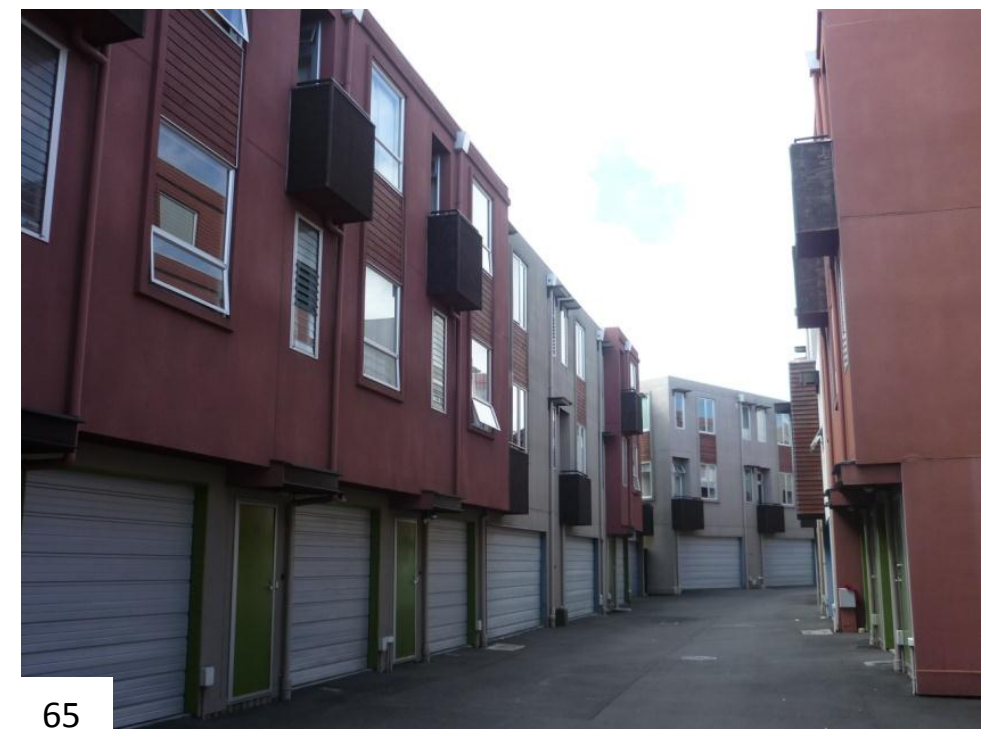

Fig.A7.3.65 - 67 Harold \& Mary Sts, Mt Eden, Auckland. Barren internal streetscapes.
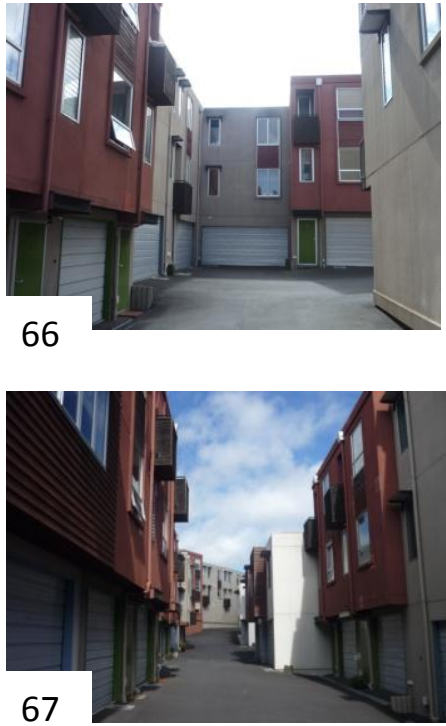

(Photos: S. J. Pattinson) 


\section{g) Picnic areas and playgrounds may be just token gestures:}

'Left-over' space often becomes token landscaping, picnic areas, and playgrounds.

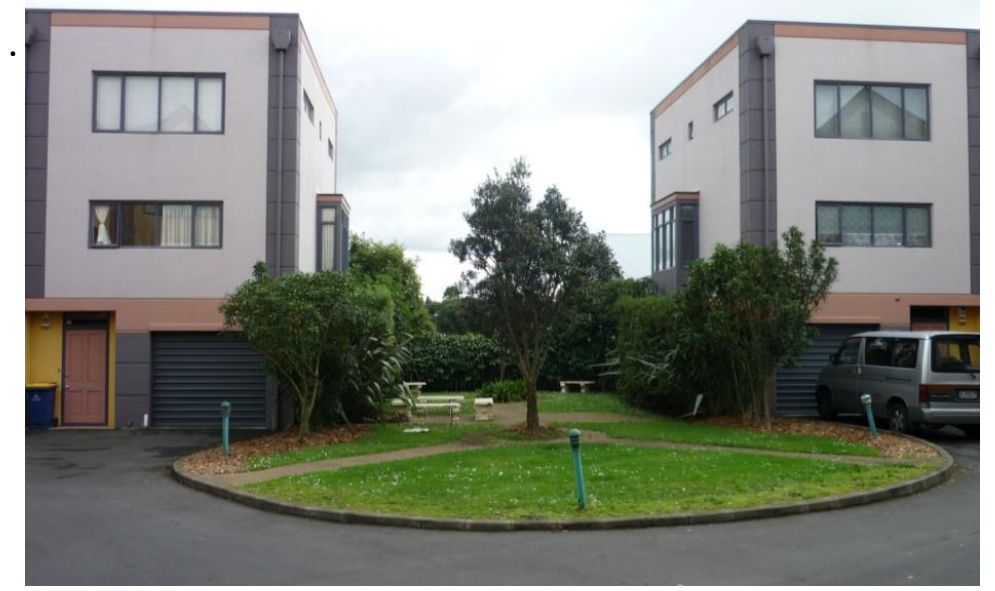

Fig.A7.3.68 Krisley Court, 6 Ambrico Place New Lynn, Auckland (Photos: S.J. Pattinson) Token picnic area i.e. 'left over' space in a carpark see also Fig.A7.3.69 below. This small green has a pinic table and seat but is surrounded by car parking and an unattractive vehicle manouevring area.

Fig.A7.3.69 (right) Krisley Court, 6 Ambrico Place New Lynn, Auckland (Photo: S.J. Pattinson) Ground-level garaging provides each unit direct vehicle access but results in the central courtyard space being car-dominated, and it is not possible for living rooms above the garages to connect directlv with outdoors.

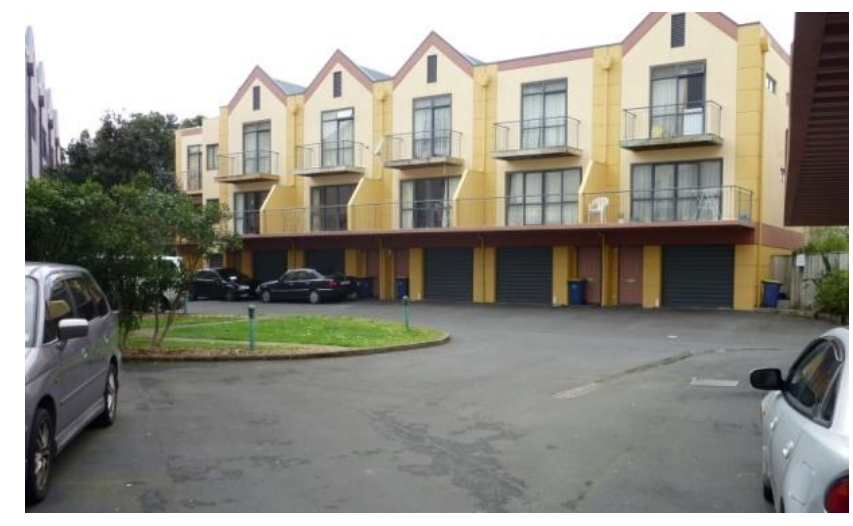

A ring road in the project below ('The Boundary', Karori) is a safety hazard for toddlers accessing the island playground; children are locked out of the tennis court.

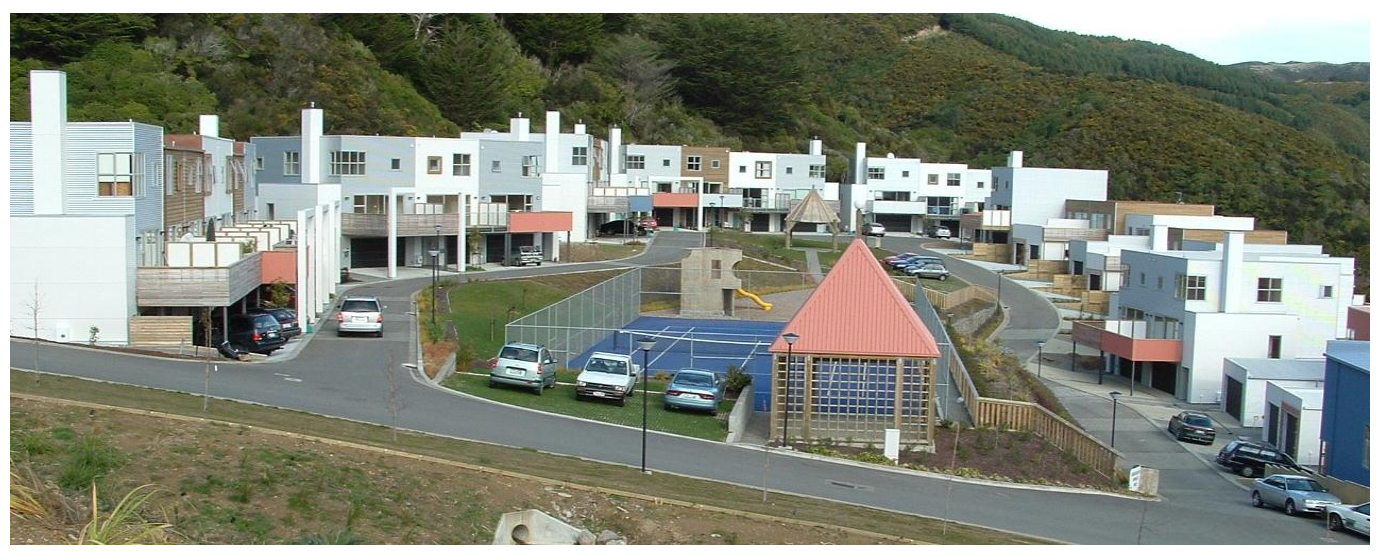

Fig.A7.3.70 'The Boundary' - Saddleback Grove, Karori, Wellington. $\quad 80$ houses, a token plavground on a sloping site and a locked tennis court. (Photo: S.J. Pattinson) 


\section{h) Pools and playgrounds can be inaccessible to residents:}

For whose use is this padlocked 'communal' pool?

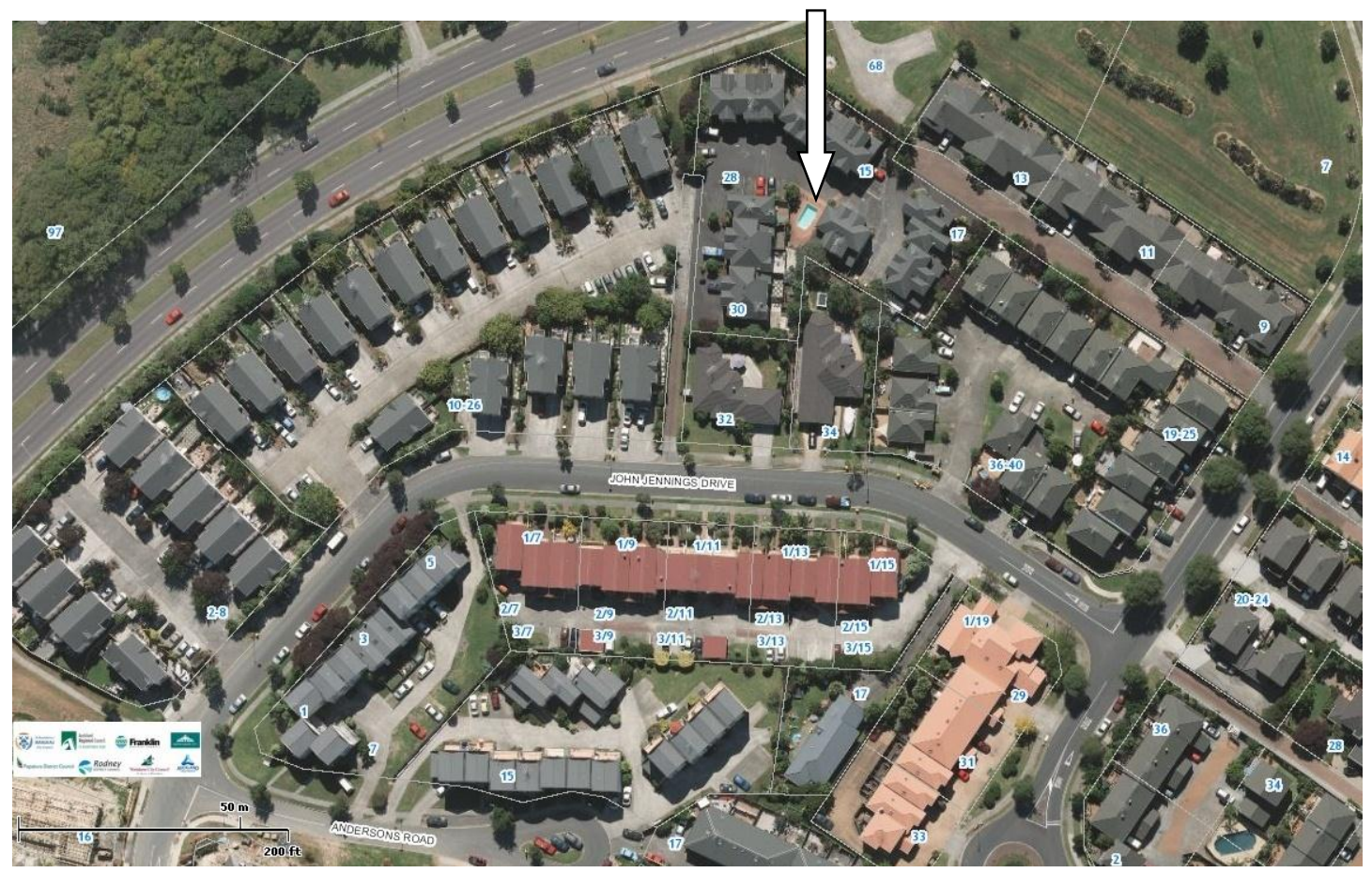

Fig.A7.3.71 Housing along John Jennings Drive, Oteha, North Shore linked by access paths, but the swimming pool (top centre) is locked and the manager unknown. (Alggi Map)

Although not a public swimming pool, this pool would be a great asset for the many children of the neighbourhood. Some residents adjacent to the pool commented to the writer that they do not know who has the key or who manages the pool.

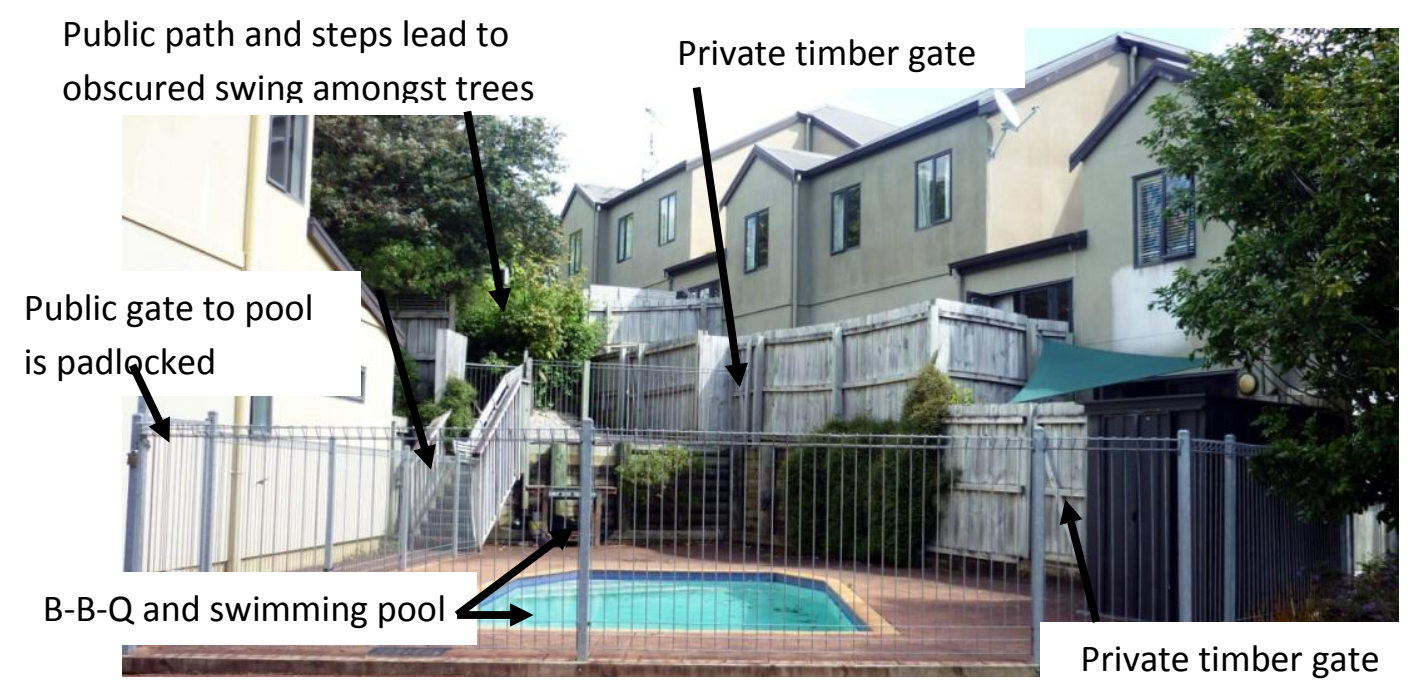

Fig.A7.3.72 - Communal swimming pool - padlocked. (Photo: S.J. Pattinson) 
In this housing at Beach Haven, North Shore, Auckland, children are not allowed to play in the central vehicle area, the pool is out of sight and out of bounds, and parents are expected to take their toddlers to the adjacent park - not a direct route because of a deep bush-filled gully in between. So casual play is confined to indoors.
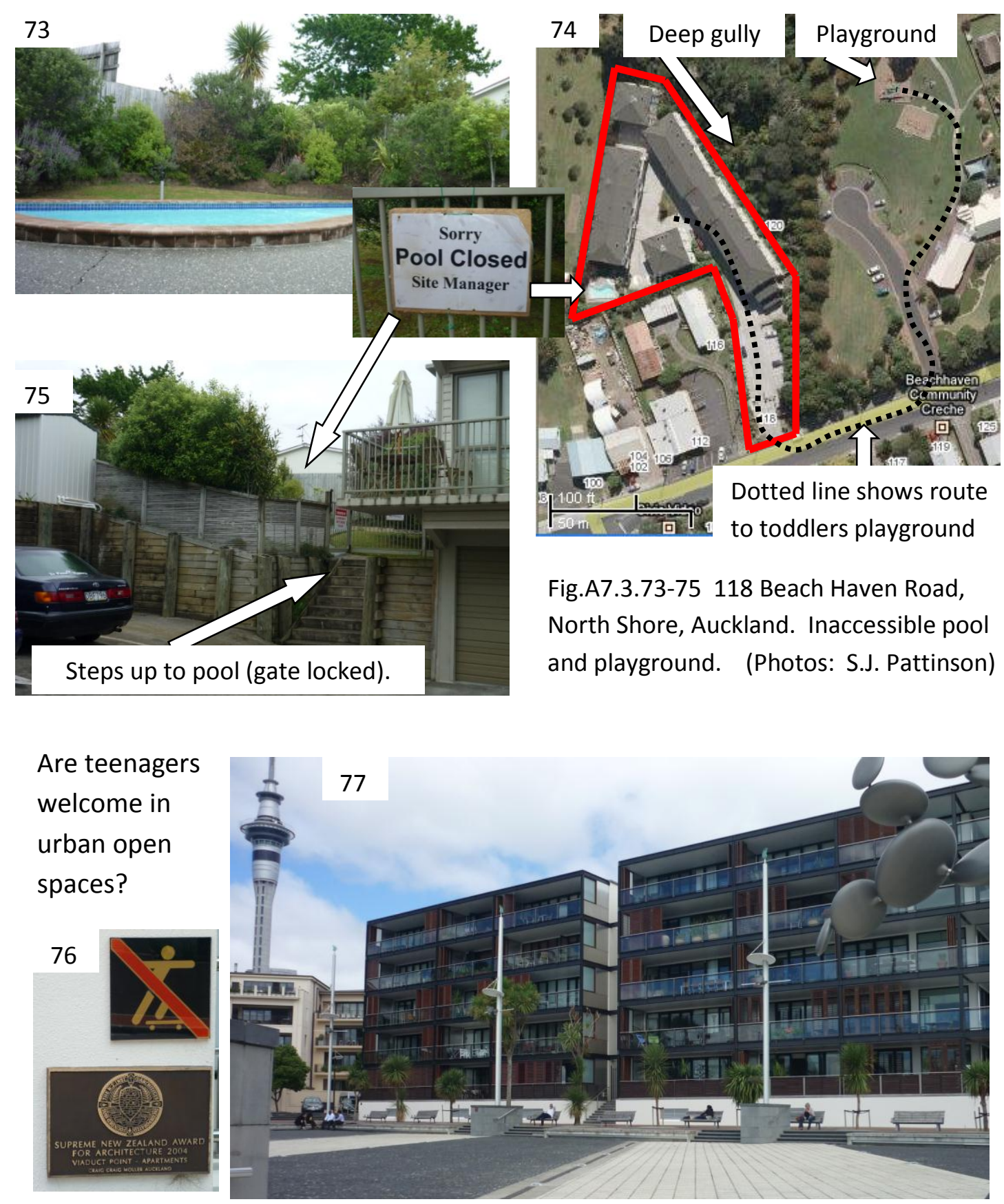

Fig.A7.3.76, 77 Sign at Entry: The Point Apartments Viaduct Harbour, Auckland Central. Can teenagers and good architecture / urban design mix?

(Photos: S.J. Pattinson) 


\section{i) Playgrounds can be unimaginative:}

Children in this medium-density neighbourhood get a standard playground while a nearby treasure trove is quarantined and turned into a roadside relic. These historic brick kilns in Ambrico Place, New Lynn, could be utilised more imaginatively.

These unique kilns have been made inactive roadside relics. Instead of separating the kilns from the playground by putting the road between them, the road could be re-routed, and the housing, playground and kilns integrated so that the fascinating kilns become centre-pieces of daily outdoor activity.

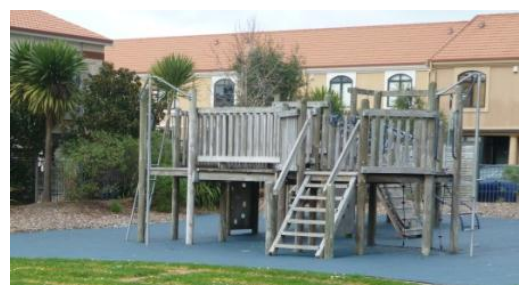

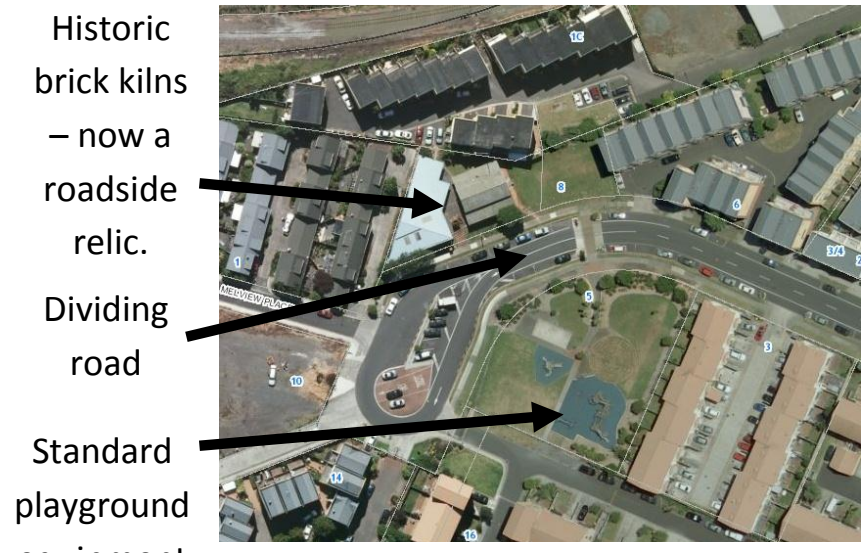

Fig.A7.3.78 Ambrico Place, New Lynn (Alggi map)

Fig.A7.3.79 Standard playground equipment at Ambrico Place (Photo: S.J.Pattinson)

Fig.A7.3.80-83 (below) Historic brick kilns, Ambrico Place, New Lynn - a missed opportunity to make the historic kilns a social and recreational community asset. (Photos: S.J.Pattinson)

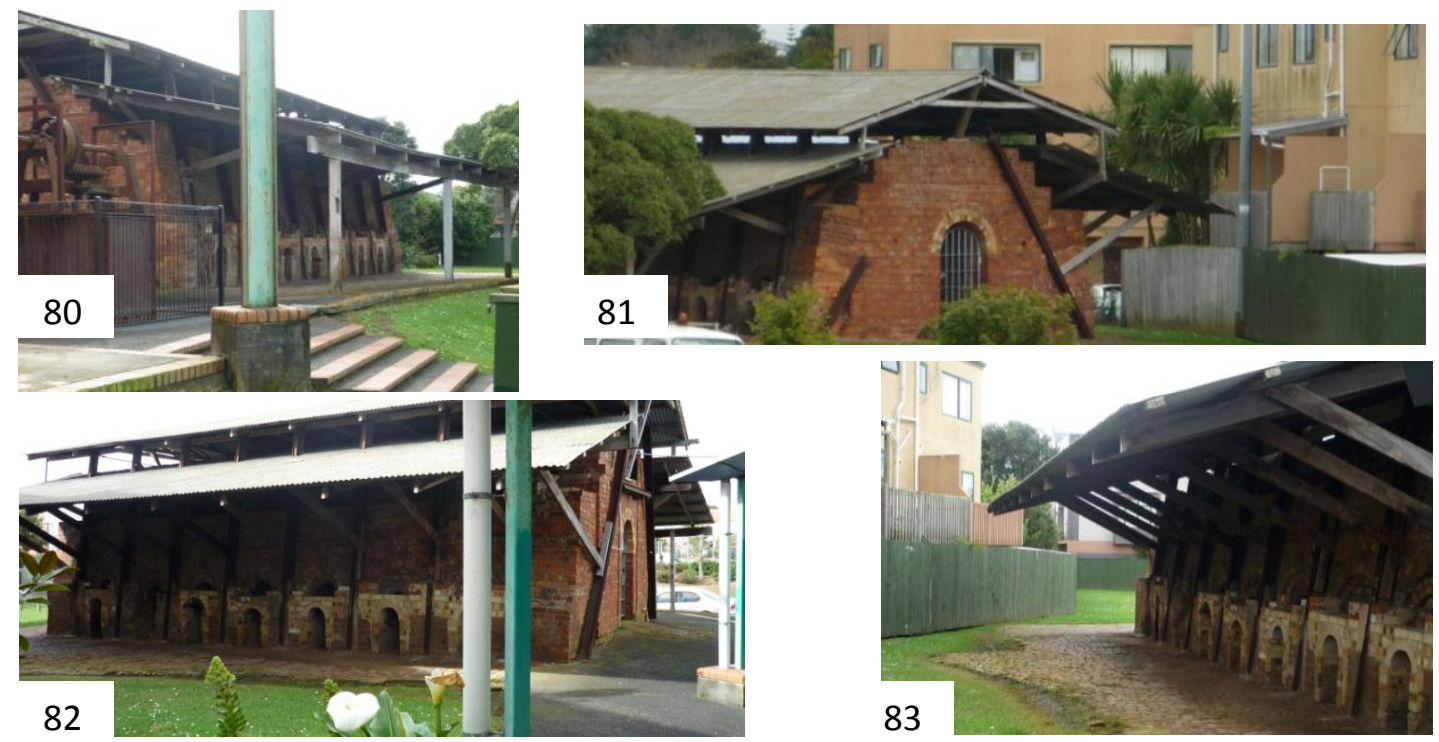




\section{j) Communal space can be ambiguous:}

What is the purpose of this green space at K-Block Apartments, Sumner Beach?

Is it for natural daylight and aesthetic outlook; recreational or social use? How suitable is it for these purposes?

Fig.A7.3.84 'K-Block' Apartments, The Esplanade, Sumner Beach, Christchurch.

(Photo: S.J. Pattinson)
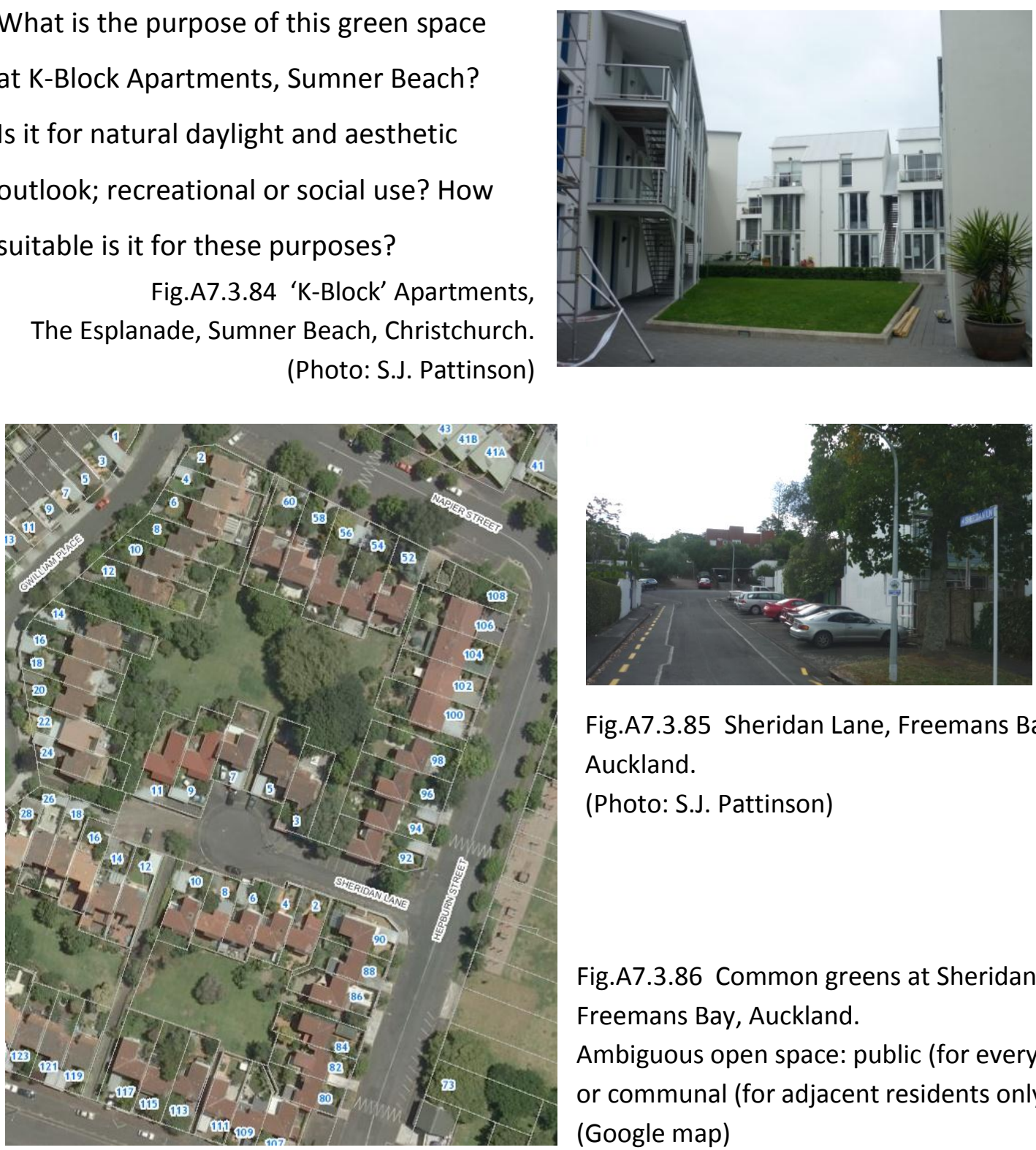

Fig.A7.3.85 Sheridan Lane, Freemans Bay, Auckland.

(Photo: S.J. Pattinson)

Fig.A7.3.86 Common greens at Sheridan Lane, Freemans Bay, Auckland.

Ambiguous open space: public (for everyone) or communal (for adjacent residents only)? (Google map)

Ambiguous open space: The common greens at the rear of these terrace houses are Councilowned and maintained. There are few children living in this area, so the greens are seldom used. A resident at Sheridan Lane commented that she never uses the common green at the back of her place; instead she interacts with neighbours in the Sheridan Lane cul-de-sac, where people come and go daily by car.

This layout (common land behind terrace housing around the perimeter of a block) is popular with designers. But this resident's comments caution that such 'commons' may lose out to 'the street' for people, activity and social interaction. Designers may inadvertently be creating unused rear 'commons' if the real action is at the front of the houses on the street. 


\section{k) Communal space may just be 'landscaped outlook' rather than social space:}

Communal spaces often seem to be landscaped outlook rather than for social space. The example below focuses on this issue in more detail. Assuming neighbourliness is desireable, the high-quality apartments at 41 Cambridge Terrace in Christchurch might benefit from reconsidering the landscaping of the internal courtyard shown below. The dimensions of the courtyard are small and intimate. The planter boxes in the middle push the circulation space hard against the doorways of the ground floor apartments. The seating faces directly into private interiors. People prefer to occupy the edges. If seating and planting were at the perimeter of the courtyard, leaving the middle free for leisurely circulation, this might facilitate more comfortable casual interaction. People would be more likely to use the seating, and maybe offer a greeting, if they could sit with their back to a wall rather than sit in the middle staring directly into someone's living space. Planting at the edges could soften the courtyard and create small, semi-private transitional spaces at the doorways. It would then be a more comfortable distance, from doorways and edgeseating, for social interaction with people strolling through the courtyard.

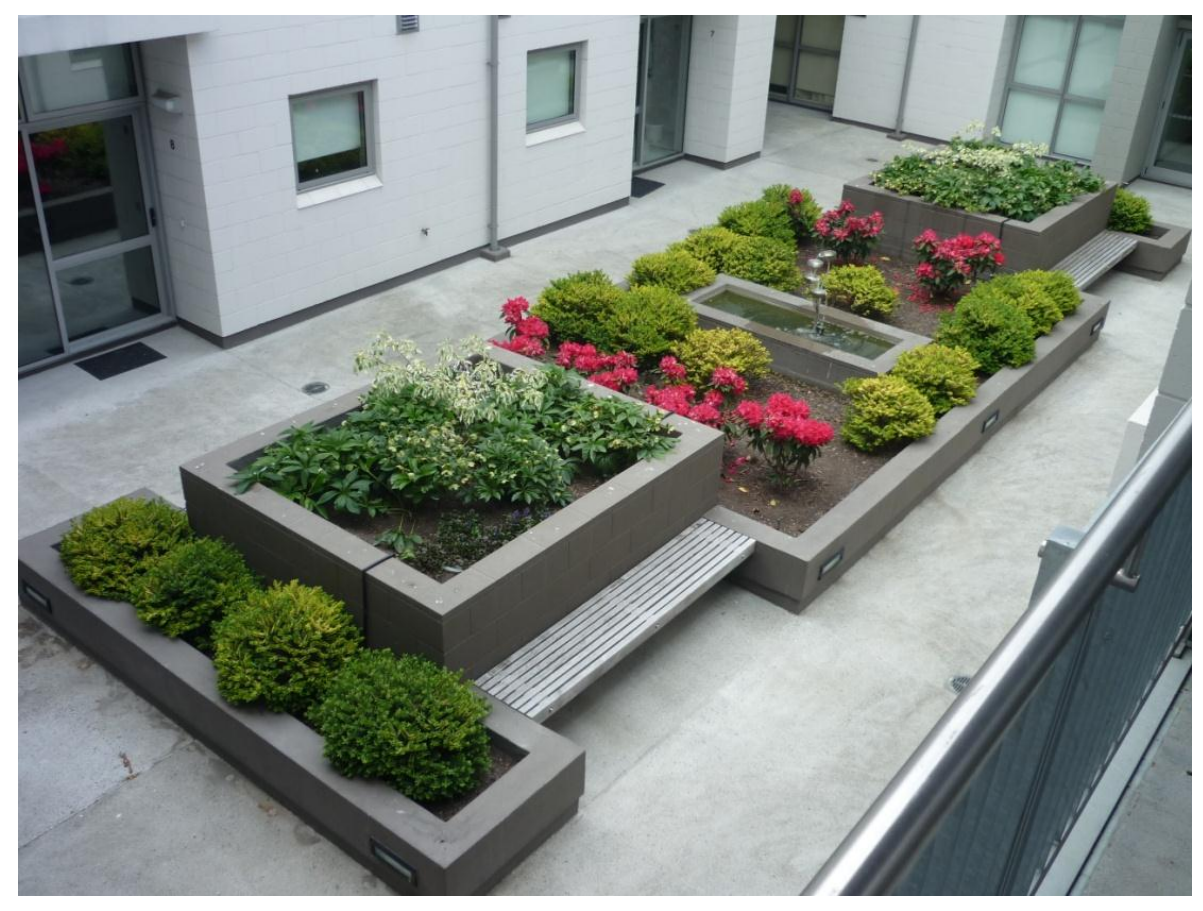

Fig.A7.3.87 Internal courtvard 41 Cambridge Terrace, Christchurch. (Photo: S.J.Pattinson) 


\section{I) Communal facilities can be inadequate:}

In the following example, an inner-city multi-unit housing development in Auckland, all 42 units share a communal laundry. The one-bedroom units (32m2) do not have their own laundries. At least one of the units houses a young family with toddlers. The washing machines in the communal laundry are out of action due to vandalism (i.e. someone was using incorrect coinage to operate the washing machines). A notice on the locked laundry room door states that the laundry facilities will be unavailable for two months while the manager awaits the arrival of spare parts and the body corporate arranges installation of security cameras. The two months has passed and still the laundry facilities are locked. Meanwhile, residents carry their washing downstairs to a commercial laundrimat down the street. A frustrated resident has posted a notice on the board requesting functional laundry facilities.

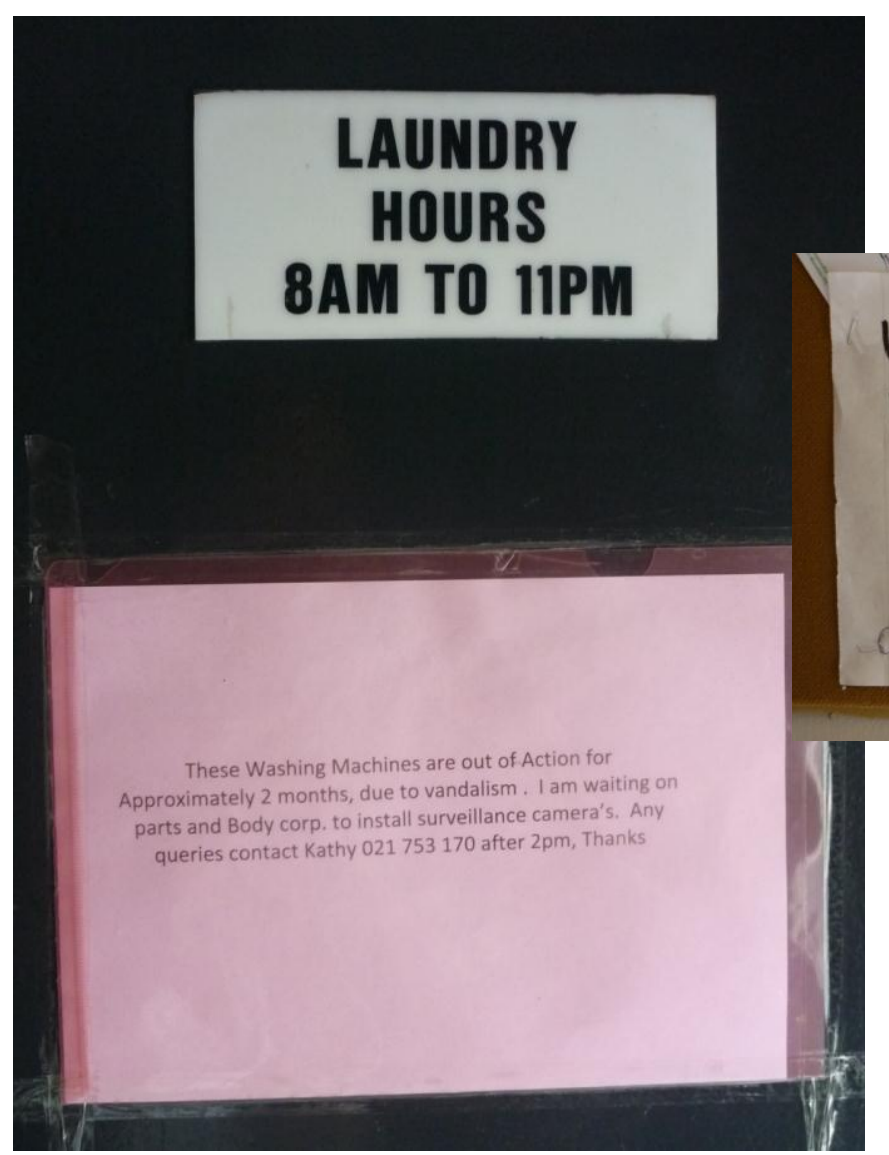

When will the Laudry function Fig.A7.3.88 Notice (left) on laundry door, posted by management, followed by resident notice (above). (Photo: S.J. Pattinson) 
The Christchurch Council's Brougham Village (below) dates from the 1970's. The standard of Council housing has improved since then (see Figure ?? - Gowerton Place). Here at Brougham Village, the units appear well designed, however the site planning lacks thought for communal facilities and single parents with children.

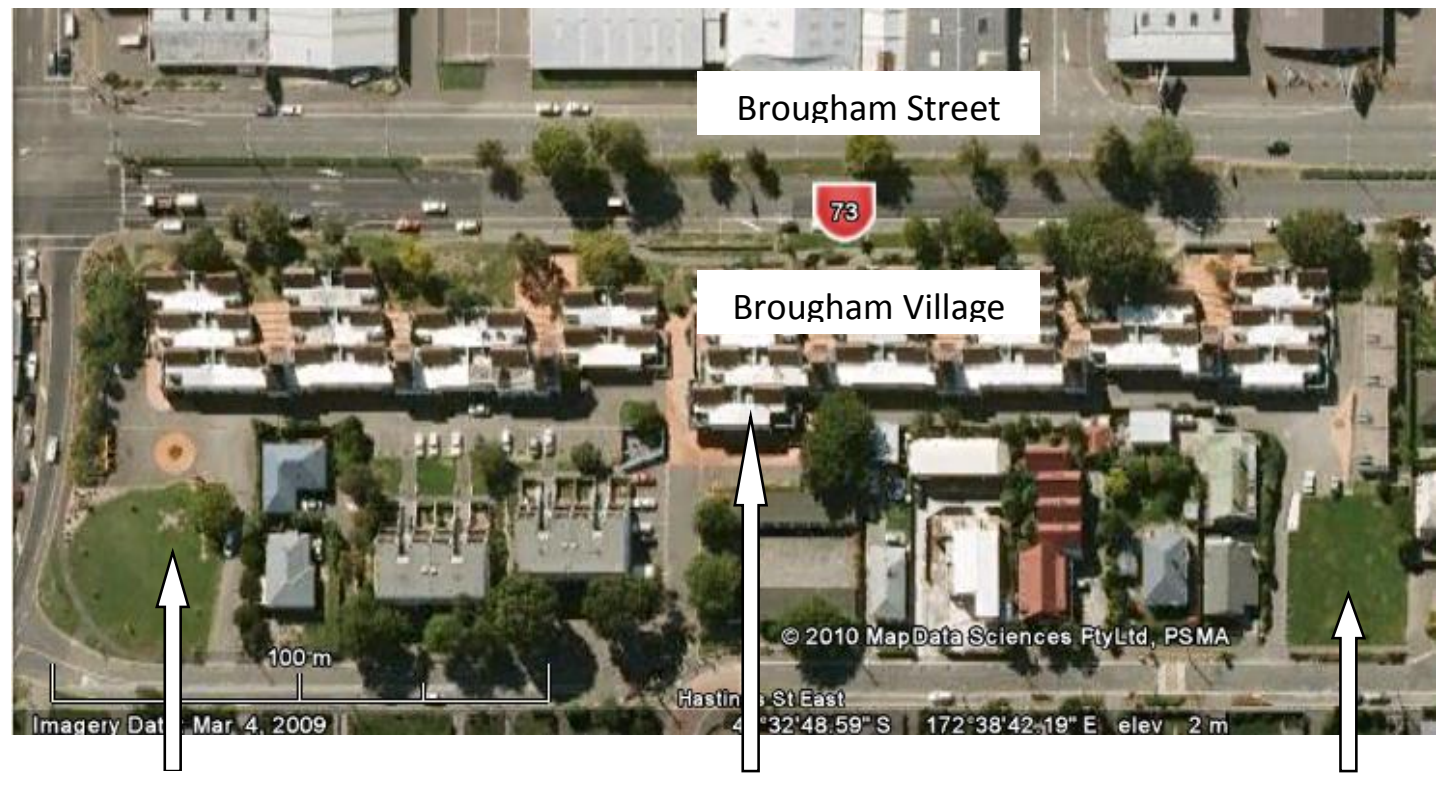

swings and seesaw

common recreation room on

small grass field

sunless side of building

Fig.A7.3.89 Brougham Village, Christchurch. (Google Maps)

The unfenced north boundary of the site means that young children are not protected from the very heavy traffic along Brougham Street, which is State Highway 73 (see Fig.A7.3.48). On-site playgrounds are separated from the housing by through-traffic. The only safe place for toddlers to play is a sunless common room on the south side of the building, or their private courtyard which is a tiny space about $4 \mathrm{~m} \times 2 \mathrm{~m}$ accessible only through a window from their Living Room (Fig.A7.3.90 \& 91)

Fig.A7.3.90, 91 Private walled courtyard - total area about $4 \mathrm{~m} \times 2 \mathrm{~m}$ accessible only via a $400 \mathrm{~mm}$ step up from the Living Room through a window. (Photo: S.I. Pattinson)
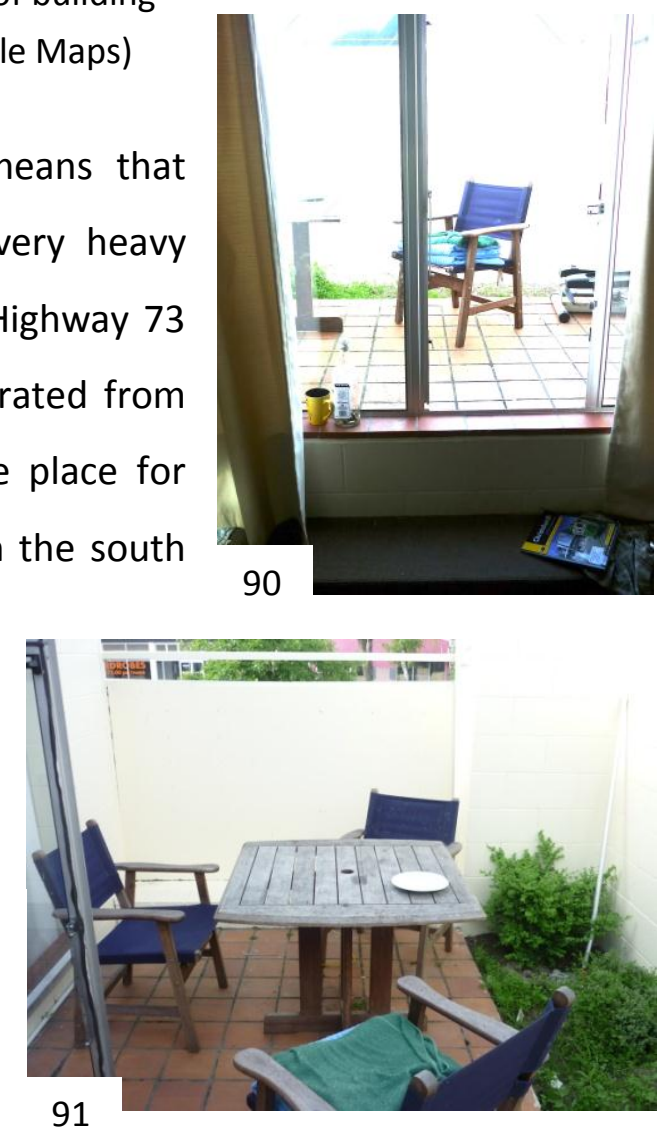
A communal recreation room is located in the middle of the village, on the south side of the complex. This basement space, with concrete floors and concrete block walls, receives little sun and is therefore cold. It has no direct link to any outdoor recreational space. The open green spaces at each end of the village are remote (120m in either direction) from the recreation room. The children's playground at the West end of the village consists of just two swings and a seesaw and is surrounded by busy roads and a vehicle manoeuvring area.

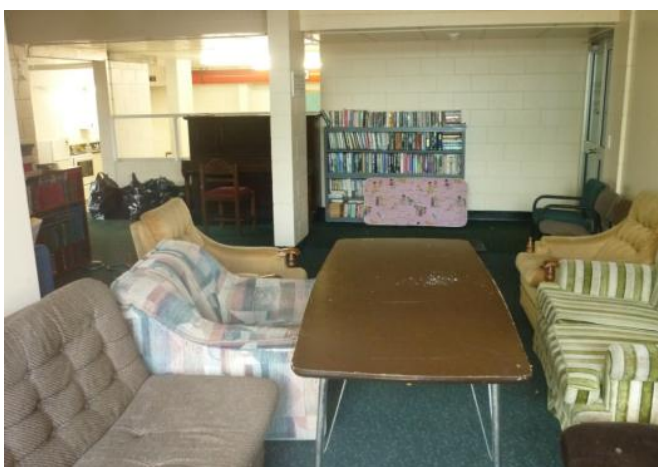

Fig.A7.3.92 Lounge space in communal recreation room (on south side of building where it gets no direct sun). (Photo: S.J. Pattinson)
The internal planning of the recreation room is poor. It is split level and broken up by walls and columns into four corners - a kitchenette corner and billiard table corner are in the lower half (behind the piano and bookcase in the photo opposite), a lounge corner (pictured), and a unisex 'accessible toilet' in the fourth corner (behind the couches on the left in the photo).

The unisex toilet has two doors for through access - it is the only link between the recreation room and the adjacent communal laundry. Not surprisingly, there are handwritten signs with arrows sellotaped to the walls indicating the direction to alternative toilets elsewhere in the complex.

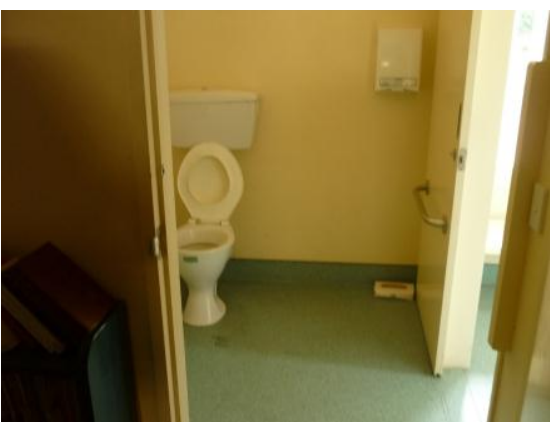

Fig.A7.3.93 Unisex toilet (viewed from lounge). (Photo: S.J. Pattinson)

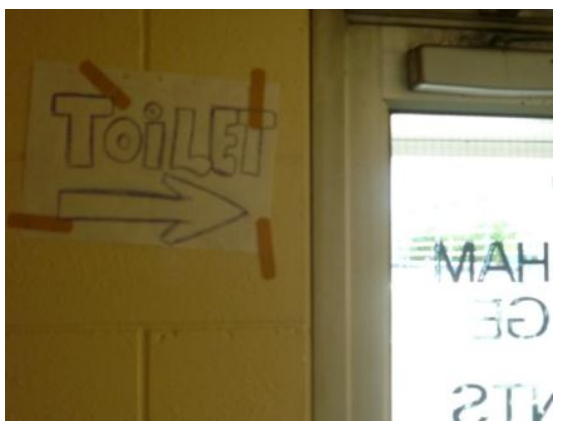

Fig.A7.3.94 Signs to alternative toilets (Photo: S.J. Pattinson) 


\section{m) The needs of children and teenagers are often overlooked:}

Examples above have already referred to inadequate consideration for children in the planning of medium-density housing. Further examples are not difficult to find. Because these developments are often located near dangerous roads, railway lines, waterways and industrial areas, and because little thought is given to child safety on-site, children in these housing environments appear to stay mostly indoors.

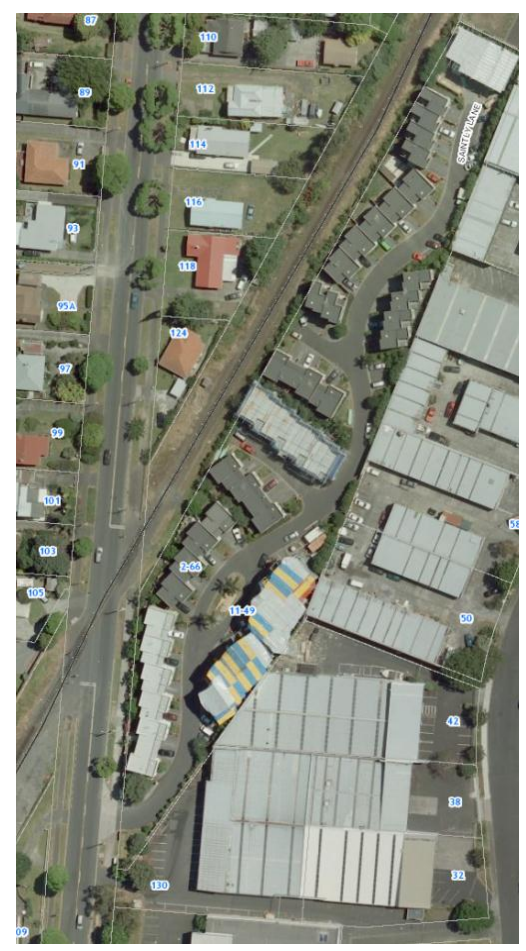

Fig.A7.3.95 Saintly Lane, Avondale, squeezed between rail and industrv. (Alggi map)

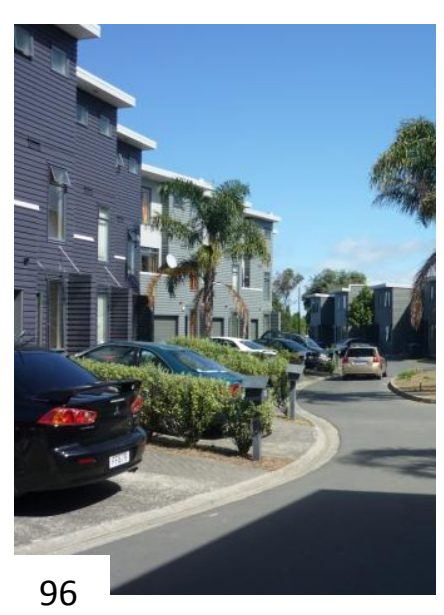

Fig.A7.3.96- 99 Saintly Lane. No footpaths and no play areas. Where can children safely play? (Photos: S.J.Pattinson)

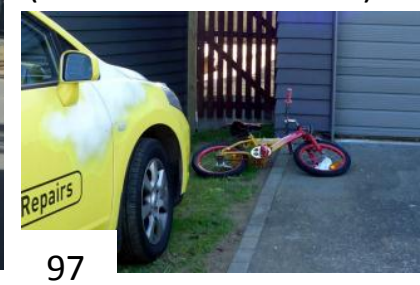

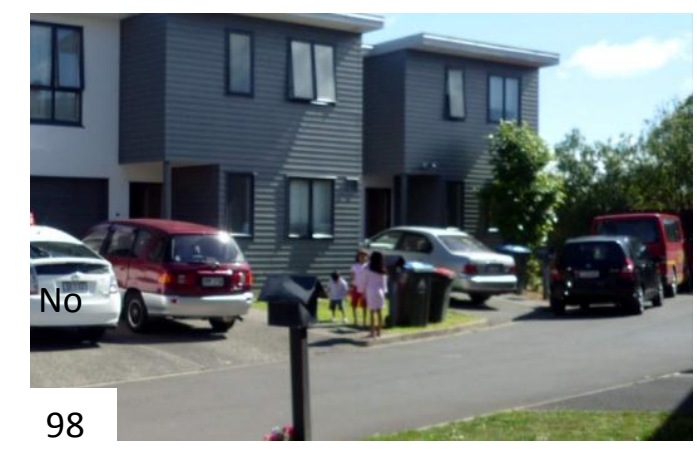

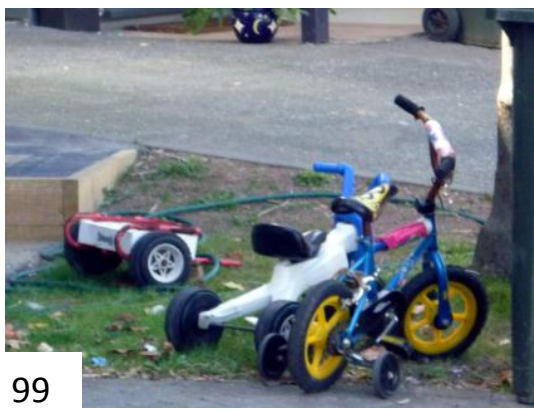


The recent emergence of 'shared streets' is a type of communal space. 'Shared streets' allow cars, slowed to walking pace, and pedestrians, including children, to safely share the use of roads and home zones as places for play and socializing. Standard streets, however, like the streets (below) in a medium-density suburb in Oteha, North Shore, Auckland, are not designed for 'shared use'.

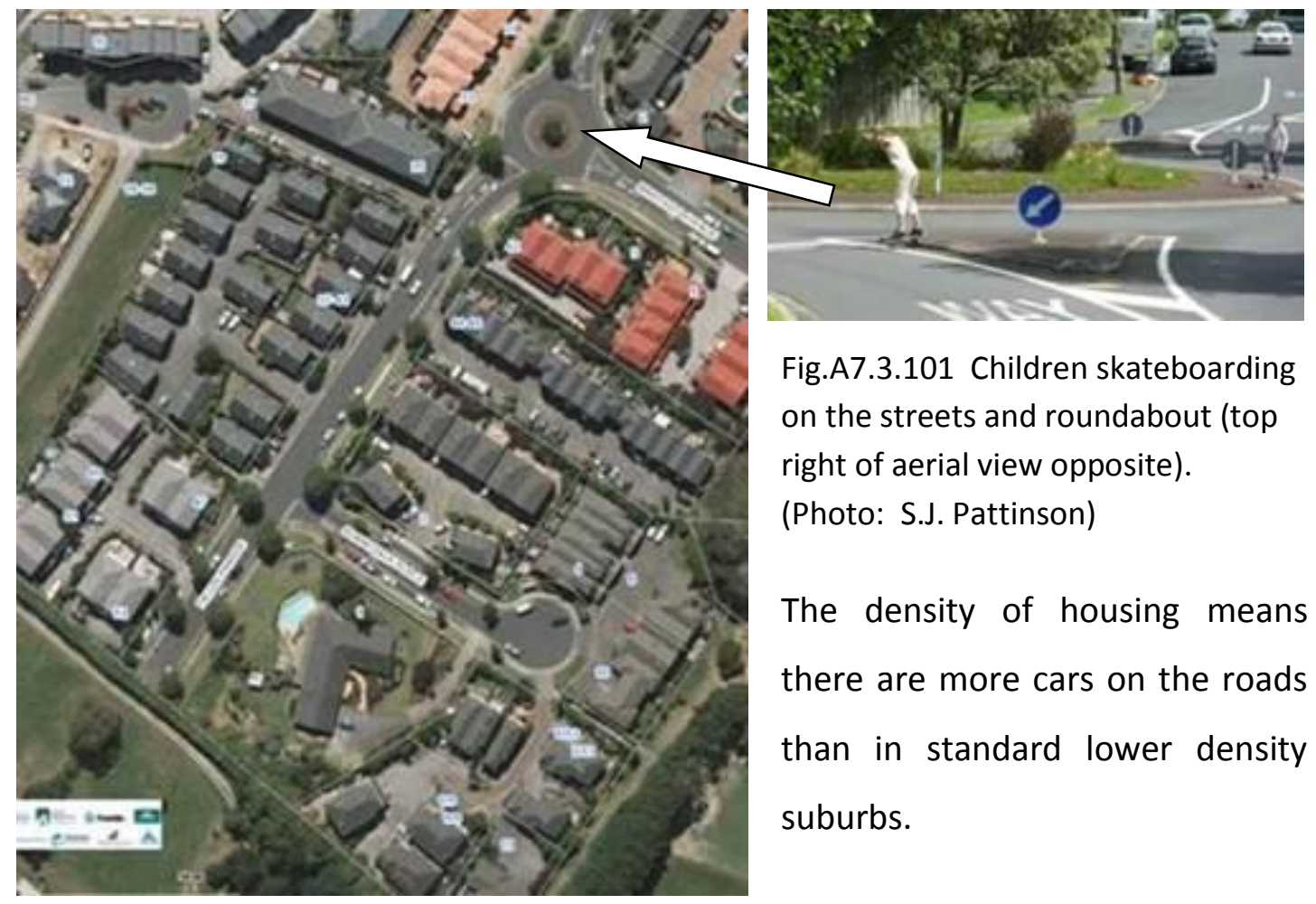

Fig.A7.3.100 Oteha, North Shore, Auckland. (Alggi map)

With tiny private yards and no play space other than roadway, it is not surprising that children play on the roads. It is not unusual for children to play on roads, however the volume of traffic generated by the number of houses in neighbourhoods of this density is significantly higher than on standard suburban streets. There are school grounds and sports fields nearby, which are good places for teenagers to hang out (but not for skateboarding). Younger children require supervision. Until they reach a suitable age to play away from home, where can they play at home other than in parking areas, on driveways, footpaths and roads? 


\section{n) The needs of the elderly may be overlooked:}

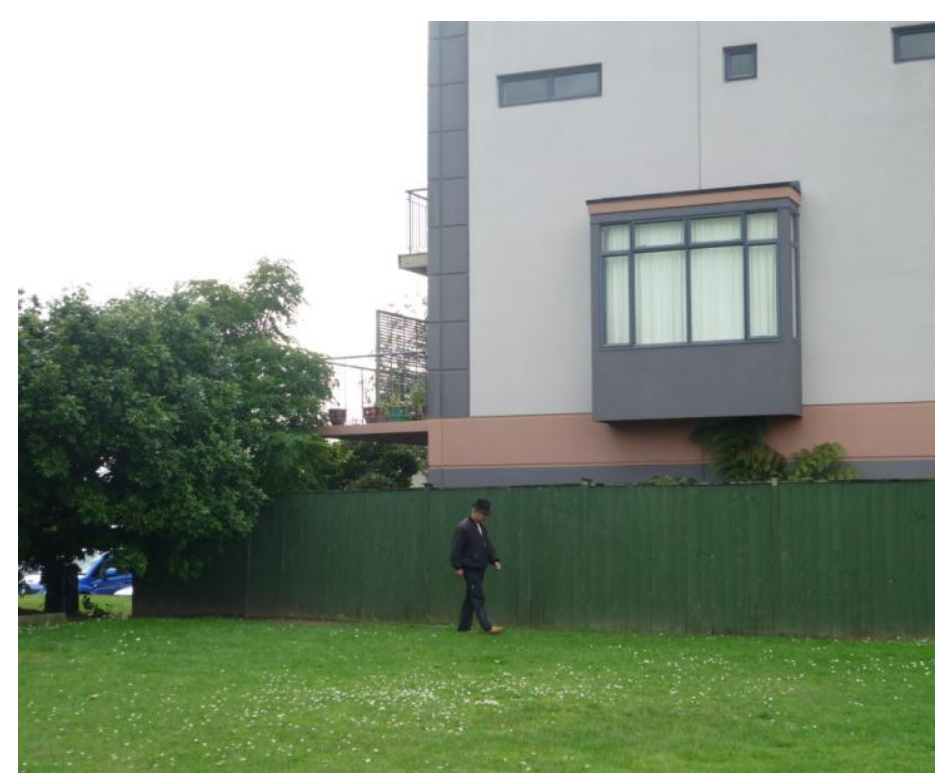

Fig.A7.3.102 An elderly man strolls the fringe of a housing development in Waitakere, Auckland. (Photo: S.J. Pattinson)

Unlike retirement villages, medium density housing developments might not give much attenetion to the needs of the elderly, even though they might house elderly people.

Some field visits were made to retirement villages as part of preliminary investigations of communal space, because retirement villages generally provide a wide variety of high quality communal spaces, both indoors and outdoors. The following photographs (taken at a well-appointed retirement village on Auckland's North Shore) show the large range of high quality communal facilities available to the residents (although on the day of the writer's visit, the only space where residents were observed together was in the card room):

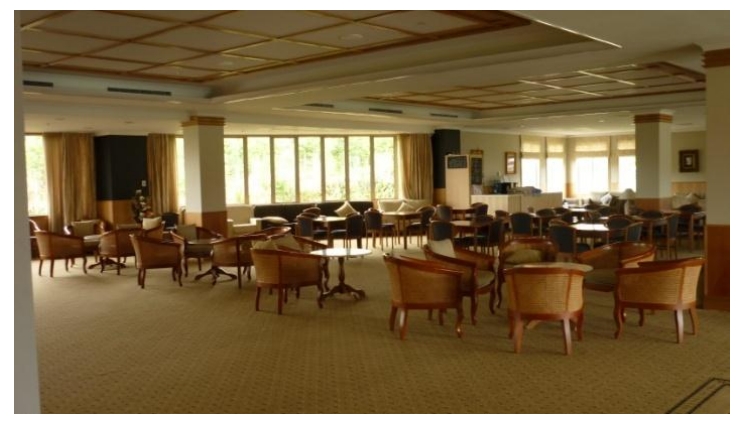

Fig.A7.3.103 Communal dining ... (Photo: S.J. Pattinson)

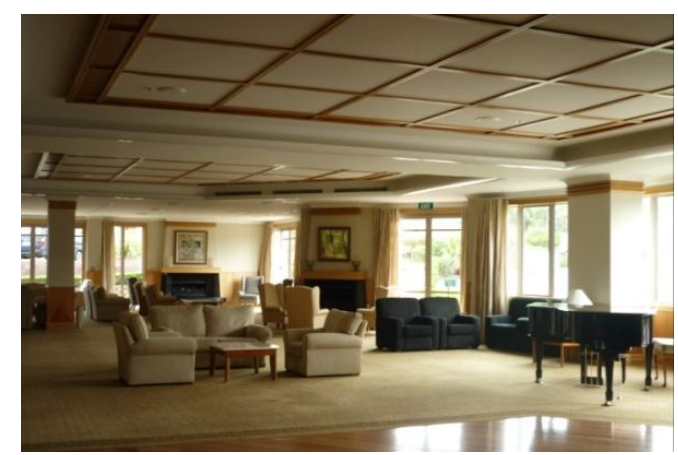

Fig.A7.3.104 ... lounge

(Photo: S.J. Pattinson) 


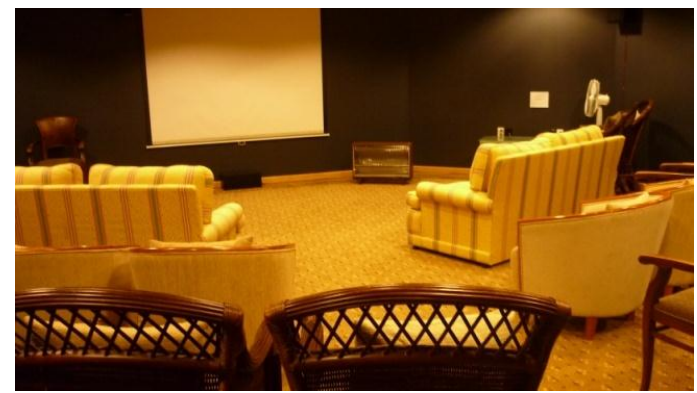

Fig.A7.3.105 ... movie room

(Photo: S.J. Pattinson)

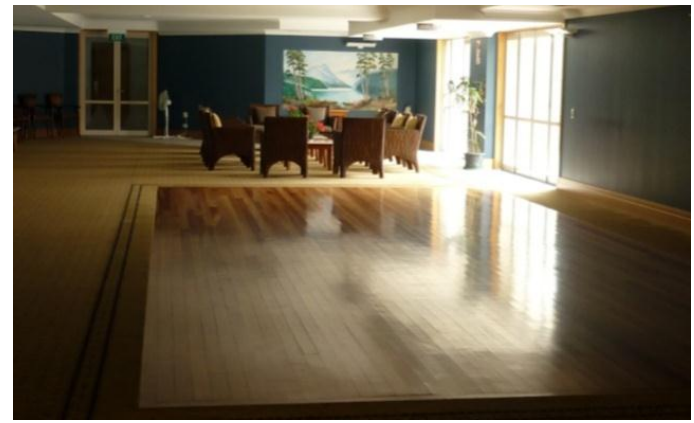

Fig.A7.3.107 ... music and dance floor (Photo: S.J. Pattinson)

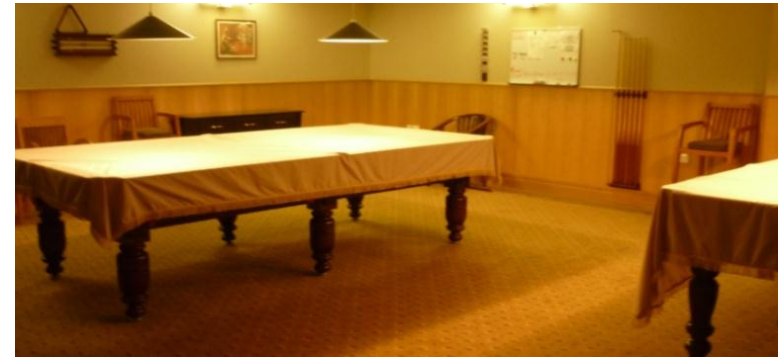

Fig.A7.3.109 ... billiard room (Photo: S.J. Pattinson)

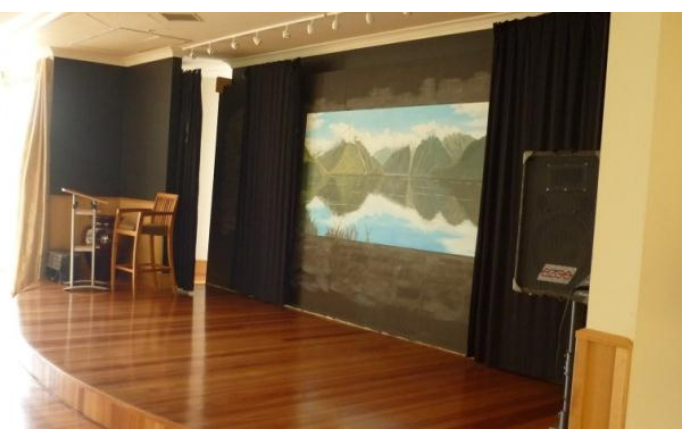

Fig.A7.3.106 ... drama / theatre / music stage (Photo: S.J. Pattinson)

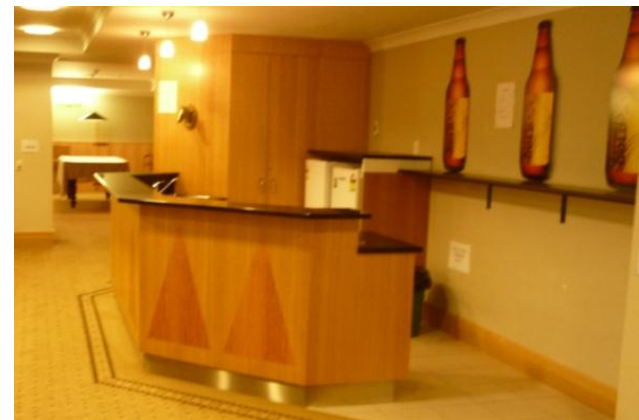

Fig.A7.3.108 .... bar room (Photo: S.J. Pattinson)

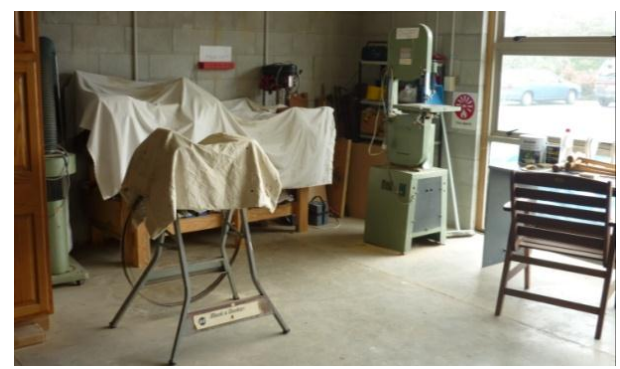

Fig.A7.3.110 ... workshop (Photo: S.J. Pattinson)

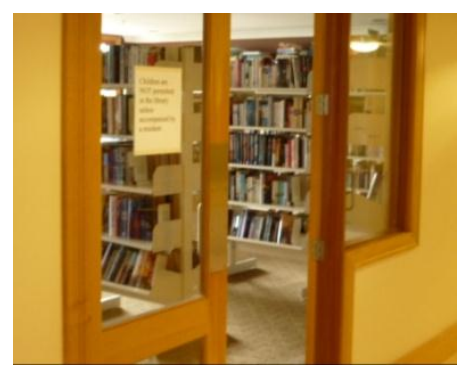

Fig.A7.3.111 ... library (Photo: S.J. Pattinson)

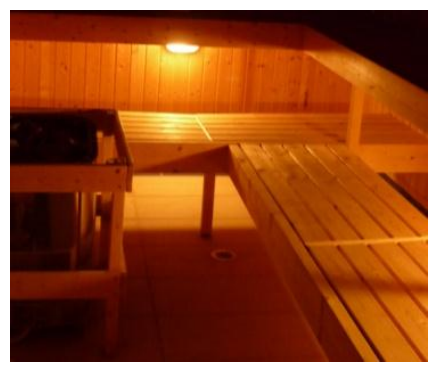

Fig.A7.3.112 ... sauna (Photo: S.J. Pattinson)

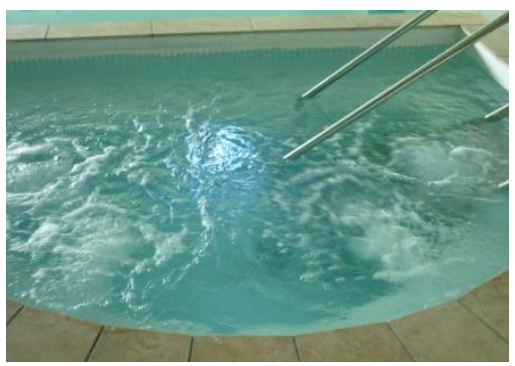

Fig.A7.3.113 ... spa pool (Photo: S.J. Pattinson) 


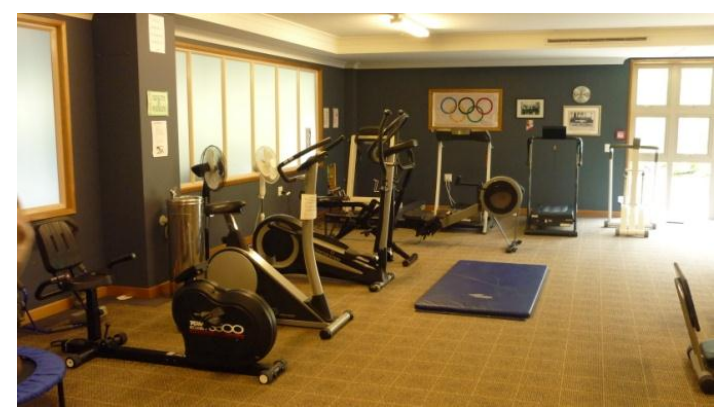

Fig.A7.3.114 ... gym

(Photo: S.J. Pattinson)

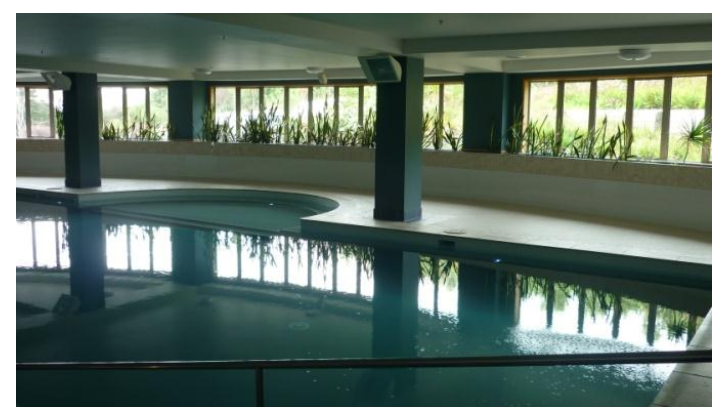

Fig.A7.3.115 ... indoor swimming pool

(Photo: S.J. Pattinson)

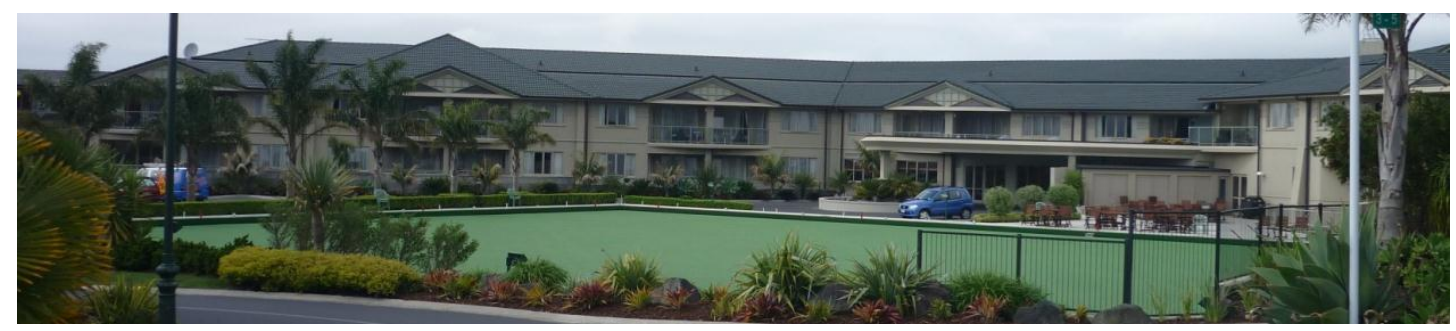

Fig.A7.3.116 ... bowling green

(Photo: S.J. Pattinson)

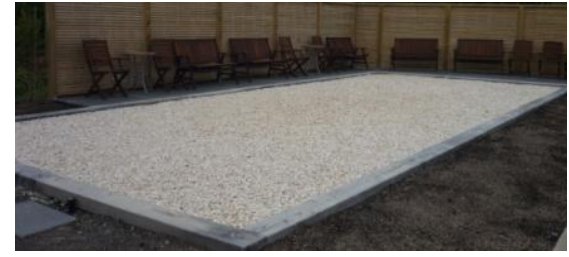

Fig.A7.3.117 ... petanque (Photo: S.J. Pattinson)

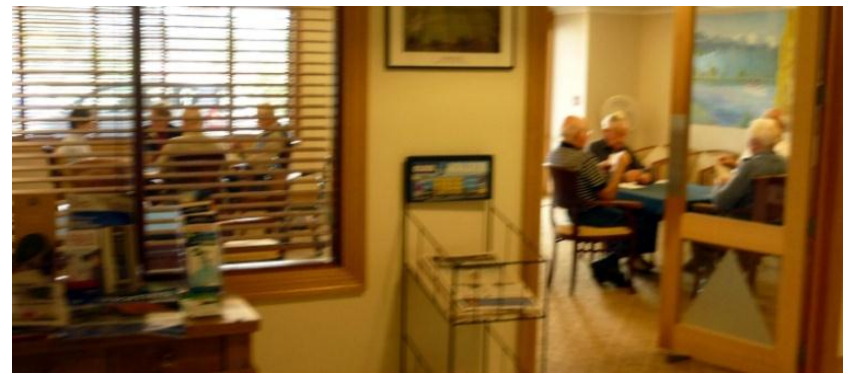

Fig.A7.3.118 ... and card room (Photo: S.J. Pattinson)

The above are all great examples of quality communal spaces, and the writer wonders why a wide range of facilities such as this is not more common in standard medium-density housing developments. After all, it is not just the elderly who can enjoy and benefit from the provision of such facilities, but all age-groups.

And are retirement villages depriving cities, including children, of regular connection with the elderly, by segregating them into an age-based fragment of society? 


\section{o) Neighbourhoods are fragmented:}

One of the issues this research explores is how multivalent communal spaces might help defragment society. A typical example of fragmentation can be seen in the example below in Christchurch. Here a retirement village is located on one side of a street and directly opposite it on the other side of the street, behind a concrete block wall, is an early childhood learning centre. The street is a busy one-way, twolane arterial road. Children and the elderly can benefit from some social contact, yet the urban design and architectural devices employed here of a busy road and a high concrete block wall provide a very effective means of separating children and the elderly. A more thoughtful approach, using multivalent communal space, would provide more opportunities for interaction between children and the elderly.

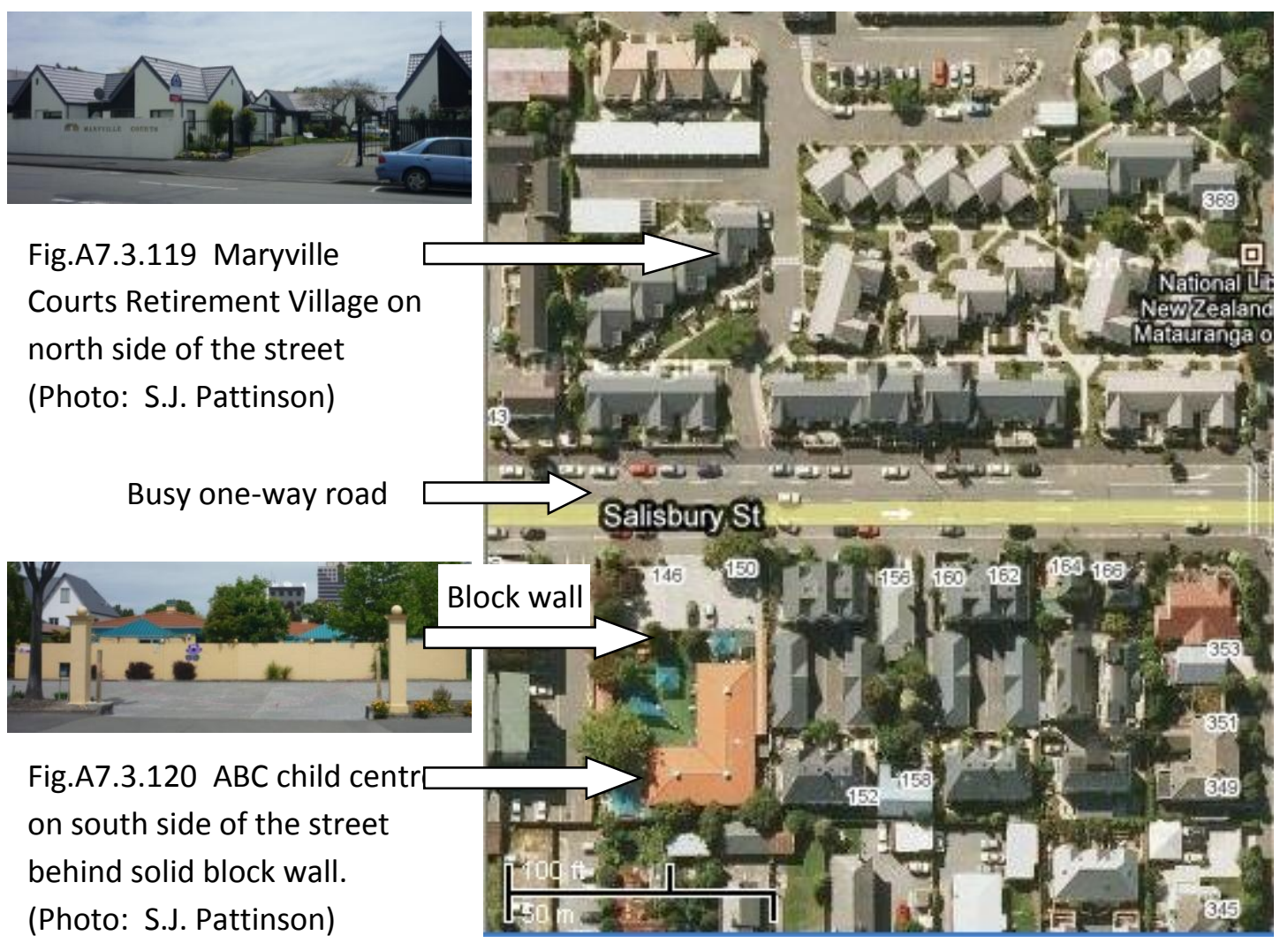

Fig.A7.3.121 Maryville Courts Retirement Village (north side of busy one-way street) and $A B C$ Child Developmental Learning Centre (south side of street).

(Google Map) 


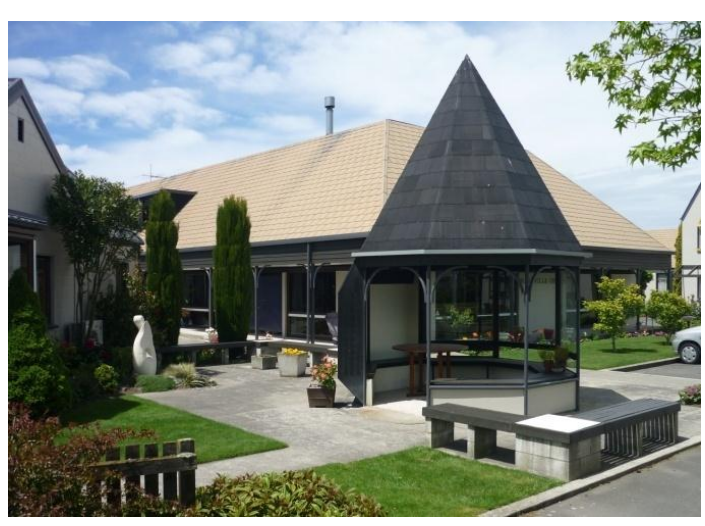

Fig.A7.3.122 A communal outdoor space in the retirement village. (Photo: S.J. Pattinson)

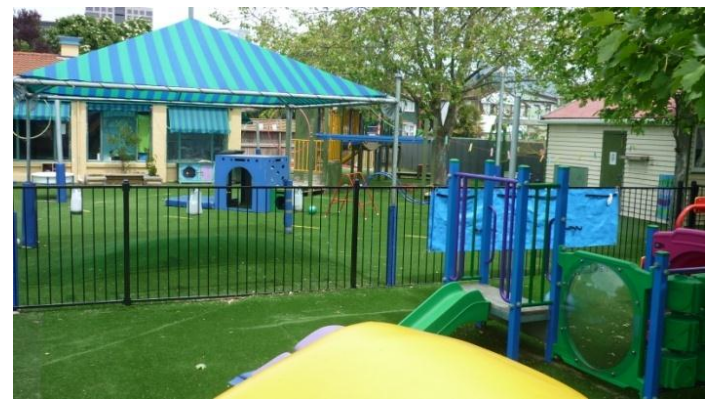

Fig.A7.3.123 A communal outdoor space in the child learning centre.

(Photo: S.J. Pattinson)

The design of cities fragments society and denies benefits of daily interaction between, say, young children and the elderly. Multivalence means exploring possibilities for intersecting or overlapping the communal outdoor requirements of, say, children and the elderly to provide opportunities for casual social interaction. In the context of medium density housing, communal spaces could be made accessible to both young and old to facilitate intergeneration social contact.

During 200 field visits to medium-density housing in Auckland, Wellington, Christchurch and Melbourne, the writer observed only one instance of children playing outdoors with adults, and that was here at Cecil Courts in Christchurch.

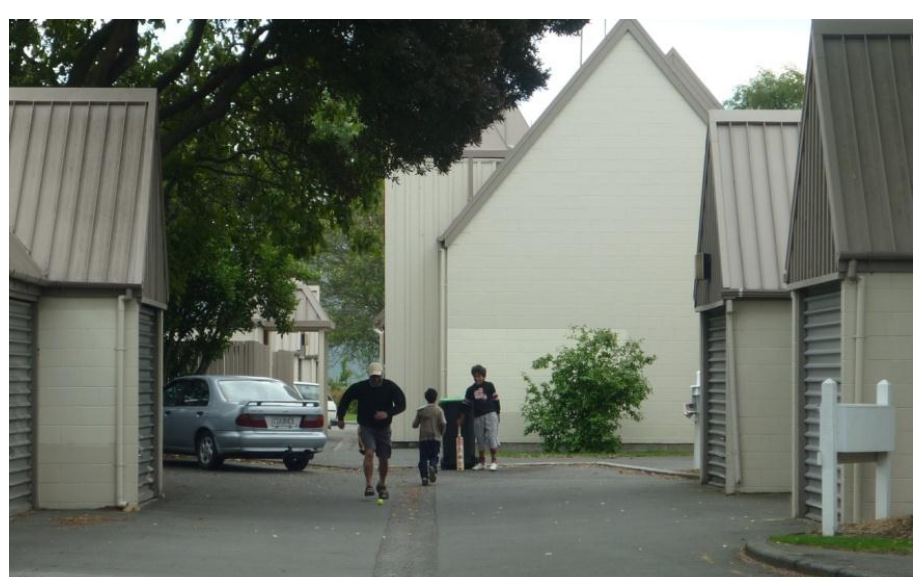

What does it take to create outdoor spaces that foster intergenerational activity?

Fig.A7.3.124 Cecil Courts, Cecil Place, Christchurch. Inter-generational cricket. (Photo: S.J. Pattinson) 


\section{p) Fragmentation of neighbourhoods may be a reflection of 'silo' thinking:}

How has the urban environment become so fragmented?

There are many people required for the creation of the built environment owners, developers, architects, landscape and urban designers, draughstpersons, town planners, land surveyors, quantity surveyors, consulting engineers of several specialties (geotechnical, traffic, fire safety, energy, acoustics, structural, electrical, hydraulics or plumbing and drainage, heating and ventilation, information and communications technology), building scientists, project managers, contractors, territorial authority approvals officers and inspectors of various specialties, landscapers, interior designers, accountants, councillors, politicians, government officials, economists, bankers, lawyers, insurers, marketing people, advertisers, real estate agents, property and facilities managers, building users and the public ....

Collectively, the efforts of all shape the built environment. Reference was made in Chapter 2 to Graham Mclndoe's comments about the negative impact on the urban environment of 'silo mentality'. Ideally, the challenge of multivalent communal space will provide a rallying call for researchers, practitioners and the community to work together to create better quality places for people to live in cities.

\section{q) Holistic, integrated, multivalent thinking is needed:}

The characteristics of univalent thinking are typified by the housing development at Alverna Heights, Whangaparaora (below) - isolated housing unconnected to shops and community facilities (except by car); repetitive house plans; ring road disconnecting houses from central open space; uncared for, undeveloped, unneeded central open space because of the emphasis on individualistic lifestyles; and no common play or recreational area (except for the barren, sloping central space). 


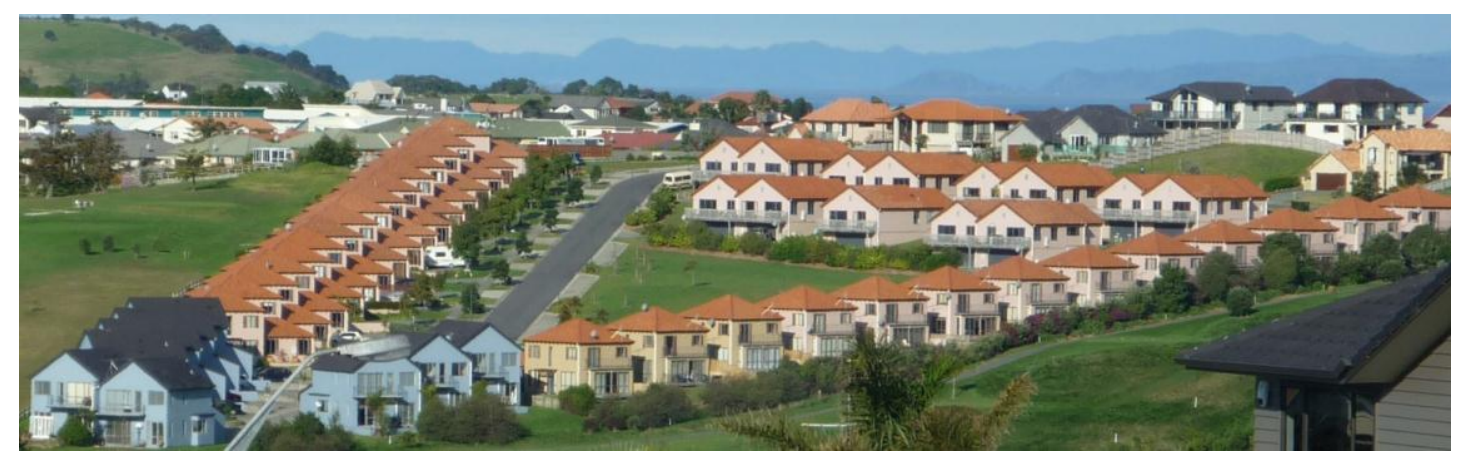

Fig.A7.3.125 Univalent development: isolated, repetitive housing with undeveloped communal space. Alverna Heights, Whangaparaoa, Auckland. (Photo: S. J. Pattinson)

Inspiration for multivalence can come from other building types - community buildings, papakainga, schools, motels and retail/office/apartment mixes.

Ropata Village, Lower Hutt, is an example of a mixed-use development. It links doctors, dentists, specialists, pharmacy and elderly care in the one development. No two of the 33 apartments is the same; the floor plans are all individual. Yet communal space abounds, indoors and outdoors a very efficient use of a small parcel of urban land.
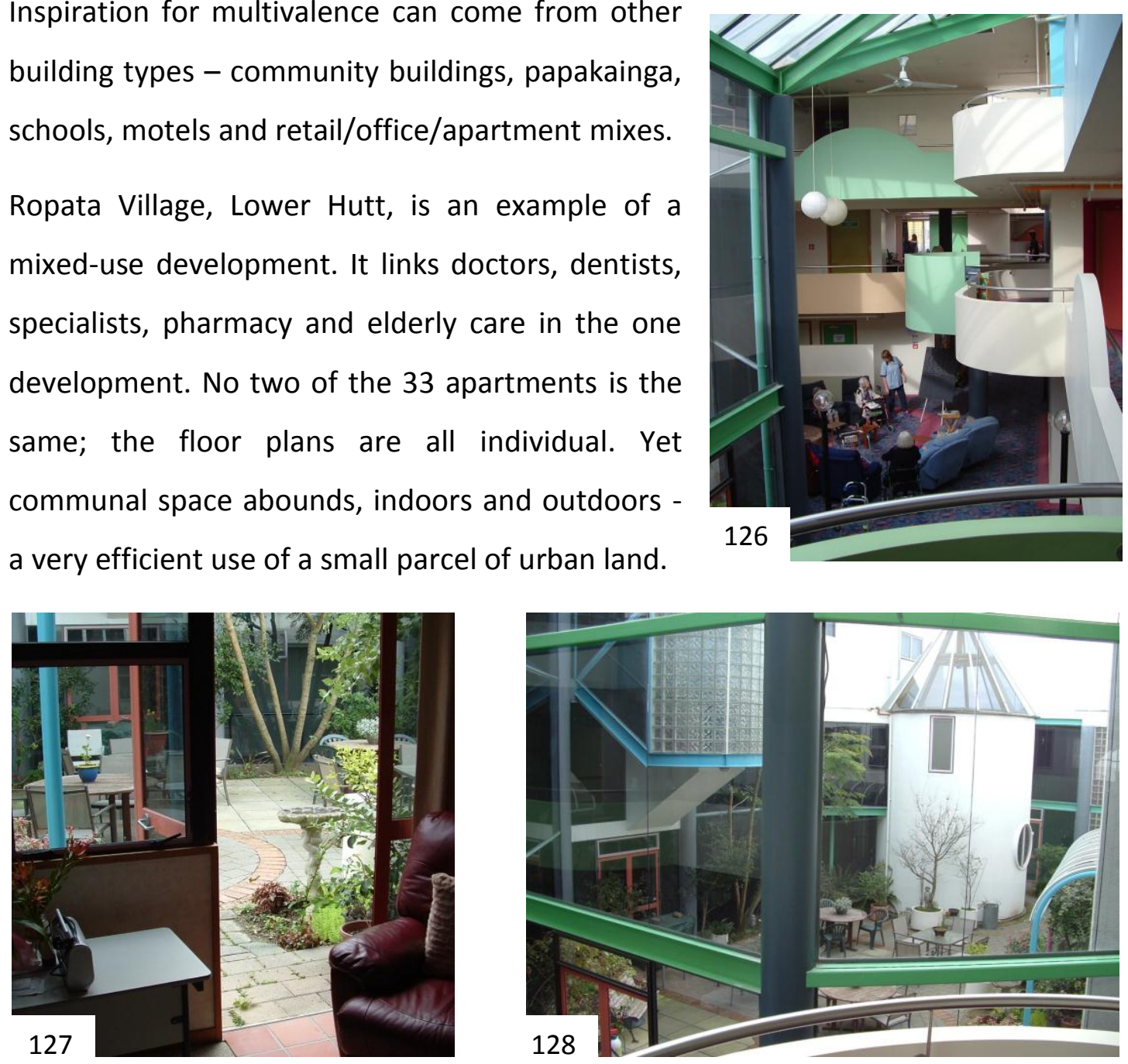

Fig.A7.3.126-128 Ropata Village, High Street, Lower Hutt. Multivalent communal spaces, indoors and outdoors.
Architect: Roger Walker (Photo: S.J.Pattinson) 


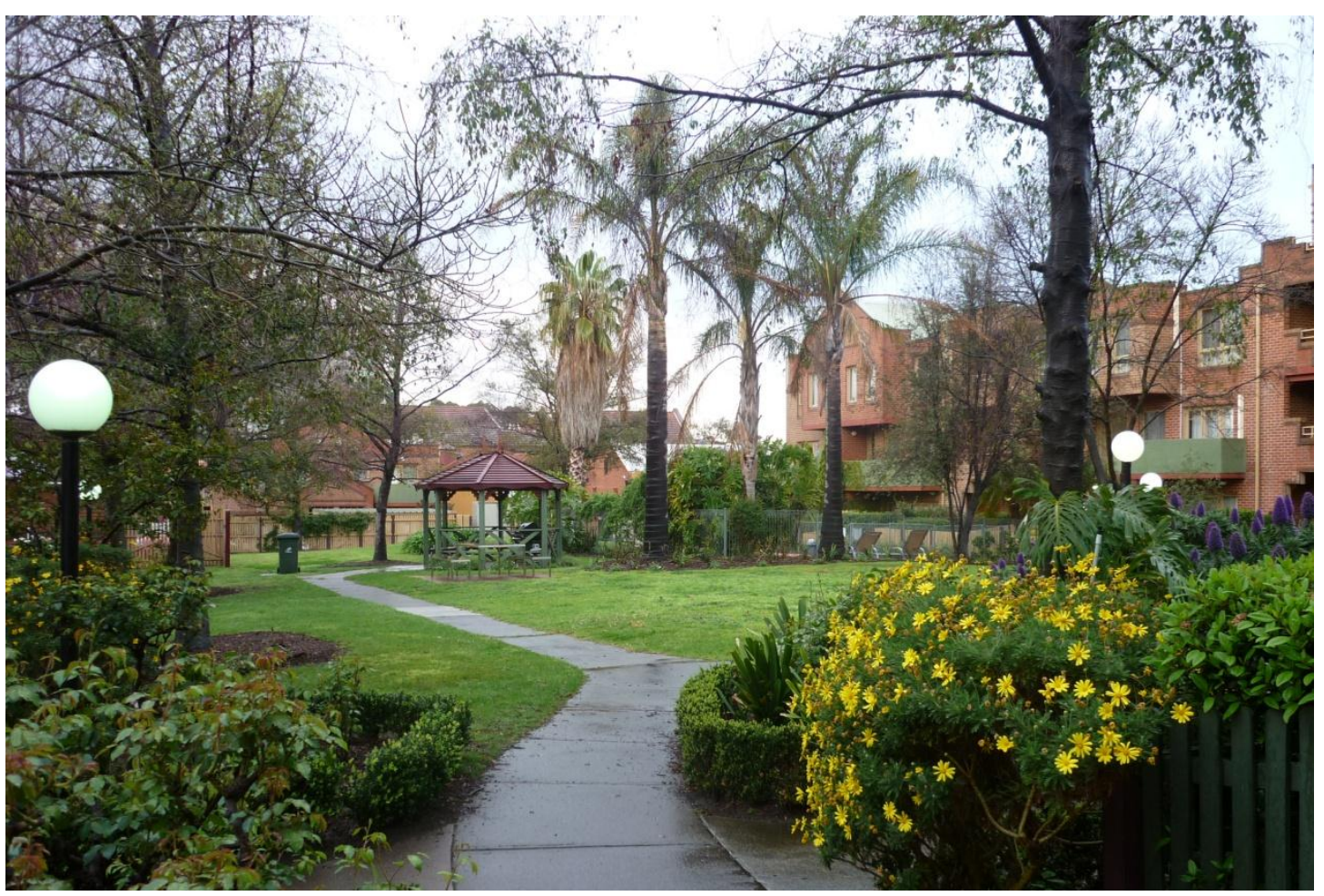

Fig.A7.3.129 Quest Royal Gardens (motel complex), Fitzroy, Melbourne. 70 units ( $1-4$ bedroom) arranged around central communal green. (Photo: S.J.Pattinson)

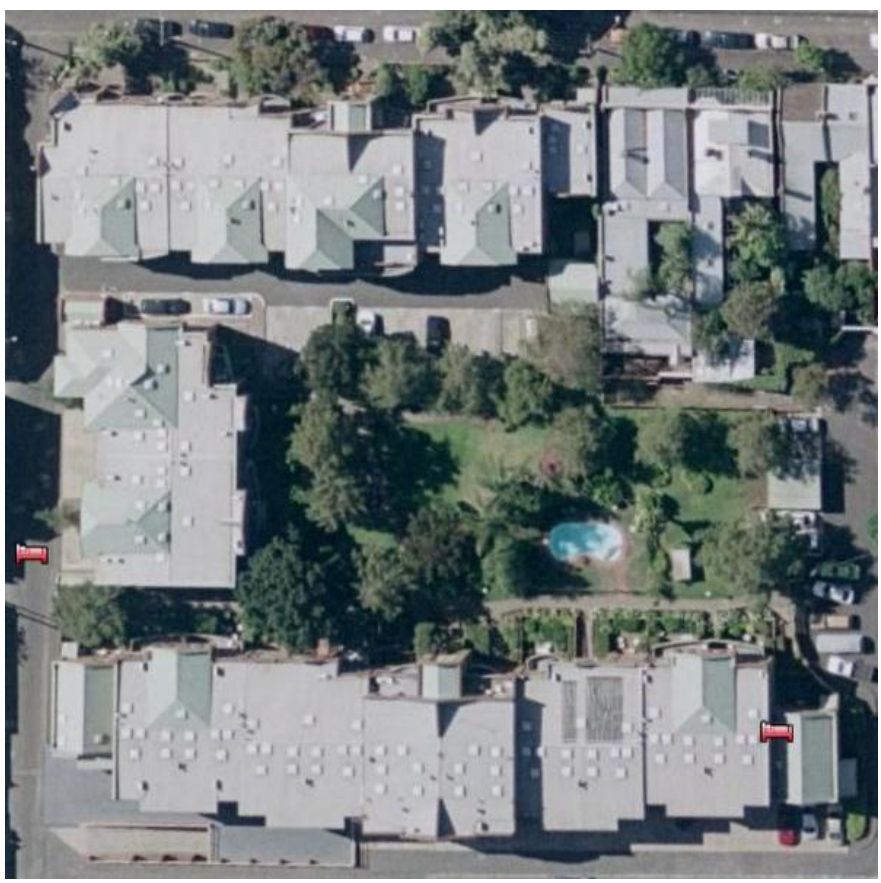

Fig.A7.3.130 Quest Royal Gardens, Fitzroy, Melbourne. (Google Earth aerial view)
This Melbourne motel complex demonstrates how 70 dwelling units, ranging in size from $1-4$ bedrooms, can be arranged at 3 - 4 storeys around the perimeter of a site to create purposeful central open space.

Signs say 'no public thoroughfare' but the attractive central green is accessible from the surrounding streets, allowing some connectivity through the neighbourhood. 


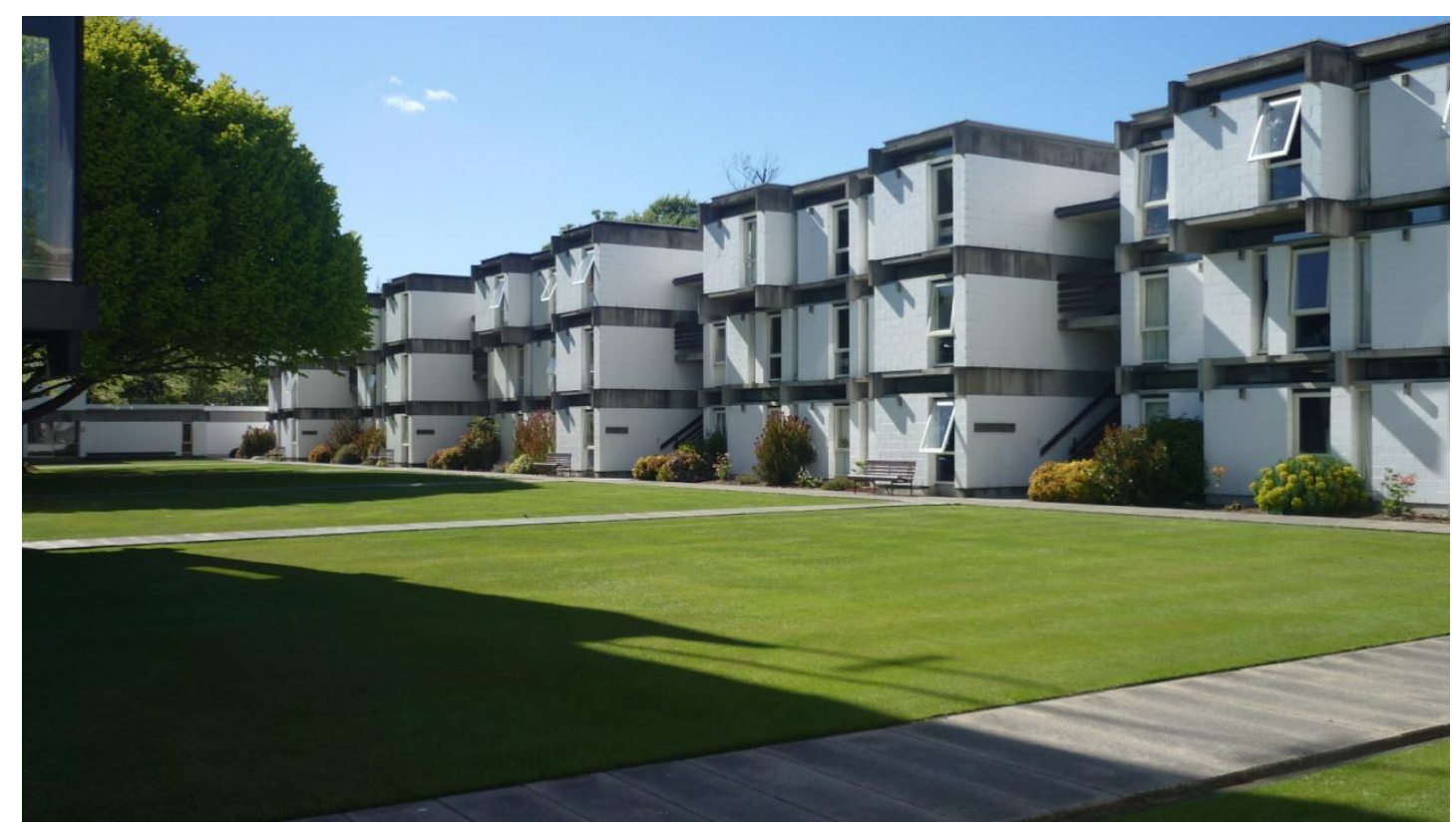

Fig.A7.3.131 College House, Waimarie Road, Christchurch. Architect: Warren \& Mahoney Student residence - includes indoor communal dining, lounges, library etc. (Photo: S.J.Pattinson)

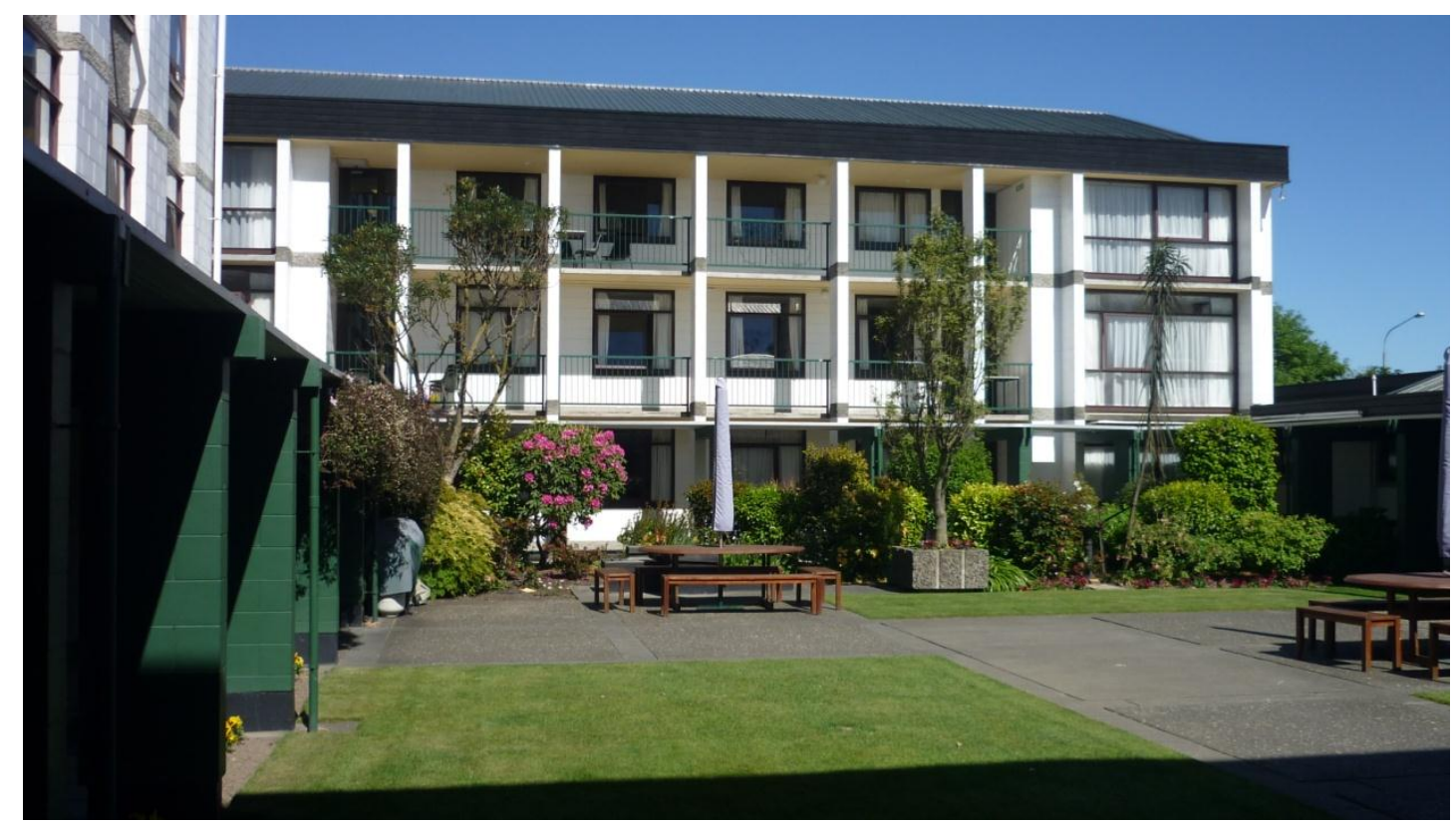

Fig.A7.3.132 Bishop Julius College, Waimarie Road, Christchurch. Student residence. Quad is outdoor assembly area - flies the national flag. (Photo: S.J.Pattinson) 
The writer has often wondered why body-corporates of medium-density housing developments do not pool resources to provide quality communal facilities for residents' benefits, such as is commonly found at motels and holiday resorts. Bayview Wairakei Resort serves as a great example of good communal facilities:
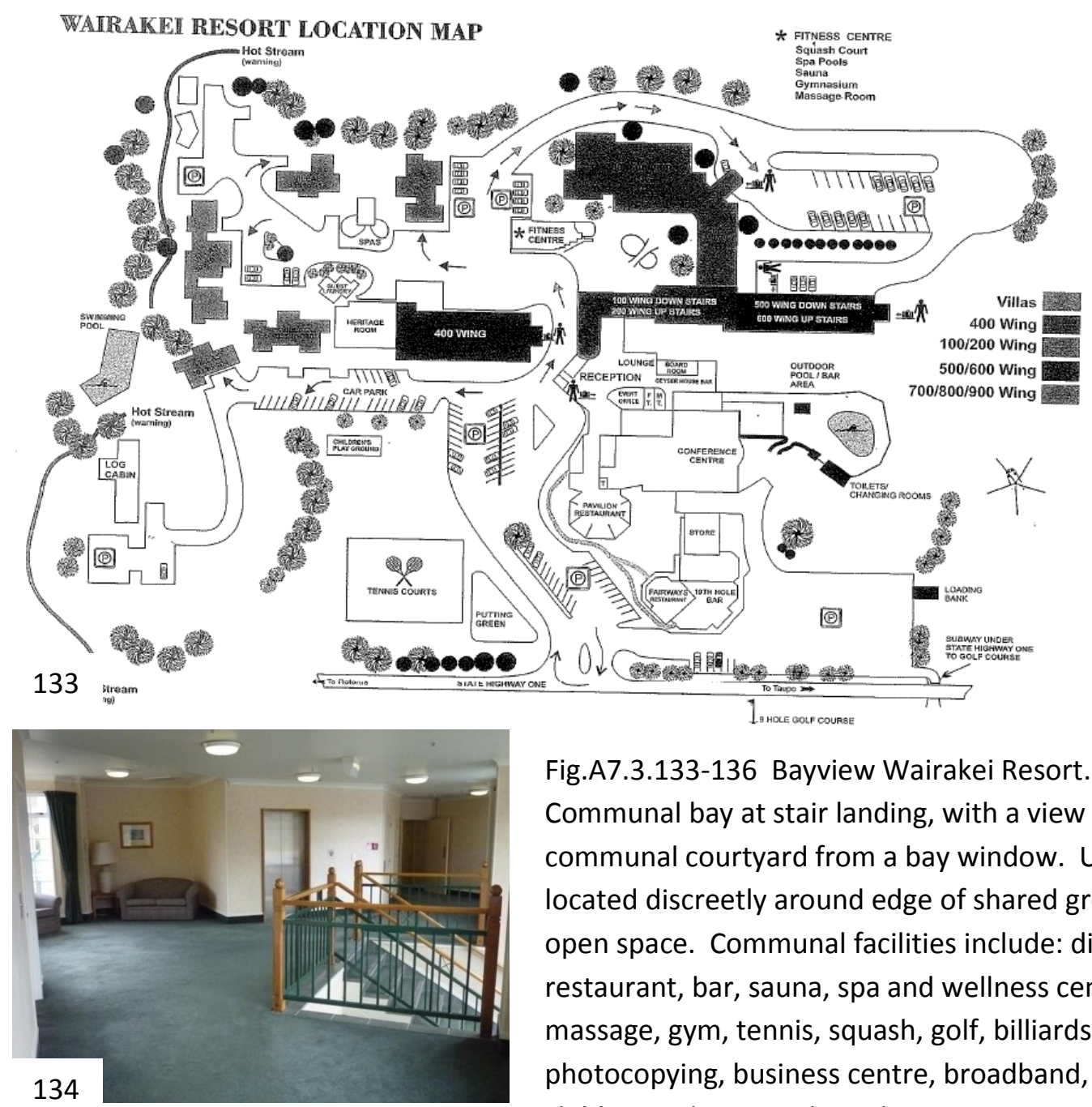

Fig.A7.3.133-136 Bayview Wairakei Resort. Communal bay at stair landing, with a view of a communal courtyard from a bay window. Units located discreetly around edge of shared green open space. Communal facilities include: dining, restaurant, bar, sauna, spa and wellness centre, massage, gym, tennis, squash, golf, billiards, fax, photocopying, business centre, broadband, childcare, playground, gardens, swimming pools ...
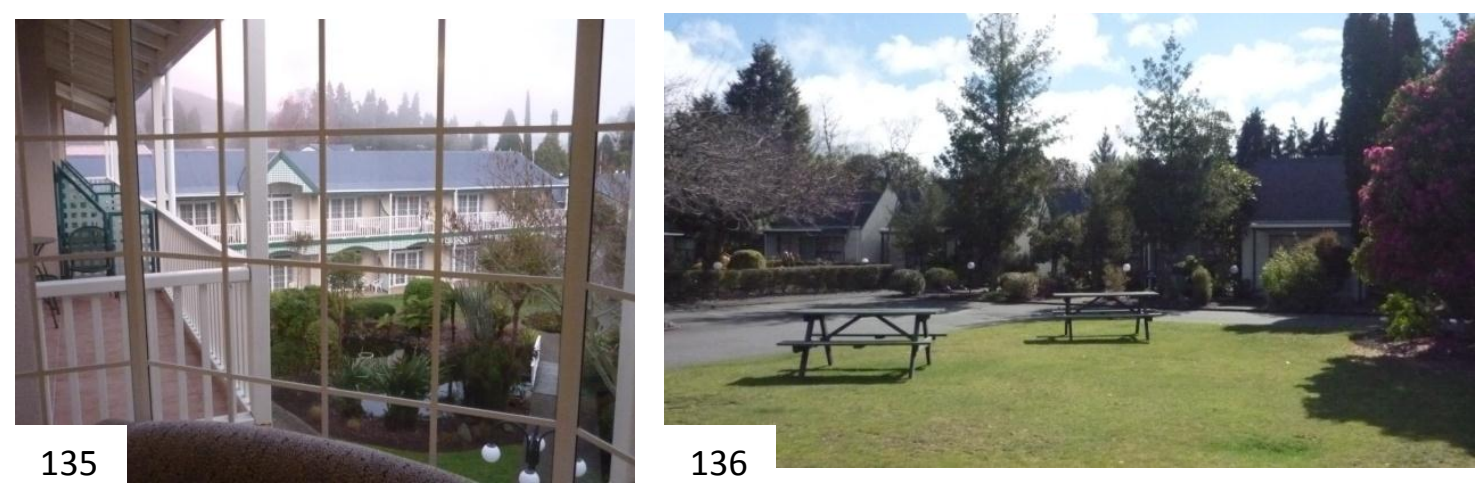
Pure bay-form for an intentional community - a primary school:

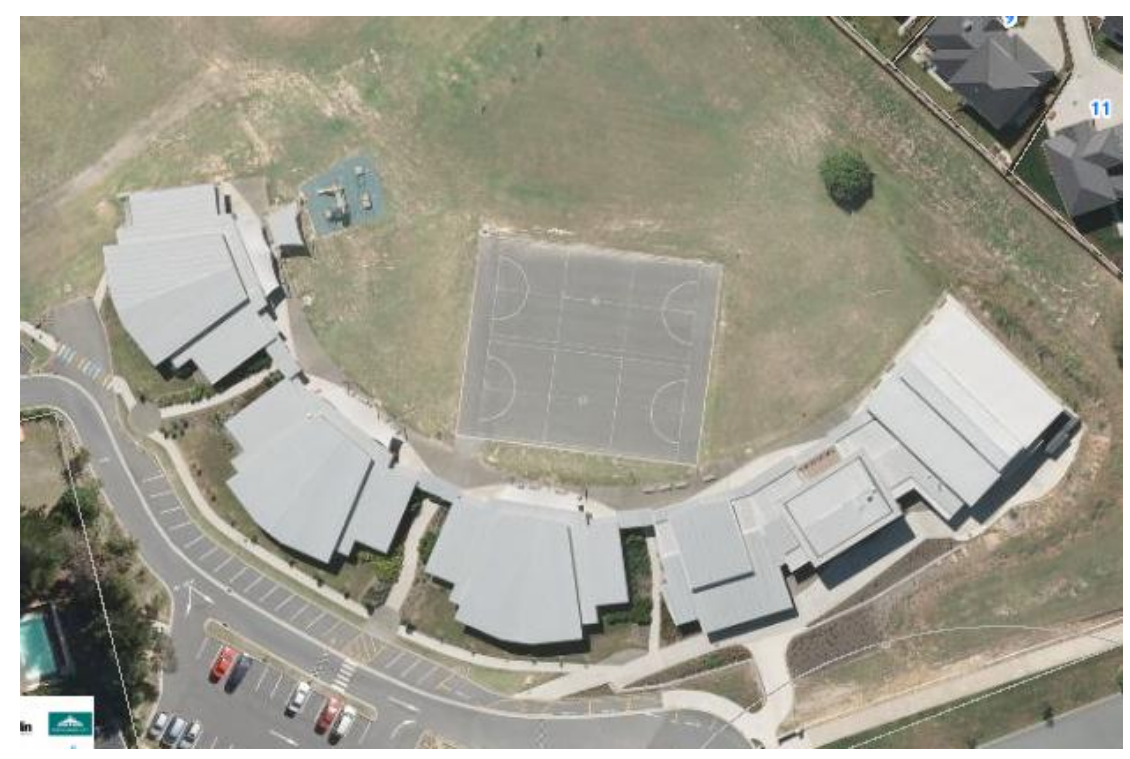

Fig.A7.3.137 Primary School at 126 Oteha Valley Road, North Shore, Auckland.

Bay-form used to group buildings around a communal outdoor space. (Alggi map)

Pure rectangular courtyard form for an intentional community - a primary school:

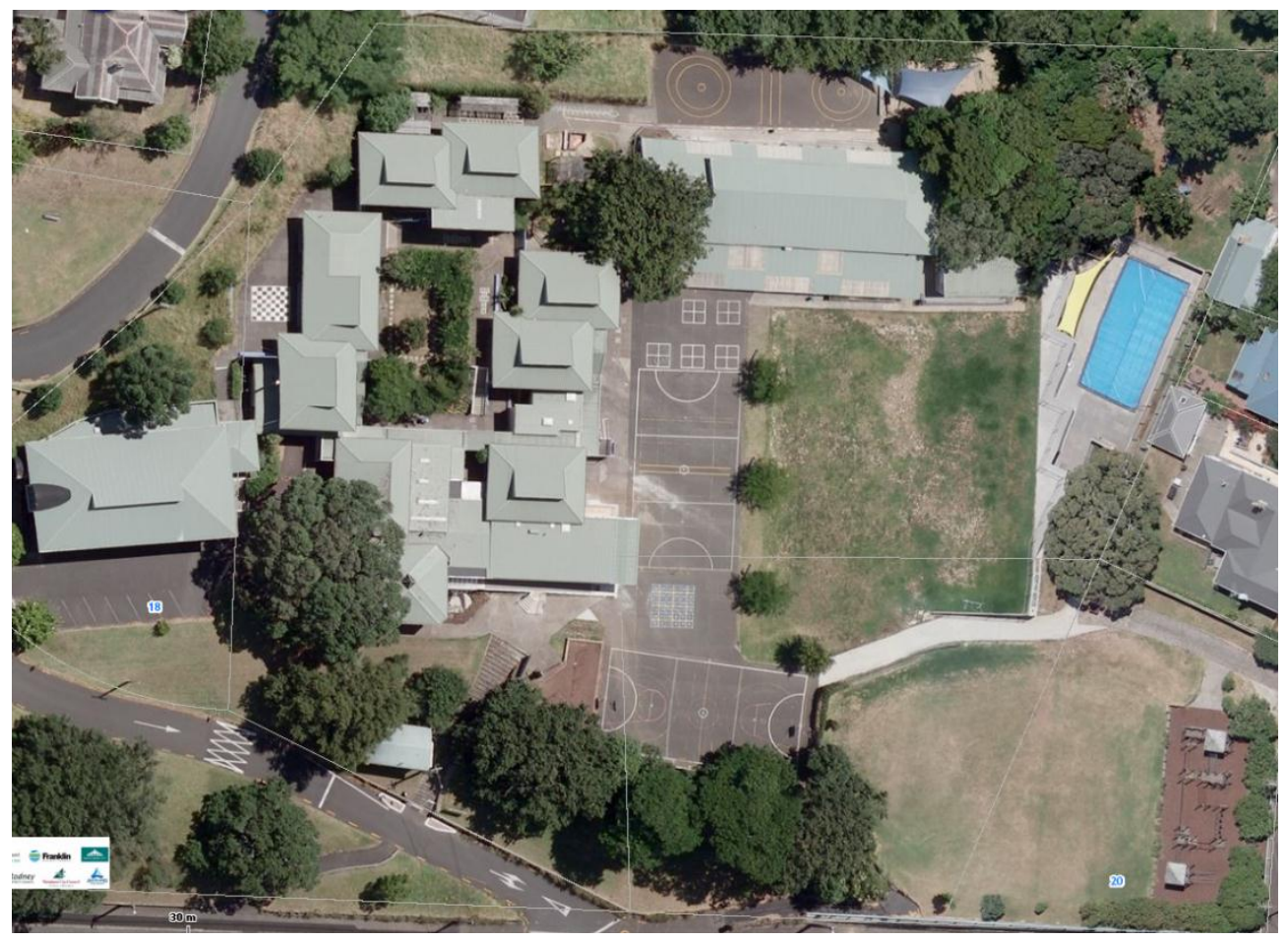

Fig.A7.3.138 Kerr Street Primary School, Devonport, North Shore, Auckland.

Rectangular courtyard forms used to define communal outdoor spaces. (Alggi map) 
Circular and rectangular forms juxtaposed for mixed-use multivalence:

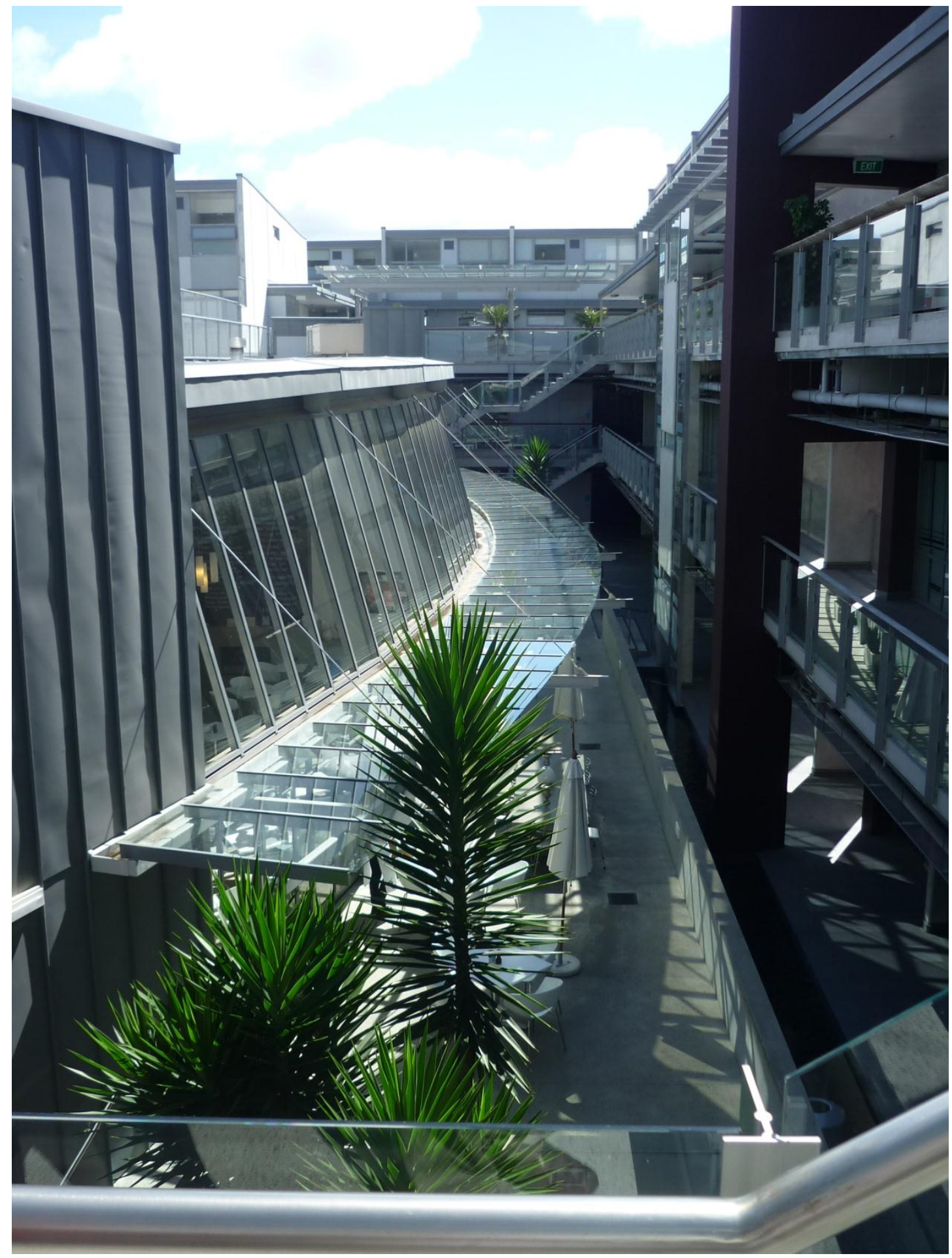

Fig.A7.3.139 Zone 23 Apartments, 21-23 Edwin Street, Mt Eden, Auckland.

Café in retail courtyard. Perimeter apartments above GFL retail/offices. (Photo: S.J.Pattinson) 


\section{Appendix 8: Analytical Framework (Wellington Schemes)}




\section{APPENDIX 8 - Analytic framework for site investigations}

This Appendix 8 considers three related issues:

1. An analytical framework for researching multivalent communal space in medium density housing;

2. Metrics - private and communal outdoor space per person;

3. Qualitative research.

The following provides some comments on these issues.

\section{An analytical framework for researching multivalent communal space}

In 1975, Amos Rapoport challenged the then prevailing view of density which was "seen largely as a matter of 'number of people per unit area'." He recognised "that density is a perceived experience and should be seen as more than 'the number of people per unit area" " and he offered "some possible guidelines for a redefinition of density. ... The first point to be made" he said, "is that while density begins with the number of people per unit area, it must go beyond it" (133-135).

Rapoport's above comments on density are applicable to the subject of this thesis research, multivalent communal space in medium density housing, since the quality of communal space is a perceived experience and more than just a matter of dwellings or people per unit area. However, the quality of communal space probably "begins with the number of [dwellings or] people per unit area." This Appendix 8 therefore selects some medium density housing developments in the Wellington region and analyses some basic metrics for each development for comparative purposes as a beginning point to understanding the quality of communal space. 
$\begin{array}{lll}\text { (C) } 2012 \text { Stephen Pattinson } & \text { Appendix } 8 & \text { M. Arch Thesis }\end{array}$

An analytical framework for the metrics of communal space would allow a systematic analysis of where and how housing developments of medium density include communal open space, and something about the quality of that space in terms of how it is used. A possible approach would be to divide developments into housing types (retirement homes; housing for rent; housing for sale; mixed) and then analyze the following aspects of each development to give a sense of what can be provided for a given number of people in a certain type of settlement:

- $\quad$ how many people (bed spaces)

- $\quad$ area of housing footprint

- area of private open space

- area of public open space

- $\quad$ use of public open space

Nine housing developments in the Wellington region (six existing, two hypothetical, and one not yet built) are selected, measured and compared.

It has not been possible in the available time for this research to analyse these selected developments in terms of tenure, i.e whether they are owner-occupied or rental housing units, or whether the common spaces are Council-owned and maintained or privately owned and managed by incorporated bodies representing the owners. Nor are the selected developmennts representative of different housing types, such as 'retirement village' or 'social housing'. The writer recognises the importance of these issues of tenure and management, and suggests they may provide a very interesting focus for future research. Regarding the following analyses, it is hoped they will indicate the usefulness of metrics as a beginning point for understanding communal space, and raise further questions that might suggest themselves as topics for future research. 


\section{Metrics - private and communal outdoor space per person}

This section consists of outdoor space metrics related to bedspaces for the following medium density housing developments in the Wellington region:

1. Existing detached housing on $400 \mathrm{~m}^{2}$ lots at Pinehill Crescent, Upper Hutt;

2. Hypothetical detached housing on $300 \mathrm{~m}^{2}$ lots, Pinehill Crescent, Upper Hutt;

3. Existing terrace housing around Pinehill Park, Upper Hutt;

4. Pinehill Crescent - hypothetical concept with cluster housing and open spaces;

5. "The Boundary" Townhousing in Karori, Wellington;

6. "Maidstone Quarter", proposal for 44 Blenheim Street, Upper Hutt;

7. "The Altair", Townhousing at Rintoul Street, Newtown, Wellington;

8. "Monterey" Townhousing at 232 Middleton Road, Glenside, Wellington;

9. 10 Park Avenue, Lower Hutt - Townhousing.

The first four developments are selected since the Pinehill Crescent subdivision and Pinehill Park are a focus for this thesis research. These developments are in the lower range of medium density, and provide a baseline for comparison with the other higher density developments. The existing detached housing in Development 1 is of a similar density to the existing terrace housing in Development 3 (15dph and $12 \mathrm{dph}$ respectively) if Pinehill Park is included in the terrace house calculations. The hypothetical detached housing in Development 2 is slightly higher density (22dph), having minimum 300m2 lots, while Development 4, hypothetical cluster housing proposed for the whole subdivision, achieves a slightly higher density again (27dph) and with the advantage of having more open space. Development 5, terrace housing clustered around large open spaces, is of a similar density to 4 .

Development 6 is not yet built; it is representative of a mid-range density of about $46 \mathrm{dph}$. Developments 7 and 8 are more compact at around $70 \mathrm{dph}$ and have 
several planned communal spaces. Development 9 has no communal space and is represntative of the upper limit for terrace house density at $88 \mathrm{dph}$.

\begin{tabular}{|c|c|c|c|c|c|}
\hline & $\begin{array}{l}\text { Medium Density } \\
\text { Housing Development }\end{array}$ & $\begin{array}{l}\text { Private } \\
\text { outdoor } \\
\text { space per } \\
\text { bedspace } \\
\text { (person) }\end{array}$ & $\begin{array}{l}\text { Shared } \\
\text { outdoor } \\
\text { space per } \\
\text { bedspace } \\
\text { (person) }\end{array}$ & $\begin{array}{l}\text { Total } \\
\text { outdoor } \\
\text { space per } \\
\text { bedspace } \\
\text { (person) } \\
\end{array}$ & $\begin{array}{l}\text { Comment: } \\
\text { (gross density } \\
\text { means all } \\
\text { open space is } \\
\text { included) }\end{array}$ \\
\hline 1. & $\begin{array}{l}\text { Existing detached } \\
\text { housing on min. } 400 \mathrm{~m} 2 \\
\text { lots at Pinehill Crescent, } \\
\text { Upper Hutt (Ch.7 Fig. 7.4) }\end{array}$ & $74 \mathrm{~m} 2$ & nil & $74 \mathrm{~m} 2$ & $\begin{array}{l}\text { Gross } \\
\text { density = } \\
15 \text { dph } \\
\text { (Fig.A8.2) }\end{array}$ \\
\hline 2. & $\begin{array}{l}\text { Hypothetical detached } \\
\text { housing on } \min .300 \mathrm{~m} 2 \\
\text { lots at Pinehill Crescent, } \\
\text { Upper Hutt (Ch.7 Fig. } 7.6 \text { ) }\end{array}$ & $45 \mathrm{~m} 2$ & nil & $45 \mathrm{~m} 2$ & $\begin{array}{l}\text { Gross } \\
\text { density = } \\
22 \mathrm{dph} \\
\text { (Fig. A8.3) }\end{array}$ \\
\hline 3. & $\begin{array}{l}14 \text { existing terraces at } \\
\text { Pinehill Park including } \\
\text { the Park as common area } \\
\text { (Ch.7 Fig. 7.4) }\end{array}$ & $49 \mathrm{~m} 2$ & $67 \mathrm{~m} 2$ & $116 \mathrm{~m} 2$ & $\begin{array}{l}\text { Gross } \\
\text { density = } \\
12 \mathrm{dph} \\
\text { (Fig.A8.4) }\end{array}$ \\
\hline 4. & $\begin{array}{l}\text { Pinehill Cres(Ch7 Fig7.10) } \\
\text { Hypothetical concept } \\
\text { with multivalent spaces }\end{array}$ & $16 \mathrm{~m} 2$ & $24 \mathrm{~m} 2$ & $40 \mathrm{~m} 2$ & $\begin{array}{l}\text { Gross } \\
\text { density = } \\
27 \mathrm{dph} \\
\end{array}$ \\
\hline 5. & $\begin{array}{l}\text { The Boundary: (Fig. A8.9) } \\
\text { Saddleback Grove, } \\
\text { Karori, Wellington }\end{array}$ & $7.6 \mathrm{~m} 2$ & 21.6 & 29.2 & $\begin{array}{l}\text { Gross } \\
\text { density = } \\
25 \text { dph }\end{array}$ \\
\hline 6. & $\begin{array}{l}\text { Maidstone Quarter, } \\
44 \text { Blenheim Street, } \\
\text { Upper Hutt (not yet built) }\end{array}$ & $8.8 \mathrm{~m} 2$ & $2.5 \mathrm{~m} 2$ & $11.3 \mathrm{~m} 2$ & $\begin{array}{l}45.8 \mathrm{dph} \\
\text { (Fig. A8.10) }\end{array}$ \\
\hline 7. & $\begin{array}{l}\text { The Altair, Rintoul Street, } \\
\text { Newtown, Wellington }\end{array}$ & $5.5 \mathrm{~m} 2$ & $1.6 \mathrm{~m} 2$ & $7.1 \mathrm{~m} 2$ & $\begin{array}{l}71 \mathrm{dph} \\
\text { (Fig.A8.18) }\end{array}$ \\
\hline 8. & $\begin{array}{l}\text { Monterey (Fig.A8.23) } \\
232 \text { Middleton Road, } \\
\text { Glenside, Wellington }\end{array}$ & $4.2 \mathrm{~m} 2$ & $4.2 \mathrm{~m} 2$ & $8.4 \mathrm{~m} 2$ & $69.3 \mathrm{dph}$ \\
\hline 9. & $\begin{array}{l}10 \text { Park Avenue, } \\
\text { Lower Hutt (Fig. A8.46) }\end{array}$ & $8.0 \mathrm{~m} 2$ & nil & $8.0 \mathrm{~m} 2$ & $88 \mathrm{dph}$ \\
\hline
\end{tabular}

Table A8.1 Summary: Outdoor space per person (i.e. bedspace). A comparison of selected housing developments in the Wellington region.

(By S. J. Pattinson) 


\section{1 - 4. PINEHILL CRESCENT, UPPER HUTT}

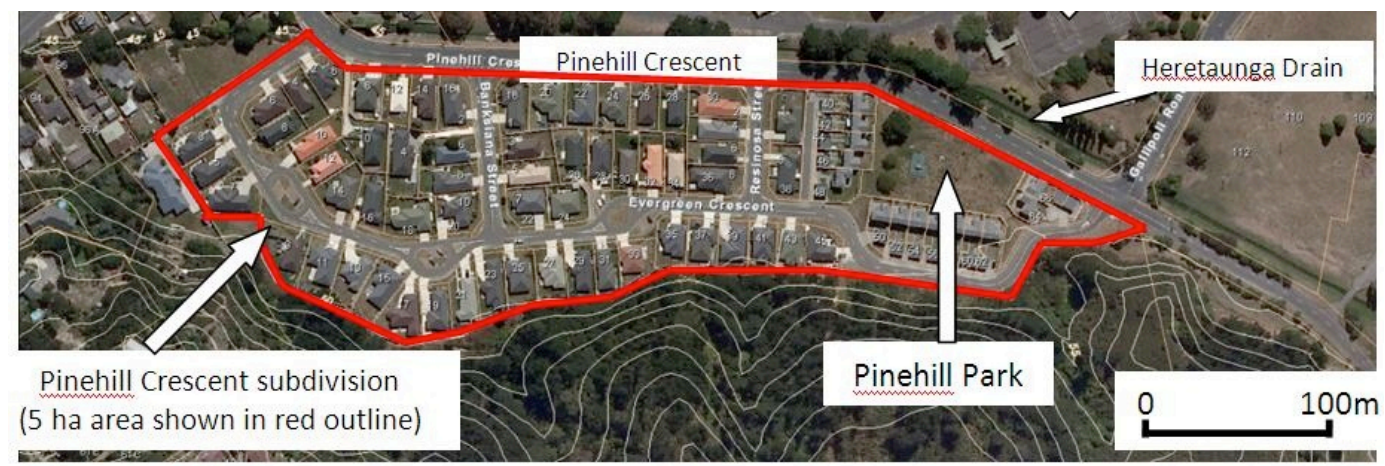

Figure A8.1 Pinehill Crescent: 75 houses on 5 hectare site (in red) (Thesis Ch. 6 Fig. 6.1)

The above aerial view of Pinehill Crescent shows the 5 ha subdivision of 75 houses outlined with a red border line. It consists of 65 detached houses on the left and centre of the subdivision and 14 terrace houses around Pinehill Park on the right. Development 1 is the existing detached housing on $400 \mathrm{~m} 2$ lots, and Development 2 looks hypothetically at detached housing on minimum 300m2 lots. Development 3 is the existing terrace housing around Pinehill Park. Development 4 looks at the whole 5ha site hypothetically with cluster housing and communal open spaces.

\section{PINEHILL CRESCENT: Existing detached housing on minimum $400 \mathrm{~m}^{2}$ lots}

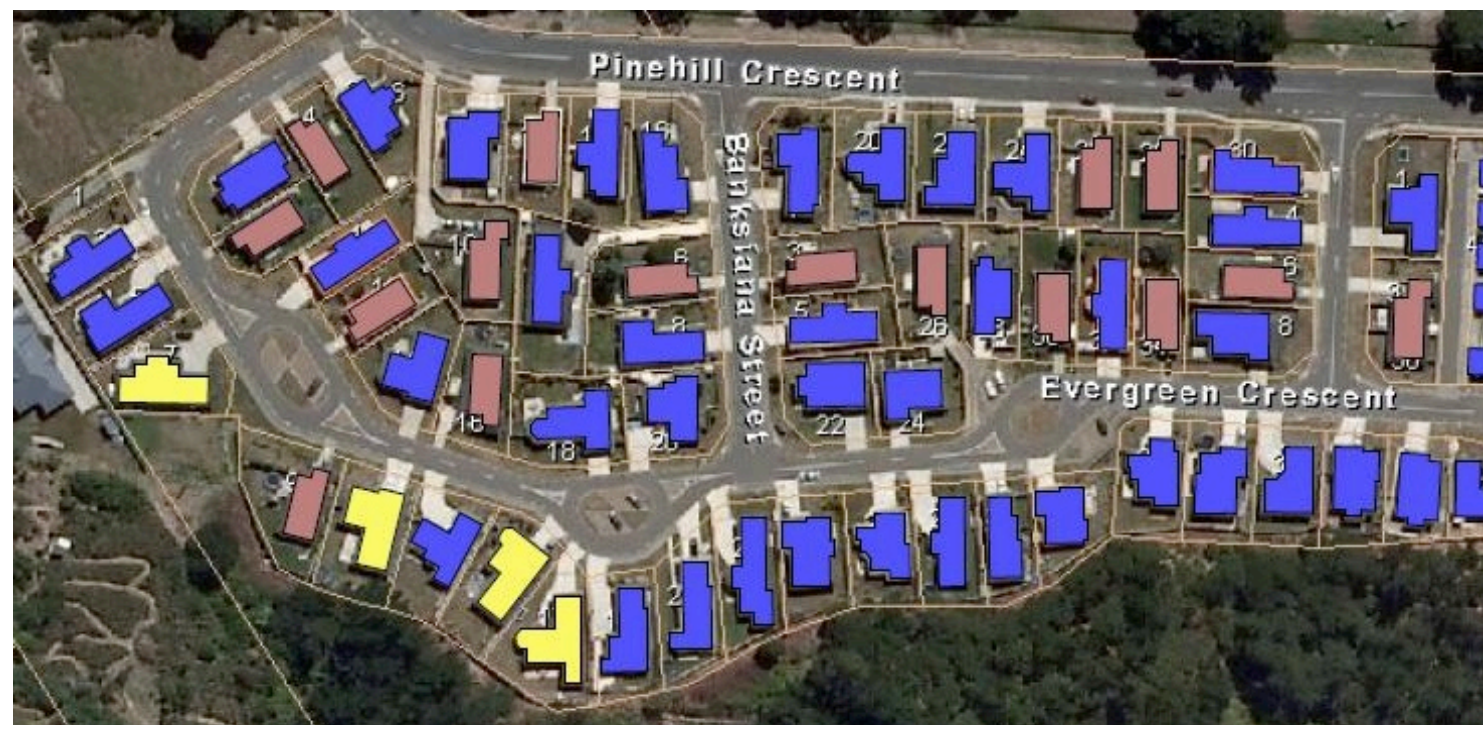

Figure A8.2 Pinehill Crescent: 65 single-storey detached houses on minimum $400 \mathrm{~m}^{2}$ lots (from thesis Ch. 7 Fig. 7.4) 

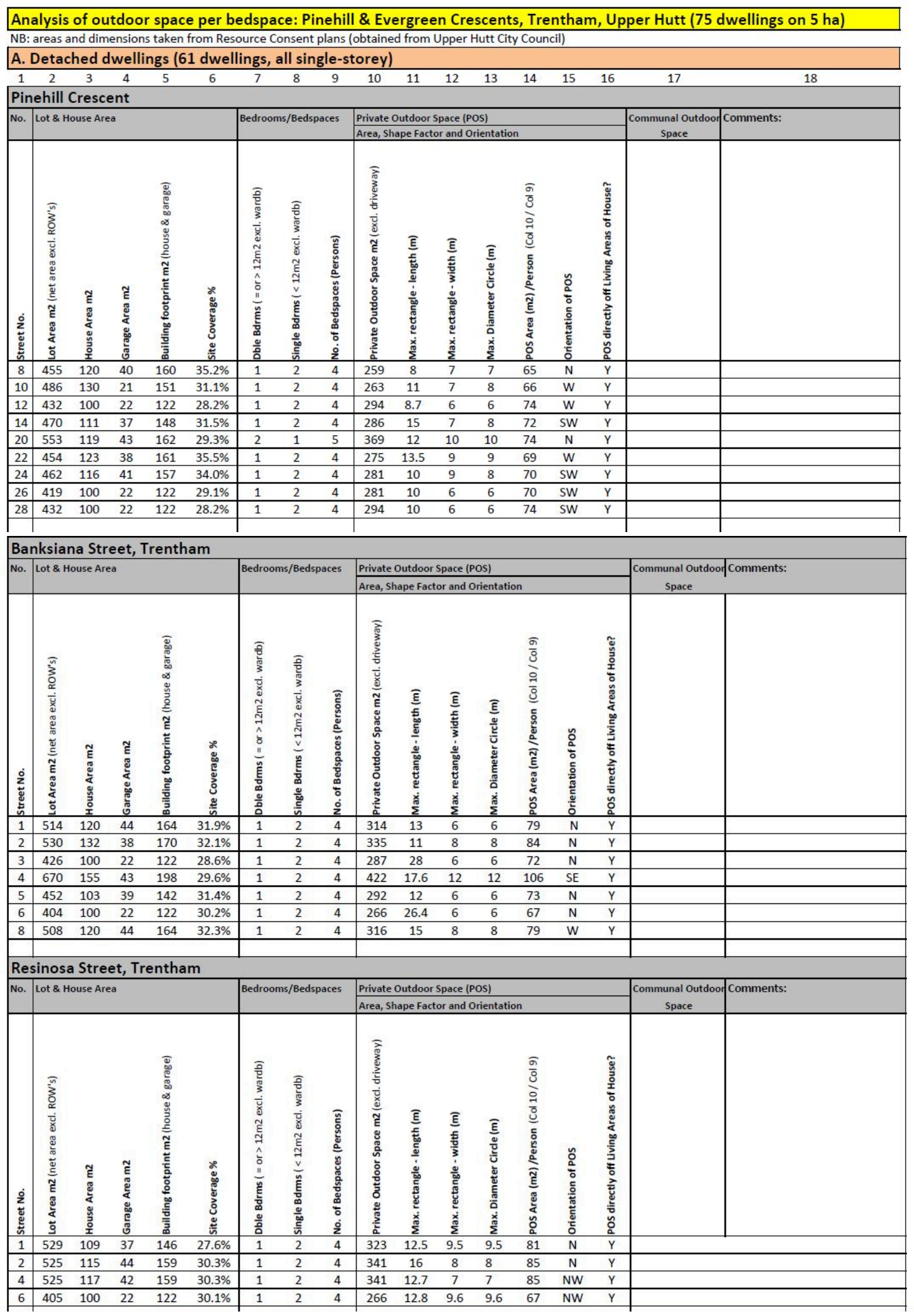

Table A8.2a Pinehill Crescent: analysis of detached houses on minimum $400 \mathrm{~m} 2$ lots 


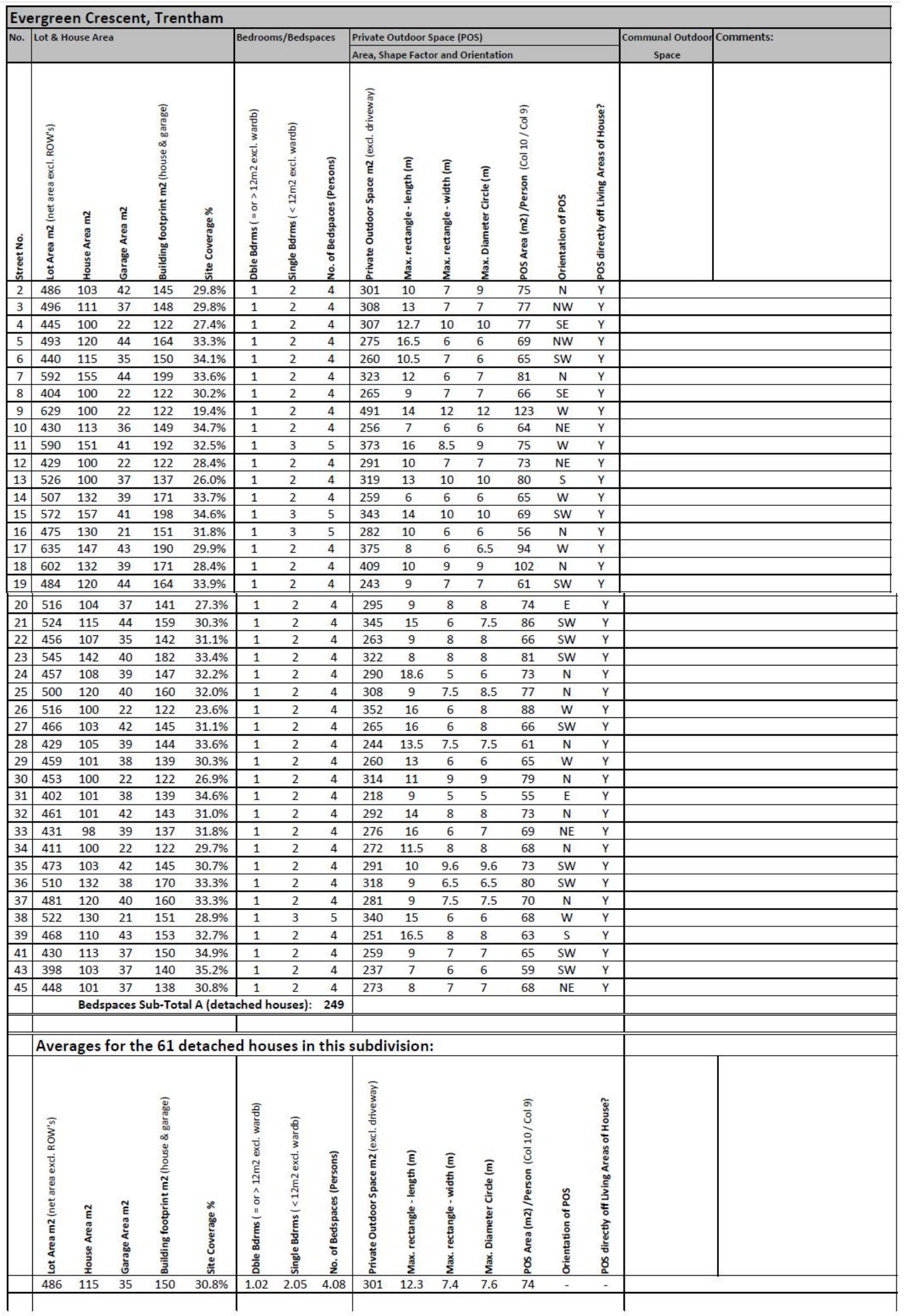

Table A8.2b Pinehill Crescent: analysis of detached houses on minimum $400 \mathrm{~m} 2$ lots 


\section{PINEHILL CRESCENT:}

\section{Hypothetical detached housing on minimum $300 \mathrm{~m}^{2}$ lots}

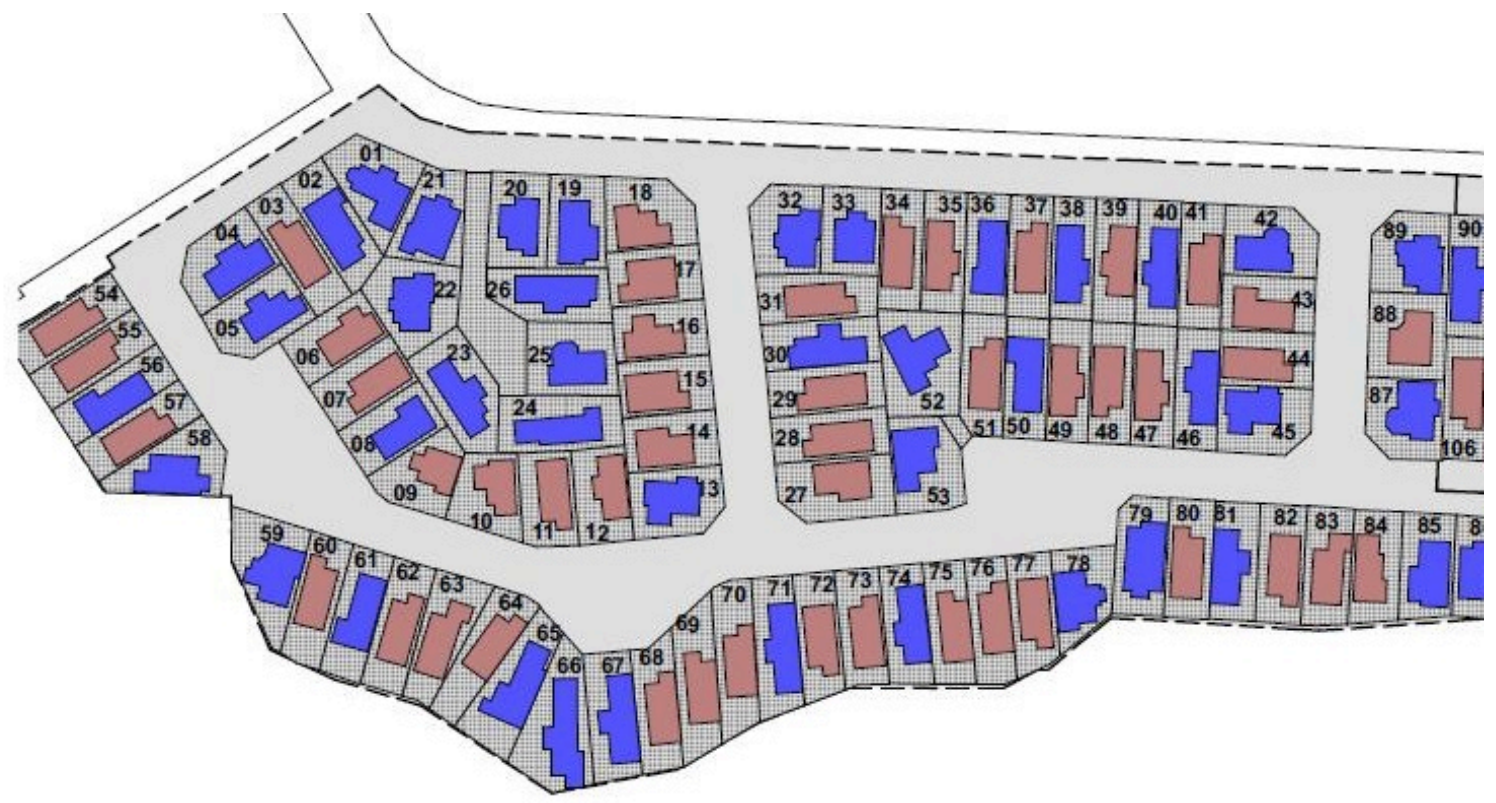

Figure A8.3 Pinehill Crescent: hypothetical single-storey, detached houses on minimum $300 \mathrm{~m}^{2}$ lots

(from thesis Ch. 7 Fig. 7.6)

This hypothetical layout assumes the following:

Average lot area:

$320 \mathrm{~m} 2$

Average house area (excl. garage):

$100 \mathrm{~m} 2$

Average garage area:

$20 \mathrm{~m} 2$

Average number of bedrooms:

1 double and 2 single

Average bedspaces (persons) per dwelling:

4

Average private outdoor space (excl. $20 \mathrm{~m} 2$ drive): $180 \mathrm{~m} 2$

Average private outdoor space / bedspace (person): $45 \mathrm{~m} 2$ 


\section{PINEHILL CRESCENT: Existing terrace housing around Pinehill Park}

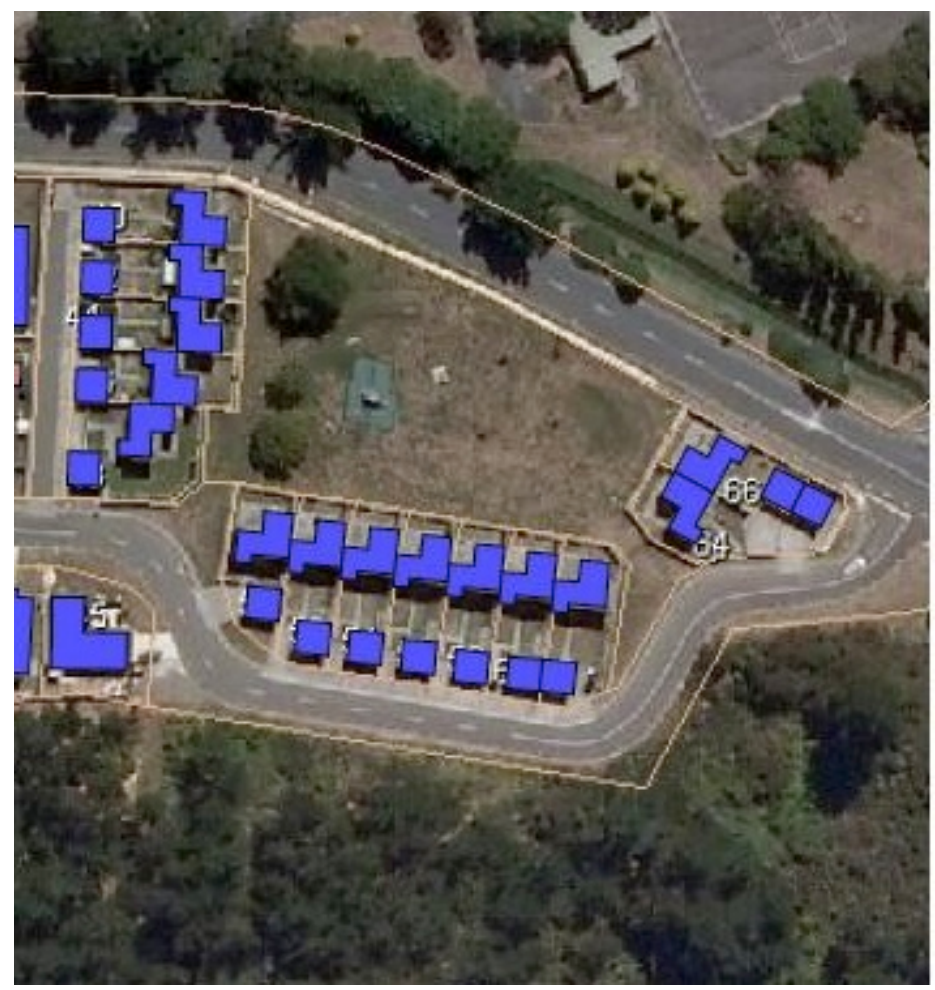

Figure A8.4 Pinehill Crescent: 14 terrace houses around three sides of Pinehill Park, Upper Hutt

(from thesis Ch. 7 Fig. 7.4)

See the following page for a table of calculations for the 14 existing terrace houses, and for notes comparing the terrace housing statistics with the statistics for the existing 65 detached houses on minimum 400m2 lots in the Pinehill Crescent subdivision. 
(C) 2012 Stephen Pattinson $\quad$ Appendix $8 \quad$ M. Arch Thesis

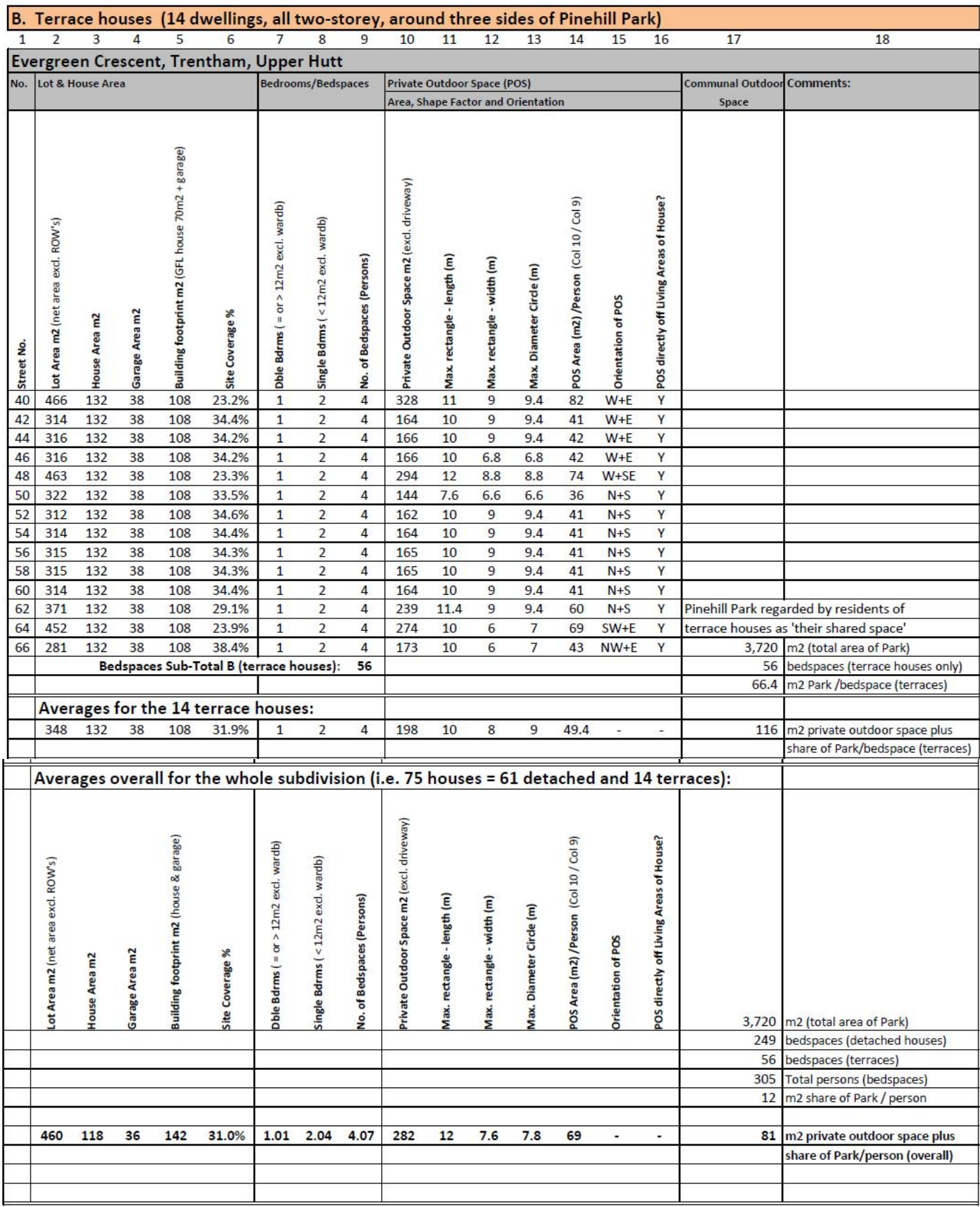

Summary:

In theory, there is a $12 \mathrm{~m} 2$ share of Pinehill Park for every bedspace in the overall development. The 75 houses consist of a total of 305 bedspaces (persons). The total area of Pinehill Park is $3,720 \mathrm{~m} 2$. This area divided by 305 bedspaces (persons) gives each person a $12 \mathrm{~m} 2$ share of the Park. However, Pinehill Park is not located centrally in the development but at the Eastern edge, flanked by the 14 terrace houses. The Case Study survey shows that Pinehill Park is considered by the residents of the terrace houses to be their communal outdoor space. Observations of the Park reveal that it is used mainly by children from the terrace houses. It was noted that some residents of the detached housing use the Park but not many and not often. A resident of the detached housing organised a neighbourhood Christmas party in early December 2010. It was held at the middle round-a-bout on the road, not in the Park. Similarly, Google street-view images show children playing on the road at a round-a-bout amongst the detached houses. So while in theory all residents might have a $12 \mathrm{~m} 2$ share in the Park, in practice the Park is shared (actively and/or passively) mainly by the 56 residents of the terrace housing, giving them each $66.4 \mathrm{~m} 2$ share of the Park in addition to their average private outdoor space of $49.4 \mathrm{~m} 2$, a total of $116 \mathrm{~m} 2$ outdoor space for each terrace house resident. Residents of the detached housing have an average private outdoor area of $74 \mathrm{~m} 2$ each, and occassionally make recreational/social use of the roads and the Park.

Table A8.3a Pinehill Crescent: analysis of terrace houses; summary for overall subdivision 


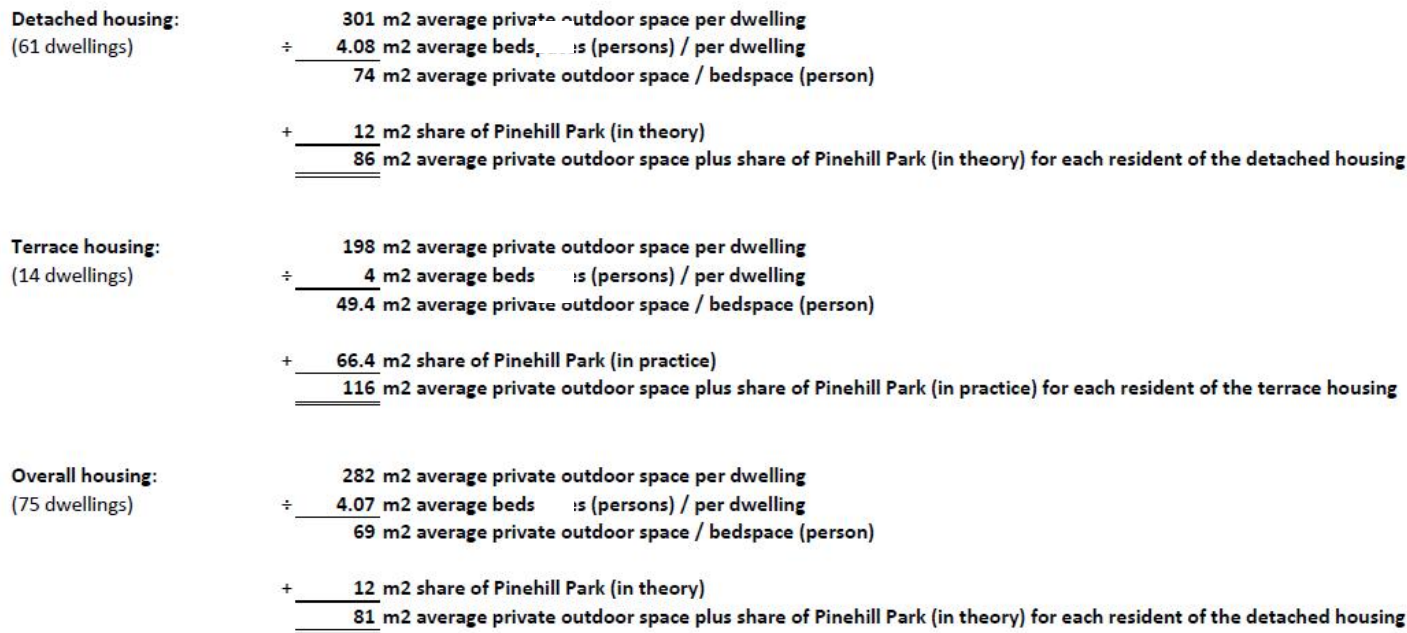

Table A8.3b Pinehill Crescent: summary for existing subdivision

\section{PINEHILL CRESCENT: Alternative hypothetical development based on the principles for multivalent communal space (see Ch.7 Fig. 7.10 and Table 7.6)}

Total site area:

$50,000 \mathrm{~m} 2$ (5ha)

Number of dwellings and bedspaces:

Dwellings: Bedspaces:

- Assume average 4 bedspaces per dwelling 140

Average private outdoor space per dwelling (approx.): $\quad 64 \mathrm{~m} 2$

Average private outdoor space / bedspace (person): $\quad 16 \mathrm{~m} 2$

Total shared outdoor space (approximate measurement):

- Orchard

- communal gardens with pools

- $\quad$ pond and landscaping

- community building 2 levels $\times 750=1,500 \mathrm{~m} 2$

$1,600 \mathrm{~m} 2$

$1,600 \mathrm{~m} 2$

$800 \mathrm{~m} 2$ 
- Playing field

- $2 x$ tennis courts plus gardens

- cluster courtyard with pool

- $\quad$ park (at eastern end of subdivision) 1,800 m2

Total shared outdoor space $\quad 13,300 \mathrm{~m} 2$
$24 \mathrm{~m} 2$

$40 \mathrm{~m} 2$

In summary, the Pinehill Crescent metrics are:

\begin{tabular}{|c|c|c|c|c|c|}
\hline & Type of Housing & $\begin{array}{l}\text { Private } \\
\text { outdoor } \\
\text { space per } \\
\text { bedspace }\end{array}$ & $\begin{array}{l}\text { Shared } \\
\text { outdoor } \\
\text { space per } \\
\text { bedspace }\end{array}$ & $\begin{array}{l}\text { Total } \\
\text { outdoor } \\
\text { space per } \\
\text { bedspace }\end{array}$ & $\begin{array}{l}\text { Comment: } \\
\text { gross is all } \\
\text { open space } \\
\text { incl. roads }\end{array}$ \\
\hline 1. & $\begin{array}{l}\text { Existing detached } \\
\text { housing on min. } 400 \mathrm{~m} 2 \\
\text { lot area (not incl. any } \\
\text { share of Pinehill Park): }\end{array}$ & $74 \mathrm{~m} 2$ & nil & $74 \mathrm{~m} 2$ & $\begin{array}{l}\text { Gross } \\
\text { density }= \\
15 \text { dph } \\
\text { (see Fig.7.4) }\end{array}$ \\
\hline 2. & $\begin{array}{l}120 \text { detached houses on } \\
\text { min. } 300 \mathrm{~m} 2 \text { lot area } \\
\text { (there is no Park in this } \\
\text { hypothetical layout): }\end{array}$ & $45 \mathrm{~m} 2$ & nil & $45 \mathrm{~m} 2$ & $\begin{array}{l}\text { Gross } \\
\text { density }= \\
22 \text { dph } \\
\text { (see Fig.7.6) }\end{array}$ \\
\hline 3. & $\begin{array}{l}14 \text { existing terrace } \\
\text { houses (including } \\
\text { Pinehill Park as common } \\
\text { area): }\end{array}$ & $49 \mathrm{~m} 2$ & $\begin{array}{c}3,720 / 56= \\
67 \mathrm{~m} 2\end{array}$ & $116 \mathrm{~m} 2$ & $\begin{array}{l}\text { Gross } \\
\text { density }= \\
12 \text { dph } \\
\text { (see Fig.7.4) }\end{array}$ \\
\hline NB. & $\begin{array}{l}14+3=17 \text { hypothetical } \\
\text { terrace houses, incl. } \\
\text { Pinehill Park as common } \\
\text { area (see Ch. } 7 \text { Fig. } 7.5 \text { ): }\end{array}$ & $49 \mathrm{~m} 2$ & $\begin{array}{c}3,720 / 68= \\
55 \mathrm{~m} 2\end{array}$ & $104 \mathrm{~m} 2$ & $\begin{array}{l}\text { Gross } \\
\text { density = } \\
15 \text { dph } \\
\text { (see Fig.7.5) }\end{array}$ \\
\hline 4. & $\begin{array}{l}140 \text { cluster houses with } \\
\text { multivalent communal } \\
\text { spaces (Ch.7 Fig. 7.10) }\end{array}$ & $16 \mathrm{~m} 2$ & $24 \mathrm{~m} 2$ & $40 \mathrm{~m} 2$ & $\begin{array}{l}\text { Gross } \\
\text { density }= \\
28 \mathrm{dph} \\
\text { (Fig.7.10) }\end{array}$ \\
\hline
\end{tabular}

Table A8.4 Pinehill Crescent: summary of analysis 


\section{THE BOUNDARY - Attached townhouses}

Saddleback Grove (off Hazelwood Avenue), Karori, Wellington

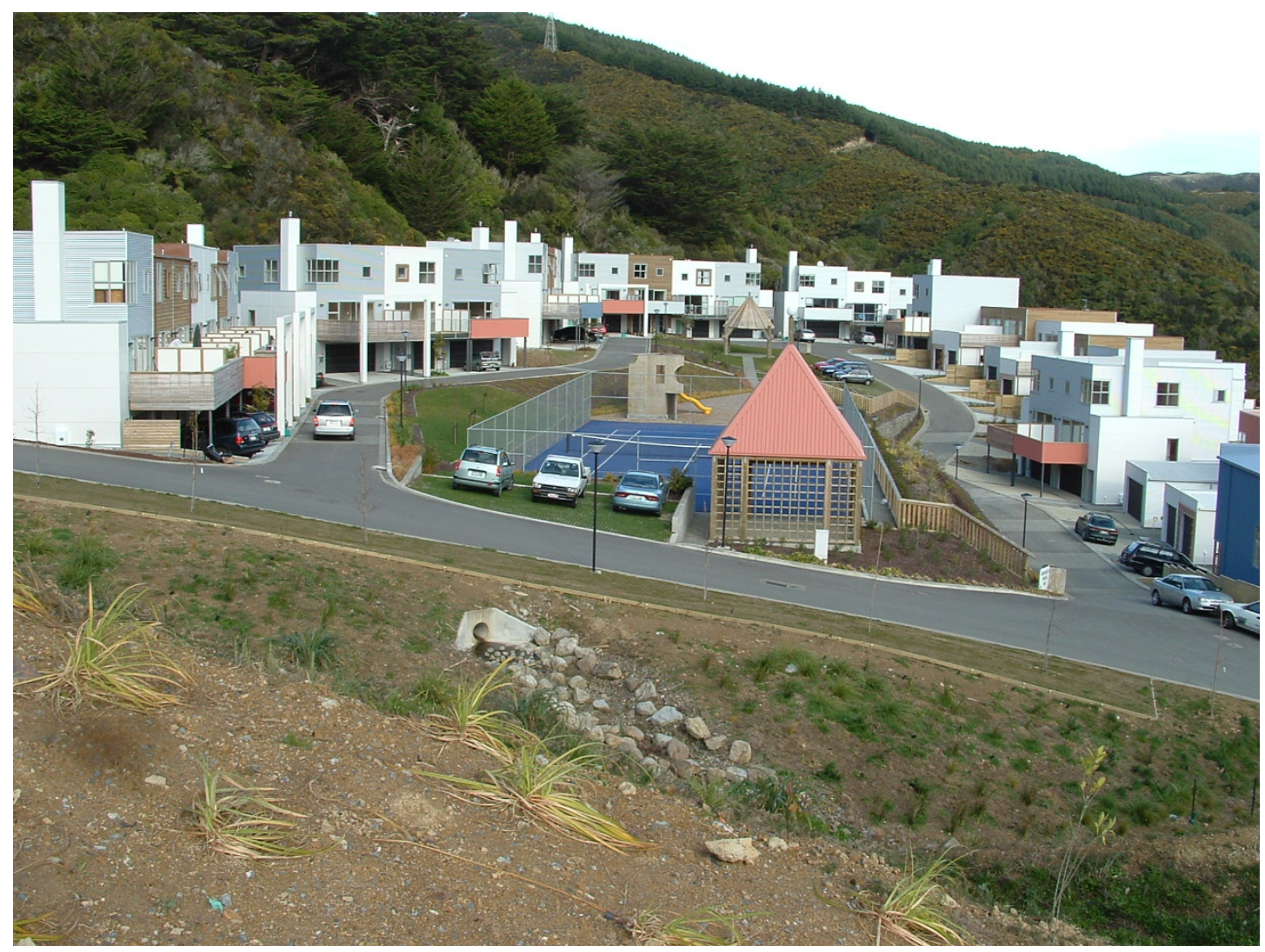

Figure A8.5 The central recreational area. The Boundary - Saddleback Grove, Karori, Wellington. This view is from the higher northern ground looking south. The large landscaped berm is in the foreground. Architect Roger Walker. (Photo: S J Pattinson)

This townhouse development consists of 82 dwellings on a 3.2856 hectare site, the density being $25 \mathrm{dph}$. There are 41 dwellings on the southern half of the site (shown in Fig. A8.5 above) arranged in a ring around a central recreation area of about 2,560m2, consisting of a summer house, tennis court, playground, gazebo, and space for a BBQ. The other 41 dwellings are on the higher ground to the north of the site, flanked by a large landscaped berm of about 3,300m2 (in the foreground of the above photo - see Fig. A8.9 for a diagrammatic plan view). 


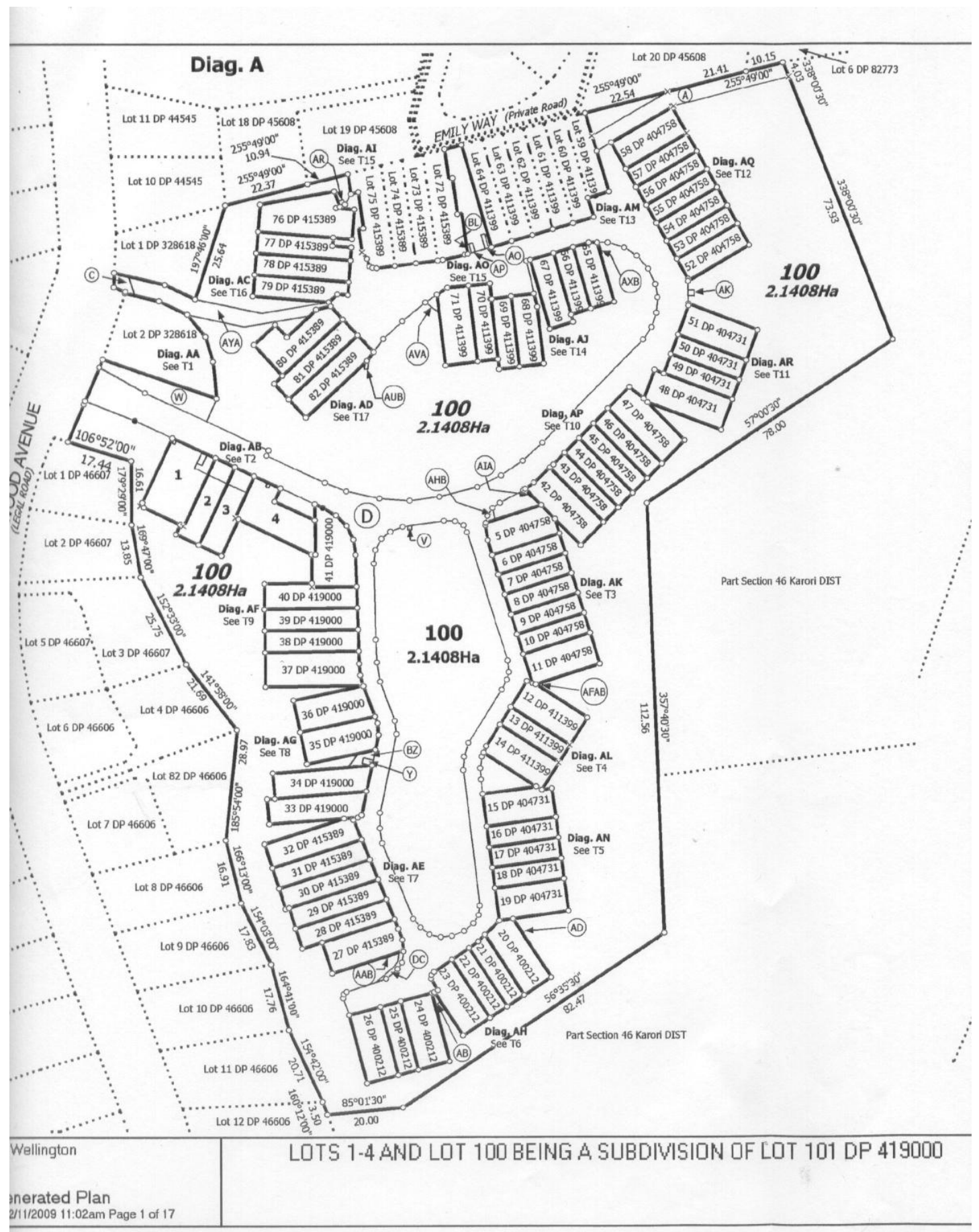

Figure A8.6 82 townhouse units at The Boundary - Saddleback Grove, Karori, Wellington (from Resource Consent documents, Wellington City Council) 


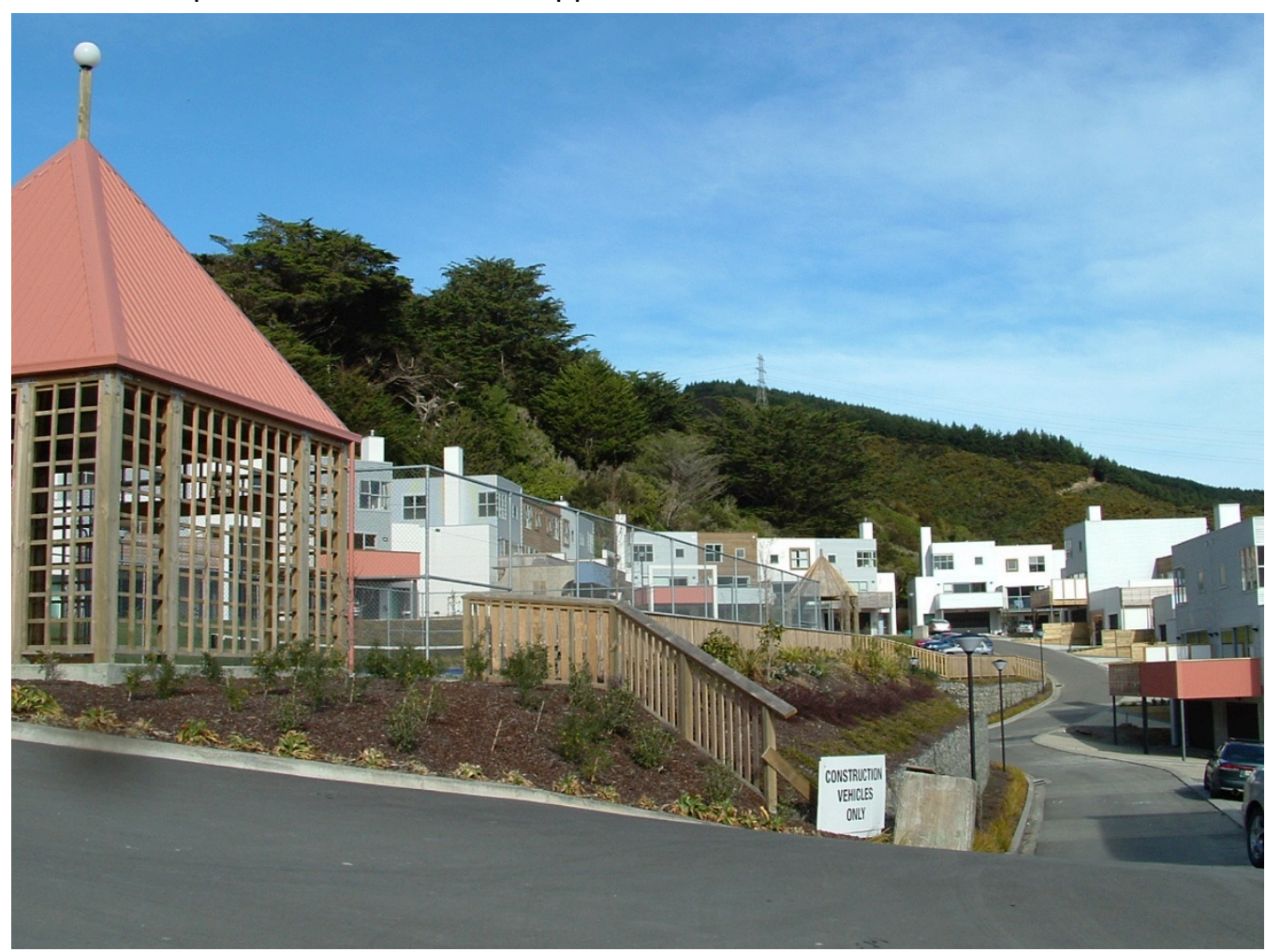

Figure A8.7 The Boundary: Houses around the recreation area. The recreational areas tend to be hilly, which may restrict the activities they could support.

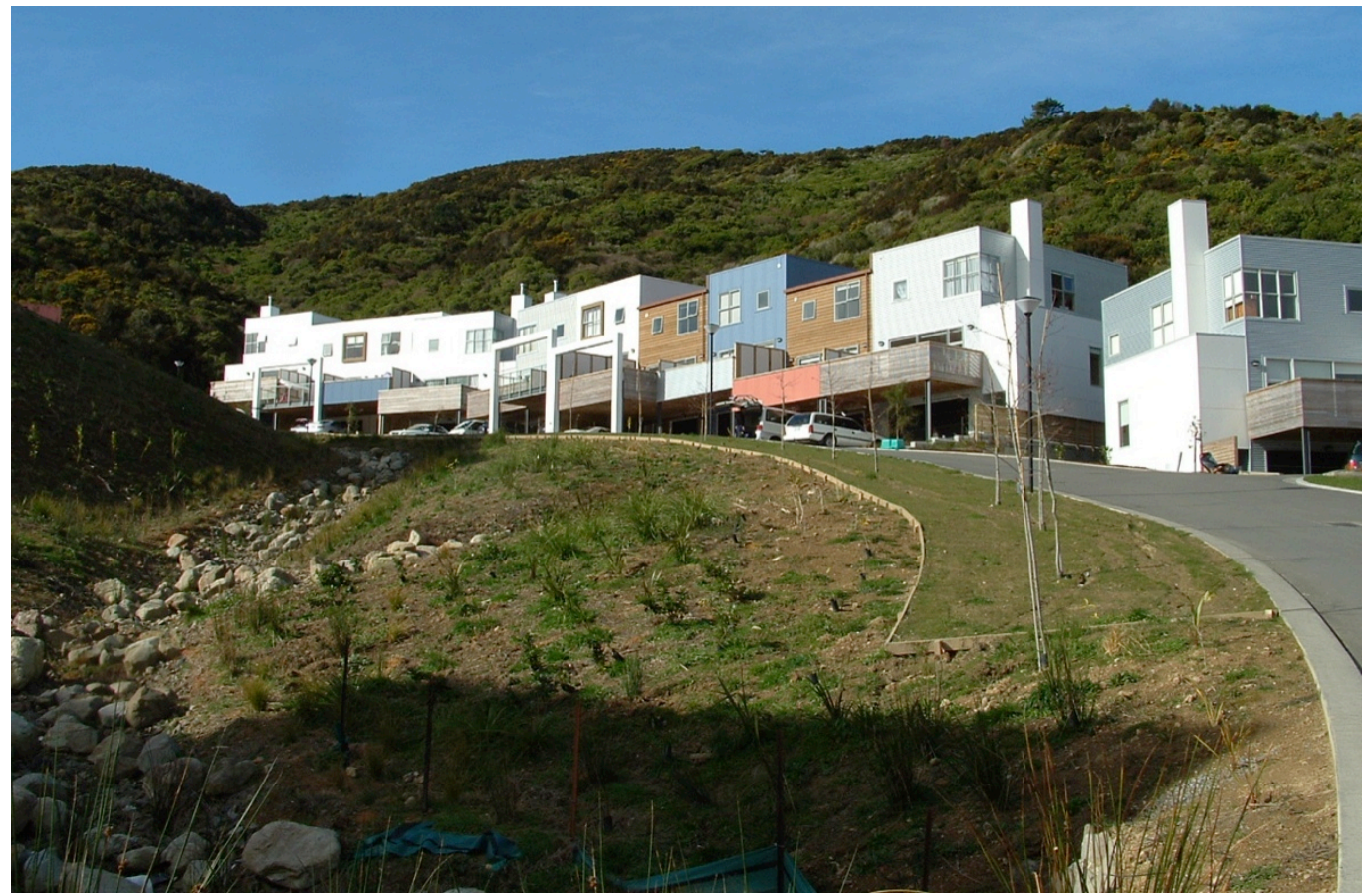

Figure A8.8 The Boundary: Houses around the landscaped berm (left and in foreground) - Saddleback Grove, Karori, Wellington. (Photo: S J Pattinson) 


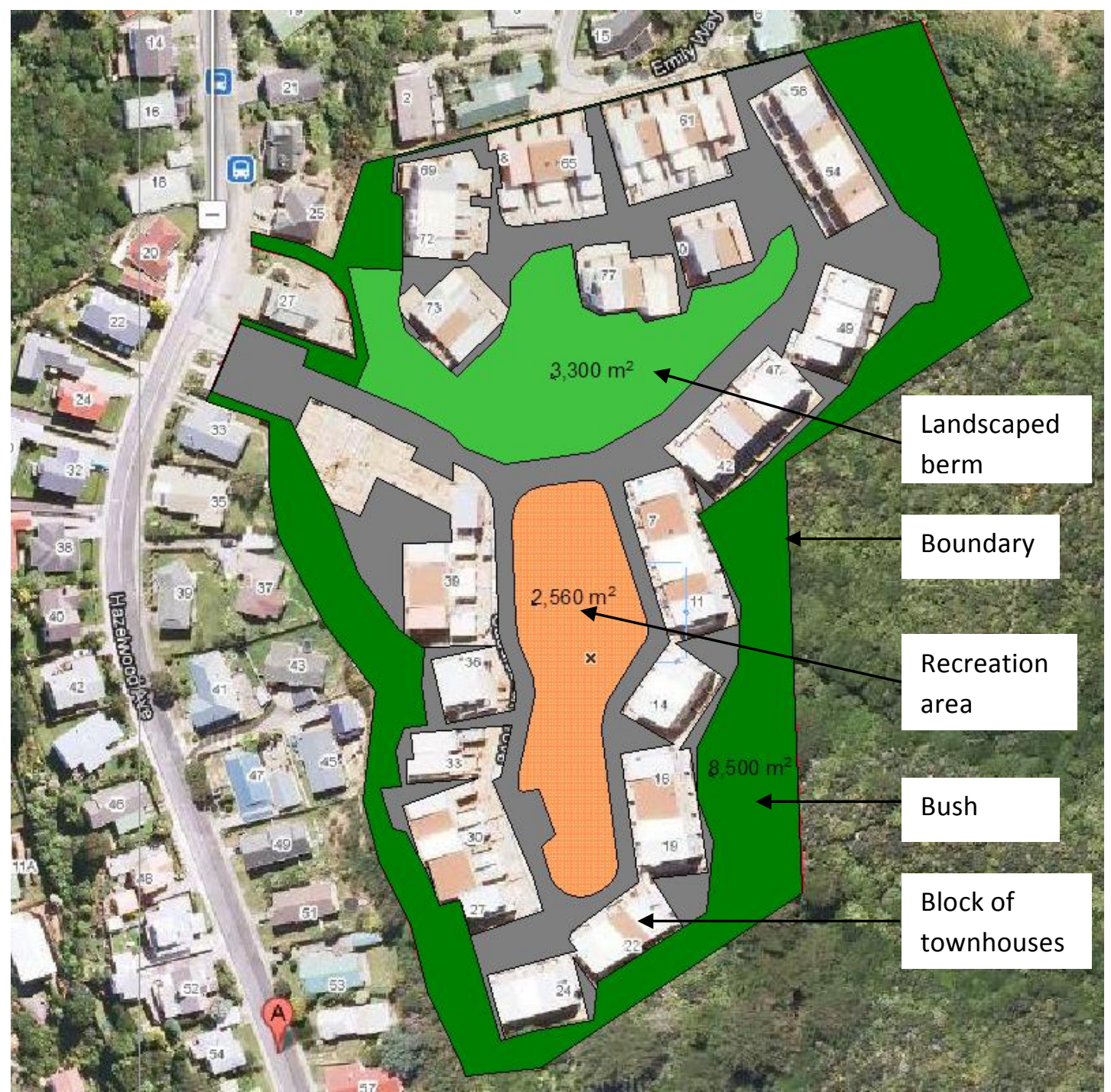

Figure A8.9 Approximate areas of communal spaces at The Boundary - Saddleback Grove, Karori, Wellington. (prepared by S J Pattinson, on Google Maps aerial view of the site)

Area of central recreational communal space

Area of landscaped berm flanking northern houses

Area of the 82 unit titles (total scaled off drawing)

Area of bush inside the boundaries of the site

Area of roading and sundry spaces

Total site area:
2,560 m2 (approx.)

3,300 m2 (approx.)

11,646 m2 (approx.)

8,500 m2 (approx.)

6,850 m2 (approx.)

$32,856 \mathrm{~m} 2$ 
Number of dwellings and bedspaces (from plans): Dwellings: Bedspaces:

- Type Aa* (1 x dble**, 2 x single bedrm) $16 \quad 64$

- Type $\mathrm{Ab}^{*}(1 \mathrm{x}$ dble**, 1 x single bedrm) $28 \quad 84$

- Type Ba $(1 \times$ dble**, $2 \times$ single bedrm $) \quad 8 \quad 32$

- Type Bb (1 x dble**, $1 \times$ single bedrm) 15

- Type C (1 1 dble**, $1 \times$ single bedrm) $12 \quad 36$

- Type D (1 x dble**, $2 \times$ single bedrm) 1 3

- Type E (1 x dble**, $1 \times$ single bedrm $) \quad 1 \quad 3$

- Type $\mathrm{F}^{*}\left(1 \times \mathrm{dble}{ }^{* *}, 1 \times\right.$ single bedrm $) \quad 1 \quad 3$

Total number of dwellings and bedspaces: $\quad 82 \quad 270$

Communal outdoor spaces***:

- Central recreational space 2,560 m2

- Landscaped berm $\quad 3,300 \mathrm{~m} 2$

Total shared space: $\quad 5,860 \mathrm{~m} 2$

Shared outdoor space / bedspace (person): $5,860 \mathrm{~m} 2 / 270=\quad 21.7 \mathrm{~m} 2$ *

Average private outdoor space per dwelling (courtyard and/or deck) (information from real estate data sheet): $\quad 25 \mathrm{~m} 2$ (average)

Total private outdoor space $82 \times 25=\quad 2,050 \mathrm{~m} 2$

Average private outdoor space / bedspace (person): 2,050m2 / $270=\quad 7.6 \mathrm{~m} 2$

Total shared and private outdoor space / bedspace: $21.7+7.6=\quad 29.3 \mathrm{~m} 2$

* Studio and study spaces not included in bedspace count. (The studios are large enough for a double bed so potentially the 45 units with studios could accommodate 90 more bedspaces).

** Double bedroom $=$ or $>12 \mathrm{~m} 2$ (Single bedroom $<12 \mathrm{~m} 2$ )

*** does not include bush areas inside the site boundaries, nor roads and sundry spaces (Fig.A8.9) 


\section{MAIDSTONE QUARTER: Stacked Townhousing}

Maidstone Quarter is a proposed housing development in Upper Hutt located on the ex-South Pacific Tyre property at 44 Blenheim Street. This proposal is not yet built. (Note that the information used in this research was obtained in 2011 from Resource Consent application documents at Upper Hutt City Council, and might not be the final documents for this proposal.)

The site is a 2.9903 hectare greenfield space in the North-west corner of the property (near the entrance to the property off Blenheim Street). The proposal consists of 127 dwellings and 10 business/storage units.

The dwellings consist of a range of types (see Fig. 13 below) all with a wide frontage $(9 \mathrm{~m})$. They are generally stacked two dwellings high in a variety of configurations.

The following tables provide an analysis of the area of the footprint of the dwellings, the number of bedspaces (persons), the area of private outdoor space per dwelling and per bedspace, the overall area of shared green space, the area of shared green space per bedspace, the total area of private and shared outdoor space per bedspace, the shape factor and orientation of such outdoor space and its relationship with indoor living areas, and an analysis of outdoor space allocated to vehicle movement and parking in this proposed development. 
Analysis of outdoor space per bedspace: Maidstone Quarter (127 dwellings, 10 business/storage units on 2.99 ha) Proposed medium density housing development on ex-South Pacific Tyre site, 44 Blenheim Street, Upper Hutt NB: 57 of the 70 residential lots (81.4\%) have two dwellings per lot, one stacked on top of the other, i.e. $57 \times 2+13=127$ total dwelling units. Areas and dimensions are approximate, based on information in Resource Consent documents (obtained from Upper Hutt City Council). A = Unit A on Ground; B = Unit B, stacked on top of Unit A House types:

Lf = "Lifestyle Home" (Ground Floor single-level 2-Bdrm flat with internal single garage) Sub-Total:

$F_{S}=$ "First Home" (Single-level 3-Bdrm flat, either on Ground or Stacked on top of another dwelling ) $\mathrm{Fm}=$ "Family Home"(Two-storey 4-Bdrm terrace house, either on Ground or Stacked on top of another dwelling ) $\mathrm{FmL}=$ "Family Home - 'L-Type' " (Single-storey 4-Bdrm home stacked on top of another dwelling) $E x=$ "Executive Home (three-level terrace house on Ground, internal double-garage, 5-Bdrms )

$$
\begin{array}{cl}
26 & 20.5 \% \\
63 & 49.6 \% \\
33 & 26.0 \% \\
4 & 3.1 \% \\
1 & 0.8 \% \\
\hline
\end{array}
$$

Total dwellings $127 \quad 100.0 \%$

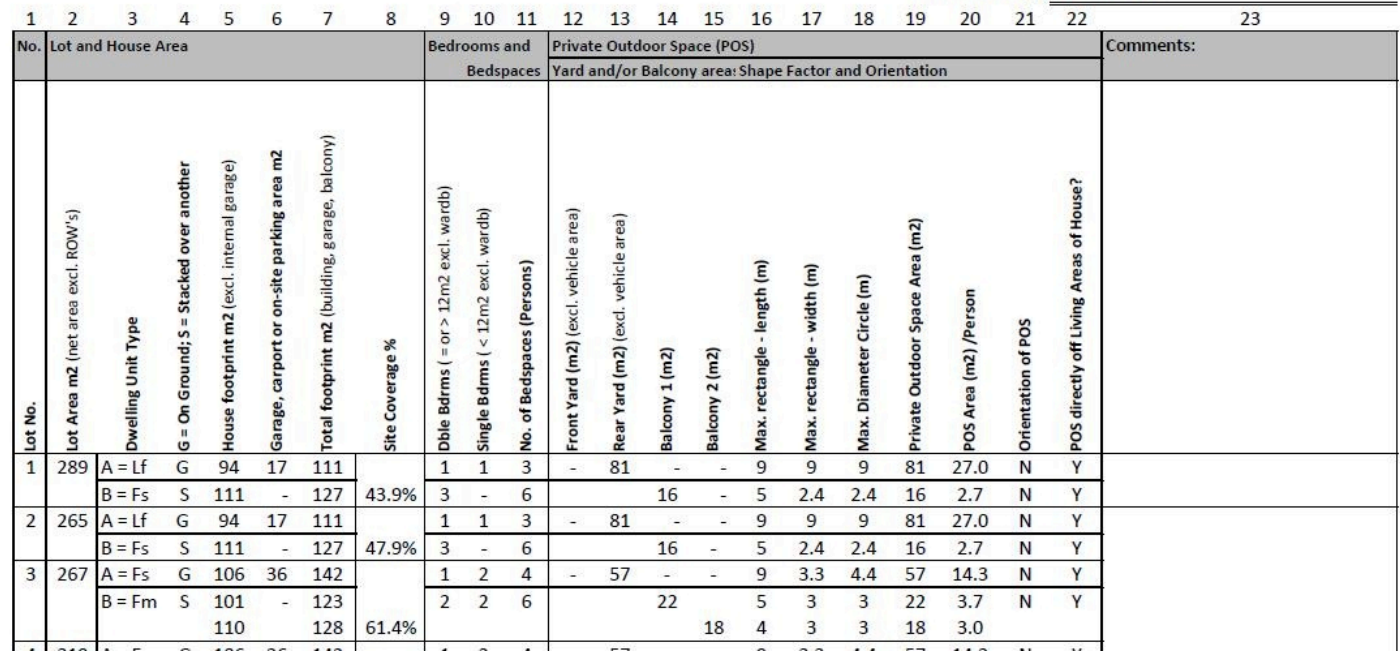

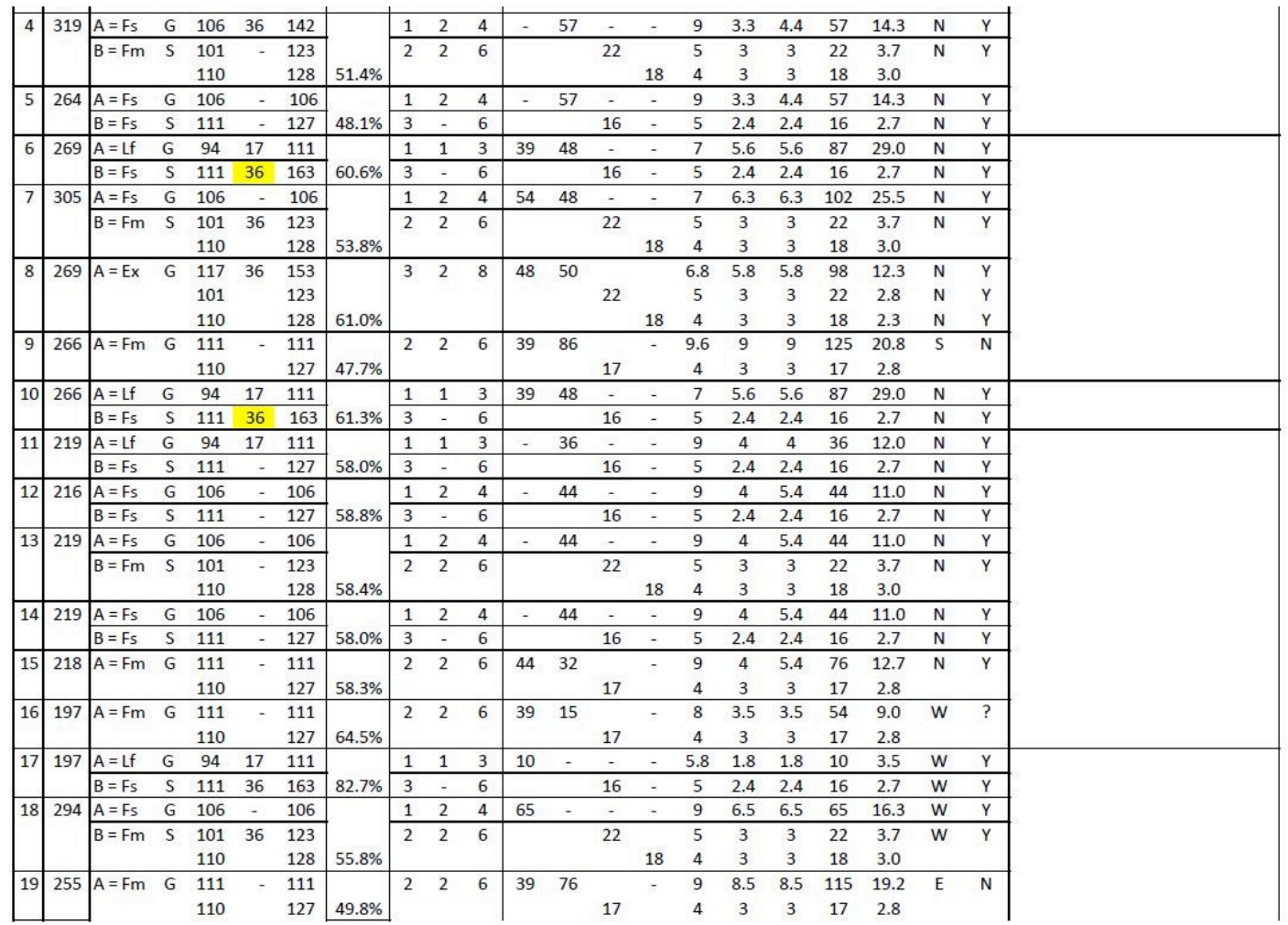

Table A8.5a - Maidstone Quarter analysis 


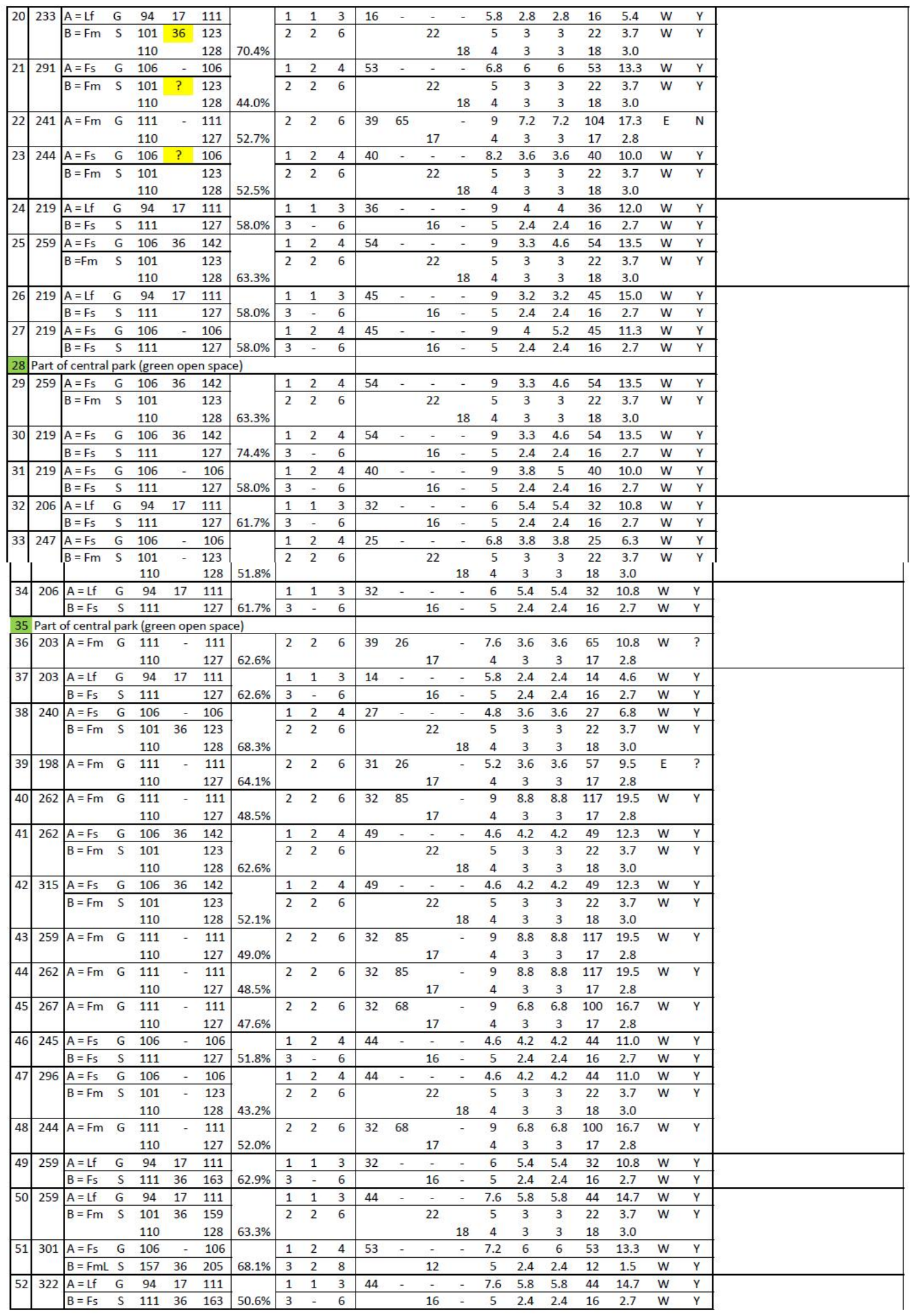

Table A8.5b - Maidstone Quarter analysis 


\begin{tabular}{|c|c|c|c|c|c|c|c|c|c|c|c|c|c|c|c|c|c|c|c|c|c|c|}
\hline \multirow[t]{2}{*}{53} & \multirow[t]{2}{*}{241} & $A=L f$ & G & 94 & 17 & 111 & \multirow[b]{2}{*}{$67.6 \%$} & 1 & 1 & 3 & 44 & - & - & - & 7.6 & 5.8 & 5.8 & 44 & 14.7 & W & $Y$ & \\
\hline & & $B=F_{s}$ & $\mathrm{~S}$ & 111 & 36 & 163 & & 3 & - & 6 & & & 16 & - & 5 & 2.4 & 2.4 & 16 & 2.7 & W & $Y$ & \\
\hline \multirow[t]{2}{*}{5} & \multirow[t]{2}{*}{281} & $A=F s$ & G & 106 & - & 106 & \multirow[b]{2}{*}{$73.0 \%$} & 1 & 2 & 4 & 53 & - & - & - & 7.2 & 6 & 6 & 53 & 13.3 & W & \multirow{2}{*}{$\begin{array}{l}Y \\
Y\end{array}$} & \\
\hline & & $\mathrm{B}=\mathrm{FmL}$ & $\mathrm{S}$ & 157 & 36 & 205 & & 3 & 2 & 8 & \multicolumn{4}{|c|}{12} & 5 & 2.4 & 2.4 & 12 & 1.5 & W & & \\
\hline \multirow[t]{2}{*}{55} & \multirow[t]{2}{*}{309} & $A=L f$ & G & 94 & 17 & 111 & \multirow[b]{2}{*}{$52.8 \%$} & 1 & 1 & 3 & 44 & - & - & - & 7.6 & 5.8 & 5.8 & 44 & 14.7 & W & \multirow{2}{*}{$\frac{Y}{Y}$} & \\
\hline & & $B=F s$ & $\mathrm{~s}$ & 111 & 36 & 163 & & 3 & - & 6 & & & 16 & - & 5 & 2.4 & 2.4 & 16 & 2.7 & W & & \\
\hline \multirow[t]{2}{*}{56} & \multirow[t]{2}{*}{236} & $A=L f$ & G & 94 & 17 & 111 & \multirow[b]{2}{*}{$69.1 \%$} & 1 & 1 & 3 & 40 & - & - & - & 6.9 & 5.8 & 5.8 & 40 & 13.3 & W & \multirow{2}{*}{$\frac{y}{y}$} & \\
\hline & & $B=F s$ & $\mathrm{~s}$ & 111 & 36 & 163 & & 3 & - & 6 & & & 16 & - & 5 & 2.4 & 2.4 & 16 & 2.7 & W & & \\
\hline 57 & 293 & $\mathrm{~A}=\mathrm{Fs}$ & G & 106 & - & 106 & & 1 & 2 & 4 & 53 & - & - & - & 7.2 & 6 & 6 & 53 & 13.3 & W & $Y$ & \\
\hline & & $\mathrm{B}=\mathrm{FmL}$ & $\mathrm{s}$ & 157 & 36 & 205 & $70.0 \%$ & 3 & 2 & 8 & & & 12 & & 5 & 2.4 & 2.4 & 12 & 1.5 & W & $\mathrm{Y}$ & \\
\hline 58 & 307 & $A=$ Lf & G & 94 & 17 & 111 & & 1 & 1 & 3 & 44 & - & - & - & 7.6 & 5.8 & 5.8 & 44 & 14.7 & W & $Y$ & \\
\hline & & $B=F s$ & $\mathrm{~S}$ & 111 & 36 & 163 & $53.1 \%$ & 3 & - & 6 & & & 16 & - & 5 & 2.4 & 2.4 & 16 & 2.7 & W & $Y$ & \\
\hline 59 & 236 & $A=L f$ & G & 94 & 17 & 111 & & 1 & 1 & 3 & 40 & - & - & - & 6.9 & 5.8 & 5.8 & 40 & 13.3 & W & $\mathrm{Y}$ & \\
\hline & & $B=F s$ & $\mathrm{~S}$ & 111 & 36 & 163 & $69.1 \%$ & 3 & - & 6 & & & 16 & - & 5 & 2.4 & 2.4 & 16 & 2.7 & W & $Y$ & \\
\hline 60 & 314 & $A=F s$ & G & 106 & - & 106 & & 1 & 2 & 4 & 53 & - & - & - & 7.2 & 6 & 6 & 53 & 13.3 & W & $Y$ & \\
\hline & & $\mathrm{B}=\mathrm{FmL}$ & $S$ & 157 & 36 & 205 & $65.3 \%$ & 3 & 2 & 8 & & & 12 & & 5 & 2.4 & 2.4 & 12 & 1.5 & W & $Y$ & \\
\hline 61 & 262 & $A=F_{s}$ & G & 106 & - & 106 & & 1 & 2 & 4 & 53 & - & - & - & 7.2 & 6 & 6 & 53 & 13.3 & W & $Y$ & \\
\hline & & $\mathrm{B}=\mathrm{Fm}$ & $\mathrm{s}$ & 101 & 36 & 159 & & 2 & 2 & 6 & & & 22 & & 5 & 3 & 3 & 22 & 3.7 & W & $Y$ & \\
\hline & & & & 110 & & 128 & $62.6 \%$ & & & & & & & 18 & 4 & 3 & 3 & 18 & 3.0 & & & \\
\hline 62 & 218 & $A=F s$ & G & 106 & - & 106 & & 1 & 2 & 4 & 46 & - & - & - & 9 & 4.4 & 5.2 & 46 & 11.5 & $\mathrm{~N}$ & $Y$ & \\
\hline & & $B=F s$ & $\mathrm{~s}$ & 111 & & 127 & $58.3 \%$ & 3 & - & 6 & & & 16 & - & 5 & 2.4 & 2.4 & 16 & 2.7 & $\mathrm{~N}$ & $Y$ & \\
\hline 63 & 216 & $A=F_{s}$ & G & 106 & - & 106 & & 1 & 2 & 4 & 46 & - & - & - & 9 & 4.4 & 5.2 & 46 & 11.5 & $\mathrm{~N}$ & $\mathrm{Y}$ & \\
\hline & & $B=F m$ & S & 101 & - & 123 & & 2 & 2 & 6 & & & 22 & & 5 & 3 & 3 & 22 & 3.7 & $\mathrm{~N}$ & $Y$ & \\
\hline & & & & 110 & & 128 & $59.3 \%$ & & & & & & & 18 & 4 & 3 & 3 & 18 & 3.0 & & & \\
\hline 64 & 216 & $A=F s$ & G & 106 & - & 106 & & 1 & 2 & 4 & 46 & - & - & - & 9 & 4.4 & 5.2 & 46 & 11.5 & $\mathrm{~N}$ & $Y$ & \\
\hline & & $B=F s$ & $\mathrm{~s}$ & 111 & & 127 & $58.8 \%$ & 3 & - & 6 & & & 16 & - & 5 & 2.4 & 2.4 & 16 & 2.7 & $\mathrm{~N}$ & $Y$ & \\
\hline 65 & 216 & $A=L f$ & G & 94 & 17 & 111 & & 1 & 1 & 3 & 40 & - & - & - & 9 & 4.4 & 4.4 & 40 & 13.2 & $\mathrm{~N}$ & $Y$ & \\
\hline & & $B=F s$ & $\mathrm{~S}$ & 111 & & 127 & $58.8 \%$ & 3 & - & 6 & & & 16 & - & 5 & 2.4 & 2.4 & 16 & 2.7 & $\mathrm{~N}$ & $Y$ & \\
\hline 66 & 217 & $A=L f$ & G & 94 & 17 & 111 & & 1 & 1 & 3 & 40 & - & - & - & 9 & 4.4 & 4.4 & 40 & 13.2 & $\mathrm{~N}$ & $Y$ & \\
\hline & & $\mathrm{B}=\mathrm{Fm}$ & $\mathrm{s}$ & 101 & & 123 & & 2 & 2 & 6 & & & 22 & & 5 & 3 & 3 & 22 & 3.7 & $\mathrm{~N}$ & $\mathrm{Y}$ & \\
\hline & & & & 110 & & 128 & $59.0 \%$ & & & & & & & 18 & 4 & 3 & 3 & 18 & 3.0 & & & \\
\hline 67 & 217 & $A=L f$ & G & 94 & 17 & 111 & & 1 & 1 & 3 & 40 & - & - & - & 9 & 4.4 & 4.4 & 40 & 13.2 & $\mathrm{~N}$ & $\mathrm{Y}$ & \\
\hline & & $B=F_{s}$ & $\mathrm{~S}$ & 111 & & 127 & $58.5 \%$ & 3 & - & 6 & & & 16 & - & 5 & 2.4 & 2.4 & 16 & 2.7 & $\mathrm{~N}$ & $Y$ & \\
\hline 68 & 216 & $A=L f$ & G & 94 & 17 & 111 & & 1 & 1 & 3 & 40 & - & - & - & 9 & 4.4 & 4.4 & 40 & 13.2 & $\mathrm{~N}$ & $Y$ & \\
\hline & & $\mathrm{B}=\mathrm{Fm}$ & $\mathrm{s}$ & 101 & & 123 & & 2 & 2 & 6 & & & 22 & & 5 & 3 & 3 & 22 & 3.7 & $\mathrm{~N}$ & $\mathrm{Y}$ & \\
\hline & & & & 110 & & 128 & $59.3 \%$ & & & & & & & 18 & 4 & 3 & 3 & 18 & 3.0 & & & \\
\hline 69 & 216 & $A=F_{s}$ & G & 106 & - & 106 & & 1 & 2 & 4 & 46 & - & - & - & 9 & 4.4 & 5.2 & 46 & 11.5 & $\mathrm{~N}$ & $\mathrm{Y}$ & \\
\hline & & $B=F s$ & $S$ & 111 & & 127 & $58.8 \%$ & 3 & - & 6 & & & 16 & - & 5 & 2.4 & 2.4 & 16 & 2.7 & $\mathrm{~N}$ & $Y$ & \\
\hline 70 & 216 & $A=L f$ & G & 94 & 17 & 111 & & 1 & 1 & 3 & 40 & - & - & - & 9 & 4.4 & 4.4 & 40 & 13.2 & $\mathrm{~N}$ & $Y$ & \\
\hline & & $B=F_{s}$ & $\mathrm{~s}$ & 111 & & 127 & $58.8 \%$ & 3 & - & 6 & & & 16 & - & 5 & 2.4 & 2.4 & 16 & 2.7 & $\mathrm{~N}$ & $Y$ & \\
\hline
\end{tabular}

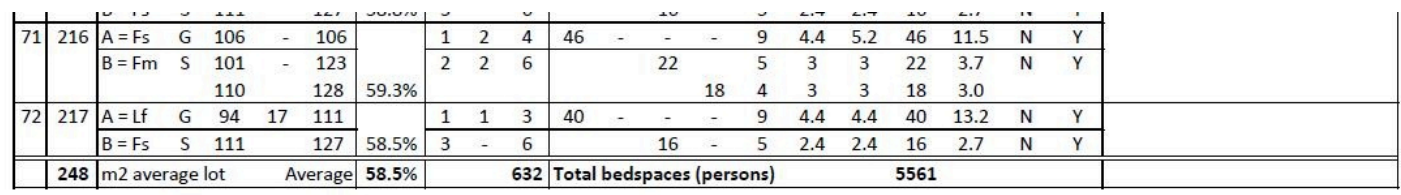

632 Total bedspaces (persons) $\div 127$ Dwellings

5.0 Average bedspaces per dwelling
$5561 \mathrm{~m} 2$ total private outdoor space

$\div 127$ Dwellings

$43.8 \mathrm{~m} 2$ average private outdoor space per dwelling

$\div 5.0$ average bedspaces (persons) per dwelling

$8.8 \mathrm{~m} 2$ ave. private outdoor space / bedspace (person)

Green Space $1110 \mathrm{~m}$ Green Space $2450 \mathrm{~m} 2$

Green Space $3436 \mathrm{~m} 2$

Green Space $4240 \mathrm{~m} 2$

Green Space $5120 \mathrm{~m}$

Green Space $6100 \mathrm{~m} 2$

Green Space $7 \quad 124 \mathrm{~m} 2$

Total Green Open Space $\overline{1580 \mathrm{~m} 2 \text { approx. }}$

(refer drawing sheet 6 for common green open space per person $=1,580 \mathrm{~m} 2 \div 632=2.5 \mathrm{~m} 2$ ) $\quad+2.5 \mathrm{~m} 2$ common green open space per person

$\overline{11.3} \mathrm{~m} 2$ total private and common outdoor space/person

Table A8.5c - Maidstone Quarter analysis 


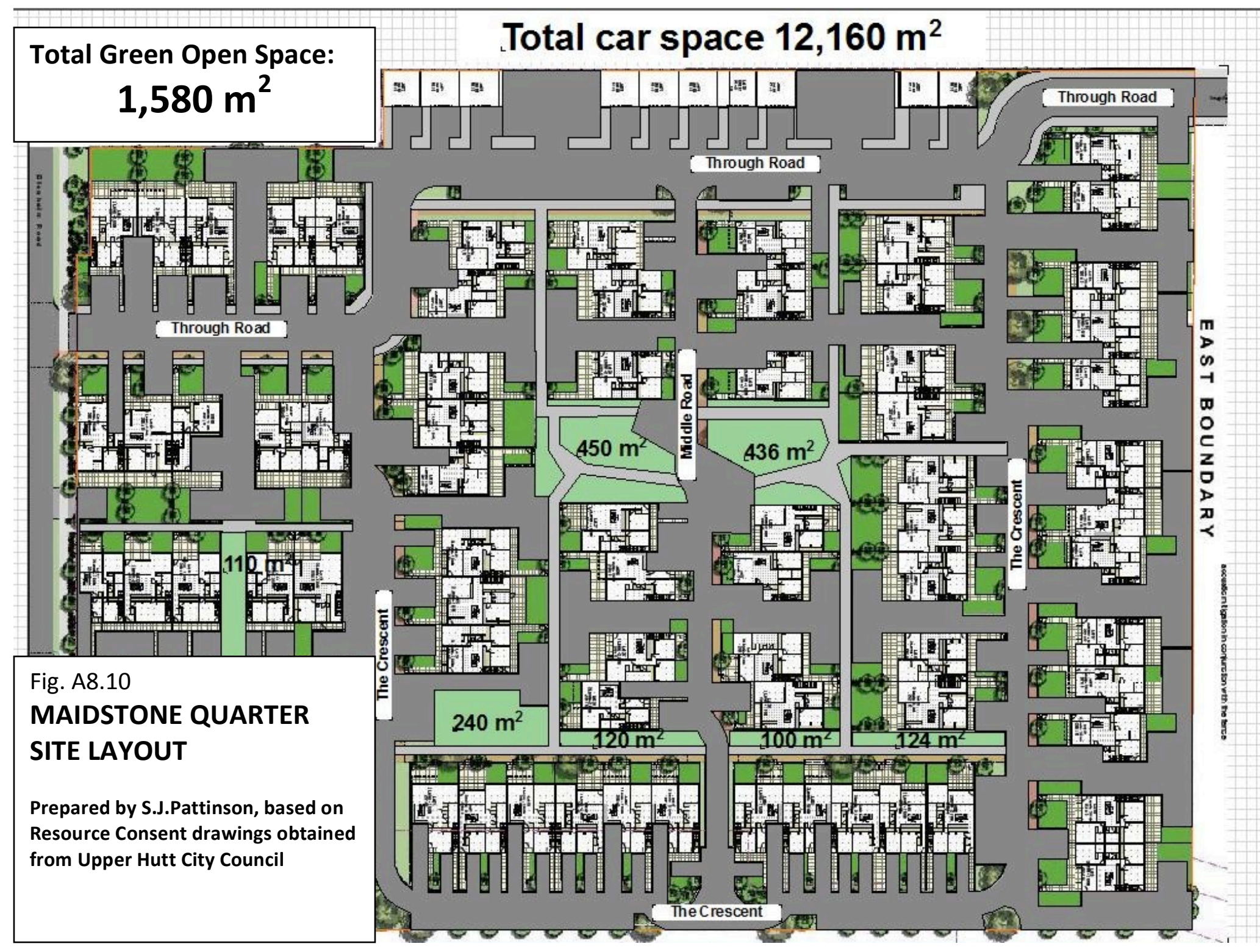




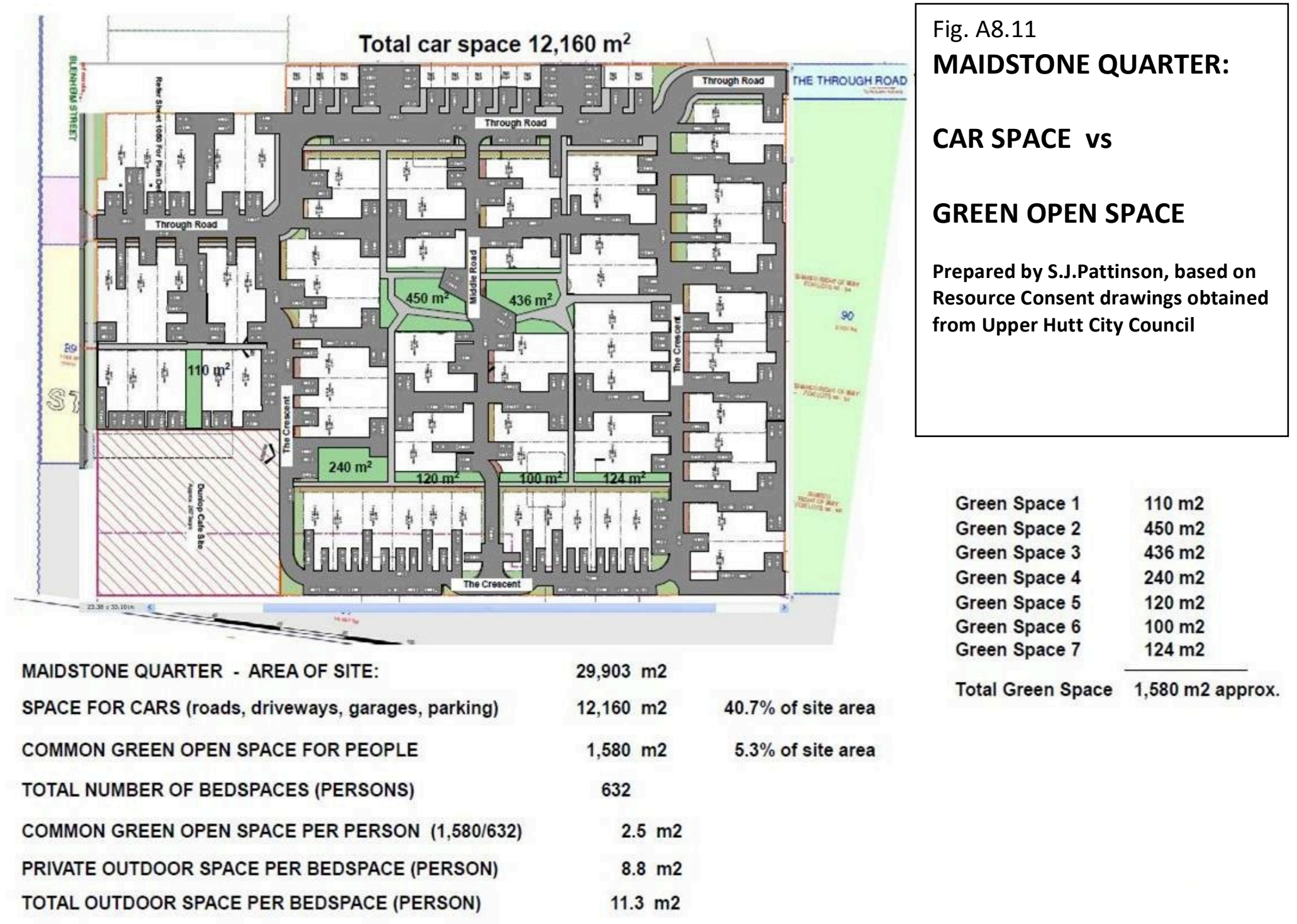




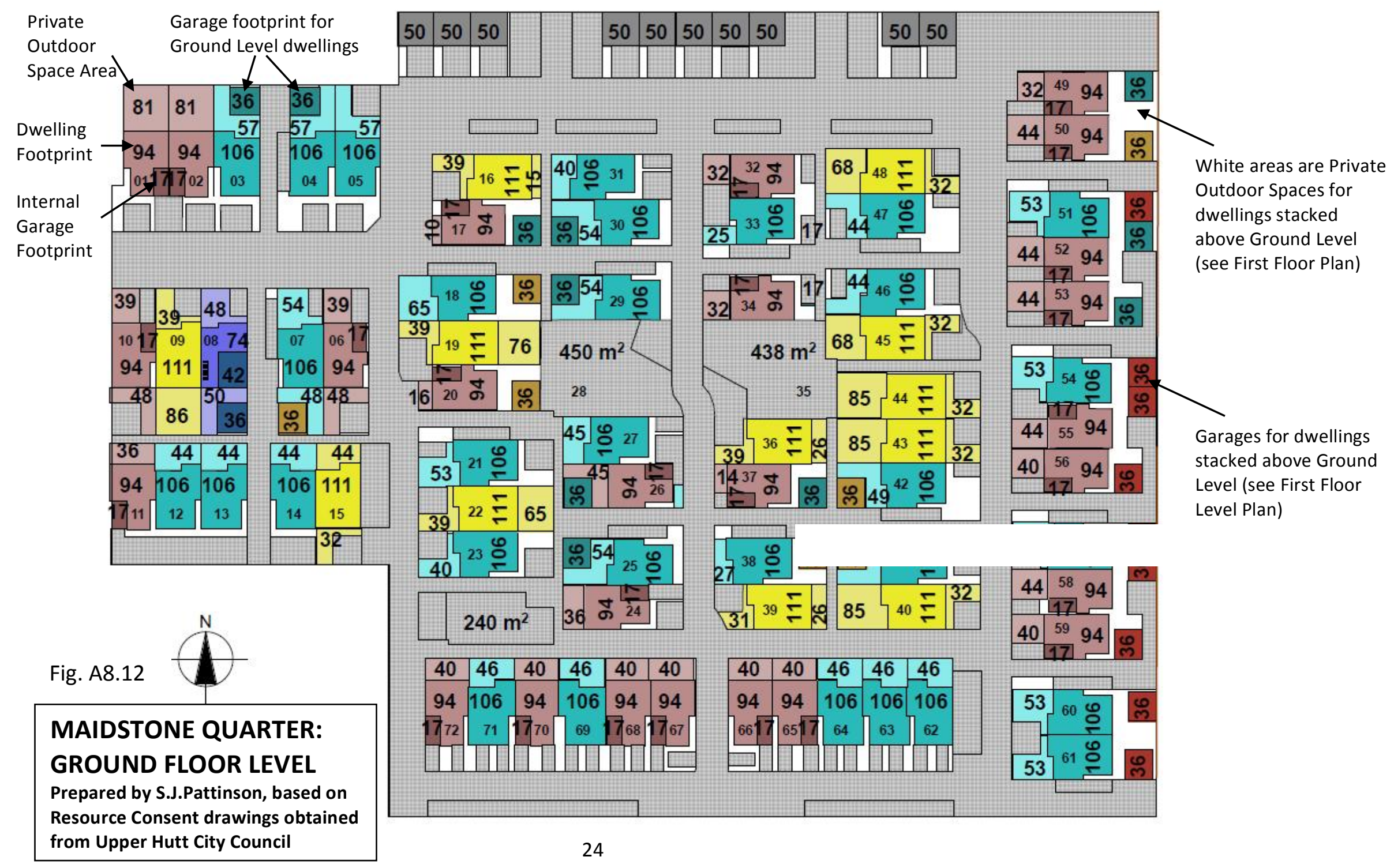




\section{KEY TO HOUSE TYPES}

Figure:
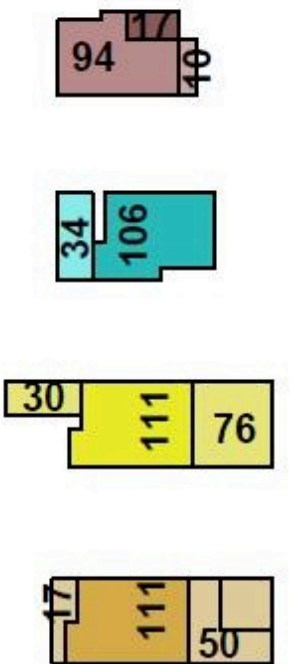

"Family Home"

(elevated $3 \mathrm{~m}$ above GL)

See 'Turnkey Plan'

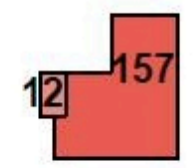

"Family Home L-Plan" (elevated $3 \mathrm{~m}$ above $\mathrm{GL}$ )

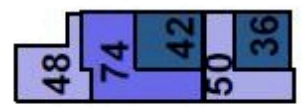

"Family Home"

(elevated $3 \mathrm{~m}$ above GL)

See 'Turnkey Plan'

Fig. A8.13 MAIDSTONE QUARTER Prepared by S.J.Pattinson,

\section{based on Resource Consent drawings obtained from Upper Hutt City Council}

Description:

Grd Floor flat 2-Bdrms $94 \mathrm{~m} 2$ internal single garage $17 \mathrm{~m} 2$ )

Grd or First Floor single storey flat 3-Bdrms 106m2

Two-storey terrace house 4-Bdrms $111 \mathrm{~m} 2 \times 2$ levels

Two-storey terrace house 4-Bdrms $111 \mathrm{~m} 2 \times 2$ levels

Single Level Dwelling on First Floor -4 or 5 Bdrms $157 \mathrm{~m} 2$

Three-storey terrace house 4 or 5 Bdrms $368 \mathrm{~m} 2 \mathrm{o} / \mathrm{a}$

Private Outdoor Space:
Private Outdoor Space varies.
The example shown has $10 \mathrm{~m} 2$
(see Lot 17 )

Private Outdoor Space varies The example shown has $40 \mathrm{~m} 2$ (see Lot 31)

Private Outdoor Space varies. The example shown has $38 \mathrm{~m} 2$ front yard and $76 \mathrm{~m} 2$ rear yard front yard and

Private Outdoor Space varies. The example shown has $38 \mathrm{~m} 2$ front yard and $76 \mathrm{~m} 2$ rear yard (see Lot 19)

Private Outdoor Space 1st FL Balcony $12 \mathrm{~m} 2$ (see Lot 54)

Private Outdoor Space includes front and reay yards and balconies (see Lot 08)
Shape Factor (Maximum rectangle that can be contained in the private outdoor space):

Varies. The available private outdoor space in the example shown can contain a rectangle $1.7 \mathrm{~m} \times 5.9 \mathrm{~m}=10 \mathrm{~m} 2$ are (see Lot 17)

Varies. The available private outdoor space in the example shown can contain a rectangle $1.7 \mathrm{~m} \times 5.9 \mathrm{~m}=10 \mathrm{~m} 2$ area (see Lot 17)

Varies. The available private Varies. The available private outdoor space in the example shown can contain a rectangle (see Lot 17)

Varies. The available private outdor space in the pruate outdoor space in the example shown can contain a rectang (see Lot 17)

Varies. $5 \mathrm{~m} \times 2.4 \mathrm{~m}=12 \mathrm{~m} 2$ area (see Lot 54)

Varies. The available private outdoor space in the front yard of the example shown can contain a rectangle $6.8 \mathrm{~m} \times 5.8 \mathrm{~m}$ $=40 \mathrm{~m} 2$ area (see Lot 08) 


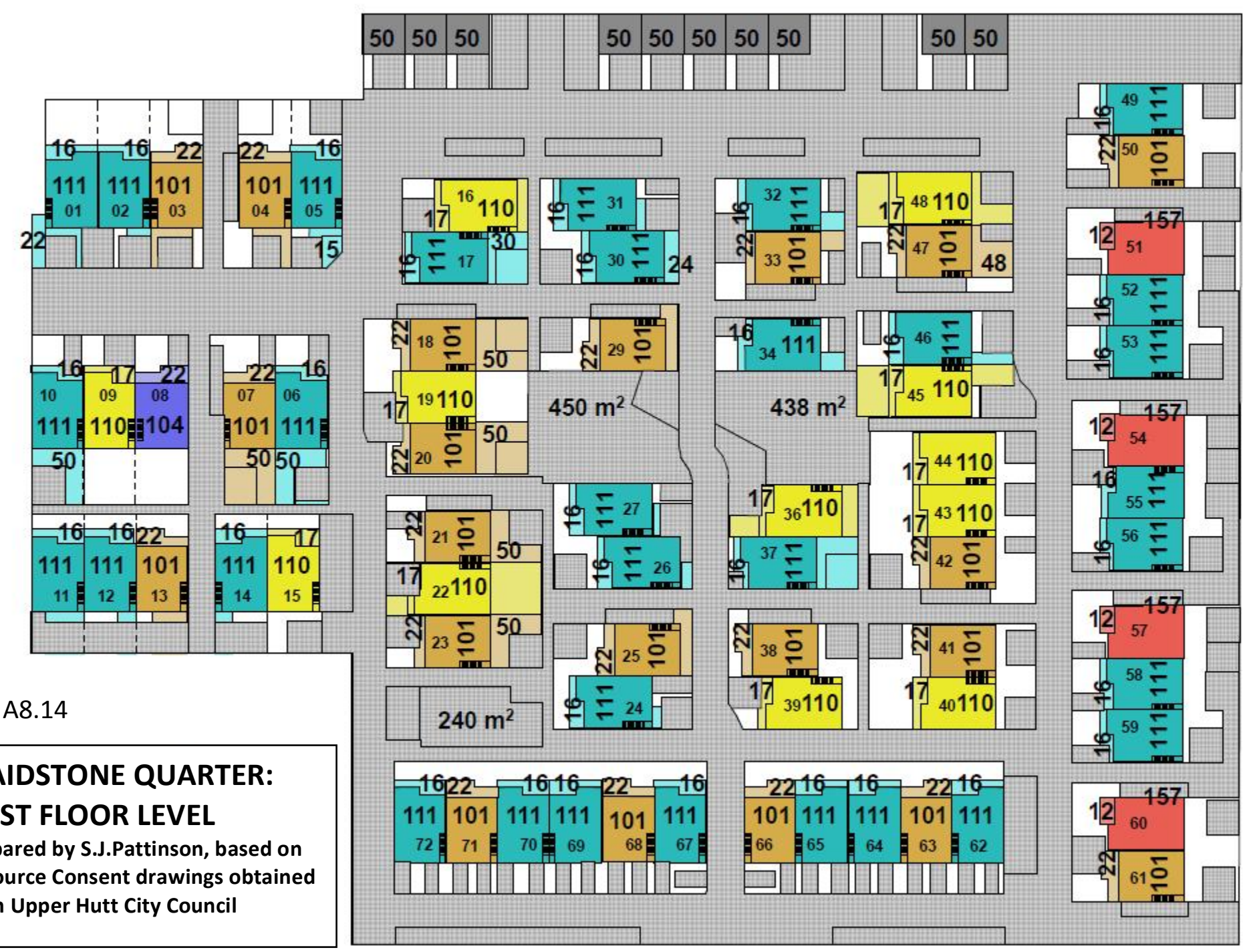




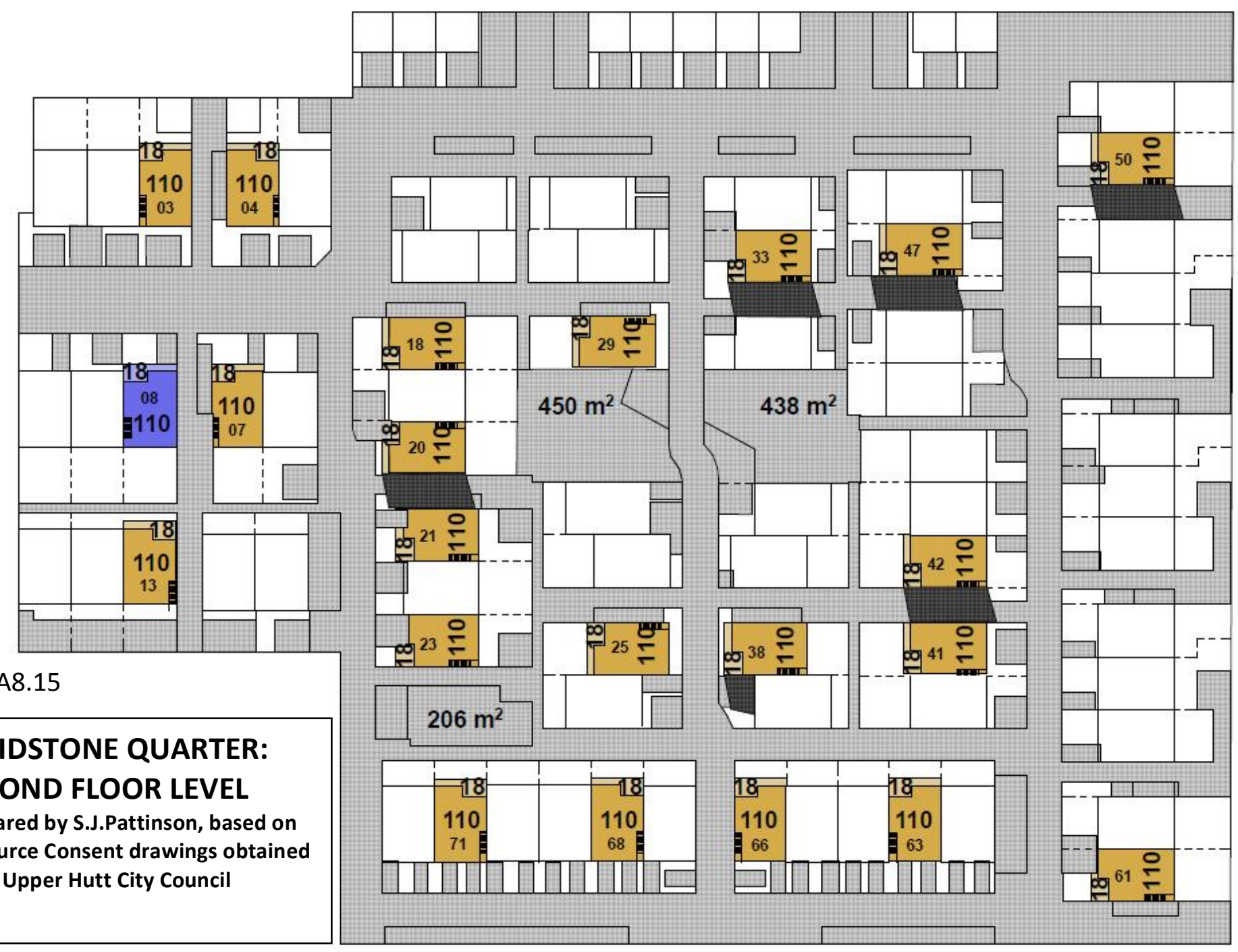




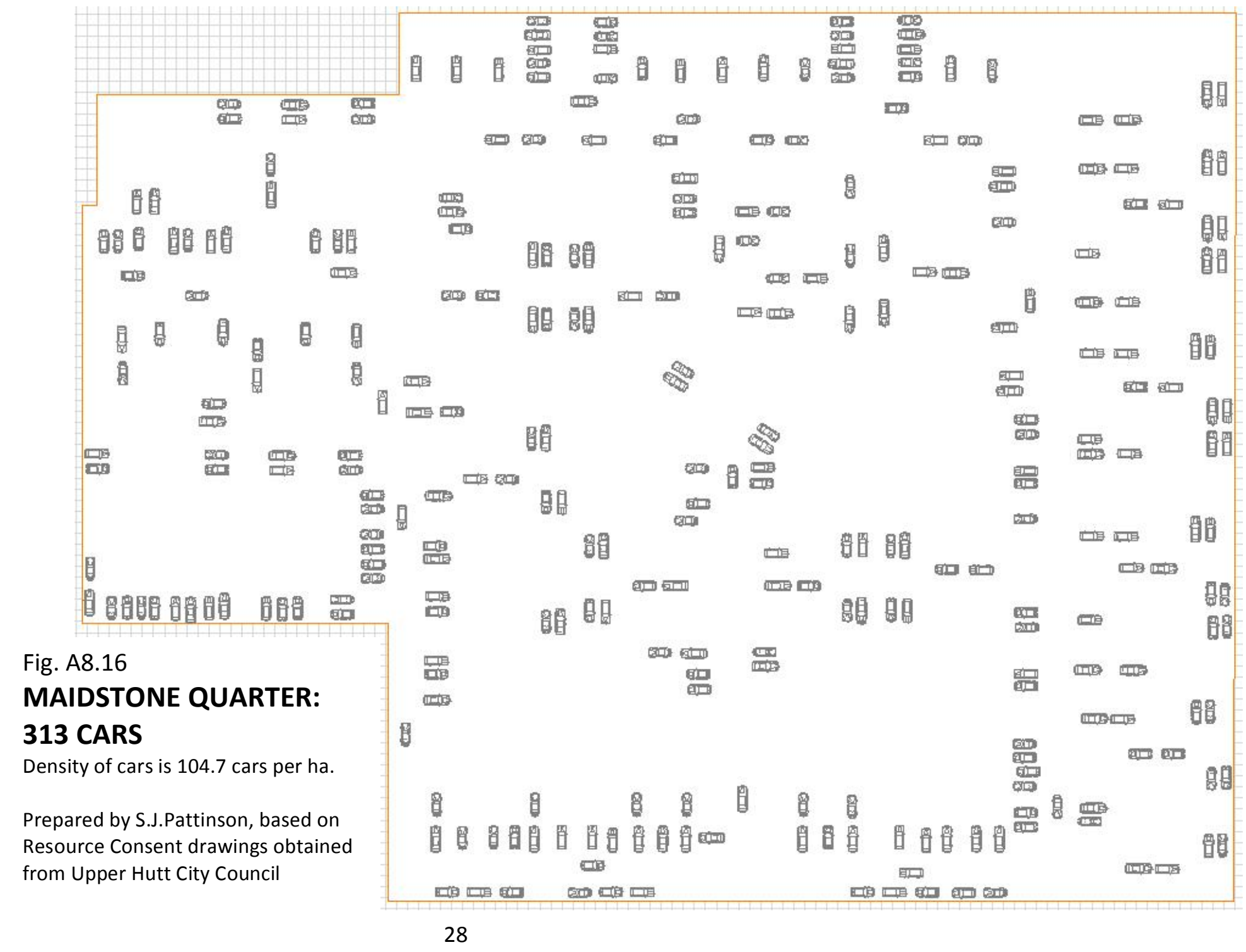




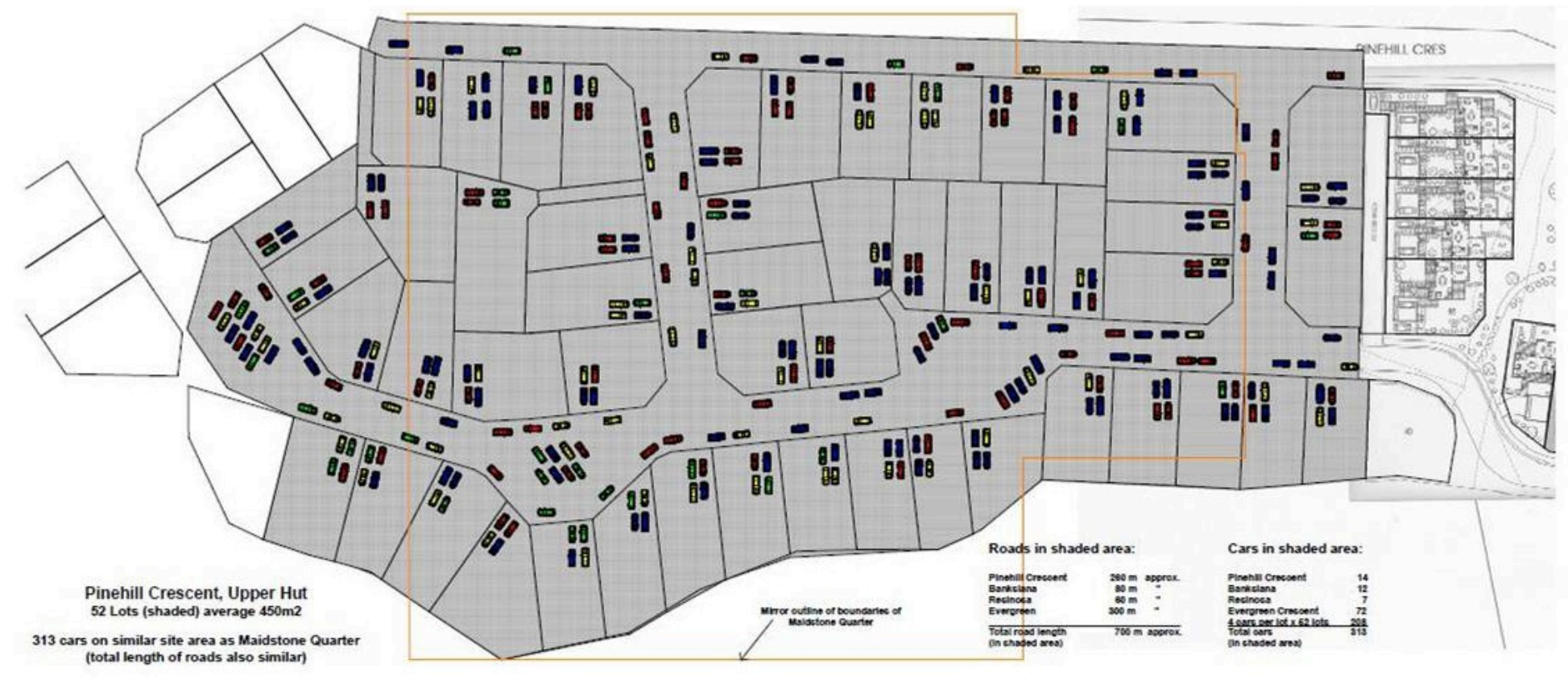

This is a plan of Pinehill Crescent subdivision, Upper Hutt.

Fig. A8.17

MAIDSTONE QUARTER:

\section{CARS}

Prepared by S.J.Pattinson, based on Resource Consent drawings obtained from Upper Hutt City Council
The shaded area on this plan is equivalent to the total area of Maidstone Quarter.

If Pinehill Crescent had the same density of cars per hectare as Maidstone Quarter (104.7 cars per ha), then the total cars on the shaded area of Pinehill Crescent would be 313, as shown above 4 cars on every lot, parking islands in roads full and all kerb space full. 
In summary, the Maidstone Quarter metrics are:

\begin{tabular}{|l|l|l|c|l|l|}
\hline Type of Housing & $\begin{array}{l}\text { Private } \\
\text { outdoor } \\
\text { space per } \\
\text { bedspace } \\
\text { (person) }\end{array}$ & $\begin{array}{l}\text { Shared } \\
\text { outdoor } \\
\text { space per } \\
\text { bedspace } \\
\text { (person) }\end{array}$ & $\begin{array}{l}\text { Total } \\
\text { outdoor } \\
\text { space per } \\
\text { bedspace } \\
\text { (person) }\end{array}$ & Comment: \\
\hline $\begin{array}{l}\text { Stacked townhousing (an as } \\
\text { yet unbuilt proposal } \\
\text { submitted to Upper Hutt } \\
\text { City Council for Resource } \\
\text { Consent): (see Fig. A8.10) }\end{array}$ & $8.8 \mathrm{~m} 2$ & $2.5 \mathrm{~m} 2$ & $11.3 \mathrm{~m} 2$ & $\begin{array}{l}127+10 \\
\text { units on } \\
2.99 \text { ha }=> \\
\text { Density }= \\
45.8 \mathrm{dph} \\
\text { (Fig. A8.10) }\end{array}$ \\
\hline
\end{tabular}

Table A8.6 Maidstone Quarter: summary of analysis 


\section{THE ALTAIR - Attached townhouses (2, 3 and 4 storeys): 108 - 126 Rintoul Street, Newtown, Wellington}

The following data for this development is taken from the Ministry for the Environment (MfE) Case Study Three: 'The Altair', 2011 (Draft). This development of $71 \mathrm{dwellings}$ on a 1.0ha site (gross density $71 \mathrm{dph}$ - includes all open spaces) has two open space landscaped areas. (Photos in this section are by S.J. Pattinson).

This development is located within 7 minutes walk of the main shopping street in Newtown. The site is $100 \mathrm{~m} \times 100 \mathrm{~m}=1.0$ ha area. All dwellings except two have lockup garages providing car-spaces for a total of 80 cars (some dwellings have double garages). Two dwellings have one car park space each, plus there are 13 visitor car park spaces. Hence the total on-site provision for cars is 95 car spaces.

Of the three case studies in this urban design investigation by Ministry for the Environment (the other two being Chester Courts, Christchurch and Stonefields, Auckland) The Altair scored the highest overall assessment (83 out of 100 points). The MfE case study methodology used for assessment consisted of evaluating the development against 20 criteria spread over four urban design 'indicators'. Each of the 20 criteria was given a score out of 5, the total possible score being 100 .

Outdoor space was assessed under the urban design 'indicator' titled "Site Context and Layout". Within this indicator, the criteria relating to outdoor space were "Landscape Coverage", "Outdoor Living Space" and "Car Parking and Access". Two further criteria from the urban design 'indicator' titled "Internal Configurations" relating to outdoor space were "Internal/External Relationships (i.e. internal connection to private outdoor space)" and "Visual Privacy (with respect to overlooking neighbouring private outdoor living areas)". 

(C) 2012 Stephen Pattinson
Appendix 8
M. Arch Thesis

Of the above-mentioned criteria, one deals specifically with communal outdoor space, viz. the criterion titled "Outdoor Living Space". Top marks (5 points) could be awarded to "Provision of usable communal open space with high quality facilities". For this criterion, "The Altair" scored 4 points, meaning that usable communal open space has been provided, but not necessarily with high quality facilities. (Chester Courts and Stonefields received the same score as The Altair for this criterion).

According to the MfE Case Study for The Altair (Draft]:

- "[t]he majority of the units have three bedrooms except units 52 to 59 which have three bedrooms plus study." (p9)

- $\quad$ "There are two communal open spaces that receive sunlight throughout the year. One is $20 \mathrm{~m}$ by $12 \mathrm{~m}$ and provides opportunities for passive recreation and circulation. The other is $26 \mathrm{~m}$ by $12 \mathrm{~m}$ and has a larger grassed area where children can play (no playground is provided). The open spaces have an interesting combination of paving materials, broken up by grassed areas, vegetation coverage and level changes. A considerable number of trees have been planted that, once mature, will enhance the local amenity." (p5)

- "The majority of units have $20 \mathrm{~m} 2$ to $24 \mathrm{~m} 2$ courtyards facing east or west, in addition to small balconies $(1.2 m \times 3 m)$ above ground level. A few units have larger courtyards of up to $36 \mathrm{~m} 2$. Landscape treatment in the courtyards is a simple composition of gravel, concrete pavers and lawn." (p.6)

- "Many of the courtyards have been treated as front yards, facing the communal open spaces, pedestrian accessways or Rintoul Street. These do not receive an adequate level of privacy. Some residents have personalised these spaces with pot plants." (p.6) 
The approximate data for The Altair, taken from the MfE Case Study, is as follows (see Figs. A8.18 - A8.22):

Total site area:

$10,000 \mathrm{~m} 2$ (1.0ha)

Number of dwellings and bedspaces:

Dwellings: Bedspaces:

- three bedroom (MfE Case Study p8 Fig.12 - typical dwelling floor plans show 2 double beds and 1 single bed)

71 355

Communal outdoor spaces:

- One is $20 m$ by $12 m$ = $240 \mathrm{~m} 2$

- $\quad$ The other is $26 m$ by $12 m=$ $312 \mathrm{~m} 2$

Total communal outdoor space: $552 \mathrm{~m} 2$

Communal outdoor space $/$ bedspace (person): $\quad 552 \mathrm{~m} 2 / 355=1.6 \mathrm{~m} 2$

Private outdoor space:

- The majority of units have $20 \mathrm{~m} 2$ to $24 \mathrm{~m} 2$ courtyards facing east or west, in addition to small balconies $(1.2 \mathrm{~m} \times 3 \mathrm{~m})$ above ground level.

Sub-Total A: say $80 \% \times 71 \times(22+3.6) \mathrm{m} 2=1,454 \mathrm{~m} 2$

- A few units have larger courtyards of up to $36 \mathrm{~m} 2$.

Sub-Total B: say $20 \% \times 71 \times 36 \mathrm{~m} 2=$

$511 \mathrm{~m} 2$

Total private outdoor space $(A+B)$ :

$1,965 \mathrm{~m} 2$

Average private outdoor space / bedspace (person): 1,965m2 / $355=5.5 \mathrm{~m} 2$

Total communal plus private outdoor space / bedspace: $\quad 1.6+5.5=7.1 \mathrm{~m} 2$ 


\section{Site Context and Layout}

Figure 3: Site Plan

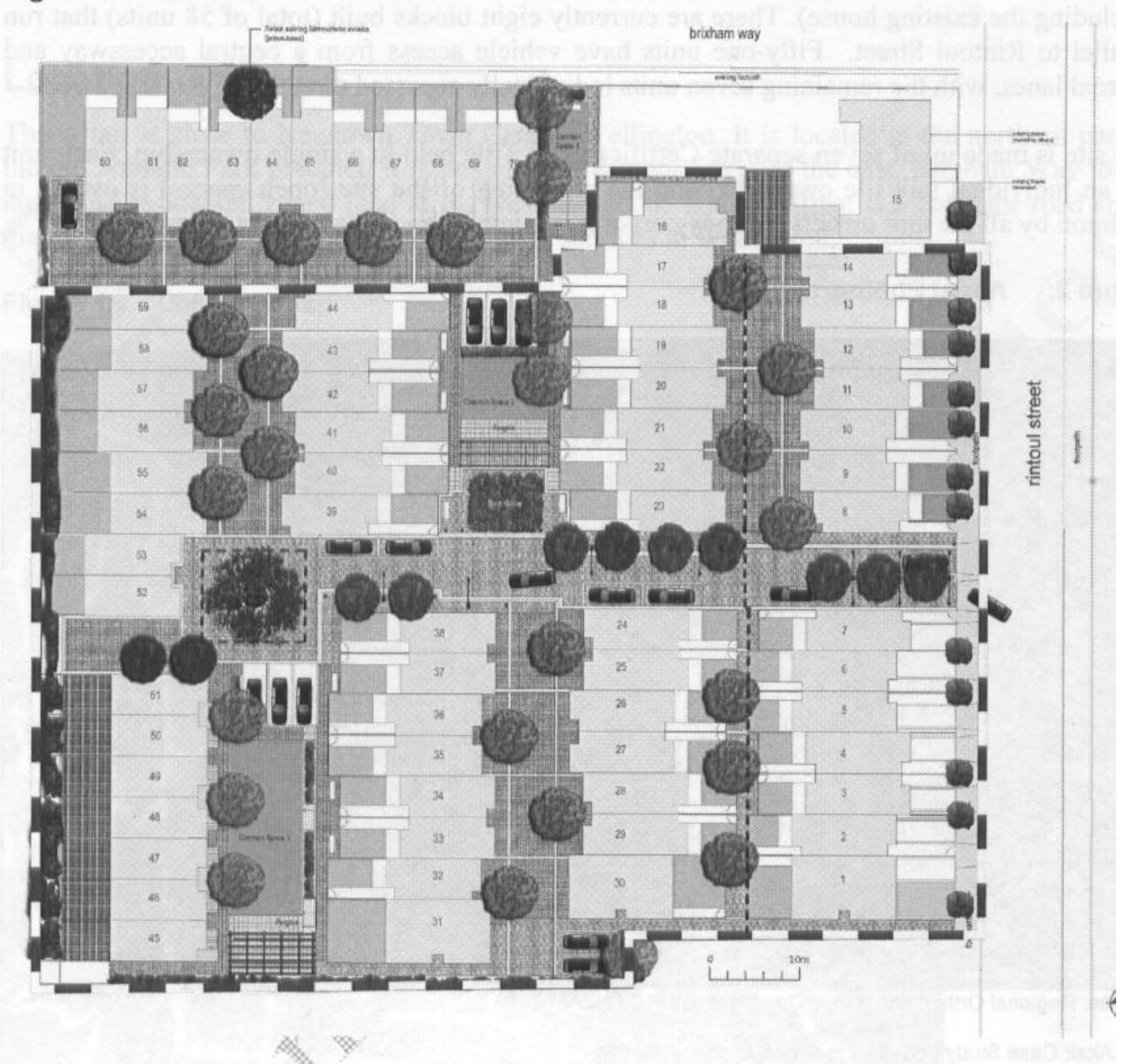

Copyright: Boffa Miskell

- The Altair Case Study

Figure A8.18 The Altair, Rintoul Street, Newtown (from MfE Case Study, Draft, 2011) 


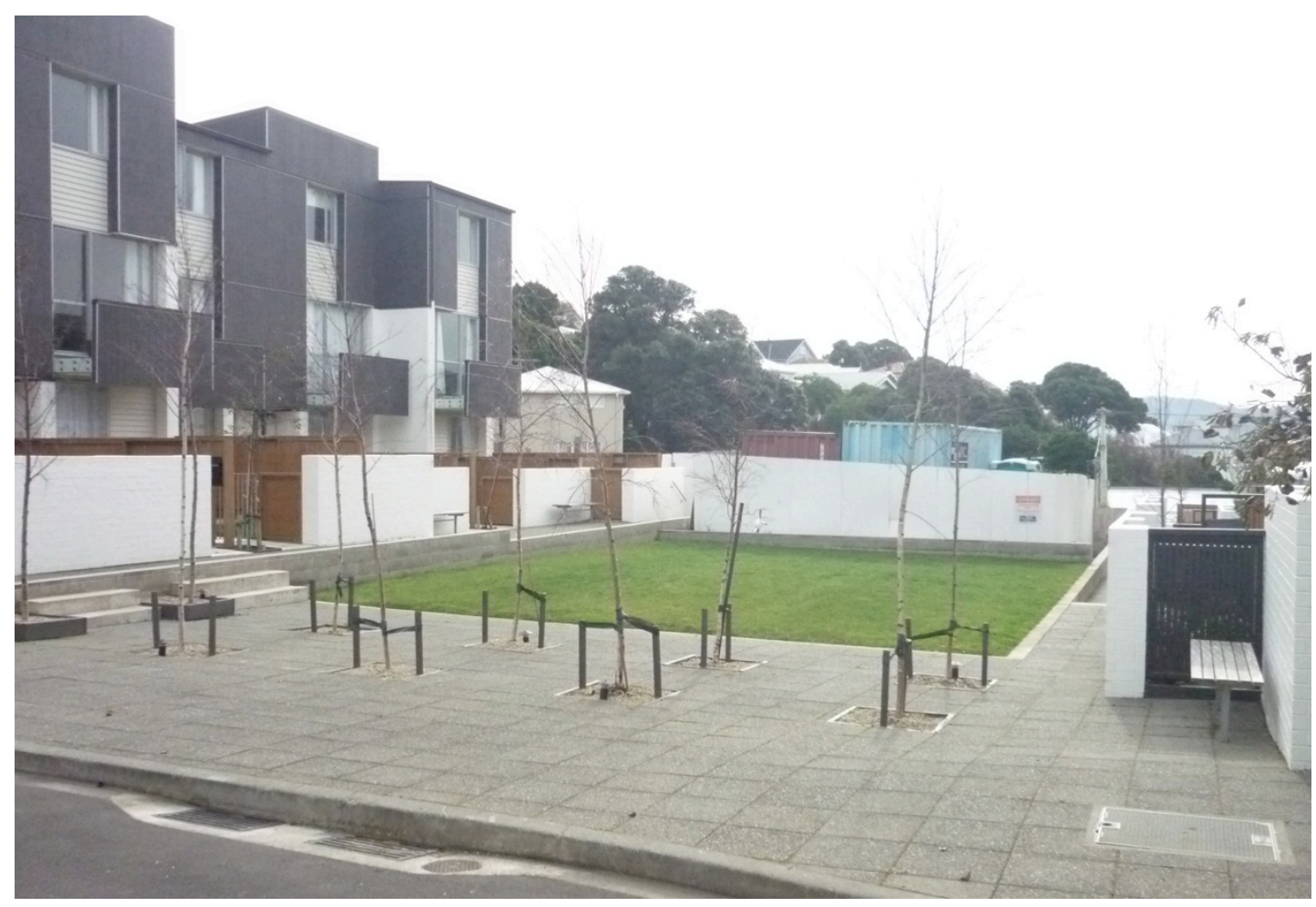

Figure A8.19 The Altair, Rintoul Street, Newtown ((Photo: S. J. Pattinson))

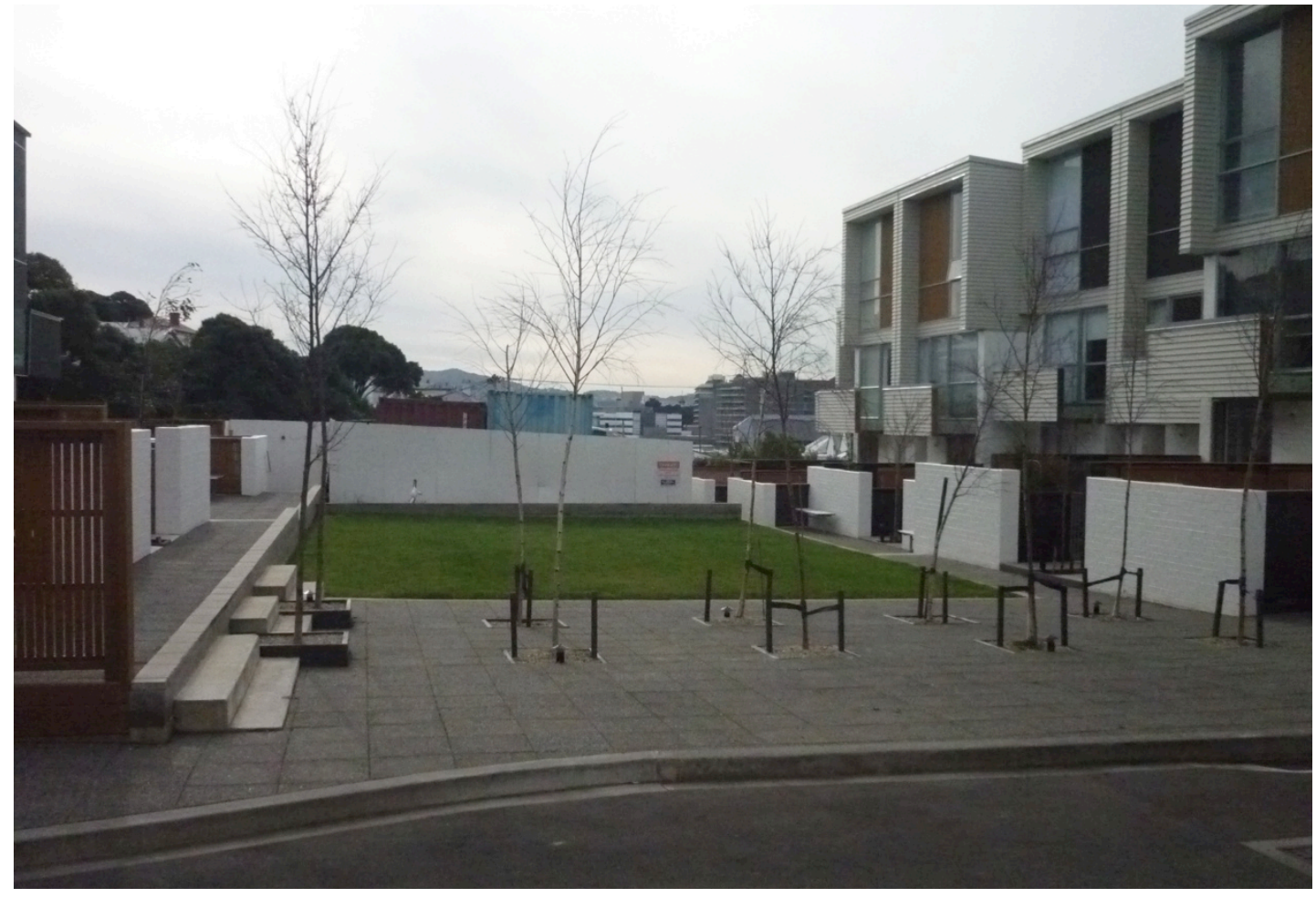

Figure A8.20 The Altair, Rintoul Street, Newtown ((Photo: S. J. Pattinson)) 


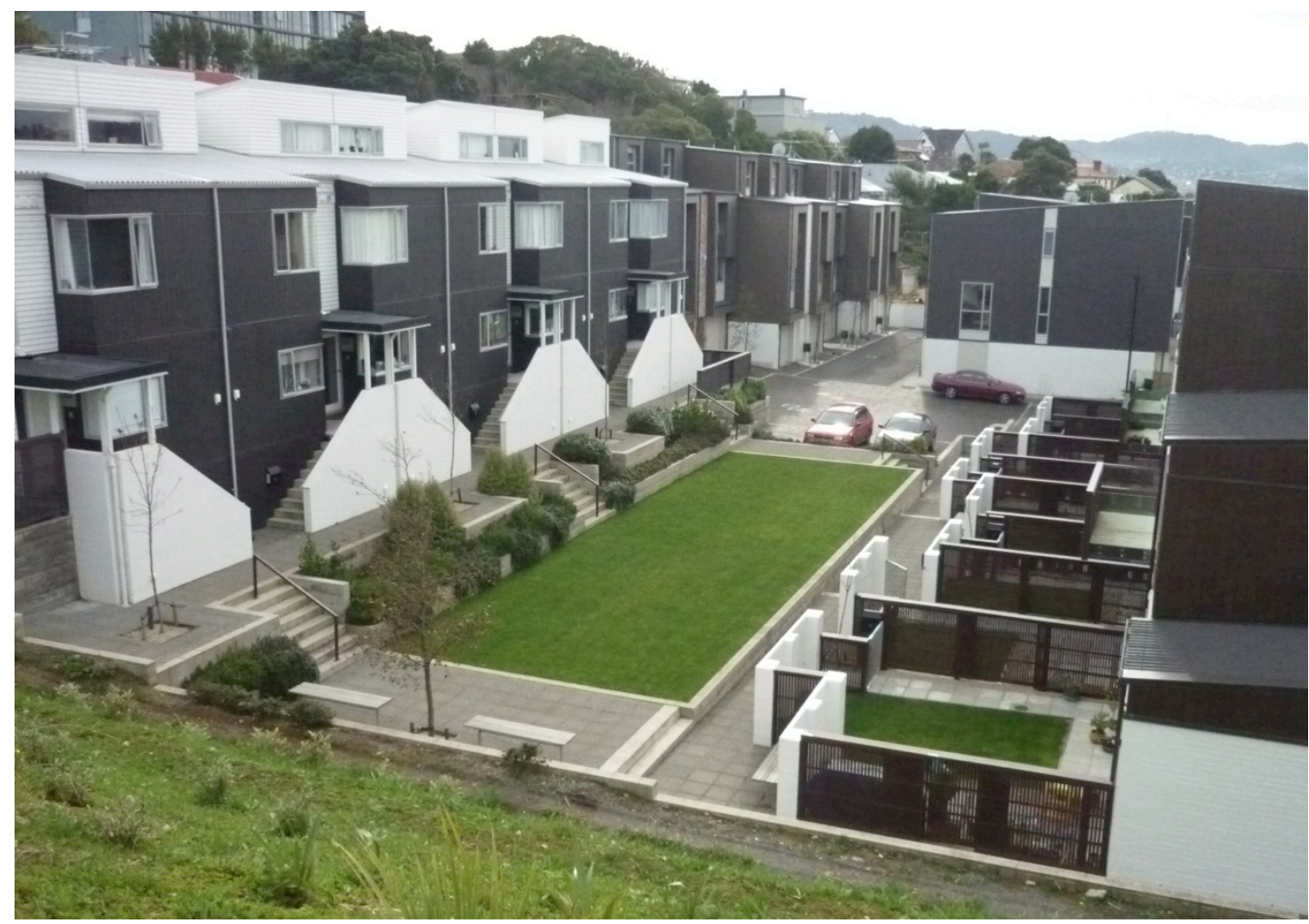

Figure A8.21 The Altair, Rintoul Street, Newtown ((Photo: S. J. Pattinson))

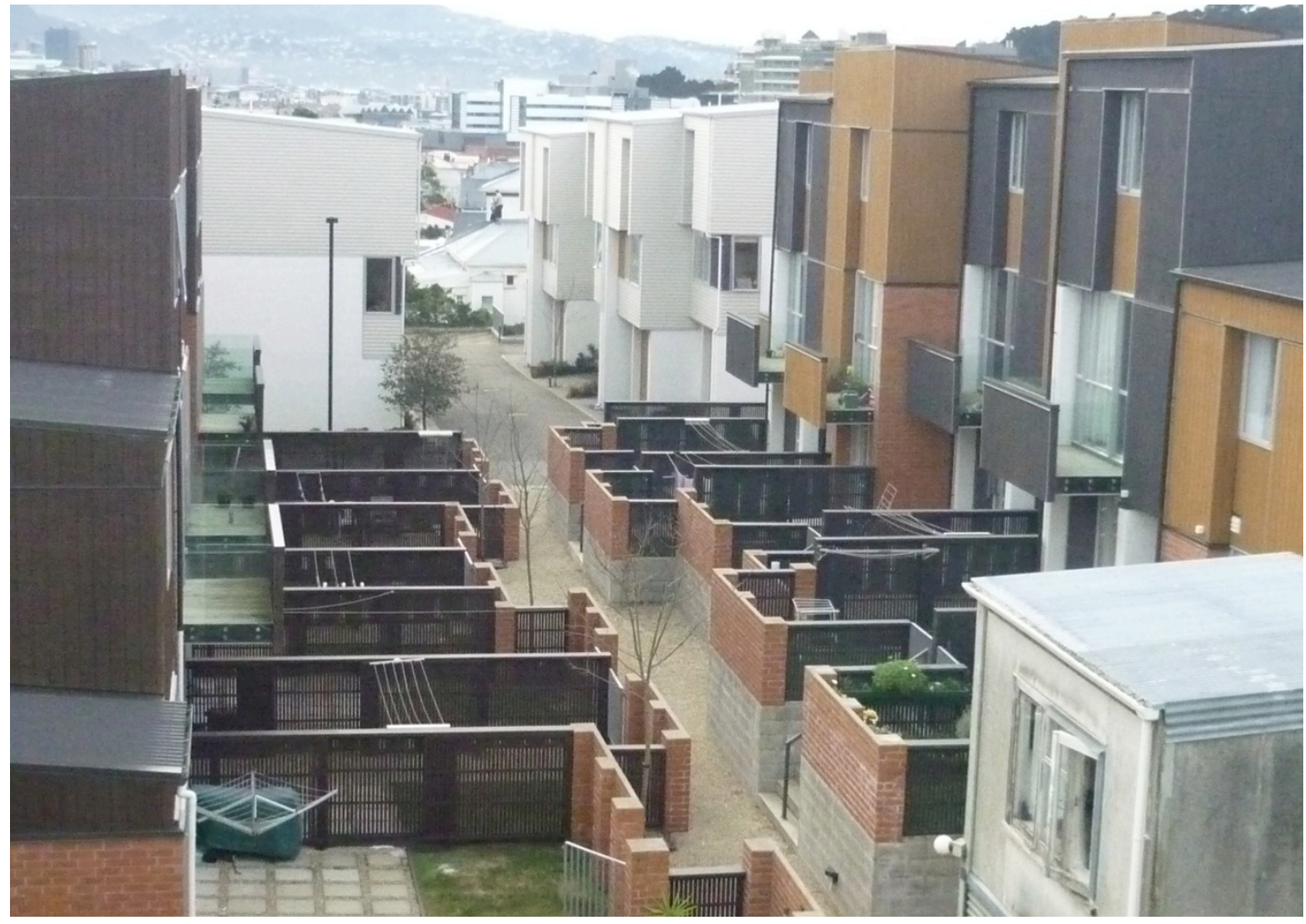

Figure A8.22 The Altair, Rintoul Street, Newtown (Photo: S. J. Pattinson) 


\section{MONTEREY - Two-storey terrace housing and one-storey stacked flats: 232 Middleton Road, Glenside, Wellington}

This development of 91 dwellings on a 1.3125ha site (gross density $69.3 \mathrm{dph}-$ includes all open spaces) has a generous provision of communal space, including:

- a tennis court

- a BBQ area and lawn with four picnic tables and seats

- an outdoor swimming pool, and

- a fitness gym

It is adjacent to open paddocks to the east, light industrial land to the north, residential to the south, and Churton Park residential subdivision to the west on the far side of Middleton Road which borders the west boundary of the site.

This development reflects Leinberger's 'neverland'. It is not low density suburban, nor is it 'walkable' urban - all the residents have to rely on a car for most trips from home. Consequently, it functions as a car-dominated living environment. The onsite manager, who has resided here for several years, commented to the writer that the central communal space is not used much - residents keep "pretty much to themselves".

Few of the "private" outdoor spaces appear private; most have visually permeable fencing and front onto either the public external road or the internal ring road. Entrance doors to the stacked units lack personalisation or offer a place to sit or chat.

It would require site observation and surveys/interviews of residents (as for Pinehill Park - Chapter 6) to find out why, with such attractive shared facilities, the central communal area is not used more by the residents of Monterey. 

(C) 2012 Stephen Pattinson
Appendix 8
M. Arch Thesis

There is a good mix of dwelling sizes, ranging from one- to three- bedrooms. According to the resident manager the dwellings have spacious bedrooms. The manager also commented that the ground consists of rock and clay, making it difficult for landscaping and gardening due to a lack of good top soil.

The approximate data for Monterey is as follows (see Fig. A8.23):

Total site area:

$13,125 \mathrm{~m} 2$ (1.3125ha)

Number of dwellings and bedspaces:

Dwellings: Bedspaces:

- one bedrm flat (plans show double bed) 20

40

- two bedrm flat (plans show 2 double beds) 28

- two bedrm 2-storey (2 double beds)

23

92

- two bedrm 2-storey + studio (2 dble beds) 5

- $\quad$ three bedroom (plans show 3 double beds) $\underline{15}$

$\underline{90}$

Total number of dwellings and bedspaces:

91

354

Central communal outdoor space:

- letter boxes, BBQ, picnic area + green space $840 \mathrm{~m} 2$

- tennis court (fenced area) $500 \mathrm{m2}$

- swimming pool (incl. paved surround) $120 \mathrm{~m} 2$

- fitness gym

$40 \mathrm{~m} 2$

Total shared central space: $\quad 1,500 \mathrm{~m} 2$

Shared outdoor space / bedspace (person): $1500 \mathrm{~m} 2 / 354=4.2 \mathrm{~m} 2$ * 
$\begin{array}{lll}\text { C } 2012 \text { Stephen Pattinson } & \text { Appendix } 8 & \text { M. Arch Thesis }\end{array}$

Private outdoor space per dwelling

(typical courtyard or deck about $9 \mathrm{~m} 2-15 \mathrm{~m} 2$ ) $^{* *}$ : $\quad 12 \mathrm{~m} 2$ (typical**)

Sub-Total A: typical private outdoor space $91 \times 12 \mathrm{~m} 2=1,092 \mathrm{~m} 2^{* *}$

Sub-Total B: non-typical "private" outdoor space

$400 \mathrm{~m} 2 * * *$

Total private outdoor space $(A+B) \quad 1,492 \mathrm{~m} 2$

Average private outdoor space / bedspace (person): $\quad 1,492 \mathrm{~m} 2 / 354=4.2 \mathrm{~m} 2$

Total shared and private outdoor space / bedspace: $\quad 8.4 \mathrm{~m} 2$

* This calculation takes into account the central communal space only; it includes the central letterbox area (which can function as a social meeting space) by the entry to the tennis court and $\mathrm{BBQ}$ area, but it does not include the rubbish bin areas and other common property around the edges of the site such as sundry left-over corners and edge strips.

** a very few properties have larger yards (say $70 \mathrm{~m} 2$ or so), eg in the corners of the site, but these are the exception and to avoid skewing the results they have been taken to be similar to the rest

*** allows for 'private' use of the mowing strip by dwellings fronting onto Middleton Rd (Fig. A8.39) 


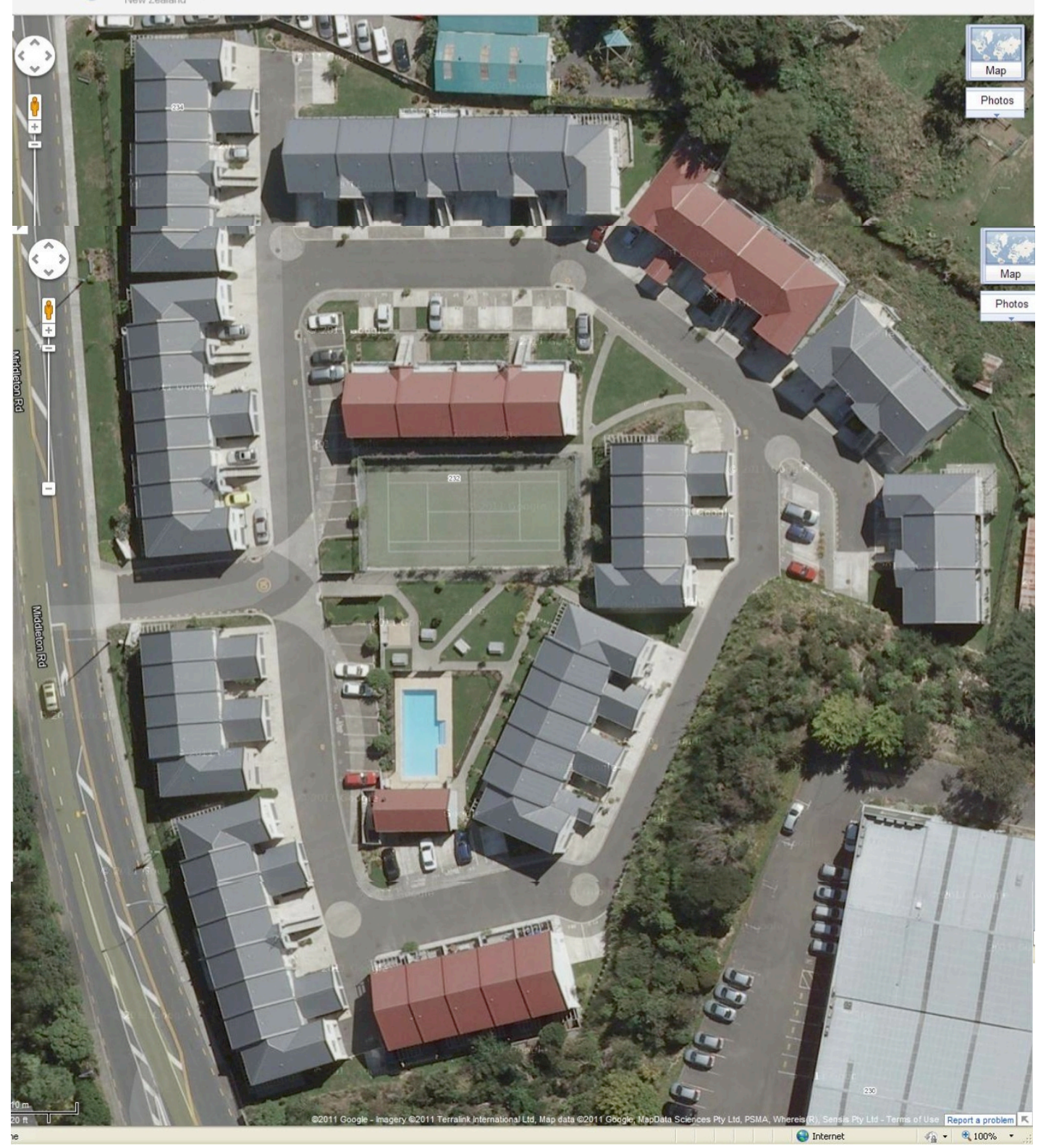

Figure A8.23 Monterey: 230-234 Middleton Road, Glenside, Wellington (Google Maps)

Central communal space includes tennis court, BBQ, lawn, pool, gym (North up the page) 


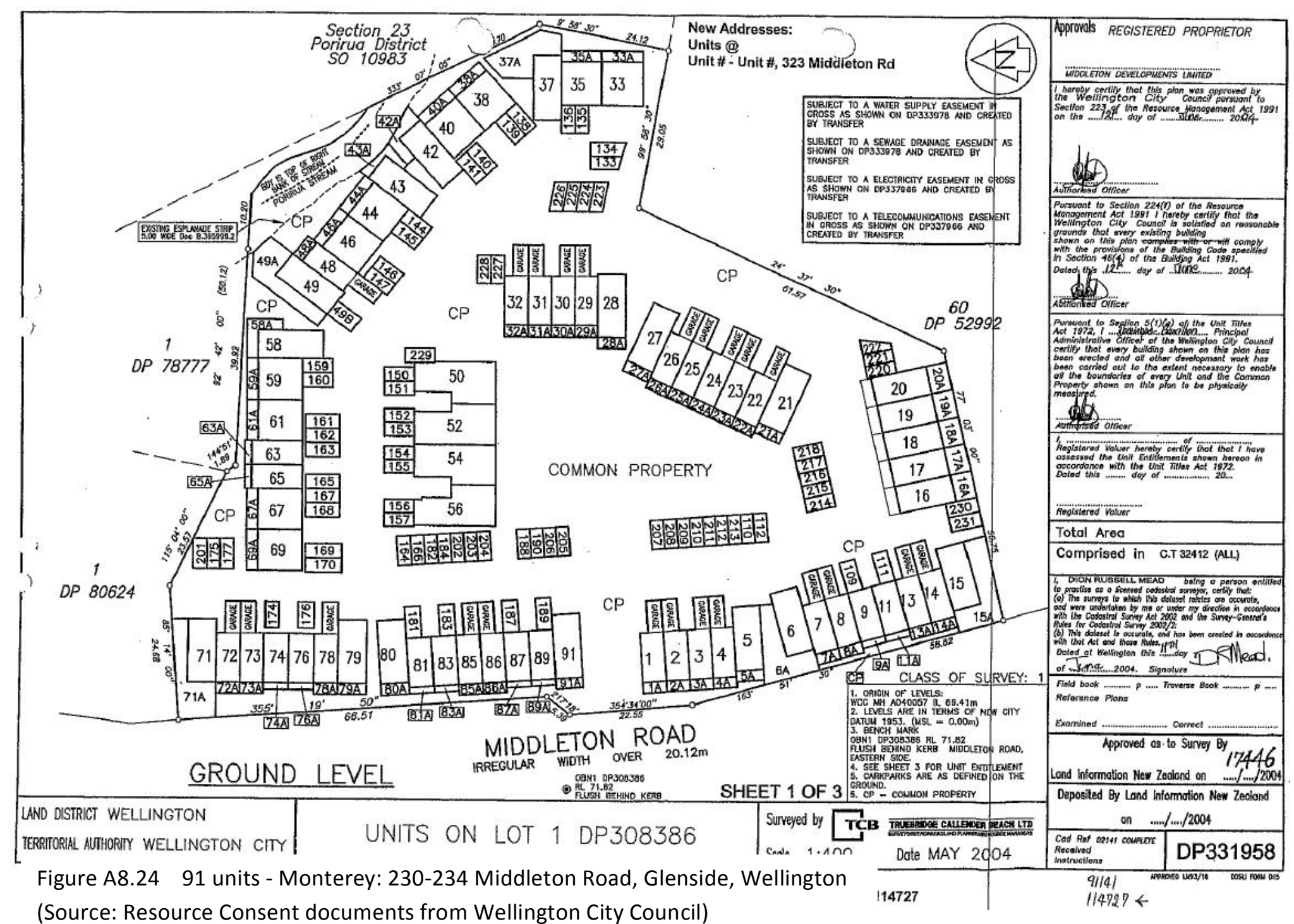




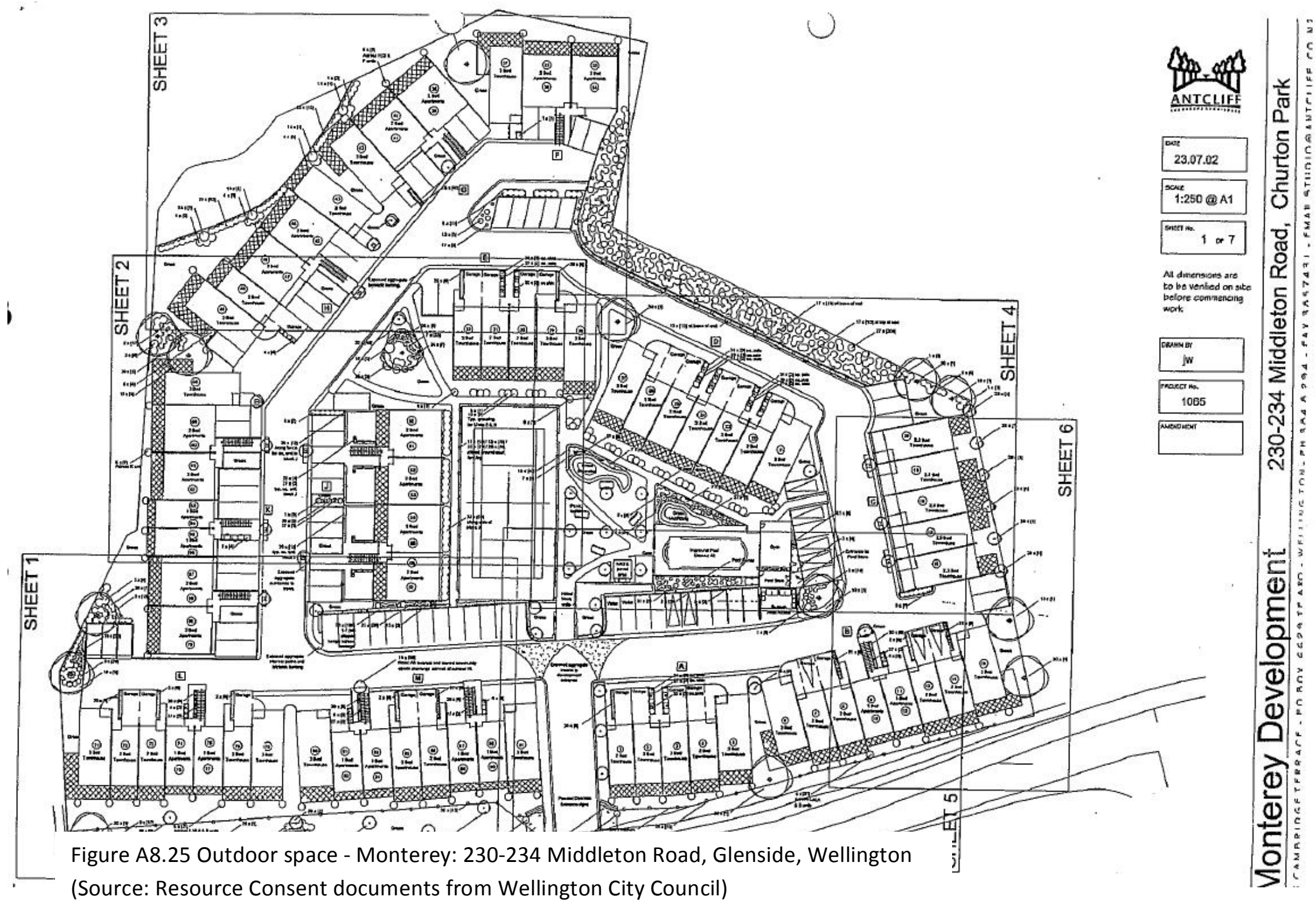




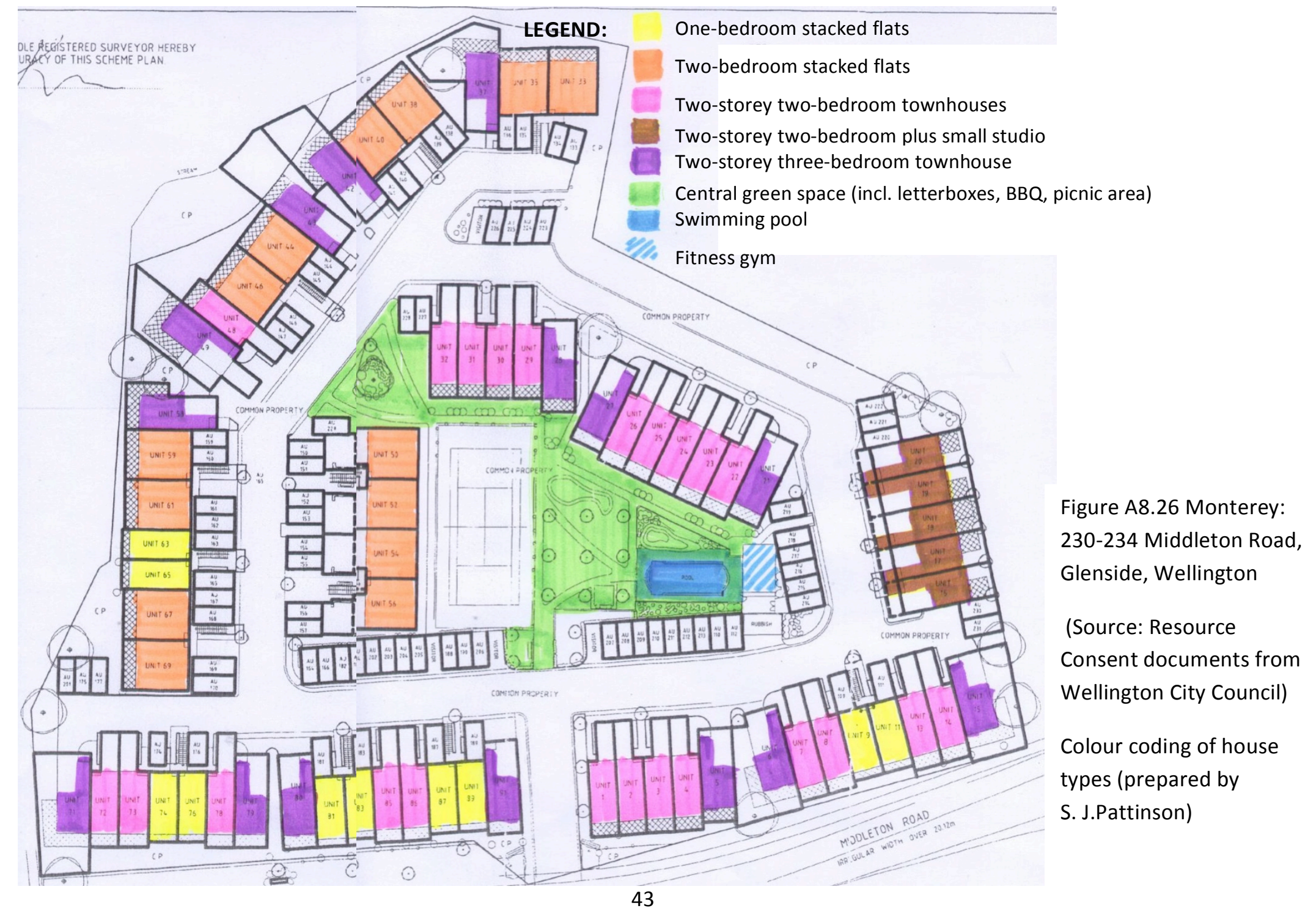




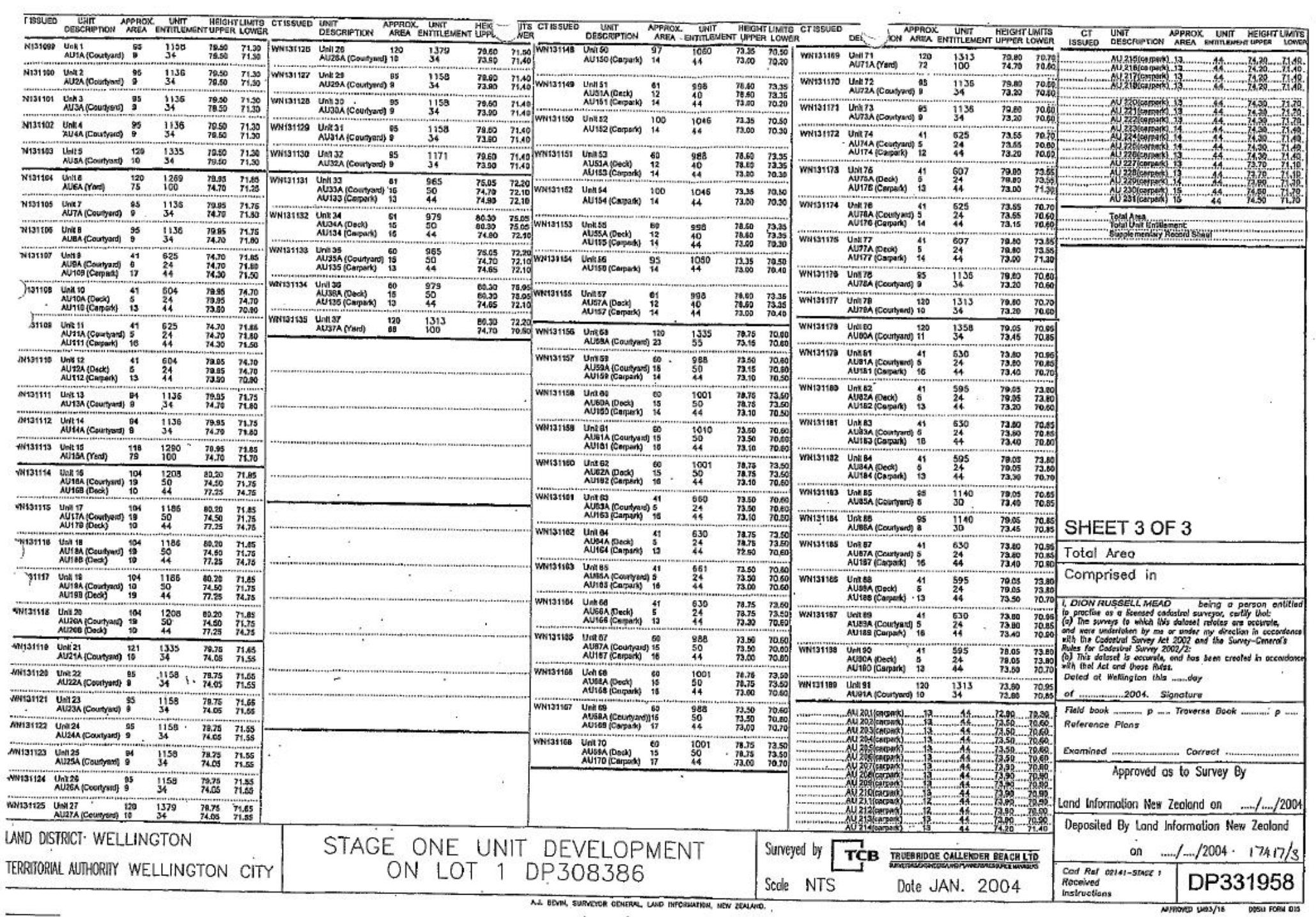

Table A8.7 Monterey, 232 Middleton Road, Wellington - Data sheet (Source: Resource Consent documents from Wellington City Council) 


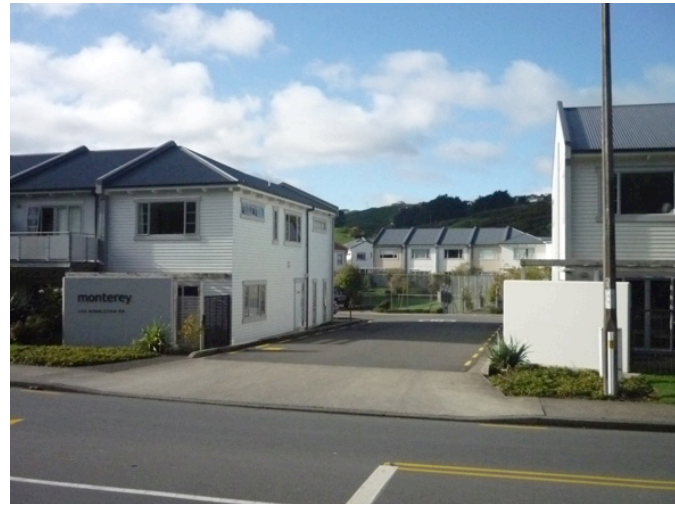

Figure A8.27 Monterey: Entrance (Photo: S J Pattinson)

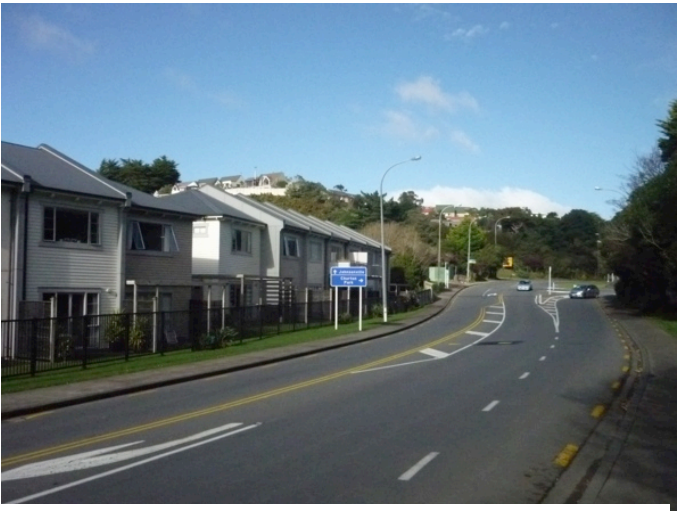

Figure A8.28 Monterey: Middleton Road (Photo: S J Pattinson)

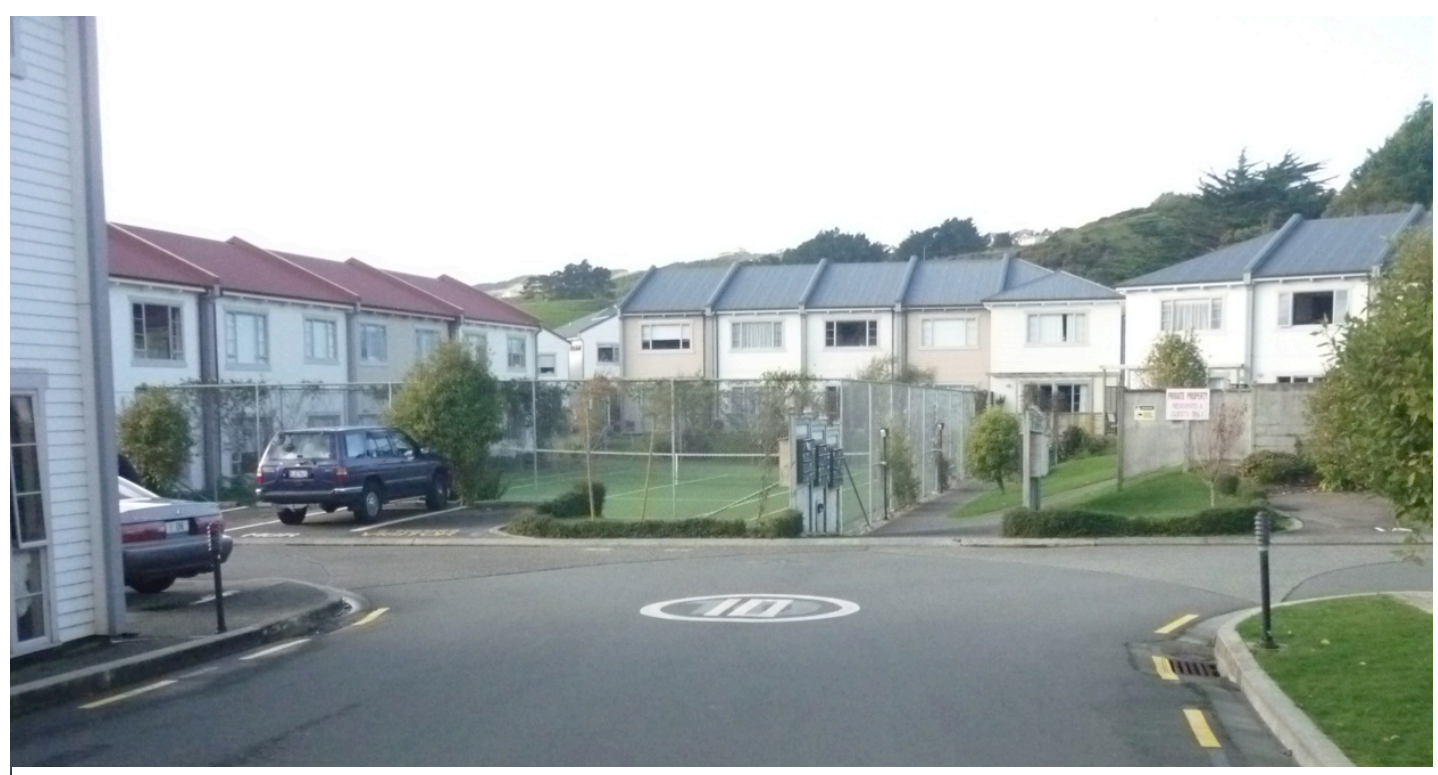

Figure A8.29 Monterey: Entrance - letterboxes in communal area by tennis court (Photo: S J Pattinson)

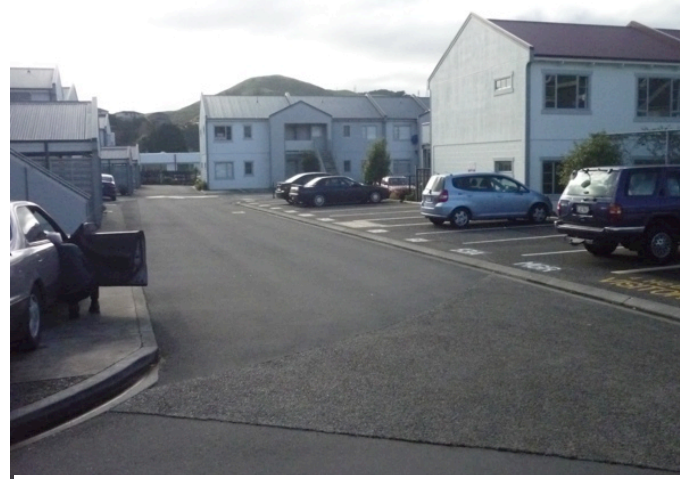

Figure A8.30 Monterey: Looking north from entrance (Photo: S J Pattinson)

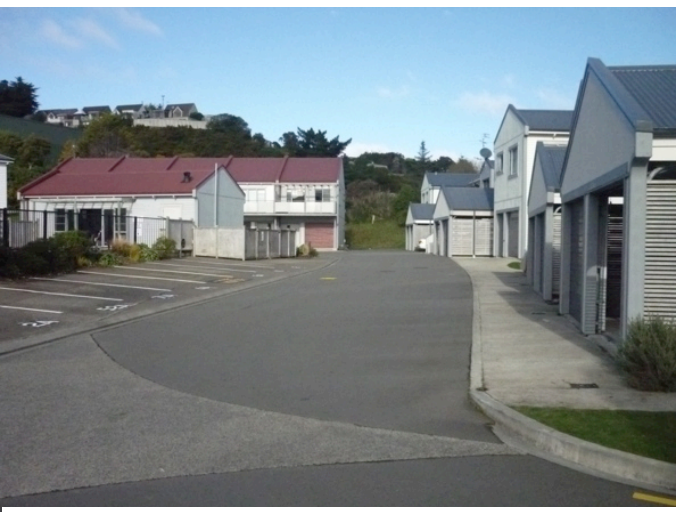

Figure A8.31 Monterey: Looking south from entrance (Photo: S J Pattinson) 

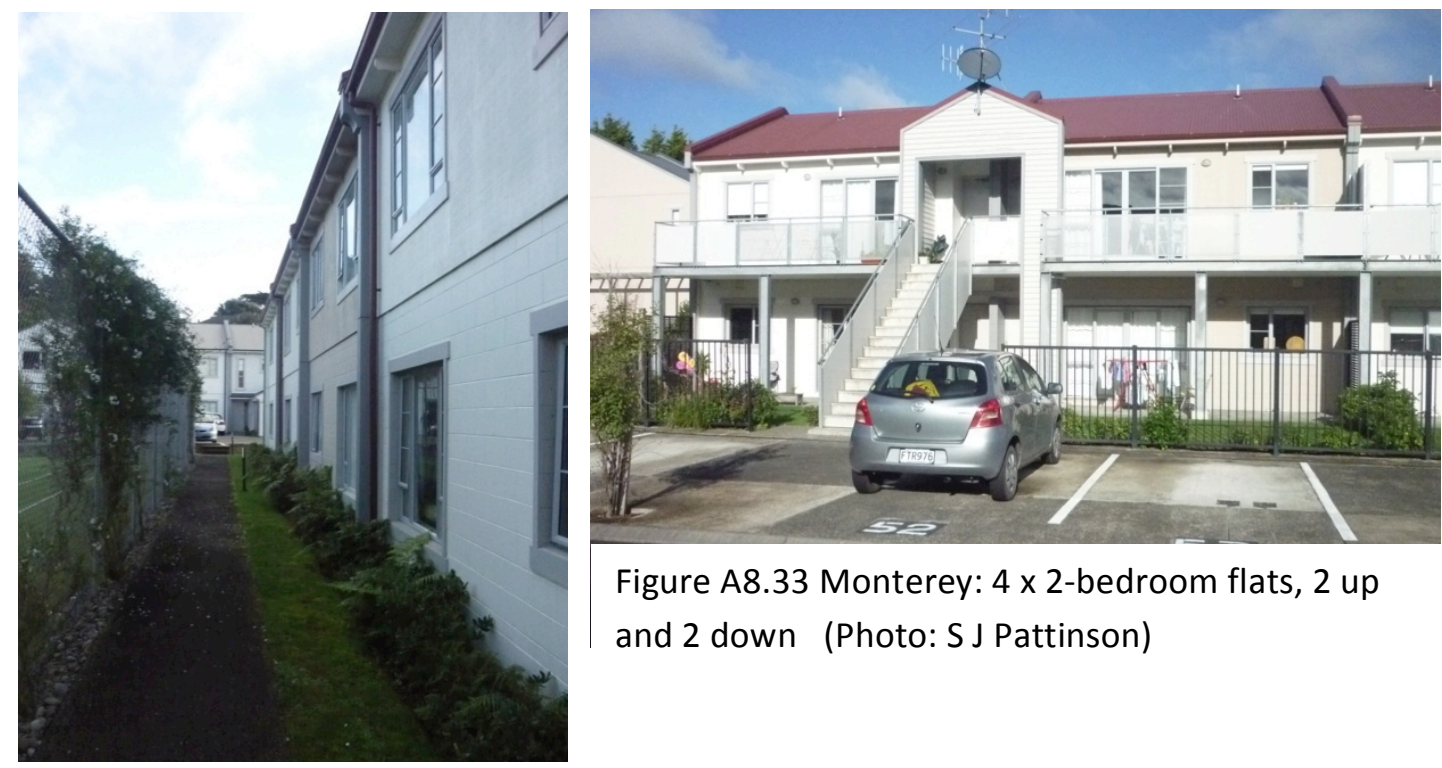

Figure A8.33 Monterey: 4 x 2-bedroom flats, 2 up and 2 down (Photo: S J Pattinson)

Figure A8.32 Monterey: Internal accessway (Photo: S J Pattinson)

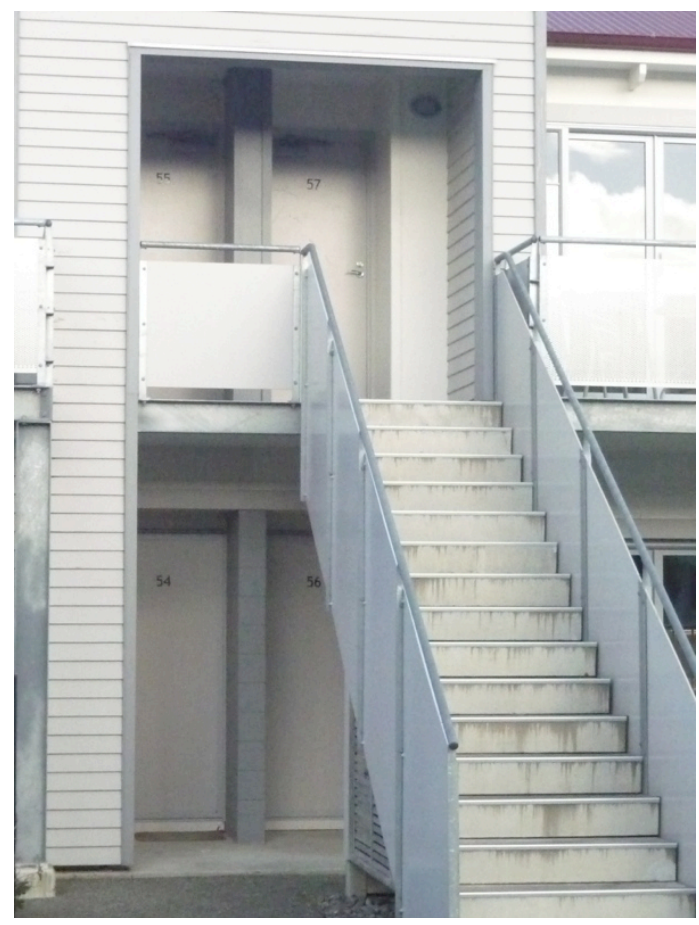

Figure A8.35 Monterey: Four entrances (Photo: S J Pattinson)

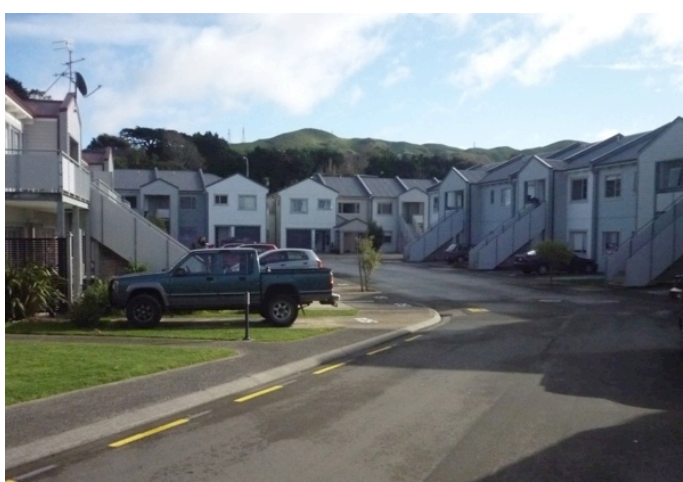

Figure A8.34 Monterey: Entrance stairs on edge of ring road (Photo: S J Pattinson)

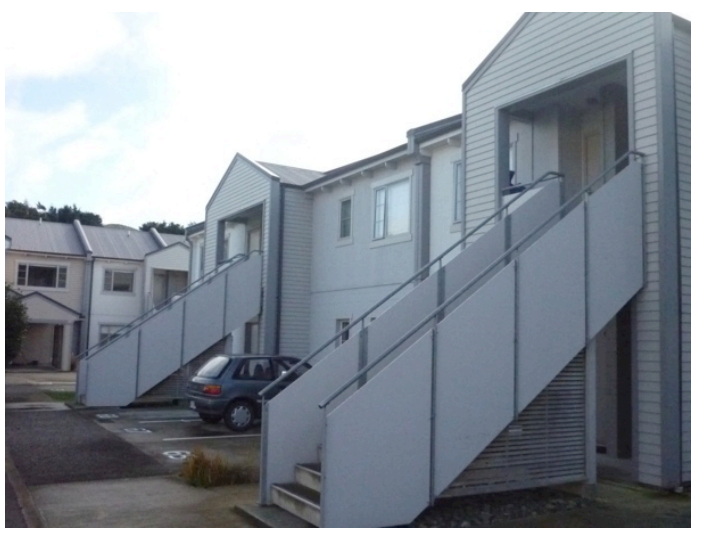

Figure A8.36 Monterey: Entrance stairs (Photo: S J Pattinson) 


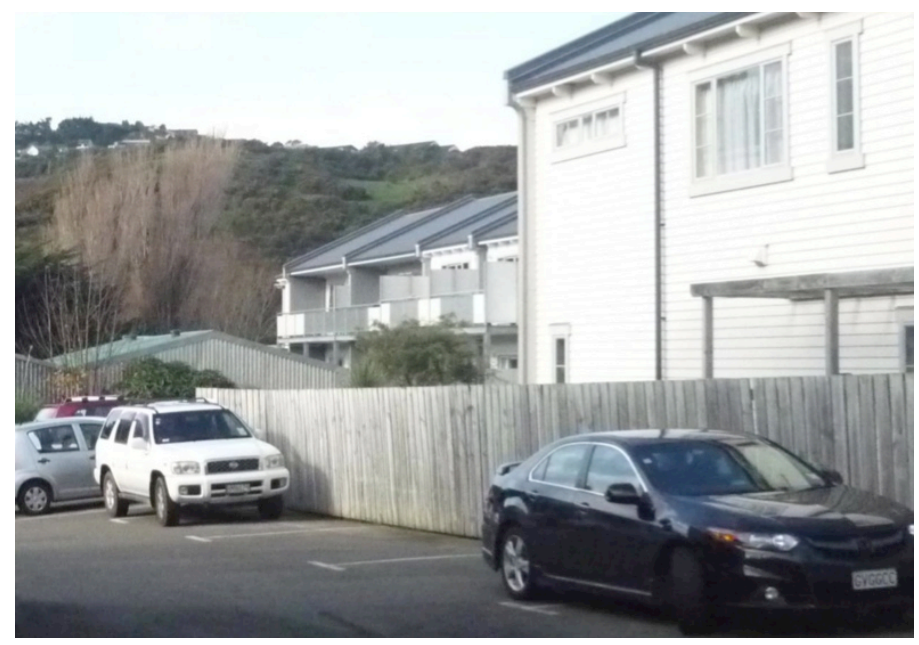

Figure A8.37 Monterey: Balconies overlooking light commercial zone, north boundary (Photo: S J Pattinson)

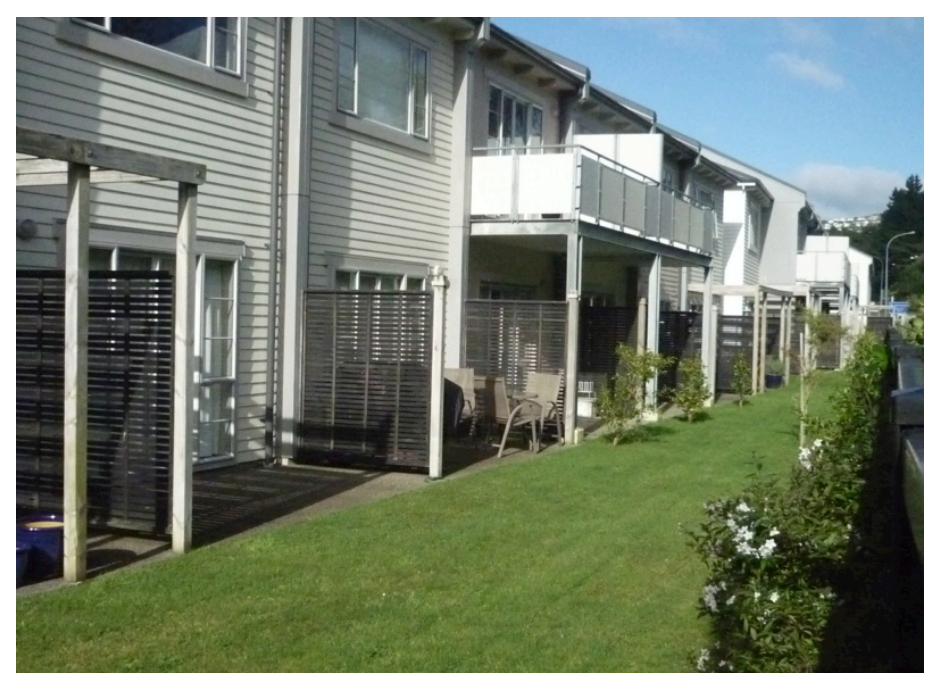

Figure A8.39 Monterey: Private outdoor spaces adjacent to common lawn by Middleton Road boundary fence (Photo: S J Pattinson)

Figure A8.40 Monterey: Private outdoor spaces adjacent to common lawn along Middleton Road boundary fence (Photo: S J Pattinson)

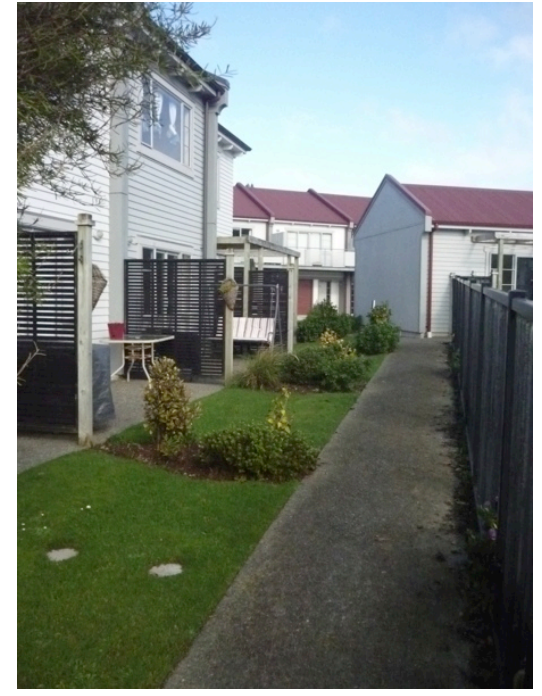

Figure A8.38 Monterey: Private outdoor space adjacent to internal common accessway (Photo: S J Pattinson)

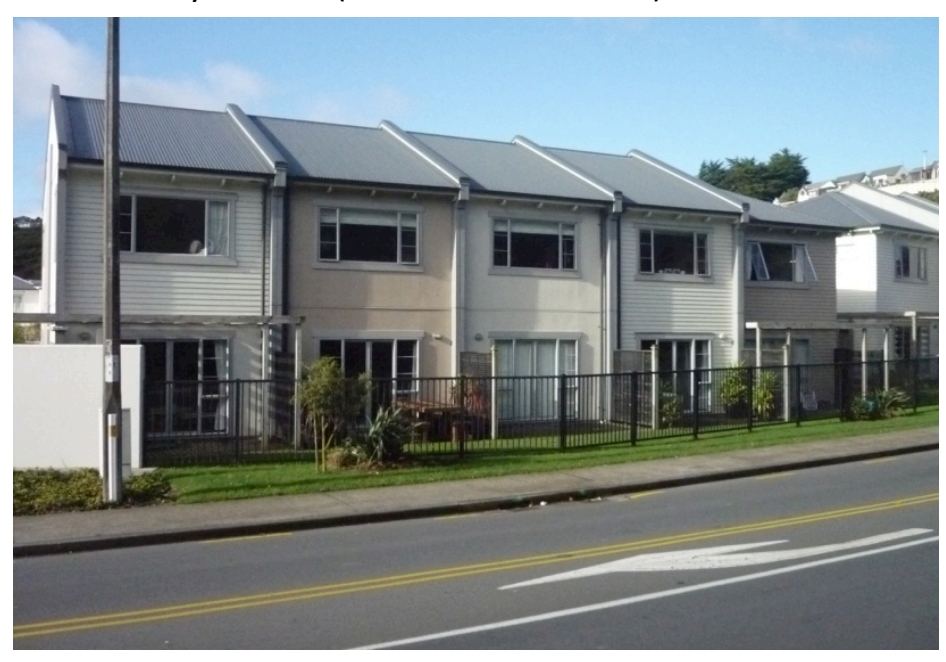




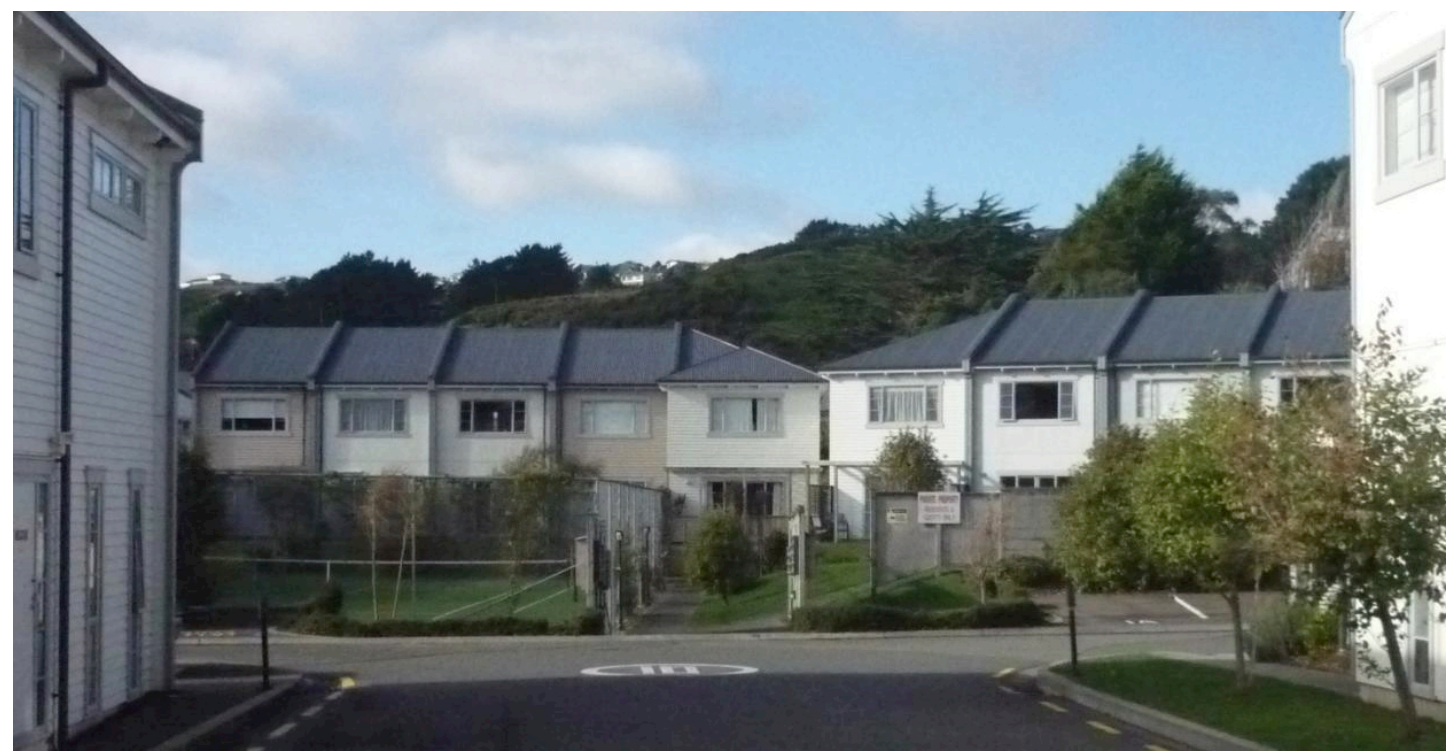

Figure A8.41 Monterey: Entrance to central communal recreation space; tennis court on left; letterboxes centre; $\mathrm{BBQ}$, picnic tables and pool to right (behind wall) (Photo: S J Pattinson)

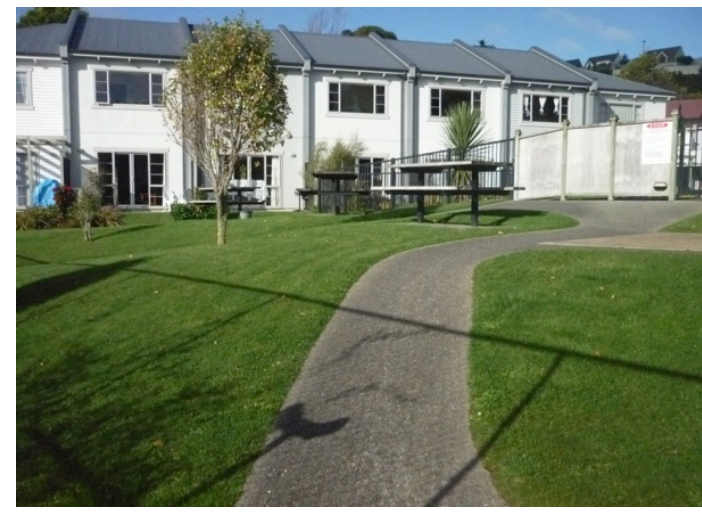

Figure A8.42 Monterey: Picnic area (Photo: S J Pattinson)

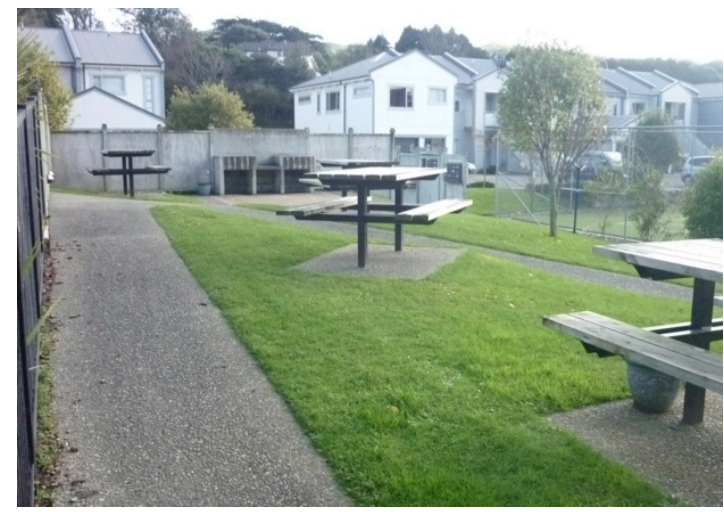

Figure A8.44 Monterey: BBQ and picnic area (Photo: S J Pattinson)

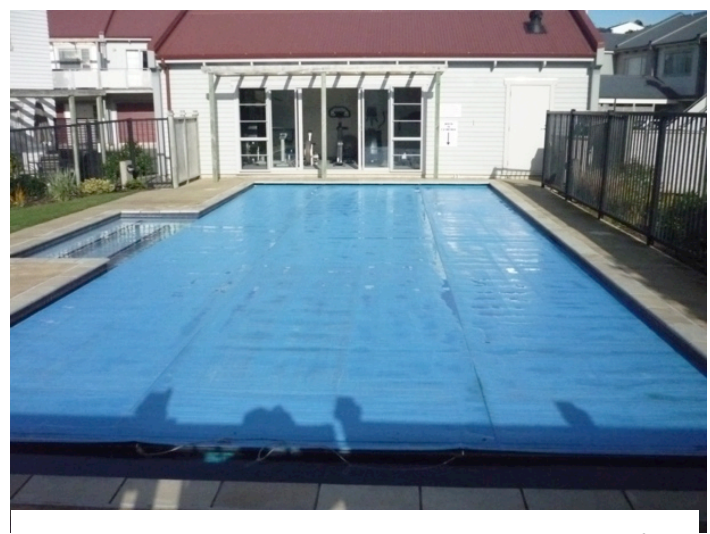

Figure A8.43 Monterey: swimming pool and fitness gym (Photo: S J Pattinson)

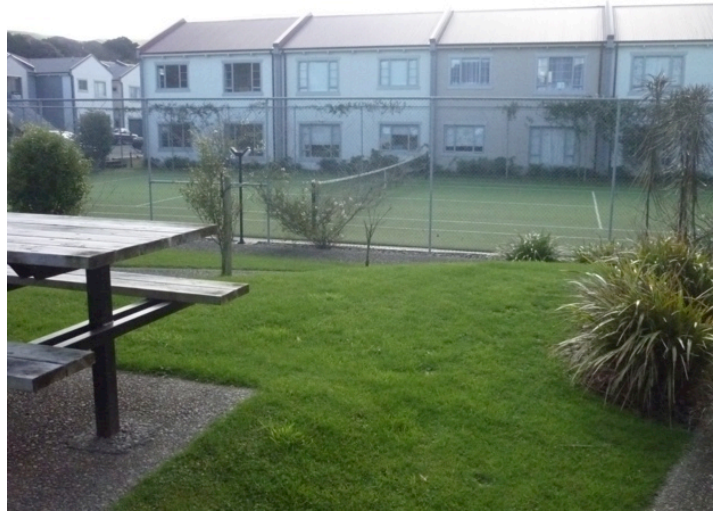

Figure A8.45 Monterey: picnic area looks over tennis court (Photo: S J Pattinson) 


\section{10 PARK AVENUE, Lower Hutt: Attached townhousing}

The approximate data for this development is as follows (see Figs. A8.46 - A8.47):

Approximate total site area (scaled off Site Plan) $\quad 2,270 \mathrm{~m} 2$ (0.227ha)

Gross density is approximately 20/0.227 = $88 \mathrm{dph}$.

Number of dwellings and bedspaces: Dwellings: Bedspaces:

- three bedrooms (5.0 bedspaces/dwelling) $20 \quad 100$

(The writer was advised by a tenant at this development that the three bedrooms were all small double bedrooms, each capable of accommodating a double bed; in their unit they had two double beds and were using the third bedroom for storage. Therefore, two double beds and one single bed5 bedspaces - is assumed in each of the twenty dwellings).

Communal outdoor space (other than driveway): nil

Private outdoor space:

- 8 units have approx $4.8 \mathrm{~m} \times 6.7 \mathrm{~m}=$ $32 \mathrm{~m} 2 \times 8=256 \mathrm{~m} 2$

- 12 units have approx. $5 \mathrm{~m} \times 9 \mathrm{~m}=$ $45 \mathrm{~m} 2 \times 12=\underline{540 \mathrm{~m} 2}$

Total private outdoor space (approx.) =

$796 \mathrm{~m} 2$

Average private outdoor space / bedspace (person): $796 \mathrm{~m} 2 / 100=8.0 \mathrm{~m} 2$

Total communal plus private outdoor space / bedspace: $\quad$ nil $+8.0=8.0 \mathrm{~m} 2$ 


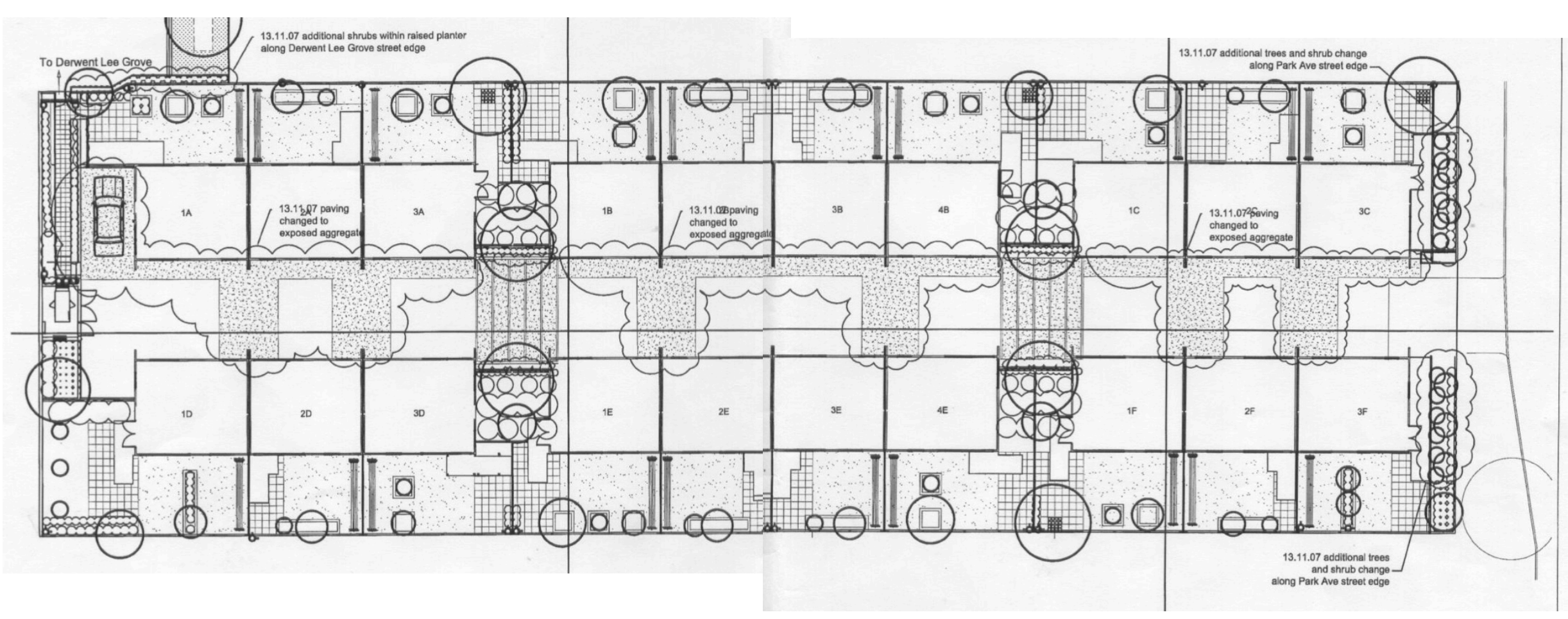

Figure A8.46 Site Plan, 10 Park Avenue, Lower Hutt (obtained from Lower Hutt City Council, Resource Consent application documents)

Twenty townhouses (three-storey). The layout is like the layout in thesis Fig. 1.6 (see also

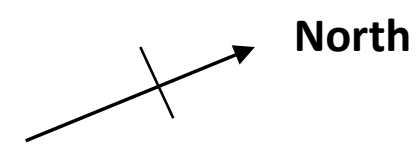
Figures 1.10a, 10b), linear blocks either side of a narrow vehicle access strip. The level of the private outdoor spaces is half-way between the sunken garage and living floor level. 


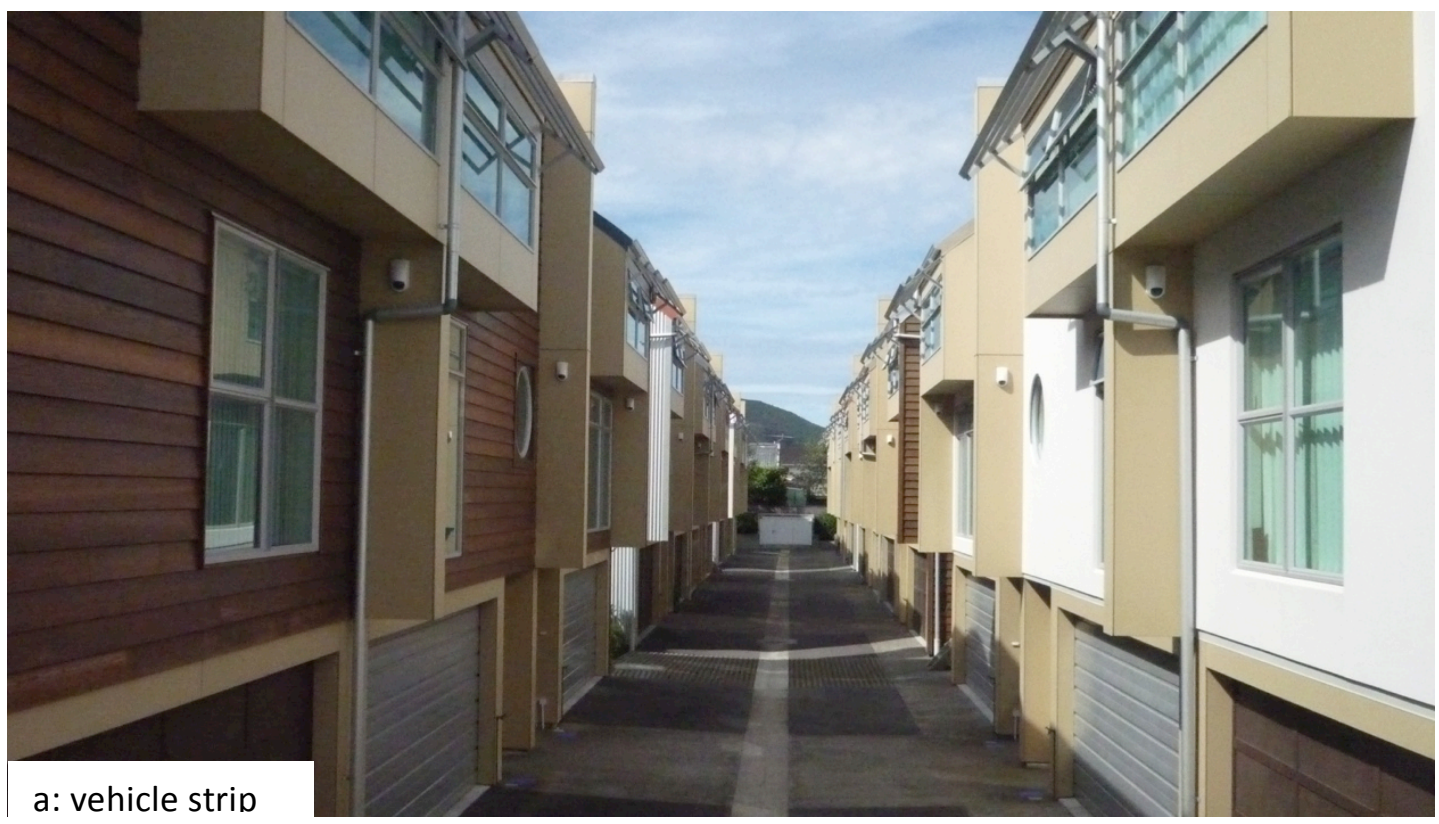

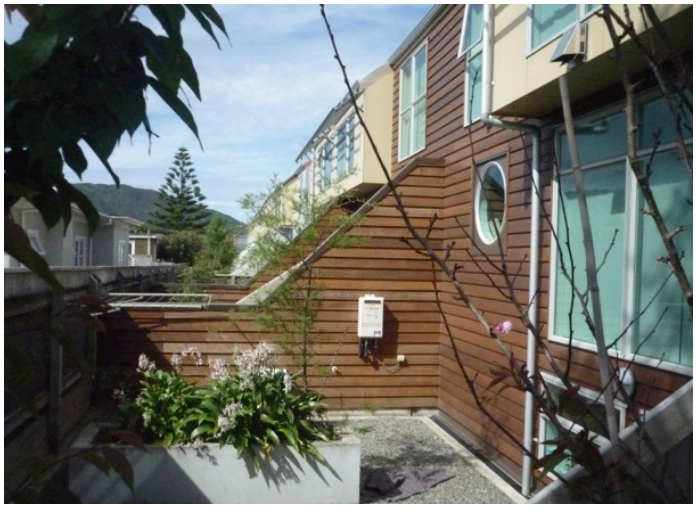

b: courtvard half a level below Living Rooms

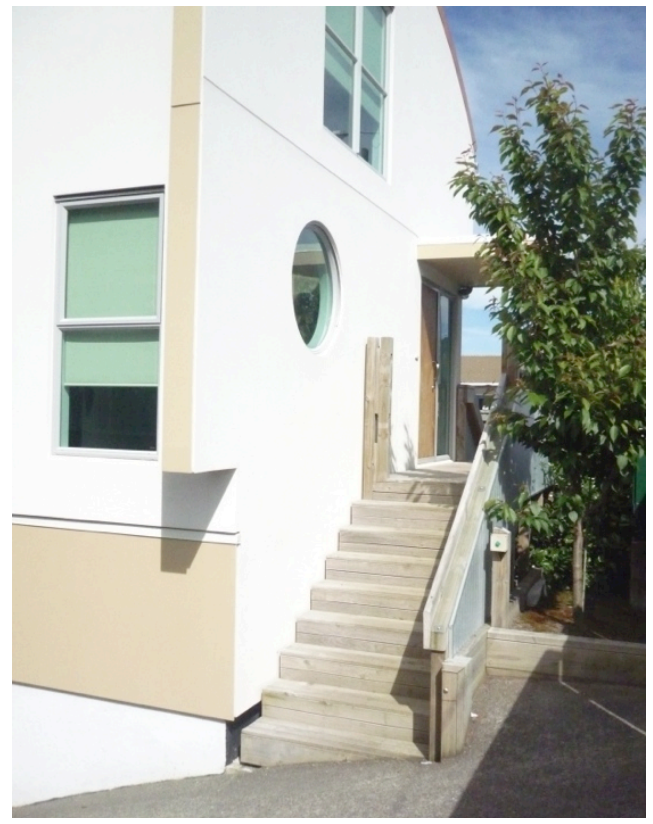

d : stair up from drivewav to front door

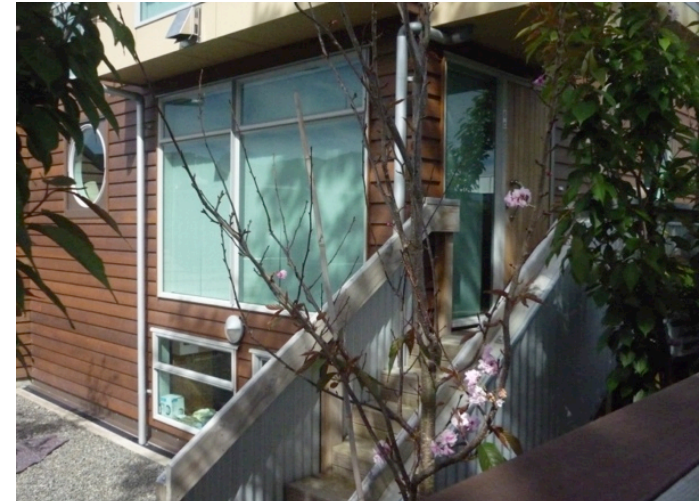

c: stair down to courtvard

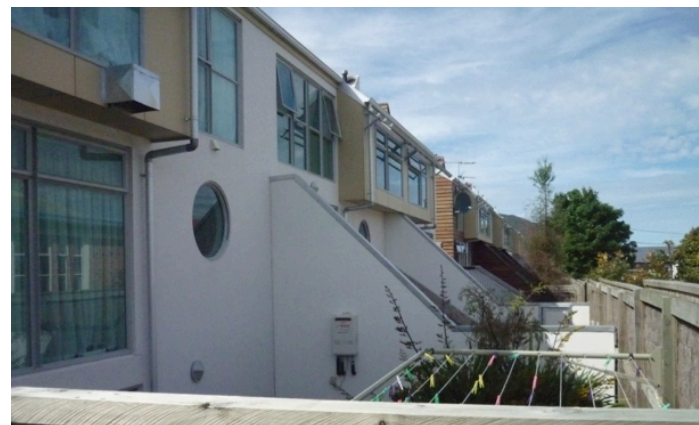

e : service or living court?

Figures A8.47 a,b,c,d,e 10 Park Avenue, Lower Hutt (Photos: S.J. Pattinson)

The level of the private outdoor spaces is half-way between the sunken Garage and Living floor levels. (3 bedrms on L3) 


\section{Qualitative Research}

There was once a time (in Britain in the 1960s) when open space for children in housing developments was prescribed in terms of area per bedspace. Standards for the provision of play areas were presented by Colquhoun and Fauset in the following table, based on the Department of the Environment Design Bulletin 27 "Children at Play" which was published in 1973:

\begin{tabular}{|l|l|}
\hline Size of scheme & Space \\
\hline $10-19$ child bedspaces & At least one play area of $30 \mathrm{~m} 2(333 \mathrm{sq} \mathrm{ft}) \mathrm{min}$. \\
\hline $20-40$ child bedspaces & At least one play area of $50 \mathrm{~m} 2(538 \mathrm{sq} \mathrm{ft}) \mathrm{min}$. \\
\hline $50-99$ child bedspaces & At least one play area of $100 \mathrm{~m} 2(1,076 \mathrm{sq} \mathrm{ft}) \mathrm{min}$. \\
\hline 100 or more child bedspaces & At least one play area of $150 \mathrm{~m} 2(1,615 \mathrm{sq} \mathrm{ft}) \mathrm{min}$. \\
\hline
\end{tabular}

Table A8.8 Space standards for the provision of children's play areas; recommendations in Design Bulletin 27 'Children at Play'.

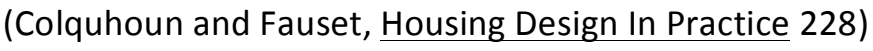

Colquhoun and Fauset explain that the number of child bedspaces in a housing development was calculated by adding up all the bedspaces in the development and then subtracting all the beds in dwellings for the elderly and in all one- and twobedroom dwellings. From the remainder, two bedspaces per dwelling (presumably for parents) were subtracted to give the final figure for the number of child bedspaces in the development. Then it was mandatory that for "all schemes containing ten or more child [bed]spaces, play space [had to be]provided on the basis of 3 m2 (32 sq ft) per child bedspace." (228) 
Colquhoun and Fauset also present standards developed by Sheffield City Council in 1974 for children's play inside housing developments, and standards for outside the developments to complement the play space inside the development:

\begin{tabular}{|c|c|c|c|c|c|}
\hline $\begin{array}{l}\text { Type of } \\
\text { play }\end{array}$ & $\begin{array}{l}\text { Major } \\
\text { user }\end{array}$ & Minor user & $\begin{array}{l}\text { Facilities } \\
\text { required }\end{array}$ & $\begin{array}{l}\text { Location } \\
\text { criteria }\end{array}$ & Size \\
\hline $\begin{array}{l}\text { Garden } \\
\text { play }\end{array}$ & $\begin{array}{l}\text { Children } \\
\text { under 3; } \\
\text { the mother } \\
\text { for clothes } \\
\text { drying, } \\
\text { sitting, } \\
\text { gardening }\end{array}$ & $\begin{array}{l}\text { The } 3-6 \text { year } \\
\text { olds; adults } \\
\text { working in } \\
\text { and sitting in } \\
\text { the sun }\end{array}$ & $\begin{array}{l}\text { Sunny area, } \\
\text { childproof fencing } \\
\text { or hedges }\end{array}$ & $\begin{array}{l}\text { Private outdoor } \\
\text { space }\end{array}$ & $\begin{array}{l}56 \mathrm{~m} 2 \text { (600 sq } \\
\text { ft) for family } \\
\text { dwelling; } \\
19-38 \mathrm{~m} 2 \\
\text { (200-400 sq ft) } \\
\text { for others }\end{array}$ \\
\hline $\begin{array}{l}\text { Doorstep } \\
\text { play }\end{array}$ & $\begin{array}{l}\text { The child } \\
\text { under } 3 . \\
\text { The } \\
\text { 3-6 year } \\
\text { olds. }\end{array}$ & $\begin{array}{l}\text { The } 6-10 \\
\text { year olds. } \\
\text { Mothers. } \\
\text { Old people }\end{array}$ & $\begin{array}{l}\text { Public area adjacent } \\
\text { to back or front of } \\
\text { house or garden } \\
\text { overlooked by main } \\
\text { room window. } \\
\text { Sheltered small- } \\
\text { scale areas with low } \\
\text { walls and balancing } \\
\text { rails, rocks, steps, } \\
\text { various surfaces. } \\
\text { Sitting areas } \\
\text { sometimes } \\
\text { including tables. }\end{array}$ & $\begin{array}{l}\text { The paved surface } \\
\text { within } 10 \mathrm{~m} \text { of } \\
\text { every house and } \\
\text { garden. This } \\
\text { includes the car } \\
\text { and circulation } \\
\text { areas near the } \\
\text { house which } \\
\text { should be carefully } \\
\text { designed to } \\
\text { emphasize the fact } \\
\text { that children play } \\
\text { there }\end{array}$ & \\
\hline $\begin{array}{l}\text { Play } \\
\text { spaces }\end{array}$ & $\begin{array}{l}\text { The } \\
3-6 \text { year } \\
\text { olds. }\end{array}$ & $\begin{array}{l}\text { The 6-10 } \\
\text { year olds. } \\
\text { Mothers } \\
\text { accompany- } \\
\text { ing children. } \\
\text { 10-15 yrs* }\end{array}$ & $\begin{array}{l}\text { Shelter, enclosure, } \\
\text { sunny area, slides, } \\
\text { climbing equipment } \\
\text { rocks, benches/seats } \\
\text { tables, hard surfaces } \\
\text { seats for mothers }\end{array}$ & $\begin{array}{l}\text { Within } 200 \mathrm{~m} \text { ( } 650 \\
\mathrm{ft} \text { ) of each house, } \\
\text { more than } 20 \mathrm{~m} \\
(65 \mathrm{ft}) \text { from the } \\
\text { nearest house. }\end{array}$ & $\begin{array}{l}\text { Minimum size } \\
100 \mathrm{~m} 2 \text { (1,076 } \\
\text { sq ft), design } \\
\text { in accordance } \\
\text { with DoE } \\
\text { Circulars ... }\end{array}$ \\
\hline
\end{tabular}

Table A8.9 Children's play provisions inside housing [developments] to complement provision outside housing areas. (Colquhoun and Fauset, Housing Design In Practice 229) (* This is where friction frequently occurs ... [so] account...for [this] in ... location and design...) 


\begin{tabular}{|c|c|c|c|c|c|}
\hline $\begin{array}{l}\text { Type of } \\
\text { play }\end{array}$ & $\begin{array}{l}\text { Major } \\
\text { user }\end{array}$ & Minor user & $\begin{array}{l}\text { Facilities } \\
\text { required }\end{array}$ & $\begin{array}{l}\text { Location } \\
\text { criteria }\end{array}$ & Size \\
\hline $\begin{array}{l}\text { Play areas (1 } \\
\text { per } 1,000 \\
\text { total } \\
\text { population) }\end{array}$ & $\begin{array}{l}\text { 5-11 year } \\
\text { olds }\end{array}$ & $\begin{array}{l}11-15 \text { year } \\
\text { olds, mothers } \\
\text { accompanying } \\
\text { children }\end{array}$ & $\begin{array}{l}\text { Slides, swings, } \\
\text { climbing } \\
\text { equipment, } \\
\text { sheltered corners } \\
\text { for sitting, rocks } \\
\text { logs, sitting areas } \\
\text { for mothers }\end{array}$ & $\begin{array}{l}\text { Within } 200-500 \\
\mathrm{~m}(650-1,600 \mathrm{ft}) \\
\text { distance of each } \\
\text { house but not } \\
\text { within } 20 \mathrm{~m} \\
(65 \mathrm{ft}) \text { of the } \\
\text { nearest dwelling }\end{array}$ & $\begin{array}{l}300 \mathrm{~mm} 2 \\
(3,200 \mathrm{sq} f t)\end{array}$ \\
\hline $\begin{array}{l}\text { Kick-about } \\
\text { areas ( } 1 \text { per } \\
1,250 \text { pop.) }\end{array}$ & $\begin{array}{l}10-15 \\
\text { year olds }\end{array}$ & $\begin{array}{l}\text { Adults. } \\
6-10 \text { year olds }\end{array}$ & $\begin{array}{l}\text { A knock up wall } \\
\text { approximately } 8 \mathrm{~m} \\
(28 \mathrm{ft}) \text { wide and } \\
2 \mathrm{~m}(6 \mathrm{ft} 6 \mathrm{in}) \text { high }\end{array}$ & $\begin{array}{l}\text { Within } 200-500 \\
\mathrm{~m} \text { of all family } \\
\text { dwellings. Ideally } \\
\text { located in } \\
\text { association with } \\
\text { play areas }\end{array}$ & $\begin{array}{l}\text { Approx. } \\
30 \mathrm{~m} \times 21 \mathrm{~m} \\
(100 \mathrm{ft} \times 71 \\
\mathrm{ft})\end{array}$ \\
\hline $\begin{array}{l}\text { Play centres/ } \\
\text { adventure } \\
\text { playgrounds/ } \\
\text { play parks }\end{array}$ & $\begin{array}{l}10-15 \\
\text { year olds }\end{array}$ & $\begin{array}{l}\text { 6-10 year } \\
\text { olds, mothers } \\
\text { accompanying } \\
\text { children, } \\
\text { adults using } \\
\text { ball games } \\
\text { areas }\end{array}$ & $\begin{array}{l}\text { Equipment listed } \\
\text { in play area; kick- } \\
\text { about areas; } \\
\text { water play, } \\
\text { hobbies yard; } \\
\text { cycling; staffing, } \\
\text { play-leaders }\end{array}$ & $\begin{array}{l}\text { More than } 30 \mathrm{~m} \\
(100 \mathrm{ft}) \text { from } \\
\text { nearest dwelling }\end{array}$ & $\begin{array}{l}0.3-1.4 \\
\text { hectares } \\
(0.75-1.0 \\
\text { acres })\end{array}$ \\
\hline
\end{tabular}

Table A8.10 Children's play provisions outside housing [developments] to complement provision inside housing areas. (Colquhoun and Fauset, Housing Design In Practice 229)

Colquhoun and Fauset comment that to "recommend standards is almost impossible because they are inevitably idealistic and cannot be applied universally." (230)

The Urban Place Supplement 2005 (UPS) to the Essex Design Guide (EDG) provides the following advice for outdoor space in higher density housing developments:

"Unlike previous guidance, this supplement does not set out a range of minimum garden sizes that escalates with the provision of bedrooms. It allows for the possibility of small, walled outside yards of around 25 sq m. ..." (UPS p75) (emphasis by the writer)

“...Within compact urban developments there are two options for designers.

"Firstly, houses can be provided without private gardens but with direct access to high quality, private communal space.... 
"Alternatively, houses could have very small private gardens or yards. At densities above $50 \mathrm{dph}$ a garden size of about $40 \mathrm{sq} \mathrm{m}$ for a limited number of houses is possible without unduly compromising the quality of the public and communal environment.

At densities above $50 \mathrm{dph}$ an outside space of at least $25 \mathrm{sq} \mathrm{m}$ would be expected of all homes. This should primarily be provided as shared community gardens. (UPS p75)

"Design criteria for private communal space:

1. Development on sites larger than 0.1 ha should provide at least $25 \mathrm{sq}$ m of private space for each home. Only space that adheres to design criteria 3, 4 and 5 [below] will be taken into account in meeting this provision.

2. Exceptionally, apartments adjacent to and overlooking a park or other large public space of high amenity value could be provided with a smaller amount of communal space. In this instance, apartments should also have balconies of $5 \mathrm{sq} m$ floor area.

3. $60 \%$ of the private communal space should receive direct sunlight for a minimum of 4 hours a day in June [mid-summer?].

4. The space should be enclosed by walls, railings and building with no public access possible.

5. The space should be designed as an extension of the built fabric and residential accommodation and contain seating and play areas with a combination of hard and soft landscape features, including trees.

"These communal living areas should be designed to be the social, outside living space and their quality of execution and management must be sufficient to develop a pride of communal ownership and occupancy." (UPS p78)

One way to facilitate the development of "a pride of communal ownership and occupancy" is to give more local autonomy to residents in the design and management of their shared open spaces. 
Specifying open space in terms of square metres per bedspace is only a starting point for making adequate provision of quality open space in medium density housing developments. But it is at least a starting point, and the above analyses have uncovered some interesting surprises.

It was surprising to the writer, for example, that the total private and communal outdoor space per bedspace at Monterey (Fig. A8.23) was so low at only $8.4 \mathrm{~m} 2$ / bedspace. This is simlar to 10 Park Avenue in Lower Hutt, where the total private and communal outdoor space per bedspace is $8.0 \mathrm{~m} 2$. There is no communal outdoor space at 10 Park Avenue so $8.0 \mathrm{~m} 2 /$ bedspace is no surprise there, but at Monterey there is a tennis court, BBQ and picnic area, swimming pool and fitness gym, so $8.4 \mathrm{~m} 2 /$ bedspace at Monterey is surprising - surprising also because of the feeling of spatial openness one experiences outdoors on site. Not only is there generous central communal space at Monterey, but the ring road is also quite wide, adding to the sense of space.

Roads have not been included in the outdoor space calculations in this Appendix. None of them appeared to the writer to have been designed as safe "shared streets". If they were it would make sense to include them as communal space. At Monterey there are strong architectural elements (the external stairs Figs. 8.34-36) right on the edge of the road suggesting the potential for human connectness with the street. By contrast, one does not get any feeling of human occupation of the central driveway at 10 Park Avenue with its edges lined by 20 closed double garage doors (Fig. A8.47a). One might consider including the street space at Monterey in the above metrics (which would significantly increase the sq. $\mathrm{m}$ per bedspace for Monterey), but not at 10 Park Avenue. There appear to be many factors beyond square metre area per bedspace to be considered in understanding the quality of communal space, and these might be worthwhile topics for future research. 